\title{
A SYSTEMATIC REVISION OF THE NEW ZEALAND GEKKONIDAE
}

\author{
Rodney Arthur Hitchmough
}

A thesis submitted for the degree of Doctor of Philosophy in Zoology

at Victoria University of Wellington

Te Whare Wananga o te Upoko o te Ika a Maui 


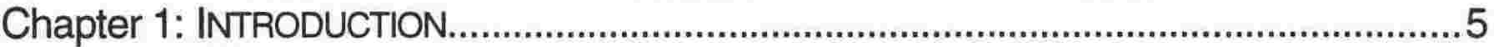

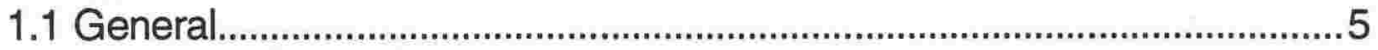

1.2 Allozyme electrophoresis........................................................................

1.3 Phylogenetic framework..............................................................................

1.4 Previous evidence for unresolved cryptic species ..................................

1.5 The importance of taxonomic resolution for conservation .....................

1.6 Structure of the thesis ..............................................................................10

Chapter 2: MATERIALS, GENERAL METHODS, and ALLOZYME LOCI.............................11

2.1 Sample collection...............................................................................11

2.2 Sample preparation ...................................................................................12

2.3 Electrophoretic methods .........................................................................12

2.4 Allozyme loci ..........................................................................................13

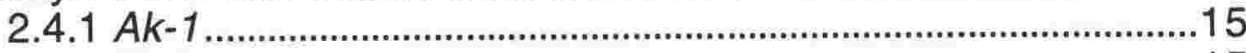

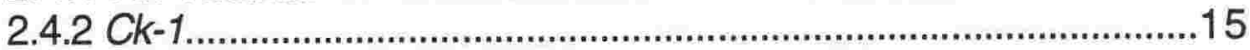

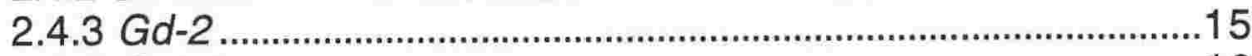

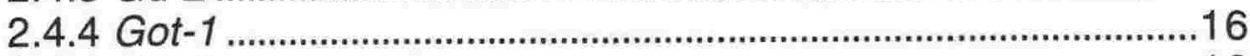

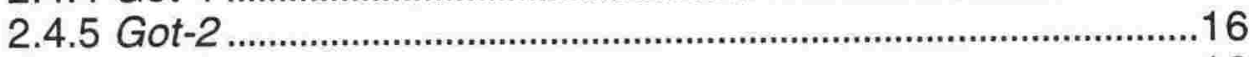

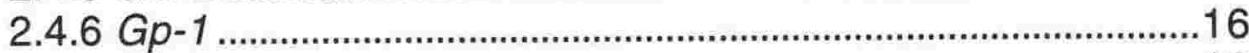

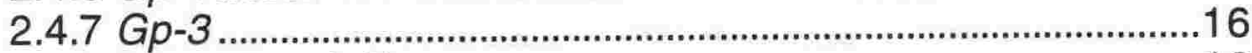

2.4.8 Gp-4 (albumin?).....................................................................16

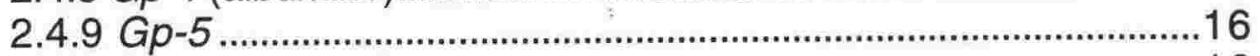

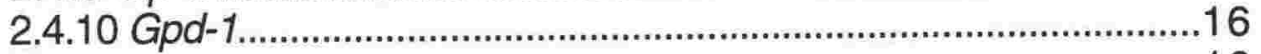

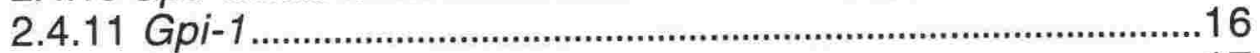

2.4.12 lcd-1 ..................................................................................17

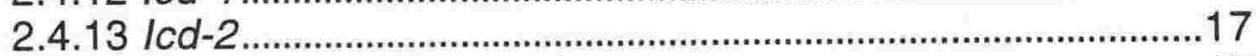

2.4.14 Ldh-1 ...................................................................................

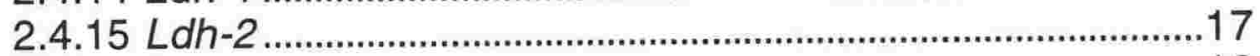

2.4.16 Mdh-1 .............................................................................18

2.4.17 Mdh-2 ….........................................................................18

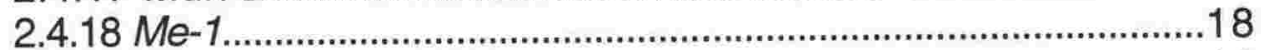

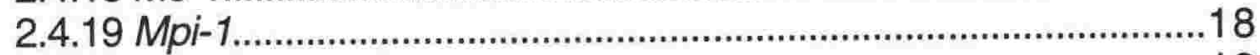

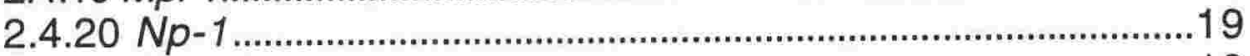

2.4.21 Pep-3..........................................................................19

2.4.22 Pgd-1 ………..................................................................19

2.4.23 Pgm-1 ……………………………...............................19

2.4.24 Pgm-2 ………………...................................................19

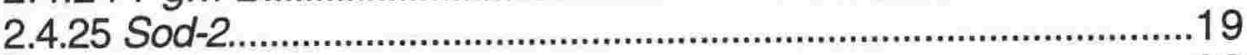

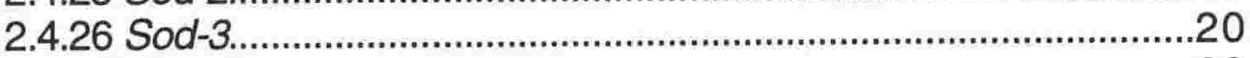

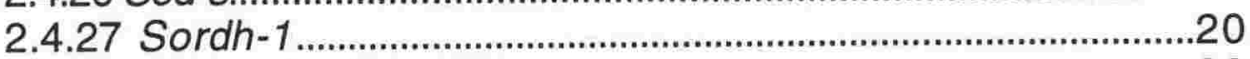

2.5 Phylogenetic methods .............................................................................20

2.6 Morphology...........................................................................................23

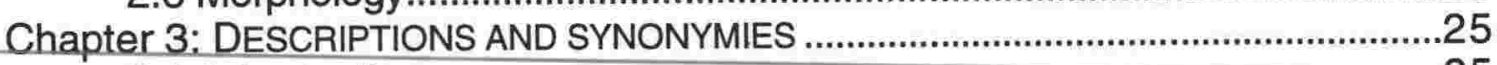

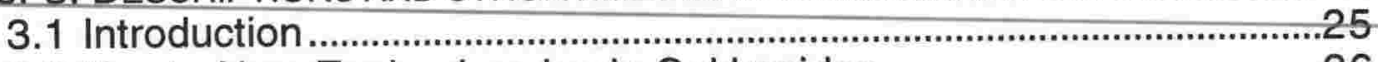

3.2 Key to New Zealand endemic Gekkonidae ............................................26

3.3 Hoplodactylus Fitzinger, 1843: ...............................................................30

The Hoplodactylus pacificus complex.........................................................30

3.3.1 Hoplodactylus pacificus (Gray, 1842).....................................30

3.3.2 Hoplodactylus n. sp. "Three Kings" .........................................34

3.3.3 Hoplodactylus n. sp. "Poor Knights" ..........................................37

3.3.4 Hoplodactylus n. sp. "Matapia" ...................................................39

3.4 Hoplodactylus Fitzinger, 1843: ...............................................................41

The Hoplodactylus maculatus complex.....................................................41 
3.4.1 Hoplodactylus maculatus (Gray, 1845) ...................................41

3.4.2 Hoplodactylus n. sp. "Mount Arthur" .......................................50

3.4.3 Hoplodactylus n. sp. "Marlborough mini" .................................52

3.4.4 Hoplodactylus n. sp. "Kaikouras".............................................55

3.4.5 Hoplodactylus brunneus (Cope 1868) ...................................57

3.4.6 Hoplodactylus n. sp. "Southern Alps" ......................................61

3.4.7 Hoplodactylus n. sp. "Cromwell Gorge" .................................64

3.4.8 Hoplodactylus n. sp. "Danseys Pass" ......................................67

3.4.9 Hoplodactylus n. sp. "Otago" .....................................................69

3.4.10 Hoplodactylus n. sp. "Southern mini" ...................................74

3.5 Hoplodactylus Fitzinger, 1843: ..............................................................77

The Hoplodactylus granulatus complex .................................................77

3.5.1 Hoplodactylus granulatus (Gray, 1843) ..................................77

3.5.2 Hoplodactylus nebulosus (McCann, 1955)...........................81

3.6 Nomen nudum ..........................................................................................83

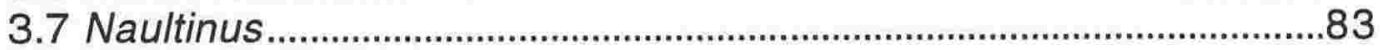

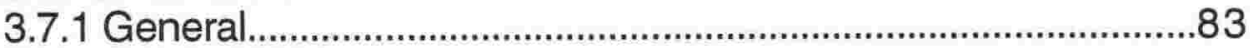

3.7.2 History.........................................................................................83

3.7.3 Nomenclature of Naultinus in the north-western

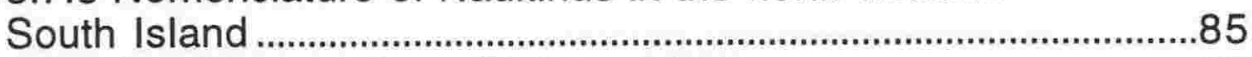

3.7.4 Naultinus stellatus (Hutton, 1872) ...........................................89

3.7.5 Naultinus tuberculatus (McCann, 1955) ................................91

Chapter 4: SPECIES IDENTIFICATION ………………...............................................94

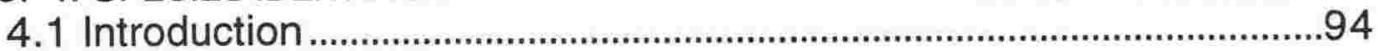

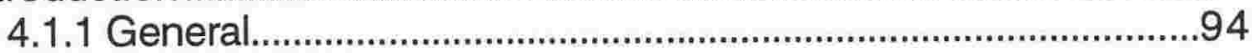

4.1.2 Species concepts ....................................................................94

4.2 The H. pacificus complex .....................................................................98

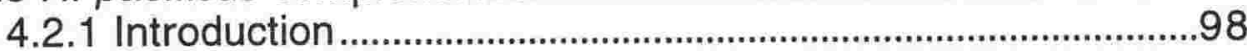

4.2.2 Samples ..................................................................................... 100

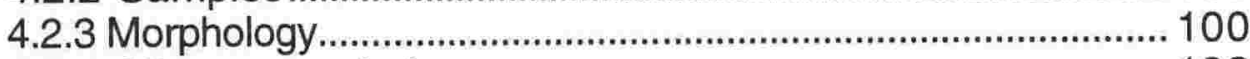

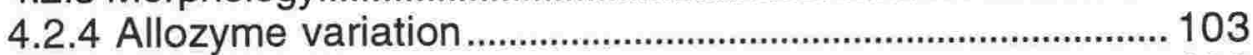

4.2.5 Discussion .............................................................................. 106

4.3 The H. maculatus complex ………………………............................. 113

4.3.1 Introduction .............................................................................. 113

4.3.2 Major groups within the complex........................................ 114

4.4 Hoplodactylus "Southern mini" ............................................................. 115

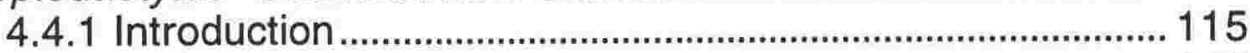

4.4.2 Sampling......................................................................................... 115

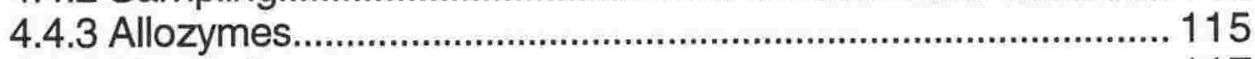

4.4.4 Morphology................................................................................ 117

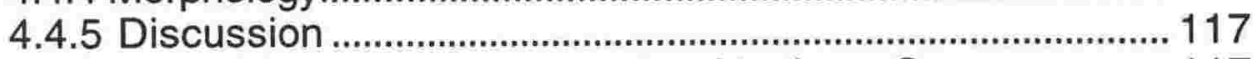

4.5 The Hoplodactylus maculatus complex, Northern Group................. 117

4.5.1 Introduction .............................................................................. 117

4.5.2 Sampling................................................................................... 118

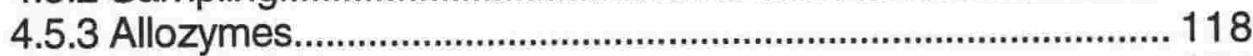

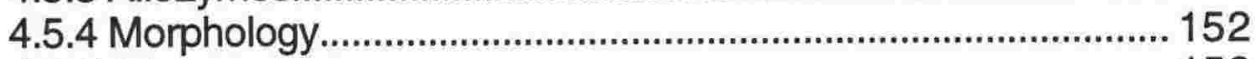

4.5.5 Discussion ................................................................................ 158

4.6 The Hoplodactylus maculatus complex, Southern Group............... 160

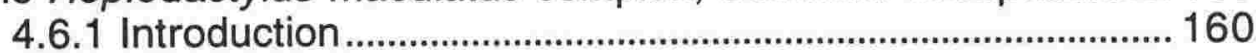

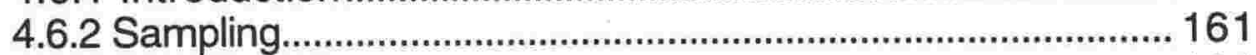

4.6.3 Allozyme Results .................................................................. 161

4.6.3.1 H. "Danseys Pass" .................................................... 199

4.6.3.2 H. "Cromwell Gorge" ................................................. 199 
4.6.3.3 H. "Otago" .................................................................... 199

4.6.3.4 H. brunneus and H. "Southern Alps" ..................... 220

4.6.3.5 H. duvaucelii .............................................................223

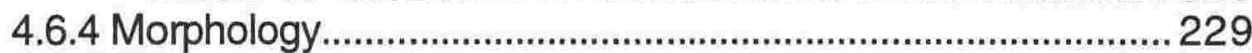

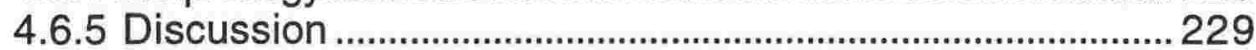

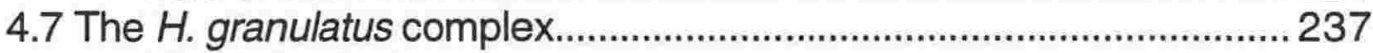

4.7.1 Introduction ................................................................................ 237

4.7.2 Sample Collection................................................................. 238

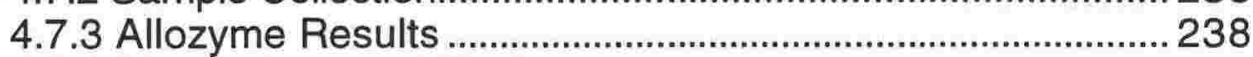

4.7.4 Morphology and Ecology ..................................................... 246

4.7.5 Discussion .............................................................................. 249

4.7.5.1 H. nebulosus.........................................................2 249

4.7.5.2 H. granulatus......................................................... 250

4.7.5.3 H. kahutarae..........................................................251

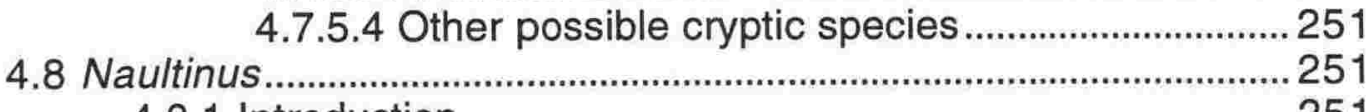

4.8.1 Introduction .............................................................................. 251

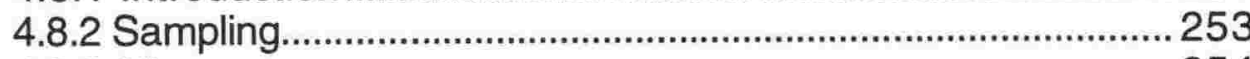

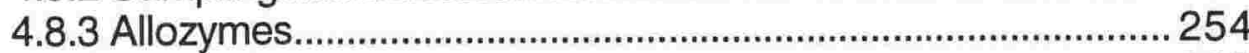

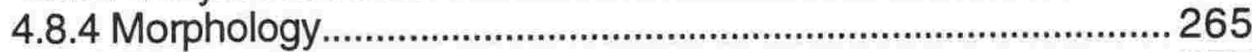

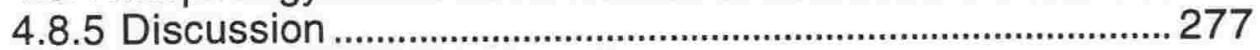

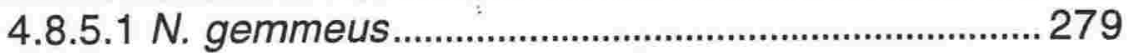

4.8.5.3 Separation of eastern and western

species pairs in the northern South Island.........................2 280

4.8.5.4 N. stellatus and N. tuberculatus..............................2. 281

4.8.5.5 N. rudis and N. manukanus................................... 282

4.8.5.6 Separation between North and South

Island populations............................................................228

4.8.5.7 Species boundaries in the North Island ...............2 283

4.9 Allozyme confirmation of the identity of..............................................28 285

Maud Island Hoplodactylus stephensi and .............................................228

Mana Island H. chrysosireticus................................................................. 285

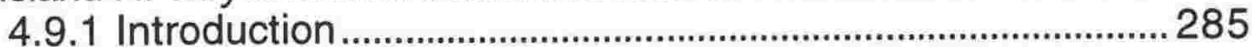

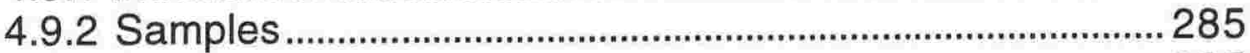

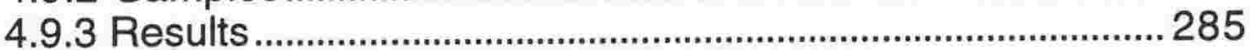

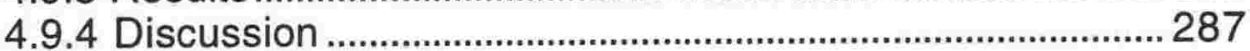

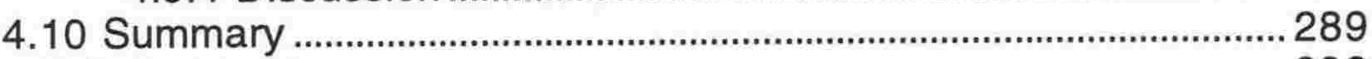

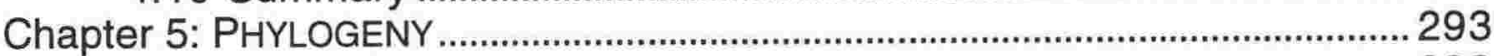

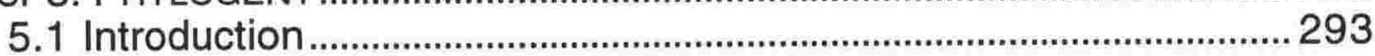

5.2 Phylogenetics of the Diplodactylinae based on

morphological analysis............................................................................. 297

5.3 A re-analysis of phylogenetic relationships among New

Zealand geckos from morphology ............................................................ 306

5.4 Phylogenetic relationships within the New Zealand gecko

fauna from allozyme data

5.5 Congruence between morphologically-based and

allozyme-based phylogenetic hypotheses.................................................. 328

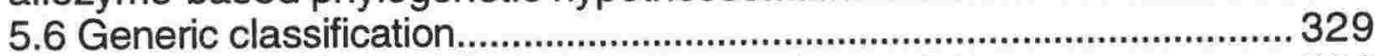

5.7 A personal view of Diplodactyline gecko evolution ...........................333

5.8 Conclusions........................................................................................... 337

Chapter 6: EVOLUTIONARY, BIOGEOGRAPHICAL, AND CONSERVATION

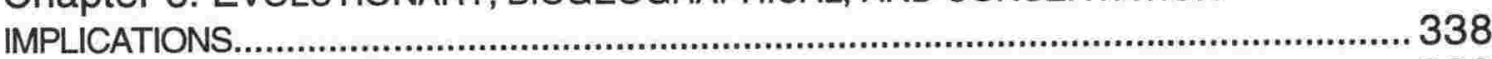

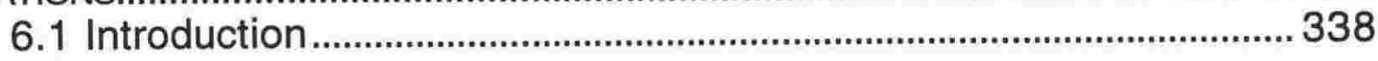


6.2 Poor correlation of rates of change in allozymes and morphology 338

6.3 Speciation mechanisms.................................................................... 341

6.5 Parallel morphological evolution correlated with habitat, and differentiation of parapatric populations within species.................. 342

6.6 Biogeography................................................................................. 343

6.6.1 The South Island as a centre for gecko evolution ............... 343

6.6.2 South Island populations of the $H$. maculatus complex

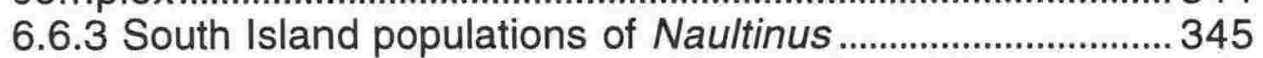

6.6.4 Island populations and rates of evolution ..............................346

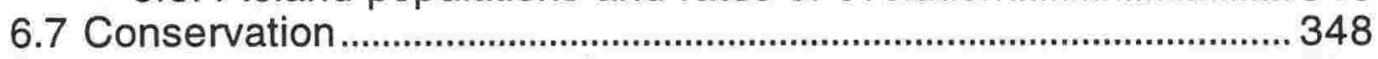

Chapter 7: CONCLUSIONS....................................................................................... 350

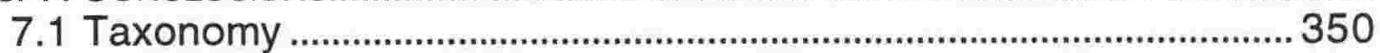

7.2 Phylogenetics........................................................................................... 351

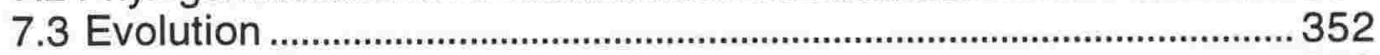

7.4 Biogeography.................................................................................... 353

7.5 Conservation ......................................................................................... 354

Acknowledgements ................................................................................... 355

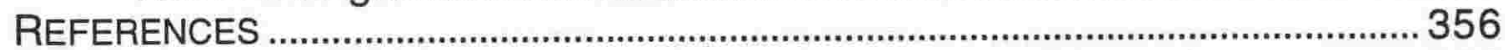

APPENDIX 1: Allozyme stain and buffer recipes used in this study.....................366

Buffers: .................................................................................................. 366

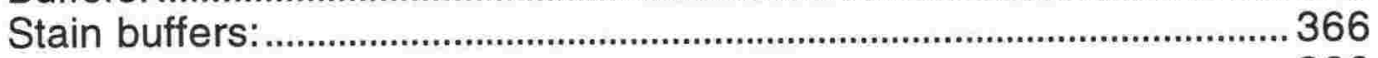

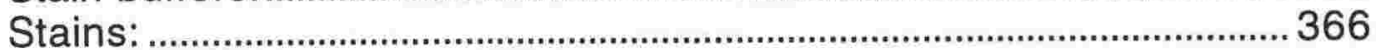




\section{ABSTRACT}

In this species I recognise 21 species of New Zealand brown geckos (Hoplodactylus), and 7 species of green gecko (Naultinus), bringing the total New Zealand gecko fauna to 28 .

The only previous comprehensive revision of the New Zealand Gekkonidae was that of McCann (1955), who recognised 4 species of brown geckos (Hoplodactylus), 6 species of South Island green geckos (Heteropholis), and one species of North Island green gecko (Naultinus). Bauer (1990) synonymised Heteropholis with Naultinus.

In this thesis I interpret the results of allozyme electrophoresis and morphological examination of specimens of all known taxa, using phylogenetic computer analysis and an Evolutionary Species Concept. Allozyme results were obtained for 686 specimens from 235 populations. I resolved 27 allozyme loci from each of these specimens, using 2 tissue types and 5 buffer systems.

The following species are recognised as a result of this study. Newly proposed species are indented below the species to which they have previously been referred:

Hoplodactylus chrysosireticus

H. duvaucelii

H. granulatus

H. nebulosus

H. kahutarae

H. maculatus

H. brunneus

H. "Mount Arthur"

H. "Kaikouras"

H. "Marlborough mini"

H. "Southern Alps"

H. "Danseys Pass"

$H$. "Cromwell Gorge"

$H$. "Otago"

$H$. "Southern mini"

H. pacificus

H. "Matapia"

$H$. "Poor Knights" 
H. "Three Kings"

\section{H. rakiurae}

H. stephensi

(H. delcourti - probably from New Zealand; presumed extinct)

Naultinus elegans (subspecies $N$. e. elegans and $N$. e. punctatus)

N. gemmeus.

N. grayii

N. manukanus

N. rudis

N. stellatus

N. tuberculatus

Hoplodactylus will include 21 extant species. Apart from one synonymy suggested by examination of type specimens, I suggest no formal changes to Naultinus, because morphological support for the status quo is strong, but allozymes identify only 5 groups ( 2 within $N$. gemmeus) rather than the 8 currently recognised.

I provide a key for the identification of all known New Zealand species.

Phylogenetic analysis of allozyme and morphological results suggests 4 major groups in the New Zealand gecko fauna:

- Naultinus

- a narrow toed group of Hoplodactylus species, including $H$. granulatus, $H$. nebulosus, $H$. kahutarae, $H$. rakiurae, and $H$. stephensi

- the $H$. pacificus complex, including $H$. pacificus, $H$. "Matapia", $H$. "Poor Knights", and $H$. "Three Kings"

- the $H$. maculatus complex, including $H$. chrysosireticus, $H$. duvaucelii, $H$. maculatus, $H$. brunneus, $H$. "Mount Arthur", $H$. "Kaikouras", $H$. "Marlborough mini", H. "Southern Alps", H. "Danseys Pass", H. "Cromwell Gorge", $H$. "Otago", and $H$. "Southern mini".

However, Naultinus is closely related to the narrow-toed group of Hoplodactylus, and falls between $H$. stephensi and the rest of the narrowtoed group of Hoplodactylus in some analyses. 
Within the $H$. maculatus complex, there are 3 species groups:

- H. chrysosireticus and $H$. "Southern mini"

- $\quad$ H. maculatus, H. "Mount Arthur", H. "Kaikouras", and $H$. "Marlborough mini"

- $\quad H$. duvaucelii, $H$. brunneus, $H$. "Southern Alps", $H$. "Danseys Pass", $H$. "Cromwell Gorge", and $H$. "Otago"

$H$. pacificus is paraphyletic with respect to the sympatric new species $H$. "Matapia" in all analyses, and $H$. granulatus is paraphyletic with respect to $H$. kahutarae in some analyses.

I disagree with many character states Bauer (1990) assigned to the New Zealand species in his morphological data matrix for the Carphodactylini. His resulting cladogram is shown to lack strong support, and alternative phylogenetic, and by inference biogeographical, relationships are possible.

Some genetically widely divergent species are so morphologically similar that traditional taxonomies had difficulty distinguishing them (e.g., $\mathrm{H}$. stephensi, all species of the $H$. pacificus complex, and all species of the $H$. maculatus complex except $H$. duvaucelii subsumed under $H$. pacificus by McCann 1955). Conversely, some morphologically highly distinct species have genetically close, previously unsuspected sister group relationships. Rates of change in allozymes and morphology therefore appear poorly correlated.

In the southern group of the $H$. maculatus complex, the overall pattern of allozyme variation is a stepped or fragmented cline, which continues across species boundaries. There is a marked absence of diagnostic derived allozyme characters in well-defined species. These allele distributions suggest parapatric speciation, with the shared polymorphisms being older than the speciation event.

On Matapia island, the sympatry of 2 morphologically distinct and reproductively isolated species, both of which fall within $H$. pacificus in trees 
suggest a double colonisation of the island, followed by morphological divergence.

There are at least 5 independent cases of coastal populations of very small $H$. maculatus-complex geckos with larger inland sister-groups, suggesting strong directional selection on body size.

There is strong circumstantial evidence for most evolution and speciation having happened in the South Island, and for all North Island species except for the $H$. pacificus complex being geologically recent colonists with South Island ancestors.

The Three Kings and Poor Knights Island groups are the only islands occupied by New Zealand geckos which are believed not to have been connected to the mainland during Pleistocene glacial periods of low sea level, and also the only islands with genetically distinct local endemics.

The recognition of these new species will alter conservation priorities, as some have very restricted known distributions. 


\section{Chapter 1: INTRODUCTION}

\subsection{GENERAL}

The New Zealand geckos (Reptilia: Gekkonidae) have been isolated from their relatives in other parts of the world for many millions of years (Towns et al. 1985), perhaps since New Zealand separated from Gondwanaland about 80 million years ago (King 1987b), and in this time have differentiated extensively. They include brilliantly coloured arboreal diurnal geckos (Naultinus) as well as nocturnal forms (Hoplodactylus) adapted to virtually every possible habitat and ecological niche, from boulder beaches to alpine rock bluffs, and semi-arid grasslands to rainforests. Mature body size ranges from c. $3 \mathrm{~g}$ to $120 \mathrm{~g}$ in extant species, and the extinct Hoplodactylus delcourti, probably from New Zealand, would have weighed more then $1 \mathrm{~kg}$. World-wide, geckos are typically regarded as tropical, but in New Zealand they are found well into the temperate zone, including the most southerly known distribution for a gecko, the Harlequin Gecko of Stewart Island in the roaring forties. The bright colours and diurnal habits of Naultinus are unusual in geckos being paralleled only by African and Indian Ocean Phelsuma, which are members of a different subfamily. Apart from one New Caledonian species, all geckos outside New Zealand are oviparous, whereas all New Zealand species are viviparous.

Despite having attracted considerable attention in the last 2 decades, lizards remain the least-known and most systematically uncertain group in New Zealand's terrestrial vertebrate fauna. Since 1970, 17 new taxa have been formally described and 3 previously synonymised taxa resurrected, yet many unresolved taxonomic problems remain. Hardy (1977) published a major taxonomic revision of the endemic Scincidae, and Patterson \& Daugherty $(1990,1994)$ added 5 further species, detected by allozyme electrophoresis. Most recently, Patterson \& Daugherty (1995) separated the New Zealand skinks formerly classified in the genus Leiolopisma into an endemic genus, Oligosoma. There has been no such thorough treatment of the Gekkonidae since McCann's (1955) monograph, although several new or resurrected taxa have been described (Robb 1980a, b; Robb \& Hitchmough 1980; Thomas 1981; Whitaker 1985). Identification of species and of important patterns of variation within species is of vital importance in conserving this increasingly threatened group in a period of limited resources. 
The geckos and Oligosoma skinks are the only extant terrestrial vertebrate groups which have speciated extensively in New Zealand, and allozyme studies have suggested biogeographical parallels both between these groups and with large endemic invertebrates. Preliminary work suggests that although the skinks have even more species than the geckos there is less geographic variation within these species, and therefore less information to be gained from them on relatively recent biogeographical changes and on short-term responses to environmental changes, a question of great theoretical interest. This suggests that improved understanding of gecko evolutionary biology would assist interpretation of the history of the entire biota.

Whitaker \& Thomas' (1989) exhaustive annotated bibliography summarised all literature published on the New Zealand herpetofauna before 1989, including all early taxonomic papers on the Gekkonidae.

\subsection{ALLOZYME ELECTROPHORESIS}

Allozyme electrophoresis is a method of assaying genetic variation within and among populations of organisms. In isozyme electrophoresis, soluble enzymes are separated in a semipermeable gel by differences in rates of migration through the gel in response to electric current. Mobility is determined by molecular weight, shape, and charge on the enzyme molecule. Isozymes are bands of activity visualised on electrophoretic gels by enzyme-specific stains. Allozymes are a subset of isozymes which express patterns consistent with allelic variation at single genetic loci. Since these allozyme bands are biparentally inherited products of nuclear loci, they are particularly useful tools to detect absence or by implication presence of gene flow between sympatric or adjacent populations.

In the absence of gene flow between populations, allozyme differences between taxa will accumulate stochastically by mutation, genetic drift, and selection. This allows allozyme variation to be used for phylogenetic reconstruction, particularly using phenetic methods. Allozymes have a high rate of homoplasy because independent mutations can lead to identical band mobilities. With this limitation, allozyme genotypes can also be used for cladistic analysis. 


\subsection{PhyLogenetic FRAMEWORK}

Kluge (1967a, b) placed the New Zealand gecko genera in the Subfamily Diplodactylinae, Tribe Carphodactylini, along with the New Caledonian Eurydactylodes, Rhacodactylus, and Bavayia and the northern Australian Pseudothecadactylus, Carphodactylus, Phyllurus, and Nephrurus. He considered the New Zealand geckos to be a "natural group" most closely related to the New Caledonian genera, which in turn are most closely related to Pseudothecadactylus.

Bauer (1990) presented results of a cladistic study of morphology of the Carphodactylini. His analysis did not include Oedura. His suggested phylogeny within the Carphodactylini was quite different to Kluge's. He considered the narrow-toed Australian genera Carphodactylus, Phyllurus, and Nephrurus to be primitive, and the New Zealand and New Caledonian genera and Pseudothecadactylus to be a monophyletic derived group (Figure 1.3.1). In their turn, the New Caledonian genera and Pseudothecadactylus were derived within the New Zealand genus Hoplodactylus, and Pseudothecadactylus (which he synonymised with Rhacodactylus) was derived from New Caledonian ancestors. Bauer's suggested phylogeny (Figure 1.3.1) for the New Zealand species, although tentative, is available as a hypothesis against which phylogenetic hypotheses based on allozymes can be compared.

\subsection{Previous EVIDENCE for UNREsolved CRYPtiC SPECIES}

Before this study began, there was strong evidence that Hoplodactylus maculatus, as then defined, contained at least 2 biological species; 2 morphologically distinct forms were known to be sympatric in the Kaikoura region (Whitaker 1984b), and Robinson (1985) found that there were 2 groups within a Central Otago population apparently breeding asynchronously. (However, Cree 1994 has since shown that the 2 groups are generated by a biennial female reproductive cycle in a single population.) R. Rowlands (pers. comm.) found circumstantial evidence for reproductive incompatibility between geographically separate $\mathrm{H}$. maculatus (sensu Robb \& Rowlands 1977) populations in captivity. Also, there are many morphologically distinct forms of this species in different geographic areas and different habitats over much of the country. 

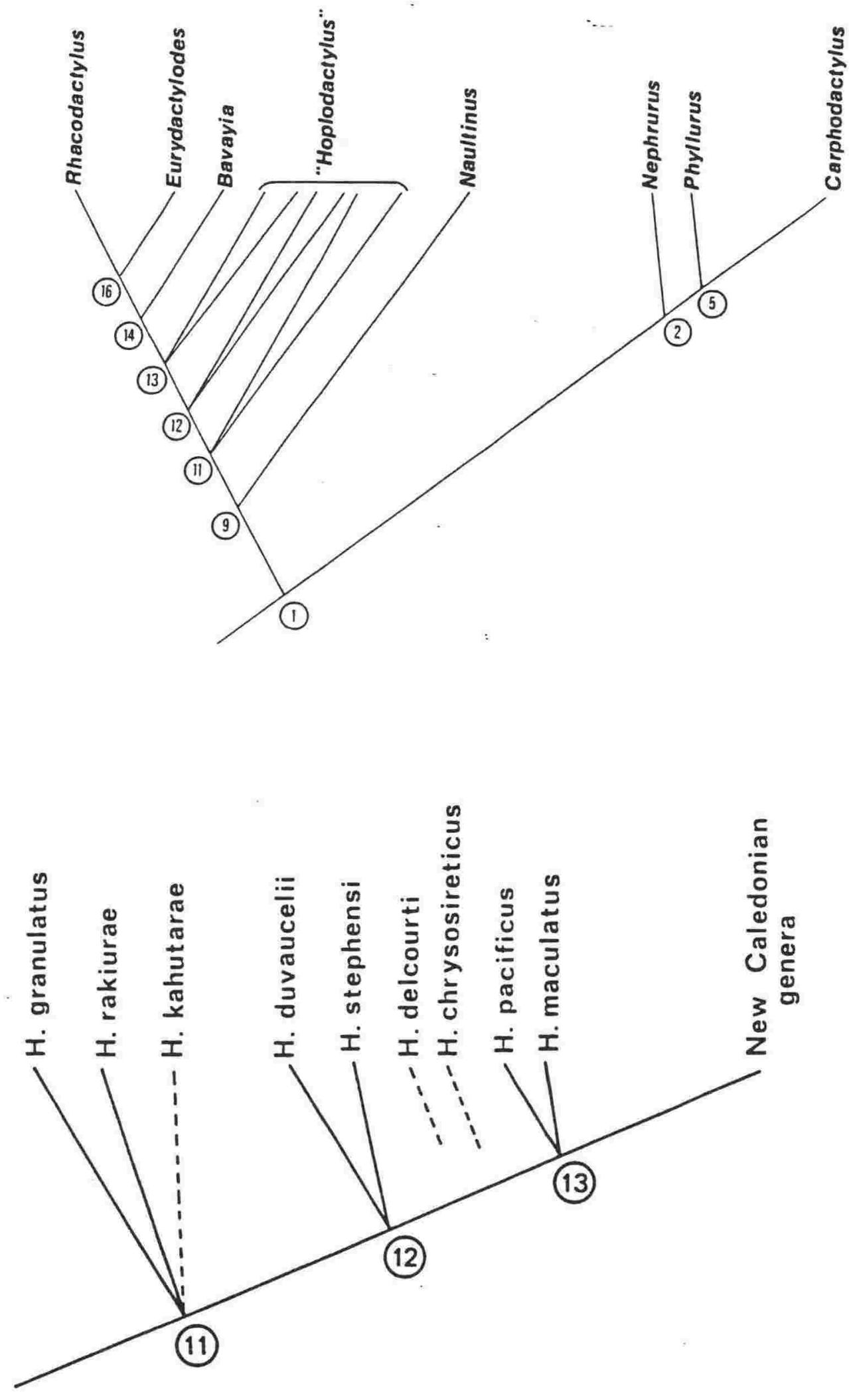

Figure 1.3.1 (After Bauer 1990, figs 16 \& 18) Bauer's (1990) consensus cladogram of (top) the Carphodactylini and (bottom) Hoplodactylus. 
H. pacificus is also morphologically variable between populations, particularly on some northern offshore islands (McCann 1955; Robb \& Rowlands 1977; Whitaker 1968).

Hoplodactylus granulatus shows little morphological variation over its almost New Zealand-wide range. However, a preliminary electrophoretic study (Vickers \& Daugherty unpublished) of 3 animals showed that one was very different biochemically from the other 2 .

In contrast, the green geckos (Naultinus spp.) are a series of morphologically distinct allopatric populations, at present recognised as 8 separate species and one subspecies. They produce fertile F1, F2, backcross, and multispecies hybrids in captivity (Meads 1982), and small-scale pilot electrophoretic studies (Vickers \& Daugherty unpublished) confirmed that they were all extremely closely related to each other.

\subsection{THE IMPORTANCE OF TAXONOMIC RESOLUTION FOR CONSERVATION}

A thorough understanding of the taxonomic status of all forms within any fauna is a prerequisite for effective conservation. Until late 1996, 4 widespread and abundant species (as then understood) of New Zealand lizards remained legally unprotected. Daugherty et al. (1990) demonstrated that the previously unprotected skink Leiolopisma nigriplantare maccanni was in fact a species complex. Six species previously referrable to $L$. $n$. maccanni have so far been described (Patterson \& Daugherty 1990, 1994); 4 of these have limited distributions. Considered separately, these species have no less need of protection than many others in the fauna. Although the combination Leiolopisma nigriplantare maccanni, used in legislation, is no longer in use, the Department of Conservation considered that the intention of the legislation is that the most common, widespread form should be unprotected, so L. n. polychroma, along with Cyclodina aenea, Hoplodactylus maculatus, and Hoplodactylus granulatus, remained unprotected (D. Newman, Department of Conservation, pers. comm.).

A major motive for the choice of this thesis topic was the likelihood that one or both of the unprotected gecko species (Hoplodactylus maculatus and $H$. granulatus) may in fact be complexes (see below), and some or all species within these complexes might be found to be rare or vulnerable. A good understanding of the alpha taxonomy, intraspecific variation, and 
phylogenetic origins of the group is necessary for interpretation of any future scientific work on these lizards, and for an understanding of priorities for conservation.

\subsection{Structure OF THE THESIS}

In this thesis I have used electrophoretic, morphometric, distributional and ecological information about the various gecko forms in New Zealand to examine the status of all recognised species, to detect cryptic species, and to provide information on the phylogeny of the whole group. I describe these methods, and the allozyme loci surveyed, in Chapter 2.

In Chapter 3 I describe 12 new and one resurrected species, redefine more narrowly 3 species formerly regarded as widespread and variable, and provide a key for the identification of these and the currently recognised species. Chapter 4 presents the evidence supporting species status for the new and redefined species described in Chapter 3.

In Chapter 5 I use phenetic and cladistic analyses of morphometric and electrophoretic data to construct phylogenetic hypotheses for the New Zealand geckos. New Caledonian and Australian Diplodactylinae are included in these analyses as outgroups. Implications of the findings of this study on the interpretation of phylogenetic relationships and classification within the subfamily as a whole are discussed.

Chapter 6 discusses the evolutionary and biogeographical implications of my results. Chapter 7 summarises the conclusions of this study. 


\section{Chapter 2: MATERIALS, GENERAL METHODS, and ALLOZYME LOCI}

\subsection{SAMPLE COLLECTION}

A large resource of preserved lizard specimens and frozen tissues suitable for electrophoresis was already available in the School of Biological Sciences at the start of this study, having been collected by $\mathrm{C}$. H. Daugherty and others in the course of other research (CD and FT collections). This collection included most of the recognised New Zealand gecko species, so further collection of some protected species (particularly Naultinus spp.) was unnecessary. Extensive series of $H$. maculatus from a few localities and smaller samples from a range of localities were also available. The FT collection was also added to during this study by specimens collected in the course of tuatara (Sphenodon spp.) distribution surveys (Daugherty et al. 1990b) and DoC fauna surveys.

I collected (RAH collection) further small samples of $H$. maculatus from as many localities as possible to clarify the geographic patterns of variation. The fine geographic scale of variation which quickly became apparent when the first samples were analysed, combined with low levels of observed polymorphism and heterozygosity within samples, meant that comprehensive geographic coverage was considered more important than large sample sizes. Published opinions that only small samples were necessary for surveying taxonomic variation (e.g., Buth 1984; Gorman \& Renzi 1979; Nei 1978; Sarich 1977) reinforced this rationale. A desire to minimise collecting impact on populations also influenced this decision. However, Archie et al. (1989) demonstrated that small sample size does decrease the stability of dendrograms calculated from allozyme-frequency data. I therefore now consider that larger sample sizes would have been desirable.

Specimens were found by turning rocks and other items of potential cover, and captured by hand. I also collected small samples of $H$. granulatus in the Auckland area, and (under permit) of $H$. pacificus and $N$. grayii in Northland and $N$. gemmeus in Otago. When collecting I kept records of the environments where populations are found, including such variables as altitude, aspect, slope, rock type, vegetation (general community type, height, dominant species), and the microhabitat the geckos were occupying. 
I obtained specimens of New Caledonian and Australian Carphodactylini for use as outgroups in cladistic analysis with the help of herpetologists in Australia.

\subsection{SAMPLE PREPARATION}

Specimens were returned to the laboratory alive, and held in terraria until they could be killed and tissues collected from them. They were killed using ether, weighed, measured, and dissected. Blood was collected by cardiac puncture, then the heart, liver, kidney, and tail were removed for storage in Autoanalyser cryotubes. Blood was centrifuged to separate plasma and cells if sufficient was collected to justify this procedure (more than about $0.5 \mathrm{ml}$ ). The tissue samples were stored at $-80^{\circ} \mathrm{C}$ until required for electrophoresis. Carcasses were labelled and stored in $70 \%$ ethanol.

\subsection{ELECTROPHORETIC METHODS}

Electrophoretic techniques were those of Allendorf et al. (1977).

Combinations of 4 tissue extracts, 5 buffer systems and 31 enzyme-specific stains, an esterase stain, and a general protein stain were screened for scorable activity, using a small number of representative specimens. Stain and buffer recipes are listed in Appendix 1.

Subsequently, those combinations which showed clear discrete bands of enzyme activity were used to screen samples from all available individuals. Subsamples of frozen tissue were thawed, then macerated by hand using a dental probe, in an autoanalyser cryotube with a drop of distilled water. They were sometimes prepared one or more days before the gel was run and refrozen at $-80^{\circ} \mathrm{C}$ until the morning of the run, thawed at room temperature, then kept on ice until loading of gels was complete. More often the sample preparation was done on the morning of the run, and the prepared samples placed straight onto a tray of chipped ice. Previously prepared subsamples could be re-used 2-3 times, with loss of intensity of activity each time, but no change in the position of stained isozyme activity.

Chromatography paper wicks c. $1 \times 10 \mathrm{~mm}$ were soaked in the supernatant and loaded at an origin cut about $5 \mathrm{~cm}$ from the cathodal edge of $\mathrm{c} .1 \times 12 \mathrm{x}$ $20 \mathrm{~cm}, 11 \%$ starch gels. Gels were loaded on a tray of chipped ice, and cooled using external ice packs while running. Samples were loaded on gels in groups of 5 , with a previously scored control sample or a cochineal 
Table 2.3.1 Running conditions for allozyme buffer systems used in this study. Dye migration distance is the distance the dye marker was allowed to run before the gel was removed.

\begin{tabular}{llcccc}
\hline & $\begin{array}{c}\text { Gel buffer } \\
\mathrm{pH}\end{array}$ & $\begin{array}{c}\text { Electrode } \\
\text { buffer } \mathrm{pH}\end{array}$ & Voltage & $\begin{array}{c}\text { Dye migration } \\
(\mathrm{cm})\end{array}$ \\
Pk & Poulik & 8.7 & 8.2 & 300 & 4 \\
Rw & Ridgeway & 8.5 & 8.1 & 300 & 4 \\
AC & Amine-Citrate & 6.0 & 6.1 & 170 & 6 \\
Ph & Phosphate & 6.7 & 6.7 & 150 & 5 \\
TC-1 & Tris-Citrate 1 & 6.7 & 6.3 & 100 & 5 \\
\hline
\end{tabular}

dye marker between alternate groups. Dye markers also marked each end of the complete row of samples. In total, including controls and markers, 40-60 wicks were loaded per gel.

Domestic "chux cloths" were used as buffer wicks to carry buffer and current between buffer trays and the gel. Running conditions for each buffer system are listed in Table 2.3.1. Sample wicks were removed after 20 minutes running time.

After running, gels were sliced horizontally into $5-7,1 \mathrm{~mm}$ slices, and each slice was stained separately for allozyme activity.

The observed patterns were assumed to represent Mendelian variation at genetic loci. I labelled enzymes, genetic loci, and alleles as recommended by Murphy \& Crabtree (1985). Multiple loci encoding the same enzyme activity were numbered sequentially beginning at the most cathodal, and alleles of polymorphic loci were identified alphabetically, beginning at the most cathodal.

Gels were run and stained in one day, and genotypes for each individual recorded the same day, in case information was lost through gels bleaching or overstaining overnight. These preliminary results were checked the next day, and most gels were also photographed to provide a long-term record for subsequent comparison with other runs.

\subsection{AlLozyme LocI}

Allozyme results were obtained for 686 New Zealand gecko specimens from 235 populations. I resolved 27 allozyme loci from each of these specimens, using 2 
Table 2.4.1 Loci scored in this study, and the buffer conditions and tissues which allowed the clearest discrimination of each. L, liver; $M$, muscle; Rw, Ridgeway buffer (Ridgeway et al. 1970); Ac, Amine-citrate buffer (Clayton \& Tretiak 1972); Ph, Phosphate buffer (Selander et al. 1971); Pk, Poulik buffer (Selander et al. 1971); Tc, Tris-citrate buffer \#1 (Selander et al. 1971).

E.C.N. Enzyme/protein Locus Tissue Buffer

\begin{tabular}{|c|c|c|c|c|}
\hline 2.7.4.3 & Adenylate kinase & $A k-1$ & M & $\mathrm{Pk}$ \\
\hline 2.7.3.2 & Creatine phosphokinase & $C k-1$ & M & Pk \\
\hline 1.1.1.49 & Glucose-6-phosphate dehydrogenase & $G d-2$ & M & $\mathrm{Ph}$ \\
\hline \multirow[t]{6}{*}{ 2.6.1.1 } & \multirow[t]{2}{*}{ Glutamate-oxalacetate transaminase } & Got-1 & M & $\mathrm{Tc}$ \\
\hline & & Got-2 & M & Tc \\
\hline & \multirow[t]{4}{*}{ General protein } & $G p-1$ & M & $\mathrm{Rw}, \mathrm{Ph}, \mathrm{Pk}$ \\
\hline & & $G p-3$ & M & $\mathrm{Rw}, \mathrm{Ph}, \mathrm{Pk}$ \\
\hline & & $G p-4$ & M & $\mathrm{Rw}, \mathrm{Ph}, \mathrm{Pk}$ \\
\hline & & $G p-5$ & M & $\mathrm{Rw}, \mathrm{Ph}, \mathrm{Pk}$ \\
\hline 1.1.1.8 & Glycerol-3-phosphate dehydrogenase & Gpd-1 & M & Ac, $R w$ \\
\hline 5.3.1.9 & Glucose phosphate isomerase & Gpi-1 & $\mathrm{L}$ & $\mathrm{Pk}$ \\
\hline \multirow[t]{2}{*}{ 1.1.1.42 } & \multirow[t]{2}{*}{ Isocitrate dehydrogenase } & $I c d-1$ & M & Ac \\
\hline & & $I c d-2$ & M & $A c$ \\
\hline \multirow[t]{2}{*}{ 1.1.1.27 } & \multirow[t]{2}{*}{ Lactate dehydrogenase } & $L d h-1$ & $\mathrm{~L}$ & $\mathrm{Pk}$ \\
\hline & & $L d h-2$ & $\mathrm{~L}$ & $\mathrm{Pk}$ \\
\hline \multirow[t]{2}{*}{ 1.1.1.37 } & \multirow[t]{2}{*}{ Malate dehydrogenase } & $M d h-1$ & M & $A c$ \\
\hline & & Mdh-2 & M & $A c$ \\
\hline 1.1.1.40 & Malic enzyme & $M e-1$ & M & $\mathrm{Ph}$ \\
\hline 5.3.1.8 & Mannose phosphate isomerase & Mpi-1 & M & Rw \\
\hline 2.4.2.1 & Purine nucleoside phosphorylase & $N p-1$ & $\mathrm{~L}$ & Rw \\
\hline 3.4.1.1 & Peptidase & Pep-3 & $\mathrm{L}$ & $R w$ \\
\hline 1.1.1.44 & Phosphogluconate dehydrogenase & Pgd-1 & M & Tc \\
\hline \multirow[t]{2}{*}{ 2.7.5.1 } & \multirow[t]{2}{*}{ Phosphoglucomutase } & Pgm-1 & $\mathrm{L}$ & Rw \\
\hline & & Pgm-2 & $\mathrm{L}$ & Rw \\
\hline \multirow[t]{2}{*}{ 1.15.1.1 } & \multirow[t]{2}{*}{ Superoxide dismutase } & Sod-2 & $L, M$ & Ac, $\mathrm{Pk}$ \\
\hline & & Sod-3 & $L, M$ & Ac, $\mathrm{Pk}$ \\
\hline 1.1 .1 .14 & Sorbitol dehydrogenase & Sordh-1 & $M$ & Tc \\
\hline
\end{tabular}


tissues and 5 buffer systems (Table 2.4.1). Allelic frequencies for each population are presented in Chapters 4 and 5 . Methods of statistical analysis of allozyme results are also presented in these chapters.

All observed banding patterns and inferred subunit structures are consistent with those previously reported for equivalent loci in humans (Harris \& Hopkinson 1976). No obvious cases of linkage disequilibrium were detected, except possibly for Pep1 and $P e p-2$, which were not included in this data set for this reason and also because of large, unexplained departures from Hardy-Weinberg equilibrium (heterozygote deficiencies) in polymorphic populations. It is, however, possible that some loci were linked, because levels of polymorphism within populations were generally low, and in these circumstances linkage is difficult to detect.

For ease of reference, samples in which only one allele was detected at a locus are referred to in this thesis as fixed for that allele, although most sample sizes were too small to allow fixation to be confirmed statistically.

\subsubsection{AK-1}

$A k-1$ encoded the polypeptide units of a monomeric enzyme, with heterozygotes appearing double-banded on stained gels. Four alleles were seen.

\subsubsection{CK-1}

Ck-1 also stained strongly on general protein gels, and could be scored as $G p-2$. It encoded the polypeptide units of a dimer, with 3-banded heterozygotes. I distinguished 4 alleles.

\subsubsection{GD-2}

In humans, $G d$ is X-linked, and encodes the polypeptide units of a dimer. In geckos, Gd-1 was a faint, cathodally-migrating locus, which often stained too faintly for reliable scoring, so was not included in this dataset. It may have resulted from cross-reaction with another dehydrogenase. $\mathrm{Gd}-2$ stained as a strong but sometimes rather streaky band of activity, migrating anodally. Few obvious heterozygotes were seen (they appeared as a more extended streak than homozygotes), and differences were mostly fixed between populations. Diplodactylini do not have visibly dimorphic sex chromosomes (King 1987a, b); their mode of sex determination is unknown. Sex linkage, as in humans, is therefore unlikely. I saw 6 alleles. 


\subsubsection{GOT-1}

Got-1 probably encoded the polypeptide units of a dimer; heterozygotes were usually blurred, but most densely stained where the middle band of a dimeric heterozygote would be expected. I found 3 alleles.

\subsubsection{GOT-2}

Got-2 was also expressed as a dimer, with rather blurred heterozygotes, and 3 alleles.

\subsubsection{GP-1}

Gp-1 encoded the polypeptide units of a cathodally-migrating dimer, with 3-banded heterozygotes clearly visible. There were 2 alleles.

\subsubsection{GP-3}

No variation was found at this locus.

\subsubsection{GP-4 (ALBUMIN?)}

Gp-4 encoded the polypeptide units of a monomer, with 2-banded heterozygotes. It was expressed strongly in plasma as well as muscle, so is probably albumin. This locus was extremely variable, with 10 alleles detected. Several alleles appeared in 2 or more distantly related species, so probably represent 2 or more different alleles of convergent mobility.

\subsubsection{GP-5}

No heterozygotes were seen in $G p-5$. There were 3 alleles.

\subsubsection{GPD-1}

Gpd-1 encoded the polypeptide units of a dimer, with 3-banded heterozygotes. However, secondary isozymes anodal to the primary band occurred in most runs. This partly obscured the banding pattern of heterozygotes, although they could be distinguished by the second band being more strongly stained than the lowest band, rather than the lowest band being the strongest as in homozygotes. Six alleles of Gpd-1 were detected in this study.

\subsubsection{GPl-1}

Gpi-1 showed prominent secondary isozymes in geckos as it does in humans (Harris \& Hopkinson 1976). This locus was also particularly prone to sideways leakage between samples across the gel. This combination partly obscured the banding pattern of heterozygotes; those for uncommon alleles cathodal to the 
common allele in any taxon were easily detected, in contrast to more anodal uncommon alleles which were probably often missed completely. For the same reason, observed heterozygosity was lower than expected in many populations. However, most taxa appeared to be fixed for one or other allele, so this was probably of little importance. I distinguished 6 alleles.

\subsubsection{ICD-1}

Icd-1 encoded the polypeptide units of a dimer, with 3 alleles. Heterozygotes were uncommon, as most taxa were fixed for one or other allele, but those heterozygotes which were seen were rather fuzzy, with densest staining in the area where the middle band of a dimeric heterozygote would be expected.

\subsubsection{3 $/ C D-2$}

Icd-2 encoded the polypeptide units of a dimer. Heterozygotes were very clearly 3banded on some gels, and very fuzzy on others. Mobility of alleles at this locus varied substantially between runs; although the relative positions of alleles remained constant, the position with respect to the origin varied greatly. The same allele could be anodal in some runs and cathodal in others, presumably depending on slight differences in buffer $\mathrm{pH}$. Nine alleles were distinguished.

\subsubsection{LDH-1}

LDH isozymes are tetramers involving both $L d h-1$ and $L d h-2$. When both these loci are homozygous, 5 isozymes are seen (Figure 2.4.14.1). The most cathodal of these bands is the tetramer with all 4 subunits derived from $L d h-1$, and the most anodal, the tetramer of $4 L d h-2$ products. The 3 intermediate bands represent the 3 possible mixtures of products of the 2 loci in ratios of $3: 1,2: 2$, and $1: 3$. If $L d h-1$ is heterozygous, 5 bands are substituted for the single most cathodal band of the homozygote, and further bands are generated by the substitution of 2 alternatives into the heterotetramers. This results in a pattern of (moving from most cathodal to most anodal), a group of 5 bands, then groups of 4,3 , and 2 bands, then a single most anodal band as in the double homozygote. No individuals which were heterozygous at both loci were found. $L d h-1$ had 3 alleles.

\subsubsection{LDH-2}

$L d h-2$ was involved with $L d h-1$ in the production of heterotetramers as explained for $L d h-1$ above. Heterozygotes at this locus showed groups of $1,2,3,4$, and 5 bands moving from most cathodal to most anodal, the reverse of the pattern for $L d h-1$ heterozygotes. I found 6 alleles. 


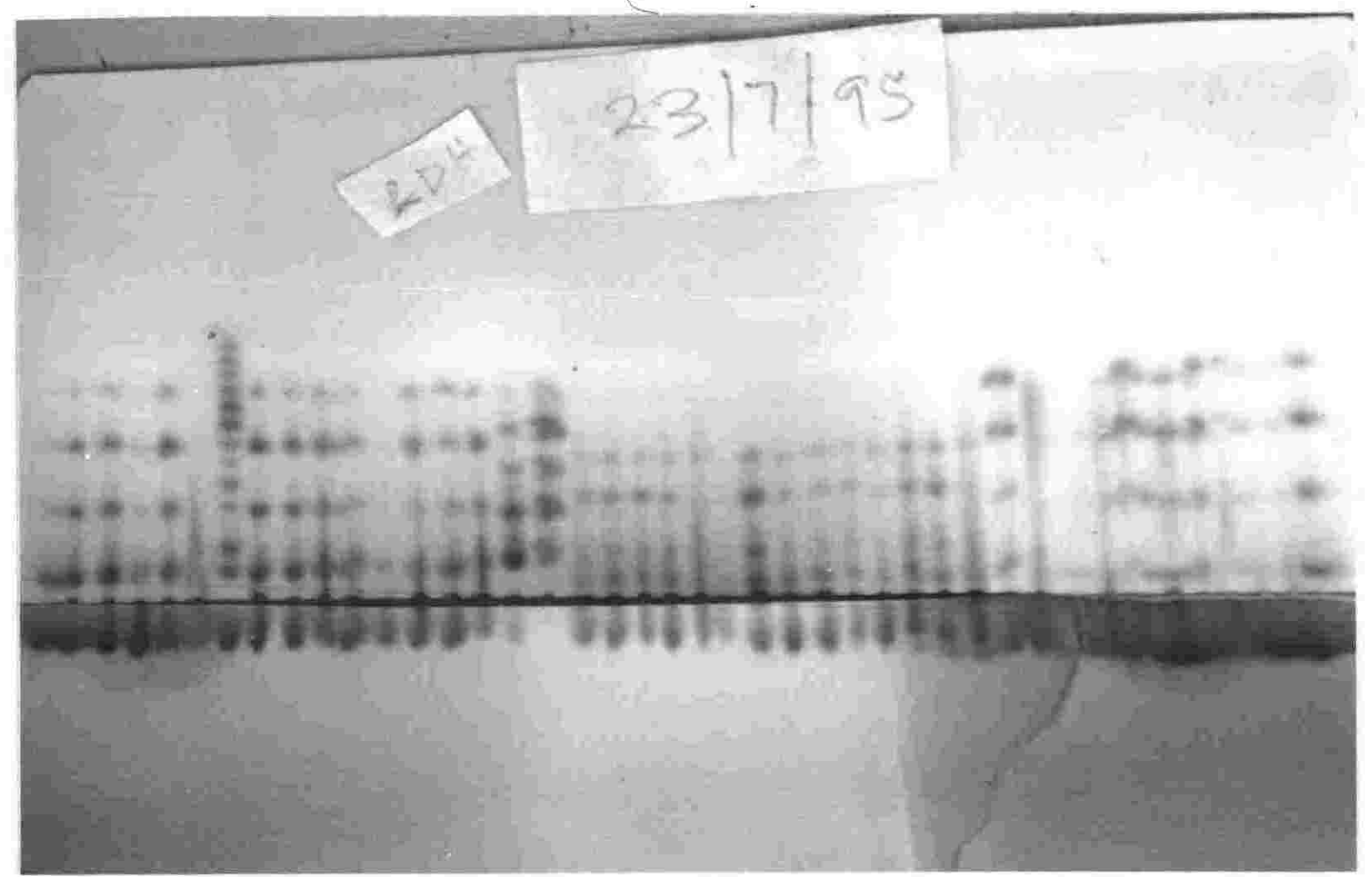

Figure 2.4.14.1 LDH gel showing 5-banded isozyme patterns of animals homozygous at both LDH loci, and 15-banded patterns from animals heterozygous at one or other locus.

\subsubsection{MDH-1}

Mdh-1 encoded the polypeptide units of a dimer, with clear 3-banded heterozygotes, although only 3 heterozygotes were seen in the whole study. There were 4 alleles.

\subsubsection{MDH-2}

I found no $M d h-2$ heterozygotes; all differences were fixed between populations or species. There were 5 alleles.

\subsubsection{ME-1}

Me-1 heterozygotes were uncommon; most differences were fixed between populations or species. Alleles $a$ and b migrated cathodally and alleles $c$ and $d$ anodally. Heterozygotes were expressed as a continuous streak between the positions of the 2 homozygotes. Four alleles were distinguished.

\subsubsection{MPl-1}

Mpi-1 encoded the polypeptide units of an anodally-migrating monomer in geckos as in humans, with very clear, 2-banded heterozygotes. I found 7 alleles. 


\subsubsection{NP-1}

Np-1 was probably a trimer, as in humans (Harris \& Hopkinson 1976), but because of strong secondary isozymes in all specimens, heterozygotes were difficult to detect. However, in situations where an uncommon allele was cathodal to the common allele in a population and was highly likely to be heterozygous, the bands of staining were more discrete and more widely spaced in the area between the most cathodal isozymes of the 2 homozygotes. Also, heterozygotes tended to have more isozyme bands in total, covering the whole spread from the most cathodal isozyme of the more cathodal allele to the most anodal isozyme of the more anodal allele. I distinguished 6 alleles.

\subsubsection{PEP-3}

This locus appeared to encode the polypeptide units of a monomer, but alleles were close together, and most populations were fixed for one or other allele. The few heterozygotes seen were 2-banded, but very blurred. There were 4 alleles.

\subsubsection{PGD-1}

$P g d-1$ in geckos encoded the polypeptide units of a dimeric enzyme, with the few heterozygotes seen staining as blurred streaks between the positions of the alternate homozygotes. All alleles were close to the origin, with some being slightly cathodal and others slightly anodal. I found 9 alleles.

\subsubsection{PGM-1}

Pgm-1 encoded the polypeptide units of a monomer, with 2-banded heterozygotes clearly visible. Secondary isozymes were sometimes also present. There were 5 alleles.

\subsubsection{PGM-2}

Pgm-2 encoded the polypeptide units of a monomer, with 2-banded heterozygotes very clearly visible, and 9 alleles.

\subsubsection{SOD-2}

Sod-2 encoded the polypeptide units of a dimer, with clear, 3-banded heterozygotes. All alleles seen were anodal; they were widely spread between the origin and the buffer front. No attempt was made to stain separately for SOD; it could be scored on slightly overstained gels from most stains on most buffers and both tissues. There were 6 alleles. 


\subsubsection{SOD-3}

Sod-3 migrated anodally, close to the buffer front in discontinuous buffers. No attempt was made to stain separately for Sod-3; it could be scored on slightly overstained gels from most stains on Ridgeway and Poulik gels. As no heterozygotes were detected, its subunit structure could not be assessed. There were 3 alleles.

\subsubsection{SORDH-1}

No variation was found at this locus.

\subsection{Phylogenetic methods}

Phylogenetic analysis aims to recover a phylogenetic tree which accurately reflects the history of evolutionary descent and divergence of the taxa under consideration. Each tree-building method has assumptions, which if not met in the dataset, compromise the result.

Phenetic clustering methods (those which group taxa by a measure of overall similarity rather than by character-state analysis) are capable of handling large datasets, and of including information from autapomorphies and frequency differences in polymorphic systems. The problem with these methods is that unless evolution is very clock-like, proceeding at an equal rate on all branches, "pseudoclades" may be generated, grouping less derived forms which resemble each other only because they are little changed from a distant common ancestor. Those phenetic techniques, such as distance Wagner and neighbour joining, which allow for variation in rate of evolution between edges in the tree, are less prone to this bias than are those, such as UPGMA and WPGMA which assume constant rates of evolution.

UPGMA or WPGMA phenograms generated from matrices of pairwise Nei's genetic distances are subject to these biases, as well as biases associated with Nei's D itself, which is highly sensitive to polymorphism (Hillis 1984). These trees are presented in this thesis, despite their poor performance in accurately recovering phylogeny, because of their familiarity to many readers, but other methods are preferred in interpreting phylogenetic patterns.

Simulation studies which test the ability of different tree-building methods to recover known phylogenies have consistently shown the neighbour joining 
method (Saitou \& Nei 1987) to perform better than other phenetic methods, and in some circumstances better than Parsimony analysis (Saitou \& Imanishi 1989; Nei 1991; Kim et al. 1993).

Parsimony analysis is the most phylogenetically rigorous of these analytical methods when analysing phylogenetic events well beyond the species level, and when numerous variable characters are available. At population to sister-species levels, where most allozyme differences are either autapomorphies or frequency differences in polymorphic systems, this method is frequently uninformative for analysis of allozyme results.

For parsimony analysis to accurately recover true phylogenies, informative characters should outnumber OTUs in the dataset being analysed. This presents problems for analysis of both morphological and allozyme data for New Zealand geckos by this technique, because of the presence of large numbers of similar taxa.

For parsimony analysis of allozyme data, either loci or alleles can be regarded as characters for analysis. The approach of regarding alleles as characters, and presence/absence of each allele as the character states was rejected, because the states for different characters are not independent of each other, and assigned ancestral states may include no alleles at some loci, which is clearly inappropriate (Murphy 1993).

Severe limitations on the size of the dataset which can be analysed by this method are imposed by the practicalities of geometric increase of computer running time with increasing numbers of taxa. The number of taxa may be limited in 3 ways: by combining the most closely related taxa to form compound "megataxa", by choosing representative taxa and deleting the others, or by running only less-rigorous heuristic searches rather than the more comprehensive branch and bound search with bootstrapping to supply a measure of confidence. None of these approaches is satisfactory, since all result in loss of important information. Deleting the most distantly related groups (potential outgroups) in the dataset reduces the ability of the programme to assess polarity of characters. Deleting ingroups which are intermediate in character composition between groups which are included may obscure the relationships between the included groups. Grouping populations is likely to generate artificial polymorphisms which are less informative than the original character set. 
Swofford \& Berlocher (1987) have suggested a technique for parsimony analysis of frequency variation in polymorphic characters among OTUs. Superficially this appears appealing for analysis of allozyme frequency data. However, the underlying assumption of this analytical technique must be that changes in allele frequencies occur only in a cladistic manner. At the levels of divergence at which frequency differences among OTUs predominate over fixed differences, and at which this technique would therefore be most useful, gene flow between populations often cannot be excluded as a possibility. This would result in patterns of allele frequency change which have not arisen by cladogenesis. Genetic substructuring of populations, highly likely in a relatively immobile animal like a gecko, particularly in patchy habitats, would result in fine-scale variation in allele frequencies in both space and time, leading to large sampling error. To obtain frequency data which represent the true population frequency with sufficient statistical confidence to be subjected to this analysis would require much larger sample sizes than were possible for this study. In small demes, frequencies may be subject to wide fluctuation because of genetic drift, and therefore the most parsimonious reconstruction of frequency changes may not accurately reflect history.

Data describing allozyme variation were analysed using the computer packages:

1) BIOSYS-1 (Swofford \& Selander 1981) - this package generated tables of allele frequency by population, measures of polymorphism and heterozygosity, pairwise distance matrices - Nei's (1978) I and D, CavalliSforza \& Edwards' (1967) arc distance, and Rogers' (1972) D, and UPGMA, WPGMA, and distance Wagner phenograms from individual, alphabeticallycoded genotype data for each specimen. Up to 89 populations at a time could be analysed.

2) MEGA (Kumar et al. 1993) - neighbour joining trees were generated by taking matrices of pairwise Cavalli-Sforza \& Edwards' arc distances generated using BIOSYS, then editing them into MEGA format, and transferring them into the MEGA package for analysis. Though developed primarily for analysis of DNA sequence data, this package can produce neighbour joining trees from any matrix of pairwise genetic distances. 
3) PAUP - parsimony analysis (Swofford 1989). Each allozyme locus was regarded as a character, and each allele as a character state. Characters could therefore be polymorphic, with multiple character states for a character; polymorphisms were not coded as separate character states.

To examine the geographic structure of allozyme variation in OtagoSouthland populations of the $H$. maculatus complex, and in particular to test for the presence of abrupt geographic boundaries, pairwise geographic and genetic differences were plotted for all pairs of populations separated by less than $60 \mathrm{~km}$. This geographic distance was chosen because large genetic distances reflecting clinal variation over longer distances are not relevant in the context of interruptions to gene flow at geographic boundaries. Large genetic distances at low geographic separations indicate absence of gene flow across parapatric boundaries, particularly when there is an obvious discontinuity between within-group and between-group pairwise genetic distances over the same range of geographic distances.

\subsection{MORPHOLOGY}

No full analysis of morphological variation was carried out independently of the allozyme analysis, but groups identified by allozyme analysis were checked for diagnostic morphological differences.

I assessed those morphological features which have formed the basis of classical taxonomies on the specimens used in the allozyme survey. Measurements taken were: total length, snout-vent length, tail length, snouteye, snout depth, eye-ear, diameter of orbit, diameter of ear opening, length of fourth digit of hind foot, and length and width of dilated portion and length of narrow distal region of this digit. The shapes of the rostral, mental, postmental, chin, head, dorsal, abdominal, and eyelid scales, the presence or absence of contact between the nostril and rostral scale, and the numbers of nasals, internasals, upper and lower labials, cloacal spurs, femoral and preanal organs, and lamellae under each toe of the hind foot were recorded for each specimen. The weight of the specimen, the overall shape of the head, and shape of the forehead, the degree of webbing and dilation of the digits, the shape of the lamellae, and of the distal region of the digits, and the skin, mouth, and eye colour were also recorded. 
Many museum specimens were examined and assigned to groups on the basis of their external morphology, but were not used in preparing the descriptions, as their identity was regarded as unconfirmed. 


\section{Chapter 3: DESCRIPTIONS AND SYNONYMIES}

\subsection{INTRODUCTION}

The following species are recognised in this thesis. Species proposed as a result of this work are indented below the species to which they have previously been referred:

Hoplodactylus chrysosireticus

H. duvaucelii

H. granulatus

H. nebulosus

H. kahutarae

H. maculatus

H. brunneus

H. "Mount Arthur"

H. "Kaikouras"

H. "Marlborough mini"

H. "Southern Alps"

H. "Danseys Pass"

H. "Cromwell Gorge"

H. "Otago"

H. "Southern mini"

H. pacificus

H. "Matapia"

H. "Poor Knights"

H. "Three Kings"

H. rakiurae

H. stephensi

(H. delcourti - probably from New Zealand; presumed extinct)

Naultinus elegans (subspecies N. e. elegans and N. e. punctatus)

N. gemmeus.

N. grayii

N. manukanus

N. rudis

N. stellatus

N. tuberculatus

In this chapter, an identification key for all the endemic New Zealand Gekkonidae is provided. Formal descriptions and synonymies are presented for the 11 new species which are proposed as a result of this study, for 2 
species which are resurrected from synonymy, and for 3 species which will be more narrowly defined after the separation from them of proposed new species, all within Hoplodactylus. Within Naultinus, descriptions and synonymies are presented for a species shown to be misnamed because of previous misidentification of type specimens, and for another for which the diagnosis is changed as a result of the same misidentification. Abbreviated references for synonyms are included in the synonymies for each species; those which are not discussed further are not included in the references section of this thesis. Species whose status has not changed are not redescribed. The new species are presented using informal tag names, to avoid any possibility that valid specific names will be used in the literature before formal publication of their descriptions.

Results and analyses justifying these decisions are presented in the following 2 chapters. The descriptions are presented before the analyses to provide names by which taxa can be discussed in the subsequent sections.

\subsection{Key to New Zealand endemic Gekkonidae}

This key will allow most New Zealand gecko specimens to be identified to species without the need to know their geographic origins or use genetic techniques. However, many morphological features, particularly scale counts and patterns, are very variable, and it is undesirable to rely on a single character for identification; atypical specimens probably crop up at times in all species.

1. Ventral skin opaque; mouth lining pigmented; toes narrow, transverse lamellae straight .21

Ventral skin translucent (particularly at night); pigment in mouth confined to diffuse patch on tongue-tip or absent; toes with expanded subdigital pads, transverse lamellae curved or chevron-shaped 2

2. Cloacal spurs pointed; no femoral pores in male; subdigital pad $<$ twothirds total length of digit 3 (H. pacificus complex) Cloacal spurs rounded; femoral pores present in male; subdigital pad $\geq$ two-thirds total length of digit

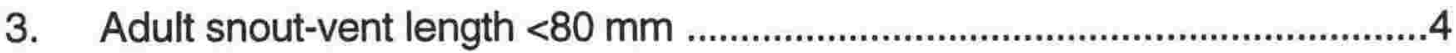

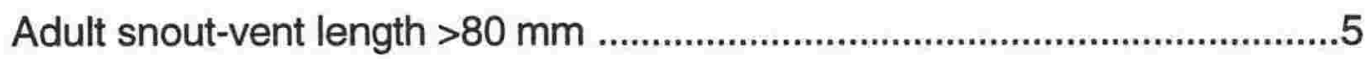

4. Adult snout-vent length $<55 \mathrm{~mm}$.............................................. "Matapia" Adult snout-vent length $>55 \mathrm{~mm}$................................................. pacificus 
5. Single large internasal, and trapezium-shaped mental separating first lower labials; 13 or more lower labials H. "Three Kings" Either 2 or more internasals, or triangular mental with first lower labials meeting behind it; 9-12 lower labials .H. "Poor Knights"

6. Rostral scale about 3 times as wide as deep; base colour pale greenish brown .7

Rostral scale $\leq 2.5$ times wider than deep; base colour brown or grey

7. Body pattern very indistinct - often appears uniform light olive colour; stocky build; distal phalanges very short H. "Southern mini" Body pattern of distinct longitudinal stripes; slender build; distal phalanges about one-quarter length of digit H. chrysosireticus

8. Rostral scale contacts or almost contacts nostril; no definite suture between first nasal and first labial

Rostral excluded from nostril by large first nasal; definite suture between first nasal and first labial

9. Large (adult snout-vent length $>100 \mathrm{~mm}$ ); $>15$ lamellae under fourth toe of hind foot

Smaller (adult snout-vent length $<80 \mathrm{~mm}$ ); $<14$ lamellae under fourth toe of hind foot

10. Adult snout-vent length $100-200 \mathrm{~mm} ; 15-20$ lamellae under fourth toe of hind foot; body pattern of transverse blotches H. duvaucelii Adult snout-vent length $>300 \mathrm{~mm}$; $>20$ lamellae under fourth toe of hind foot; body pattern of longitudinal stripes H. delcourti

11. Predominantly grey in colour; eyes brown or greenish, small, not prominent in profile; striped morph very uncommon; in open, usually montane-subalpine habitats .12 Predominantly brown in colour; eyes gold or brown, large, somewhat prominent in profile; in lower altitude open or forested habitats .16

12. Adults $>68$ (usually $>70$ ) $\mathrm{mm}$ snout-vent .13 $<68$ (usually <65) mm snout-vent 14

13. Single internasal; belly speckled H. "Danseys Pass" Usually 2 or more internasals; belly uniformly pale .H. "Otago" (part)

14. Snout $<1.35 x$ longer than deep; $\leq 11$ (usually $\leq 10$ ) broad lamellae under fourth toe 15 Snout $>1.37 x$ longer than deep; $\geq 11$ narrow lamellae under fourth toe ...H. "Mt Arthur" 
15. Distal phalange tapering gradually from dilated portion of digit H. "Southem Alps" Distal phalange narrowing abruptly from dilated portion of digit H. "Cromwell Gorge" (part)

16. Body and head somewhat depressed; distal phalanges strongly curved and largely subsumed in pad, not narrowing abruptly.

.H. "Otago" (part)

Body and head not depressed; prominent black spots and blotches on original tail; distal phalanges narrow abruptly from expanded subdigital pads

H. brunneus

17. Toes narrow abruptly from pad to narrow, moderately curved distal phalanx, occupying $>1 / 4$ length of toe

Toes narrow gradually from pad to strongly curved distal phalanx, occupying $<1 / 4$ length of toe 19

18. Snout shallow; eye prominent, brown to yellowish in colour, usually with several posterior superciliary denticles spinose; $\geq 11$ lamellae under fourth toe H. maculatus Snout more conical; eye distinctly green, with $\leq 2$ small spines at rear end of supraciliary fringe; $\leq 10$ lamellae under fourth toe

H. "Cromwell Gorge" (part)

19. Body and head somewhat depressed; small to large body size; original tail longer than head + body H. "Otago" (part) Body and head not depressed; snout deep, conical, eye not prominent in profile; small body size; original tail about equal to snout-vent length 20

20. Usually no pigment on tongue; $\geq 10$ (usually 11-12) lamellae under fourth toe; mental triangular H. "Kaikouras" Diffuse patch of dark pigment on tongue-tip; 9-10 lamellae under fourth toe; mental trapezium-shaped H. "Marlborough mini"

21. Dorsal scales of snout much larger than dorsals of body; pupil straight edged; diurnal; subdigital lamellae continue uninterrupted to claw 26 (Naultinus)

Dorsal scales of snout only slightly larger than dorsals of body; pupil with crenulate edges, particularly after preservation; primarily nocturnal or crepuscular; subdigital lamellae differentiated into scansorial pad not continuing onto distal phalanx 22

22. Eye black; pupil not visible in daylight H. kahutarae Eye brownish, with visible pupil .23 
23. Body warm tan or buff in colour, with prominent longitudinal stripes of uniform width H. stephensi

Body colour/pattern not as above.

24. Inside of mouth and tongue purple; distinctive body pattern of rows of chevrons H. rakiurae Inside of mouth and tongue yellow/orange with dark purple palate and throat

25

25. Belly heavily blotched with dark grey; snout shallow, somewhat concave in profile, longer than eye-ear distance H. granulatus Belly finely spotted with greyish brown; snout shorter than eye-ear distance, deep, blunt, not concave in profile H. nebulosus

26. Dorsal body scales heterogenous, some considerably enlarged ........27 Dorsal scales homogenous

27. Enlarged scales over entire dorsum; tongue greenish yellow; mouth dark blue; foot-pads grey; many mature males brown or grey rather than green N. rudis

Enlarged scales confined to nape and pelvic area, or to dorso-lateral rows; tongue yellow; mouth pale lavender or orange; foot-pads yellowish; belly green in females, pale blue in males ........ . manukanus

28. Markings, if present, large circular blotches; mouth lining yellow .N. stellatus (except northernmost populations) Markings not large and circular; mouth lining blue or lavender ............29

29. Scales moderately enlarged, conical ....................................................30 Scales minute, granular to somewhat conical ......................................31

30. ????????? .N. tuberculatus ????????? N. gemmeus [because of the substantial geographic variation within N. gemmeus in such characters as tongue, mouth, and pad colour and contact between rostral and nostril (possibly indicative of undescribed specific variation), no diagnostic differences exist between these 2 species as a whole, but pairwise comparisons between $N$. tuberculatus and any individual population of $N$. gemmeus reveal several differences]

31. Mouth lining lavender; orange markings around lips and nostrils ........32 Mouth lining blue; lips and nostrils white or green

32. Foot-pads yellow N. stellatus (northernmost populations) Foot-pads grey-green N. grayii (North Cape)

33. Scales on snout flattened, polygonal; tongue orange N. grayii Scales on snout domed; tongue blue 
34. Snout-vent length $<75 \mathrm{~mm}$; adult male with pale blue belly; foot pads grey-green; cloacal spurs green N. e. elegans Snout-vent length of adults $>80 \mathrm{~mm}$; adult male with pale blue band along flanks and pale green belly; foot pads yellow; cloacal spurs yellow N. e. punctatus

\subsection{HOPLODACTYLUS FITZINGER, 1843:}

\section{THE HOPLODACTYLUS PACIFICUS COMPLEX}

The members of this complex are distinguished from other members of Hoplodactylus by most of the features used by Robb \& Rowlands (1977) to distinguish $\mathrm{H}$. pacificus from $\mathrm{H}$. maculatus. Of particular importance in defining this group are the restriction of preanal organs to a small central patch, with no extension to the thighs, the moderately dilated toe pads extending no more than two-thirds of the length of the digit, and the fan of pointed cloacal spurs (shared with Naultinus and narrow-toed Hoplodactylus, but not with any other broad-toed Hoplodactylus). The broad contact between nostril and rostral, emphasised by Robb \& Rowlands (1977) as distinguishing $H$. pacificus from $H$. maculatus, is shared with $H$. duvaucelii and $H$. delcourti, and some South Island members of the $H$. maculatus complex.

\subsubsection{HOPLODACTYLUS PACIFICUS (GRAY, 1842)}

(FIGURE 3.3.1.1)

1842 Naultinus pacificus Gray. Zool. Misc.: 58.

Type locality: South Sea Islands.

Lectotype: BMNH 1946.8.22.67, designated by Bauer 1990.

Paralectotype: BMNH 1946.8.22.65.

1842 Naultinus pacifica Gray. ibid.: 72.

1843 Naultinus pacificus (part) Gray. Travels in New Zealand: 203.

1843 Platydactylus duvaucelii Gray. Ibid.: 203. (nec Platydactylus duvaucelii Duméril \& Bibron, 1836).

1845 Naultinus pacificus (part) Gray. Catalogue of the specimens of lizards in the British Museum: 169.

1851 Platydactylus pacificus Duméril. Catalogue méthodique de la collection des Reptiles: 35 (nec Platydactylus pacificus Duméril, 1851 ad Bavay 1869).

1857 Hoplodactylus pomarii Girard. Proc. Acad. Nat. Sci. Philadelphia 8:197.

Type locality: New Zealand.

Holotype: USNM 5690. 
1858 Gehyra oceanica (part) Girard. Herpetology of the United States exploring expedition: 273.

1867 Dactylocnemis pacificus Steindachner. Reptilien. Reise der Fregatte Novara: 11.

1871 Naultinus pacificus (part) Buller. Trans. New Zealand Inst. 3: 7. 1872 Naultinus pacificus Hutton. Trans. New Zealand Inst. 4: 172. 1885 Hoplodactylus pacificus Boulenger. Catalogue of the lizards in the British Museum, vol. 1: 173.

1954 Hoplodactylus duvaucelii (part) Hard. Tane 6: 143.

1955 Hoplodactylus pacificus (part) McCann. Dominion Museum bull. 17: 44.

1961 Naultinus pacificus (part) Chrapliwy et al. Herpetologica 17: 7. 1961 Hoplodactylus pacificus (part) Myers. Herpetologica 17: 169. 1977 Hoplodactylus pacificus (part) Robb \& Rowlands. Rec. Auckland Institute \& Museum 14: 137; figs 1, 3, 5, 7, 9.

1980 Hoplodactylus pacificus (part) Robb. Rec. National Museum of New Zealand 1: 308.

1990 Hoplodactylus pacificus (part) Bauer. Bonner zoologische Monographien, Nr. 30: 120-123; fig. 43, 44.

DIAGNOSIS: A medium-sized Hoplodactylus (60-75 mm snout-vent); rostral contacts the nostril for about one quarter of its circumference; rostral twice as wide as deep, subquadrangular; distance between snout and eye distinctly greater than the distance between eye and ear opening; distal phalanx of fourth toe usually at least half the length of the dilated portion; 3-4 pointed cloacal spurs forming a fan-like group on each side of the base of the tail.

DESCRIPTION: Head oviform, snout distinctly longer than the distance between the eye and the ear opening; forehead slightly concave; ear opening large, oval, oblique; body and limbs moderate to robust; digits somewhat dilated; the length of the dilated portion of the fourth toe being about 2.6 times its width, and about 1.7 times the length of the distal phalanx; distal inferior lamellae slightly angular; proximal ones straight; 10-16 lamellae under fourth toe; a distinct web between toes 2,3 , and 4 , but web slight between toes 1 and 2, and absent between toes 4 and 5; head covered with granular scales, of moderate size on the snout, and tiny on the remainder of the head; rostral broad, subquadrangular, in contact with the nostril, short median cleft from upper border; nostril pierced between rostral, first upper labial and 3-6 (usually 4-5) nasals, the anterior-most of which is 


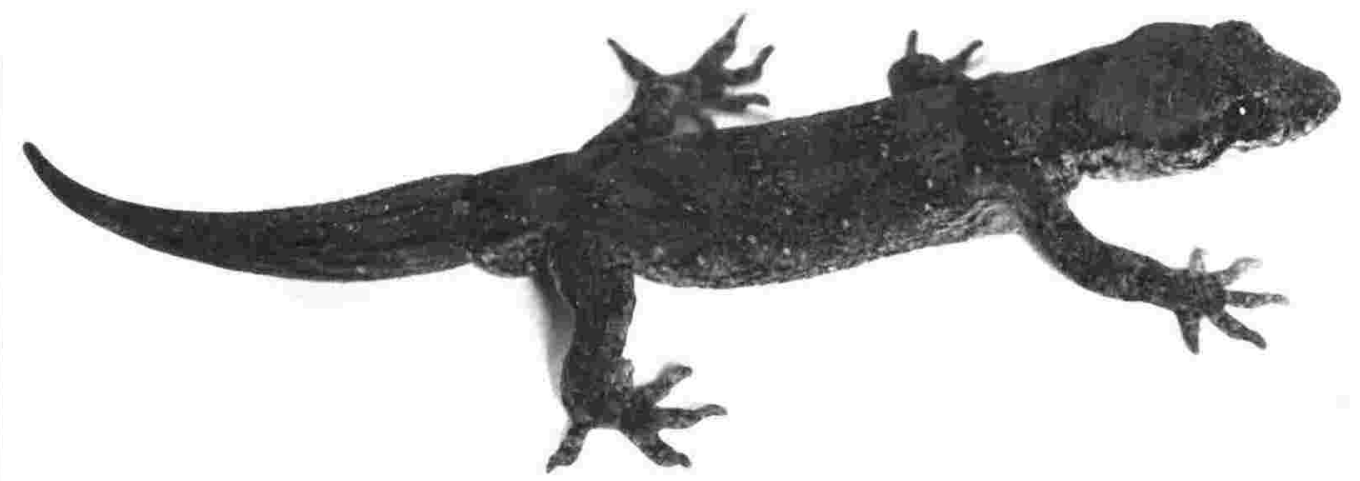

Figure 3.3.1.1 H. pacificus (RAH483, Matapia Island).

enlarged; 1 large internasal; 9-16 upper labials, and 8-14 lower labials; mental subtriangular, shorter than, or occasionally equal to, first lower labials; dorsal scales minute, granular; abdominal scales small, juxtaposed or subimbricate; in the males 1-4 series of preanal pores forming a roughly triangular patch, the number of pores very variable; no femoral pores; in females a patch of enlarged preanal scales, but no pores; tail cylindrical, tapering, covered with small, quadrangular, juxtaposed scales arranged in whorls; in the males, the base of the tail swollen to accommodate the hemipenes, and with 3-4 pointed cloacal spurs in a fan-like group on each side of the swelling; females with vestigial cloacal spurs.

COLOUR: Highly variable; longitudinal stripes, transverse blotches, or chevronshaped markings on a dull brown background on dorsum of body; head with dark V-shaped marking on top of head behind eyes, diffuse, dark-edged pale stripe from eye to ear, often with diffuse denticulate markings on lips; venter uniformly pale. About $20-25 \%$ of individuals in many populations have a bright mustard-yellow crescent on the nape of the neck, often with blotches of the same colour along the rest of the body and tail. This marking is believed to be caused by a single-locus recessive allele (Rowlands 1976). 
MATERIAL EXAMINED: RAH194, 210, 247-49, 361, 483, 484, CD853, 859-863,, 983, 1078-1080, FT303, 307, 547, 553, 558, 568, 571, 577, 579, 615, 616, 618, 620, 622, 2477, 2488-2491, 2494, 2495.

DISTRIBUTION AND GEOGRAPHIC VARIATION: Mainland North Island, and offshore islands to the east and north of the North Island. Most common on mammal-free offshore islands; on mainland patchily distributed; commonest in the north, but localities thinly scattered on the southern half of the island, extending to Whiteman's Valley east of Upper Hutt. Specimens from populations on the Aupori and Karikari Peninsulas are smaller ( $\leq 65 \mathrm{~mm}$ snout-vent) than those from further south (65-75 mm snout-vent), and have a lower incidence of the mustard nape marking.

HABITAT: Forest and shrubland. By day found under rocks, under loose bark, in hollows in trees, and particularly in crevices in clay banks. At night often forages up trees.

REPRODUCTION: Annual reproductive cycle, with ovulation in spring, and birth of (usually) twins in late summer.

COMMENTS: Hoplodactylus pacificus was the second species of New Zealand gecko to be described (Gray 1842a, b), but its identity has been unclear for much of its history. All species of Hoplodactylus except those discovered and described in the last 3 decades have been confused with $H$. pacificus at times, and $H$. pacificus appears in the formal synonymy of most of them; even the very much larger $H$. duvaucelii and morphologically very distinct $H$. granulatus were at times confused with this species (Smith 1933; Hard 1954; Hutton 1872). All known members of the Hoplodactylus maculatus complex (Robb \& Rowlands 1977; see below), along with $H$. chrysosireticus and H. stephensi (Robb 1980a, b), were included in McCann's (1955) concept of $H$. pacificus.

Girard (1857) described Hoplodactylus pomarii from "New Zealand". Robb \& Rowlands (1977) included $H$. pomarii in the synonymy of $H$. pacificus without explanation. The Holotype (USNM 5690) is extant and was measured at $72 \mathrm{~mm}$ snout-vent and photographed by Bauer (1986). Bauer's photograph shows a typical $H$. pacificus. All the New Zealand lizard collections of the US Exploring Expedition, which provided Girard's material, are believed to be from the Bay of Islands (Whitaker \& Thomas 1989), well within the range of 
$H$. pacificus. There is no evidence that the expedition could have had access to specimens of any of the new species I propose. The type specimen is too large and the wrong colour to be identified with $H$. "Matapia", and too small to be an adult of either $H$. "Poor Knights" or $H$. "Three Kings", though it is not certain that the specimen is mature.

Bauer (1990) included Pentadactylus brunneus Cope, 1868 in the synonymy of $H$. pacificus (synonymy fide Kluge 1965). Kluge established this synonymy during the period when $H$. maculatus was regarded as a synonym of $H$. pacificus. I consider that the type specimen of $P$. brunneus is referable to the species of the $H$. maculatus complex found in lowland Canterbury (section 3.3.2.5). The original description of $P$. brunneus clearly agrees with $H$. maculatus sensu Robb \& Rowlands (1977). Robb \& Rowlands (1977) did not include $P$. brunneus in the synonymy of either $H$. pacificus or $H$. maculatus. Aelurosaurus brunneus (Boulenger, 1885a) and Aeluroscalobotes brunneus (Boulenger, 1885b) are generic reassignments of the same taxon, and therefore are also removed from the synonymy of $H$. pacificus.

Dactylocnemis wullerstorfii Fitzinger, 1861 is a nomen nudum. Bauer (1990) listed it as such, but included it in the synonymy of $H$. pacificus. I consider that there is insufficient evidence associated with this name to associate it with this or any other species, and therefore do not include it in the synonymy of $H$. pacificus.

CONSERVATION STATUS: Although comparison of population densities between mammal-free islands and the mainland indicates that $H$. pacificus has probably declined greatly since the introduction of terrestrial mammals, it remains widespread, though very patchily distributed, on the mainland North Island, and is found on many offshore islands. It is therefore not endangered, although the fragmented nature of its mainland distribution must mean it is very vulnerable to continued attrition by local extinction. The separation of 3 new species $(H$. "Matapia", H. "Poor Knights", and H. "Three Kings") from H. pacificus has not significantly altered its conservation status, as all 3 have very localised distributions. 
1977 Hoplodactylus pacificus (part) Robb \& Rowlands. Rec. Auckland Institute \& Museum 14: 137.

1980 Hoplodactylus pacificus (part) Robb. Rec. National Museum of New Zealand 1: 308.

1990 Hoplodactylus pacificus (part) Bauer. Bonner zoologische Monographien, Nr. 30: 120-123; fig 44.

DIAGNOSIS: A large Hoplodactylus (up to $90 \mathrm{~mm}$ snout-vent); rostral contacts the nostril for about one quarter of its circumference; rostral twice as wide as deep, subquadrangular; distance between snout and eye distinctly greater than the distance between eye and ear opening; one large internasal; 12-14 upper and 13-14 lower labials; webbing between toes 2, 3, and 4 distinct; distal phalanx of fourth toe usually at least half the length of the dilated portion; 3 pointed cloacal spurs forming a fan-like group on each side of the base of the tail.

DESCRIPTION: Head oviform, snout distinctly longer than the distance between the eye and the ear opening; forehead slightly concave; ear opening large, oval, oblique; body and limbs moderate to robust; digits somewhat dilated; the length of the dilated portion of the fourth toe being about 2.6 times its width, and about 1.7 times the length of the distal phalanx; distal inferior lamellae slightly angular; proximal ones straight; 15-16 lamellae under fourth toe; head covered with granular scales, of moderate size on the snout, and tiny on the remainder of the head; rostral broad, subquadrangular, in contact with the nostril, short median cleft from upper border; nostril pierced between rostral, first upper labial and 4 nasals, the anterior-most of which is enlarged; 1 large internasal; $12-14$ upper labials, and 13-14 lower labials; mental subtriangular, shorter than, or occasionally equal to, first lower labials; dorsal scales minute, granular; abdominal scales small, juxtaposed or subimbricate; in the males 1-4 series of preanal pores forming a roughly triangular patch, the number of pores very variable; no femoral pores; in females a patch of enlarged preanal scales, but no pores; tail cylindrical, tapering, covered with small, quadrangular, juxtaposed scales arranged in whorls; in the males, the base of the tail swollen to accommodate the hemipenes, and with 3-4 pointed cloacal spurs in a fanlike group on each side of the swelling; females with vestigial cloacal spurs.

COLOUR: Highly variable; longitudinal stripes, transverse blotches, or chevronshaped markings on a dull brown background on dorsum of body; head with dark 


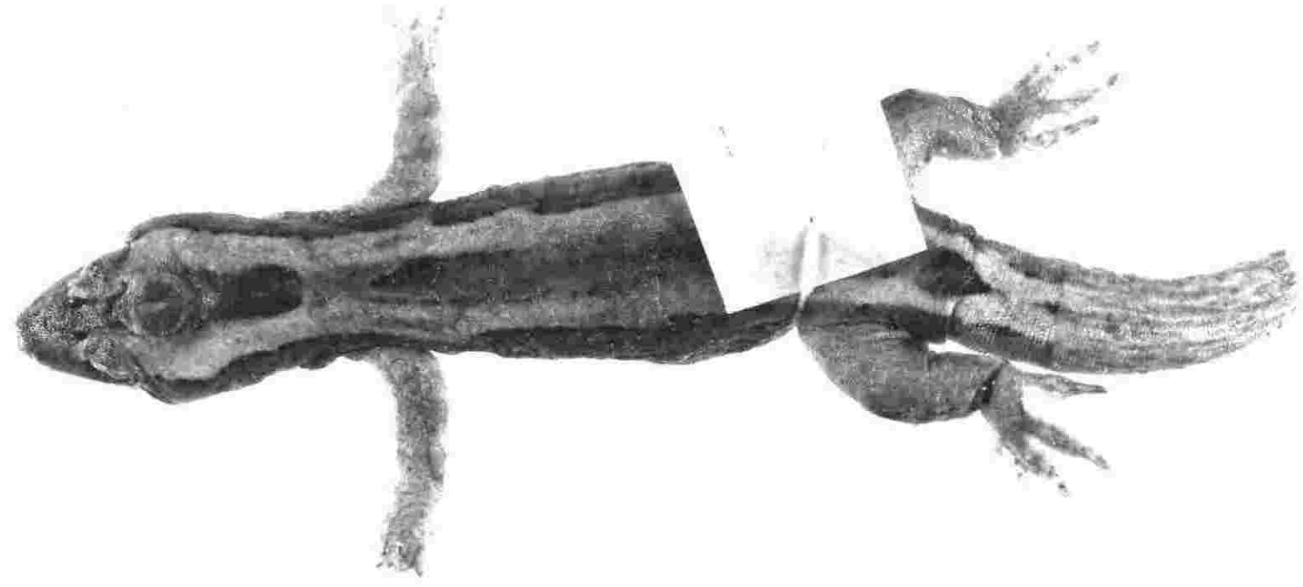

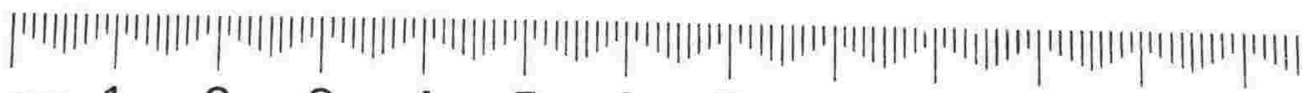

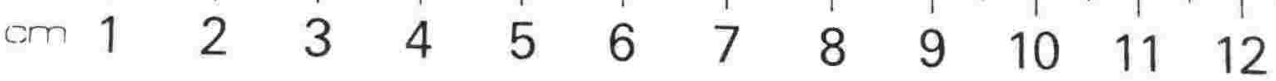

Figure 3.3.2.1 H. "Three Kings" (FT603, Great King Island).

V-shaped marking on top of head behind eyes, diffuse, dark-edged pale stripe from eye to ear, often with diffuse denticulate markings on lips; venter uniformly pale. As in $H$. pacificus, a substantial minority have a bright mustard-yellow crescent on the nape of the neck, often with blotches of the same colour along the rest of the body and tail.

MATERIAL EXAMINED: FT603-605.

DISTRIBUTION AND GEOGRAPHIC VARIATION: The Three Kings Islands; abundant on Great King Island, the largest and best known of the group, and also recorded from North East, West, and South West Islands and Archway and Hinemoa Rocks (McCallum 1985; McCallum et al. 1986). Specimens examined are all from Great King, therefore the identity of populations on other islands of the group requires confirmation.

HABITAT: Coastal forest and shoreline (McCallum et al. 1986)

REPRODUCTION: Unknown.

COMMENTS: McCann (1955) appears to have been the first taxonomist to have examined Hoplodactylus specimens from the Three Kings Islands. 
McCann (1955) commented that "specimens from the Great King Island and the Mokohinau-Great Barrier collections exhibit a remarkable increase in size, in the number of lamellae under the fourth toe, and a notable decrease in the number of preanal pores. Femoral pores are virtually absent. On the average these specimens are larger in all dimensions than others from the rest of the area". When Robb \& Rowlands (1977) reinstated H. maculatus and redescribed $H$. pacificus, they noted in a footnote that, in comparison with other $\mathrm{H}$. pacificus, "specimens from Three Kings and Mokohinau Islands are particularly robust in all dimensions".

CONSERVATION STATUS: Abundant on the Three Kings Islands, which are Nature Reserves with landing allowed by permit only.

3.3.3 HOPLODACTYLUS N. SP. "POOR KNIGHTS"

(FIGURE 3.3.3.1)

1968 Hoplodactylus pacificus (part) Whitaker. New Zealand journal of science 11(4): 623-651.

1977 Hoplodactylus pacificus (part) Robb \& Rowlands. Rec. Auckland Institute \& Museum 14: 137.

1980 Hoplodactylus pacificus (part) Robb. Rec. National Museum of New Zealand 1: 308.

1990 Hoplodactylus pacificus (part) Bauer. Bonner zoologische Monographien, Nr. 30: 120-123; fig 44.

DIAGNOSIS: A large Hoplodactylus (up to $88 \mathrm{~mm}$ snout-vent); rostral contacts the nostril for about one quarter of its circumference; rostral twice as wide as deep, subquadrangular; distance between snout and eye distinctly greater than the distance between eye and ear opening; either with 2 or more internasals and a trapezium-shaped mental separating the first lower labials, or with one large internasal, and a narrow, triangular mental, with the first lower labials meeting behind it; 8-13 upper and 9-12 lower labials; webbing between toes 2,3 , and 4 distinct; distal phalanx of fourth toe usually at least half the length of the dilated portion; 3-4 pointed cloacal spurs forming a fanlike group on each side of the base of the tail.

DESCRIPTION: Head oviform, snout distinctly longer than the distance between the eye and the ear opening; forehead slightly concave; ear opening large, oval, oblique; body and limbs moderate to robust; digits somewhat dilated; the length of the dilated portion of the fourth toe being about 2.6 times its width, and about 1.7 times the length of the distal phalanx; 

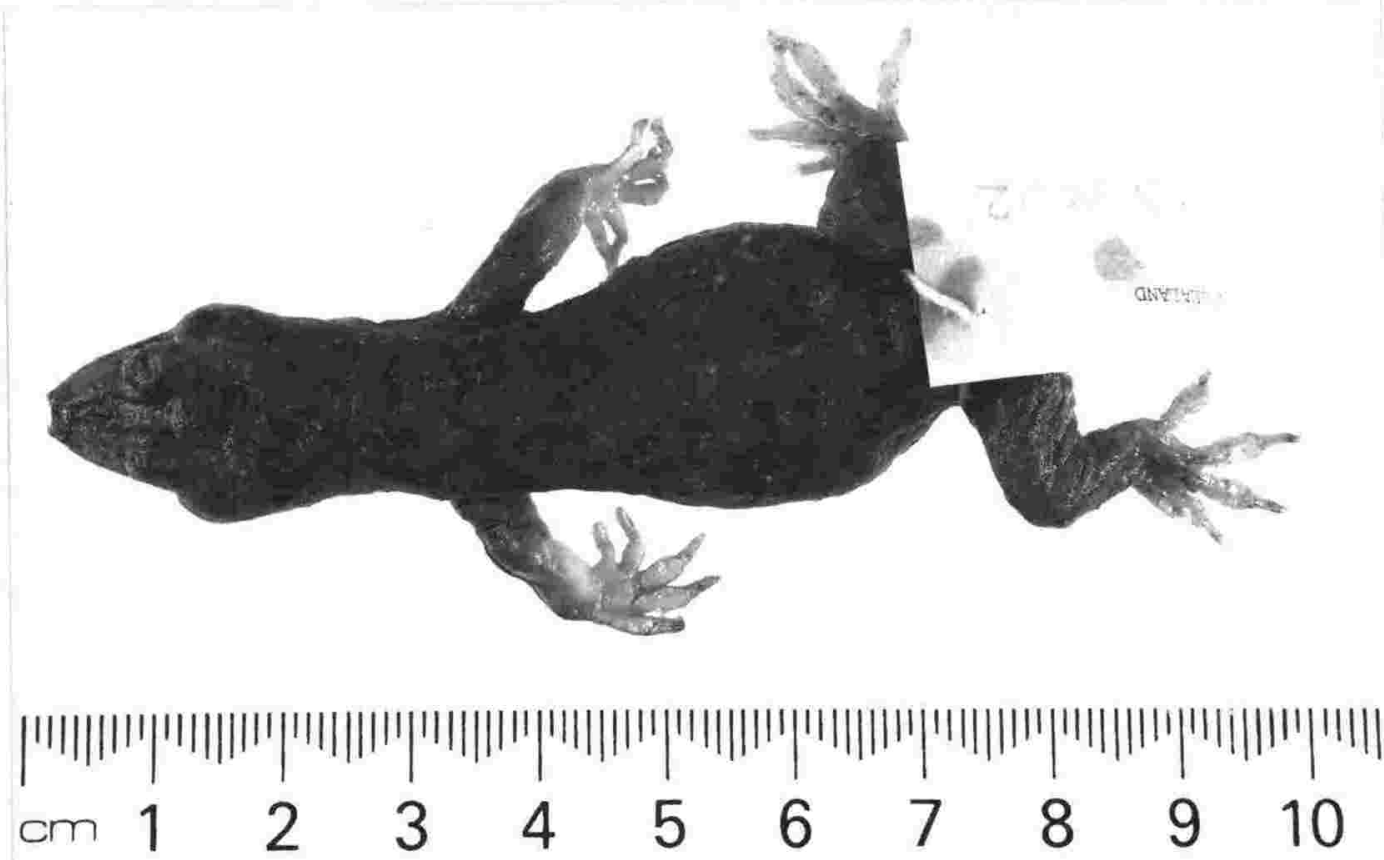

Figure 3.3.3.1 H. "Poor Knights" (CD1022, Aorangi Island).

distal inferior lamellae slightly angular; proximal ones straight; 11-16 lamellae under fourth toe; head covered with granular scales, of moderate size on the snout, and tiny on the remainder of the head; rostral broad, subquadrangular, in contact with the nostril, short median cleft from upper border; nostril pierced between rostral, first upper labial and 4-5 nasals, the anterior-most of which is enlarged; usually 2 or more small, occasionally one large internasal; 8-13 upper labials, and 9-12 lower labials; mental trapezium-shaped or triangular, shorter than, or occasionally equal to, first lower labials; dorsal scales minute, granular; abdominal scales small, juxtaposed or subimbricate; in the males 1-4 series of preanal pores forming a roughly triangular patch, the number of pores very variable; no femoral pores; in females a patch of enlarged preanal scales, but no pores; tail cylindrical, tapering, covered with small, quadrangular, juxtaposed scales arranged in whorls; in the males, the base of the tail swollen to accommodate the hemipenes, and with 3-4 pointed cloacal spurs in a fanlike group on each side of the swelling; females with vestigial cloacal spurs.

COLOUR: Highly variable; longitudinal stripes, transverse blotches, or chevronshaped markings on a dull brown background on dorsum of body; head with dark $\mathrm{V}$-shaped marking on top of head behind eyes, diffuse, dark-edged pale stripe from eye to ear, often with diffuse denticulate markings on lips; venter uniformly pale. As in $H$. pacificus, a substantial minority have a bright mustard-yellow crescent on the 
nape of the neck, often with blotches of the same colour along the rest of the body and tail.

SPECIMENS EXAMINED: CD1018-1023 (Aorangi Island), CD1024-1026 (Sugarloaf Rock).

DISTRIBUTION: The Poor Knights Islands, on all islands of the group.

COMMENTS: Robb \& Rowlands (1977) were the first to examine Poor Knights specimens in the context of a taxonomic review, although Whitaker (1968) had earlier published ecological and morphological observations on this population. Whitaker (1968) noted that $H$. pacificus specimens on the Poor Knights resembled those from the Three Kings and Mokohinaus in being larger than those on the mainland and having higher lamellae counts. However, it should be noted that at the time Whitaker did this work $H$. maculatus was regarded as conspecific with $H$. pacificus, and most of the populations he would have been familiar with would have been $H$. maculatus.

HABITAT: Coastal forest and shoreline.

REPRODUCTION: Unknown.

CONSERVATION STATUS: Abundant on the Poor Knights Islands, which are Nature Reserves with landing allowed by permit only.

3.3.4 HOPLODACTYLUS N. SP. "MATAPIA"

(FIGURE 3.3.4.1)

1988 Hoplodactylus chrysosireticus (part) Pickard \& Towns, Atlas of the amphibians and reptiles of New Zealand: 33.

DIAGNOSIS: A small Hoplodactylus (45-55 mm snout-vent); rostral contacts the nostril for about one quarter of its circumference; rostral twice as wide as deep, subquadrangular; distance between snout and eye distinctly greater than the distance between eye and ear opening; webbing between toes 2, 3, and 4 distinct; distal phalanx of fourth toe usually at least half the length of the dilated portion; 3-4 pointed cloacal spurs forming a fan-like group on each side of the base of the tail. 


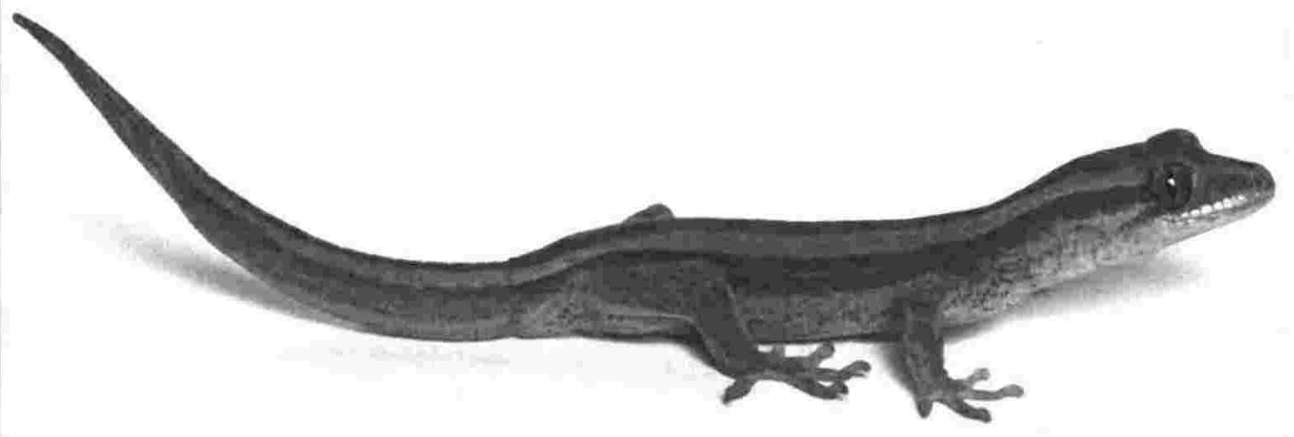

Figure 3.3.4.1 H. "Matapia" (RAH486, Matapia Island).

DESCRIPTION: Head oviform, snout 1.3-1.4 times longer than the distance between the eye and the ear opening; forehead slightly concave; ear opening large, oval, oblique; body and limbs moderately slender; digits somewhat dilated; the length of the dilated portion of the fourth toe being about 2.6 times its width, and about 1.7 times the length of the distal phalanx; distal inferior lamellae slightly angular; proximal ones straight; 12-15 lamellae under fourth toe; head covered with granular scales, of moderate size on the snout, and tiny on the remainder of the head; rostral broad, subquadrangular, in contact with the nostril, short median cleft from upper border; nostril pierced between rostral, first upper labial and 4-5 nasals, the anterior-most of which is enlarged; 1 large internasal; 10-13 upper labials, and 8-12 lower labials; mental subtriangular, shorter than, or occasionally equal to, first lower labials; dorsal scales minute, granular; abdominal scales small, juxtaposed or subimbricate; in the males 3-4 series of preanal pores forming a roughly triangular patch, the number of pores very variable; no femoral pores; in females a patch of enlarged preanal scales, but no pores; tail cylindrical, tapering, covered with small, quadrangular, juxtaposed scales arranged in whorls; in the males, the base of the tail swollen to accommodate the hemipenes, and with 3-4 pointed cloacal spurs in a fanlike group on each side of the swelling; females with vestigial cloacal spurs. 
COLOUR: Reddish tan with paler dorso-lateral longitudinal stripes (Figure 3.3.4.1).

DISTRIBUTION: Known only from Matapia (Motupia) Island, in shallow water off Ninety Mile Beach.

HABITAT: Cyperus ustulatus herbfield on Matapia Island.

REPRODUCTION: Unknown.

SPECIMENS EXAMINED: RAH251, 485, 486.

CONSERVATION STATUS: Confined to Cyperus ustulatus on Matapia Island, which is Maori-owned. The entire island is only 2 ha in area, and the herbfield covers about half of this. The species must be regarded as very vulnerable to any environmental perturbation, such as the introduction of any exotic mammal, or modification of the vegetation by adventive weeds.

COMMENTS: There is no evidence that any previous taxonomic worker had specimens of $H$. "Matapia" available to them. The first specimen of this species to be collected (see Chapter 5) was referred to $H$. chrysosireticus (Pickard \& Towns 1988).

\subsection{HOPLODACTYLUS FITZINGER, 1843:}

\section{THE HOPLODACTYLUS MACULATUS COMPLEX}

The species formerly included in $\mathrm{H}$. maculatus do not form a diagnosable group exclusive of all other previously recognised species, but these species plus $H$. chrysosireticus can be distinguished from the $H$. pacificus complex by the criteria discussed under that complex above. $H$. duvaucelii and $H$. delcourti fall into the $H$. maculatus complex according to all these morphological features except for the broad rostral-nostril contact which they share with the $H$. pacificus complex.

3.4.1 HOPLODACTYLUS MACULATUS (GRAY, 1845)

(FIGURE 3.4.1.1)

1845 Naultinus pacificus (part) Gray. Catalogue of the Specimens of Lizards in the British Museum: 169.

1845 Naultinus maculatus Gray. Catalogue of the Specimens of Lizards in the British Museum: 273.

Type locality: New Zealand. 
Lectotype: BMNH1946.9.8.14 (formerly XXII.75h), designated by

Bauer (1990).

Paralectotype: BMNH1946.9.8.15 (formerly XXII.75i). 1871 Naultinus pacificus (part) Buller. Trans. New Zealand Inst. 3: 7. 1871 Naultinus granulatus (?) Buller. Ibid: 9. (fide Robb \& Rowlands 1977). 1872 Naultinus pacificus (part) Hutton. Trans. New Zealand Inst. 4: 172. 1885 Hoplodactylus maculatus (part?) Boulenger. Catalogue of the lizards in the British Museum, vol. 1: 171; pl. XIV (fig. 1).

1897 Hoplodactylus maculatus (part?) Lucas \& Frost. Trans. Proc. New Zealand Inst. 29: 271.

1901 Woodworthia digitata Garman. Bull. Mus. Comp. Zool. 39: 4; pl. 1 (figs 2, 2a-f).

Type locality: New Zealand.

Syntypes: MCZ 6153, 152218.

1955 Hoplodactylus pacificus (part) McCann. Dominion Museum Bulletin 17:44; fig. 6.

1961 Naultinus pacificus (part) Chrapliwy et al. Herpetologica 17: 7. 1961 Hoplodactylus pacificus (part) Myers. Herpetologica 17: 169. 1965 Hoplodactylus digitatus Wermuth. Das Tierreich 80: 94. 1965 Hoplodactylus pacificus (part) Wermuth. Ibid.: 95. 1977 Hoplodactylus maculatus (part) Robb \& Rowlands. Rec. Auckland Inst. Mus. 14: 139; figs 2, 4, 6, 8, 10. 1990 Hoplodactylus maculatus (Part) Bauer. Bonner zoologische Monographien, Nr. 30: 117-120; fig. 40-42.

DIAGNOSIS: A small to medium sized Hoplodactylus ; nostril excluded from the rostral by the anterior nasal and first upper labial; rostral may be twice as long as deep, subpentagonal; distance between snout and eye equal to, or slightly greater than, distance between eye and ear opening; often with a series of spinose scales at the posterior end of the superciliary fringe; peritoneum lightly pigmented; tongue with diffuse patch of dark pigment at tip; digits scansorial, broadly expanded; distal phalanx of fourth toe about half the length of the dilated portion; 11-15 transverse lamellae under fourth toe of hind foot; in males, 1-2 bluntly conical enlarged scales on the base of the tail; preanal organs extending onto thighs.

DESCRIPTION: Head short, oviform; snout equal to, or slightly longer than the distance between the eye and the ear-opening; forehead flat or very slightly concave; ear-opening moderate, oval, oblique; body and limbs slender to 

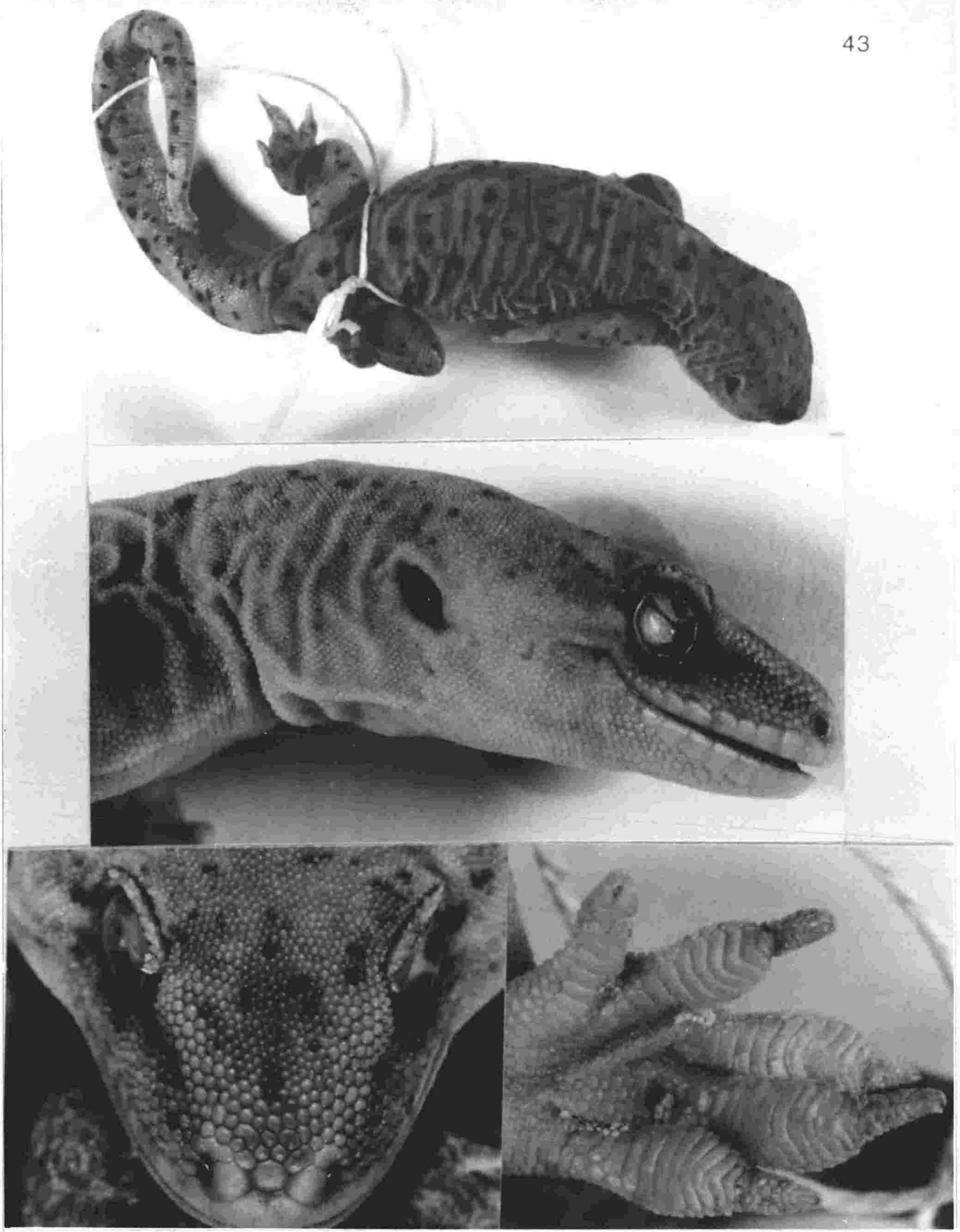

Figure 3.4.1.1 BMNH1946.9.8.14, lectotype of $N$. maculatus. Top, whole animal, dorsal view; centre, lateral view of head, showing large ear opening and spinose superciliary fringe; bottom left, head, showing exclusion of nostril from rostral; bottom right, ventral surface of hind foot, showing narrow, curved lamellae, and relatively long, narrow distal phalanges. 
moderate; digits somewhat dilated, the length of the dilated portion being about twice the length of the distal phalanx; distal inferior lamellae chevron-shaped, the proximal ones straight; 11-15 (usually 12-15) under fourth toe; a small web between digits 2, 3, and 4 but slight between digits 1 and 2 , and absent between digits 4 and 5 ; head covered with granular scales, of moderate size on the snout, tiny on the remainder of the head; rostral broad, subpentagonal, with a short median cleft from the upper border, rostral excluded from the nostril by the first upper labial and anterior nasal; nostril pierced between first upper labial and 4-5 (usually 4) nasals, the anterior-most of which is considerably enlarged; 1-3 (usually 1 large) internasals; 9-12 upper labials; 8-11 lower labials; mental quadrangular, shorter than first lower labial; 1-3 postmentals; ventral scales medium-sized, flat, subimbricate; original tail cylindrical, tapering, longer than snout-vent length, covered with small, sub-hexagonal juxtaposed scales arranged in whorls, with autotomy septa visible; in males, preanal organs in 3-8 angular series forming a roughly triangular patch, with 2-4 rows of about 5-14 pores extending to the base of each thigh; in females preanal organs absent; in males base of tail swollen to accommodate hemipenes, and with one large, bluntly conical cloacal spur on each side of swelling; females with cloacal spurs vestigial.

COLOUR: Dull brown with highly variable markings, including longitudinal or transverse stripes, blotches, and chevron patterns.

REPRODUCTION: Annual reproductive cycle, with young born in late summer (Robinson 1985).

SPECIMENS EXAMINED: RAH1a, 33, 34, 74-76, 78, 92, 93, 133-136, 137a, 139-142, 146, 177-187, 229-232, 286-288, 292, 296, 297, 343-358, 398-402, 414-416, 445, $446,475,478,479,482,498-501$, CD227, 228, 273-278, 285, 286, 290-296, 434$441,564,603-611,664-673,1228$, FT150-153, 185, 187, 275, 276, 279-288, 304, $305,308,309,523,541,542,545,546,548,552,556,557,559,612,613,2053$, 2054, 2057, 2492, 2901, 2902.

DISTRIBUTION, HABITAT, AND GEOGRAPHIC VARIATION: Marlborough, Nelson, islands in Cook Strait, North Island mainland as far north as Whangarei Heads, some northern offshore islands as far north as the Cavalli group. 
H. maculatus from populations in the Hauraki Gulf between Port Jackson on the Coromandel Peninsula and Great Barrier Island in the south-east and Whangarei Heads in the north-west are small (<65 mm SVL), with a distinctive pattern of many small spots arranged in paravertebral lines, frequently fusing to form crenulate longitudinal stripes. These populations are found mainly on boulder beaches, and are very uncommon on the mainland.

On islands off the east coast of the Coromandel Peninsula from Cuvier Island south and in the Bay of Plenty, and in mainland North Island localities from the Bay of Plenty south to the Wairarapa, animals are larger (up to $75 \mathrm{~mm}$ snout-vent) and highly polymorphic in colour pattern. The Hauraki Gulf-type pattern is seen occasionally, most commonly in the Coromandel island populations, but many animals have broad, straight or irregular longitudinal stripes, broad, straight transverse bands, large paired blotches, or W-shaped transverse markings on the dorsal body surface. Animals of these populations are commonly found in lowland forest under loose bark or in hollows in standing trees, and occasionally under stones in screes or in crevices in bluffs. The scattered coastal populations have a similar range of markings, but are often much smaller at all ages.

Populations from the southern coast of the North Island are distinguished by allozyme differences at 2 loci from others of the species, and a central population of this group, that at Cape Turakirae, is also distinctive in containing very small animals, with linear measurements only about twothirds of those of adjacent populations, and with sexual dimorphism in size (Whitaker 1982). However there seems to be a steep cline rather than an abrupt boundary between these populations and typical North Island-type populations, indicating that they are probably not reproductively isolated, despite their differences.

Populations from islands in Cook Strait and a few adjacent localities on both main islands form another distinct group in allozyme-based phenograms (see Chapters 4 and 5). This group of populations forms a transition zone between the typical North Island and South Island forms.

South Island animals of this species are more slender than those from the North Island, with less expanded digital pads, and are very dark in colour, with paler blotchy or chevron-shaped transverse markings on an almost 
black background. Some mainland South Island populations contain allozymes at some loci shared with South Island species; others have allozymes indistinguishable from Cook Strait populations. They are found from Cape Farewell at the north-western corner of the South Island, to the Cloudy Range in southern Marlborough, in lowland forest and rock bluffs and screes, and occasionally on boulder beaches, although the common boulder beach gecko of Nelson and especially Marlborough is $H$. "Marlborough mini".

COMMENTS: Gray's (1845) catalogue includes the type specimens of $H$. maculatus twice. They are first mentioned on page 169 along with specimens now included in $H$. pacificus, $H$. granulatus, and $H$. duvaucelii, under the name Naultinus pacificus, but on p. 273 , having apparently reexamined this material and acquired additional New Zealand specimens, he separated Naultinus maculatus, N. granulatus, and a species he named $N$. brevidactylus from $N$. pacificus. Although these new names had only minimal descriptions, and the layout of the descriptions (Figure 3.4.1.2) is unusual in listing the specimen numbers before the name, the intention is clear, and the new published name was associated with identifiable type specimens. I therefore have no hesitation in following Bauer (1990) in regarding Gray (1845) as the first valid author of the name Naultinus maculatus. Robb \& Rowlands (1977), when resurrecting $H$. maculatus from synonymy, chose the fuller description by Boulenger (1885a) of Hoplodactylus maculatus from the same type specimens as Gray's (1845) as the first valid use of this specific name. None of the subsequent authors (Boulenger 1885a, Robb \& Rowlands 1977, Bauer 1990) who have published papers of taxonomic importance on this species since that of Gray (1845) have made it clear that Gray's specimen h, Boulenger's specimen a, and Robb \& Rowlands' and Bauer's BMNH1946.9.8.14 are the same specimen, as are Gray's specimen i, Boulenger's specimen b, and Robb \& Rowlands' and Bauer's BMNH1946.9.8.15 (C. McCarthy, BMNH, pers. comm.).

Despite the lack of locality data associated with them, the types of Hoplodactylus maculatus (Gray, 1845) can be unambiguously identified with the most northern species of the $H$. maculatus complex. This species has a range of sub-digital lamellae counts overlapping only slightly with those of most other species in the complex, and usually much higher. Western populations of $H$. "Otago" have a range of lamellae counts also 
Page 144, after 9. Hemidactylus, insert:-

9."Nubilia. Tail mther depressed, subangular above, with cross rings of spines, the lower edge denticulated. Toes nearly free. Sides simple.

Page 154, line 31, for Inhab. — ? insert: -

a. In spirits. ?

Page 156, before 10. Velermesia, insert :-

$$
\text { 9." Nubilia. }
$$

Toes very slightly webbed at the base, rather dilated, last jnint compressed, clawed. Sides and binder part of the thighs simple. Tail rather depressed, lat beneath, sharp-edgecl, with a series of spines on the edge, and distant rings of spines with a series of broad shields beneath. Sides of body and limbs roundeil, simple. The back with a series of larger triangular tubercles. Femoral and preanal pores (uone?)

Argent's Nunilia. Nubilia Argentii.

Pale brown, with irregular dark cross bands; face with a hlack streak on each side; back with large bigh angular tubercles.

a. In spirits. Singapore. From Mr. Argent's cullection.

Page 157, line 34, after acales add, and larger tubercles.

Page 161, line 18, arld after P. Jamori, Fauna Japon. Saur. t. 2, f. $1-4$.

Page 169. Naultinus pacificus, erase specimen $b$. add scales minute; and add :-

$e-g$. Pale brown, with 3 more or less divided and longitudinal black streaks. New Zealand. From Mrr. Earl's collection. Zool. Erebus \& Terror, $t$. f. Toes vary in leugth, perhaps sexual.

N. brevidactylus, MISS.

$h, i$. Brown, with scattered black spots. New Zealand. From Mr. Earl's collection. Zuol. Erebus \& Terror, t. f. Toes short, perhaps sexual.

N. saaculatus, MISS.

The Gravular Naultinus. Naultinus grauulatus. N. paeificus, var. Gray, Dieffienbuch, and spec. b. Catal.p. 169.

Pale brown, with irregular darker cross bands, with white edges in front; scales granular, moderate, of under side larger; labial shields gradually smaller.

a. In spirits. New Zealind, Dr. Dieffenbacb. Presented by R. Owen, Евq.

b. In spirits. New Zealand. From Mr. Earl's collection.

Figure 3.4.1.2 Copy of Gray's (1845) original description of $N$. maculatus, showing the unusual layout which has caused debate on the validity of this description.

lower than, but overlapping more extensively with, $H$. maculatus, but lack the numerous spinose superciliary denticles which can be seen on the $H$. maculatus type, and have very short distal phalanges, unlike the $H$. maculatus type. The northern species is highly variable in colour pattern (see below), and includes specimens of the same colour patterns as both $H$. maculatus types, whereas western $H$. "Otago" show little variation in 
their typical pattern of transverse stripes, unlike either of the $H$. maculatus types.

Robb \& Rowlands (1977) and Bauer (1990) regarded Woodworthia digitata Garman, 1901 as the only junior synonym of $H$. maculatus. The 2 syntypes of this name are very small juveniles, which makes identification more difficult. However, the combination of rostral excluded from the nostril by a large first nasal with a definite suture with the first upper labial, rather long, narrow, fairly straight distal phalanges, high lamellae counts, and multiple internasals and postmentals (Figure 3.4.1.3), suggests that these specimens belong to the same species as the types of $H$. maculatus.

Gray's (1845) name $N$. brevidactylus requires further investigation. The description provided with this name is inadequate to permit identification of the taxon it applied to. Of the 3 specimens listed, 2 cannot now be traced; the third is currently catalogued as $H$. duvaucelii (C. McCarthy, BMNH, pers. comm.). The name has never been used since its original publication. If the name is synonymous with $H$. duvaucelii, it would be a junior synonym, as that species was described in 1836.

PUBLISHED STUDIES: Most studies of common geckos $(H$. maculatus sensu Boulenger 1885a, H. pacificus sensu McCann 1955, H. maculatus sensu Robb \& Rowlands 1977) in the North Island concern this species. However, a few Wellington-based studies may have included the North Island populations of $H$. "Marlborough mini", found abundantly along the coast between Island Bay and Makara. All references to $H$. maculatus or $H$. pacificus on Cook Strait islands refer to $H$. maculatus, except for rare references to $H$. stephensi from Stephens Island as $H$. pacificus; the latter usually making the identity of the species under discussion clear. References from the period between 1955 and 1977 relating to populations of $H$. pacificus from the northern half of the North Island or northern offshore island refer either to $H$. maculatus or $H$. pacificus. There are no identifiable references to South Island populations of this species.

CONSERVATION STATUS: Abundant in the southern North Island, and on many offshore islands, most of which are securely reserved; elsewhere in the North Island and in the northern South Island, patchily distributed, and locally common. In some areas (e.g., the Wanganui River valley and the Gisborne area) populations have survived in highly modified agricultural 

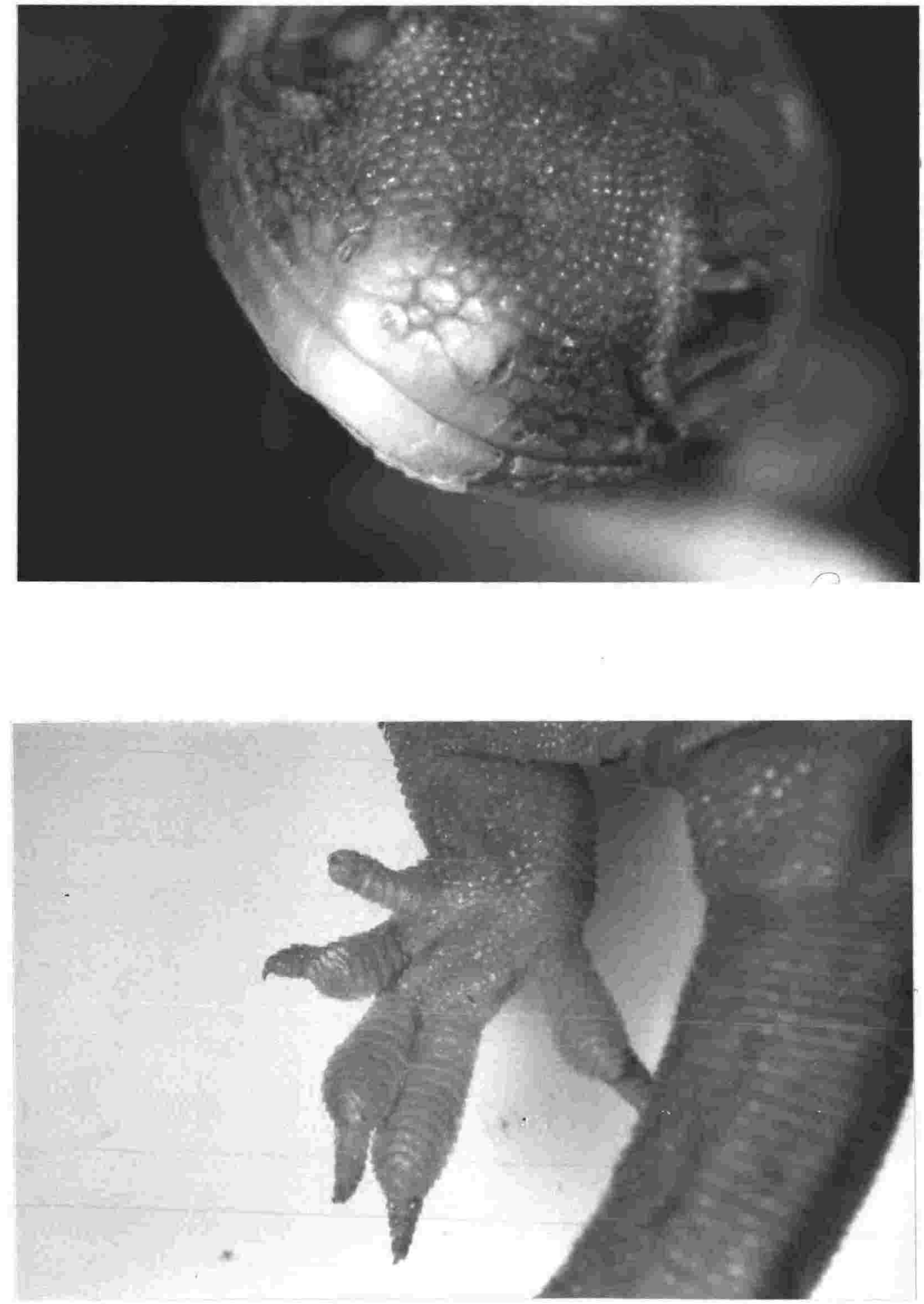

Figure 3.4.1.3 MCZ 152218, Syntype of Woodworthia digitata Garman 1901, showing diagnostic features which identify it with $H$. maculatus. Top, snout, showing rostral excluded from nostril; bottom, hind foot, showing narrow lamellae, and relatively long, narrow distal phalanges. 
landscapes by occupying old hollow cabbage trees (Cordyline australis). The recent death of many of these trees in the epidemic of cabbage tree sudden decline disease must doom these populations of $\mathrm{H}$. maculatus, although the lack of recruitment to these cabbage tree populations would have had the same effect in the longer term. Coastal populations are vulnerable to habitat degradation by human activity, as they occupy a narrow littoral zone, and are isolated from other populations.

\subsubsection{HOPLODACTYLUSN. SP. "MOUNT ARTHUR"}

(FIGURE 3.4.2.1)

DIAGNOSIS: A small Hoplodactylus (63-64 mm snout-vent; $\mathrm{n}=2$ adults); nostril just touching rostral; rostral about twice as long as deep, subpentagonal; distance between snout and eye slightly greater than distance between eye and ear opening; 3-5 spinose scales at the posterior end of the superciliary fringe; tongue with diffuse patch of dark pigment at tip; digits scansorial, broadly expanded; distal phalanges short (about one quarter of length of toe), on fourth toe about 0.8 times width of dilated portion; 11-12 transverse lamellae under fourth toe of hind foot; in males, one bluntly conical enlarged scale at each side of the base of the tail; small triangular patch of preanal organs, with only one row extending to the base of the thighs, surrounded by non-porate but enlarged scales.

DESCRIPTION: Head oviform; snout longer than distance between eye and earopening, 1.8 times diameter of ocular disc in fresh specimens; forehead flat or slightly concave; ear opening small, round; body and limbs moderate; digits moderately dilated, length of dilated portion of fourth toe being about 2.25 times its width; distal phalanx very short, 0.8 times width of dilated portion; distal inferior lamellae chevron-shaped, remainder curved; 11-12 lamellae under fourth toe; dorsal surface of head and body covered with small granular scales; rostral about twice as broad as deep, with a short median cleft from upper border, just touching nostril; anterior nasal considerably enlarged; one medium-large roughly circular internasal; 7-10 upper labials and 5-8 lower labials; mental quadrangular, shorter than first lower labial; 1-3 postmentals; ventral scales medium-sized, flat, somewhat overlapping; tail cylindrical, tapering, about equal in length to snout-vent length, covered with small, sub-hexagonal juxtaposed scales arranged in whorls, with autotomy septa visible; in males, preanal organs in 3 angular series forming a roughly triangular patch, with 2 rows of about 5 pores extending to the base of each thigh; in females preanal organs absent; in males base of tail swollen to accommodate hemipenes, and with one large, bluntly conical cloacal spur on each side of swelling; females with cloacal spurs vestigial. 


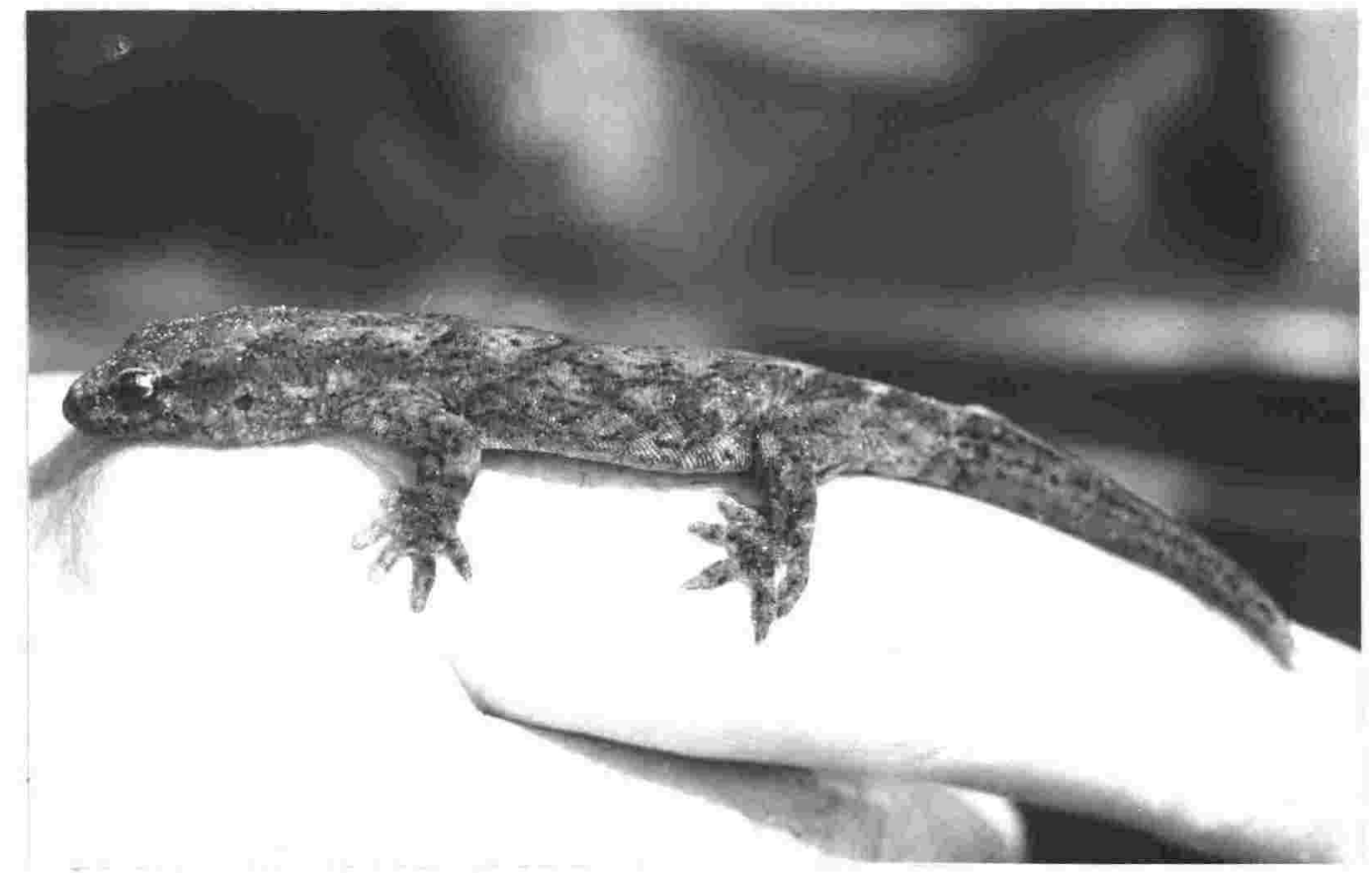

Figure 3.4.2.1 $\mathrm{H}$. "Mount Arthur" (RAH 405, Mount Arthur)

COLOUR: Dark grey with chevron-shaped transverse markings.

MATERIAL EXAMINED: RAH 405 and 407.

DISTRIBUTION: Above bushline on Mount Arthur, Nelson.

HABITAT: Marble scree.

REPRODUCTION: A female dissected in April had roughly half-term foetuses. At this time of year, most Hoplodactylus have given birth and are yolking ovarian follicles. $H$. "Mt Arthur" may therefore have a biennial reproductive cycle like high altitude populations of $H$. "Otago" (Cree 1994).

COMMENTS: There are no identifiable references to this species.

CONSERVATION STATUS: Known from about 2-3 hectares of scree on Mount Arthur in Kahurangi National Park, but common in this area, and with no immediate threat. A specimen which appears to be referable to this species has been photographed in 
similar habitat on Mount Owen (S. Courtney, Department of Conservation, pers. comm.). Similar habitats nearby should be searched urgently.

3.4.3 HOPLODACTYLUSN. SP. "MARLBOROUGH MINI"

(FIGURE 3.4.3.1)

1955 Hoplodactylus pacificus (part) McCann. Dominion Museum Bulletin 17:44. 1961 Naultinus pacificus (part) Chrapliwy et al. Herpetologica 17: 7.

1961 Hoplodactylus pacificus (part). Myers. Herpetologica 17: 169.

1965 Hoplodactylus pacificus (part) Wermuth. Das Tierreich 80: 95.

1977 Hoplodactylus maculatus (part) Robb \& Rowlands. Rec. Auckland Inst. Mus. 14: 139. 1990 Hoplodactylus maculatus (part) Bauer. Bonner zoologische Monographien, Nr. 30: 117-120; fig. 42.

DIAGNOSIS: A small Hoplodactylus (most populations $<60 \mathrm{~mm}$ snout-vent; Glenduan population $<65 \mathrm{~mm}$ ); nostril excluded from the rostral by the anterior nasal and first upper labial; rostral may be twice as long as deep, subpentagonal; mental trapezium-shaped; distance between snout and eye about equal to distance between eye and ear opening; peritoneum lightly pigmented; mouth lining pinkish, tongue often without diffuse patch of dark pigment at tip; digits scansorial, broadly expanded; distal phalanx of fourth toe seldom more than half the length of the dilated portion, $\leq 10$ lamellae under fourth toe; in males, 1 bluntly conical enlarged scale on the base of the tail; preanal organs extending onto thighs.

DESCRIPTION: Head oviform; snout about equal to distance between eye and ear-opening; forehead flat or slightly concave; ear opening small, round; body and limbs moderately slender; digits moderately dilated, length of dilated portion of fourth toe being about 2.25 times its width; distal phalanx short, 0.8 times width of dilated portion; distal inferior lamellae chevron-shaped, remainder curved; 9 to 10 lamellae under fourth toe; dorsal surface of head and body covered with small granular scales; rostral about twice as broad as deep, with a short median cleft from upper border, excluded from nostril by contact between anterior nasal and first upper labial; anterior nasal considerably enlarged; 1 medium-large roughly circular internasal; 10-12 upper labials and 10-15 (usually 10-12) lower labials; mental quadrangular or bluntly triangular, shorter than first lower labial; 1-3 postmentals; ventral scales medium-sized, flat, imbricate; tail cylindrical, tapering, about equal in length to snout-vent length, covered with small, sub-hexagonal juxtaposed scales arranged in whorls, with autotomy septa visible; in males, preanal organs in 2-4 angular series forming a roughly triangular patch, with 2 rows of about 6 pores extending to the base of 


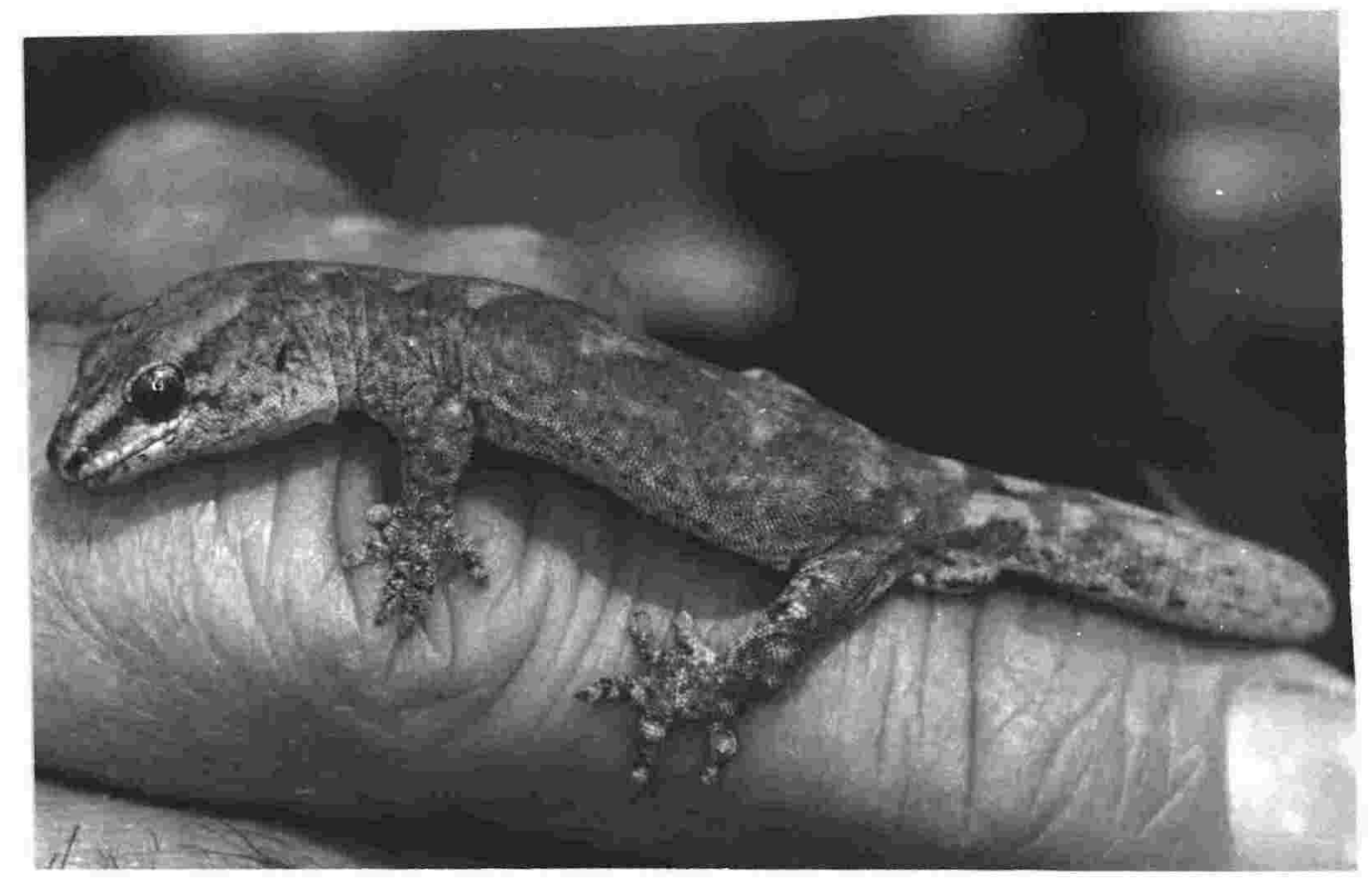

Figure 3.4.3.1 H. "Marlborough mini" (RAH411, Acheron Road).

each thigh; in females preanal organs absent; in males base of tail swollen to accommodate hemipenes, and with 1 large, bluntly conical cloacal spur on each side of swelling; females with cloacal spurs vestigial.

CoLOUR: $H$. "Marlborough mini" is brown, almost uniform or with very variable markings (blotches or transverse or longitudinal stripes). There is a distinct stripe down side of head through eye. The top of head sometimes has a dark $V$ behind the eyes but has no other markings.

DISTRIBUTION, HABITAT, AND GEOGRAPHIC VARIATION: $H$. "Marlborough mini" is found along the coastline between Island Bay and Makara to the south and west of Wellington in the North Island, under driftwood and in screes and creviced coastal bluffs; under boulders and Muehlenbeckia on the Nelson Boulder Bank and adjacent coastline at Glenduan in Nelson; under boulders or driftwood at many sites along the Marlborough east coast; and inland on rock bluffs associated with such vegetation as Marlborough tree daisies, Sophora prostrata, matagouri, and Muehlenbeckia in Marlborough south of the Wairau Valley and North Canterbury. 
Morphologically, the most distinct population is the Nelson Boulder BankGlenduan one. Animals there are larger and darker in colour than elsewhere. Animals from the east coast of Marlborough tend to be somewhat bigger and more boldly patterned than those from inland sites. North Island specimens are very small, but otherwise resemble coastal Marlborough ones.

COMMENTS: $H$. "Marlborough mini" was first recognised as distinct from sympatric $H$. "Southern Alps" (as $H$. maculatus) during searches for $H$. kahutarae in the late 1970s (Whitaker 1984b). However, it has not previously been considered distinct from other small species of the $H$. maculatus complex such as $H$. "Southern mini", $H$. "Kaikouras", and some populations of $H$. "Southern Alps".

No published name is available for this species. It is very difficult to determine whether early authors included this species in their concept of Hoplodactylus maculatus without examining all material listed in their papers; however, the ranges of size and scale counts published suggest that they did not. More recent authors, including McCann (1955) and Robb \& Rowlands (1977), however, certainly included this species in their concepts of $H$. pacificus and later $H$. maculatus, as many of the specimens they list belong to this species.

$H$. "Marlborough mini" populations from the coastline south and west of Wellington have been included in several studies of $H$. maculatus. Usually this species cannot be distinguished from nearby populations of $H$. maculatus in these accounts.

MATERIAL EXAMINED: RAH91 198, 199, 214, 215, 221-224, 252, 267-272, 276-282, 289-292, 303, 320-325, 359, 372-375, 380-382, 383a, 409-412, 480, FT332, CD571, 573, 574, 2127, 2129-2131.

REPROduction: Like the rest of the genus, $H$. "Marlborough mini" is viviparous. Females collected in December gave birth in February to twin offspring, which is typical for the genus.

CONSERVATION STATUS: $H$. "Marlborough mini" is widely distributed and locally abundant in patches of suitable habitat, even where this habitat is readily accessible from adjacent roads; a great deal of suitable habitat must 
be less accessible. Much of the species' habitat is too high in altitude to be at risk of development. Some coastal populations are at risk from roading, or crushing by vehicles, pedestrians and dumped spoil. Few populations are in protected areas.

\subsubsection{HOPLODACTYLUSN. SP. "KAIKOURAS"}

(FIGURE 3.4.4.1)

DIAGNOSIS: A small Hoplodactylus ( $<65 \mathrm{~mm}$ snout-vent); nostril excluded from the rostral by the anterior nasal and first upper labial; rostral may be twice as long as deep, subpentagonal; distance between snout and eye about equal to distance between eye and ear opening; mental triangular; peritoneum lightly pigmented; mouth lining pinkish, tongue often with diffuse patch of dark pigment at tip; digits scansorial, broadly expanded; distal phalanx of fourth toe seldom more than half the length of the dilated portion; $\geq 10$ (usually 11-12) lamellae under fourth toe; in males, 1-2 bluntly conical enlarged scales on the base of the tail; preanal organs extending onto thighs.

DESCRIPTION: Head oviform; snout about equal to distance between eye and ear-opening; forehead flat or slightly concave; ear opening small, round; body and limbs moderate; digits moderately dilated, length of dilated portion of fourth toe being about 2.25 times its width; distal phalanx short, about equal to width of dilated portion; distal inferior lamellae chevron-shaped, remainder curved; 1012 lamellae under fourth toe; dorsal surface of head and body covered with small granular scales; rostral about twice as broad as deep, with a short median cleft from upper border, just touching nostril; anterior nasal considerably enlarged; one medium-large roughly circular internasal; 10-13 upper labials and 9-13 lower labials; mental triangular, shorter than first lower labial, first lower labials sometimes in contact behind mental; 1 postmental; ventral scales medium-sized, flat, imbricate; tail cylindrical, tapering, about equal in length to snout-vent length, covered with small, sub-hexagonal juxtaposed scales arranged in whorls, with autotomy septa visible; in males, preanal organs in 4-7 angular series forming a roughly triangular patch, with 2 rows of 7-10 pores extending to the base of each thigh; in females preanal organs absent; in males base of tail swollen to accommodate hemipenes, and with 1-2 small, bluntly conical cloacal spurs on each side of swelling; females with cloacal spurs vestigial.

COLOUR: $H$. "Kaikouras" is brown, with distinct paler transverse stripes, and a distinct dark stripe down the side of the head through the eye. The top of the head often has a dark $V$ behind the eyes but has no other markings. 

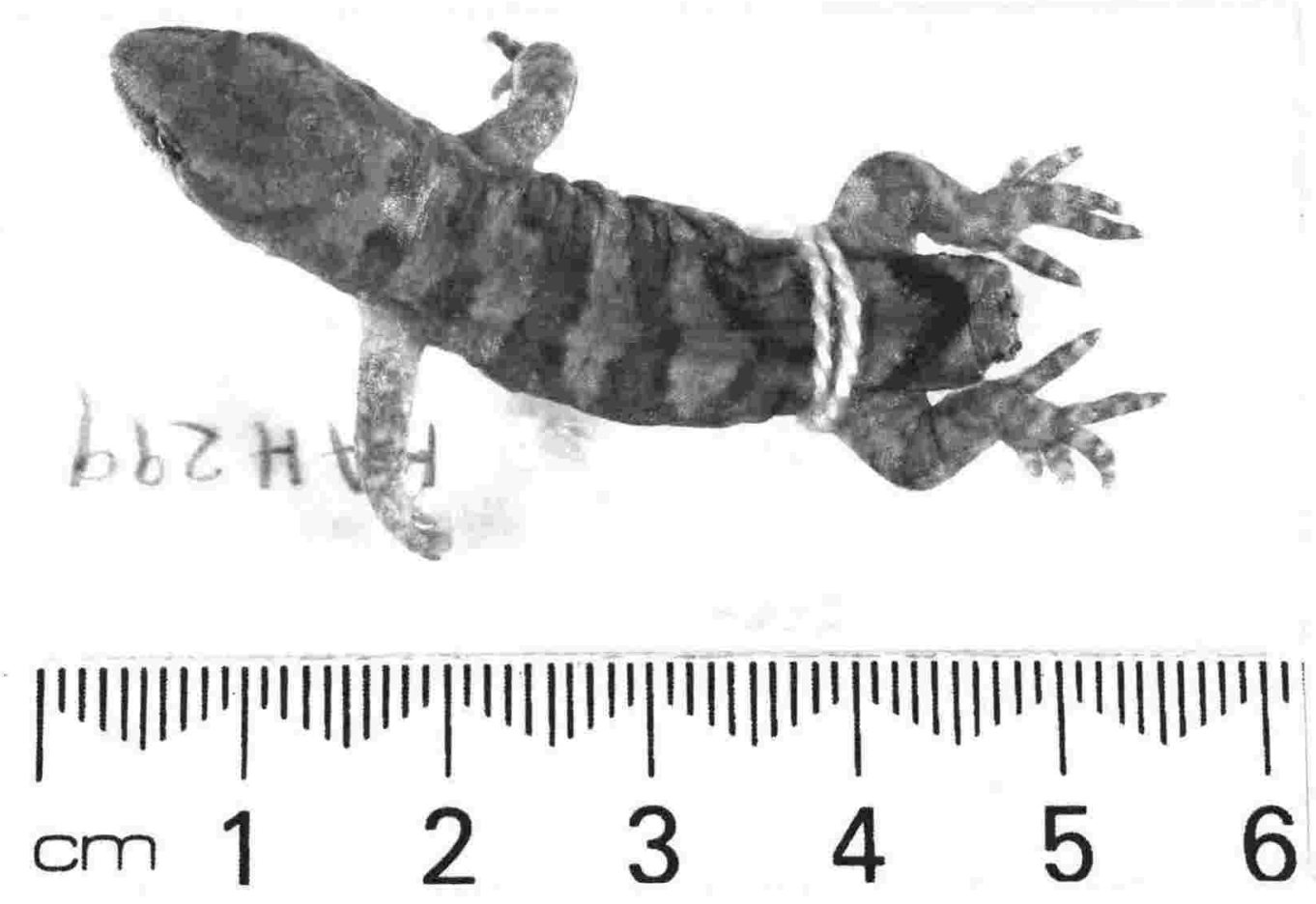

Figure 3.4.4.1 H. "Kaikouras" (RAH299, "Batty", Upper Blue Duck catchment).

DISTRIBUTION, HABITAT, AND GEOGRAPHIC VARIATION: Northern Seaward Kaikoura Ranges north of about the latitude of Kaikoura town, and ranges west of Ward township.

MATERIAL EXAMINED: RAH218-220, 225 (Taylor Farm, inland of Ward); RAH 298303 (Seaward Kaikoura Range).

COMMENTS: No published name is available for this species. It is very difficult to determine whether early authors included this species in their concept of Hoplodactylus maculatus without examining all material listed in their papers, some of which is now unavailable or unidentifiable; however, the ranges of size and scale counts published, the inaccessible habitat of the species, and the absence of material from presently available collections suggests that they did not. More recent authors since McCann (1955) cannot be held to have included this species in their concepts of $H$. pacificus and later $H$. maculatus, as no specimens were available to them.

CONSERVATION STATUS: The species has a restricted distribution, and is superficially extremely similar to its close relative $H$. "Marlborough mini", which makes assessment of its status difficult. 
3.4.5 HOPLODACTYLUS BRUNNEUS (COPE 1868)

(FIGURE 3.4.5.1)

1868 Pentadactylus brunneus Cope. Proc. Acad. Nat. Sci. Philadelphia 20: 320

Type locality: Australia.

Holotype: ANSP 7385 (fide Kluge 1965).

1871 Naultinus pacificus (part) Buller. Trans. New Zealand Inst. 3: 7.

1872 Naultinus pacificus (part) Hutton. Trans. New Zealand Inst. 4:172.

1885 Aelurosaurus brunneus Boulenger. Catalogue of lizards in the British Museum vol. 1: 74.

1885 Hoplodactylus maculatus (part?) Boulenger. Catalogue of the lizards in the British Museum, vol. 1: 171.

1885 Aeluroscalobotes brunneus Boulenger. Ann. Mag. Nat. Hist. (5)16: 387.

1897 Hoplodactylus maculatus (part?) Lucas \& Frost. Trans. Proc. New Zealand Inst. 29: 271.

1955 Hoplodactylus pacificus (part) McCann. Dominion Museum Bulletin 17: 44.

1961 Naultinus pacificus (part) Chrapliwy et al. Herpetologica 17: 7.

1961 Hoplodactylus pacificus (part) Myers. Herpetologica 17: 169.

1965 Hoplodactylus pacificus (part) Wermuth. Das Tierreich 80: 95.

1977 Hoplodactylus maculatus (Part) Robb \& Rowlands. Rec. Auckland Inst. Mus. 14: 139.

1990 Hoplodactylus maculatus (Part) Bauer. Bonner zoologische Monographien, Nr. 30: 117-120; fig. 42.

DIAGNOSIS: A small to medium Hoplodactylus (adults $55-75 \mathrm{~mm}$ snoutvent); anterior nasal and first upper labial usually not in contact, occasionally just touching at their corners; nostril usually just touching the rostral, occasionally just excluded; the rostral may be twice as long as deep, subpentagonal; distance between snout and eye equal to, or slightly greater than, distance between eye and ear opening; usually without spinose scales at the posterior end of the superciliary fringe (occasionally with one); peritoneum lightly pigmented; mouth lining pinkish, tongue with diffuse patch of dark pigment at tip; terminal scansors on digit one only; digits scansorial, broadly expanded; webbing between toes 2,3 , and 4 slight; distal phalanx of fourth toe about one quarter the length of the dilated portion; 9-12 (usually 9-11) broad transverse lamellae under fourth toe of hind foot; in males, 1 or 2 bluntly conical enlarged scale on each side of the base of the tail; preanal organs extending onto thighs. 


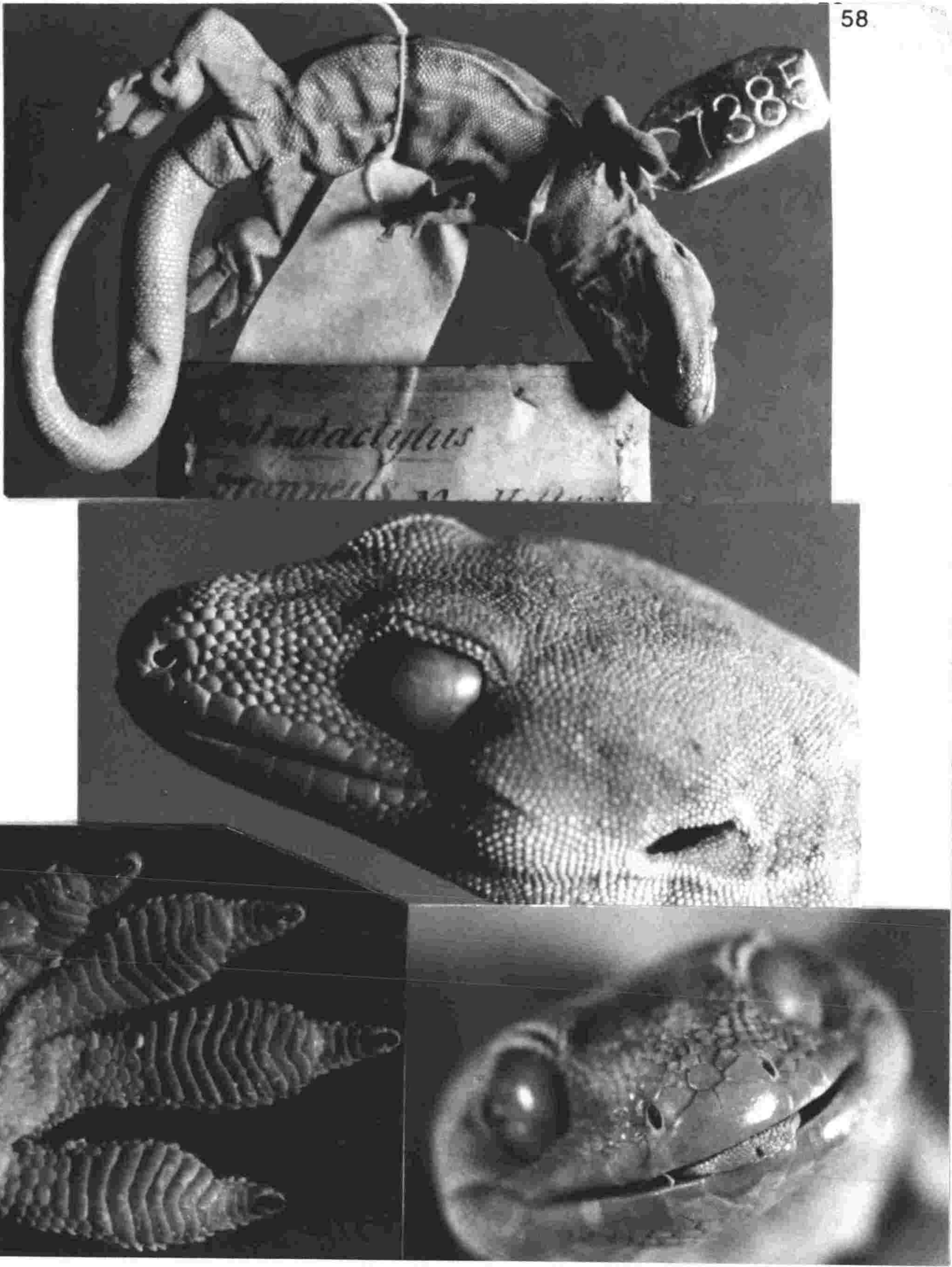

Figure 3.4.5.1 H. brunneus, type specimen (ANSP 7385).Top, whole animal, ventral view; centre, lateral view of head, showing absence of spines from superciliary fringe, and near contact of rostral and nostril; bottom left, ventral surface of hind foot; bottom right, head, showing near contact of rostral and nostril. 
DESCRIPTION: Head short, oviform; snout equal to, or slightly longer than the distance between the eye and the ear-opening; forehead slightly concave; ear-opening moderate, oval, oblique; body and limbs moderate to stout; digits broadly dilated, the length of the dilated portion being about 4 times the length of the distal phalanx; anterior inferior lamellae chevron-shaped, the posterior ones straight; 9-12 (usually 9-11) under fourth toe; head covered with granular scales, of moderate size on the snout, tiny on the remainder of the head; rostral broad, subpentagonal, with a short median cleft from the upper border, anterior nasal and first upper labial usually not in contact, occasionally just touching at their corners; nostril usually just touching the rostral, occasionally just excluded; 4 nasals, the anterior-most of which is considerably enlarged; usually 1 large internasal (occasionally 2 , smaller); 8-14 upper labials; 7-12 lower labials; mental usually bluntly triangular, sometimes quadrangular, shorter than first lower labial; 1 postmental; ventral scales medium-sized, flat, imbricate; tail cylindrical, tapering, longer than snout-vent length, covered with small, sub-hexagonal juxtaposed scales arranged in whorls, with autotomy septa visible; in males, preanal organs in 3-4 angular series forming a roughly triangular patch, with 1-3 (usually 2) rows of 3-11 pores extending to the base of each thigh; in females preanal organs absent; in males base of tail swollen to accommodate hemipenes, and with 1-2 bluntly conical cloacal spurs on each side of swelling; females with cloacal spurs vestigial.

COLOUR: $H$. brunneus is brown, with black spots and blotches, particularly on the tail, and with prominent paler stripes or blotches. The dorsal pattern is very variable, from longitudinal or transverse stripes to paired blotches and chevron patterns. The venter is uniformly pale.

MATERIAL EXAMINED: RAH14, 21, 77, 79, 80, 100, 107, 116-120, 202-208, 406, 406a, 487, CD565.

DISTRIBUTION: H. brunneus is distributed from Kaitorete Spit south of Banks Peninsula, through the north Canterbury plains to southern Marlborough. It shows substantial geographic variation in size (Freeman 1993).

HABITAT: $H$. brunneus is associated with coastal divaricating shrubs on Kaitorete Spit, rock bluffs and screes on Banks Peninsula, and standing dead trees on the Canterbury Plains. It is nocturnal. 
REPRODUCTION: Like the rest of the genus, $H$. brunneus is viviparous. Females collected in December gave birth in February-March to twin offspring, which is typical for the genus.

COMMENTS: The type specimen of Pentadactylus brunneus Cope, 1868 was obtained by the Academy of Natural Sciences, Philadelphia as part of an exchange, from the Musee Jardin des Plantes, Paris, in 1868. $H$. brunneus is abundant on and around Banks Peninsula, including the 19th Century French settlement at Akaroa.

Bauer (1990) included Pentadactylus brunneus Cope, 1868 in the synonymy of $H$. pacificus (synonymy fide Kluge 1965). Kluge established this synonymy during the period when $H$. maculatus was regarded as a synonym of $H$. pacificus. The original description of $P$. brunneus clearly agrees with $H$. maculatus sensu Robb \& Rowlands (1977), explicitly stating that the rostral is excluded from the nostril. However, Kluge (1965) found that the rostral just contacted the nostril at its corner, the condition seen in many South island species of the $H$. maculatus complex, but not in $H$. maculatus itself. Robb \& Rowlands (1977) did not include $P$. brunneus in the synonymy of either $H$. pacificus or $H$. maculatus.

Aelurosaurus brunneus (Boulenger, 1885), and Aeluroscalabotes brunneus (Boulenger, 1885) are generic reassignments of the same taxon (Kluge 1991), and therefore are also clearly synonyms of $H$. maculatus sensu Robb \& Rowlands (1977), not H. pacificus.

The description of the colour as "brown" is consistent only with the Canterbury and eastern populations of the Otago/Southland species of the southern group of the $H$. maculatus complex; the other species in which the rostral and nostril just contact are grey. The relatively long tail, low lamellae counts, and narrow distal phalanges all exclude one or more other species, but are all consistent with the Canterbury species.

$H$. brunneus was included in McCann's (1955) $H$. pacificus. The species was also included in Robb \& Rowlands' (1977) and all subsequent concepts of $H$. maculatus, although Robb \& Rowlands (1977) did not include $P$. brunneus in the synonymy of either $H$. pacificus or $H$. maculatus. Earlier descriptions contained insufficient information to assess whether or not they included this 
species without examining the specimens listed, which, if extant, are all in northern hemisphere museums.

CONSERVATION STATUS: $H$. brunneus is locally abundant in patches of suitable habitat, even where this habitat is readily accessible from adjacent roads; a great deal of suitable habitat must be less accessible, particularly on Banks Peninsula. However, collecting and habitat degradation have greatly reduced the formerly extremely abundant population of very small animals at Birdlings Flat (pers. obs.; Freeman 1993), and recently intensive collection for the pet shop trade has taken place in Christchurch's Port Hills. The species' distribution presumably formerly included the Canterbury Plains, now so severely modified that almost no suitable habitat remains. In the hills of north Canterbury, the distribution seems to be very patchy. Animals which agree morphologically with this species, but have not been sampled for electrophoresis, are common on Motunau Island (Freeman 1993), and the gecko population on Quail Island in Lyttelton Harbour (Mclvor 1972) is very likely to belong to this species.

PUBLISHED STUDIES: Most studies of common geckos ( $H$. maculatus sensu Boulenger 1885a, H. pacificus sensu McCann 1955, H. maculatus sensu Robb \& Rowlands 1977) in Canterbury concern this species. In particular, Mclvor (1972) studied the ecology of the population on Quail Island in Lyttelton Harbour, Tocher $(1992,1993)$ compared the thermal physiology of this species with $H$. "Southern Alps", and Freeman (1993) discussed geographic variation in body size within $H$. brunneus.

3.4.6 HOPLODACTYLUS N. SP. "SOUTHERN ALPS" (FIGURE 3.4.6.1) ?1845 Naultinus pacificus (part) Gray. Catalogue of the Specimens of Lizards in the British Museum: .

?1845 Naultinus maculatus (part) Gray. Catalogue of the Specimens of Lizards in the British Museum: 273.

1871 Naultinus pacificus (part) Buller. Trans. New Zealand Inst. 3: 7. 1871 Naultinus granulatus (?) Buller. Ibid: 9. (fide Robb \& Rowlands 1977). 1872 Naultinus pacificus (part) Hutton. Trans. New Zealand Inst. 4:172. ?1885 Hoplodactylus maculatus (part?) Boulenger. Catalogue of the lizards in the British Museum, vol. 1: 171.

?1897 Hoplodactylus maculatus (part) Lucas \& Frost. Trans. Proc. New Zealand Inst. 29: 271. 


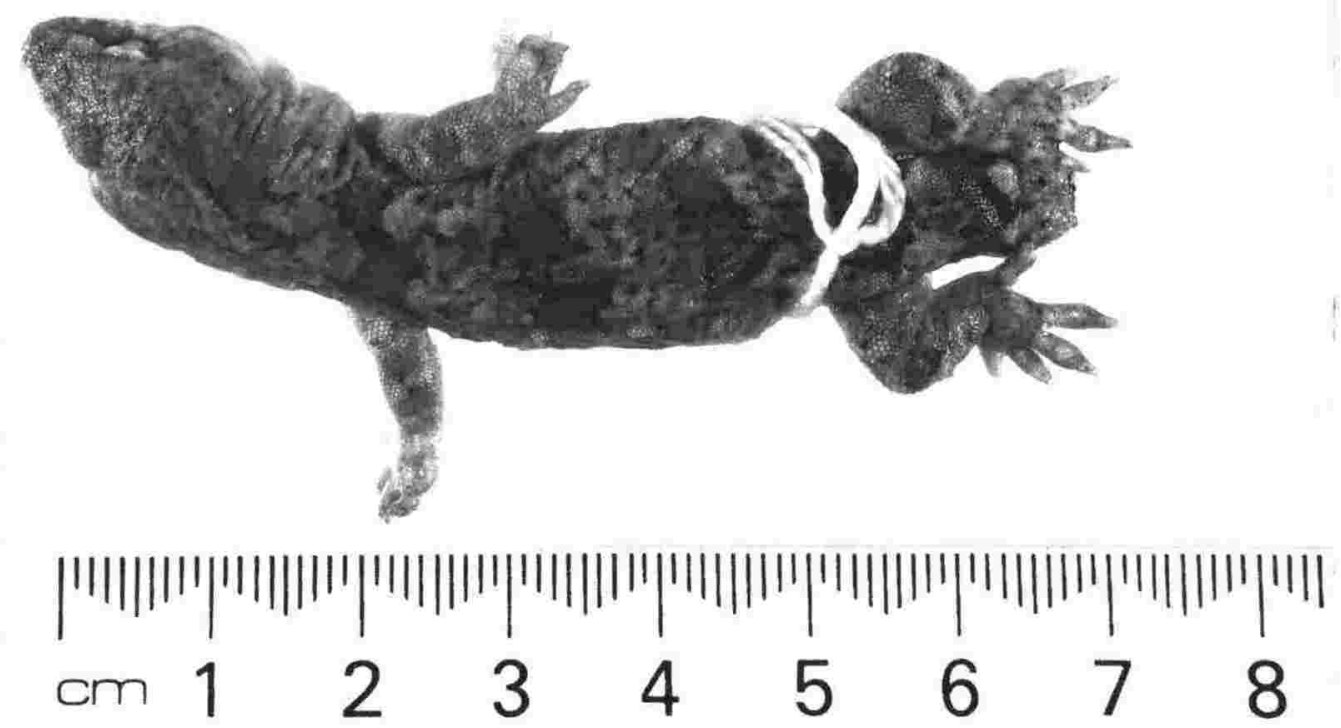

Figure 3.4.6.1 H. "Southern Alps" (RAH306, Hakataramea Pass).

1955 Hoplodactylus pacificus (part) McCann. Dominion Museum Bulletin 17:44.

1961 Naultinus pacificus (part) Chrapliwy et al. Herpetologica 17: 7. 1961 Hoplodactylus pacificus (part) Myers. Herpetologica 17: 169. ?1965 Hoplodactylus digitatus Wermuth. Das Tierreich 80: 94. 1965 Hoplodactylus pacificus (part) Wermuth. Das Tierreich 80: 95. 1977 Hoplodactylus maculatus (part) Robb \& Rowlands. Rec. Auckland Inst. Mus. 14: 139.

1990 Hoplodactylus maculatus (part) Bauer. Bonner zoologische Monographien, Nr. 30: 117-120; fig. 42.

DIAGNOSIS: A small to medium but robust Hoplodactylus ( $<68$, usually $<65$ $\mathrm{mm}$ snout-vent); anterior nasal and first upper labial usually not in contact, occasionally iust touchina at their corners: nostril usuallv iust touchina the 
one quarter the length of the dilated portion, tapering gradually from dilated portion; 9-11 broad transverse lamellae under fourth toe of hind foot; in males, 1 or occasionally 2 bluntly conical enlarged scale on each side of the base of the tail; preanal organs extending only to base of thighs.

DESCRIPTION: Head short, oviform; snout equal to, or slightly longer than the distance between the eye and the ear-opening; forehead slightly concave; ear-opening moderate, oval, oblique; body and limbs moderate to stout; digits broadly dilated, the length of the dilated portion being about 3 times the length of the distal phalanx; anterior inferior lamellae chevron-shaped, the posterior ones straight; 9-11 under fourth toe; a web between digits 2, 3, and 4 but slight between digits 1 and 2, and absent between digits 4 and 5; head covered with granular scales, of moderate size on the snout, tiny on the remainder of the head; rostral broad, subpentagonal, with a short median cleft from the upper border, anterior nasal and first upper labial usually not in contact, occasionally just touching at their corners; nostril usually just touching the rostral, occasionally just excluded; 4-5 (usually 4) nasals, the anterior-most of which is considerably enlarged; 1 large internasal; 8-12 upper labials; 8-10 lower labials; mental usually bluntly triangular, sometimes quadrangular, shorter than first lower labial; 1-3 (usually 1) postmentals; ventral scales medium-sized, flat, imbricate; tail cylindrical, tapering, about equal to or slightly longer than snout-vent length, covered with small, sub-hexagonal juxtaposed scales arranged in whorls, with autotomy septa visible; in males, preanal organs in 3-4 angular series forming a roughly triangular patch, with 1-3 (usually 2) rows of 3-11 pores extending to the base of each thigh; in females preanal organs absent; in males base of tail swollen to accommodate hemipenes, and with 1-2 bluntly conical cloacal spurs on each side of swelling; females with cloacal spurs vestigial.

COLOUR: H. "Southern Alps" is grey, sometimes with dull green and/or pink shadings. It usually has transverse bands (rarely longitudinal stripes) across the dorsum, often with fine longitudinal streaks superimposed. The venter is heavily speckled with dark scales on a paler background.

MATERIAL EXAMINED: RAH7, 9-13, 15, 24, 25, 83, 84, 108, 109, 111-113, $152-$ 158, 200-201, 211-213, 217, 226-228, 257, 258, 263-266, 275, 305-308, $313,314,326-328,330-337,403,404,408,447,452$, CD548-563, 569, 570, 572, 782-790, 2128, FT587-591, 627, 628. 
DISTRIBUTION: $H$. "Southern Alps" is distributed from northern Otago, along the eastern slopes of the axial ranges through Canterbury to southern Marlborough.

It shows geographic variation in allozymes (see Chapter 4), size, and colour pattern. Populations from Arthur's Pass north are medium sized $(60-65 \mathrm{~mm}$ snout-vent), and have simple transverse bands. Many populations in the McKenzie Basin are similar in size, but are very silvery grey, often with streaky markings and a vertebral stripe. Populations around Tekapo are smaller $(55-60 \mathrm{~mm})$, and very dark. Populations in the Waitaki and Hakataramea Valleys include some striped individuals.

HABITAT: $H$. "Southern Alps" is associated with both rock bluffs and screes in subalpine tussock grassland. It is nocturnal.

REPRODUCTION: Like the rest of the genus, $H$. "Southern Alps" is viviparous. Females collected in December gave birth in March to twin offspring, which is typical for the genus.

COMMENTS: This species was included in Robb \& Rowlands' (1977) and all subsequent definitions of $H$. maculatus. It was included in McCann's (1955) $H$. pacificus. Earlier descriptions contained insufficient information to assess whether or not they included this species.

PUBLISHED STUDIES: Tocher $(1992,1993)$ compared the thermal physiology of this species with $H$. brunneus, and found substantial differences between them.

CONSERVATION STATUS: $H$. "Southern Alps" is widely distributed and locally abundant in patches of suitable habitat, even where this habitat is readily accessible from adjacent roads; a great deal of suitable habitat must be less accessible. Much of the species' habitat is within the DoC estate, and much of the rest is too high in altitude to be currently at risk of development.

\subsubsection{HOPLODACTYLUS N. SP. "CROMWELL GORGE"} (FIGURE 3.4.7.1) DIAGNOSIS: A small to medium Hoplodactylus ( $<65 \mathrm{~mm}$ snout-vent); anterior nasal and first upper labial either not in contact, with the nostril contacting the rostral for about $1 / 8$ to one quarter its circumference, or broadly in contact, 


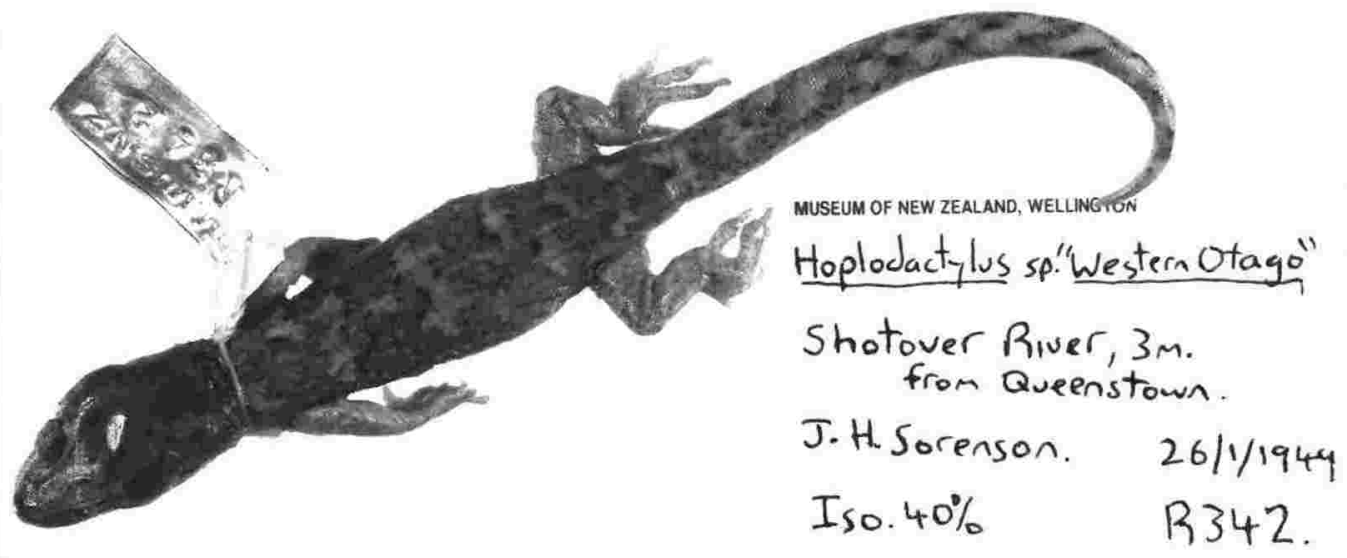

|

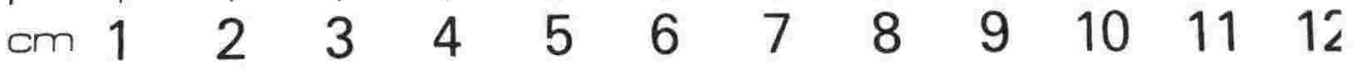

Figure 3.4.7.1 H. "Cromwell Gorge", NMNZR342, from the Shotover Valley.

with the nostril excluded from the rostral; the rostral may be twice as long as deep, subpentagonal; distance between snout and eye equal to, or slightly greater than, distance between eye and ear opening; usually with 1 or 2 spinose scales at the posterior end of the superciliary fringe; peritoneum lightly pigmented; mouth lining pinkish, tongue with diffuse patch of dark pigment at tip; terminal scansors on digit one only; digits scansorial, moderately expanded; webbing between toes 2,3 , and 4 slight; distal phalanx of fourth toe about one quarter the length of the dilated portion, narrowing abruptly from expanded portion; 9-11 broad transverse lamellae under fourth toe of hind foot; in males, 1 or occasionally 2 bluntly conical enlarged scales on each side of the base of the tail; preanal organs extending only to base of thighs.

DESCRIPTION: Head short, oviform; snout equal to, or slightly longer than the distance between the eye and the ear-opening; forehead slightly concave; ear-opening moderate, oval, oblique; body and limbs moderate; digits broadly dilated, the length of the dilated portion being about 3 times the length of the distal phalanx; anterior inferior lamellae chevron-shaped, the posterior ones straight; 9-11 under fourth toe; a web between digits 2, 3, and 4 but slight between digits 1 and 2, and absent between digits 4 and 5 ; head covered with granular scales, of moderate size on the snout, tiny on the 
remainder of the head; rostral broad, subpentagonal, with a short median cleft from the upper border, anterior nasal and first upper labial either not in contact, or broadly in contact; nostril either broadly in contact with the rostral (c. $1 / 8$ to one quarter its circumference), or completely excluded; 4-5 (usually 4) nasals, the anterior-most of which is considerably enlarged; 1 large internasal; 8-12 upper labials; 8-10 lower labials; mental usually bluntly triangular, sometimes quadrangular, shorter than first lower labial; 1-3 (usually 1) postmentals; ventral scales medium-sized, flat, imbricate; tail cylindrical, tapering, about equal to or slightly longer than snout-vent length, covered with small, sub-hexagonal juxtaposed scales arranged in whorls, with autotomy septa visible; in males, preanal organs in 3-4 angular series forming a roughly triangular patch, with 1-3 (usually 2) rows of 3-11 pores extending to the base of each thigh; in females preanal organs absent; in males base of tail swollen to accommodate hemipenes, and with 1-2 bluntly conical cloacal spurs on each side of swelling; females with cloacal spurs vestigial.

COLOUR: $H$. "Cromwell Gorge" is grey, with transverse bands across the dorsum. The venter is very heavily speckled with dark scales on a paler background.

MATERIAL EXAMINED: RAH38-40, 61, 62, 443, 444, 455, 458.

DISTRIBUTION: This proposed species has been collected only from the Cromwell and Kawarau Gorges, in western Otago. Two distinct populations are included in it. The population from Nevis Bluff, on the south bank of the Kawarau River, has the rostral quite broadly in contact with the nostril, and reaches at least $64 \mathrm{~mm}$ in length. Animals from populations on the north bank of the Clutha River in the Cromwell Gorge are smaller (c. $55 \mathrm{~mm}$ snoutvent), have narrower toes, and have the first nasal and first upper labial in broad contact, excluding the rostral from the nostril.

The area south of the Gorge was surveyed for reptiles by Whitaker (1986) and colleagues, who found only $H$. "Otago" and $H$. "Southern mini". My own collecting revealed only $H$. "Otago" in the Dunstan Ranges, and in the Clutha Valley from Alexandra east. I have collected $H$. "Southern Alps" near Lindis Pass, but the distributions of $H$. "Southern Alps", $H$. "Otago" and $H$. "Cromwell Gorge" in the area between Lindis Pass, Wanaka and Cromwell are unknown. Whitaker $(1984 a, 1987)$ recorded "common geckos" from this area, 
most of which he identified as the "maxi" type (= H. "Otago"?). Unfortunately, none of these were collected. NMNZR342, from the Shotover Valley, agrees morphologically with $H$. "Cromwell Gorge" from the Kawarau Gorge.

HABITAT: $H$. "Cromwell Gorge" is associated with both schist tors and screes. It is nocturnal.

REPRODUCtion: Like the rest of the genus, $H$. "Cromwell Gorge" is viviparous. Females collected in December gave birth in March to twin offspring, which is typical for the genus.

COMMENTS: The point near Queenstown in Robb \& Rowlands' (1977) distribution map for $H$. maculatus probably relates to NMNZR342 (from the Shotover Valley), which agrees in morphology with $H$. "Cromwell Gorge".

CONSERVATION STATUS: $H$. "Cromwell Gorge" is locally abundant in patches of suitable habitat, even where this habitat is readily accessible from adjacent roads, but its known distribution is quite restricted, and must have been severely reduced by construction of the Clyde Dam.

\subsubsection{HOPLODACTYLUS N. SP. "DANSEYS PASS"}

(FIGURE 3.4.8.1)

DIAGNOSIS: A medium to large, robust Hoplodactylus (70-80 mm snout-vent); anterior nasal and first upper labial usually not in contact, occasionally just touching at their corners; nostril usually just touching the rostral, occasionally just excluded; the rostral may be twice as long as deep, subpentagonal; distance between snout and eye equal to, or slightly greater than, distance between eye and ear opening; usually without spinose scales at the posterior end of the superciliary fringe, occasionally with 1 or 2; peritoneum lightly pigmented; mouth lining pinkish, tongue with diffuse patch of dark pigment at tip; terminal scansors on digit one only; digits scansorial, broadly expanded; webbing between toes 2,3 , and 4 slight; distal phalanx of fourth toe about one quarter the length of the dilated portion; 11-15 narrow transverse lamellae under fourth toe of hind foot; in males, 1 bluntly conical enlarged scale on each side of the base of the tail; preanal organs extending only to base of thighs.

DESCRIPTION: Head short, oviform; snout equal to, or slightly longer than the distance between the eye and the ear-opening; forehead slightly concave; ear-opening moderate, oval, oblique; body and limbs moderate to stout; 


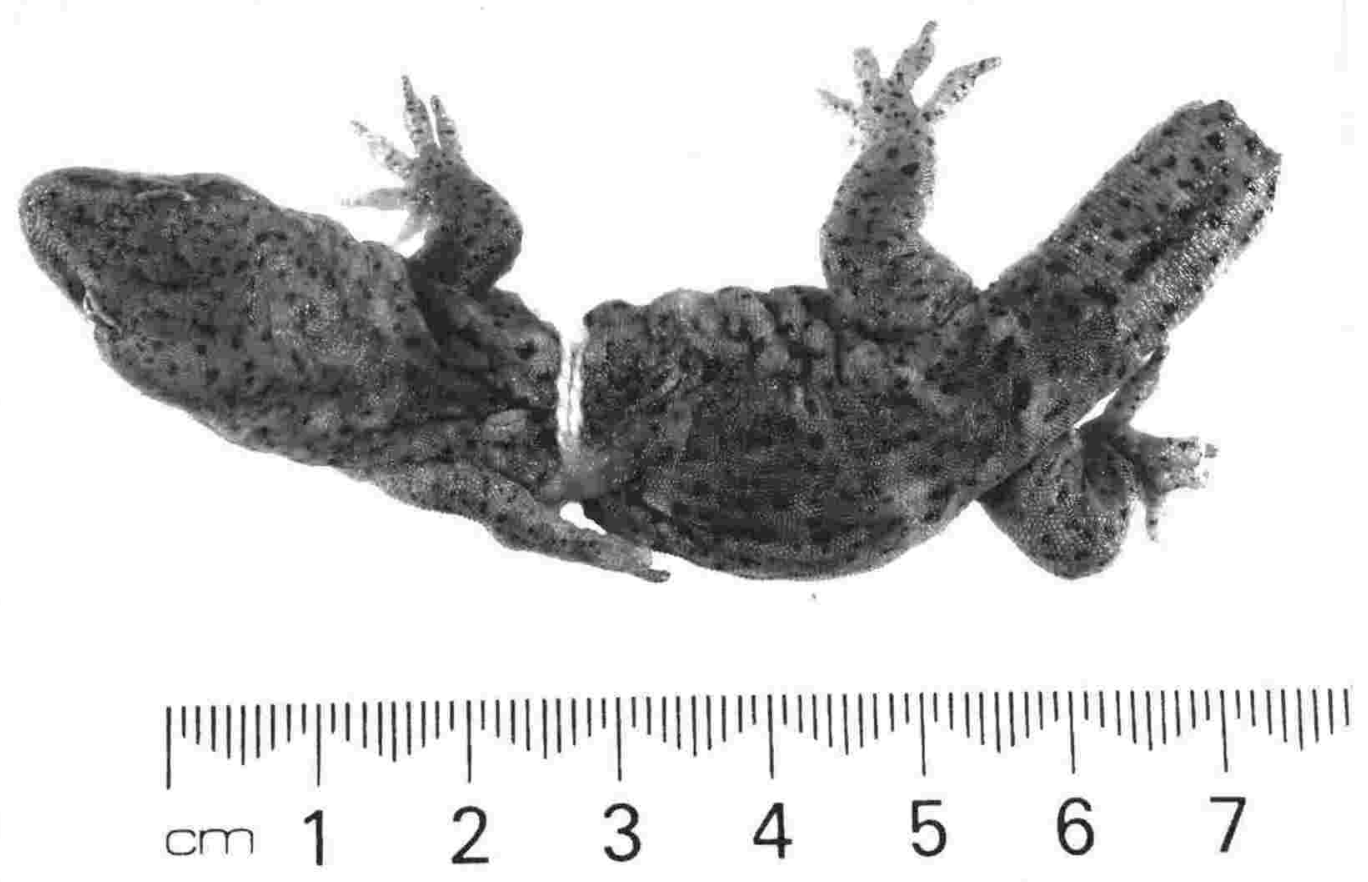

Figure 3.4.8.1 H. "Danseys Pass", RAH316, Kyeburn Diggings.

digits broadly dilated, the length of the dilated portion about 3 times the length of the distal phalanx; anterior inferior lamellae chevron-shaped, the posterior ones straight; 11-15 under fourth toe; a small web between digits 2, 3 , and 4 but slight between digits 1 and 2 , and absent between digits 4 and 5 ; head covered with granular scales, of moderate size on the snout, tiny on the remainder of the head; rostral broad, subpentagonal, with a short median cleft from the upper border, anterior nasal and first upper labial usually not in contact, occasionally just touching at their corners; nostril usually just touching the rostral, occasionally just excluded; 4-5 (usually 4) nasals, the anterior-most of which is considerably enlarged; 1 internasal; $9-13$ upper labials; 10-12 lower labials; mental triangular to quadrangular, shorter than first lower labial; 1-3 postmentals; ventral scales medium-sized, flat, imbricate; tail cylindrical, tapering, longer than snout-vent length, covered with small, sub-hexagonal juxtaposed scales arranged in whorls, with autotomy septa visible; in males, preanal organs in 5 angular series forming a roughly triangular patch, with 1-2 rows of about 6 pores extending to the base of each thigh; in females preanal organs absent; in males base of tail swollen to accommodate hemipenes, and with 1-2 bluntly conical cloacal spurs on each side of swelling; females with cloacal spurs vestigial. 
ColouR: $H$. "Danseys Pass" is grey, sometimes with dull green and/or pink shadings, and with streaky transverse bands across the dorsum. The venter is heavily speckled with dark scales on a paler background.

MATERIAL EXAMINED: RAH283-285, 304, 315-319.

DISTRIBUTION: $H$. "Danseys Pass" has a restricted distribution in north-eastern Otago. I found it at Kyeburn Diggings and at the top of Danseys Pass. It shows no detectable geographic variation in either allozymes or morphology, but its relationship to populations further east in the Kakanui and Horse Ranges requires clarification.

HABITAT: $H$. "Danseys Pass" is closely associated with rock bluffs in subalpine tussock grassland. It is nocturnal.

REPRODUCTION: Like the rest of the genus, $H$. "Danseys Pass" is viviparous. Females collected in December gave birth in March to twin offspring, which is typical for the genus.

COMMENTS: There is no evidence that any previous taxonomist had access to any specimens of this species. However, it is sufficiently similar morphologically to other species of the $H$. maculatus complex that it would certainly have been included in Robb \& Rowlands' (1977) concept of this species, and in McCann's (1955) much broader concept of $H$. pacificus.

CONSERVATION STATUS: Despite its limited geographic range, $H$. "Danseys Pass" appears to be secure at present. It is locally abundant in patches of suitable habitat, even where this habitat is readily accessible from adjacent roads; a great deal of suitable habitat must be less accessible. Much of the species' habitat is too high in altitude to be at risk of development.

3.4.9 HOPLODACTYLUS N. SP. "OTAGO"

(FIGURES 3.4.9.1, 3.4.9.2)

1871 Naultinus pacificus (part) Buller. Trans. New Zealand Inst. 3: 7.

1871 Naultinus granulatus (?) Buller. Ibid: 9. (fide Robb \& Rowlands 1977). ?1885 Hoplodactylus maculatus (part) Boulenger. Catalogue of the lizards in the British Museum, vol. 1: 171.

?1897 Hoplodactylus maculatus (part) Lucas \& Frost. Trans. Proc. New Zealand Inst. 29: 271. 
70

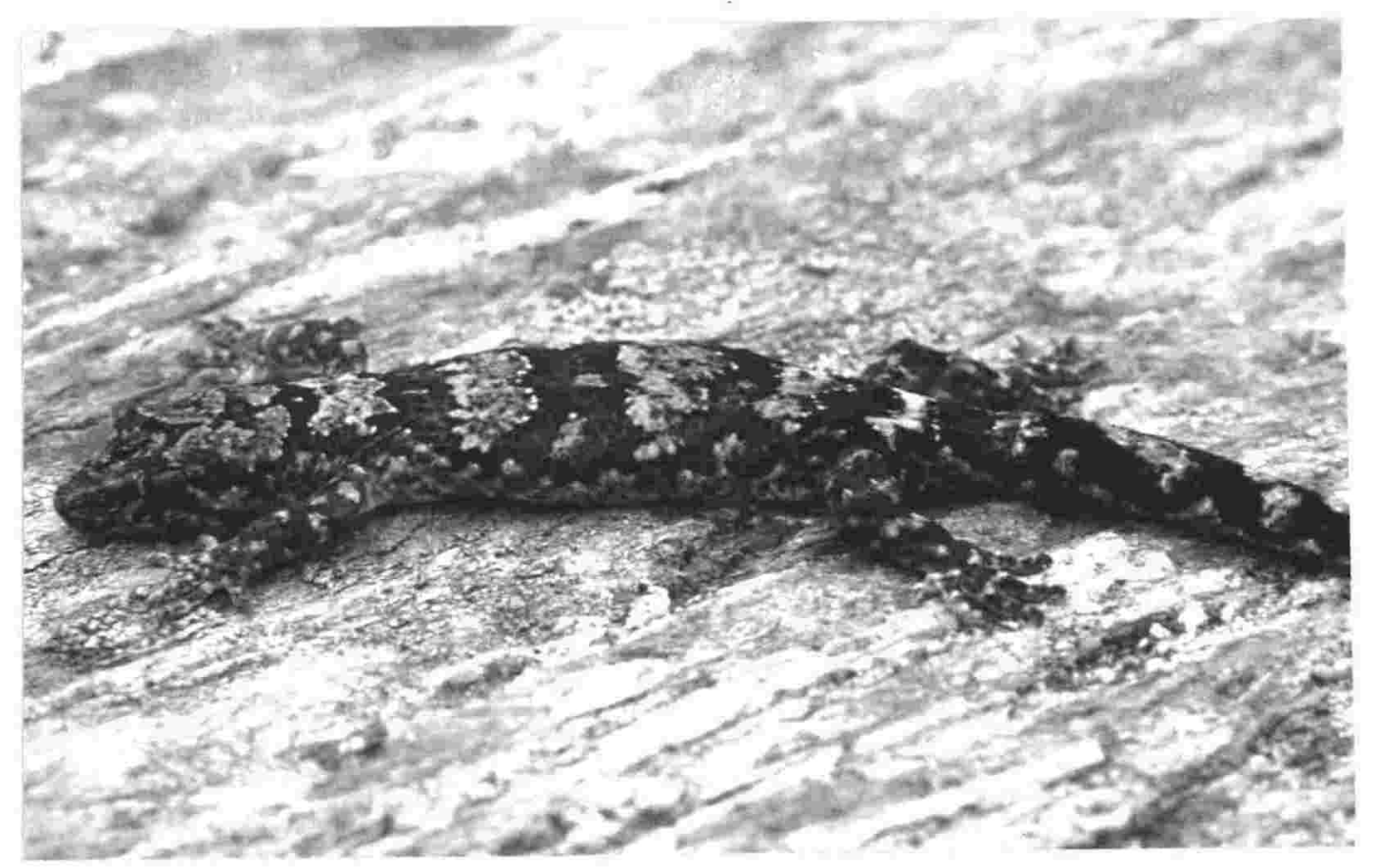

Figure 3.4.9.1 H. "Otago", Taieri Ridge (photographed by Graeme Loh).

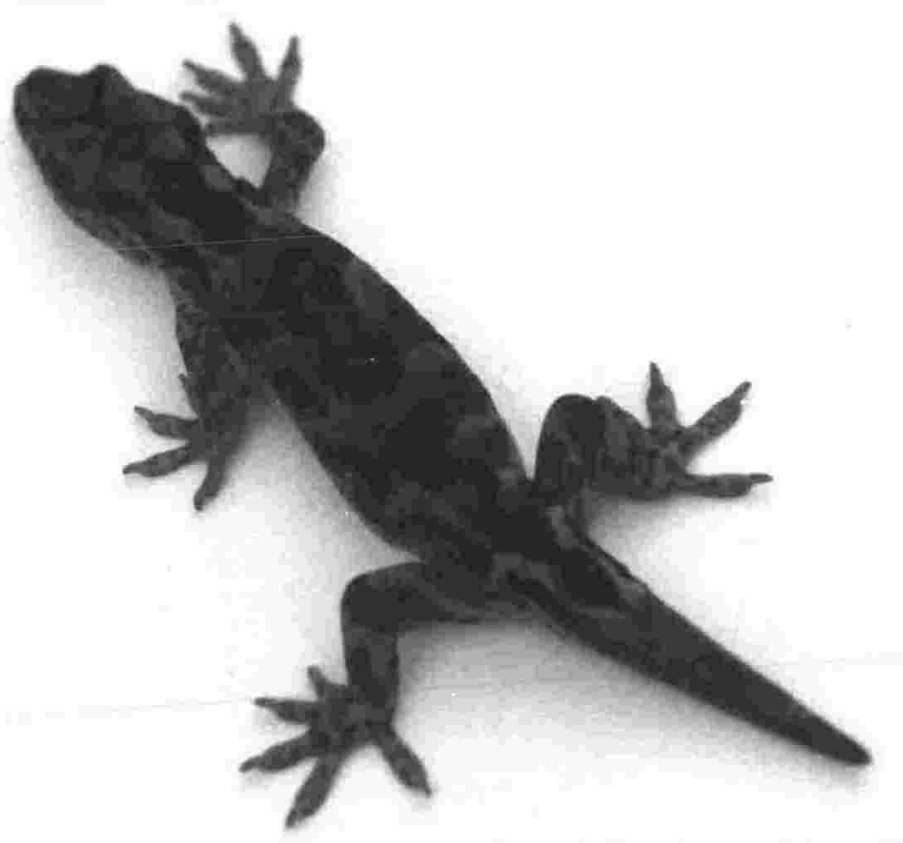

Figure 3.4.9.2 H. "Otago", RAH481, Te Anau (note the very large feet). 
1955 Hoplodactylus pacificus (part) McCann. Dominion Museum Bulletin 17: 44.

1961 Naultinus pacificus (part) Chrapliwy et al. Herpetologica 17: 7. 1961 Hoplodactylus pacificus (part) Myers. Herpetologica 17: 169. 1965 Hoplodactylus pacificus (part) Wermuth. Das Tierreich 80: 95. 1977 Hoplodactylus maculatus (Part) Robb \& Rowlands. Rec. Auckland Inst. Mus. 14: 139. 1990 Hoplodactylus maculatus (Part) Bauer. Bonner zoologische Monographien, Nr. 30: 117-120; fig. 42.

DIAGNOSIS: A small to large, slender to robust Hoplodactylus (adults $55-80$ $\mathrm{mm}$ snout-vent); anterior nasal and first upper labial touching or not in contact; nostril either excluded from the rostral or just touching; the rostral may be twice as long as deep, subpentagonal; distance between snout and eye equal to, or slightly greater than, distance between eye and ear opening; usually without spinose scales at the posterior end of the superciliary fringe (occasionally with 1 or 2); peritoneum lightly pigmented; mouth lining pinkish, tongue with diffuse patch of dark pigment at tip; terminal scansors on digit one only; digits scansorial, broadly expanded; webbing between toes 2 , 3 , and 4 slight; distal phalanx of fourth toe about one quarter the length of the dilated portion; 9-13 narrow-broad transverse lamellae under fourth toe of hind foot; in males, 1 or 2 bluntly conical enlarged scales on each side of the base of the tail; preanal organs extending onto thighs.

DESCRIPTION: Head oviform; snout equal to, or slightly longer than the distance between the eye and the ear-opening; forehead slightly concave; ear-opening moderately large, oval, oblique; body and limbs slender to stout; digits moderately to broadly dilated, the length of the dilated portion being about 4 times the length of the distal phalanx; anterior inferior lamellae chevron-shaped, the posterior ones straight; 9-13 under fourth toe; head covered with granular scales, of moderate size on the snout, tiny on the remainder of the head; rostral broad, subpentagonal, with a short median cleft from the upper border, anterior nasal and first upper labial may touch or not; nostril excluded from the rostral, or just touching; 4 nasals, the anteriormost of which is considerably enlarged; $1-3$ internasals, or occasionally anterior nasals meet; 10-14 upper labials; 9-13 lower labials; mental triangular to trapezium-shaped, shorter than first lower labial; 1-3 postmentals, or first labials in contact behind mental; ventral scales mediumsized, flat, imbricate; tail cylindrical, tapering, longer than snout-vent length, 
covered with small, sub-hexagonal juxtaposed scales arranged in whorls, with autotomy septa visible; in males, preanal organs in 3-5 angular series, with 2-3 rows of 6-10 pores extending to the base of each thigh; in females preanal organs absent; in males base of tail swollen to accommodate hemipenes, and with 1 large, bluntly conical cloacal spur on each side of swelling; females with cloacal spurs vestigial.

ColouR: Central and eastern Otago and Southland populations of $H$. "Otago" are brown, with prominent paler stripes or blotches. The dorsal pattern is very variable, from longitudinal or transverse stripes to paired blotches and chevron patterns. The venter is uniformly pale to lightly speckled. The eye is usually deep golden-yellow in eastern populations, more brown in Central and Southland populations. Western populations are coloured grey, sometimes with dull green and/or pink shadings, and with prominent transverse bands across the dorsum. The venter is uniformly pale. The eye is greenish or brown flecked with green.

MATERIAL EXAMINED: RAH16-20, 26-32, 35-37, 50-60, 81, 82, 94-97, 110, $114,115,121-132,143-145,147-151,159-165,173-175,259,260,274$, $338,339,418-442,448-451,453,454,456,457,459-463,467-474,481$, CD942, 943, 945, 946, 1910, 1911, FT585, 586, 588, 2065, 2066.

DISTRIBUTION: $H$. "Otago" is distributed south of the schist/greywacke boundary in Otago, in scattered localities in eastern Southland, and on the Hazelburgh Group of islands in Foveaux Strait. A single very immature specimen (NMNZR1826 = EDG314) of the common gecko complex, probably from this species, is known from Pearl Island in Port Pegasus. $H$. "Otago" shows substantial geographic variation in size, with Central Otago animals being small and slender, and western populations particularly large and robust.

HABITAT: $H$. "Otago" is nocturnal. It is closely associated with strongly foliated schist in Central Otago, where it is most abundant. However, populations in other areas are found in association with basalt (Otago Peninsula), plutonic igneous rocks (Foveaux Strait), and greywacke (Southland). Although it is currently known almost entirely from open habitats, much of its current range would have been forested or at least wooded 1000 years ago. Western populations are usually closely associated with rock bluffs in subalpine tussock grassland. However, the Eglinton Valley specimen was crossing the 
road at night, in beech forest, and the Te Anau specimen was collected under driftwood near the shoreline of the lake.

REPRODUCTION: Like the rest of the genus, $H$. "Otago" is viviparous. Cree (1994) has demonstrated a biennial reproductive cycle in females from cool, high-altitude habitats in the Middlemarch area, but females from the Alexandra area have an annual cycle similar to most other species in the genus (MacAvoy 1976). Females from large-bodied, high-altitude western populations collected in February gave birth in March to twin offspring.

COMMENTS: This species was included in Robb \& Rowlands' (1977) and all subsequent definitions of $H$. maculatus. It was included in McCann's (1955) $H$. pacificus. Earlier descriptions contained insufficient information to assess whether or not they included this species.

Robb (1982, p. 319, \& pers. comm.) excluded the Otago Museum specimen from 5000 feet in the Old Man Range (morphologically typical of largebodied western populations) from her concept of $H$. maculatus, and regarded it as an undescribed species, although it carries a label indicating that she identified it as $H$. maculatus at some stage.

The thermal biology of $H$. "Otago" was studied by Morris (1965), Evetts (1978), Grimmond \& Evetts (1981), and Evetts \& Grimmond (1982). MacAvoy (1976) and Pollock \& MacAvoy $(1973,1978)$ studied reproductive cycles in populations near Alexandra. Shelton (1978) examined the effects of 3 light regimes on activity of animals from near Middlemarch.

There are no other identifiable references to this species, apart from unpublished internal Wildlife Service reports of lizard distribution surveys which record $H$. maculatus sensu Robb \& Rowlands (1977), which cannot be reliably identified to species.

CONSERVATION STATUS: $H$. "Otago" is abundant in most parts of Central Otago which have exposed schist tors, but is much less common where the tussock vegetation has given way to intensive agriculture. In some places removal of loose rocks from tors by gardeners has also reduced habitat quality very noticeably during the period of this study. H. "Otago" is more patchily distributed in the other parts of its range, but is locally abundant in patches of suitable habitat, even where this habitat is readily accessible from 
adjacent roads; a great deal of suitable habitat must be less accessible. Much of the species' habitat is too high in altitude to be at risk of development. The Remarkables and Eyre Mountains, both with healthy populations, are protected areas.

\subsubsection{HOPLODACTYLUS N. SP. "SOUTHERN MINI"}

(FIGURE 3.4.10.1)

DIAGNOSIS: A small Hoplodactylus (53-59 mm snout-vent; $\mathrm{n}=8$ adults); nostril excluded from the rostral by the anterior nasal and first upper labial; rostral at least 2.5 times as long as deep, subpentagonal; distance between snout and eye slightly greater than distance between eye and ear opening; 3-5 spinose scales at the posterior end of the superciliary fringe; peritoneum lightly pigmented; mouth lining pinkish, tongue with diffuse patch of dark pigment at tip; distal phalanges very short, on fourth toe 0.5-0.6 times width of dilated portion; 10-13 transverse lamellae under fourth toe of hind foot; in males, 1 bluntly conical enlarged scale at each side of the base of the tail; small triangular patch of preanal organs, with only one row extending to the base of the thighs, surrounded by non-porate but enlarged scales.

DESCRIPTION: Head oviform; snout longer than distance between eye and ear-opening, twice diameter of ocular disc in fresh specimens; forehead flat or slightly concave; ear opening medium, oval, oblique; body and limbs moderate to stout; digits moderately dilated, length of dilated portion of fourth toe being about 2.6 times its width; distal phalanx very short, 0.5-0.6 times width of dilated portion; distal inferior lamellae chevron-shaped, remainder curved; 10-13 lamellae under fourth toe; slight webbing between toes 2, 3 , and 4; dorsal surface of head and body covered with small granular scales; rostral broad and shallow, 2.5-3 times as broad as deep, with a short median cleft from upper border, excluded from nostril by enlarged anterior nasal; nostril surrounded by first upper labial and 4 nasals; anterior nasal considerably enlarged; one small, roughly circular internasal; 8 or 9 upper labials and 8-10 lower labials; mental subtriangular, or with very narrow posterior face, shorter than first lower labial; postmental large, roughly circular; ventral scales medium-sized, flat, somewhat overlapping; tail cylindrical, tapering, about equal in length to snout-vent length, covered with small, sub-hexagonal juxtaposed scales arranged in whorls, with autotomy septa visible; in males, preanal organs in 2 to 4 angular series forming a roughly triangular patch, with a single row of 3 to 6 pores extending to the base of each thigh; scales immediately adjacent to the preanal organs about twice as large as other ventral scales; in females an indefinite patch of 


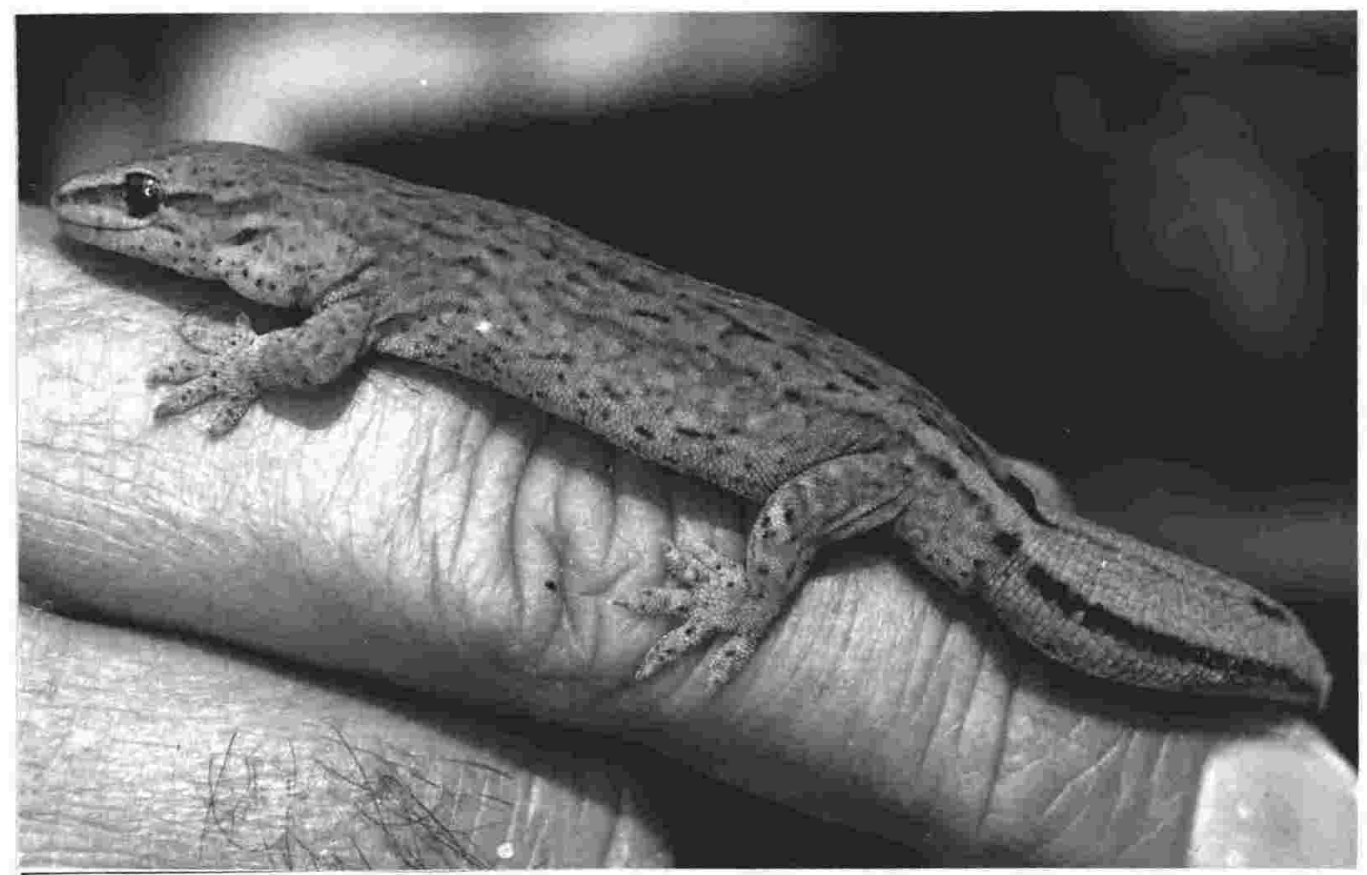

Figure 3.4.10.1 H. "Southern mini", live captive specimen, Gorge Burn, Eyre Mountains.

enlarged preanal scales, preanal organs absent; in males base of tail swollen to accommodate hemipenes, and with one large, bluntly conical cloacal spur on each side of swelling; females with cloacal spurs vestigial.

COLOUR: $H$. "Southern mini" is coloured light, drab, somewhat translucent olive-brown or greyish-brown, with scattered darker streaks. There are hints of vestigial longitudinal dorsal stripes in the pectoral and pelvic regions, and obscure darker stripes along the sides of the head, but the predominant effect is of almost uniform colouring. The belly is off-white, with scattered dark scales. The tail may be yellowish below.

MATERIAL EXAMINED: RAH85, 86, 166, 167, 168, 169, 170, 171, 172, CD1211, Gorge Burn, Eyre Mountains; CD944, Nokomai Station, Mataura River; CD947, 948, Remarkables.

DISTRIBUTION: $H$. "Southern mini" has a restricted distribution; it is known so far only from The Remarkables Range, the Hector Mountains, the Slate Range, and the Eyre Mountains, all in south-western Otago and northwestern Southland (Livingstone, Eyre, Remarkables and Nokomai Ecological Districts). Areas to the north and east of this range have been well 
surveyed for lizards (RAH, pers. obs; Whitaker 1984a, 1987), and $H$.

"Southern mini" has not been found. The country to the south and west is less well known herpetologically, but lizard records from Fiordland are very sparse, and none seems to refer to this species. The Takitimu Range has recently been searched for lizards, and no $H$. "Southern mini" were found ( $T$. Jewell, pers. comm.). It shows no detectable geographic variation in either allozymes or morphology.

HABITAT: $H$. "Southern mini" is closely associated with rock screes and boulder piles in subalpine tussock grassland, where it can be extremely abundant. It is less common on rock bluffs. It is nocturnal.

REPRODUCTION: Like the rest of the genus, $H$. "Southern mini" is viviparous. Females collected in February gave birth in March to twin offspring, which is typical for the genus.

COMMENTS: There is no evidence that any previous taxonomist had access to any specimens of this species. Robb \& Rowlands' (1977, fig. 11) localities of specimens examined include a locality for $\mathrm{H}$. maculatus which appears to be close to or within the known range of $H$. "Southern mini". However, I believe this locality relates to NMNZR342, a specimen of $H$. "Cromwell Gorge" from the lower Shotover Valley. Unfortunately Robb \& Rowlands (1977) did not list specimen numbers for the material they examined, simply total numbers of specimens examined at each institution.

There are no other identifiable references to this species, apart from Whitaker's (1986) unpublished internal Wildlife Service report of a lizard distribution survey in the Queenstown area.

CONSERVATION STATUS: Despite its limited geographic range, $H$. "Southern mini" appears to be secure at present. It is locally abundant in patches of suitable habitat, even where this habitat is readily accessible from adjacent roads; a great deal of suitable habitat must be less accessible. The Remarkables and Eyre Mountains, which include the largest known populations, are protected areas. 


\subsection{HOPLODACTYLUS FITZINGER, 1843:}

\section{THE HOPLODACTYLUS GRANULATUS COMPLEX}

The narrow-toed group of Hoplodactylus spp. includes $H$. stephensi, $H$. rakiurae, $H$. kahutarae, $H$. granulatus, and $H$. nebulosus, which is resurrected from the synonymy of $H$. granulatus. $H$. nebulosus is redescribed, and $H$. granulatus redefined to exclude $H$. nebulosus. The status of the other species of the group is unchanged.

3.5.1 HOPLODACTYLUS GRANULATUS (GRAY, 1843) (FIGURES 3.5.1.1, 3.5.1.2) 1843 Naultinus pacificus (part) Gray. Travels in New Zealand, vol 2: 203. 1845 Naultinus granulatus Gray. Catalogue of the specimens of lizards in the catalogue of the British Museum: 273.

Type locality: New Zealand.

Lectotype: BMNH 1946.8.22.71, designated by Bauer 1990.

Paralectotypes: BMNH 1946.8.22.70, BMNH 1946.8.22.72, BMNH 1946.9.8.13.

1863 Hoplodactylus (Naultinus) granulatus Hochstetter. Neu-Seeland: 429. 1870 Naultinus greyii Knox. Trans. New Zealand Inst. 2: 20. (lapsus pro Naultinus grayii Bell, 1843; nec Naultinus grayii Bell, 1843 - fide Bauer 1990).

1871 Naultinus granulatus Buller. Trans. New Zealand Inst. 3: 9.

1872 Naultinus pacificus (part) Hutton. Trans. New Zealand Inst. 4: 172. 1875 Naultinus granulatus Günther. The zoology of the voyage of HMS Erebus and Terror, vol. 2: 17.

1881 Naultinus sylvestris Buller. Trans New Zealand Inst. 13: 149.

Type locality: Wooded country of the Wanganui District, North Island (New Zealand).

Holotype: not located by Bauer 1990.

1885 Naultinus versicolor Colenso Trans New Zealand Inst. 17: 149.

Type locality : Forests near Norsewood, County of Waipawa and

Glenross, County of Hawke's Bay (New Zealand).

Syntypes: CMC; NMNZ (specimens not located by Bauer 1990). 1885 Naultinus elegans (part) Boulenger. Catalogue of lizards in the British Museum vol. 1: 169.

1885 Naultinus silvestris Boulenger ibid., vol. 1: 169 (ex errore in synonymy of Naultinus elegans Gray, 1842 pro Naultinus sylvestris Buller, 1881 - fide Bauer 1990) 1885 Hoplodactylus granulatus Boulenger ibid., vol. 1: 171; pl. XV (fig. 1). 


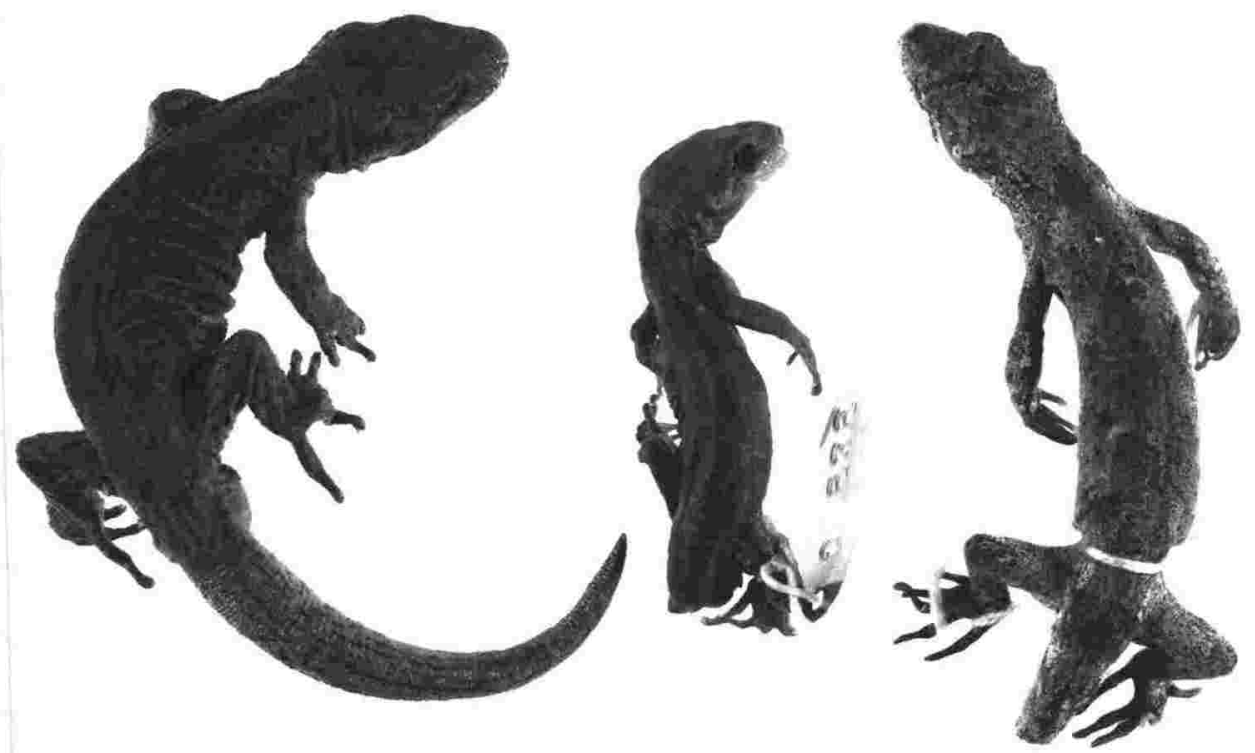

|
$\mathrm{cm} 1$
23
45
7
8910
$\begin{array}{lllll}11 & 12 & 13 & 14 & 15\end{array}$

Figure 3.5.1.1 Adult $H$. granulatus (RAH496, Reefton, right), compared with $H$. nebulosus (NMNZ DM 1569, left), and a gecko from the Open Bay Islands (NMNZ G535, centre).

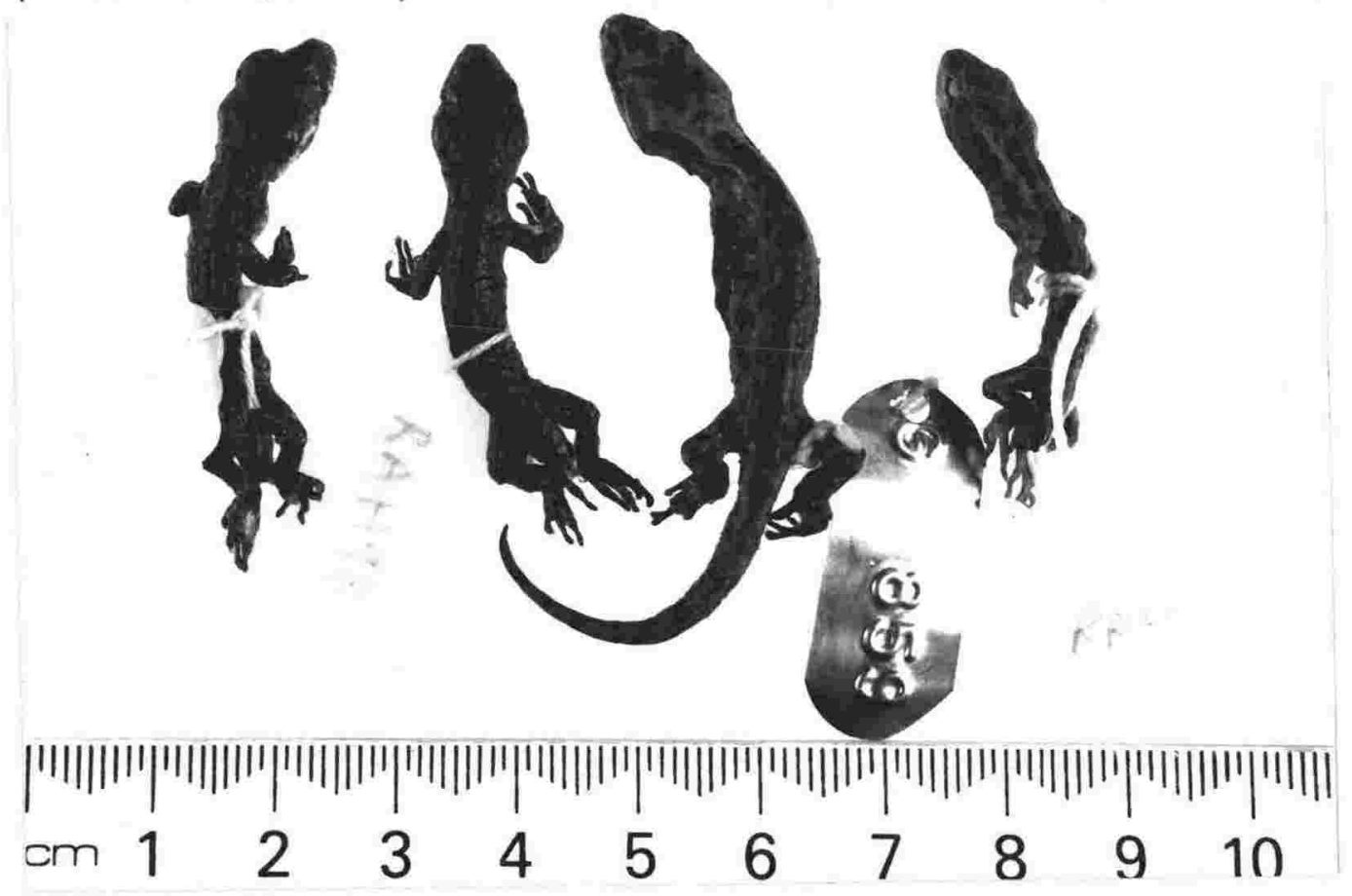

Figure 3.5.1.2 Juvenile (new-born) $H$. granulatus (2 animals at left, RAH98 and RAH99, Northcross) compared with a juvenile $H$. granulatus from Esperence Valley, Fiordland (NMNZ G859), and a juvenile H. rakiurae (RAH364, right). 
1904 Dactylocnemis granulatus Hutton \& Drummond. The animals of New Zealand: 439.

1904 Hoplodactylus granulatus Hutton. Index Faunae Novae Zelandiae: 39. 1961 Naultinus granulatus Chrapliwy et al. Herpetologica 17: 7.

1961 Naultinus grayi Chrapliwy et al. ibid.: 7. (lapsus pro Naultinus greyii (Knox, 1870); nec Naultinus grayii Bell, 1843).

1961 Naultinus brevidactylus Chrapliwy et al. ibid: 7. (nec Naultinus brevidactylus Gray, 1845).

1961 Naultinus maculatus Chrapliwy et al. ibid: 7. (ex errore).

1961 Hoplodactylus granulatus Myers Herpetologica 17: 169.

1981 Hoplodactylus granulatus (part) Thomas. New Zealand journal of zoology: 33-47.

1990 Hoplodactylus granulatus (part) Bauer. Bonner zoologische Monographien, Nr. 30: 117-120.

DIAGNOSIS: A large Hoplodactylus (80-95 mm snout-vent); rostral contacts the nostril for about one quarter of its circumference; rostral twice as wide as deep, subquadrangular; distance between snout and eye distinctly greater than the distance between eye and ear opening, snout concave in profile; tongue and mouth lining yellow, throat blue-black; 2-4 pointed cloacal spurs forming a fan-like group on each side of the base of the tail; tail longer than head + body, tapering, sometimes lacking visible autotomy planes.

DESCRIPTION: Head ovate, elongate, depressed, snout distinctly longer than the distance between the eye and the ear opening; forehead concave; ear opening large, oval, oblique; body and limbs moderate to robust; digits feebly dilated; inferior lamellae straight; 11-14 lamellae under fourth toe; toes unwebbed; head covered with granular scales, of moderate size on the snout, and small on the remainder of the head; rostral about twice as broad as deep, subtruncate, with median cleft, in contact with the nostril, short median cleft from upper border; nostril pierced between rostral, first upper labial and 4-6 nasals; the anterior-most nasal enlarged; 1 large or 2 smaller internasals; 9-13 upper labials, and 9-12 lower labials; mental rhomboid or triangular, shorter than, or occasionally equal to, first lower labials; dorsal scales small, granular to conical; abdominal scales juxtaposed or subimbricate; in the males 5-7 series of preanal pores forming a roughly triangular patch, the number of pores very variable; $3-4$ series of femoral pores; in females a patch of enlarged preanal scales, but no pores; tail cylindrical, tapering, covered with small, quadrangular, juxtaposed scales 
arranged in whorls; in the males, the base of the tail swollen to accommodate the hemipenes, and with 2-4 pointed cloacal spurs in a fanlike group on each side of the swelling; females with vestigial cloacal spurs.

COLOUR: Chevron-shaped markings on a brown, grey, dull green, or brick red background on dorsum of body; head with dark V-shaped marking on top of head behind eyes, dark-edged pale stripe from eye to ear, denticulate markings on lips; venter cream heavily blotched with dark grey. Some specimens with white, yellow, or brick-red blotches

MATERIAL EXAMINED: RAH65-67, 71-73, 87, 88, 90, 209, 261, 262, 340-342, $360,363,395,396,496,497$, CD989-991.

DISTRIBUTION AND GEOGRAPHIC VARIATION: Throughout the North Island except for northern Northland and the South Island except for the rain shadow areas east of the main dividing ranges. $H$. granulatus is found only on the largest or very sheltered offshore islands - Great Barrier, Little Barrier, Waiheke, Kapiti, and Maud. Specimens from Westland and Southland are more slender and more brightly coloured than those from Nelson, Marlborough, and the North Island. Specimens from the northern North Island lack visible autotomy septa in the tail. There is substantial allozyme variation among populations (see Chapter 4), suggesting possible cryptic species, but samples are too widely scattered to allow taxonomic decisions to be made on this basis. A female collected near Reefton gave birth in November (R Morris, pers. comm.), about 3 months earlier than the birth season in North Island and Nelson populations. A small juvenile specimen from subalpine scrub in Fiordland has markings somewhat suggestive of $H$. rakiurae (see Chapter 4).

HABITAT: Forest and shrubland. By day found under loose bark, or in hollows in trees, or among epiphytes. Often basks directly in the late afternoon. At night forages in trees or shrubs.

REPRODUCTION: Annual reproductive cycle, with ovulation in spring, and viviparous birth of (usually) twins in late summer in North Island populations. Other birth seasons have been recorded for other populations (e.g., the Reefton specimen which gave birth in November - R Morris, pers. comm.).

COMMENTS: Thomas (1981) presented a detailed discussion of the synonymy of $H$. granulatus and $H$. nebulosus. The absence of visible 
autotomy septa in the tail unambiguously identifies the type of $H$. granulatus as northern in origin. The name $H$. granulatus would therefore apply to the most northern species if future evidence justifies recognition of further species within the $H$. granulatus complex.

CONSERVATION STATUS: Abundant in forest and shrubland throughout. It is usually inaccessible in the canopy, so its abundance is probably often underestimated; in the past it was often seen during logging operations.

3.5.2 HOPLODACTYLUS NEBULOSUS (MCCANN, 1955)

(FIGURE 3.5.1.1) 1955 Heteropholis nebulosus McCann. Dominion Museum Bulletin 17:69, 71; plate VII, fig. 8-11.

1980 Hoplodactylus nebulosus Robb. New Zealand amphibians and reptiles: $60,109,111,121$.

1981 Hoplodactylus granulatus (part) Thomas. New Zealand journal of zoology: 33-47.

1982 Hoplodactylus granulatus (part)Thomas. New Zealand herpetology: 336.

1986 Hoplodactylus granulatus (part) Gill. Collins Handguide to the Frogs and Reptiles of New Zealand: 36.

1990 Hoplodactylus granulatus (part) Bauer. Bonner zoologische Monographien, Nr. 30: 117-120.

DIAGNOSIS: A medium to large-sized, stout Hoplodactylus; nostril contacts the rostral for about one quarter of its circumference; snout short, blunt, not concave in profile; mouth lining and tongue yellow, throat blackish; colour pattern of predominantly transverse chevron markings, belly speckled with dark brown but not heavily blotched; tail shorter than head + body, tapering, with visible autotomy planes.

DESCRIPTION: Head ovate, snout slightly longer than the distance between the eye and the ear opening; forehead concave; ear opening large, oval, oblique; body and limbs moderate to robust; digits feebly dilated; inferior lamellae straight; 11-14 lamellae under fourth toe; toes unwebbed; head covered with granular scales, of moderate size on the snout, and small on the remainder of the head; rostral about twice as broad as deep, subtruncate, with median cleft, in contact with the nostril, short median cleft from upper border; nostril pierced between rostral, first upper labial and 4-5 nasals, the anterior-most of which is enlarged; 1-3 internasals; 11-13 upper labials, and 
10-13 lower labials; mental rhomboid or triangular, shorter than, or occasionally equal to, first lower labials; dorsal scales small, granular to conical; abdominal scales juxtaposed or subimbricate; in the males 5-7 series of preanal pores forming a roughly triangular patch, the number of pores very variable; 2-4 series of femoral pores; in females a patch of enlarged preanal scales, but no pores; tail cylindrical, tapering, covered with small, quadrangular, juxtaposed scales arranged in whorls; in the males, the base of the tail swollen to accommodate the hemipenes, and with 2-4 pointed cloacal spurs in a fan-like group on each side of the swelling; females with vestigial cloacal spurs.

COLOUR: Chevron-shaped markings on a brown or grey background on dorsum of body, often running together to form indistinct longitudinal stripes over the front half of the body; head with dark V-shaped marking on top of head behind eyes, dark-edged pale stripe from eye to ear, denticulate markings on lips; venter cream, speckled with dark brown.

MATERIAL EXAMINED: CD1092.

DISTRIBUTION AND GEOGRAPHIC VARIATION: Islands around Stewart Island, but there are no recent specimens from Stewart Island itself. It is found on the Muttonbird Islands north-east of Stewart island, but is apparently absent from the Hazelburgh Group in central Foveaux Strait. Recent collections of sloughed skins indicate that it is probably found on Codfish Island; the skins were from this species or $H$. granulatus; specimens are required to confirm their identity.

HABITAT: Forest and shrubland. By day, found under loose bark, or in hollows in trees, or among epiphytes. Often basks directly in late afternoon. At night, forages in trees or shrubs.

REPRODUCTION: Unknown.

COMMENTS: Thomas (1981) has presented a detailed discussion of the synonymy of $H$. granulatus and $H$. nebulosus. McCann (1955) described Heteropholis nebulosus from the Stewart Island region, as part of a major revision of the New Zealand lizard fauna. The other species of Heteropholis recognised or described by McCann (1955) are all green geckos, now included in Naultinus Gray, 1842 (Bauer 1990). However, subsequent 
examination of the types of $H$. nebulosus indicated that they fitted more comfortably into Hoplodactylus than Heteropholis or Naultinus (Robb 1982). Robb (1980a) included $H$. nebulosus in Hoplodactylus in her popular monograph, while retaining its separate identity.

Thomas (1981) could find no diagnostic morphological differences between $H$. nebulosus and Hoplodactylus granulatus, and reduced the former to synonymy with the latter.

CONSERVATION STATUS: Abundant on small, rodent-free offshore islands around Stewart Island. Codfish Island, where sloughed skins were recently collected, has kiore (Rattus exulans), but no other mammals; it also has much taller, more diverse forest than the islands where $H$. nebulosus has apparently disappeared since the introduction of mammals such as $R$. rattus and cats.

\subsection{NOMEN NUDUM}

1861 Dactylocnemis wullerstorfii Fitzinger. Österr. Akad. Wissensch. Math.nat. Klasse 42: 400.

COMMENTS: This name appeared in a listing of species as a house gecko from New Zealand. There is no evidence to link it to any individual species. Individuals of several New Zealand species very occasionally enter and live in houses, but none is commonly found in this situation as several tropical species are.

\subsection{NAULTINUS}

\subsubsection{GENERAL}

The diagnosis and synonymy of Naultinus are unchanged by my study. The identity of $N$. tuberculatus has been clarified, reducing $N$. poecilochlorus to a junior synonym, and necessitating minor changes to the description and synonymy of $N$. stellatus.

\subsubsection{HISTORY}

Naultinus was the first genus erected for New Zealand Gekkonidae (Gray 1842a). Initially it included all the endemic geckos, but the nocturnal brown species were removed to Hoplodactylus by Boulenger (1885a). The latter move was disputed by Chrapliwy et al. (1961) on the basis of page priority for N. pacificus (a brown gecko) over N. elegans (a green gecko) in Gray's 
(1842a) original publication; they proposed to transfer the generic name Naultinus to the New Zealand brown geckos and erect a new genus, Naultinulus, for the green geckos. This proposal was quickly rejected, following argument by Myers (1961) in favour of the status quo.

Fischer (1882) proposed the generic name Heteropholis for the South Island rough-scaled green gecko $H$. rudis, on the basis of its "thicker" toes, and particularly its mixture of normal and greatly enlarged scales. This generic name was little used until McCann (1955) used it in his revision of the New Zealand lizard fauna to encompass all the South Island green geckos. From then until 1990 the former name was in almost universal use for the South Island species of green geckos. In the latest systematic revision of Naultinus, Bauer (1990) synonymised Heteropholis, previously used for the South Island species, with Naultinus, previously limited to North Island species. There had been widespread agreement in the New Zealand herpetological community before 1990 that a change was justified; Thomas (1982) and Meads (1982) had argued in favour of this synonymy, and Gill (1986) had presented it as a fait accompli, without supporting evidence or authority.

There has been considerable dispute about the justification for species-level splitting of the New Zealand green geckos. Last century many species names were proposed, some, such as $N$. sulphureus (Buller 1871), for colour morphs apparently determined by single-locus mendelian polymorphisms and freely interbreeding with the more common colour morph(s) in the area, and others for distinct regional populations. Most of these names were little used, and until McCann's (1955) revision, all green geckos were usually called $N$. elegans. McCann (1955) recognised one species of Naultinus ( $N$. elegans), and 6 of Heteropholis ( $H$. gemmeus, $H$. manukanus, $H$. nebulosus, $H$. rudis, $H$. stellatus, and $H$. tuberculatus). $H$. nebulosus has subsequently been removed to Hoplodactylus (Thomas 1981; see above). Robb \& Hitchmough (1980) reviewed the North Island species, and recognised 2 species, $N$. grayi and $N$. elegans, the latter with 2 subspecies, $N$. e. elegans and $N$. e. punctatus. Robb $(1980 a, b)$ described $H$. poecilochlorus from the Lewis Pass area. Bauer (1990) recognised 8 species, N. grayii and $N$. elegans from the North Island and $N$. gemmeus, $N$. manukanus, $N$. poecilochlorus, N. rudis, N. stellatus, and N. tuberculatus from the South Island. 
Bull \& Whitaker (1975) discussed the biogeography of South Island green geckos, and suggested that their morphological diversity had arisen as they dispersed from Pleistocene ice-age refugia. They pointed out that populations in the coastal extremities of the various species' ranges were similar to each other and to the North Island species, whereas the inland populations were much more distinct.

Bauer (1990) included Naultinus spp. in his cladistic study of the morphology of the Carphodactylini, but found few morphological characters of determinable polarity within the genus.

\subsubsection{NOMENCLATURE OF NAULTINUS IN THE NORTH-WESTERN SOUTH ISLAND} Bruce Thomas and I have examined the types of $H$. tuberculatus (McCann), Rep 199 (C. McCann's G111, West Coast) and Rep 171 (C. McCann's G110, Ikamatua) in the collection of Canterbury Museum. This examination indicates that they are not conspecific with the north-western populations which have been known by this name for at least the last 25 years. I therefore examined specimens of these and geographically intermediate populations to reassess their identities and relationships.

The first description of a green gecko from Nelson was $N$. elegans stellatus (Hutton 1872), described from a very small specimen from Mount Arthur. Hutton's (1872) description was very inadequate, concentrating almost entirely on colour, despite the colours obviously having been altered by preservation.

McCann (1955), in a review of all New Zealand lizards, raised the taxon to species status (as Heteropholis stellatus), as well as resurrecting the generic name Heteropholis for all the South Island green geckos. He designated a neotype, the original type having been lost. His neotype is from Lake Rotoiti.

McCann (1955) also described $H$. tuberculatus. He did not explicitly designate a type specimen, simply stating "type in the collection of the Canterbury Museum". The type locality was listed as "Westland, South Island", and a Canterbury Museum paratype was listed as coming from "Ikamaroa, West Coast". These were the only specimens mentioned. McCann's (1955) map 1 indicated localities for this species which appear to be near Lake lanthe and Lake Paringa in southern Westland; whether these were intended to represent the specific localities of the specimens 
mentioned in the description is not clear. McCann (1955) distinguished Heteropholis tuberculatus from all other Heteropholis by differences in size and colour, as well as 2 features which he appears to have regarded as diagnostic: the scales are tuberculate or conical throughout, and the nostril is not in contact with the rostral scale.

In the 1960s green geckos collected from the north-western corner of the South Island (Cape Farewell area and western Golden Bay) were identified as $H$. tuberculatus on the basis of their western distribution, their plain green or lightly spotted colour, and their rostral being excluded from the nostril, despite the facts that these populations are about $400 \mathrm{~km}$ north of McCann's (1955, map 1) closest locality for $H$. tuberculatus, and that the animals were much smaller than those McCann described. These populations became well known, and animals from them were widely kept and bred in captivity. These, rather than the Canterbury Museum type specimens or McCann's (1955) localities, seem to have been the subject of all recent publications mentioning the species (e.g., Bull \& Whitaker 1975; Robb 1980a; Meads 1982; Thomas 1982; Gill 1986; Towns 1985; Pickard \& Towns 1988). Distribution maps (e.g., Bull \& Whitaker 1975, fig. 2; Robb 1980a, page 124; Pickard \& Towns 1988, fig. 16) included only these northern populations.

Populations of green geckos from Nelson other than western Golden Bay and Cape Farewell have been referred to $H$. stellatus in all recent publications (e.g., Bull \& Whitaker 1975; Robb 1980a; Meads 1982; Thomas 1982; Gill 1986; Towns 1985; Pickard \& Towns 1988).

In the early 1970s green geckos with large body size and a unique intricate colour pattern were collected in the area of Lewis Pass. They were clearly distinct from the neighbouring species $N$. stellatus, $N$. rudis, and $N$. gemmeus, and also from " $N$. tuberculatus" as then understood based on the northern populations, so Robb (1980a, b) described them as a new species, Heteropholis poecilochlorus, with types from Lewis Pass. An accidental reversal of planned publication dates meant that the name must be regarded as having been published in Robb's (1980a) popular monograph, rather than in the formal paper (Robb 1980b) which designated the types. The monograph (Robb 1980a) included animals from the Reefton area in the discussion of $H$. poecilochlorus, but the paper (Robb 1980b) mentioned only the Lewis Pass population, from which the type specimens were obtained. 
Rep 171 (C. McCann's G110, syntype of $H$. tuberculatus) is from Ikamatua (misprinted as Ikamaroa by McCann 1955), only about $20 \mathrm{~km}$ from Reefton, and about $125 \mathrm{~km}$ north of the nearer locality in McCann's (1955) Map 1 (B W Thomas, Landcare New Zealand Manaaki Whenua, pers. comm.). Reefton is within the range of N. poecilochlorus (Robb 1980a). If Robb's (1980b) formal description of $N$. poecilochlorus is compared with McCann's (1955) descriptions or redescriptions of the South Island Naultinus spp., $N$. poecilochlorus shares with $N$. tuberculatus sensu McCann (1955) tuberculate body scales (unique to these species in Naultinus) and nostrils not touching the rostral. The latter character is shared with North Island Naultinus spp. but not with any other South Island species according to McCann, but shared by all species except $N$. rudis and $N$. manukanus according to Bauer (1990). The 2 species, as described, differ only in the more complex colour pattern of most $N$. poecilochlorus, and in the presence of a large internasal in $N$. poecilochlorus, which is described as absent in $N$. tuberculatus. However, Rep 171, the Ikamatua syntype of N. tuberculatus, has a large internasal, and the absence of this scale in Rep 199 appears to be the result of an earlier injury or developmental abnormality involving the entire right side of the upper jaw, which has also caused the upper labials on this side to be very abnormal in size and shape. The absence of this scale is therefore not a taxonomically valid character. Occasional specimens of $N$. poecilochlorus lack the full complexity of the typical colour pattern of this species (Robb 1980a, b), so this character too cannot be regarded as diagnostic.

Therefore Naultinus poecilochlorus (Robb, 1980a) should be regarded as a junior synonym of Naultinus tuberculatus (McCann, 1955). The name poecilochlorus (Robb, 1980a) will of course be available if at any time the Lewis Pass population with its more distinctive colour pattern is considered to warrant formal taxonomic separation, but I consider that, in the absence of other differences, further investigation of the distribution of this colour form in the field is required before this can be justified.

Further investigation of $N$. tuberculatus, and particularly of the relationship between the Lewis Pass population and others is at present impossible, as there are no recent specimens from known localities other than Lewis Pass.

The north-west Nelson populations which have been assigned to $N$. tuberculatus for the past 25 years or so share with the types of that species 
tuberculate scales and nostrils not contacting the rostral. However, these characters are also shared by all (tuberculate scales) or many (nostrils excluded from rostral) $N$. stellatus. The north-west Nelson animals are much smaller than the Westland ones: 45-55 mm snout-vent (Robb 1980a), cf. 62$77 \mathrm{~mm}$ snout-vent for the types of $N$. tuberculatus (McCann 1955) and $79 \mathrm{~mm}$ snout-vent for the type of $N$. poecilochlorus (Robb 1980b).

The possibility that the West Coast and north-west Nelson populations are connected by intermediate populations in the northern part of the West Coast to form a cline has recently been shown to be unlikely by records which have become available from this area. No specimens are available, but photographs of a Naultinus specimen from the North Branch, Mohikinui River by lan Henderson (22 February 1982) show a typical specimen of $N$. stellatus, similar to those from the Maitai Valley near Nelson. A photograph by Mary Morgan Richards of a yellowish-green Naultinus specimen from Denniston is of poorer quality, but is also consistent with $N$. stellatus.

The north-west Nelson populations are very similar to adjacent populations of $N$. stellatus (see Table 4.5.3.1). Bull \& Whitaker (1975) commented "populations of $H$. tuberculatus merge with those of $H$. stellatus in Golden Bay". Pickard \& Towns' (1988) distribution maps for these 2 species show some overlap, and their distributions are somewhat different to those illustrated by Robb (1980a). This confusion results from the impossibility of drawing an artificial line separating clinally varying populations into 2 species, rather than from the 2 species being found in sympatry. I therefore consider that the north-western populations should be included in $N$. stellatus. Even within $N$. stellatus as previously recognised there are considerable differences between populations (Robb 1980a). These populations (including the north-western ones formerly misidentified) form a north-south cline of increasing body size, decreasing relative tail length, and increasing complexity of colour pattern, with little or no overlap between, for example, the western Golden Bay and Maitai Valley populations. However, the geographically intermediate Sandy Bay population overlaps in size and colour with both these other well-known populations. The few well-known populations are widely separated geographically, so whether this cline is stepped or gradual is unknown. 
SUMMARY:

1) The types of $N$. tuberculatus are from Westland and near Reefton, and are large robust animals. They differ from Robb's (1980a, b) N. poecilochlorus only in colour pattern, and this difference is not diagnostic, so I reduce $N$. poecilochlorus to synonymy with $N$. tuberculatus.

2) Populations of Naultinus from the north-west corner of the South Island, formerly misidentified as $N$. tuberculatus, are one extreme of a cline in size and colour pattern within N. stellatus.

3) N. stellatus is separated from N. tuberculatus by several diagnostic morphological differences, but a morphologically intermediate specimen is known from Shenandoah. This may represent contact at a hybrid zone, or a short and dramatic morphological cline. I consider this insufficient evidence to justify reduction of these taxa from species to a lower taxonomic level.

\subsubsection{NAULTINUS STELLATUS (HUTTON, 1872)}

1872 Naultinus elegans stellatus Hutton. Trans. Proc. New Zealand Inst. (1872) 4: (171).

Type locality: near the top of Mount Arthur, New Zealand.

Holotype: NMNZ (specimen "not available" fide McCann 1955). 1877 Naultinus pulcherrimus Buller. Trans. Proc. New Zealand Inst. 9: 326; pl. 17 top and middle.

Type locality: One of the Nelson and Foxhill railway stations, in the

Waimea District, South Island, New Zealand.

Holotype: NMNZ (specimen "not available" fide McCann 1955). 1955 Heteropholis stellatus McCann. Dominion Mus. Bull. 17: 66; pl. 7 (figs 1-7).

Neotype locality: Lake Rotoiti, Nelson District, South Island, New Zealand.

Neotype: NMNZ R458. 1982 Naultinus elegans stellatus Meads. New Zealand herpetology: 324. 1982 Naultinus elegans tuberculatus Meads. New Zealand herpetology: 324.

1982 Naultinus stellatus Thomas. New Zealand herpetology: 336. 1982 Naultinus tuberculatus Thomas. New Zealand herpetology: 336. 1986 Naultinus elegans stellatus Gill. Collins handguide to the frogs and reptiles of New Zealand: 48.

1986 Naultinus elegans tuberculatus Gill. Collins handguide to the frogs and reptiles of New Zealand: 48. 
DIAGNOSIS: Small to large Naultinus (55-80 mm snout-vent). Nostril excluded from rostral by contact between anterior nasal and anterior supralabial except in many specimens from the Nelson Lakes district, in which the nostril may contact the nostril. Scales conical-tuberculate. Tongue yellow; mouth lining yellow-orange or pale lavender; no dark blue pigment in mouth.

DESCRIPTION: Head oviform; snout bluntly rounded, about 1.3 times longer than distance between eye and ear opening, 2.0-2.6 times diameter of ocular disc; forehead flat or very slightly concave; ear-opening small to minute, oval; body and limbs moderate to robust; digits feebly dilated, tapering towards distal extremity, lamellae straight-edged or very slightly curved, in an uninterrupted series, decreasing in width distally; 9-12 (usually 10) larger and 5-7 (usually 5) smaller lamellae beneath fourth toe; no or very slight webbing between toes 2,3 , and 4 ; in southern populations snout covered with enlarged, domed scales of irregular size, but especially large on the canthus rostralis and tip of the snout; in northern populations snout scales small, domed; remainder of head covered with a mixture of large and small tuberculate scales; rostral 2.0-2.6 (usually 2.0) times as broad as deep, lateral borders converge gradually (rather than abruptly) anteriorly, usually lacking median cleft; nostril surrounded by first upper labial and 3-4 nasals, the anterior-most of which is very large, roughly square or oblong, about equal in size to internasal; single, very large internasal, deeper than rostral, occasionally excluded from rostral by extended bases of anterior nasals; 9-12 upper and 8-11 lower labials; mental 4-sided, anterior wider than posterior, depth about equal to or slightly less than that of first lower labial; usually 3 small postmentals; dorsal scales large, conical; ventral scales large, flat, subimbricate; in males preanal pores in 5-7 angular series forming a roughly triangular patch; femoral pores in $2-3$ series, extending about half the length of the femur; in females a patch of enlarged preanal scales with scattered vestigial pores; a few scattered vestigial femoral pores; tail cylindrical, tapering, slightly longer than snout-vent length, covered with scales similar to those of dorsal and ventral body surfaces; in males, base of tail swollen to accommodate hemipenes, and with 2-4 large, pointed scales on each side of swelling; females with vestiges of enlarged scales.

COLOUR: In northernmost populations, bright green, uniform or with greenishyellow longitudinal dorso-lateral streaks or rows of spots; in the central part of the species' range, grey-green or olive green, uniform or more commonly with roughly diamond-shaped blotches, outlined, if at all, in slightly darker 
shades of the background colour. In the south of the species' range, males dark sepia-brown or silvery grey, with circular green blotches dorsally, cream mottled with dark sepia ventrally; females as in the centre of the range.

Tongue orange-yellow, mouth lining light lavender-blue in northern populations, changing clinally to orange-yellow in southern populations. Orange markings around nostrils and corners of mouth in northern populations.

MATERIAL EXAMINED: RAH368, 387, 397 (Nelson Lakes), RAH370, 371 (Maitai Valley), RAH367, CD995 (Sandy Bay), CD994 (Adele Island), RAH466 (Golden Bay).

DISTRIBUTION AND GEOGRAPHIC VARIATION: See section 3.7.3.

HABITAT: Leptospermum shrubland and beech forest. $N$. stellatus, like other members of the genus, inhabits the canopy of the vegetation, and is diurnal.

REPRODUCTION: Annual reproductive cycle, with ovulation and mating in spring, and birth in autumn (March-May). Robb (1980a) noted betweenpopulation variation in birth season, which is maintained when specimens from these populations are kept in captivity at the same locality (B. Goertz, Reptile Research Centre, pers. comm.), indicating that there is a genetic basis for this difference.

COMMENTS: See section 3.7.3.

CONSERVATION STATUS: Widely distributed in Nelson, but very cryptic, therefore often escapes notice. Several populations are within protected areas: North-west Nelson National Park, Abel Tasman National Park (including an island population on Adele Island), and Nelson Lakes National Park all have populations. The impact of dramatically increased wasp populations since the establishment of Vespula vulgaris should be investigated.

\subsubsection{NAULTINUS TUBERCULATUS (MCCANN, 1955)}

1955 Heteropholis tuberculatus McCann. Dominion Mus. Bull. 17:61.

Type locality: Westland, South Island.

Holotype: Canterbury Museum Rep 199 (C. McCann G111) 
Syntype: Canterbury Museum Rep 171 (C. McCann G110); locality Ikamaroa (lapsus pro Ikamatua).

1980 Heteropholis poecilochlorus Robb. New Zealand amphibians and reptiles in colour: 67; pl. 19.

1980 Heteropholis poecilochlorus Robb. Rec. Nat. Mus. New Zealand 1: 309; fig. $1 \mathrm{C}$.

Type Locality: Lewis Pass, South Island, New Zealand.

Holotype: NMNZ R1862.

1982 Naultinus elegans poecilochlorus Meads. New Zealand herpetology: 324.

1986 Naultinus elegans poecilochlorus Gill. Collins handguide to the frogs and reptiles of New Zealand: 48 .

DIAGNOSIS: Large Naultinus (70-80 mm snout-vent). Nostril excluded from rostral by contact between anterior nasal and anterior supralabial. Scales conical-tuberculate, but not greatly enlarged. Upper eyelid fringed with strongly conical scales. Tongue black, mouth lining dark blue.

DESCRIPTION: Head oviform; snout bluntly rounded, about 1.3 times longer than distance between eye and ear opening, 2.0-2.6 times diameter of ocular disc; forehead flat or very slightly concave; ear-opening small to minute, oval; body and limbs moderate to robust; digits feebly dilated, tapering towards distal extremity, lamellae straight-edged or very slightly curved, in an uninterrupted series, decreasing in width distally; 9-12 (usually 10) larger and 5-7 (usually 5) smaller lamellae beneath fourth toe; no or very slight webbing between toes 2,3 , and 4; snout covered with enlarged, domed scales of irregular size, but especially large on the canthus rostralis and tip of the snout; remainder of head covered with a mixture of large and small tuberculate scales; rostral 2.0-2.6 (usually 2.0) times as broad as deep, lateral borders converge gradually (rather than abruptly) anteriorly, usually lacking median cleft; nostril surrounded by first upper labial and 3 (occasionally 4) nasals, the anterior-most of which is very large, roughly square or oblong, about equal in size to internasal; single, very large internasal, deeper than rostral, occasionally excluded from rostral by extended bases of anterior nasals; 8-11 (usually 9-10) upper and 8-11 (usually 9) lower labials; mental 4-sided, anterior wider than posterior, depth about equal to or slightly less than that of first lower labial; 2-4 small postmentals; dorsal scales large, conical; ventral scales large, flat, subimbricate; in males preanal pores in 5-7 angular series forming a roughly 
triangular patch; femoral pores in 2-3 series, extending about half the length of the femur; in females a patch of enlarged preanal scales with scattered vestigial pores; a few scattered vestigial femoral pores; tail cylindrical, tapering, slightly longer than snout-vent length, covered with scales similar to those of dorsal and ventral body surfaces; in males, base of tail swollen to accommodate hemipenes, and with 2-3 large, pointed scales on each side of swelling; females with vestiges of enlarged scales.

COLOUR: Bright green to olive green, uniform or with greenish-yellow dorsolateral rows of spots, or with dorso-lateral, lateral, and ventro-lateral rows of small yellow-green spots or streaks and a pale mid-dorsal stripe; markings outlined, if at all, in slightly darker shades of the background colour. Tongue black, mouth lining dark blue.

MATERIAL EXAMINED: RAH365, 366 (Lewis Pass).

DISTRIBUTION AND GEOGRAPHIC VARIATION: See section 3.7.3.

HABITAT: Leptospermum shrubland and beech forest. N. tuberculatus, like other members of the genus, inhabits the canopy of the vegetation, and is diurnal.

REPRODUCTION: Poorly known; captive specimens have given birth in autumn (April-May - Robb 1980b).

COMMENTS: See section 3.7.3.

CONSERVATION STATUS: Possibly widely distributed in Westland, but very cryptic, and its range is very poorly known. The only well-known population is at Lewis Pass, in the Lewis Pass National Reserve. The impact of dramatically increased wasp populations since the establishment of Vespula vulgaris should be investigated. 


\section{Chapter 4: SPECIES IDENTIFICATION}

\subsection{INTRODUCTION}

\subsubsection{GENERAL}

As described in Section 1.4, there was strong evidence before this work commenced that the alpha (species-level) taxonomy of the New Zealand geckos did not accurately reflect the variation seen in nature, with undescribed species clearly existing in $H$. maculatus sensu Robb \& Rowlands (1977), and possibly in H. granulatus and $H$. pacificus. Naultinus, on the other hand, was possibly oversplit.

In this chapter I justify the taxonomic changes presented in Chapter 3. Only taxa with changed or controversial taxonomic status are discussed here. The broader phylogenetic context into which these taxonomic changes fit is discussed in Chapter 5.

\subsubsection{SPECIES CONCEPTS}

The modern era of biology has been dominated by, and largely built around, the Biological Species Concept of Dobzhansky (1937) and Mayr (1942, 1969), which rapidly displaced older, typological species concepts in the zoological, although not the botanical literature. Templeton (1989) has pointed out that all species concepts are biological, and that the basic species criterion of Mayr (1963) was reproductive isolation, and hence suggested that Isolation Species Concept (ISC) was a more accurate term for Mayr's and similar concepts.

Only in the last decade have serious alternatives to the ISC been proposed. As systematics advanced rapidly with the availability of modern biochemical and molecular techniques to solve previously intractable problems, and of computer-based analytical procedures for these and other data, more and more situations were found where the ISC could not unambiguously categorise taxa into species. The ISC occasionally suggested nonsensical results because closely related taxa were reproductively isolated, but more distant relatives were not (e.g., the Rana pipiens complex, Hillis 1988), but in particular it could not provide useful criteria to assign to species populations separated in space or time (Frost \& Hillis 1990). Captive hybridisation experiments were supported by some workers, but others recognised that they gave misleading results, as some sympatric species which are reproductively isolated in nature hybridise quite readily in 
captivity, and some other species simply cannot be bred in captivity. In these situations a de facto typological species concept was often used.

Several alternative species concepts have been discussed in the literature since about 1980. Of these, the Recognition Species Concept (RSC, Paterson 1985) is closest to the Isolation Species Concept, to the extent that in practical terms, it can be considered one of the ISC group of concepts (Echelle 1990). It differs from Mayr's concept in considering reproductive inclusiveness (those animals which are considered potential mates by the organism itself) rather than exclusiveness (partners with which successful reproduction is not possible). However, it is largely behaviourally based, and behaviour is labile in many animals, so unrepeatable and uninterpretable results may occur, and appropriate data may be difficult to obtain for many taxa. The problems of interpreting relationships among allopatric populations are the same as those for the ISC except when animals are transferred between populations (not possible or desirable in many situations), and assessment of the status of extinct populations is impossible using this concept. The concept is therefore unusable in practical terms in a taxonomic revision such as this, and has not been taken up by many workers. This does not mean, however, that specific mate recognition systems are not of vital importance in maintaining the integrity of many species.

The Phylogenetic Species Concept (PSC - Rosen 1978, 1979; Nelson \& Platnick 1981; Donoghue 1985), despite its emphasis on cladistic monophyly, is basically typological, defining the species as the smallest diagnosable taxonomic unit, and has many of the problems of the old-style typological concepts. Species need not have any unique characters; unique combinations of characters are sufficient, but there must be no sharing of the diagnostic character or character combination with even a very small proportion of individuals of another species. In practical terms this concept would result in a huge proliferation of recognised species, as most subspecies and a great many populations with no formal status currently would be eligible for recognition as species. Many of these would differ only in one or 2 very trivial characters, and there would be little taxonomic stability as natural gene flow between populations could cause loss of species status. Detection of very low-frequency polymorphic variants resembling another species would also cause loss of species status. Problems such as 
narrow hybrid zones are also dealt with very poorly by this concept (Frost \& Hillis 1990).

The Evolutionary Species Concept (ESC - Wiley 1978, 1980, 1981) defines species as groups of organisms on permanently independent evolutionary trajectories. It is an improvement on the ISC group of concepts because it provides criteria for the assessment of specific status of taxa which the ISC supplies no data on, such as allopatric, extinct, or parthenogenetic organisms, but most species defined by the ISC will be unchanged under this concept. Some will have strongly differentiated subspecies raised to species status, but because it requires evidence of permanent separation of evolutionary trajectories it avoids the problems posed by the PSC of unmanageably huge numbers of often ephemeral, trivially distinct, typologically defined species. The major problem with the ESC is the subjective decision required to determine whether taxa in those situations not adequately covered by the ISC are on permanently independent evolutionary trajectories. Frost \& Hillis's (1990) review of herpetological applications of species concepts strongly favoured an Evolutionary Species Concept.

The Cohesion Species Concept (CSC - Templeton 1989) has much in common with the ESC, and shares many of its advantages, such as applicability to hybridising and asexual taxa. However, it defines species in terms of the mechanisms yielding cohesion rather than the manifestation of cohesion over evolutionary time. In this respect it is related to aspects of the RSC. However, the cohesion mechanisms included in this concept are much broader than those of the RSC, including genetic drift, natural selection, and demographic exchangeability, all of which can be applied to non-sexual as well as sexually reproducing populations. While data on the strength of these cohesion mechanisms are often not available to systematists, the operation of the mechanisms can be inferred from the patterns of variation in the populations under study. Note that the cohesion and evolutionary concepts are mutually compatible, and should usually generate very similar taxonomies.

Finally, Avise \& Ball (1990) emphasised concordance among character distributions in assessing species status for those taxa not amenable to assessment using the ISC. This is very close to my interpretation in practical terms of the ESC. 
As a working guideline, Thorpe (1982) demonstrated that the great majority of populations separated by genetic distances of Nei's $D \geq 0.15$ could be shown to be separate species using evidence independent of the allozyme data. Similarly, Highton (1989), in a survey of Plethodon salamanders, found that a genetic distance of about 0.15 circumscribed groups of populations which were geographically continuous and morphologically distinct from other such groups, whereas larger genetic distances resulted in the inclusion of geographically disjunct and morphologically dissimilar populations, and smaller genetic distances grouped populations in a manner which could not be related to geography or morphology. $\mathrm{He}$, too, took Nei's $D \geq 0.15$ to be a suitable criterion for species status.

However, the inverse argument, that populations separated by Nei's $\mathrm{D}<0.15$ must be conspecific, is absolutely dependent on an assumption of gradualism. If speciation occurs as a result of a period of rapid change, species might be diagnosably different (phylogenetic species concept), on separate evolutionary trajectories (evolutionary species concept), and/or reproductively isolated from each other (biological/isolation and related species concepts), at genetic distances very much lower than those generated by assumptions of gradualism. Values such as the well-supported $\mathrm{D}=0.15$ value quoted above should therefore be regarded as maxima above which populations are unlikely to be conspecific, but below which species status is unresolved by the genetic technique alone. However, measures of genetic distance from allozyme studies can vary subtstantially depending on the mix of rates of evolution in the particular loci included (Sarich 1977), so genetic distance criteria for species status must be considered in the context of each individual study.

In this study I have chosen to use an Evolutionary Species Concept, as my data are unsuitable for assessment using the Cohesion Species Concept; the mechanisms underlying the observed distributions of electromorphs cannot be directly inferred from these distributions. I have used several lines of evidence to assess whether a taxon is on a separate evolutionary trajectory from its relatives.

1) Any operational taxonomic units (OTUs) which are reproductively isolated in sympatry (as per the ISC) clearly lack cohesion with each other and are on separate evolutionary trajectories. 
2) If a hybrid zone is narrow relative to the distributions of the hybridising OTUs, is congruent for at least 2 genetically unlinked characters, and appears to be stable, the OTUs forming it are judged to be on separate evolutionary trajectories.

3) Any pair of sister OTUs which are genetically (as assayed by allozymes), morphologically, and/or ecologically more distinct from each other than are sister species defined by criteria 1 and 2 above are considered to be demonstrably on separate evolutionary trajectories.

4) Any OTU which is the sister group of a clade of 2 or more distinct species under criteria 1-3 above is also judged to be a distinct species on a unique evolutionary trajectory.

\subsection{THE H. PACIFICUS COMPLEX}

\subsubsection{INTRODUCTION}

In this section I justify recognition of three new species, $H$. "Matapia", $H$. "Three Kings", and H. "Poor Knights".

There is a history of informal comment in literature suggesting possible taxonomic subdivision within H. pacificus sensu Robb \& Rowlands (1977).

McCann (1955), when he synonymised $H$. maculatus with $H$. pacificus, commented that "specimens from the Great King Island and the MokohinauGreat Barrier collections exhibit a remarkable increase in size, in the number of lamellae under the fourth toe, and a notable decrease in the number of preanal pores. Femoral pores are virtually absent. On the average these specimens are larger in all dimensions than others from the rest of the area".

Robb \& Rowlands (1977) noted that, in comparison with other H. pacificus, "specimens from Three Kings and Mokohinau Islands are particularly robust in all dimensions".

Specimens larger than those on the mainland and with higher lamellae counts are also found on the Poor Knights Islands (Whitaker 1968). They have attracted particular attention on Sugarloaf Rock, south of the main Poor Knights group, where a very large population is associated with a gannet (Sula bassana) breeding colony. This population shows the unusual 


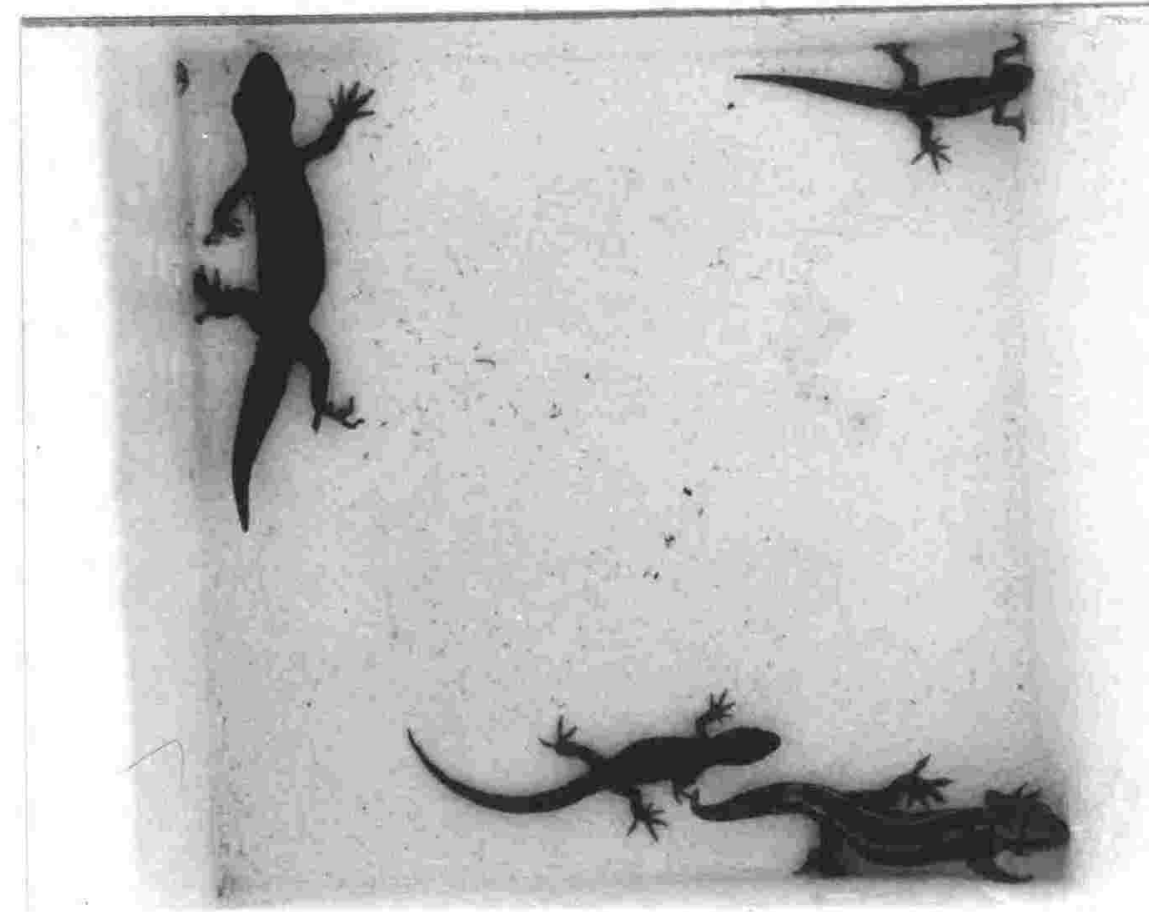

Figure 4.2.1.1 H. pacificus (2 larger specimens) and $\mathrm{H}$. "Matapia" (2 smaller specimens) collected in sympatry on Matapia Island.

behaviour of avidly scavenging fish scraps dropped by the birds, and has become diurnal to utilise this resource (A. H. Whitaker pers. comm.). Other populations of $H$. pacificus are strictly nocturnal (Benson 1976); this was one of the features used by Robb \& Rowlands (1977) to separate H. pacificus and $H$. maculatus.

Because of this morphological variation, samples of $H$. pacificus sensu Robb \& Rowlands (1977) were collected from as many localities as possible, including Great King Island and Aorangi Island and Sugarloaf Rock in the Poor Knights Group, and their morphology and electrophoretic profiles compared. Unfortunately no material from the Mokohinau population suitable for electrophoresis has been available. The taxonomic status of this population remains uncertain.

Small, longitudinally striped geckos were seen by P. Anderson (pers. comm.) on Matapia Island (often incorrectly spelt Motupia) in 1986. They are sympatric on Matapia Island with typical $H$. pacificus (Figure 4.2.1.1). They were found among plants of the robust New Zealand endemic sedge Cyperus ustulatus, and were remarkably active and agile, leaping from plant to plant when disturbed, quite unlike the much larger, normal-looking $H$. pacificus which were found nearby under stones among shrubs. Anderson 
tentatively identified the striped geckos as $H$. chrysosireticus on the basis of their colour pattern. A specimen was sent alive to New Zealand Department of Conservation herpetologists in Wellington for confirmation of this identification. The identification as $H$. chrysosireticus was accepted, and on this basis Matapia Island appeared as a locality for this species in the "Atlas of the amphibians and reptiles of New Zealand" (Pickard \& Towns 1988).

\subsubsection{SAMPLES}

An attempt was made to keep the first Matapia Island specimen alive in captivity, but it died after only a few days. The body was found and placed in a $-80^{\circ} \mathrm{C}$ freezer a day or 2 later. Subsequently, further small samples of small, striped geckos and morphologically typical $H$. pacificus were collected from Matapia Island.

Other members of the $H$. pacificus complex were collected mainly from offshore islands, with only 4 mainland localities sampled (Figure 4.2.2.1, Table 4.2.2.1). This reflects their much lower abundance and patchy distribution in habitats with mammalian predators compared to offshore islands free of introduced mammals. $H$. pacificus is known from scattered localities throughout the North Island (the southernmost record is from Whiteman's Valley near Wellington), but the most southern samples available for this study were from islands in the Bay of Plenty.

In total 53 individuals from 19 populations were sampled.

\subsubsection{MORPHOLOGY}

Several characters clearly were inconsistent with the original identification of the striped Matapia Island specimen as $H$. chrysosireticus. The specimen had its nostril broadly in contact with its rostral scale, rather than excluded by the anterior nasal; 3 pointed cloacal spurs on each side, rather than a single rounded one; tapering toes with the narrow distal portion about one-third of the total length of the toes, rather than broadly expanded pads with short distal phalanges; and a small central patch of preanal organs with no extension onto the thighs, rather than with 2 rows extending onto the thighs. This set of characters clearly placed the specimen in $\mathrm{H}$. pacificus rather than $H$. chrysosireticus. The lack of preanal organs on the thighs, curved rather than straight lamellae, and absence of belly markings also exclude $H$. stephensi, the only other longitudinally striped species. 


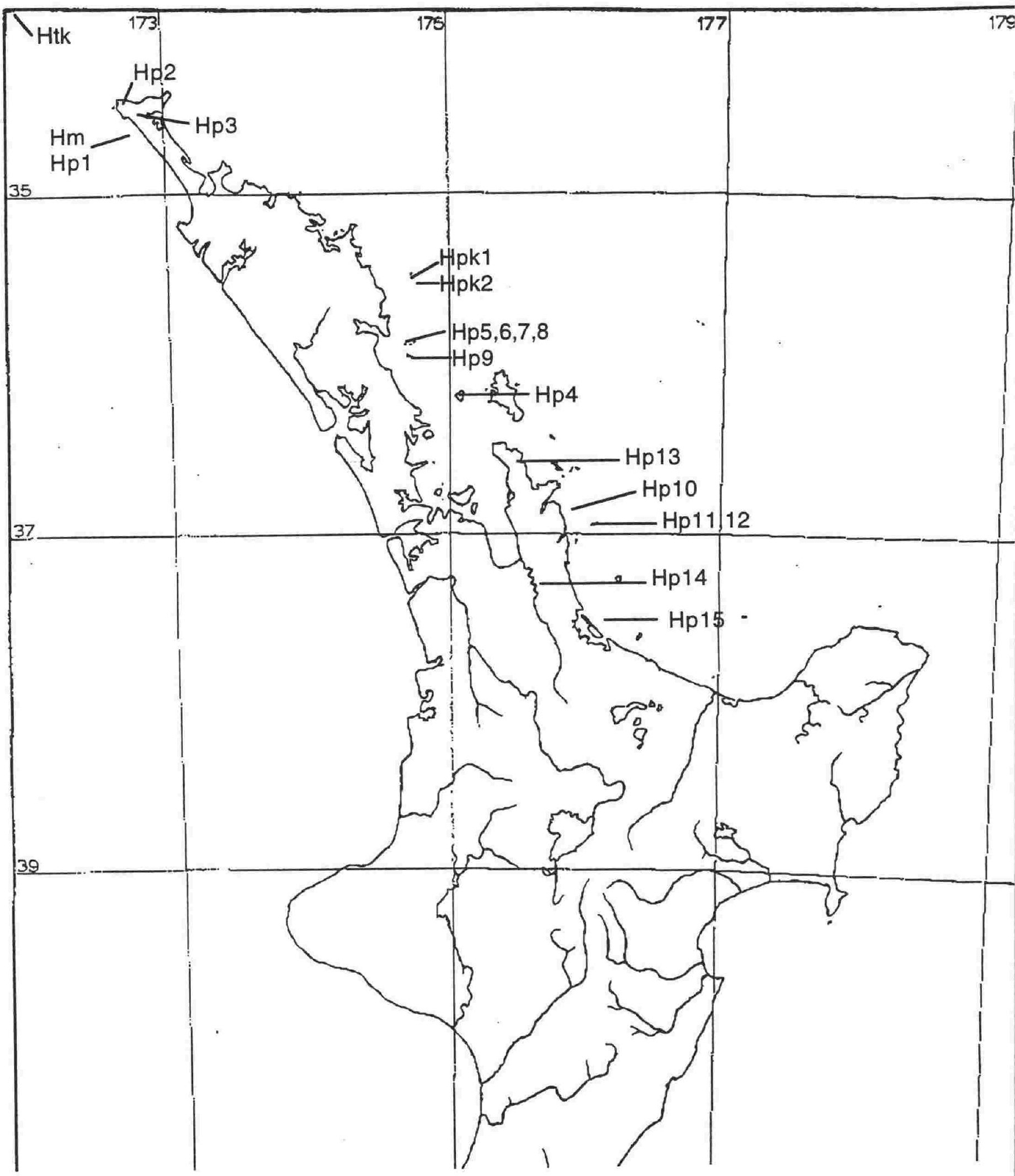

Figure 4.2.2.1 Collecting localities for specimens of the $H$. pacificus complex surveyed for allozyme variation. For key see Table 4.2.2.1. 
Table 4.2.2.1 Localities and number key for each population of the $H$. pacificus complex surveyed in this thesis.

\begin{tabular}{lll}
\hline $\begin{array}{l}\text { Population } \\
\text { number }\end{array}$ & Species & Locality \\
\hline Htk & H. n.sp. "Three Kings" & Great King Island \\
Hpk1 & H. n.sp. "Poor Knights" & Aorangi Island, Poor Knights group \\
Hpk2 & H. n.sp. "Poor Knights" & Sugarloaf Rock, Poor Knights group \\
Hm & H. n.sp. "Matapia" & Matapia Island \\
Hp1 & H. pacificus & Matapia Island \\
Hp2 & H. pacificus & Cape Reinga \\
Hp3 & H. pacificus & Waitiki Landing \\
Hp4 & H. pacificus & Little Barrier Island \\
Hp5 & H. pacificus & Pupuha Island, Chickens group \\
Hp6 & H. pacificus & One Island, Chickens group \\
Hp7 & H. pacificus & Muriwhenua Island, Chickens group \\
Hp8 & H. pacificus & Wareware Island, Chickens group \\
Hp9 & H. pacificus & Hen Island \\
Hp10 & H. pacificus & Castle Island \\
Hp11 & H. pacificus & Hongiora Island, Aldermen group \\
Hp12 & H. pacificus & Raumahuanui Island, Aldermen group \\
Hp13 & H. pacificus & Waikawau Bay, Coromandel Peninsula \\
Hp14 & H. pacificus & Paeroa \\
Hp15 & H. pacificus & Karewa Island \\
& & \\
\hline
\end{tabular}

The 4 specimens (all sexually mature) subsequently collected on Matapia Island fell very clearly into 2 groups, as did other specimens seen but not collected (P. Anderson, Department of Conservation, pers. comm.). Two were 46 and $48 \mathrm{~mm}$ in snout-vent length, about $3 \mathrm{~g}$ in weight, and light golden brown in colour with straight-edged longitudinal stripes, like the first specimen collected. The other 2 were 72 and $76 \mathrm{~mm}$ snout-vent, 9 and $11 \mathrm{~g}$ weight, and dark brown in colour, one with zig-zag transverse stripes and a mustard nape marking, the other with very irregular longitudinal stripes. The latter sizes and colours fall within the typical range in many $H$. pacificus populations.

Three Kings and Poor Knights specimens are substantially larger than those from other $H$. pacificus-complex populations (up to $90 \mathrm{~mm}$ snout-vent and $19 \mathrm{~g}$ weight). $H$. "Poor Knights" and $H$. "Three Kings" can be distinguished morphologically by the lower number of lower labials (9-12 vs 13-14) in $H$. "Poor Knights". Within the Poor Knights, in the Sugarloaf Rock specimens the first lower labials were in contact behind the narrow, triangular mental, whereas the Aorangi specimens had the same condition as in other 
populations of the $H$. pacificus complex - a bluntly triangular mental and rounded postmental separating the first lower labials. Aorangi specimens also usually had 3 (occasionally 2 ) small internasals, whereas Sugarloaf specimens had one large internasal, like $H$. "Three Kings".

\subsubsection{ALLOZYME VARIATION}

Analysis of allozyme genotypes confirmed the conclusion from morphological evidence that $H$. "Matapia" was much more similar to $H$. pacificus than to any other species and was very clearly not $H$. chrysosireticus or the other strongly striped species, $H$. stephensi. It differed from both of these at 14 loci (not the same loci in each case - see Chapter 5).

Populations of the $H$. pacificus complex varied at 10 out of 27 allozyme loci (Table 4.2.4.1). As far as could be detected from the small samples available, levels of polymorphism were very low (Table 4.2.4.2). There was no evidence of genetic structuring within samples suggestive of cryptic sympatric species; direct-count and Hardy-Weinberg expected heterozygosity were similar in all populations (Table 4.2.4.2; sample sizes were too small to permit tests for significance). Populations in the Chickens Island group showed higher levels of observed polymorphism then most others. All 4 populations sampled in this island group showed polymorphism, including the 2 highest levels of polymorphism detected in the complex.

$H$. "Three Kings" and $H$. "Poor Knights" each had one diagnostic allele (Icd-2(a) and Pgd-1(d) respectively), and these 2 species and $H$. "Matapia" all had the Gp4(c) allele, not seen in any $H$. pacificus. No fixed allozyme differences were found between the diurnal fish-scavenging population from Sugarloaf Rock and those from Aorangi in the main Poor Knights group, but each population had one allele which was absent from the other.

$H$. "Matapia" had 2 apparently fixed differences from the sympatric $H$. pacificus population, but differed from the Hen Island, Little Barrier, Coromandel, and Paeroa populations of $H$. pacificus only by 2 polymorphisms: at Pgm-1 H. "Matapia" is polymorphic for the Pgm-1(a) allele, not seen in any other $H$. pacificus-complex population, and at Gp-4, for the Gp-4(c) allele, also found in $H$. "Three Kings" and H. "Poor Knights", but not in H. pacificus. 


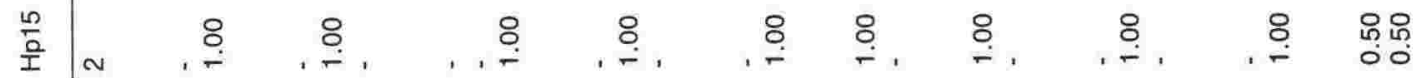

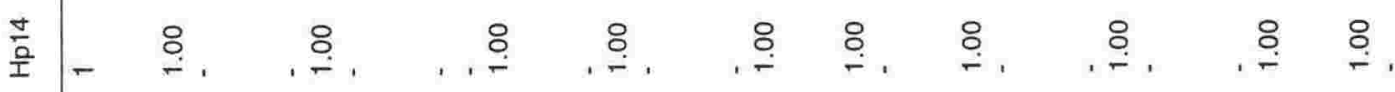

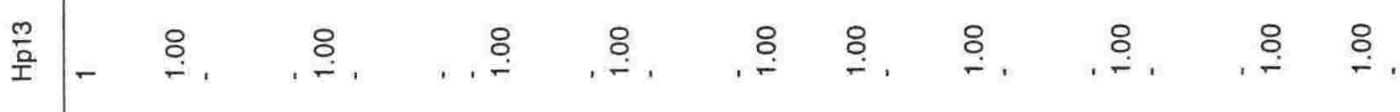

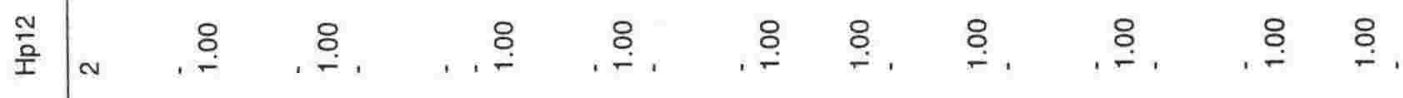

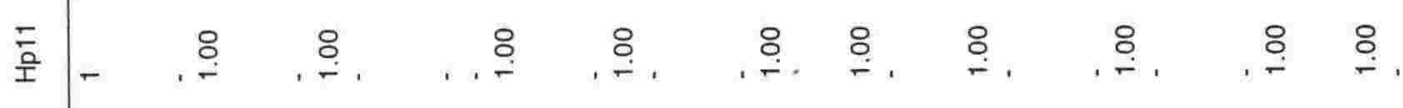

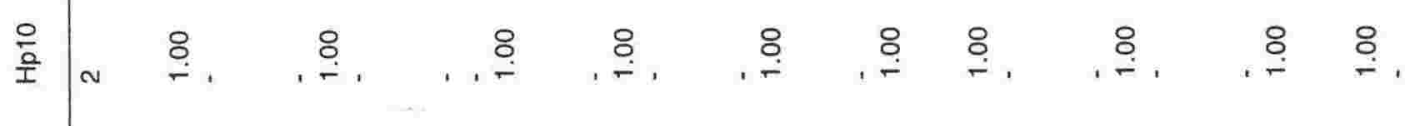

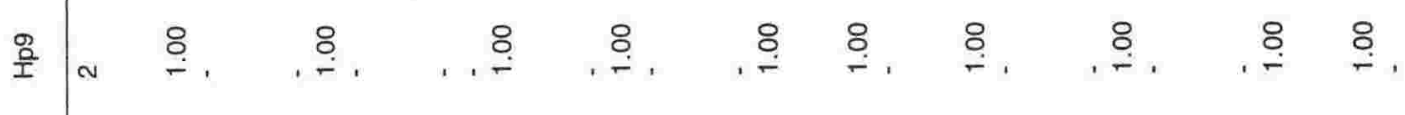

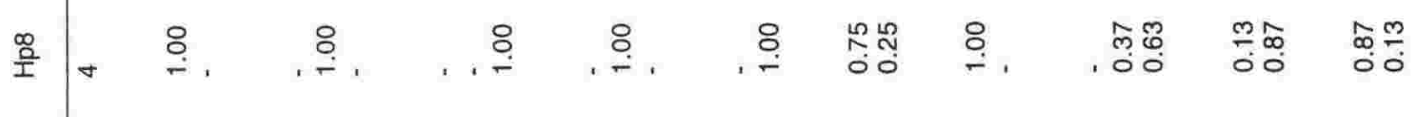

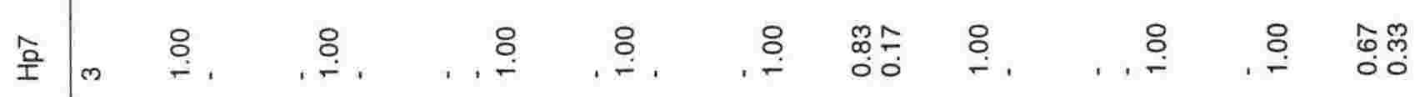

迢

亭鲑

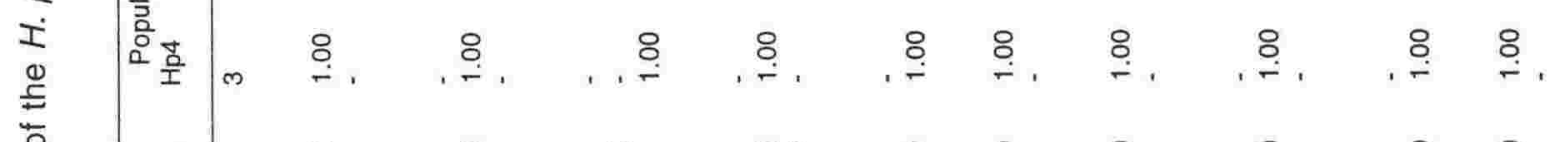

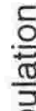

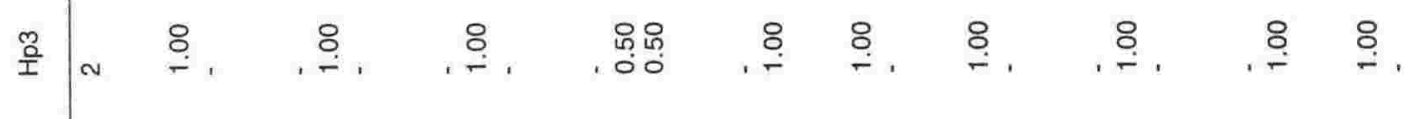

옫

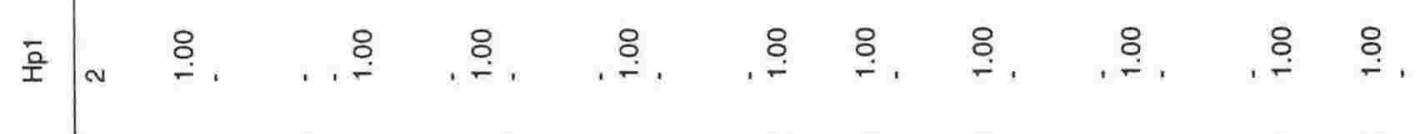

王

童

童

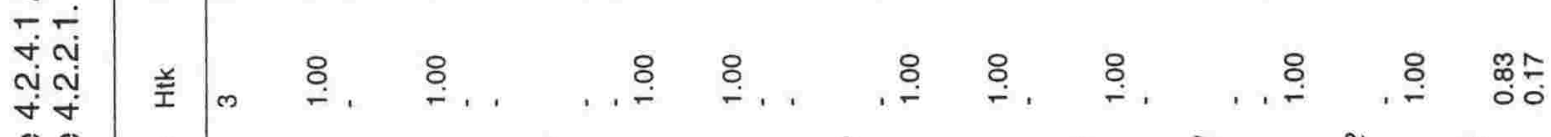

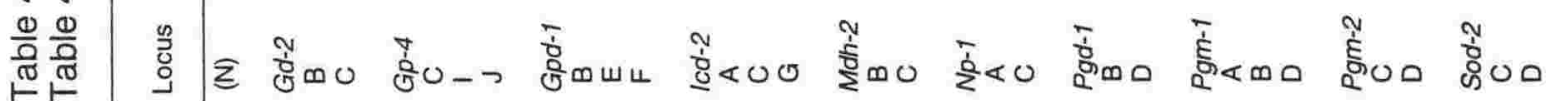


Table 4.2.4.2 measures of polymorphism and heterozygosity in populations of the $H$. pacificus complex (standard errors in parentheses). H. pacificus populations are identified by location only.

\begin{tabular}{|c|c|c|c|c|c|}
\hline \multirow[b]{2}{*}{ Population } & \multirow{2}{*}{$\begin{array}{l}\text { Mean } \\
\text { sample } \\
\text { size per } \\
\text { locus }\end{array}$} & \multirow{2}{*}{$\begin{array}{l}\text { Mean no. } \\
\text { of alleles } \\
\text { per locus }\end{array}$} & \multirow{2}{*}{$\begin{array}{l}\text { Percentage } \\
\text { of loci } \\
\text { polymorphic }\end{array}$} & \multicolumn{2}{|c|}{ Mean heterozygosity } \\
\hline & & & & $\begin{array}{l}\text { direct- } \\
\text { count }\end{array}$ & $\begin{array}{l}\text { hdywbg } \\
\text { expected }\end{array}$ \\
\hline H. "Three Kings" & $\begin{array}{l}3.0 \\
(0.0)\end{array}$ & $\begin{array}{l}1.0 \\
(0.0)\end{array}$ & 3.7 & $\begin{array}{l}0.01 \\
(0.01)\end{array}$ & $\begin{array}{l}0.01 \\
(0.01)\end{array}$ \\
\hline H. "Poor Knights" (Aorangi) & $\begin{array}{l}5.0 \\
(0.0)\end{array}$ & $\begin{array}{l}1.0 \\
(0.0)\end{array}$ & 3.7 & $\begin{array}{c}0.01 \\
(0.01)\end{array}$ & $\begin{array}{l}0.02 \\
(0.02)\end{array}$ \\
\hline H. "Poor Knights" (Sugarloaf) & $\begin{array}{l}3.0 \\
(0.0)\end{array}$ & $\begin{array}{l}1.0 \\
(0.0)\end{array}$ & 3.7 & $\begin{array}{c}0.01 \\
(0.01)\end{array}$ & $\begin{array}{l}0.02 \\
(0.02)\end{array}$ \\
\hline H. "Matapia" & $\begin{array}{l}3.0 \\
(0.0)\end{array}$ & $\begin{array}{l}1.1 \\
(0.1)\end{array}$ & 7.4 & $\begin{array}{c}0.03 \\
(0.03)\end{array}$ & $\begin{array}{l}0.04 \\
(0.03)\end{array}$ \\
\hline Matapia Island & $\begin{array}{l}2.0 \\
(0.0)\end{array}$ & $\begin{array}{l}1.0 \\
(0.0)\end{array}$ & 0.0 & $\begin{array}{c}0.00 \\
(0.00)\end{array}$ & $\begin{array}{l}0.00 \\
(0.00)\end{array}$ \\
\hline Cape Reinga & $\begin{array}{l}3.0 \\
(0.0)\end{array}$ & $\begin{array}{l}1.0 \\
(0.0)\end{array}$ & 3.7 & $\begin{array}{c}0.01 \\
(0.01)\end{array}$ & $\begin{array}{l}0.01 \\
(0.01)\end{array}$ \\
\hline Waitiki Landing & $\begin{array}{l}2.0 \\
(0.0)\end{array}$ & $\begin{array}{l}1.0 \\
(0.0)\end{array}$ & 3.7 & $\begin{array}{c}0.04 \\
(0.04)\end{array}$ & $\begin{array}{l}0.03 \\
(0.03)\end{array}$ \\
\hline Little Barrier Island & $\begin{array}{l}3.0 \\
(0.0)\end{array}$ & $\begin{array}{l}1.0 \\
(0.0)\end{array}$ & 0.0 & $\begin{array}{c}0.00 \\
(0.00)\end{array}$ & $\begin{array}{l}0.00 \\
(0.00)\end{array}$ \\
\hline Pupuha Island (Chickens) & $\begin{array}{l}8.0 \\
(0.0)\end{array}$ & $\begin{array}{l}1.0 \\
(0.0)\end{array}$ & 3.7 & $\begin{array}{c}0.01 \\
(0.01)\end{array}$ & $\begin{array}{l}0.01 \\
(0.01)\end{array}$ \\
\hline Stack One (Chickens) & $\begin{array}{l}3.0 \\
(0.0)\end{array}$ & $\begin{array}{l}1.0 \\
(0.0)\end{array}$ & 3.7 & $\begin{array}{c}0.00 \\
(0.00)\end{array}$ & $\begin{array}{l}0.02 \\
(0.02)\end{array}$ \\
\hline Muriwhenua Island (Chickens) & $\begin{array}{l}3.0 \\
(0.0)\end{array}$ & $\begin{array}{l}1.1 \\
(0.1)\end{array}$ & 7.4 & $\begin{array}{c}0.04 \\
(0.03)\end{array}$ & $\begin{array}{l}0.03 \\
(0.02)\end{array}$ \\
\hline Wareware Island (Chickens) & $\begin{array}{l}4.0 \\
(0.0)\end{array}$ & $\begin{array}{l}1.1 \\
(0.1)\end{array}$ & 14.8 & $\begin{array}{c}0.03 \\
(0.02)\end{array}$ & $\begin{array}{l}0.05 \\
(0.03)\end{array}$ \\
\hline Hen Island & $\begin{array}{l}2.0 \\
(0.0)\end{array}$ & $\begin{array}{l}1.0 \\
(0.0)\end{array}$ & 0.0 & $\begin{array}{c}0.00 \\
(0.00)\end{array}$ & $\begin{array}{l}0.00 \\
(0.00)\end{array}$ \\
\hline Castle Island & $\begin{array}{l}2.0 \\
(0.0)\end{array}$ & $\begin{array}{l}1.0 \\
(0.0)\end{array}$ & 0.0 & $\begin{array}{l}0.00 \\
(0.00)\end{array}$ & $\begin{array}{l}0.00 \\
(0.00)\end{array}$ \\
\hline Hongiora Island (Aldermen) & $\begin{array}{l}1.0 \\
(0.0)\end{array}$ & $\begin{array}{l}1.0 \\
(0.0)\end{array}$ & 0.0 & $\begin{array}{c}0.00 \\
(0.00)\end{array}$ & $\begin{array}{l}0.00 \\
(0.00)\end{array}$ \\
\hline Raumahuanui Is (Aldermen) & $\begin{array}{l}2.0 \\
(0.0)\end{array}$ & $\begin{array}{l}1.0 \\
(0.0)\end{array}$ & 0.0 & $\begin{array}{c}0.00 \\
(0.00)\end{array}$ & $\begin{array}{l}0.00 \\
(0.00)\end{array}$ \\
\hline Coromandel & $\begin{array}{l}1.0 \\
(0.0)\end{array}$ & $\begin{array}{l}1.0 \\
(0.0)\end{array}$ & 0.0 & $\begin{array}{c}0.00 \\
(0.00)\end{array}$ & $\begin{array}{l}0.00 \\
(0.00)\end{array}$ \\
\hline Paeroa & $\begin{array}{l}1.0 \\
(0.0)\end{array}$ & $\begin{array}{l}1.0 \\
(0.0)\end{array}$ & 0.0 & $\begin{array}{l}0.00 \\
(0.00)\end{array}$ & $\begin{array}{l}0.00 \\
(0.00)\end{array}$ \\
\hline Karewa Island & $\begin{array}{l}2.0 \\
(0.0)\end{array}$ & $\begin{array}{l}1.0 \\
(0.0)\end{array}$ & 3.7 & $\begin{array}{l}0.04 \\
(0.04)\end{array}$ & $\begin{array}{l}0.03 \\
(0.03)\end{array}$ \\
\hline
\end{tabular}


$H$. "Matapia" showed minimal allozyme separation from $H$. pacificus overall (Nei's $D=0.01-0.07$ ). Some between-population genetic distances within $H$. pacificus were as high (Nei's $D=0.00-0.14$ ) as distances between $H$. "Three Kings" and $H$. pacificus populations (Nei's $D=0.08-0.16$ ), $H$. "Poor Knights" and $H$. pacificus populations (Nei's $D=0.04-0.19$ ), and $H$. "Three Kings" and H. "Poor Knights" (Nei's D = 0.11-0.12) (Nei's I and D, Table 4.2.4.3, and Cavalli-Sforza \& Edwards' arc distance and Rogers' D, Table 4.2.4.4).

Within the $H$. pacificus complex, $H$. "Three Kings" and $H$. "Poor Knights" are sister groups in WPGMA (Figure 4.2.4.1), distance Wagner (Figure 4.2.4.2) (BIOSYS package), and neighbour joining (Figure 4.2.4.3) (MEGA package) phenograms, and a maximum parsimony cladogram (Figure 4.2.4.4) (PAUP package), but the edge uniting this pair to the exclusion of others is short. This pair is the sister group of $H$. pacificus plus $H$. "Matapia". H. pacificus is paraphyletic with respect to $H$. "Matapia" in all analyses.

\subsubsection{DISCUSSION}

The H. pacificus complex is well differentiated from the rest of Hoplodactylus both genetically and morphologically (Chapter 5 ). Within the group there is little morphological or allozyme diversity relative to that found in the $H$. maculatus or $\mathrm{H}$. granulatus complexes. Only the sympatry of the 2 morphologically distinct populations on Matapia Island, and the 2 apparently fixed allozyme differences between them, suggest that the group represents anything other than a somewhat geographically variable species with divergent allopatric populations on the long-isolated Poor Knights and Three Kings groups. However, the separate species status of the 2 Matapia populations is indisputable, regardless of species concept. The problem is to decide which of these 2 populations should be regarded as $H$. pacificus, and which as a new species.

The patterns of genetic and morphological divergence of these 2 populations from the rest of $H$. pacificus appear contradictory. Both Matapia populations fall within the cluster of $H$. pacificus from North Island mainland and landbridge island populations in phenograms, and the allozyme evidence shows no division within this group of populations corresponding with an obvious species boundary. $H$. "Matapia" is genetically very similar (D $=0.01$ ) to $H$. pacificus populations from Little Barrier Island, Wareware Island (Chickens), Hen Island, Coromandel, and Paeroa. It is morphologically distinct from these in body size, colour, and in most cases colour pattern, 


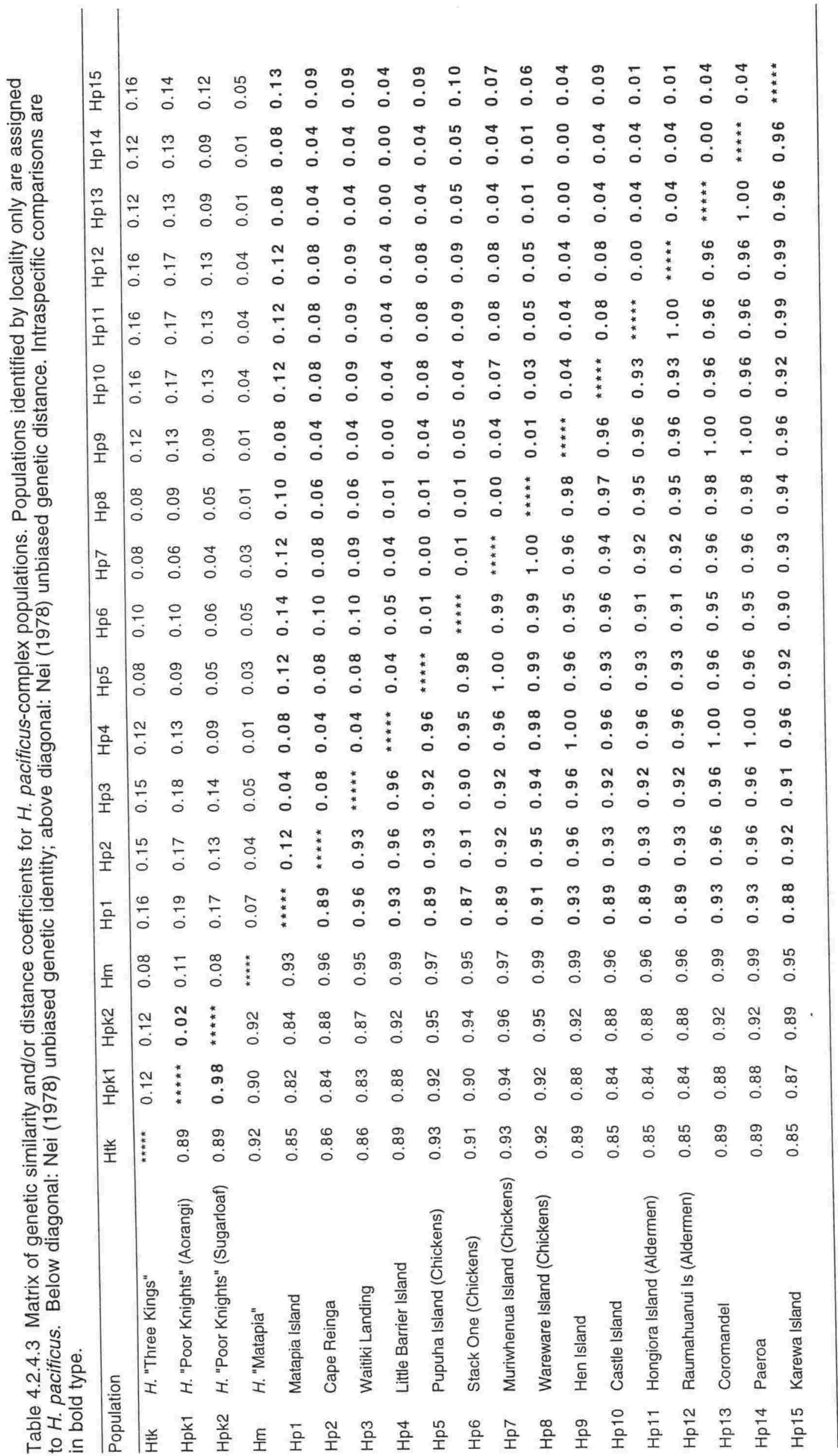


일

要

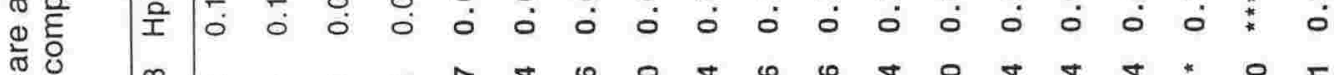

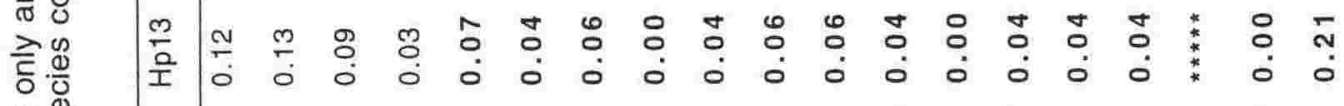

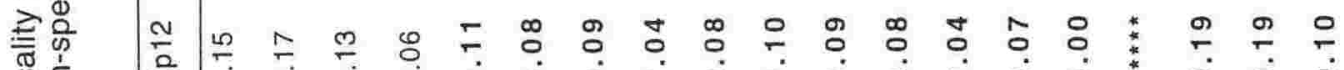
응

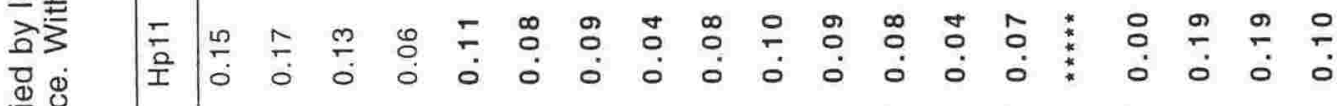
窇党

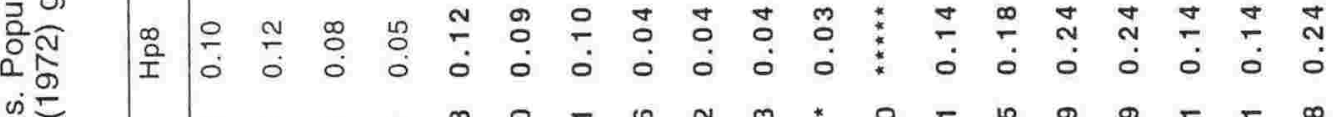

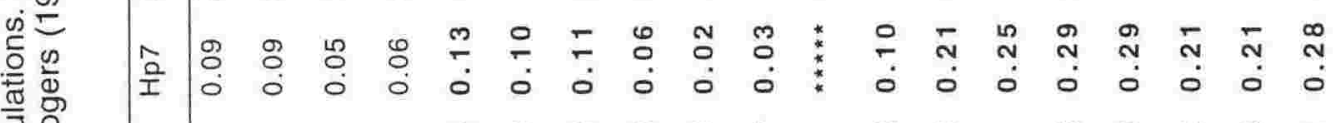

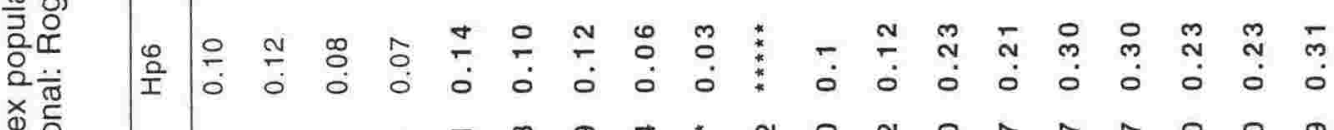
U. 结雚 I茎

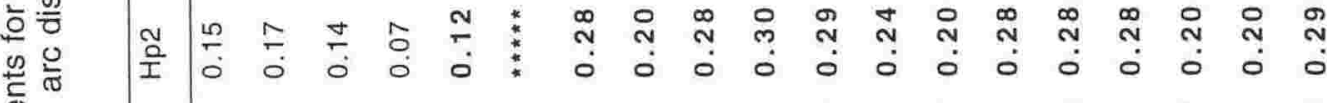
恶余

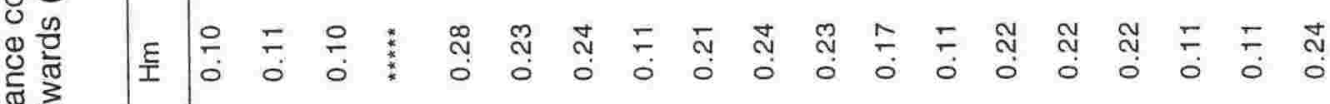

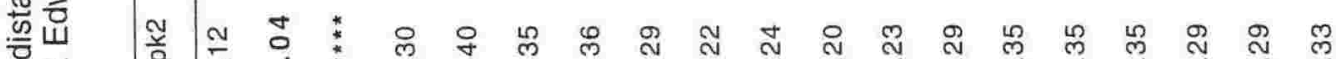

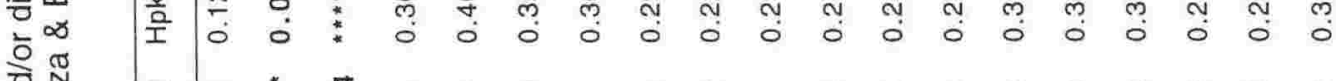

可苟 ह

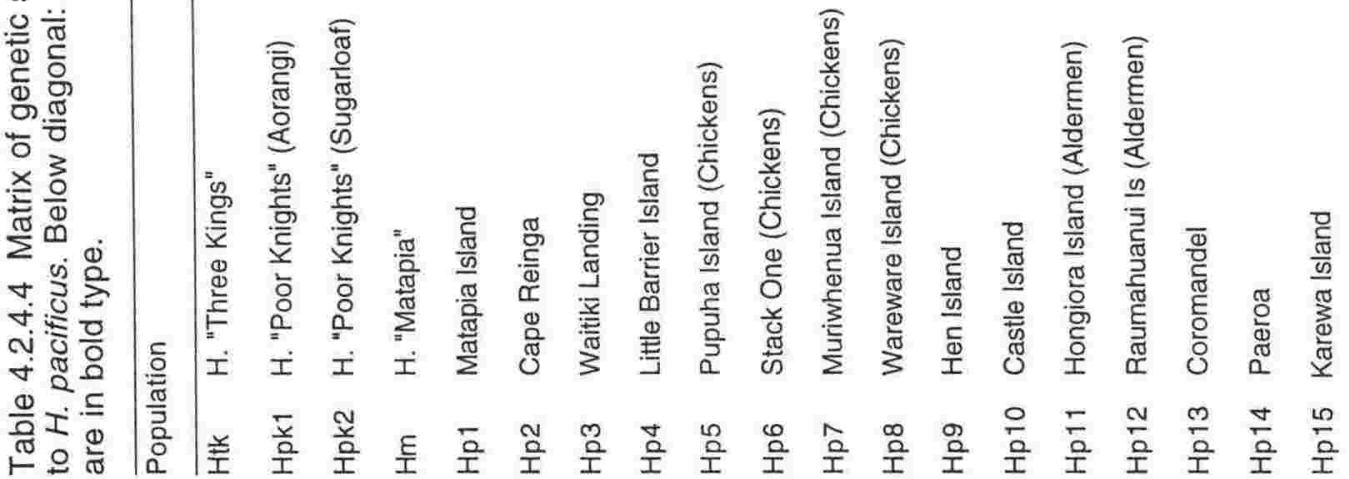




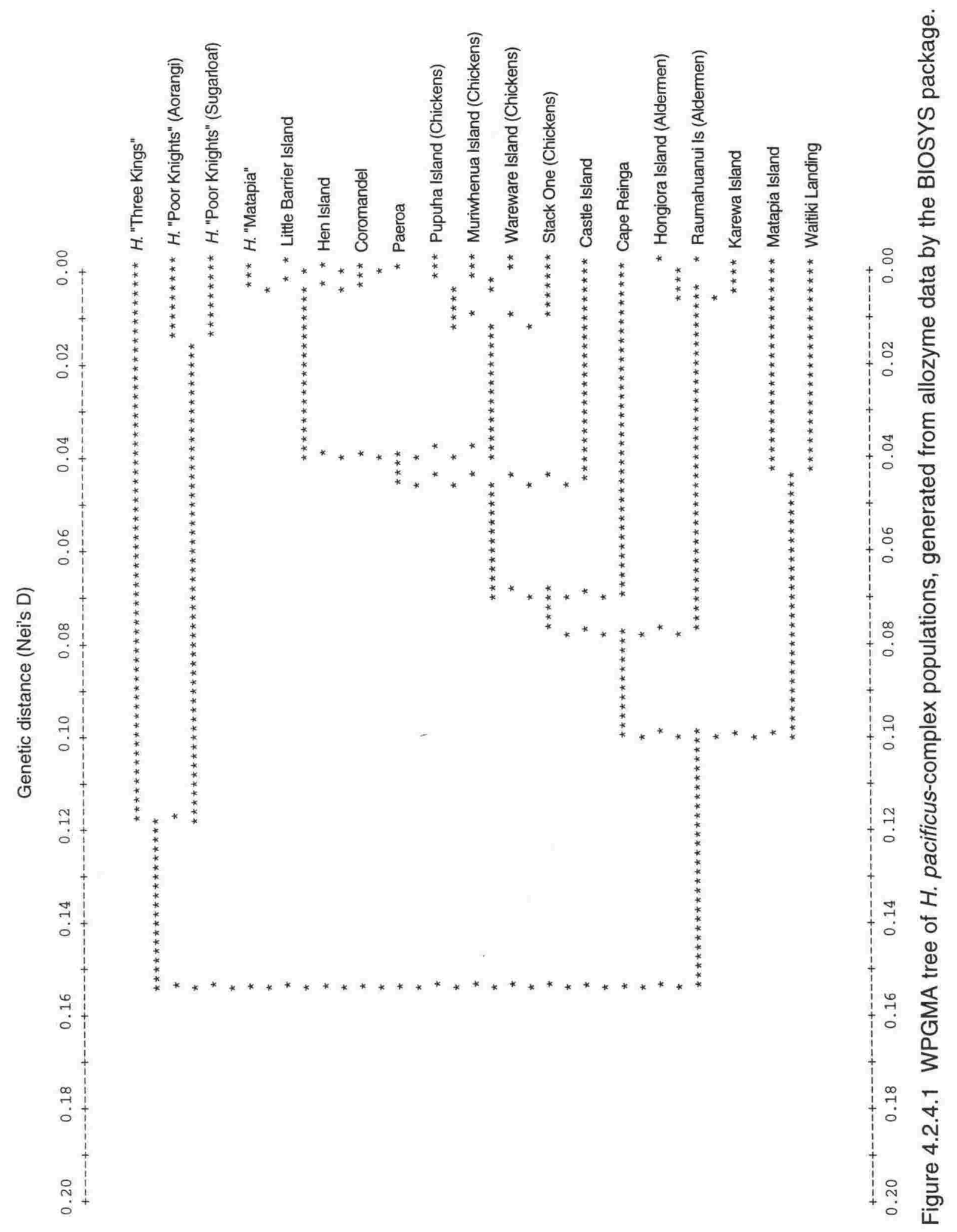




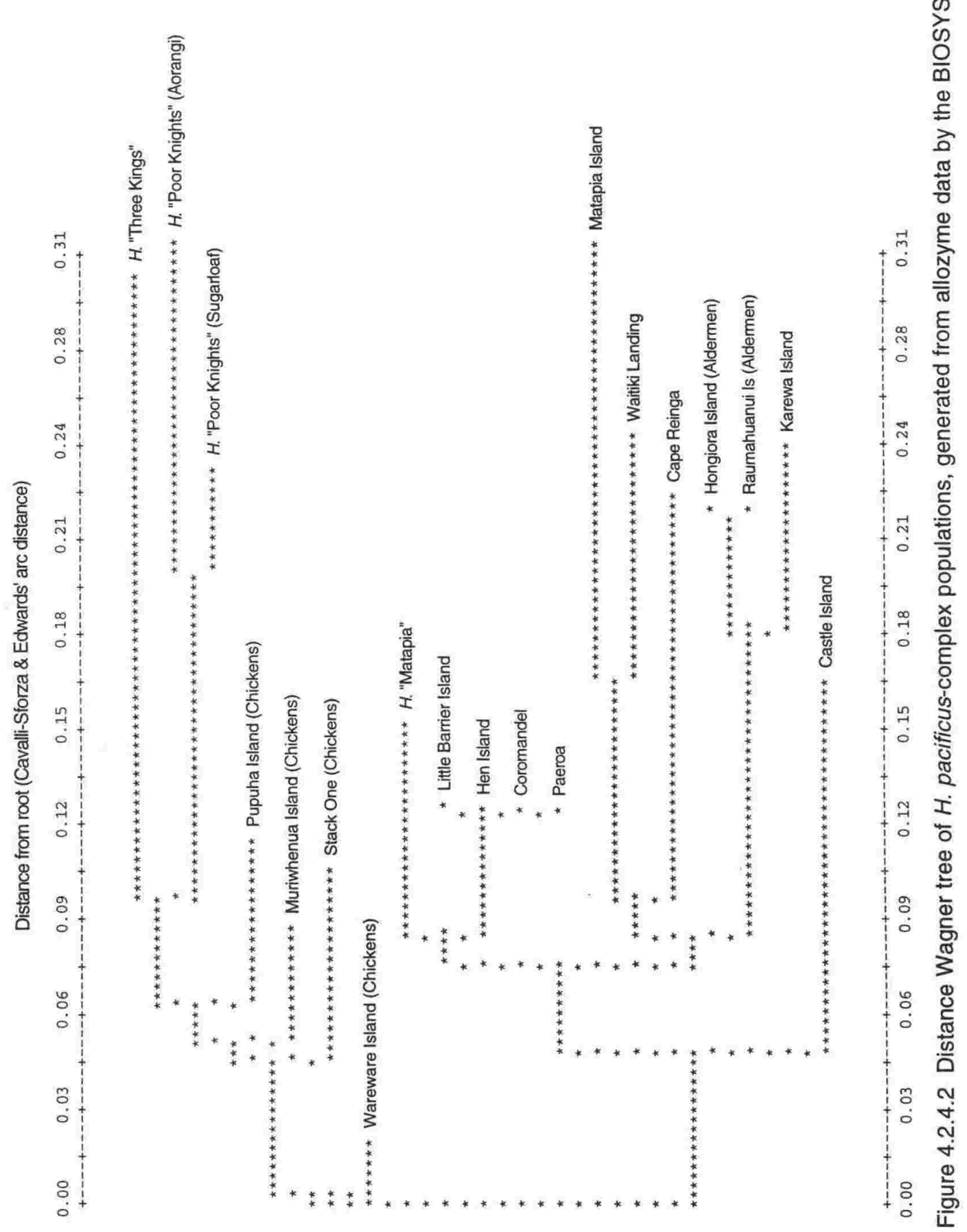




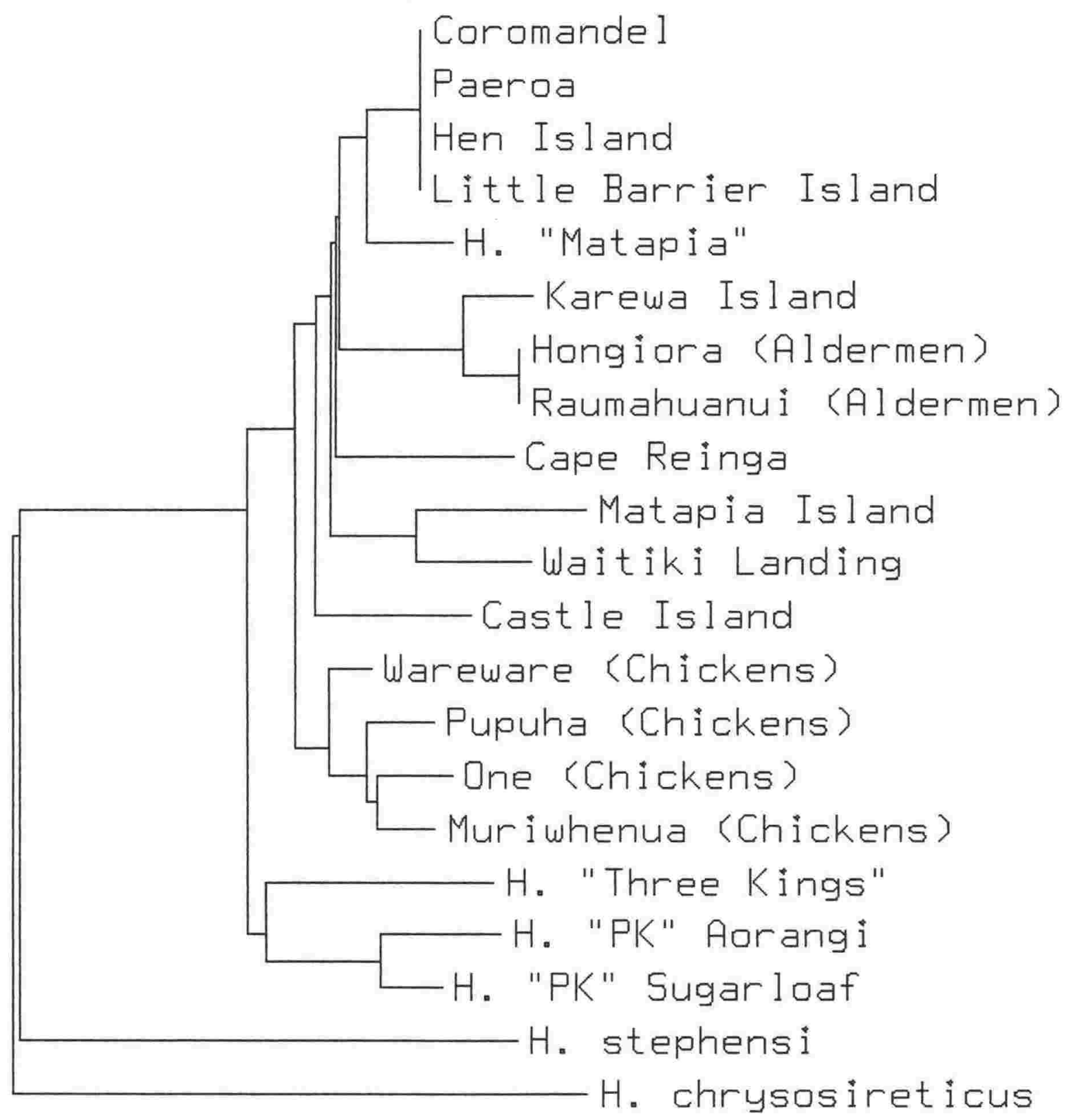

Figure 4.2.4.3 neighbour joining tree of $H$. pacificus-complex populations, generated from allozyme data by the MEGA package. $H$. stephensi and the Taranaki population of $H$. chrysosireticus are included as outgroups. 


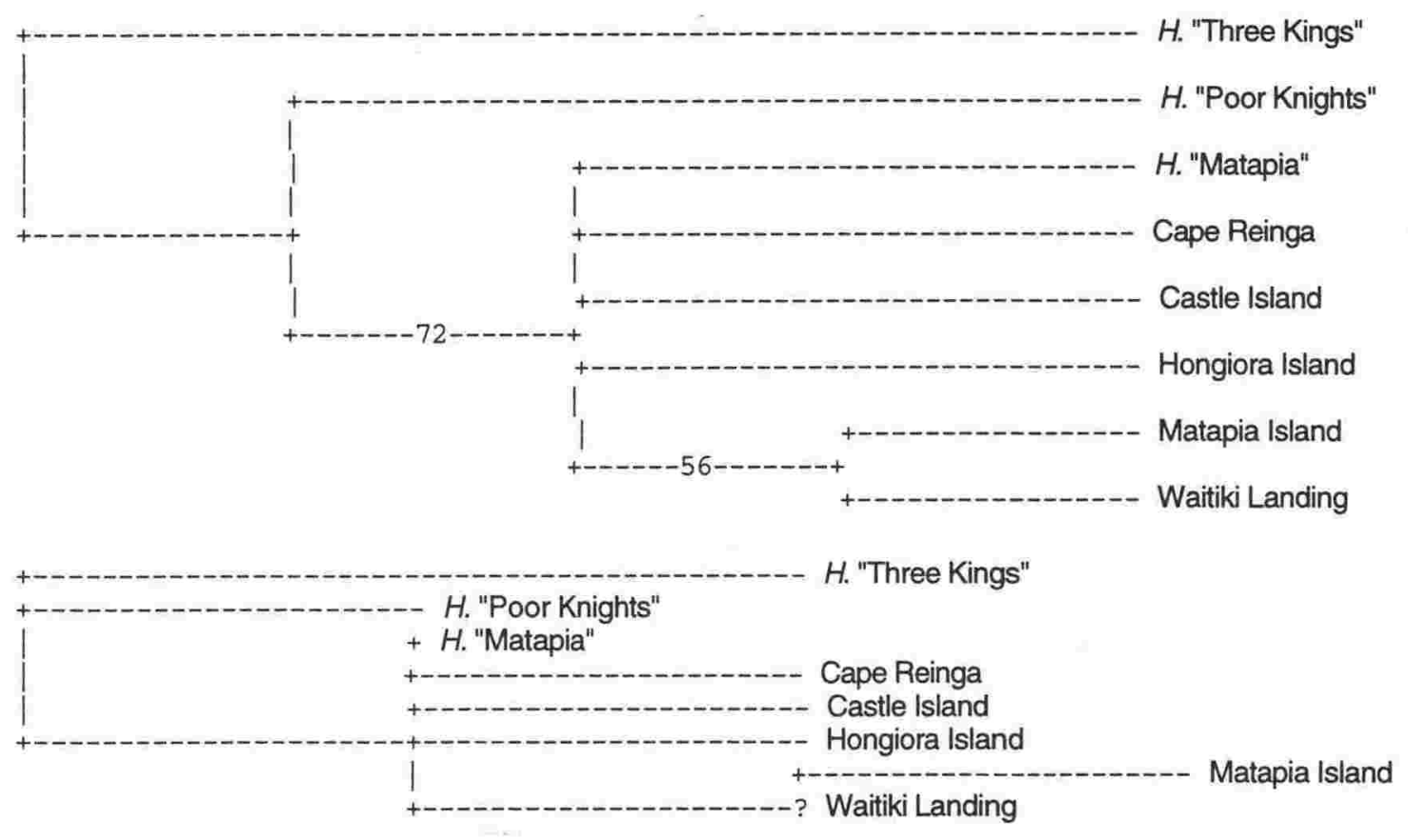

Figure 4.2.4.4 Bootstrap 50\% majority-rule consensus maximum parsimony cladogram and phylogram of $H$. pacificus-complex populations, generated from allozyme data by the PAUP package. H. pacificus populations are identified by location only. Bootstrap values are indicated on branches of the cladogram. Tree length $=16$; Consistency index $(\mathrm{Cl})=0.750 ; \mathrm{Cl}$ excluding uninformative characters $=0.667$; Retention index $(\mathrm{RI})=0.333$; Rescaled consistency index $(R C)=0.250 ; f$ value $=0 ; f$-ratio $=0.0000 ;$ (multistate unordered and stepmatrix characters excluded from f-value calculations).

although one specimen from Wareware Island had a very similar striped pattern. Matapia Island $H$. pacificus are separated by a slightly larger genetic distance $(D=0.04)$ from the genetically most similar population, which is the geographically closest mainland population (Waitiki Landing), and are morphologically typical $H$. pacificus. This genetic separation is generated by one apparently fixed difference and one locus where 2 alleles were detected in the Waitiki Landing population but only one of them in the Matapia population, a degree of genetic difference common among allopatric populations within species in this and other studies.

In the absence of significant genetic evidence for specific separation between either of the sympatric Matapia populations and the rest of $H$. pacificus, the morphological and ecological/behavioural distinctiveness of the smaller striped taxon justifies its recognition as H. n. sp. "Matapia". 
Is $H$. pacificus paraphyletic with respect to $H$. "Matapia"? The phenetic trees generated from allozyme data certainly indicate this. However, the presence in H. "Matapia" of the Pgm-1(a) and Gp-4(c) alleles, both shared with outgroups, but with no $H$. pacificus population, suggests that a sister-group relationship might be revealed by more sensitive analyses using more informative datasets such as DNA sequences.

If the root of the group is assumed to be between $H$. "Poor Knights" plus $H$. "Three Kings" and $H$. pacificus plus $H$. "Matapia" (or between $H$. "Poor Knights" and $H$. "Three Kings"), the most basal group of $H$. pacificus populations in neighbour-joining and DISWAG phenograms is the Chickens Islands group, and $H$. pacificus must be paraphyletic to $H$. "Matapia". However, if $H$. "Matapia" is the sister group of $H$. pacificus, then the basal position of the Chickens group of populations cannot represent an ancestral condition. The basal position of this group, its high levels of polymorphism compared with other populations, and its allelic composition are all consistent with introgression from $\mathrm{H}$. "Poor Knights" or a closely related population. Removing these populations from clustering analyses did not change the topology of the trees, except to increase slightly the separation between $H$. "Poor Knights" $+H$. "Three Kings" and H. pacificus $+H$. "Matapia".

Although the relationship between $H$. pacificus and $H$. "Matapia" is uncertain, there is strong evidence from the allozyme analyses that $H$. "Three Kings" and $H$. "Poor Knights" are the sister group of this pair. These 2 populations are genetically only slightly more similar to each other than they are to $H$. pacificus, and they are each morphologically diagnosable, so each is considered to justify separate species status.

\subsection{THE H. MACULATUS COMPLEX}

\subsubsection{INTRODUCTION}

Since at least the late 1970s it has been clear that in several areas of New Zealand 2 taxa of geckos, distinct in size and colour but both referable to Hoplodactylus maculatus (sensu Robb \& Rowlands 1977), are sympatric or parapatric. During field research on Hoplodactylus kahutarae in the Seaward Kaikoura Ranges, Whitaker (1984b) found sympatric, morphologically distinct large and small taxa of this complex. G. Patterson (pers. comm.) found similar small geckos at Gorge Burn in the Eyre Mountains in 1982, and this form was later found in other nearby areas of 
north-western Southland and south-western Otago (Whitaker 1986). In other areas morphologically distinct populations are parapatric but not sympatric.

Robb (1982, p. 319, and pers. comm.) considered that a specimen from 5000 feet $(\mathrm{c} .1500 \mathrm{~m}$ ) altitude in the Old Man Range in the collection of the Otago Museum was sufficiently distinct morphologically from $\mathrm{H}$. maculatus (sensu Robb \& Rowlands 1977) that it could not be referred to that species, and represented a new species.

Because of this morphological diversity, and the presence of 2 sympatric morphospecies in at least 2 widely separated areas, allozyme electrophoresis was used to detect patterns of genetic relationships between populations. Congruence between genetic and morphological divergence in sympatry or at parapatric boundaries was used to detect groups of populations which were judged to be on separate evolutionary trajectories, and therefore separate species under the concept followed in this thesis.

Analysis of allozyme variation has confirmed the existence of a complex of at least 10 undescribed species within Hoplodactylus maculatus sensu Robb \& Rowlands (1977). This variation is described below. There is a hierarchy of levels of differentiation within the complex, and whereas some species are readily identifiable by morphology (e.g., $H$. "Southern mini"), others, although distinct genetically, are more difficult to diagnose morphologically because of very high levels of within-species, between-population variation.

\subsubsection{MAJOR GROUPS WITHIN THE COMPLEX}

Analysis of allozyme data from populations of the complex reveals that $H$. "Southern mini" is more closely related to $H$. chrysosireticus than to other members of the $H$. maculatus complex (Chapter 5). Among the other populations there is a primary split between northern and southern groups (Table 4.3.2.1; Chapter 5). That the northern and southern species groups of the complex are not conspecific by any species concept is apparent from their broad sympatry, including microsympatry in parts of southern Marlborough and northern Canterbury. The groups do not correspond on any broad geographic scale to the small/large dichotomy detected in sympatric populations. Where they are sympatric, the northern and southern groups are differentiated by fixed differences at at least 6 loci. These 3 groups are considered separately below. 
Table 4.3.2.1 Species groups of the $H$. maculatus complex.

H. chrysosireticus group:
H. chrysosireticus
$H$. "Southern mini"

Northern group:

H. maculatus

H. "Mount Arthur"

H. "Kaikouras"

H. "Marlborough mini"

Southern group:

H. duvaucelii

H. brunneus

H. "Southern Alps"

H. "Danseys Pass"

H. "Cromwell Gorge"

H. "Otago"

\subsection{HOPLODACTYLUS "SOUTHERN MINI"}

\subsubsection{INTRODUCTION}

As described in section 4.3.1, small, dull olive-brown geckos are sympatric with more typical $H$. maculatus sensu Robb \& Rowlands (1977) at Gorge Burn (Eyre Mountains) and in nearby areas of north-western Southland and south-western Otago (G. Patterson pers. comm.; Whitaker 1986). As this sympatry made it clear that this form was a separate species from $H$. maculatus, its relationships were investigated.

\subsubsection{SAMPLING}

$H$. "Southern mini" was collected from the Eyre, Remarkables, and Slate Ranges (Figure 4.4.2.1) $(n=13)$.

\subsubsection{ALLOZYMES}

No polymorphism or geographic variation was found within $H$. "Southern mini". It is distantly related to other species of the $H$. maculatus complex (7-10 fixed allozyme differences from 26 loci - see Table 5.5.2; Nei's D =0.40-0.66). H. "Southern mini" has 3 diagnostic alleles (Gp-5(b), Np-1(e), Sod-3(c)). 


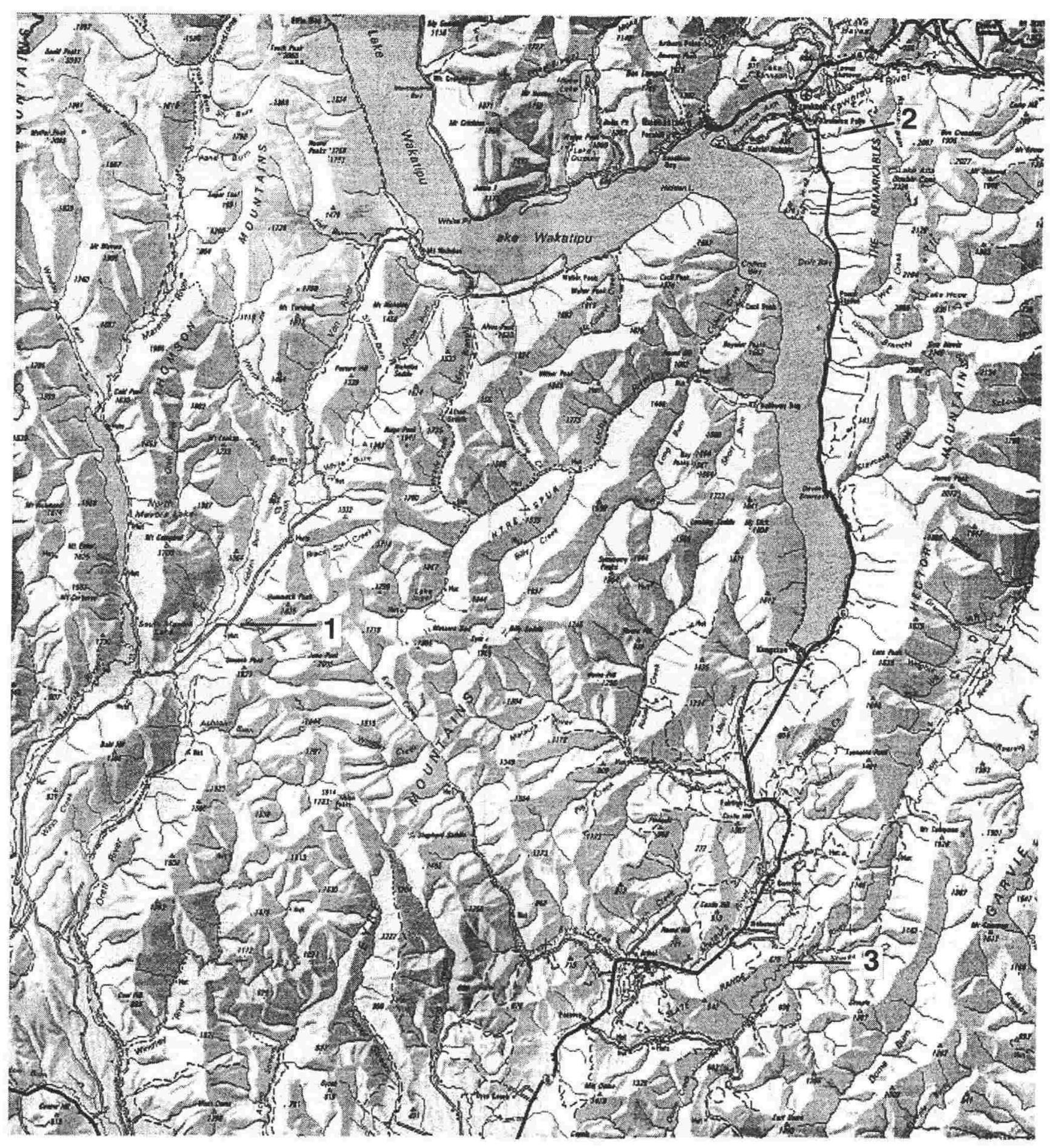

Figure 4.4.2.1.. Collecting localities for $H$. "Southern mini". 1, Gorge Burn, Eyre Mountains; 2, Remarkables Range; 3, Nokomai Station. 
Allozyme electrophoresis supports a sister-group relationship with $H$. chrysosireticus, but the allozyme distance between them is substantial (Nei's $D=$ $0.38-0.40 ; 10$ apparently fixed differences- Table 5.5.2), and only $G d-2(a)$ is diagnostic of this pair.

\subsubsection{MORPHOLOGY}

Morphologically, $H$. "Southern mini" is most similar to $H$. chrysosireticus rather than other species of the $H$. maculatus complex. They share the same basic colour pattern (although $H$. "Southern mini" never shows the bold longitudinal stripes seen in many $\mathrm{H}$. chrysosireticus), broad, shallow rostral, single conical cloacal spur in males, and broadly dilated toe-pads. They differ in the smaller size, stockier build, shorter distal phalanges, and lower number of preanal organs in $H$. "Southern mini".

\subsubsection{DISCUSSION}

$H$. "Southern mini" is sympatric throughout its known range with $H$. "Otago", from which it can be readily distinguished by its small size, drab colour pattern, and broad, shallow rostral. It has been collected less than $20 \mathrm{~km}$ from the closest known population of $\mathrm{H}$. "Cromwell Gorge", and the distribution of the 2 in the area between these locations is unknown. Apart from size, the characters separating it from $\mathrm{H}$. "Otago" also distinguish it from $H$. "Cromwell Gorge". It and its probable sister species $H$. chrysosireticus are allopatric, but the substantial allozyme divergence between them, coupled with diagnostic morphological differences justify recognition of $H$. "Southern mini" as a new species.

\subsection{The Hoplodactylus maculatus complex, Northern Group}

\subsubsection{INTRODUCTION}

I include 4 species, $H$. "Mount Arthur", $H$. "Kaikouras", $H$. "Marlborough mini", and $H$. maculatus itself in this group. They form a monophyletic clade within the $H$. maculatus complex (Chapter 5).

Within the northern group of the $H$. maculatus complex, the presence of substantial size differences between populations in different habitats separated by small geographic distances has been noted in the popular literature (Waddington 1970), but the only suggestion of specific-level subdivision has been the informal separation of inland Marlborough populations of $H$. "Marlborough mini" on the basis of their sympatry with populations of $H$. "Southern Alps" (Whitaker 1984b). Their close relationship with some coastal populations of small animals was not recognised. To 
reduce possible confusion in the presentation of results below, populations are referred to by their final species identity as well as locality. This does not imply that a priori decisions about species identity preceded allozyme analysis.

\subsubsection{SAMPLING}

$H$. maculatus complex, northern group populations were sampled from South Island localities from the Hurunui River valley north, from many southern North Island localities, and from offshore islands in Cook Strait and north-east of the North Island (Figures 4.5.2.1, 4.5.2.2; Table 4.5.2.1).

Samples were taken from 219 specimens from 72 populations.

\subsubsection{ALLOZYMES}

Within the northern group of the $H$. maculatus complex, variation was found in 18 of the 27 allozyme loci surveyed (Table 4.5.3.1).

At 6 of these 18 loci, variation was not taxonomically informative. Ak-1, Got-1, $\mathrm{Ldh}-2$, and $\mathrm{Me}-1$ showed only rare alleles in single individuals, and are not considered further. Gpi-1 variation was detected only in the Ward population of $H$. "Kaikouras", in which 3 of the 4 individuals sampled were homozygous for the allele common in the southern group of the $H$. maculatus complex. $P g m-1$ varied only in the D'Urville Island and nearby Raihonui Stack populations, where $3 \mathrm{Pgm}-1(\mathrm{bd})$ heterozygotes were found. All other populations in the group were fixed for Pgm-1(b).

Three loci showed fixed or almost fixed differences among proposed species of the northern group. Got-2 showed a fixed difference between $H$. "Mount Arthur", which shared the Got-2(a) allele common in the southern group of the $H$. maculatus complex, and the rest of the northern group (Got-2(b)). Pep3 showed an almost fixed difference between $H$. maculatus (Pep-3(c)) and the other 3 species, which shared the $P e p-3(b)$ allele common in the southern group. The single exception was the Red Hills animal, which was homozygous for Pep-3(b). Pgd-1 showed a fixed difference within this group between $H$. "Kaikouras" (Pgd-1(f)) and the other 3 species, which were all fixed for $P g d-1(e)$. Both these alleles were also found in the southern group.

Ck-1, Gd-2, Gp-4, Icd-1, Icd-2, Ldh-1, Np, Pgm-2, and Sod-2 all showed more widespread polymorphisms, with geographic and taxonomic structuring (Figures 4.5.3.1-9). 


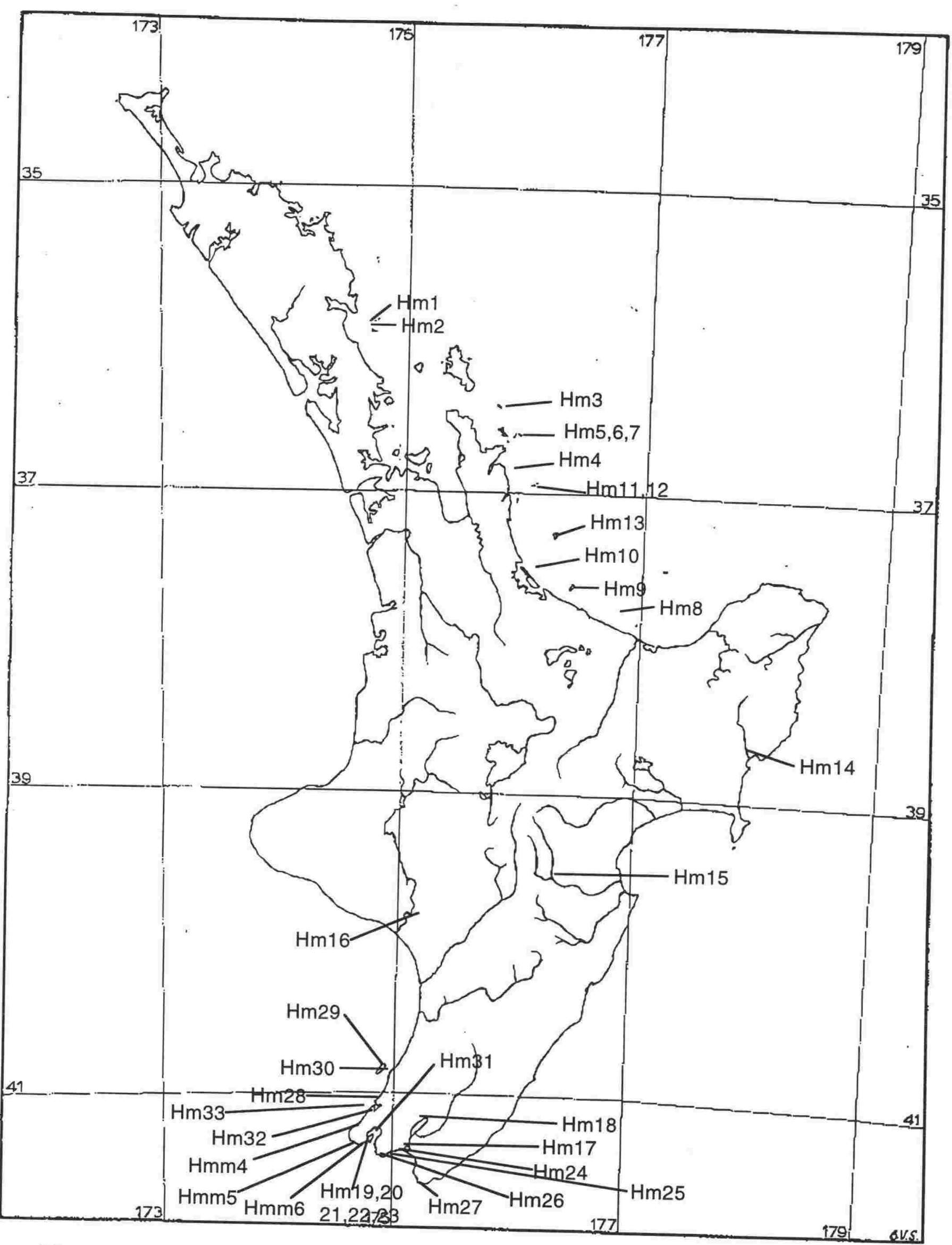

Figure 4.5.2.1 Sampling localities for species of the northern group of the $H$. maculatus species complex in the North Island. For key see Table 4.5.2.1. 


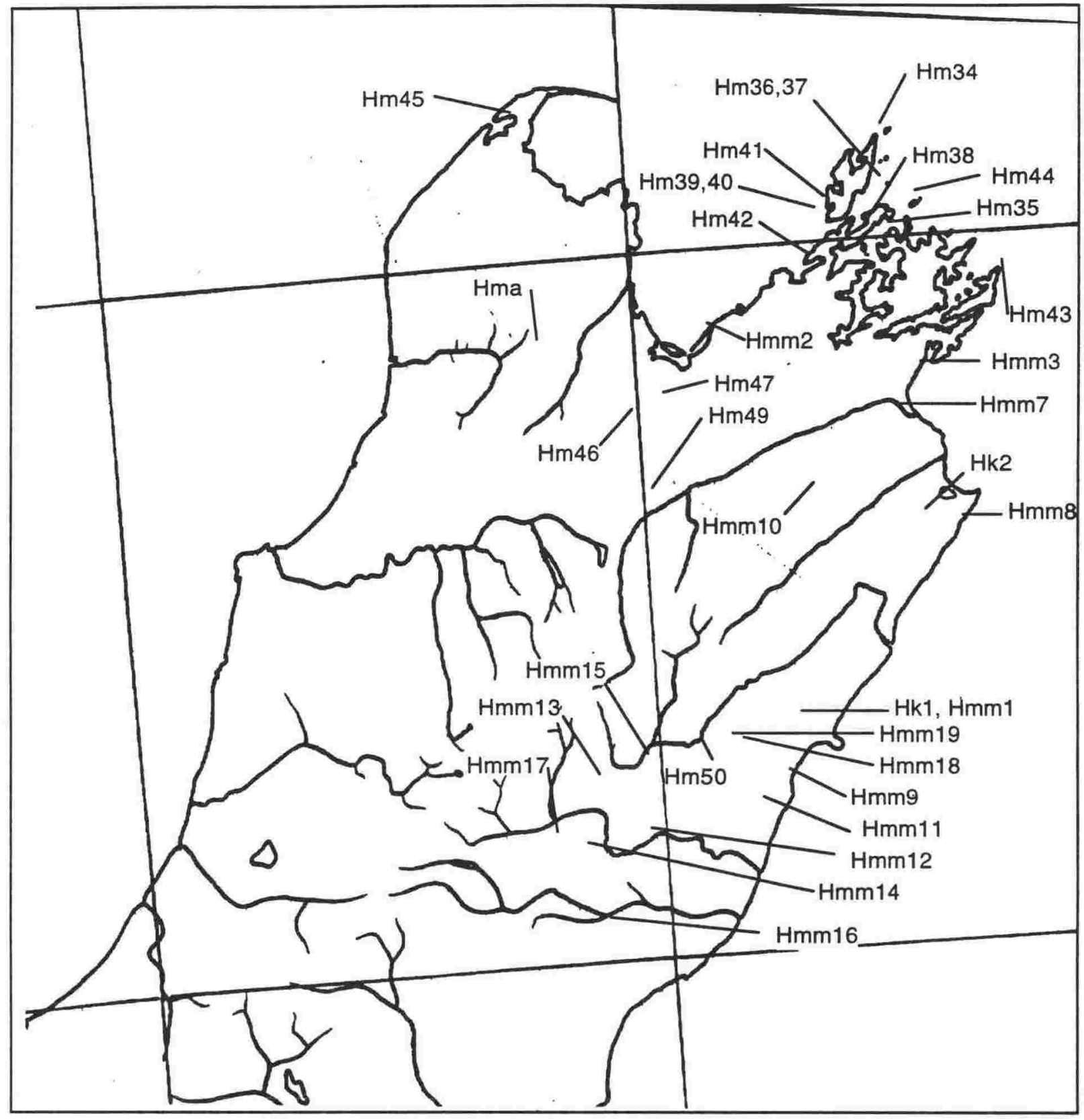

Figure 4.5.2.2 Sampling localities for species of the northern group of the $H$. maculatus species complex in the South Island. For key see Table 4.5.2.1. 
Table 4.5.2.1 Localities and population codes for samples of $H$. maculatus complex, northern group species (refer to Figure 4.5.2.1).

\begin{tabular}{|c|c|c|}
\hline $\begin{array}{l}\text { Population } \\
\text { code }\end{array}$ & Species & Locality \\
\hline $\begin{array}{l}\mathrm{Hma} \\
\mathrm{Hk} 1\end{array}$ & $\begin{array}{l}\text { H. "Mount Arthur" } \\
\text { H. "Kaikouras" }\end{array}$ & Mount Arthur \\
\hline $\begin{array}{l}\text { Hk1 } \\
\text { Hk2 }\end{array}$ & $\begin{array}{l}\text { H. "Kaikouras" } \\
\text { H. "Kaikouras" }\end{array}$ & $\begin{array}{l}\text { Batty } \\
\text { Ward }\end{array}$ \\
\hline Hmm1 & $\begin{array}{l}\text { H. "Kaikouras" } \\
\text { H. "Marlborough mini" }\end{array}$ & $\begin{array}{l}\text { Ward } \\
\text { Batty }\end{array}$ \\
\hline $\mathrm{Hmm} 2$ & H. "Marlborough mini" & $\begin{array}{l}\text { Batty } \\
\text { Glenduan, Nelson }\end{array}$ \\
\hline $\mathrm{Hmm3}$ & H. "Marlborough mini" & $\begin{array}{l}\text { Glenduan, Nelson } \\
\text { Robin Hood Bay }\end{array}$ \\
\hline $\mathrm{Hmm4}$ & H. "Marlborough mini" & $\begin{array}{l}\text { Robin Hood Bay } \\
\text { Makara }\end{array}$ \\
\hline $\begin{array}{l}\text { Hmm5 } \\
\text { Hmm6 }\end{array}$ & H. "Marlborough mini" & $\begin{array}{l}\text { Makara } \\
\text { Red Rocks }\end{array}$ \\
\hline $\begin{array}{l}\mathrm{Hmm6} \\
\mathrm{Hmm} 7\end{array}$ & H. "Marlborough mini" & $\begin{array}{l}\text { Red Rocks } \\
\text { Island Bay }\end{array}$ \\
\hline $\begin{array}{l}\mathrm{Hmm7} \\
\mathrm{Hmm8}\end{array}$ & H. "Marlborough mini" & $\begin{array}{l}\text { Island Bay } \\
\text { Wairau River Mouth }\end{array}$ \\
\hline $\begin{array}{l}\mathrm{Hmm8} \\
\mathrm{Hmm9}\end{array}$ & H. "Marlborough mini" & $\begin{array}{l}\text { Wairau River Mouth } \\
\text { Ward Beach }\end{array}$ \\
\hline $\begin{array}{l}\text { Hmm9 } \\
\text { Hmm10 }\end{array}$ & H. "Marlborough mini" & Goose Bay, Kaikoura \\
\hline $\begin{array}{l}\text { Hmm10 } \\
\text { Hmm11 }\end{array}$ & $\begin{array}{l}\text { H. "Marlborough mini" } \\
H \text {. "Marlborough mini" }\end{array}$ & Waihopai Valley \\
\hline Hmm12 & $\begin{array}{l}\text { H. "Marlborough mini" } \\
\text { H. "Marlborough mini" }\end{array}$ & Hundalee \\
\hline $\mathrm{Hmm13}$ & H. "Marlborough mini" & $\begin{array}{l}\text { Waiau } \\
\text { Jacks Pass }\end{array}$ \\
\hline $\mathrm{Hmm} 14$ & H. "Marlborough mini" & $\begin{array}{l}\text { Jacks Pass } \\
\text { Montrose }\end{array}$ \\
\hline Hmm15 & H. "Marlborough mini" & $\begin{array}{l}\text { Montrose } \\
\text { Acheron Road }\end{array}$ \\
\hline $\begin{array}{l}\mathrm{Hmm} 16 \\
\mathrm{Hmm} 17\end{array}$ & H. "Marlborough mini" & $\begin{array}{l}\text { Acheron Road } \\
\text { Hurunui }\end{array}$ \\
\hline $\begin{array}{l}\mathrm{Hmm17} \\
\mathrm{Hmm} 18\end{array}$ & H. "Marlborough mini" & $\begin{array}{l}\text { Hurunui } \\
\text { Horseshoe Lake }\end{array}$ \\
\hline $\begin{array}{l}\mathrm{Hmm18} \\
\mathrm{Hmm} 19\end{array}$ & H. "Marlborough mini" & Horseshoe Lake 1 , Seaward Kaikoura Range \\
\hline $\begin{array}{l}\mathrm{Hmm19} \\
\mathrm{Hm1}\end{array}$ & H. "Marlborough mini" & Kahutara Saddle 2, Seaward Kaikoura Range \\
\hline $\begin{array}{l}\mathrm{Hm1} \\
\mathrm{Hm} 2\end{array}$ & H. maculatus & Muriwhenua Island, Chickens group \\
\hline $\begin{array}{l}\mathrm{Hm} 2 \\
\mathrm{Hm} 3\end{array}$ & $\begin{array}{l}\text { H. maculatus } \\
\text { H. maculatus }\end{array}$ & Wareware Island, Chickens group \\
\hline $\mathrm{Hm}$ & $\begin{array}{l}\text { H. maculatus } \\
\text { H. maculatus }\end{array}$ & $\begin{array}{l}\text { Cuvier Island } \\
\text { Castle Island }\end{array}$ \\
\hline Hm5 & $\begin{array}{l}\text { H. macularus } \\
\text { H. maculatus }\end{array}$ & $\begin{array}{l}\text { Castle Island } \\
\text { Middle Island, Mercury group }\end{array}$ \\
\hline Hm6 & H. maculatus & $\begin{array}{l}\text { Middle Island, Mercury group } \\
\text { Green Island, Mercury group }\end{array}$ \\
\hline $\mathrm{Hm7}$ & H. maculatus & $\begin{array}{l}\text { Green Island, Mercury group } \\
\text { Korapuke Island, Mercury group }\end{array}$ \\
\hline $\mathrm{Hm} 8$ & H. maculatus & $\begin{array}{l}\text { Korapuke Island, Mercury group } \\
\text { Moutoki Island }\end{array}$ \\
\hline $\mathrm{Hm9}$ & H. maculatus & $\begin{array}{l}\text { Moutoki Island } \\
\text { Plate Island }\end{array}$ \\
\hline Hm10 & H. maculatus & Karewa Island \\
\hline $\begin{array}{l}\mathrm{Hm} 11 \\
\mathrm{Hm} 12\end{array}$ & H. maculatus & Hernia Island \\
\hline $\begin{array}{l}\mathrm{Hm} 12 \\
\mathrm{Hm} 13\end{array}$ & H. maculatus & Hongiora Island, Aldermen group \\
\hline $\begin{array}{l}\mathrm{Hm} 13 \\
\mathrm{Hm} 14\end{array}$ & H. maculatus & Mayor Island \\
\hline $\begin{array}{l}\mathrm{H} \text { H14 } \\
\mathrm{Hm} 15\end{array}$ & H. maculatus & Gisborne \\
\hline $\begin{array}{l}\operatorname{Hm} 15 \\
\mathrm{Hm} 16\end{array}$ & H. maculatus & Hawkes Bay \\
\hline $\begin{array}{l}\mathrm{Hm} 16 \\
\mathrm{Hm} 17\end{array}$ & $\begin{array}{l}\text { H. maculatus } \\
\text { H. maculatus }\end{array}$ & Wanganui \\
\hline $\begin{array}{l}\mathrm{Hm} 17 \\
\mathrm{Hm} 18\end{array}$ & $\begin{array}{l}\text { H. maculatus } \\
\text { H. maculatus }\end{array}$ & $\begin{array}{l}\text { Lake Pounui } \\
\text { Featherston }\end{array}$ \\
\hline Hm19 & $\begin{array}{l}\text { H. maculatus } \\
\text { H. maculatus }\end{array}$ & $\begin{array}{l}\text { Featherston } \\
\text { Miramar, Wellington }\end{array}$ \\
\hline $\mathrm{Hm} 20$ & H. maculatus & $\begin{array}{l}\text { Miramar, Wellington } \\
\text { Seatoun Heights, Wellington }\end{array}$ \\
\hline $\begin{array}{l}\mathrm{H} \text { H'21 } \\
\mathrm{H} \text { m22 }\end{array}$ & H. maculatus & $\begin{array}{l}\text { Hataitai, Wellington } \\
\text { Heation }\end{array}$ \\
\hline $\begin{array}{l}\mathrm{Hm} 22 \\
\mathrm{Hm} 23\end{array}$ & H. maculatus & Lyall Bay, Wellington \\
\hline $\begin{array}{l}\mathrm{Hm} 23 \\
\mathrm{H} m 24\end{array}$ & H. maculatus & Moa Point, Wellington \\
\hline $\begin{array}{l}\mathrm{H} m 24 \\
\mathrm{H} m 25\end{array}$ & H. maculatus & Ocean Beach, south Wairarapa \\
\hline $\begin{array}{l}\mathrm{Hm} 25 \\
\mathrm{H} \text { H26 }\end{array}$ & H. maculatus & Fishermans Rock, south Wairarapa \\
\hline $\begin{array}{l}\mathrm{H} \text { m26 } \\
\mathrm{Hm} 27\end{array}$ & H. maculatus & Cape Turakirae, south Wairarapa \\
\hline $\begin{array}{l}\mathrm{H} m 27 \\
\mathrm{Hm} 28\end{array}$ & H. maculatus & Cape Palliser, south Wairarapa \\
\hline $\begin{array}{l}\mathrm{Hm} 28 \\
\mathrm{Hm} 29\end{array}$ & H. maculatus & Pukerua Bay \\
\hline $\begin{array}{l}\operatorname{Hm} 29 \\
\mathrm{Hm} 30\end{array}$ & H. maculatus & Kapiti Island \\
\hline $\begin{array}{l}\mathrm{Hm} 30 \\
\mathrm{Hm} 31\end{array}$ & H. maculatus & Aeroplane Island \\
\hline $\begin{array}{l}\mathrm{Hm} 31 \\
\mathrm{Hm} 32\end{array}$ & H. maculatus & Ward Island \\
\hline $\begin{array}{l}\mathrm{Hm} 32 \\
\mathrm{Hm} 33\end{array}$ & H. maculatus & Titahi Bay \\
\hline $\begin{array}{l}\mathrm{Hm} 33 \\
\mathrm{Hm} 34\end{array}$ & H. maculatus & Mana Island. \\
\hline $\begin{array}{l}\mathrm{H} \text { m34 } \\
\mathrm{Hm} 35\end{array}$ & H. maculatus & Stephens Island \\
\hline $\begin{array}{l}\mathrm{H} 3 \mathrm{H} 35 \\
\mathrm{Hm} 36\end{array}$ & $\begin{array}{l}\text { H. maculatus } \\
\text { H. maculatus }\end{array}$ & $\begin{array}{l}\text { Bird Island } \\
\text { South Trio Island }\end{array}$ \\
\hline Hm37 & & $\begin{array}{l}\text { South Trio Island } \\
\text { North Trio Island }\end{array}$ \\
\hline $\mathrm{Hm} 38$ & $\begin{array}{l}\text { H. maculatus } \\
\text { H. maculatus }\end{array}$ & $\begin{array}{l}\text { North Trio Island } \\
\text { Maud Island }\end{array}$ \\
\hline $\mathrm{Hm} 39$ & H. maculatus & Raihonui Stack, D'Urville Island \\
\hline $\mathrm{Hm} 40$ & H. maculatus & Paddock Rock 1, D'Urville Island \\
\hline $\mathrm{Hm} 41$ & H. maculatus & D'Unille Island \\
\hline $\mathrm{Hm} 42$ & H. maculatus & Whangarae Bay \\
\hline $\mathrm{Hm} 43$ & H. maculatus & North Brother Island \\
\hline $\mathrm{Hm} 44$ & H. maculatus & Outer Chetwode Island \\
\hline $\mathrm{Hm} 45$ & H. maculatus & Kaihoka Lakes \\
\hline $\mathrm{Hm} 46$ & H. maculatus & Trass Valley \\
\hline $\mathrm{Hm} 47$ & H. maculatus & Wairoa Valley \\
\hline $\mathrm{Hm} 48$ & H. maculatus & unknown \\
\hline $\mathrm{Hm} 49$ & H. maculatus & Red Hills \\
\hline $\mathrm{Hm} 50$ & H. maculatus & Cloudy Hut, Clarence River \\
\hline
\end{tabular}


Table 4.5.3.1 Allele frequencies at variable loci in populations of the northern group of the $H$. maculatus complex.

\begin{tabular}{|c|c|c|c|c|c|c|c|c|c|c|c|c|}
\hline Locus & $\begin{array}{l}\text { Popl } \\
\text { Hima }\end{array}$ & $\begin{array}{l}\text { tion } \\
\text { Hk1 }\end{array}$ & HK2 & Hmm1 & $\mathrm{Hmm} 2$ & $\mathrm{Hmm3}$ & Hmm4 & Hmm5 & Hmm6 & $\mathrm{Hmm7}$ & Hmm8 & $\mathrm{Hmm} 9$ \\
\hline$(\mathrm{N})$ & 2 & 5 & 4 & 1 & 4 & 2 & 13 & 6 & 1 & 2 & 4 & 4 \\
\hline \multicolumn{13}{|l|}{$A k-1$} \\
\hline $\mathrm{a}$ & 1.00 & 1.00 & 1.00 & 1.00 & 1.00 & 1.00 & 1.00 & 1.00 & 1.00 & 1.00 & 1.00 & 1.00 \\
\hline d & - & $\cdot$ & $\cdot$ & $\cdot$ & $\cdot$ & - & $\cdot$ & $\cdot$ & - & - & - & - \\
\hline \multicolumn{13}{|l|}{$C k-1$} \\
\hline $\begin{array}{l}a \\
b\end{array}$ & $:$ & 1.00 & 0.88 & 1.00 & 1.00 & 1.00 & 1.00 & 1.00 & 1.00 & 1.00 & 1.00 & 1.00 \\
\hline d & 1.00 & $\cdot$ & 0.12 & . & . & . & . & 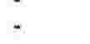 & $:$ & $\therefore$ & . & 5 \\
\hline \multicolumn{13}{|l|}{$G d-2$} \\
\hline b & - & - & - & - & . & - & . & - & . & - & . & - \\
\hline $\mathrm{c}$ & 1.00 & 1.00 & 1.00 & 1.00 & 1.00 & 1.00 & 1.00 & 1.00 & 1.00 & 1.00 & 1.00 & 1.00 \\
\hline \multicolumn{13}{|l|}{ Got-1 } \\
\hline a & 1.00 & 1.00 & 1.00 & 1.00 & 1.00 & 1.00 & 1.00 & 1.00 & 1.00 & 1.00 & 1.00 & 1.00 \\
\hline b & $\cdot$ & $\cdot$ & - & - & - & - & $\cdot$ & $\cdot$ & $\cdot$ & $\cdot$ & $\cdot$ & - \\
\hline \multicolumn{13}{|l|}{ Got-2 } \\
\hline $\begin{array}{l}\mathrm{a} \\
\mathrm{b}\end{array}$ & . & 1.00 & 1.00 & 1.00 & 1.00 & 1.00 & 1.00 & 1.00 & 1.00 & $i_{100}$ & 1.00 & 1.00 \\
\hline \multicolumn{13}{|l|}{$G p-4$} \\
\hline $\mathrm{b}$ & - & - & - & - & - & - & - & - & - & - & - & . \\
\hline c & 1.00 & - & - & 1.00 & 1.00 & 1.00 & 1.00 & 1.00 & 1.00 & 1.00 & 1.00 & 1.00 \\
\hline e & - & 1.00 & 1.00 & - & - & - & - & $\cdot$ & - & - & - & - \\
\hline $\mathrm{h}$ & $\cdot$ & - & $\cdot$ & - & - & - & - & - & - & $\cdot$ & $\cdot$ & $\cdot$ \\
\hline \multicolumn{13}{|l|}{ Gpi-1 } \\
\hline b & 1.00 & 1.00 & 0.25 & 1.00 & 1.00 & 1.00 & 1.00 & 1.00 & 1.00 & 1.00 & 1.00 & 1.00 \\
\hline d & $\cdot$ & $\cdot$ & 0.75 & $\cdot$ & $\cdot$ & - & $\cdot$ & $\cdot$ & $\cdot$ & $\cdot$ & $\cdot$ & - \\
\hline \multicolumn{13}{|l|}{$\mid c d-1$} \\
\hline $\mathrm{b}$ & 1.00 & . & - & - & . & - & - & - & - & . & - & - \\
\hline c & $\cdot$ & 1.00 & 1.00 & 1.00 & 1.00 & 1.00 & 1.00 & 1.00 & 1.00 & 1.00 & 1.00 & 1.00 \\
\hline \multicolumn{13}{|l|}{$1 c d-2$} \\
\hline a & - & - & . & . & . & - & - & - & - & - & - & - \\
\hline $\begin{array}{l}c \\
d\end{array}$ & 1.00 & 1.00 & 0.88 & 1.00 & 0.88 & 0.75 & 1.00 & 1.00 & 0.50 & 0.75 & 0.38 & 1.00 \\
\hline$f$ & - & . & . & . & . & . & 5 & $\therefore$ & $\therefore$ & $\dot{0}_{0.25}$ & : & 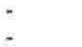 \\
\hline $\mathrm{h}$ & - & - & 0.12 & - & 0.12 & - & - & - & 0.50 & - & 0.25 & - \\
\hline $\mathrm{i}$ & - & $\cdot$ & $\cdot$ & - & $\cdot$ & 0.25 & - & - & 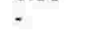 & - & 0.38 & - \\
\hline \multicolumn{13}{|l|}{$L d h-1$} \\
\hline$b$ & - & - & - & - & - & - & . & - & . & - & - & - \\
\hline$c$ & 1.00 & 1.00 & 1.00 & 1.00 & 1.00 & 1.00 & 1.00 & 1.00 & 1.00 & 1.00 & 1.00 & 1.00 \\
\hline \multicolumn{13}{|l|}{$L d h-2$} \\
\hline e & 1.00 & 1.00 & 1.00 & 1.00 & 1.00 & 1.00 & 1.00 & 1.00 & 1.00 & 1.00 & 1.00 & 1.00 \\
\hline f & - & $\cdot$ & - & - & - & - & - & - & $\cdot$ & - & - & $\cdot$ \\
\hline $\mathrm{Me-1}$ & & & & & & & & & & & & \\
\hline a & 1.00 & 1.00 & 0.75 & 1.00 & 1.00 & 1.00 & 1.00 & 1.00 & 1.00 & 1.00 & 1.00 & 1.00 \\
\hline$d$ & $\cdot$ & - & 0.25 & $\cdot$ & $\cdot$ & $\cdot$ & - & - & $\cdot$ & $\cdot$ & $\cdot$ & - \\
\hline$N p-1$ & . & & & & & & & & & & & \\
\hline $\mathrm{c}$ & - & - & - & . & - & - & - & - & - & - & - & . \\
\hline$f$ & 1.00 & 1.00 & 1.00 & 1.00 & 1.00 & 1.00 & 1.00 & 1.00 & 1.00 & 1.00 & 1.00 & 1.00 \\
\hline Pep-3 & & & & & & & & & & & & \\
\hline $\begin{array}{l}b \\
c\end{array}$ & 1.00 & 1.00 & 1.00 & 1.00 & 1.00 & 1.00 & 1.00 & 1.00 & 1.00 & 1.00 & 1.00 & 1.00 \\
\hline c & $\cdot$ & $\cdot$ & - & - & - & - & - & - & . & - & - & - \\
\hline$P g d-1$ & & & & & & & & & & & & \\
\hline e & 1.00 & ; & - & 1.00 & 1.00 & 1.00 & 1.00 & 1.00 & 1.00 & 1.00 & 1.00 & 1.00 \\
\hline f & - & 1.00 & 1.00 & - & $\cdot$ & $\cdot$ & - & $\cdot$ & - & - & $\cdot$ & \\
\hline$P g m-1$ & & & & & & & & & & & & \\
\hline & 1.00 & 1.00 & 1.00 & 1.00 & 1.00 & 1.00 & 1.00 & 1.00 & 1.00 & 1.00 & 1.00 & 1.00 \\
\hline d & $\cdot$ & - & - & - & $\cdot$ & - & $\cdot$ & $\cdot$ & - & $\cdot$ & $\cdot$ & - \\
\hline$P g m-2$ & & & & & & & & & & & & \\
\hline$b^{\circ}$ & - & - & - & $\therefore$ & - & - & - & 0.33 & - & - & - & - \\
\hline $\begin{array}{l}c \\
e\end{array}$ & 1.00 & 0.70 & 0.88 & 1.00 & 0.12 & 1.00 & 0.67 & 0.08 & 1.00 & 1.00 & 0.25 & 1.00 \\
\hline $\mathrm{g}$ & . & 0.30 & 0.12 & $:$ & $\begin{array}{l}0.25 \\
0.50\end{array}$ & : & 0.33 & 0.58 & $\vdots$ & : & 0.75 & : \\
\hline $\mathrm{h}$ & - & $\cdot$ & $\cdot$ & - & 0.12 & - & - & - & . & . & . & . \\
\hline Sod-2 & & & & & & & & & & & & \\
\hline b & $\therefore$ & - & - & - & - & - & - & - & - & - & - & - \\
\hline c & 1.00 & - & - & - & - & 0.75 & 0.92 & - & - & 0.50 & 0.62 & - \\
\hline d & - & 1.00 & 1.00 & 1.00 & 1.00 & 0.25 & 0.08 & 1.00 & 1.00 & 0.50 & 0.38 & 1.00 \\
\hline
\end{tabular}


Table 4.5.3.1 (continued)

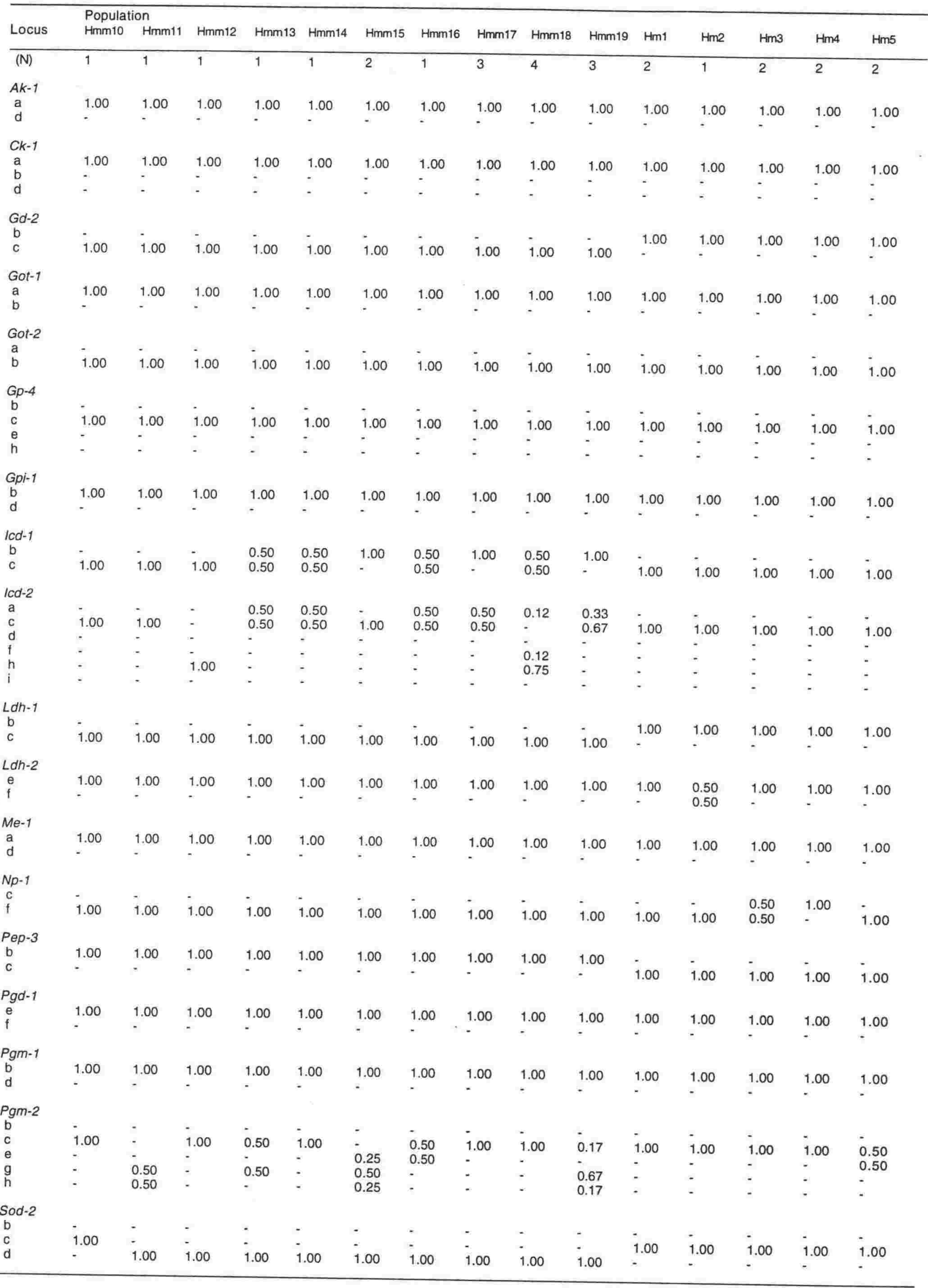


Table 4.5.3.1 (continued)

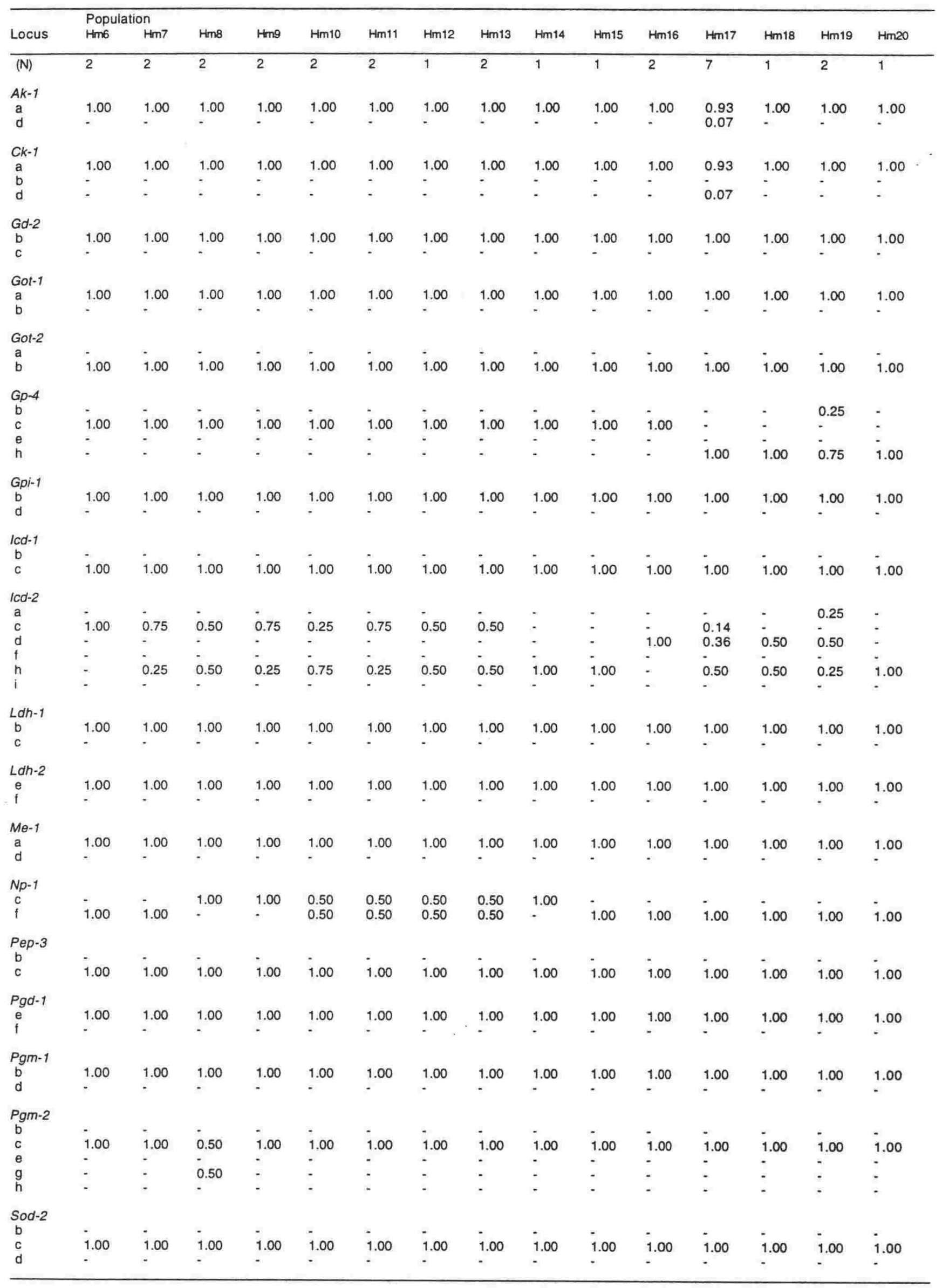


Table 4.5.3.1 (continued)

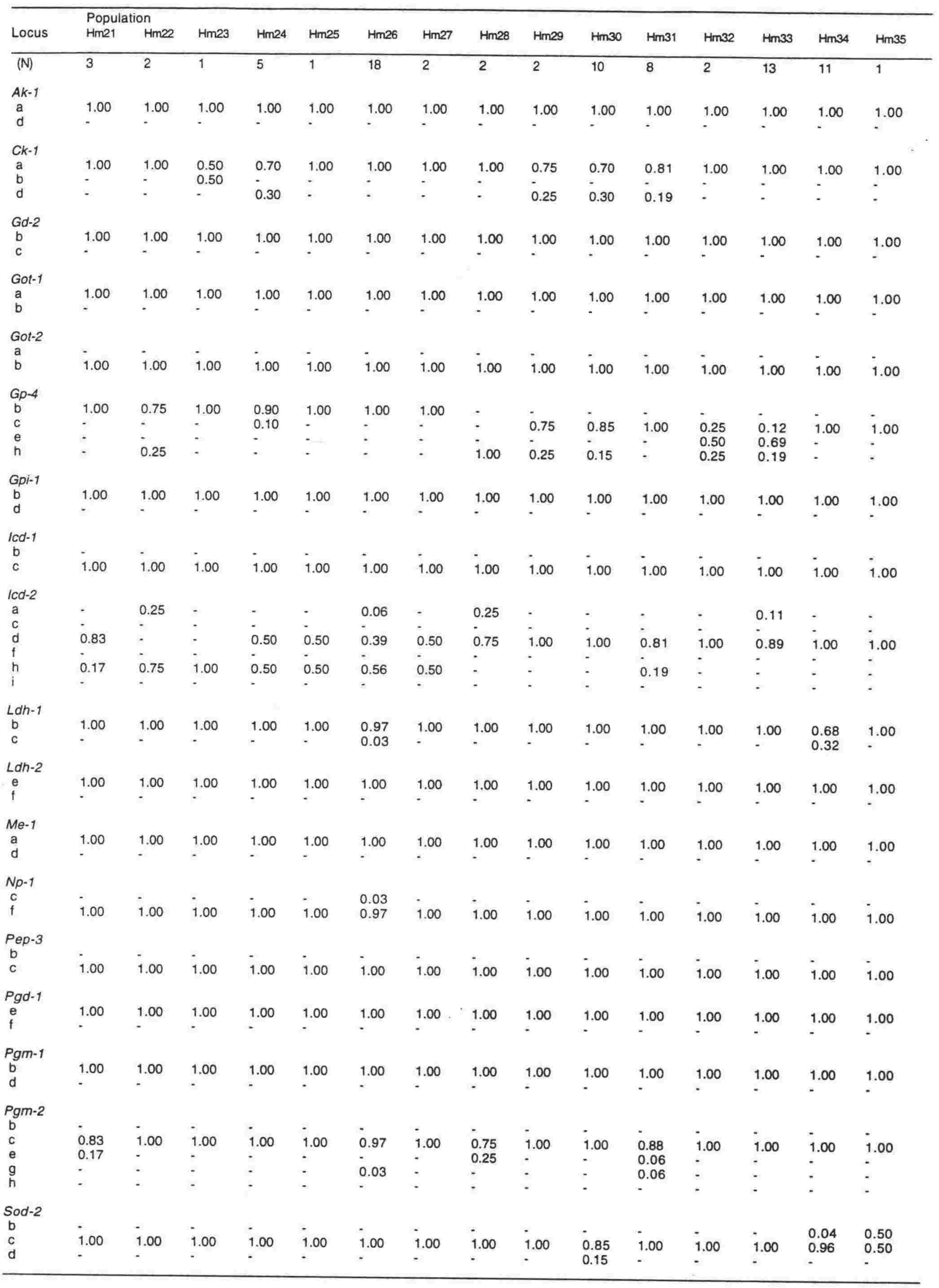


Table 4.5.3.1 (continued)

\begin{tabular}{|c|c|c|c|c|c|c|c|c|c|c|c|c|c|c|c|}
\hline LOCUS & $\begin{array}{l}\text { Popula } \\
\text { Hm36 }\end{array}$ & Hm37 & $\mathrm{Hm} 38$ & $\mathrm{Hm} 39$ & $\mathrm{Hm} 40$ & $\mathrm{Hm} 41$ & $\mathrm{Hm} 42$ & $\mathrm{Hm} 43$ & $\mathrm{Hm} 44$ & Hin45 & $\mathrm{Hm} 46$ & $\mathrm{Hm} 47$ & $\operatorname{Hin} 48$ & $\mathrm{Hm} 49$ & Hm50 \\
\hline (N) & 1 & 2 & 2 & 3 & 2 & 5 & 3 & 2 & 2 & 3 & 2 & 3 & 1 & 1 & 1 \\
\hline \multicolumn{16}{|l|}{$\begin{array}{l}A k-1 \\
a\end{array}$} \\
\hline a & 1.00 & 1.00 & 1.00 & 1.00 & 1.00 & 1.00 & 1.00 & 1.00 & 1.00 & 1.00 & 1.00 & 1.00 & 1.00 & 1.00 & 1.00 \\
\hline d & - & - & - & $\cdot$ & $\cdot$ & - & - & $\cdot$ & - & - & - & - & - & $\cdot$ & \\
\hline \multicolumn{16}{|l|}{$C_{k-1}$} \\
\hline $\begin{array}{l}a \\
b\end{array}$ & 1.00 & 1.00 & 1.00 & 1.00 & 1.00 & 1.00 & 0.83 & 1.00 & 1.00 & 1.00 & 1.00 & 1.00 & 1.00 & 1.00 & 1.00 \\
\hline $\begin{array}{l}\text { D } \\
\text { d }\end{array}$ & $:$ & : & $\vdots$ & $:$ & : & . & 0.17 & : & . & . & : & : & 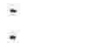 & $\therefore$ & $\therefore$ \\
\hline \multicolumn{16}{|l|}{ Gd-2 } \\
\hline$b$ & 1.00 & 1.00 & 1.00 & 1.00 & 1.00 & 1.00 & 1.00 & 1.00 & 1.00 & 1.00 & - & . & - & - & 1.00 \\
\hline$c$ & - & $\cdot$ & $\cdot$ & $\cdot$ & - & $\cdot$ & $\cdot$ & $\cdot$ & - & - & 1.00 & 1.00 & 1.00 & 1.00 & - \\
\hline \multicolumn{16}{|l|}{$\begin{array}{c}\text { Got-1 } \\
a\end{array}$} \\
\hline a & 1.00 & 1.00 & 1.00 & 1,00 & 1.00 & 1.00 & 1.00 & 1.00 & 1.00 & 0.83 & 1.00 & 1.00 & 1.00 & 1.00 & 1.00 \\
\hline b & - & - & - & - & $\cdot$ & $\cdot$ & $\cdot$ & $\cdot$ & - & 0.17 & $\cdot$ & $\cdot$ & $\cdot$ & & \\
\hline \multicolumn{16}{|l|}{$\begin{array}{c}\text { Got-2 } \\
a\end{array}$} \\
\hline a & - & : & - & - & - & - & - & - & - & - & - & - & - & - & . \\
\hline b & 1.00 & 1.00 & 1.00 & 1.00 & 1.00 & 1.00 & 1.00 & 1.00 & 1.00 & 1.00 & 1.00 & 1.00 & 1.00 & 1.00 & 1.00 \\
\hline \multirow{2}{*}{\multicolumn{16}{|c|}{$\begin{array}{c}G p-4 \\
\mathrm{~b}\end{array}$}} \\
\hline $\begin{array}{l}b \\
c\end{array}$ & 1.00 & 100 & & & & & & $\bar{j}_{75}$ & is & - & - & - & - & - & - \\
\hline $\begin{array}{l}c \\
e\end{array}$ & $\begin{array}{l}1.00 \\
-\end{array}$ & $\begin{array}{l}1.00 \\
.\end{array}$ & 1.00 & 1.00 & 1.00 & 1.00 & 1.00 & 0.75 & 1.00 & 1.00 & 1.00 & 1.00 & 1.00 & 1.00 & 1.00 \\
\hline h & - & - & - & - & - & . & . & 0.25 & . & . & $:$ & : & $\therefore$ & : & : \\
\hline \multicolumn{16}{|l|}{$\underset{b}{G p i-1}$} \\
\hline $\mathrm{b}$ & 1.00 & 1.00 & 1.00 & 1.00 & 1.00 & 1.00 & 1.00 & 1.00 & 1.00 & 1.00 & 1.00 & 1.00 & 1.00 & 1.00 & 1.00 \\
\hline d & $\cdot$ & $\cdot$ & $\cdot$ & $\cdot$ & $\cdot$ & $\cdot$ & $\cdot$ & $\cdot$ & $\cdot$ & $\cdot$ & - & - & $\cdot$ & - & $\cdot$ \\
\hline \multirow{2}{*}{\multicolumn{16}{|c|}{$\begin{array}{l}1 c d-1 \\
\text { b }\end{array}$}} \\
\hline & in & & & - & - & - & - & - & - & - & - & - & - & 0.50 & - \\
\hline c & 1.00 & 1.00 & 1.00 & 1.00 & 1.00 & 1.00 & 1.00 & 1.00 & 1.00 & 1.00 & 1.00 & 1.00 & 1.00 & 0.50 & 1.00 \\
\hline \multicolumn{16}{|l|}{$\begin{array}{c}I c d-2 \\
a\end{array}$} \\
\hline $\begin{array}{l}a \\
c\end{array}$ & : & : & : & : & : & $\therefore$ & : & : & : & : & $\cdot$ & - & - & - & - \\
\hline $\begin{array}{l}c \\
d\end{array}$ & 1.00 & 1.00 & 1.00 & 1.00 & 1.00 & 1.00 & 1.00 & 1.00 & 1.00 & 1.00 & 1.00 & 1.00 & 100 & in & \\
\hline$t$ & . & - & - & - & - & - & - & - & - &. & .00 & $\begin{array}{l}1.00 \\
-\end{array}$ & .00 & $\begin{array}{l}1.00 \\
-\end{array}$ & 1.00 \\
\hline$n$ & - & - & - & - & - & - & - & - & - & - & - & - & - & - & - \\
\hline $\mathrm{i}$ & $\cdot$ & $\cdot$ & , & $\cdot$ & $\cdot$ & - & - & - & & - & - & - & $\cdot$ & - & - \\
\hline \multicolumn{16}{|l|}{ Ldh-1 } \\
\hline b & - & 0.75 & 1.00 & 1.00 & 1.00 & 0.70 & 1.00 & 0.75 & 1.00 & 1.00 & 0.25 & 0.17 & - & 1.00 & 0.50 \\
\hline c & 1.00 & 0.25 & $\cdot$ & $\cdot$ & $\cdot$ & 0.30 & $\cdot$ & 0.25 & - & - & 0.75 & 0.83 & 1.00 & - & 0.50 \\
\hline \multicolumn{16}{|l|}{$\operatorname{ldoh}_{\mathrm{e}}$} \\
\hline $\begin{array}{l}e \\
f\end{array}$ & 1.00 & 1.00 & 1.00 & 1.00 & 1.00 & 1.00 & 1.00 & 1.00 & 1.00 & 1.00 & 1.00 & 1.00 & 1.00 & 1.00 & 1.00 \\
\hline M & 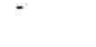 & 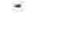 & - & $\cdot$ & - & - & $\cdot$ & " & & - & - & $\cdot$ & & $\cdot$ & - \\
\hline \multicolumn{3}{|l|}{$\begin{array}{l}\text { Me-1 } \\
\text { a }\end{array}$} & 1.00 & 1.00 & 1.00 & 1.00 & 1.00 & 1.00 & 1.00 & 1.00 & 1.00 & 1.00 & 1.00 & & \\
\hline d & - & . & - & $\cdot$ & - & $\cdot$ & - & $\cdot$ & $\cdot$ & - & $\cdot$ & - & $\therefore$ & .00 & 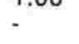 \\
\hline $\begin{array}{l}N p-1 \\
C\end{array}$ & . & . & . & - & . & . & . & . & - & - & & & & & \\
\hline f & 1.00 & 1.00 & 1.00 & 1.00 & 1.00 & 1.00 & 1.00 & 1.00 & 1.00 & 1.00 & 1.00 & 1.00 & 1.00 & 1.00 & 1.00 \\
\hline Pep-3 & & & & & & & & & & & & & & & \\
\hline b & - & - & - & - & - & . & - & - & - & . & - & - & - & 1.00 & - \\
\hline c & 1.00 & 1.00 & 1.00 & 1.00 & 1.00 & 1.00 & 1.00 & 1.00 & 1.00 & 1.00 & 1.00 & 1.00 & 1.00 & $\cdot$ & 1.00 \\
\hline $\begin{array}{l}P g d-1 \\
e^{-1}\end{array}$ & 1.00 & 1.00 & 1.00 & 1.00 & 1.00 & 1.00 & 1.00 & 1.00 & 1.00 & 1.00 & 1.00 & 1.00 & 1.00 & 1.00 & 1.00 \\
\hline f & 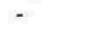 & $\cdot$ & $\cdot$ & - & - & - & - & $\cdot$ & $\cdot$ & - & $\cdot$ & - & $\cdot$ & & \\
\hline Pgm-1 & & & 1.00 & 0.83 & 1.00 & 0.80 & 1.00 & 1.00 & 1.00 & 1.00 & 1.00 & 1.00 & 1.00 & 1.00 & 1.00 \\
\hline d & - & $\because .00$ & $\because$ & 0.17 & - & 0.20 & - & - & - &. & - & - & - & 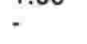 & .00 \\
\hline Pgm-2 & & & & & & & & & & & & & & & \\
\hline ? & $i .00$ & 1.00 & $\overline{1} 00$ & ino & - & : & - & - & - & $\therefore$ & - & - & - & - & - \\
\hline 6 & $\begin{array}{l}1.00 \\
-\end{array}$ & $\begin{array}{l}1.00 \\
-\end{array}$ & 1.00 & $\begin{array}{l}1.00 \\
-\end{array}$ & $\begin{array}{l}1.00 \\
.\end{array}$ & $\begin{array}{l}1.00 \\
.\end{array}$ & $\begin{array}{l}1.00 \\
-\end{array}$ & 0.25 & 1.00 & 1.00 & : & 0.83 & 1.00 & 1.00 & 1.00 \\
\hline 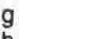 & - & - & . & - & - & - & - & 0.75 & . & . & 1.00 & 0.17 & $\therefore$ & $:$ & 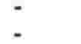 \\
\hline$n$ & - & $\cdot$ & $\cdot$ & - & $\cdot$ & - & $\cdot$ & - & $\cdot$ & $\cdot$ & - & $\cdot$ & - & - & - \\
\hline 500 & - & - & - & - & - & - & . & - & 0.25 & - & - & - & $\cdot$ & - & - \\
\hline 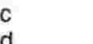 & 1.00 & 1.00 & 0.75 & 1.00 & 1.00 & 1.00 & 1.00 & 1.00 & 0.50 & 0.83 & 1.00 & 0.33 & 1.00 & 1.00 & 1.00 \\
\hline & - & $\cdot$ & 0.25 & - & - & - & - & - & 0.25 & 0.17 & - & 0.67 & - & & - \\
\hline
\end{tabular}




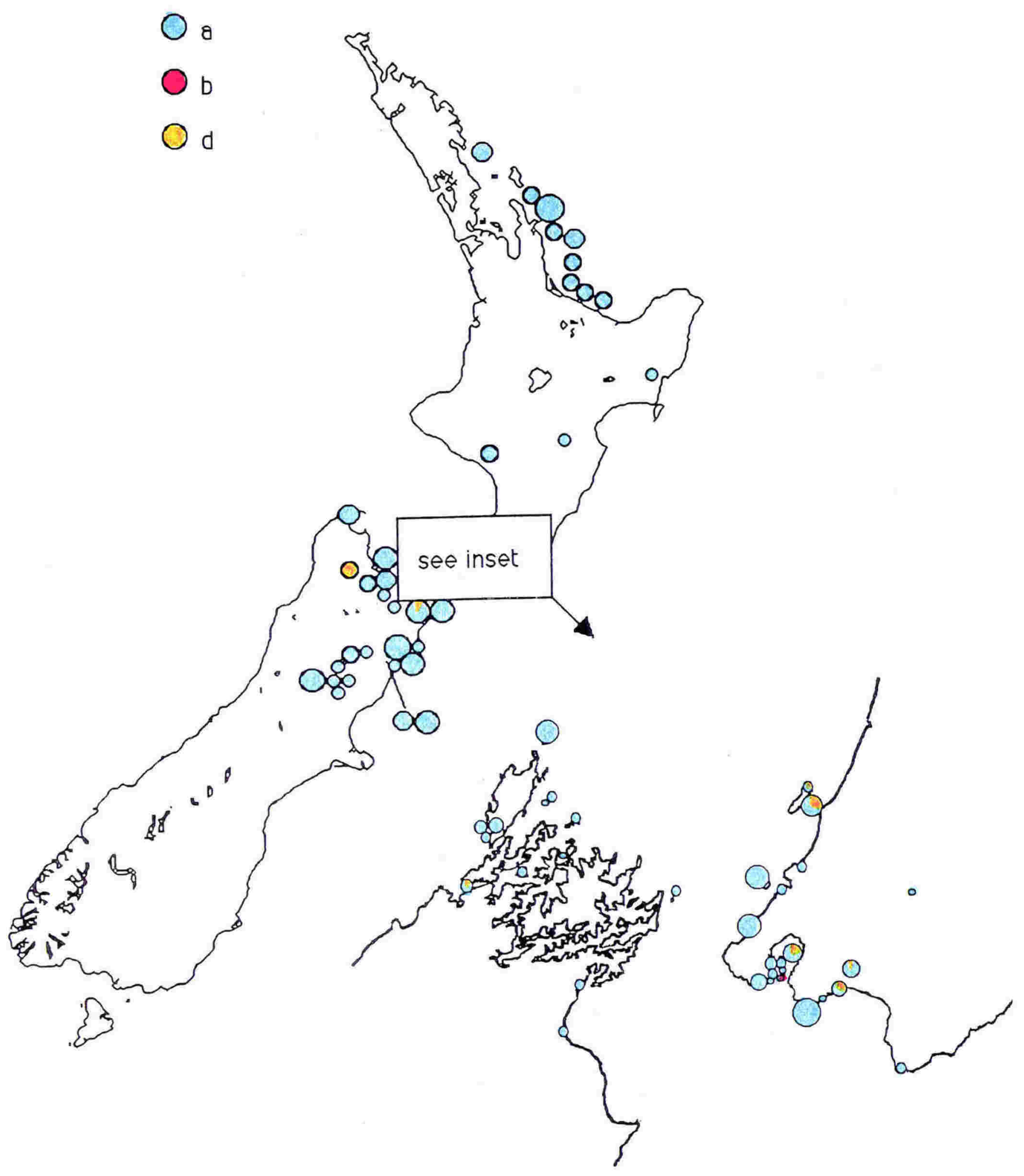

Figure 4.5.3.1 $C k-1$ allele distributions among populations of the northern species group of the $H$. maculatus complex. The total area of the pie chart for each sample is directly proportional to sample size (see Table 4.5.3.1), but pie charts on the inset are at a different scale to those on the main map. 


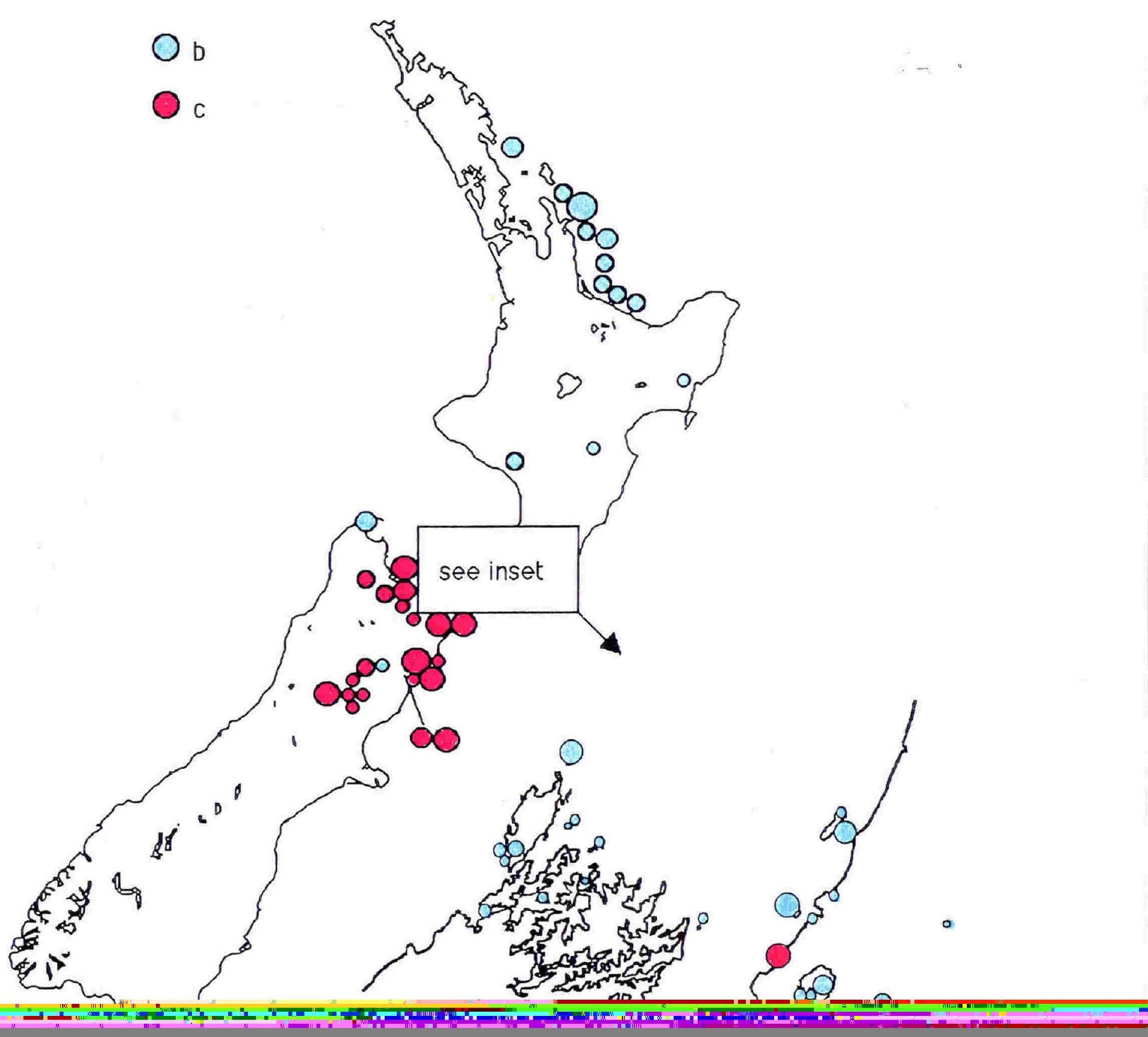




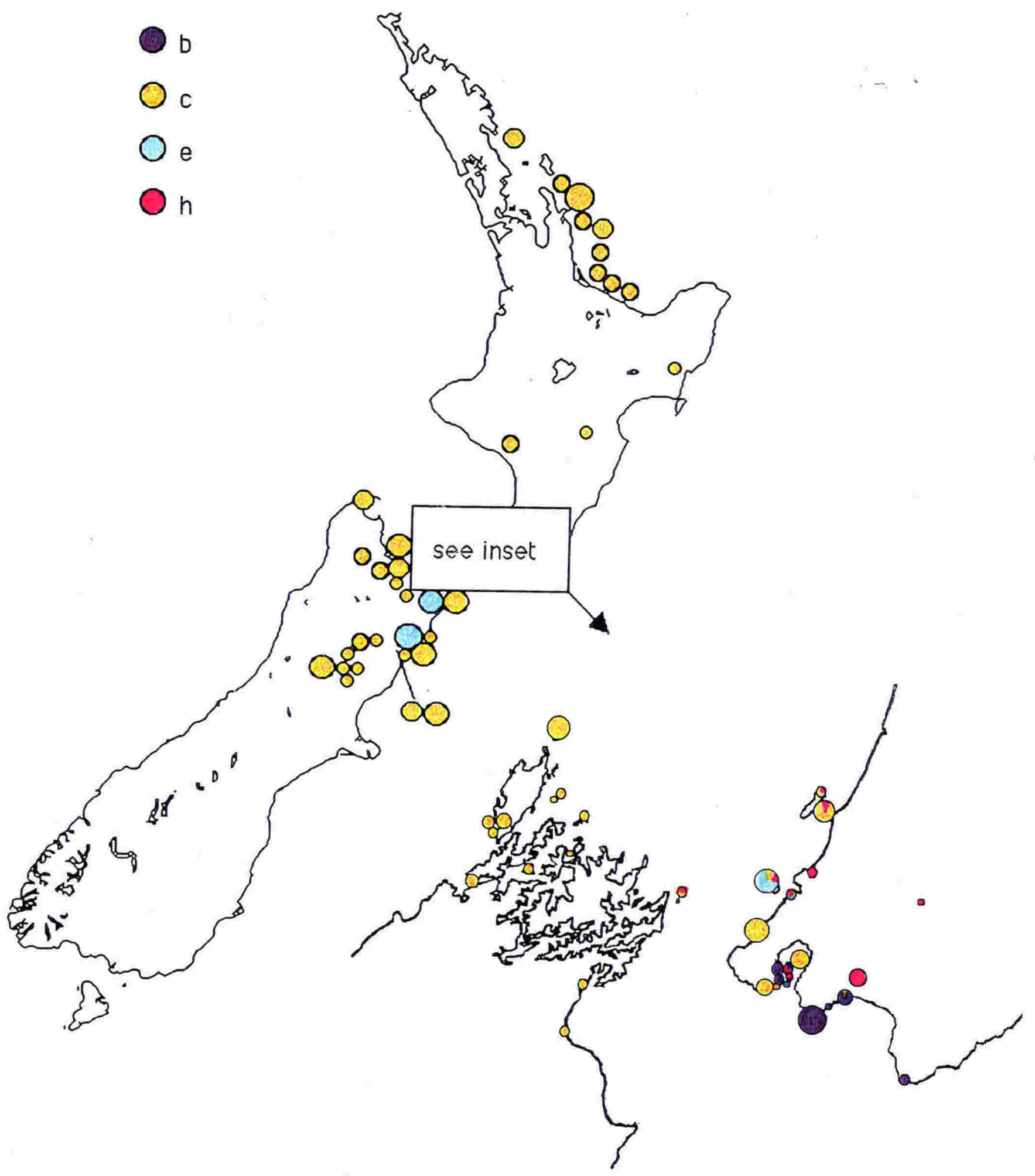

Figure 4.5.3.3 Gp-4 allele distributions among populations of the northern species group of the $H$. maculatus complex. The total area of the pie chart for each sample is directly proportional to sample size (see Table 4.5.3.1), but pie charts on the inset are at a different scale to those on the main map. 


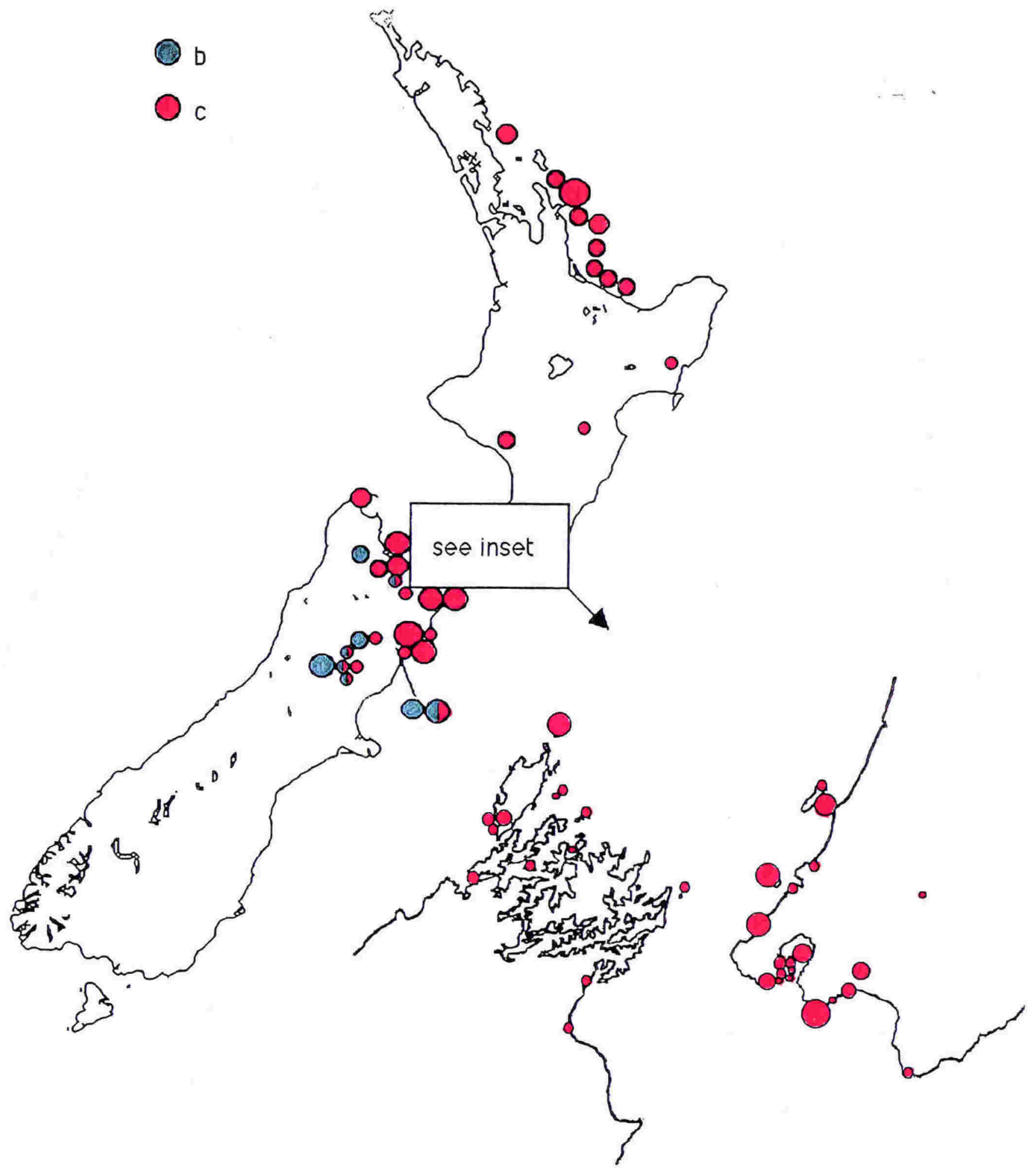

Figure 4.5.3.4 lcd-1 allele distributions among populations of the northern species group of the $H$. maculatus complex. The total area of the pie chart for each sample is directly proportional to sample size (see Table 4.5.3.1), but pie charts on the inset are at a different scale to those on the main map. 


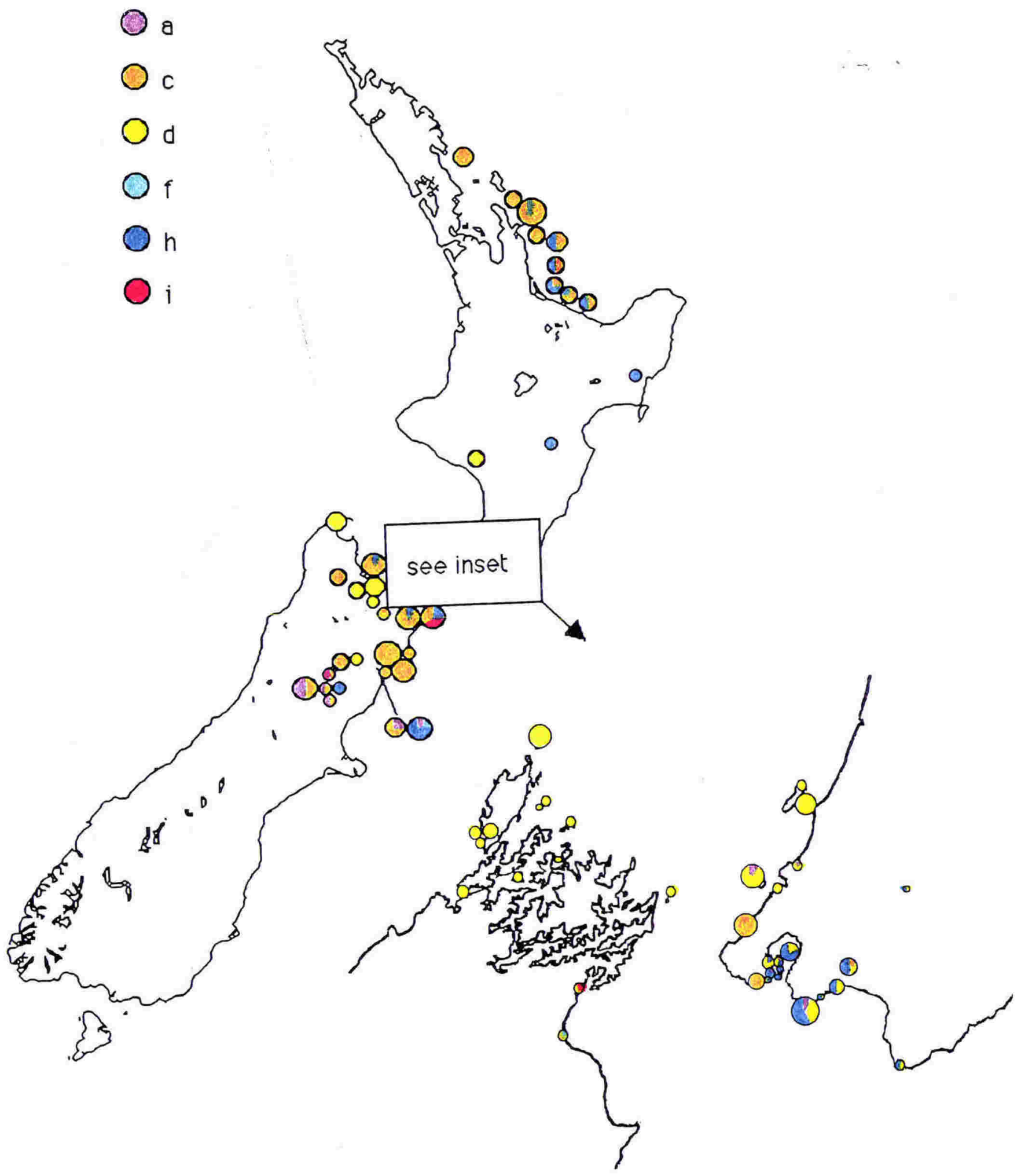

Figure 4.5.3.5 Icd-2 allele distributions among populations of the northern species group of the $H$. maculatus complex. The total area of the pie chart for each sample is directly proportional to sample size (see Table 4.5.3.1), but pie charts on the inset are at a different scale to those on the main map. 


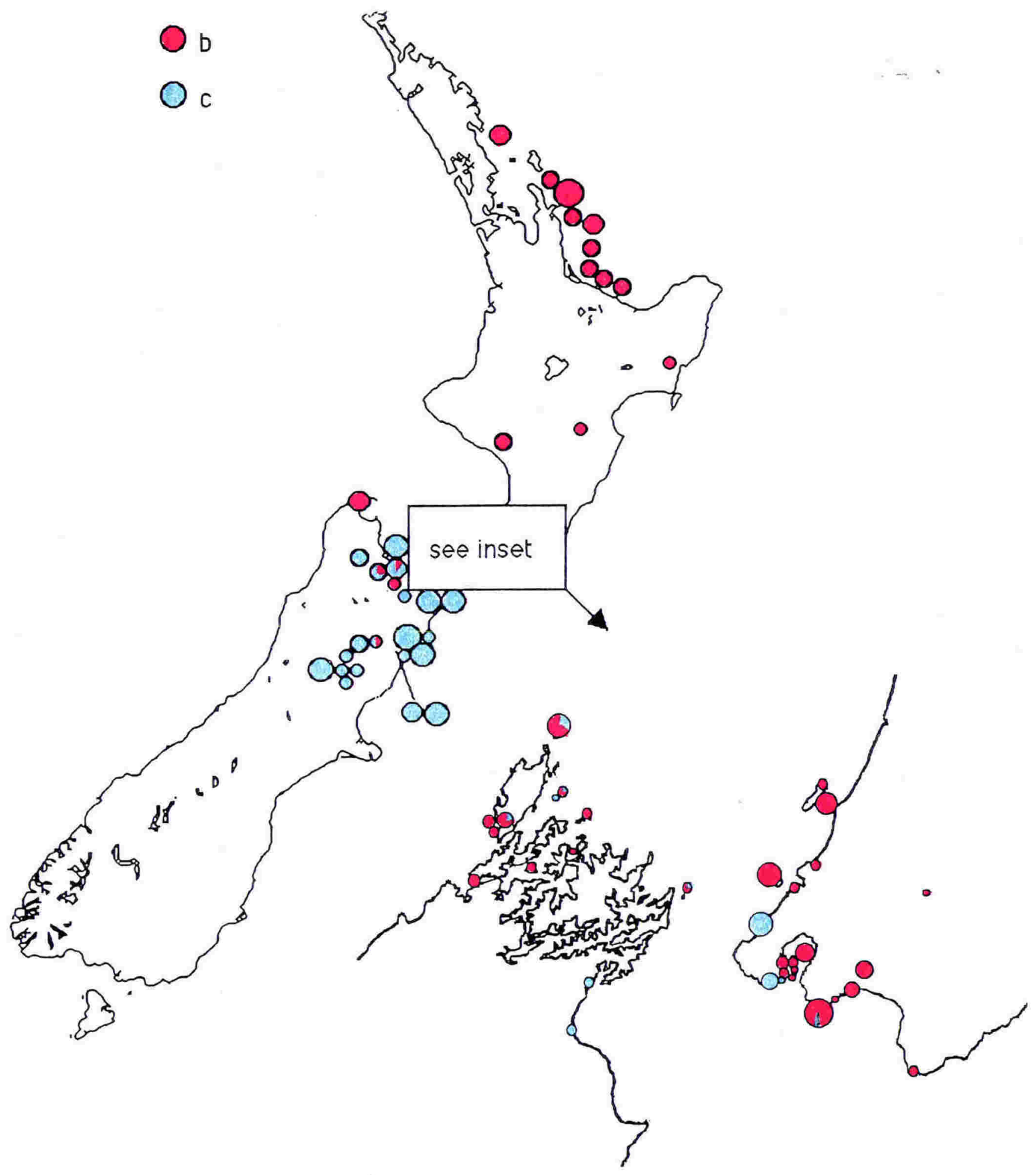

Figure 4.5.3.6 Ldh-1 allele distributions among populations of the northern species group of the $H$. maculatus complex. The total area of the pie chart for each sample is directly proportional to sample size (see Table 4.5.3.1), but pie charts on the inset are at a different scale to those on the main map. 


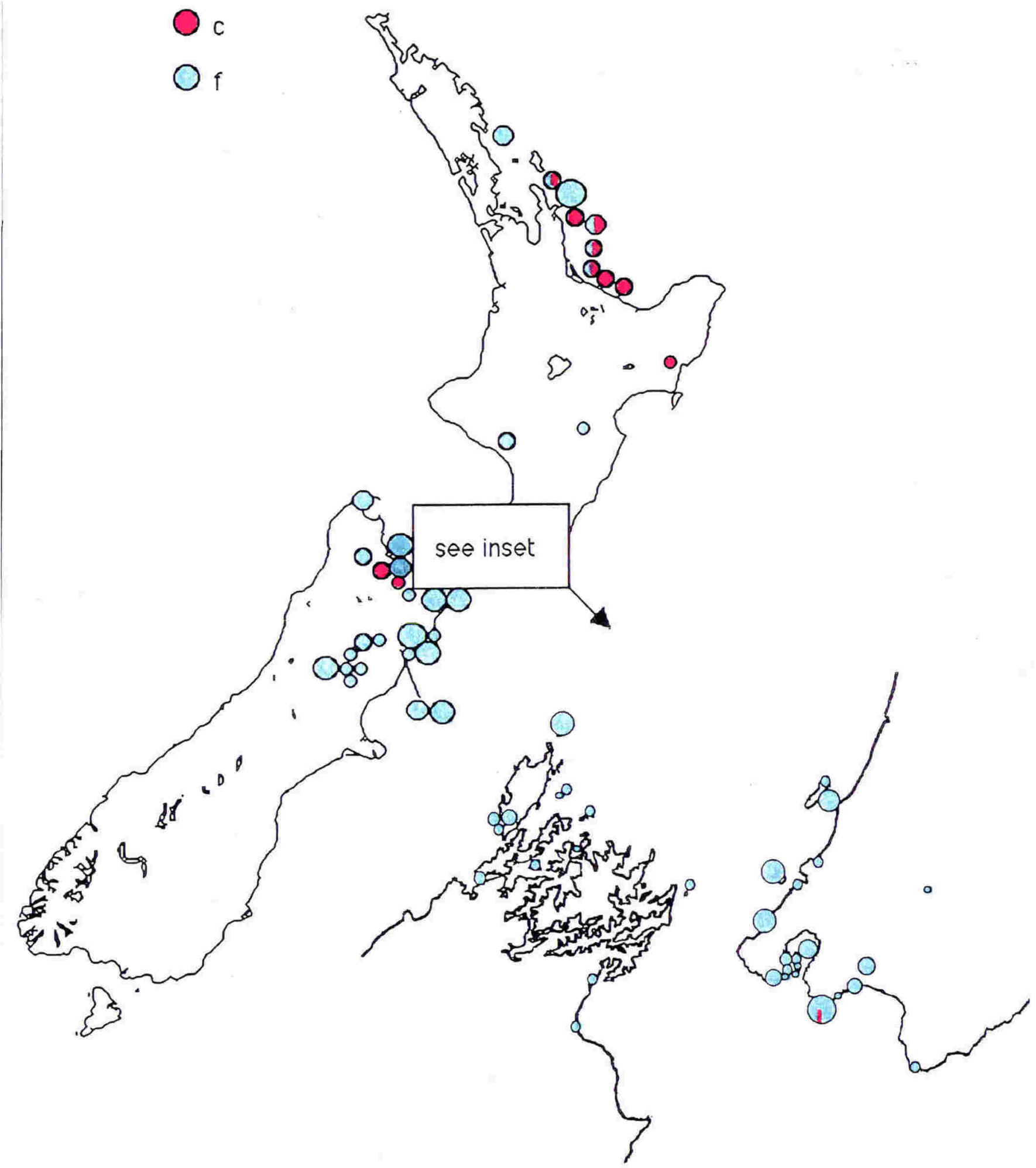

Figure 4.5.3.7 $\mathrm{Np}$ allele distributions among populations of the northern species group of the $H$. maculatus complex. The total area of the pie chart for each sample is directly proportional to sample size (see Table 4.5.3.1), but pie charts on the inset are at a different scale to those on the main map. 


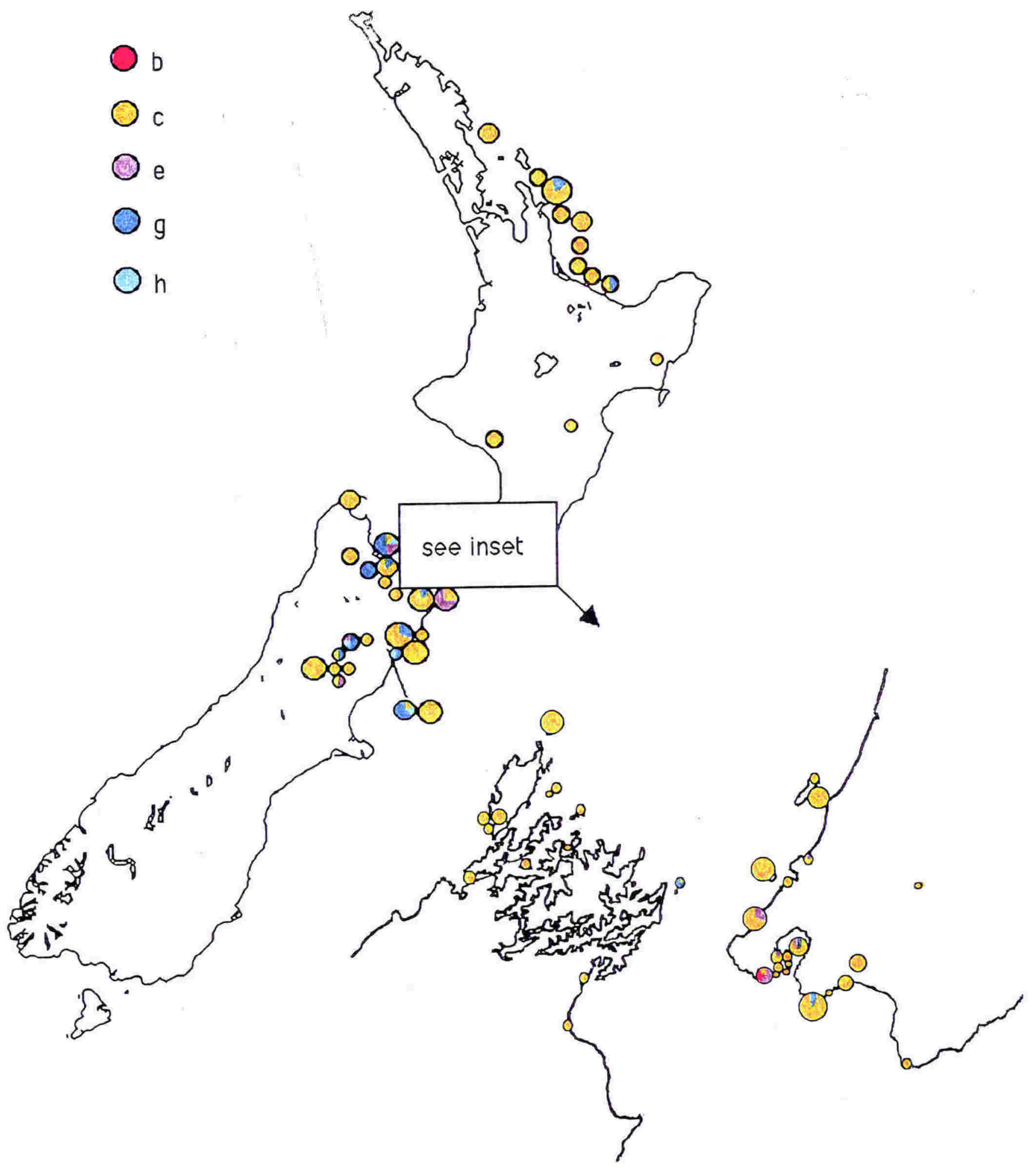

Figure 4.5.3.8 Pgm-2 allele distributions among populations of the northern species group of the $H$. maculatus complex. The total area of the pie chart for each sample is directly proportional to sample size (see Table 4.5.3.1), but pie charts on the inset are at a different scale to those on the main map. 


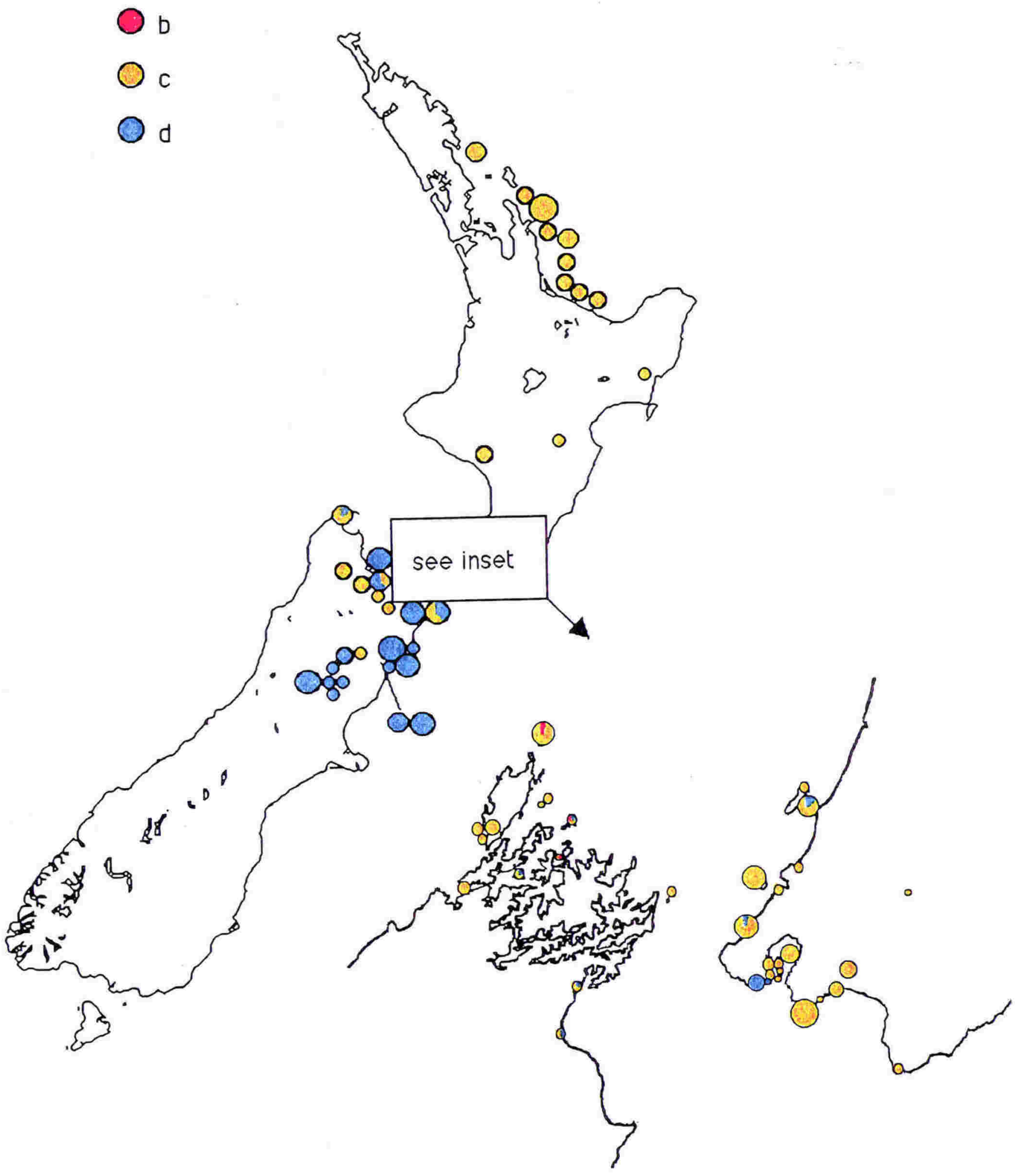

Figure 4.5.3.9 Sod-2 allele distributions among populations of the northern species group of the $H$. maculatus complex. The total area of the pie chart for each sample is directly proportional to sample size (see Table 4.5.3.1), but pie charts on the inset are at a different scale to those on the main map. 
As far as could be detected from the small samples available, levels of polymorphism were very low (Table 4.5.3.2). The original sample from Batty (Kaikouras) showed substantial departure from Hardy-Weinberg equilibrium, with one animal (RAH303) differing from the other 5 by apparent fixed differences at 2 loci. When this animal was treated as a separate sample, observed levels of heterozygosity were very close to expected levels in both groups (Table 4.5.3.2). These 2 sympatric samples differed by Nei's $D=0.08$. RAH303 was indistinguishable from neighbouring populations of $H$. "Marlborough mini" in both allozymes (Table 4.5.3.1) and morphology (Section 4.5.4). The remaining sample of 5 animals was very similar to the Ward sample in both allozymes and morphology. The Batty sample was therefore interpreted as including sympatric specimens of 2 reproductively isolated species, and these 2 samples were subsequently analysed separately.

The Ward population of $H$. "Kaikouras" also showed lower than expected heterozygosity (Table 4.5.3.2). The departure from Hardy-Weinberg equilibrium in this population is probably an artefact of my inability to detect some heterozygotes of Gpi-1 because of secondary isozymes (see Chapter 3). There was no evidence of genetic structuring within other samples; observed heterozygosity was close to Hardy-Weinberg expectation (sample sizes were too small to permit tests for significance).

Geographically adjacent populations which showed no fixed allozyme differences were grouped to make the analysis more manageable and increase sample sizes. Distance matrices for both grouped and ungrouped populations (Nei's D, Tables 4.5.3.3 and 4.5.3.4 for ungrouped and grouped samples respectively, and CavalliSforza \& Edwards' arc distance and Rogers' D, Tables 4.5.3.5 and 4.5.3.6) showed 4 groups of populations with lower genetic distances between populations within groups, and larger distances between groups. Distances within and between these groups are summarised in Table 4.5.3.7. Within-species and between-species distances overlap to some extent. One of the lowest between-species values $(0.08)$ was for the sympatric samples at Batty, Kaikouras.

Populations of $H$. maculatus from inland Nelson (Wairoa Valley, Trass, and Red Hills) show more allozyme divergence from the rest of the $H$. maculatus populations and from each other than do any other populations within proposed species of this group. At $G d-2$ they are homozygous for the (c) allele, which they share with other species in both the northern and southern groups, but with no other $H$. maculatus. At 
Table 4.5.3.2 measures of polymorphism and heterozygosity in grouped populations of the Northern Group of the $H$. maculatus complex.

\begin{tabular}{|c|c|c|c|c|c|}
\hline \multirow[b]{2}{*}{ Population } & \multirow{2}{*}{$\begin{array}{l}\text { Mean } \\
\text { sample } \\
\text { size per } \\
\text { locus }\end{array}$} & \multirow{2}{*}{$\begin{array}{l}\text { Mean no. } \\
\text { of alleles } \\
\text { per locus }\end{array}$} & \multirow{2}{*}{$\begin{array}{l}\text { Percentage } \\
\text { of loci } \\
\text { polymorphic }\end{array}$} & \multicolumn{2}{|c|}{ Mean heterozygosity } \\
\hline & & & & $\begin{array}{l}\text { direct- } \\
\text { count }\end{array}$ & $\begin{array}{l}\text { hdywbg } \\
\text { expected }\end{array}$ \\
\hline Mount Arthur & $\begin{array}{l}2.0 \\
(0.0)\end{array}$ & $\begin{array}{l}1.0 \\
(0.0)\end{array}$ & 0.0 & $\begin{array}{l}0.00 \\
(0.00)\end{array}$ & $\begin{array}{l}0.00 \\
(0.00)\end{array}$ \\
\hline Batty 1 & $\begin{array}{c}5.0 \\
(0.0)\end{array}$ & $\begin{array}{l}1.0 \\
(0.0)\end{array}$ & 3.7 & $\begin{array}{l}0.02 \\
(0.02)\end{array}$ & $\begin{array}{l}0.02 \\
(0.02)\end{array}$ \\
\hline Ward & $\begin{array}{l}4.0 \\
(0.0)\end{array}$ & $\begin{array}{l}1.2 \\
(0.1)\end{array}$ & 18.5 & $\begin{array}{l}0.03 \\
(0.02)\end{array}$ & $\begin{array}{l}0.06 \\
(0.03)\end{array}$ \\
\hline Batty 2 & $\begin{array}{l}6.0 \\
(0.0)\end{array}$ & $\begin{array}{l}1.0 \\
(0.0)\end{array}$ & 3.7 & $\begin{array}{l}0.01 \\
(0.01)\end{array}$ & $\begin{array}{l}0.01 \\
(0.01)\end{array}$ \\
\hline Glenduan, Nelson & $\begin{array}{c}4.0 \\
(0.0)\end{array}$ & $\begin{array}{l}1.1 \\
(0.1)\end{array}$ & 7.4 & $\begin{array}{l}0.04 \\
(0.03)\end{array}$ & $\begin{array}{l}0.04 \\
(0.03)\end{array}$ \\
\hline Red Rocks & $\begin{array}{c}6.0 \\
(0.0)\end{array}$ & $\begin{array}{l}1.1 \\
(0.1)\end{array}$ & 3.7 & $\begin{array}{l}0.02 \\
(0.02)\end{array}$ & $\begin{array}{l}0.02 \\
(0.02)\end{array}$ \\
\hline Hundalee & $\begin{array}{l}1.0 \\
(0.0)\end{array}$ & $\begin{array}{l}1.0 \\
(0.0)\end{array}$ & 3.7 & $\begin{array}{l}0.04 \\
(0.04)\end{array}$ & $\begin{array}{l}0.04 \\
(0.04)\end{array}$ \\
\hline Marlborough Coast & $\begin{array}{l}22.0 \\
(0.0)\end{array}$ & $\begin{array}{l}1.2 \\
(0.1)\end{array}$ & 11.1 & $\begin{array}{l}0.03 \\
(0.02)\end{array}$ & $\begin{array}{l}0.04 \\
(0.02)\end{array}$ \\
\hline Waiau & $\begin{array}{l}1.0 \\
(0.0)\end{array}$ & $\begin{array}{l}1.0 \\
(0.0)\end{array}$ & 0.0 & $\begin{array}{l}0.00 \\
(0.00)\end{array}$ & $\begin{array}{l}0.00 \\
(0.00)\end{array}$ \\
\hline Inland Marlborough & $\begin{array}{l}11.0 \\
(0.0)\end{array}$ & $\begin{array}{l}1.2 \\
(0.1)\end{array}$ & 11.1 & $\begin{array}{l}0.06 \\
(0.03)\end{array}$ & $\begin{array}{l}0.05 \\
(0.03)\end{array}$ \\
\hline Kahutara 1 & $\begin{array}{l}4.0 \\
(0.0)\end{array}$ & $\begin{array}{l}1.1 \\
(0.1)\end{array}$ & 7.4 & $\begin{array}{l}0.04 \\
(0.03)\end{array}$ & $\begin{array}{l}0.04 \\
(0.03)\end{array}$ \\
\hline Chickens + Mercuries Islands & $\begin{array}{l}9.0 \\
(0.0)\end{array}$ & $\begin{array}{l}1.1 \\
(0.1)\end{array}$ & 11.1 & $\begin{array}{l}0.02 \\
(0.01)\end{array}$ & $\begin{array}{l}0.02 \\
(0.01)\end{array}$ \\
\hline Bay of Plenty Islands & $\begin{array}{c}15.0 \\
(0.0)\end{array}$ & $\begin{array}{l}1.1 \\
(0.1)\end{array}$ & 11.1 & $\begin{array}{l}0.02 \\
(0.02)\end{array}$ & $\begin{array}{l}0.04 \\
(0.02)\end{array}$ \\
\hline Gisborne & $\begin{array}{l}1.0 \\
(0.0)\end{array}$ & $\begin{array}{l}1.0 \\
(0.0)\end{array}$ & 0.0 & $\begin{array}{l}0.00 \\
(0.00)\end{array}$ & $\begin{array}{l}0.00 \\
(0.00)\end{array}$ \\
\hline Hawkes Bay & $\begin{array}{c}1.0 \\
(0.0)\end{array}$ & $\begin{array}{l}1.0 \\
(0.0)\end{array}$ & 0.0 & $\begin{array}{l}0.00 \\
(0.00)\end{array}$ & $\begin{array}{l}0.00 \\
(0.00)\end{array}$ \\
\hline Wairarapa & $\begin{array}{l}12.0 \\
(0.0)\end{array}$ & $\begin{array}{l}1.3 \\
(0.1)\end{array}$ & 18.5 & $\begin{array}{l}0.03 \\
(0.02)\end{array}$ & $\begin{array}{l}0.04 \\
(0.03)\end{array}$ \\
\hline Seatoun Heights & $\begin{array}{c}1.0 \\
(0.0)\end{array}$ & $\begin{array}{l}1.0 \\
(0.0)\end{array}$ & 0.0 & $\begin{array}{l}0.00 \\
(0.00)\end{array}$ & $\begin{array}{l}0.00 \\
(0.00)\end{array}$ \\
\hline South coast, North Island & $\begin{array}{r}32.0 \\
(0.0)\end{array}$ & $\begin{array}{l}1.4 \\
(0.1)\end{array}$ & 22.2 & $\begin{array}{l}0.03 \\
(0.02)\end{array}$ & $\begin{array}{l}0.03 \\
(0.02)\end{array}$ \\
\hline Cook Strait & $\begin{array}{r}57.0 \\
(0.0)\end{array}$ & $\begin{array}{l}1.4 \\
(0.1)\end{array}$ & 29.6 & $\begin{array}{l}0.02 \\
(0.01)\end{array}$ & $\begin{array}{l}0.03 \\
(0.01)\end{array}$ \\
\hline Brothers Island & $\begin{array}{l}2.0 \\
(0.0)\end{array}$ & $\begin{array}{l}1.1 \\
(0.1)\end{array}$ & $11: 1$ & $\begin{array}{l}0.06 \\
(0.03)\end{array}$ & $\begin{array}{l}0.06 \\
(0.03)\end{array}$ \\
\hline Titahi Bay, Mana Island & $\begin{array}{l}15.0 \\
(0.0)\end{array}$ & $\begin{array}{l}1.1 \\
(0.1)\end{array}$ & 7.4 & $\begin{array}{l}0.02 \\
(0.02)\end{array}$ & $\begin{array}{l}0.03 \\
(0.02)\end{array}$ \\
\hline Trass Valley & $\begin{array}{l}2.0 \\
(0.0)\end{array}$ & $\begin{array}{l}1.0 \\
(0.0)\end{array}$ & 3.7 & $\begin{array}{l}0.02 \\
(0.02)\end{array}$ & $\begin{array}{l}0.02 \\
(0.02)\end{array}$ \\
\hline Wairoa Valley & $\begin{array}{l}3.0 \\
(0.0)\end{array}$ & $\begin{array}{l}1.1 \\
(0.1)\end{array}$ & 11.1 & $\begin{array}{l}0.05 \\
(0.03)\end{array}$ & $\begin{array}{l}0.04 \\
(0.03)\end{array}$ \\
\hline Red Hills & $\begin{array}{l}1.0 \\
(0.0)\end{array}$ & $\begin{array}{l}1.0 \\
(0.0)\end{array}$ & 3.7 & $\begin{array}{l}0.04 \\
(0.04)\end{array}$ & $\begin{array}{l}0.04 \\
(0.04)\end{array}$ \\
\hline Cloudy Hut & $\begin{array}{c}1.0 \\
(0.0)\end{array}$ & $\begin{array}{l}1.0 \\
(0.0)\end{array}$ & 3.7 & $\begin{array}{l}0.04 \\
(0.04)\end{array}$ & $\begin{array}{l}0.04 \\
(0.04)\end{array}$ \\
\hline
\end{tabular}




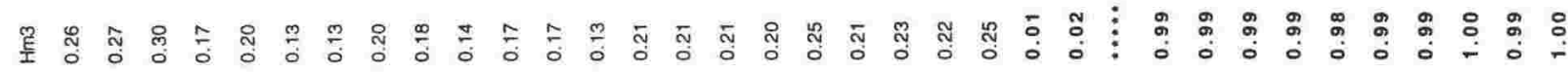

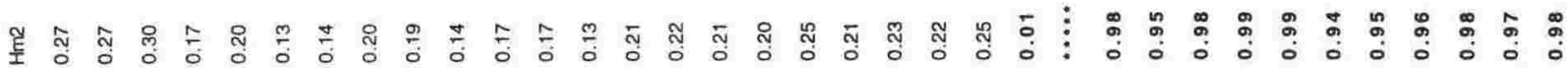

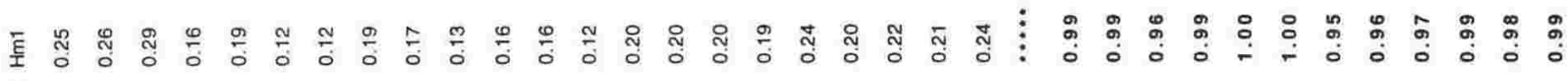

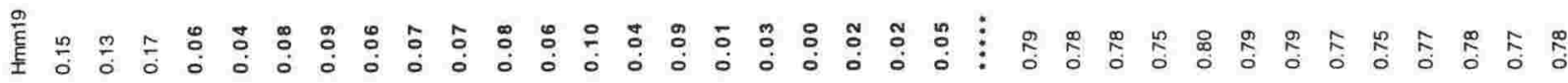
閏

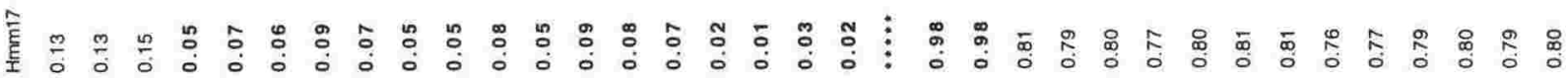

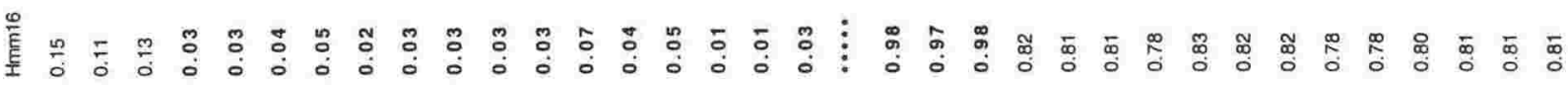

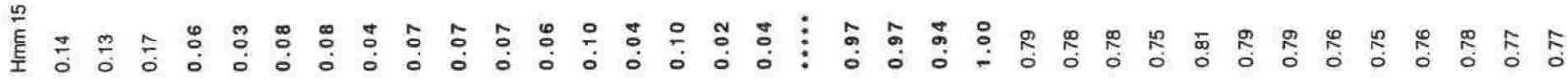
董 重 带

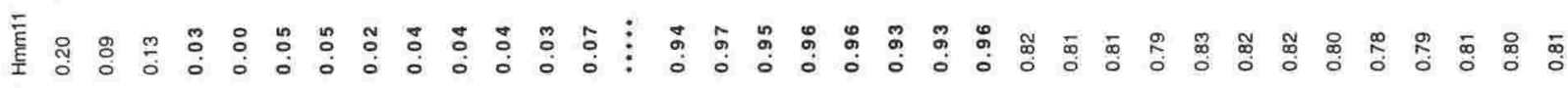

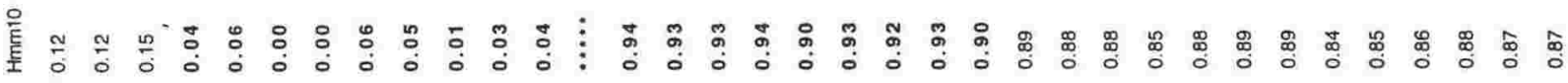

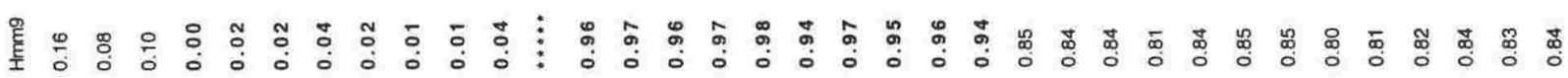

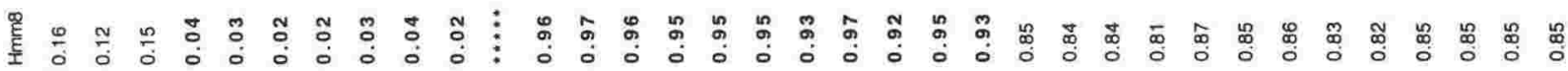

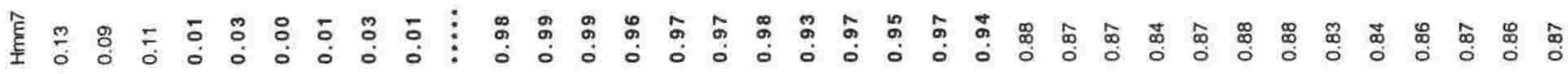

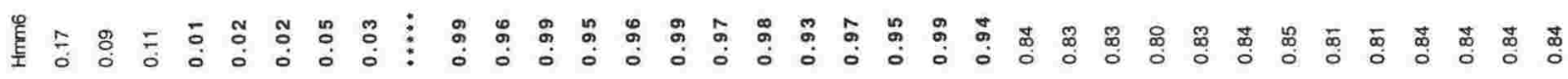
崖

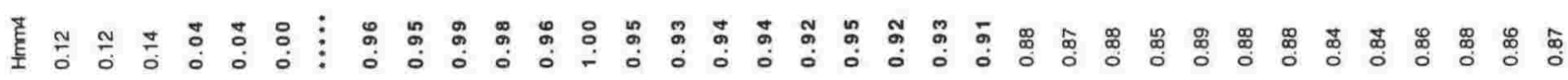

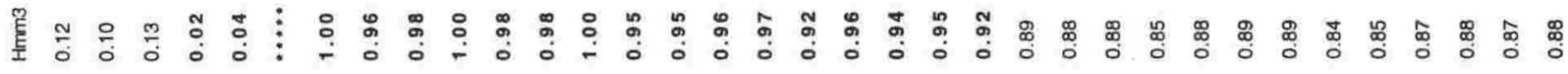

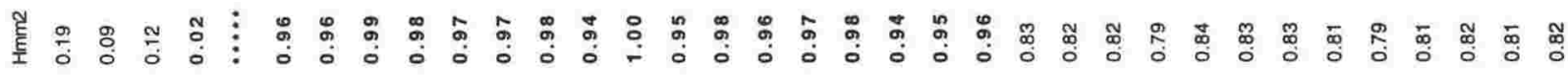
重 赵

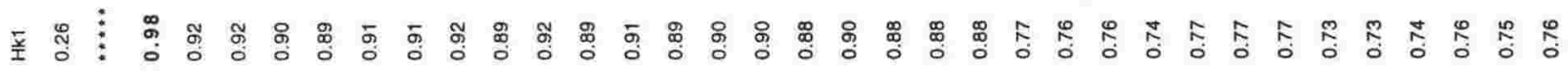
寽 


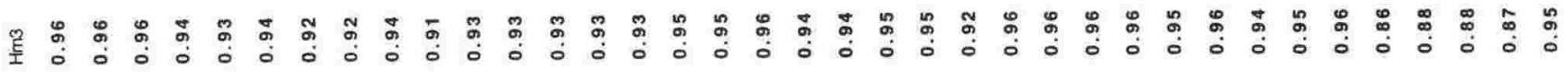

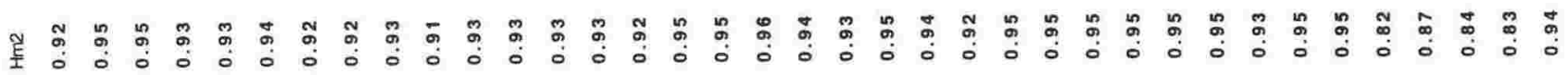

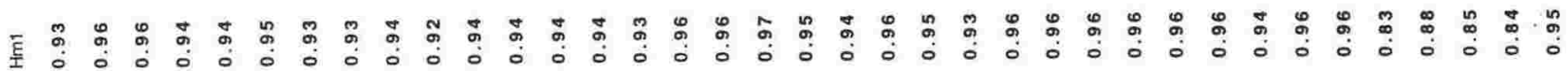

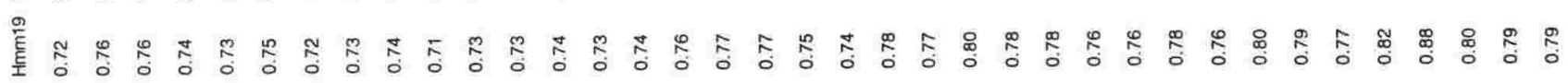

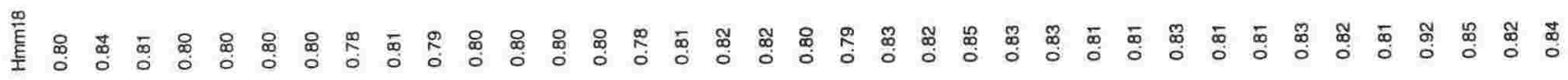

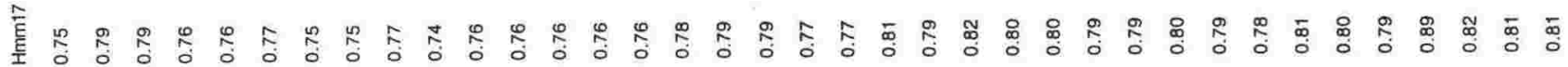

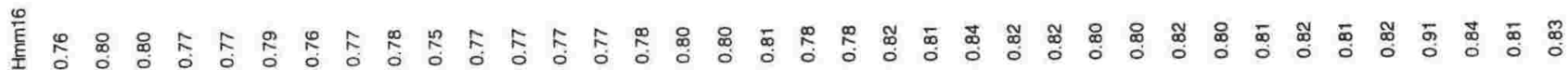

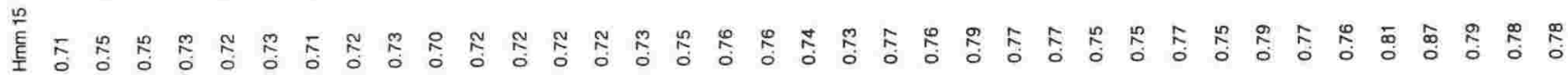

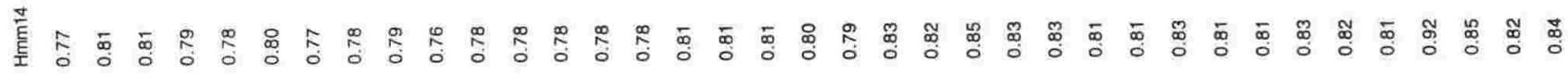

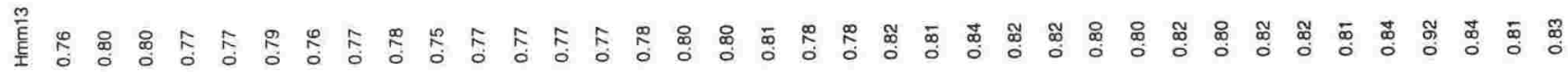

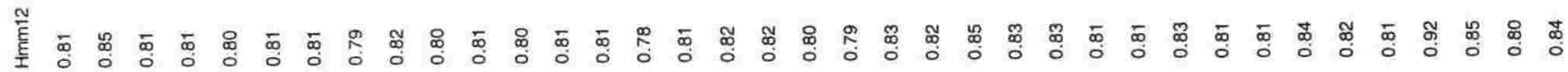

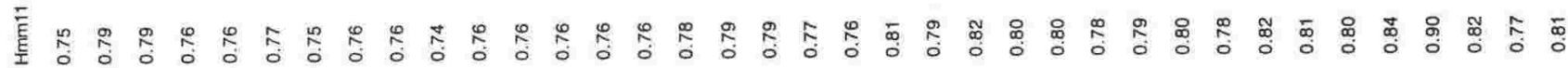
衰

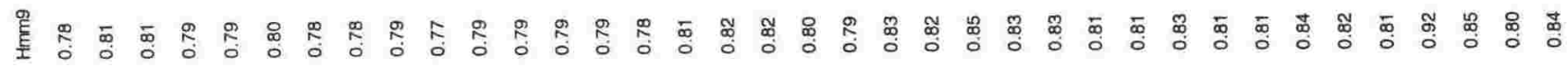

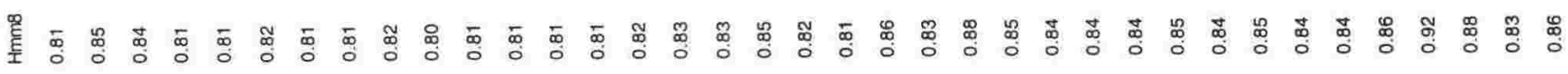
衰

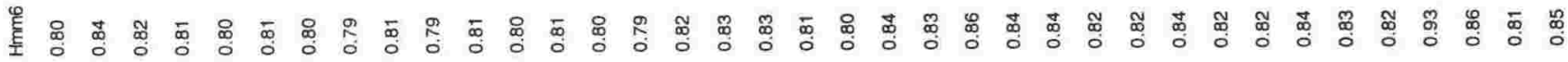

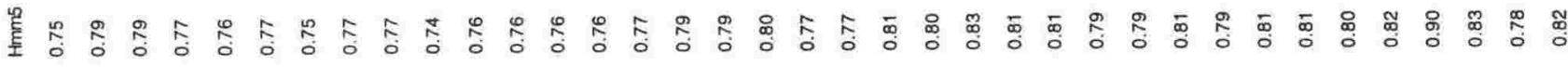

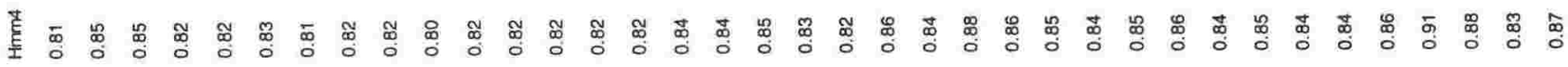

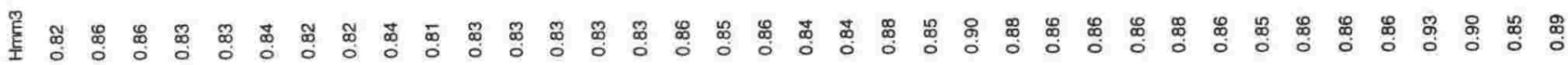

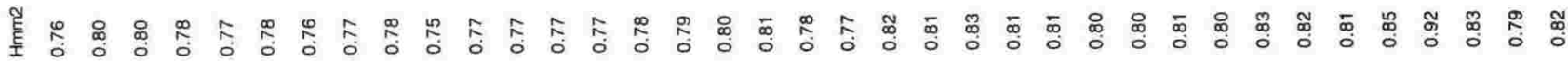

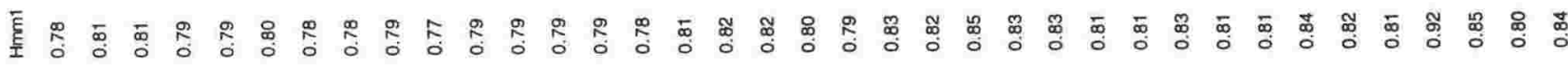
至

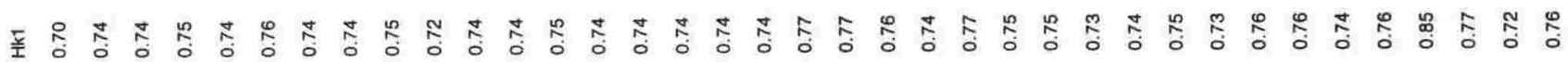

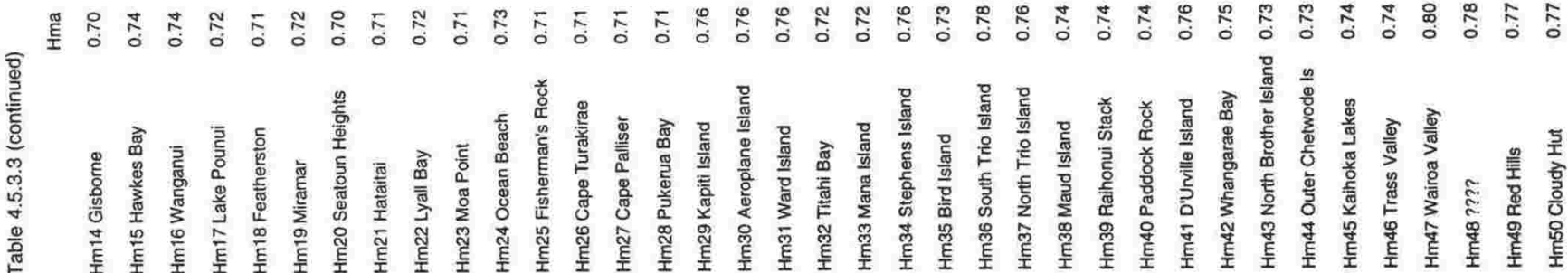




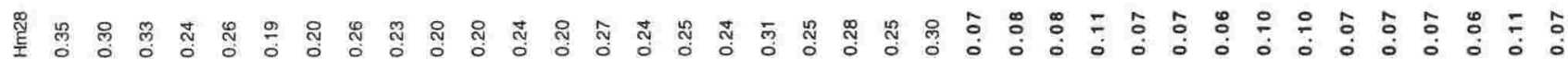
矛

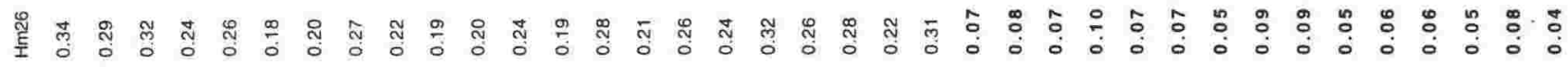
藍 荰

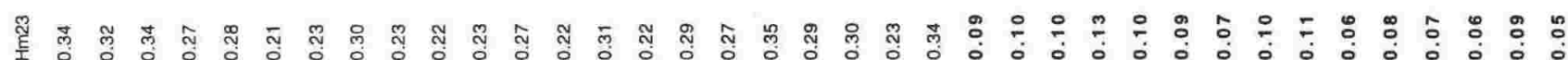
蛋

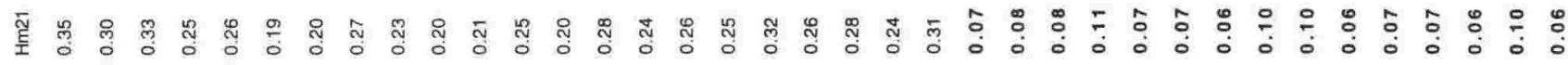

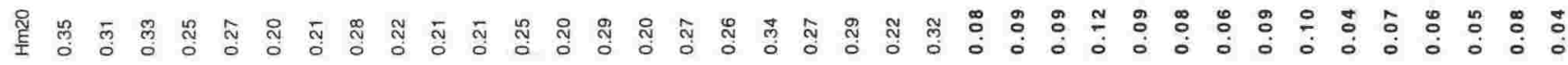

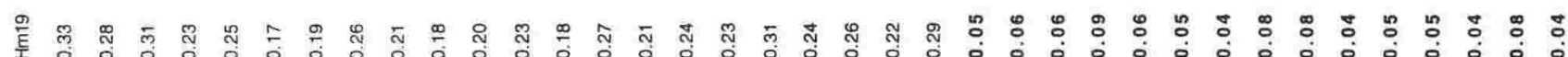
瑴 范 言 重

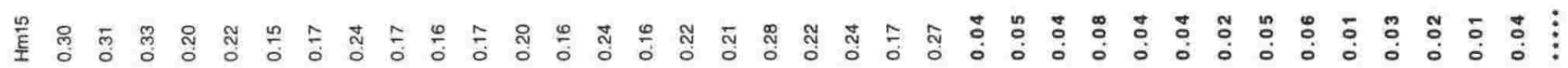

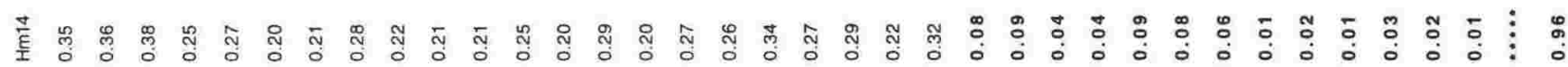

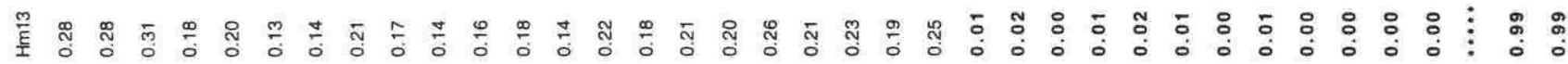

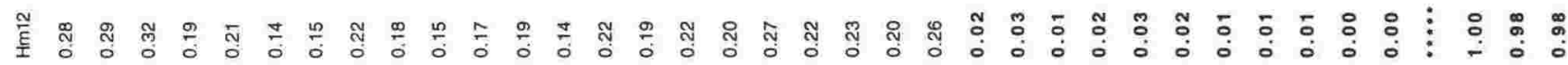

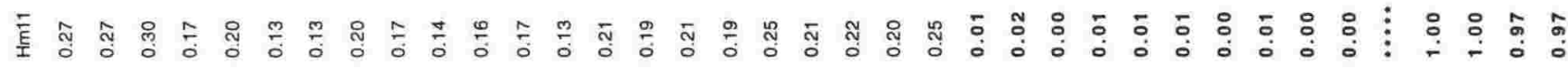

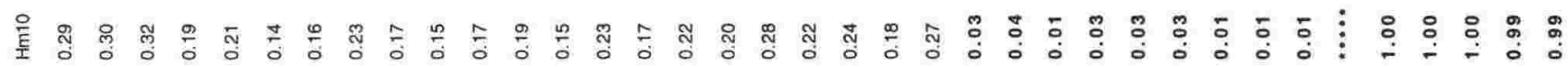
星 齿业 全

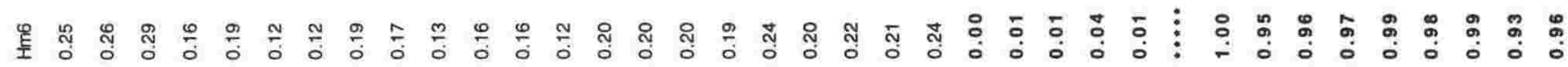

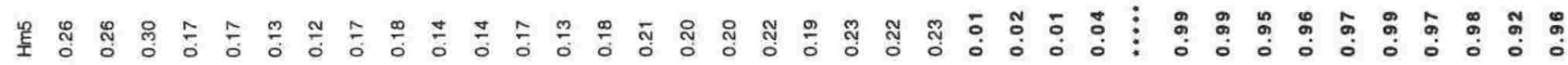
壱

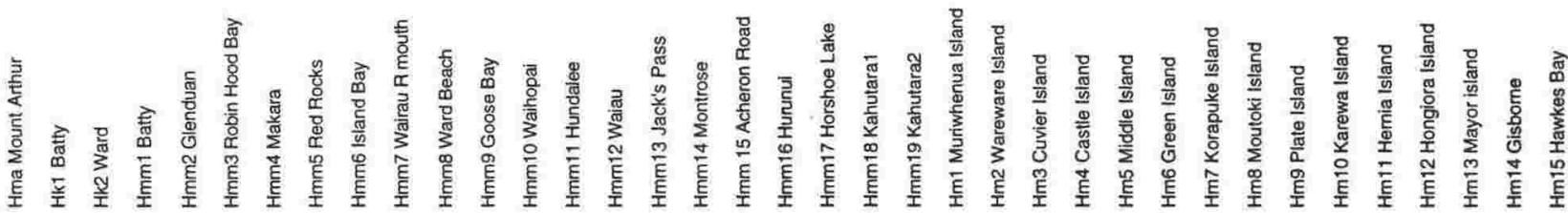




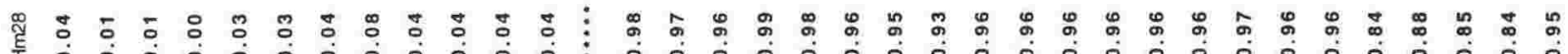
忒

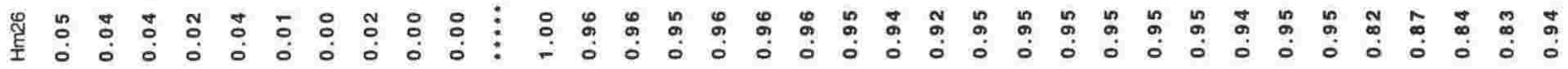

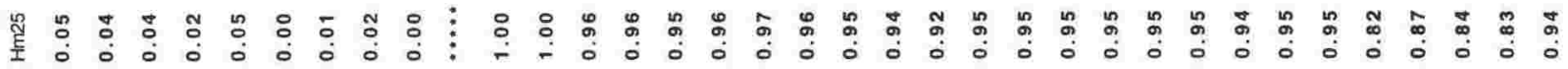

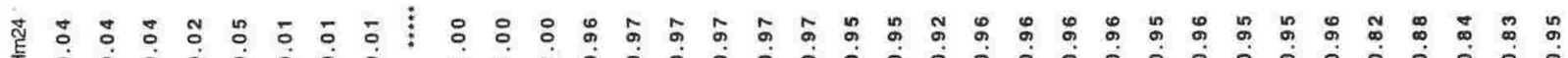

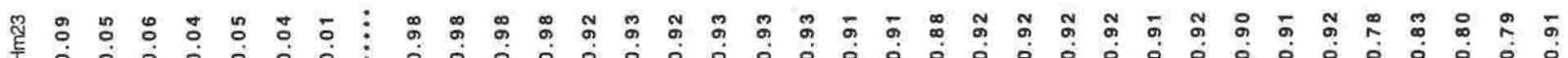

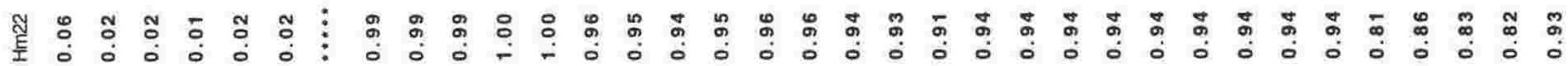

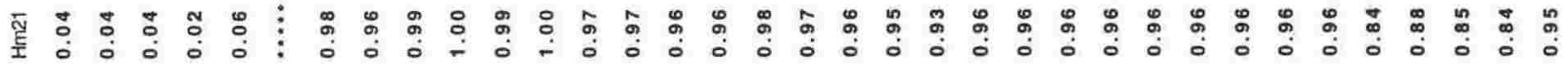

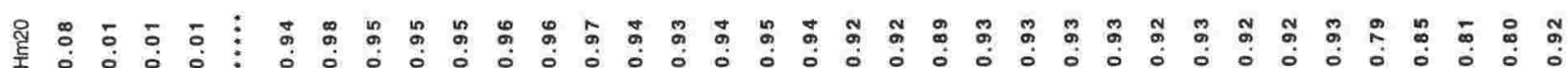
हீ

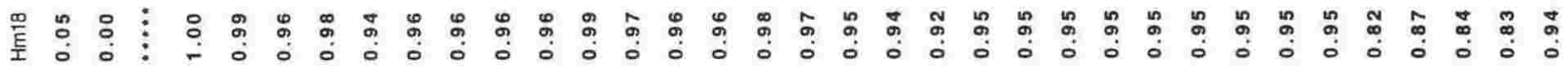

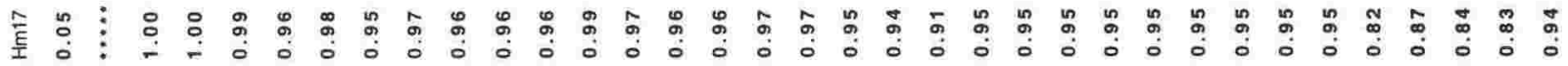

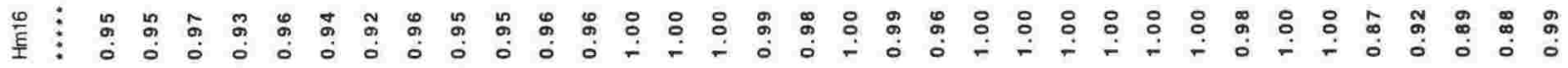
卢

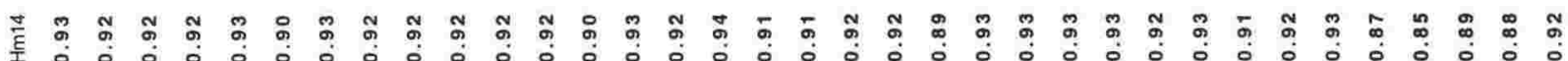
絰 $\begin{aligned} & 0 \\ & \dot{0}\end{aligned}$ Е

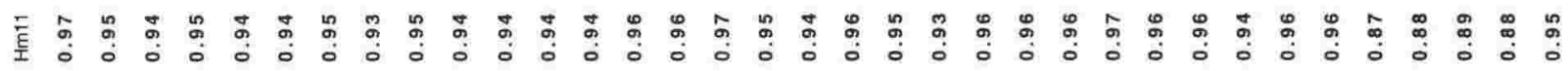
底

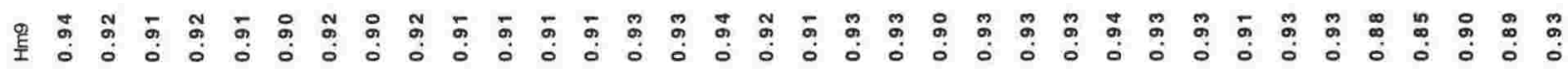

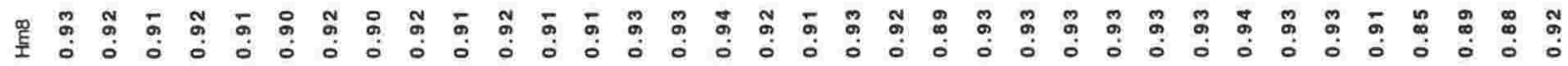
全 $\begin{aligned} & \hat{1} \\ & 0\end{aligned}$ 星 导

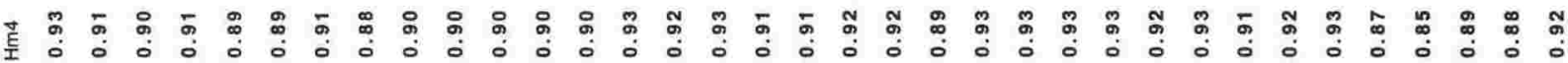


旿令

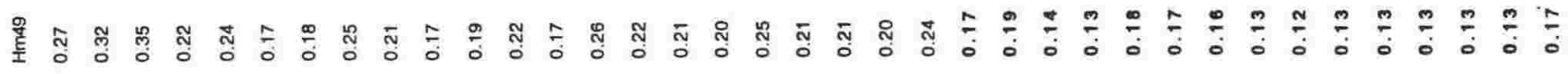
晕品

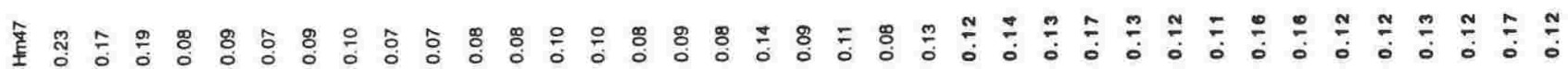
星竞

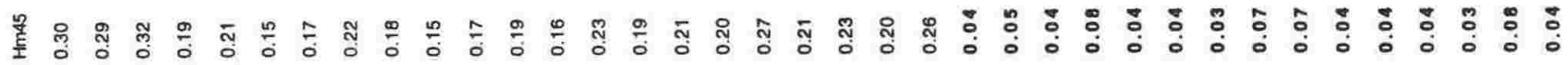

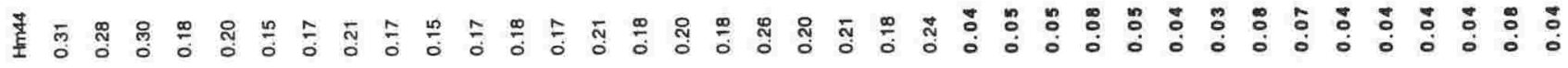

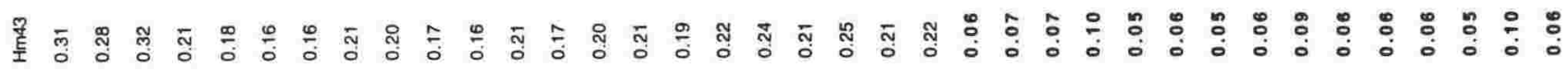

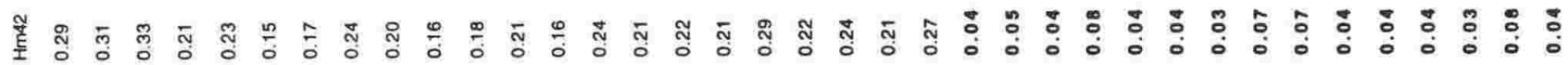

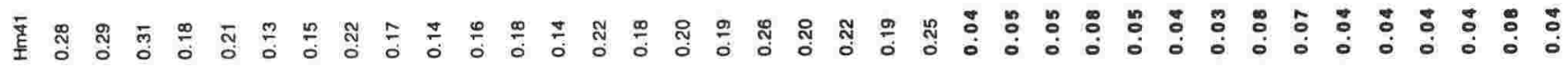

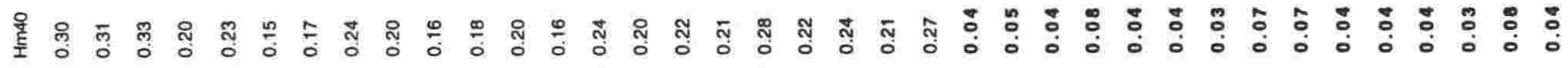

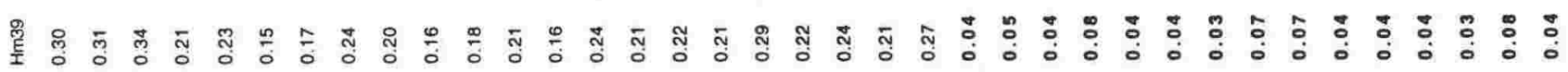

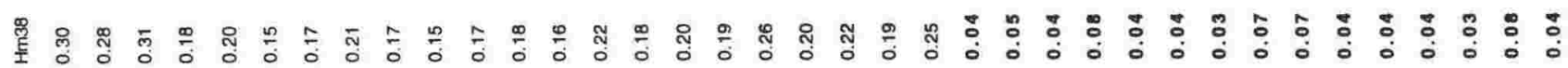
音跑

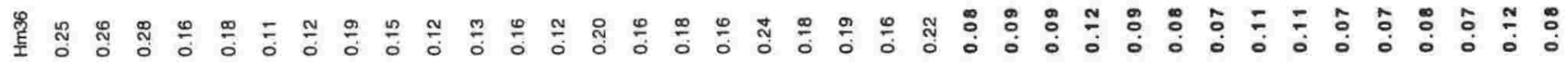

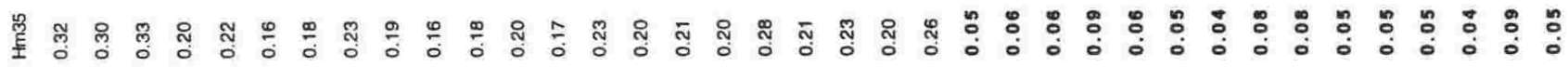

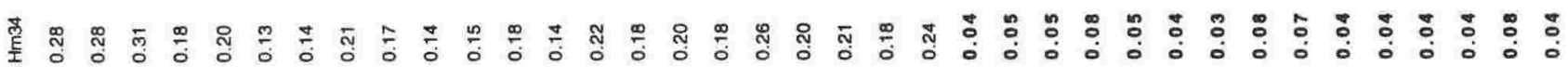

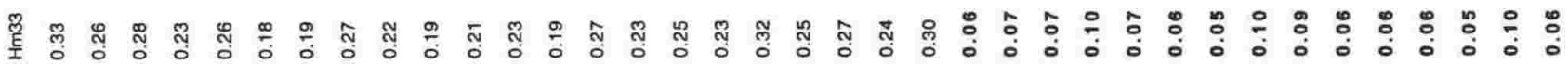

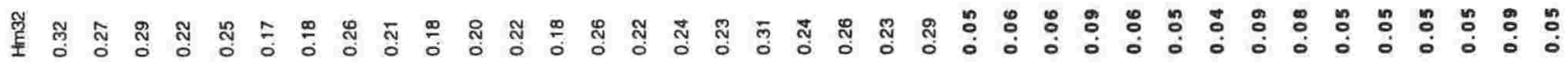

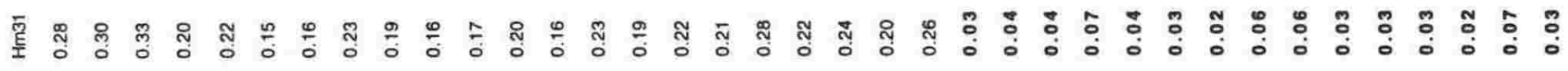

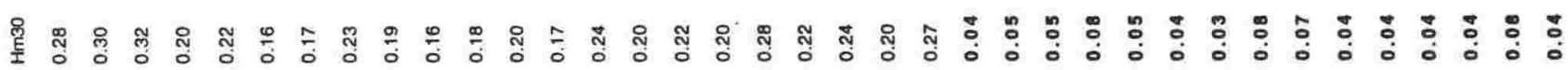

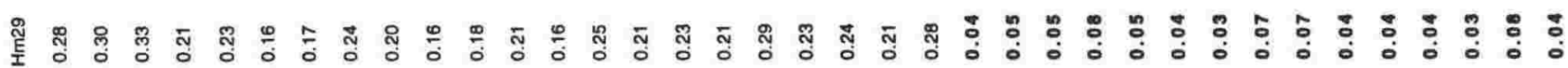


皇

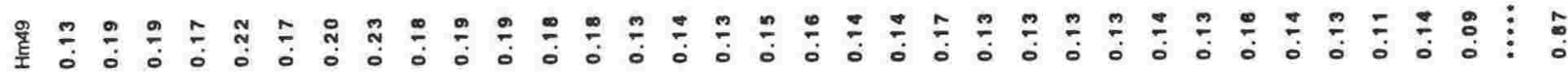

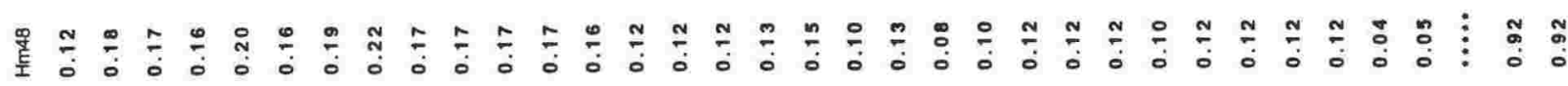

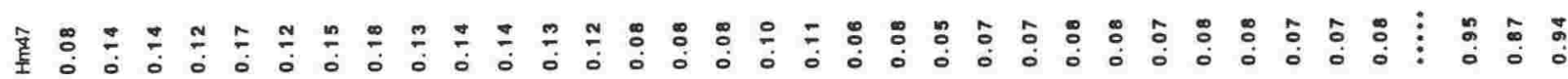

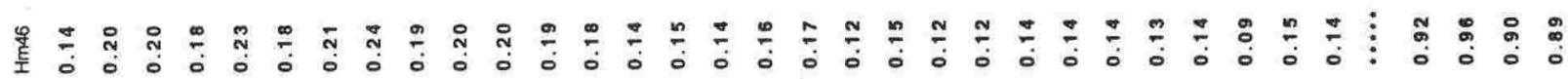

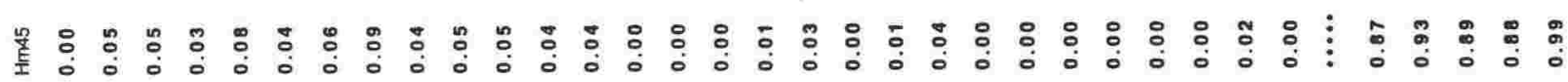

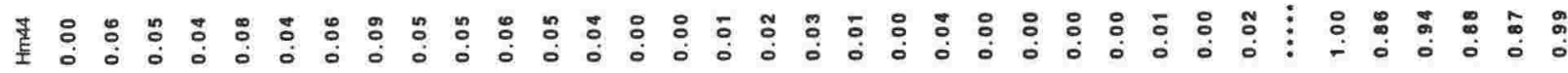

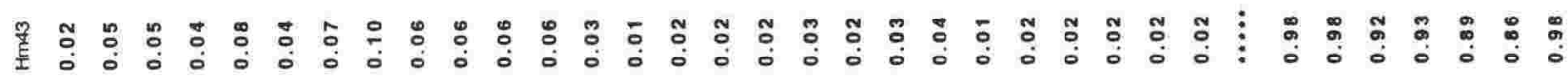

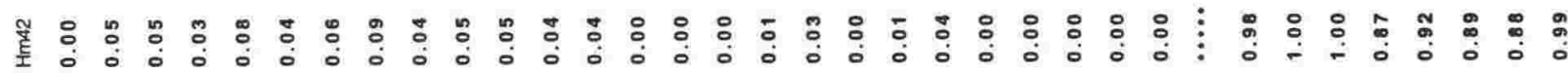

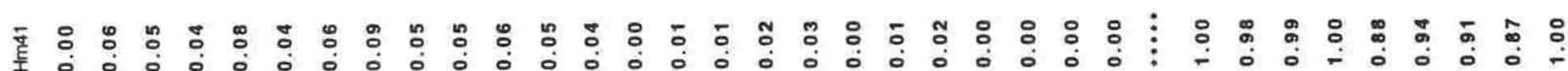

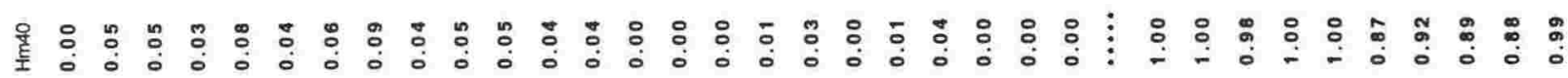

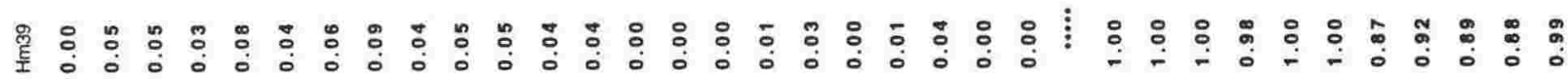

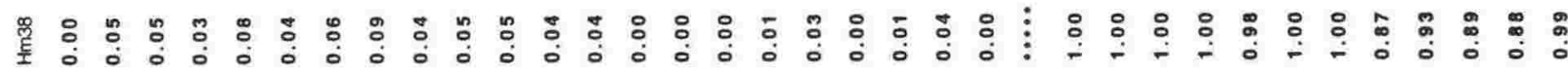

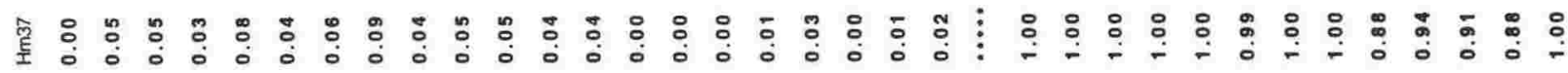

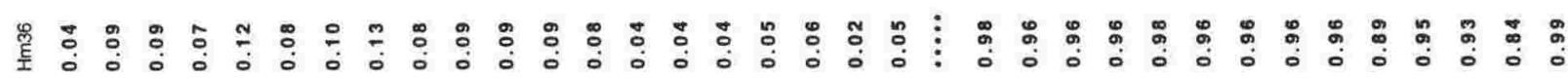

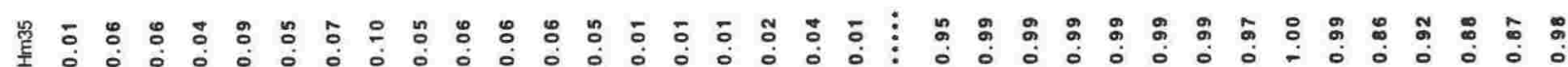

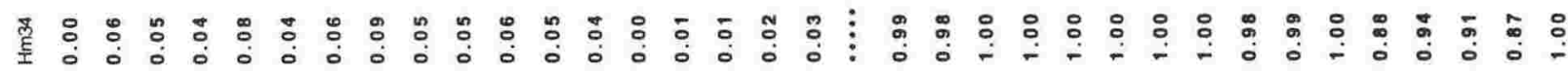
對 菣

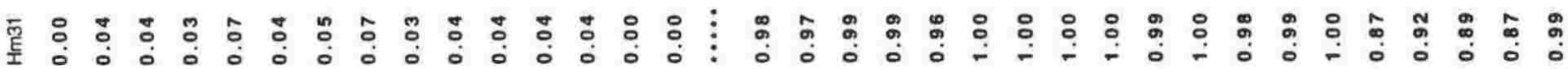

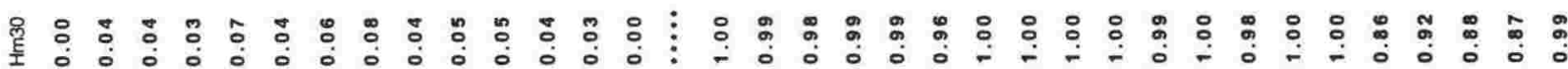
疍 


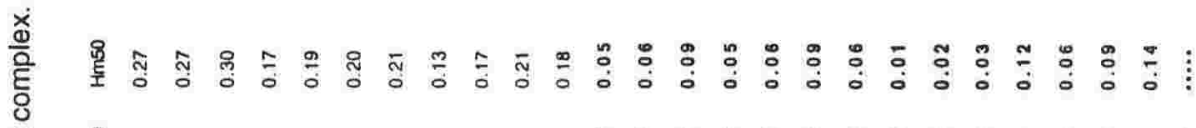

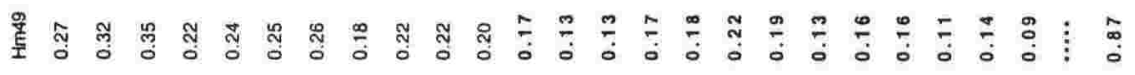

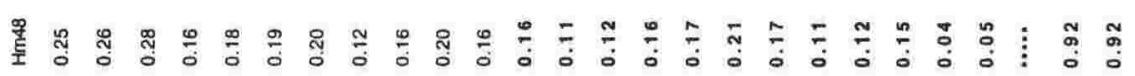

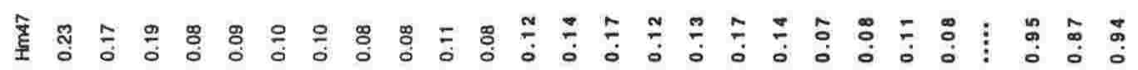

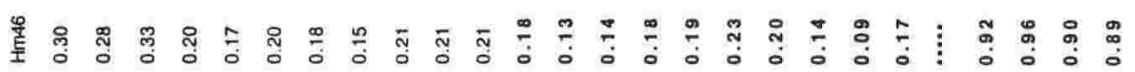

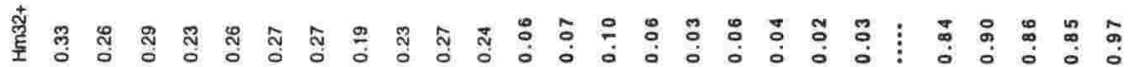

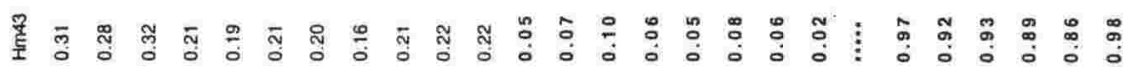

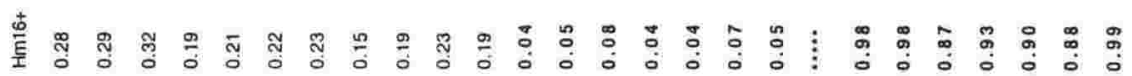

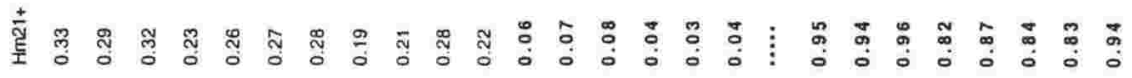

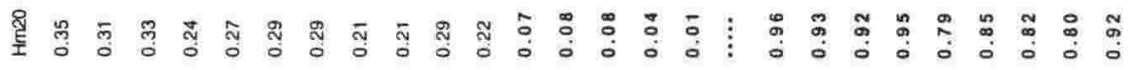

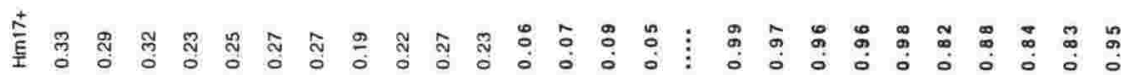

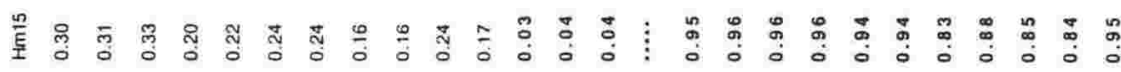

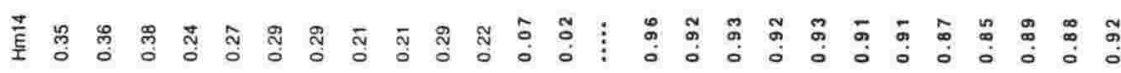

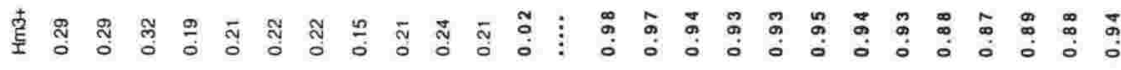
至

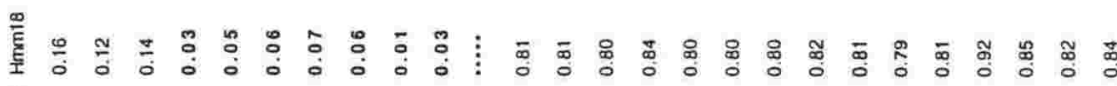

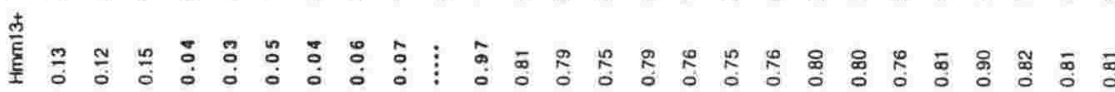

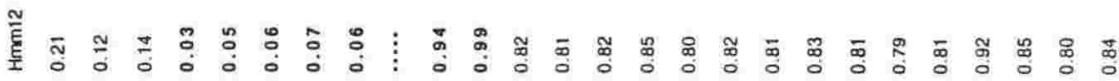

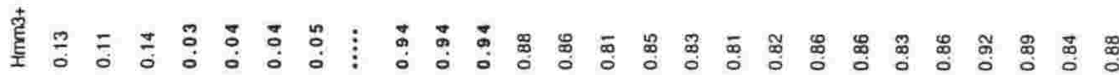
㐮

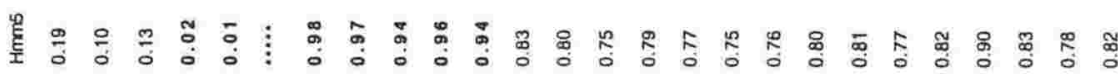

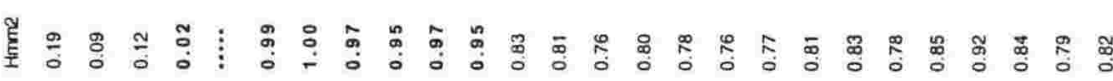

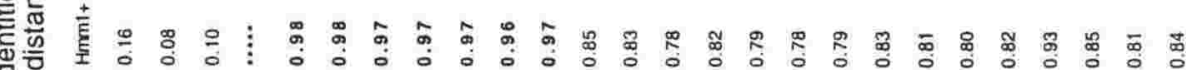

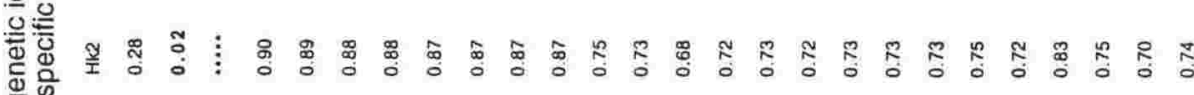

㐘焉至总 结 든.ㄴำ

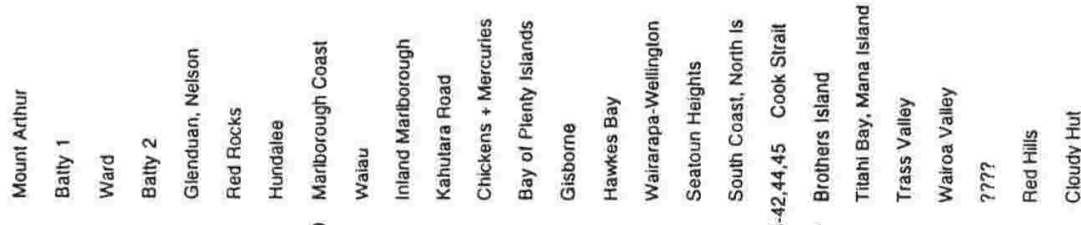


흉

苼

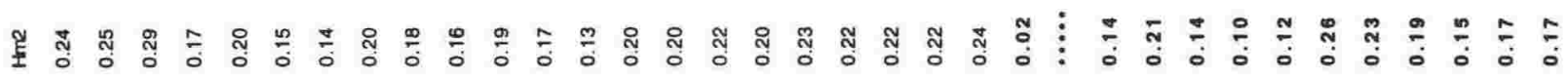

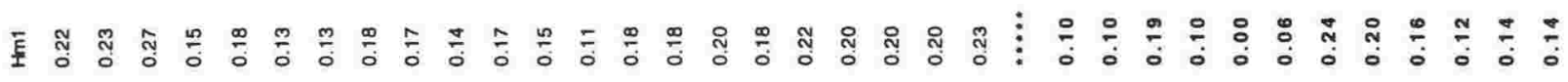

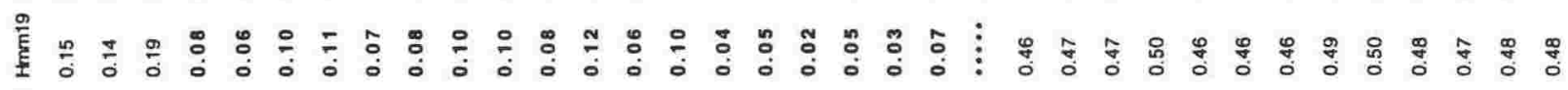

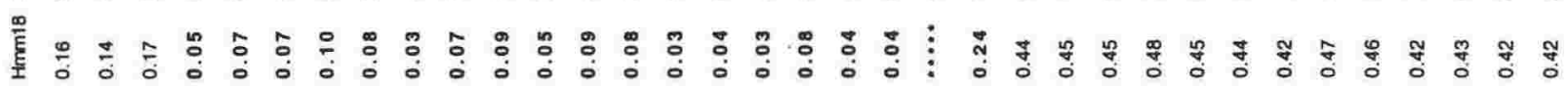

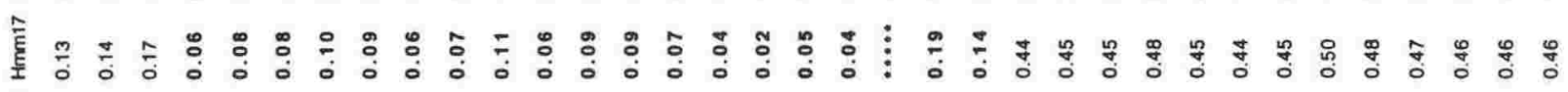
雳 毫

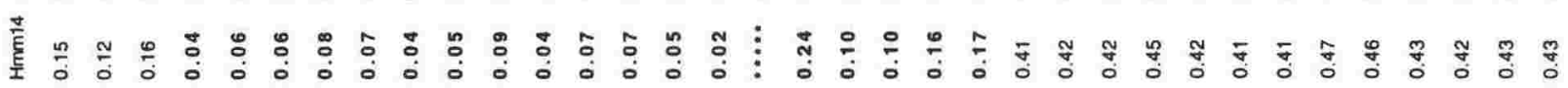

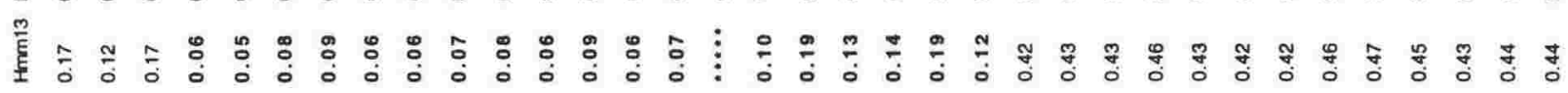

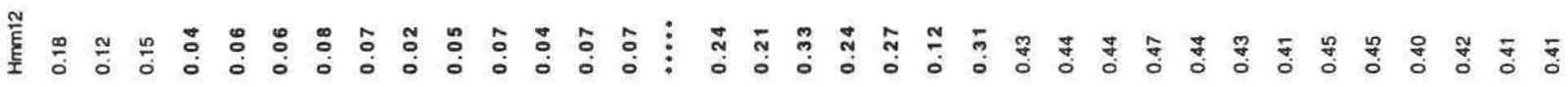

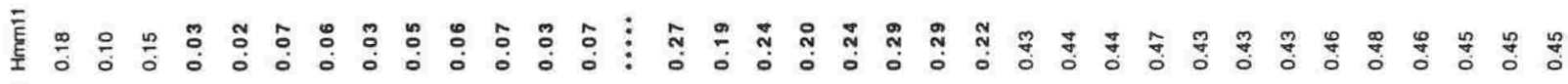

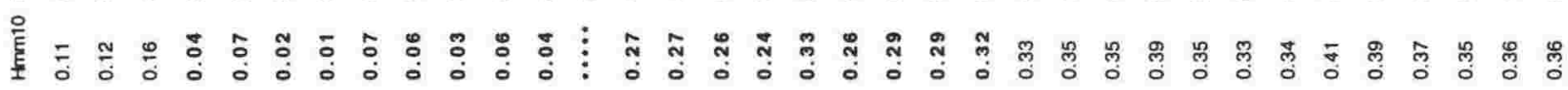

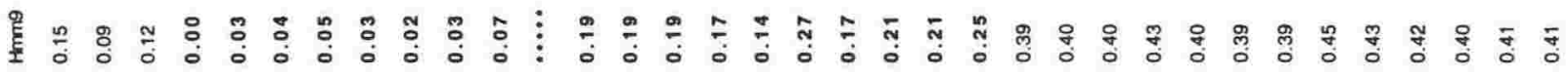

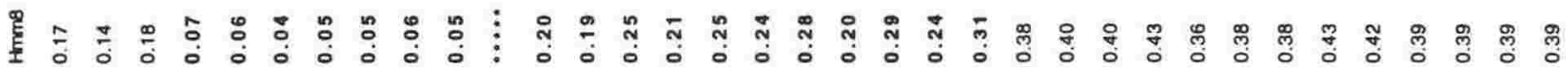
自

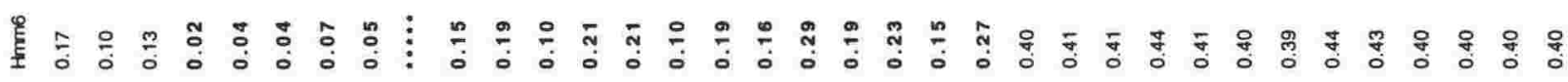

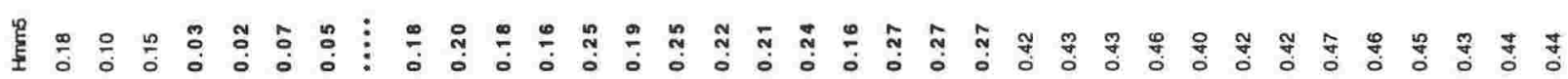

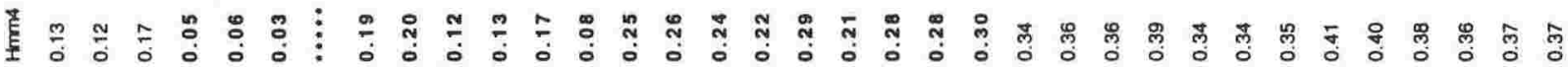

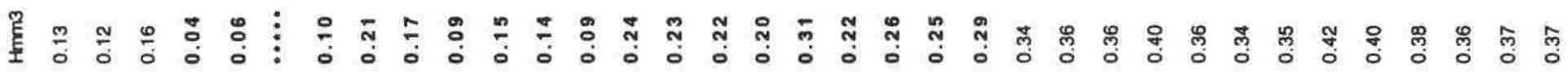

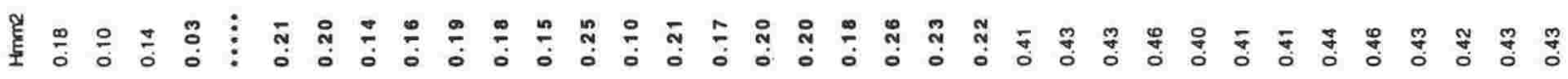

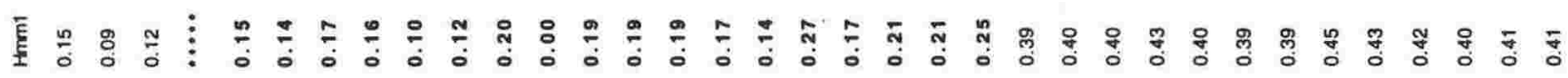

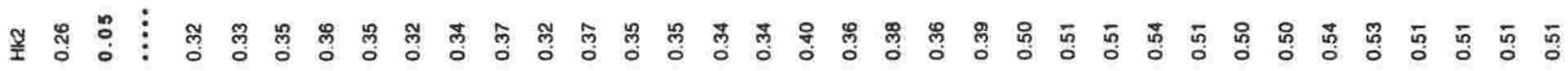

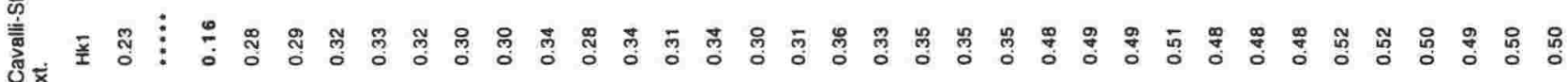

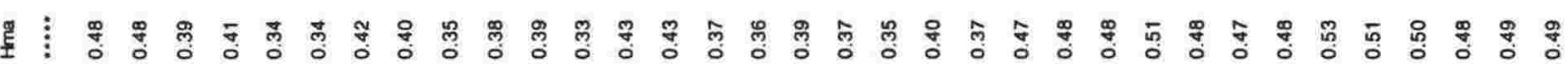

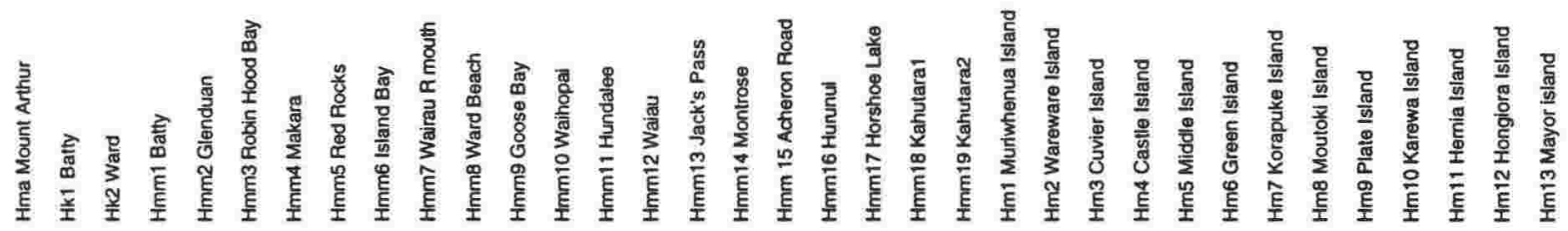




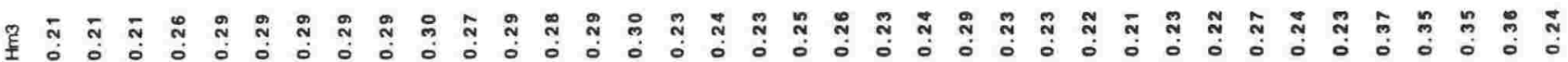
ข

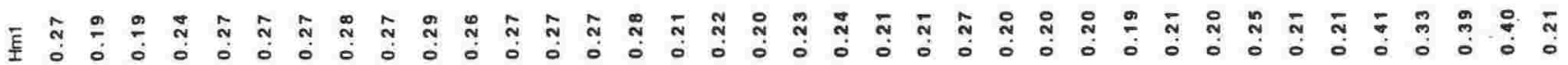

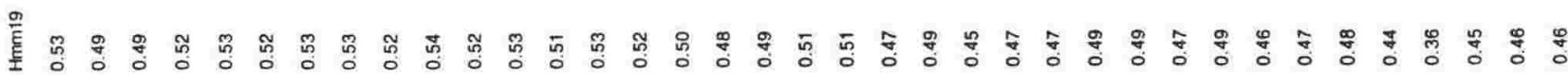

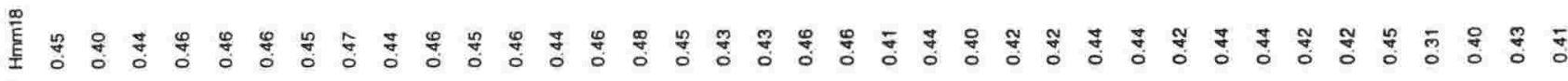

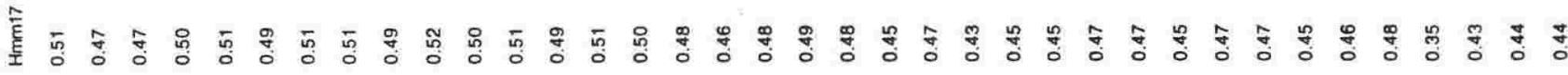

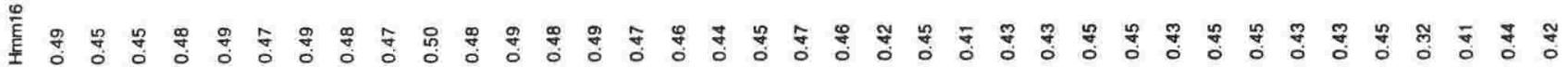

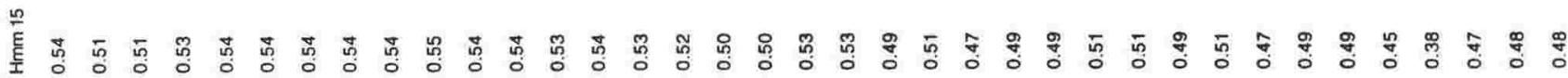

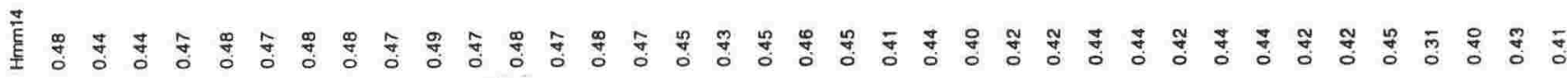

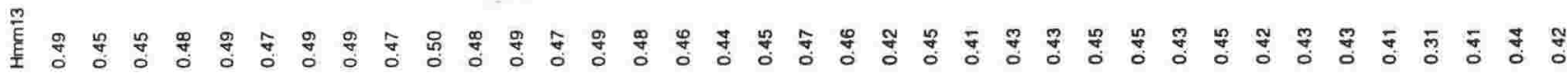

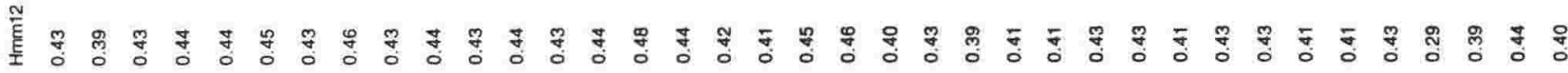

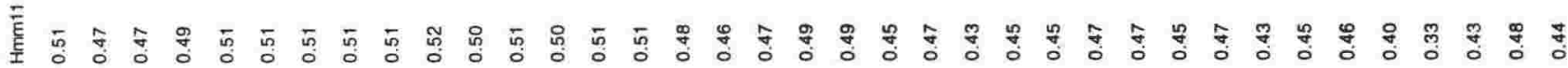

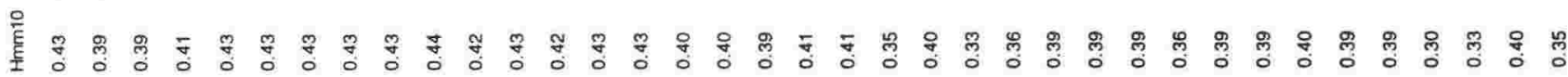

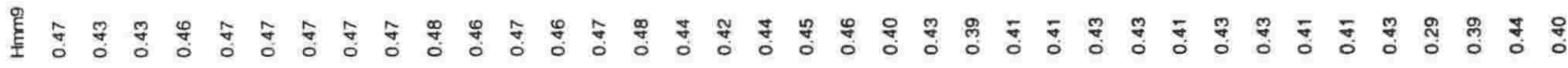

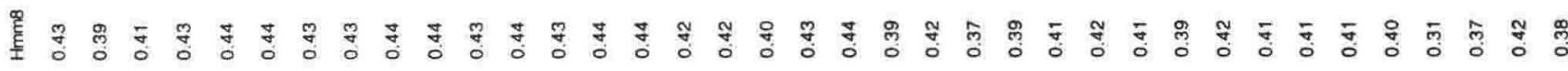

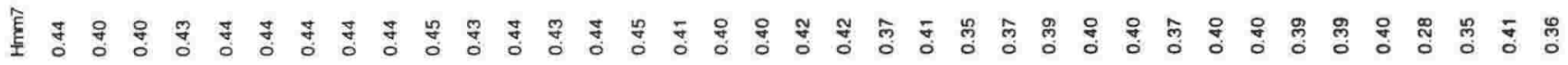

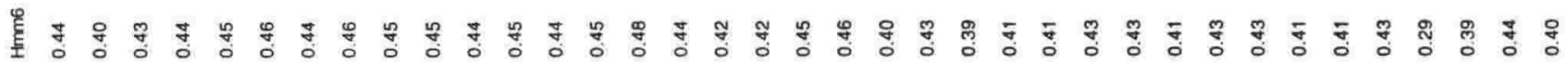

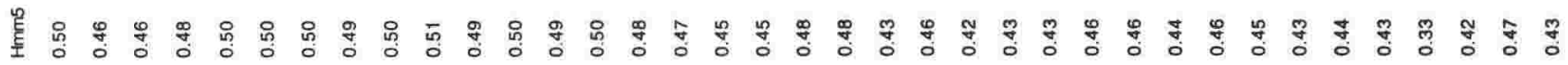

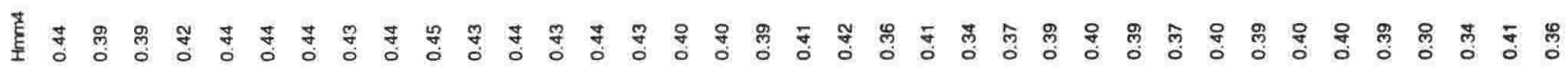
岳 孚

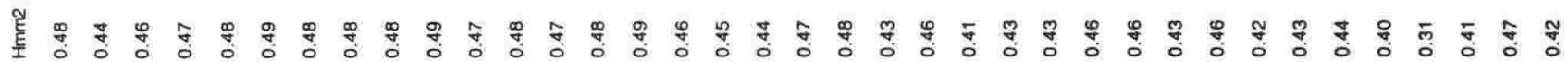

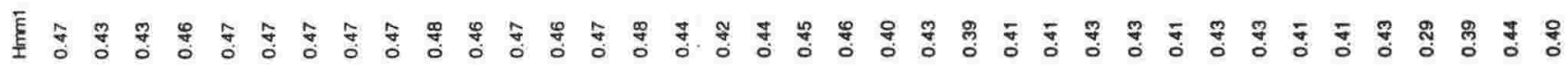

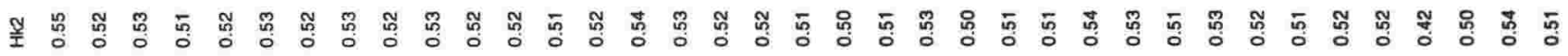

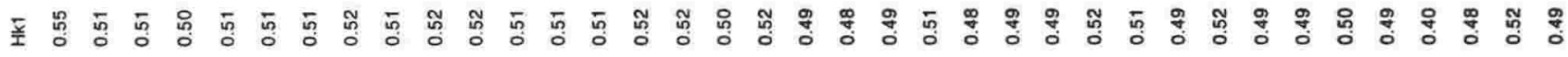

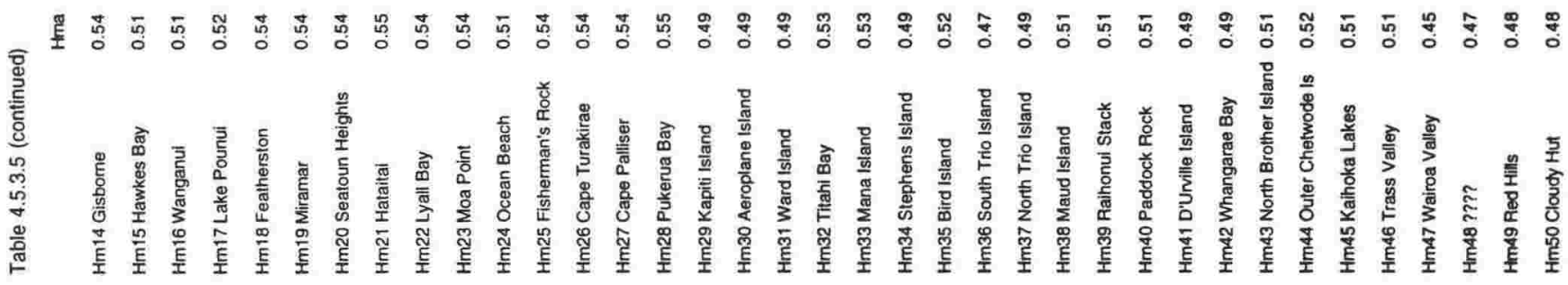




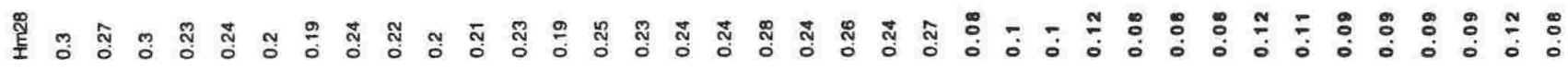

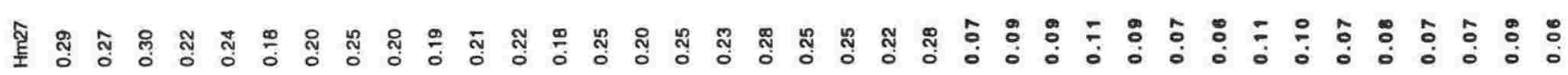

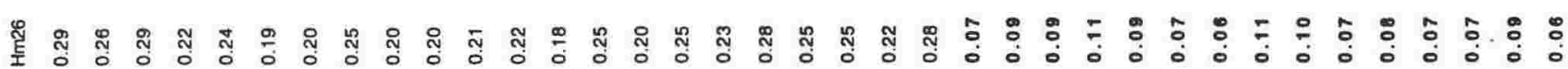

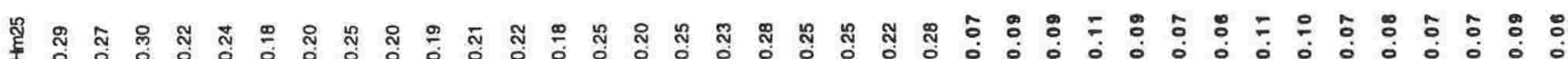

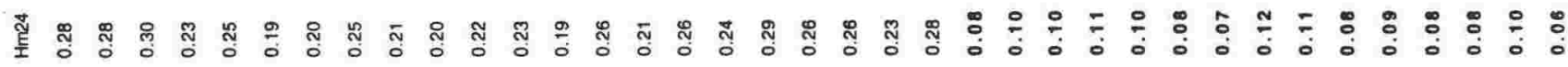

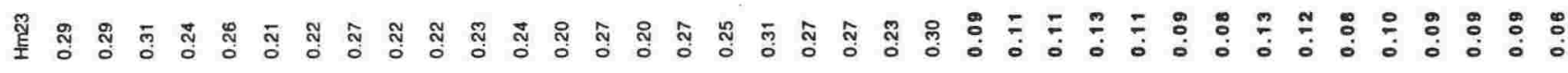

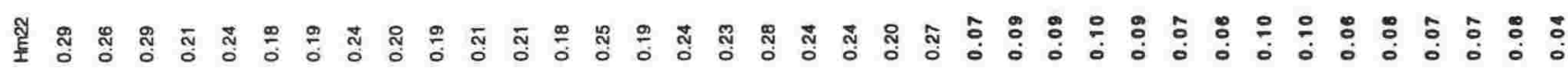

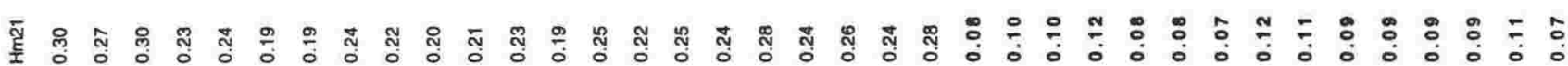

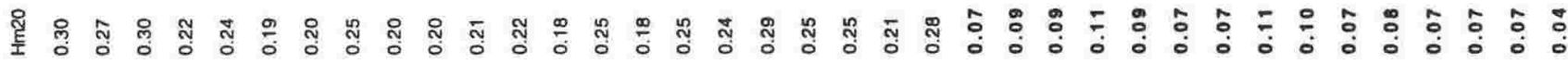

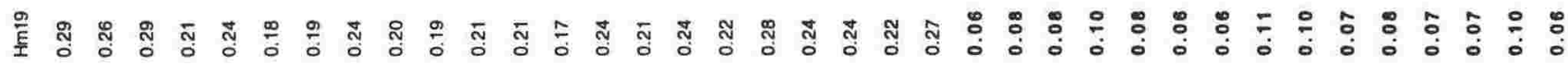

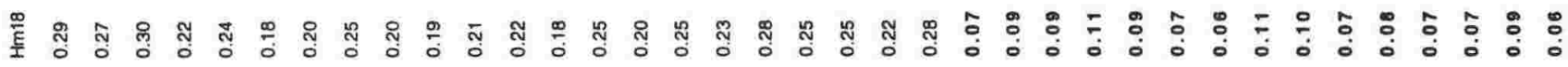

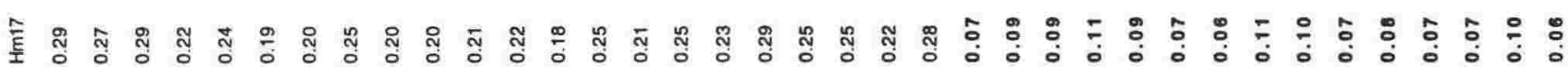

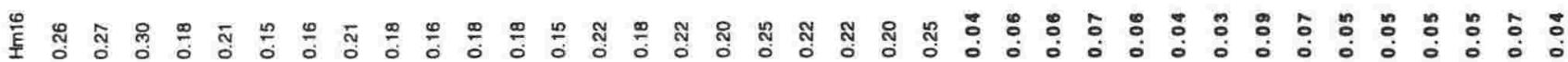

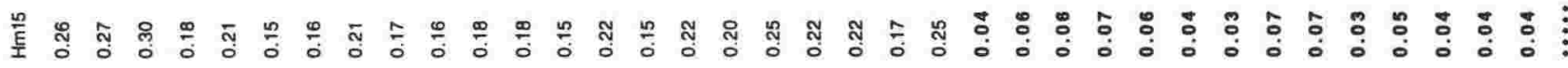

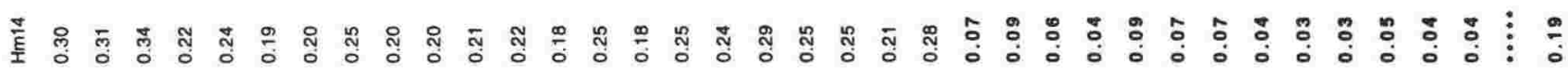

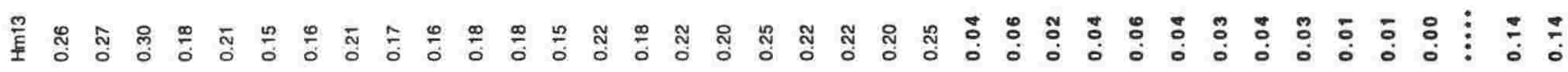
茞 㐮 尽

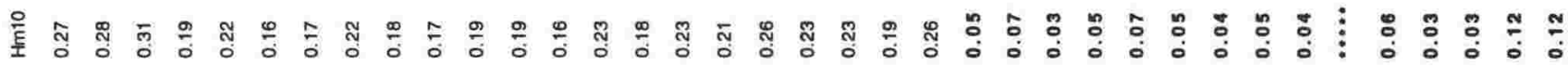

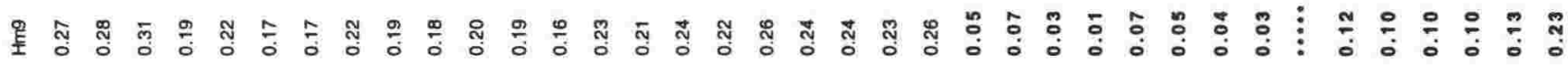
龇

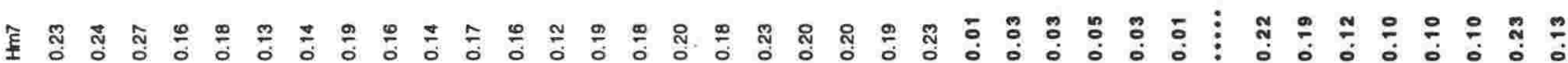

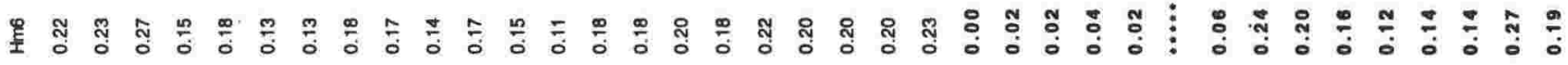

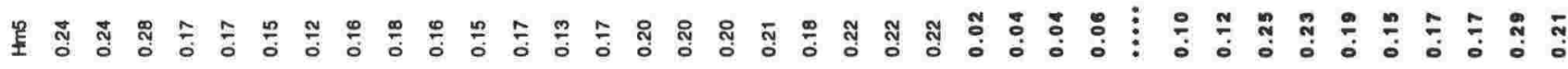

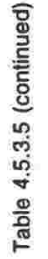

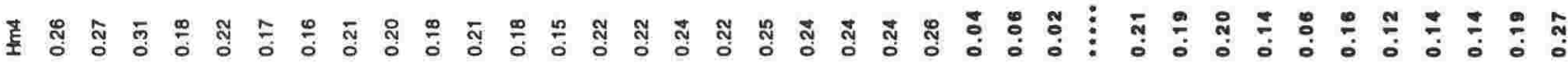

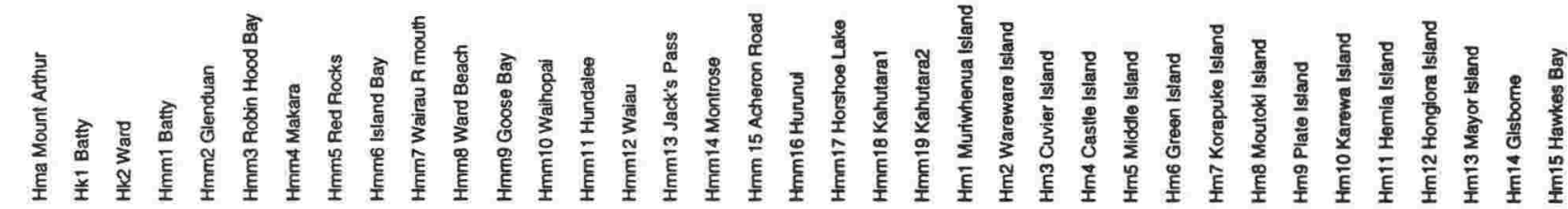




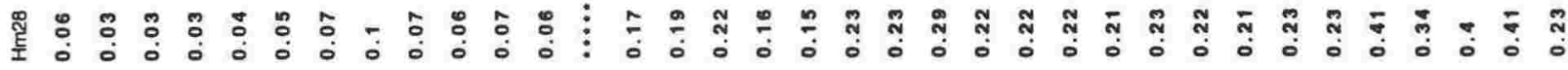

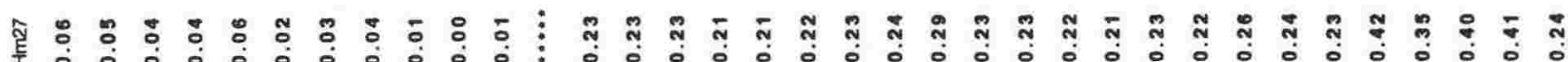
蛋:

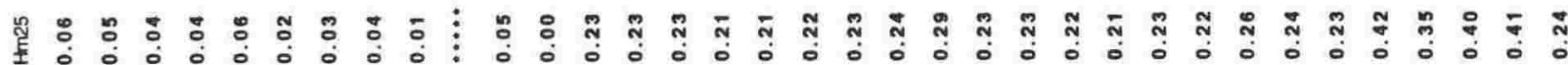
荇

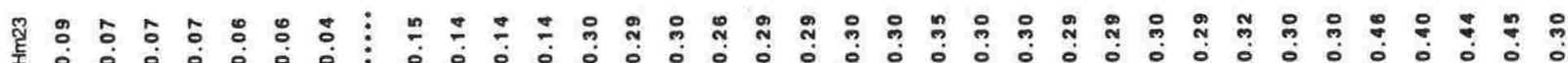
สูก

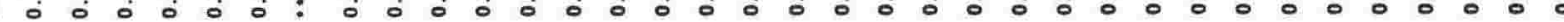

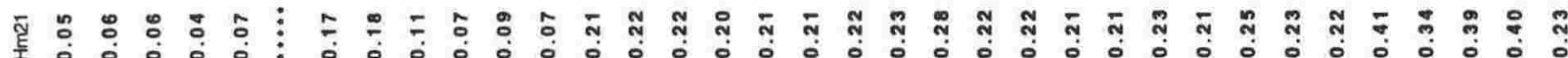

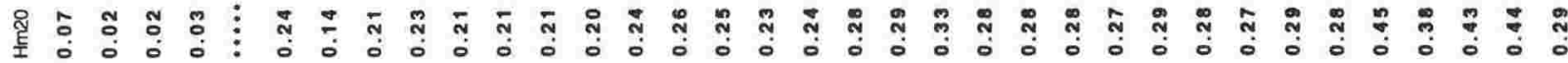
宝 产 : 弯

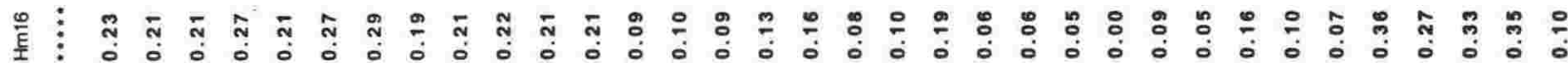

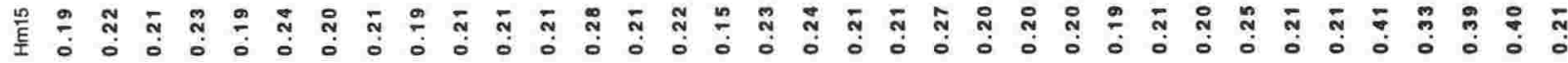

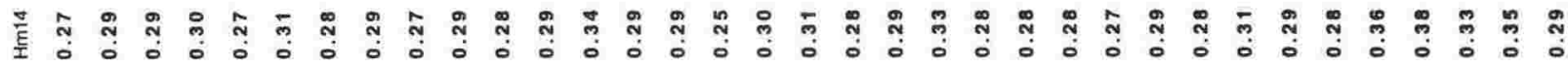
冓

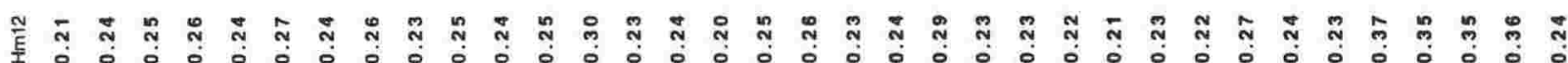

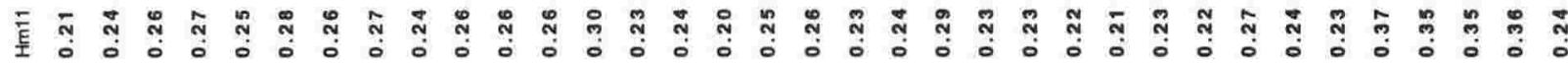

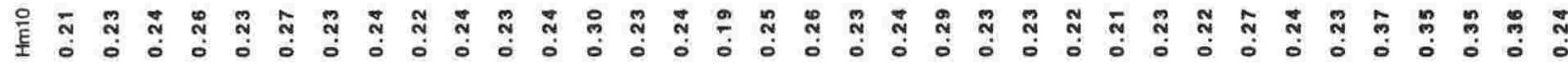

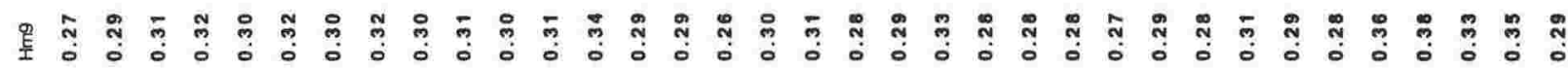
零 金 里 $\frac{0}{0}$ 过 茎

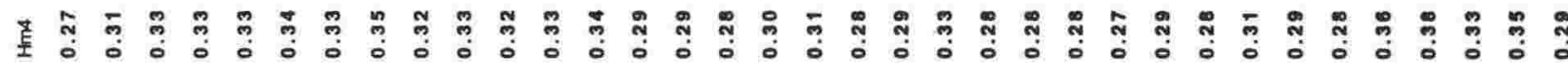


尊 范

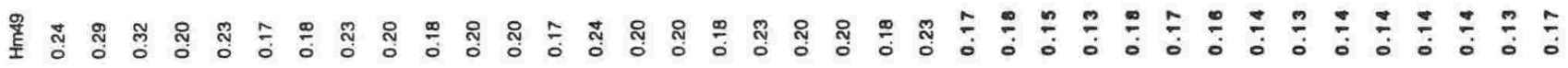

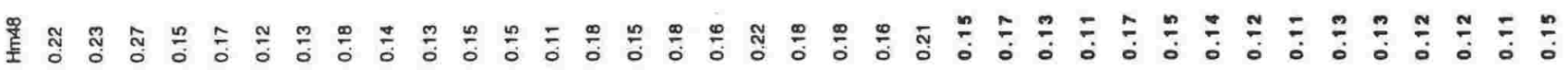

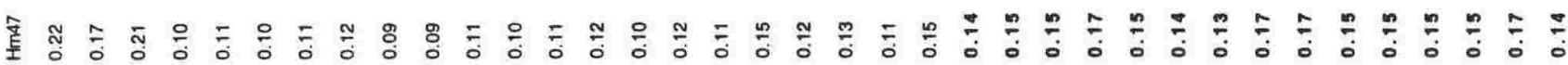

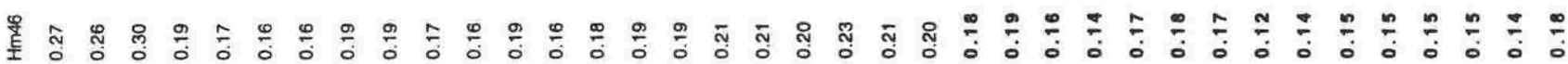

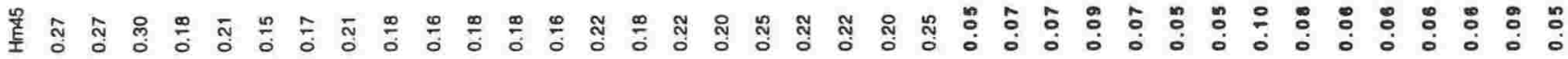
禾

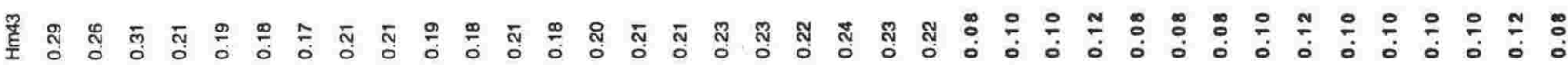
芷

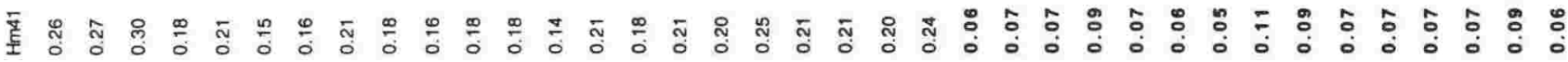
异

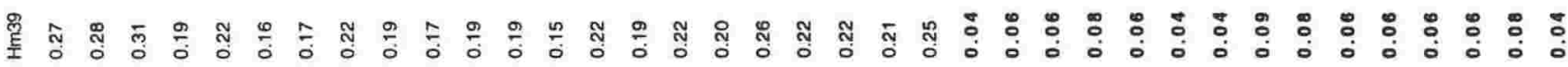

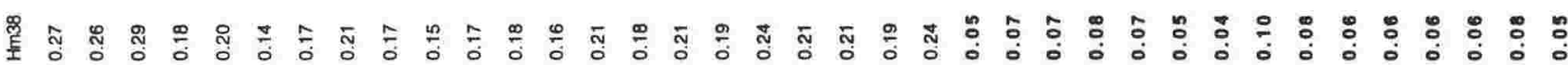

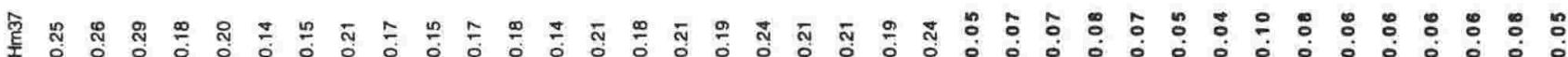

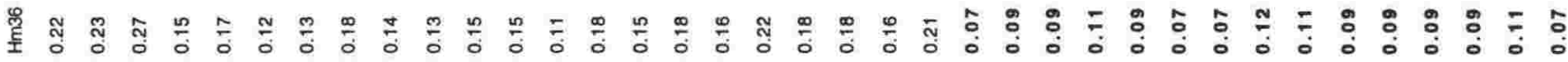

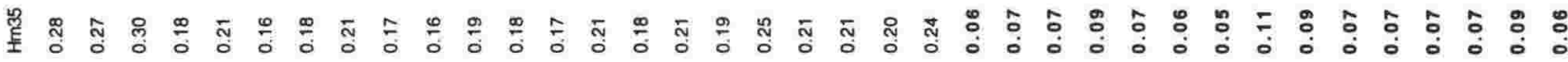

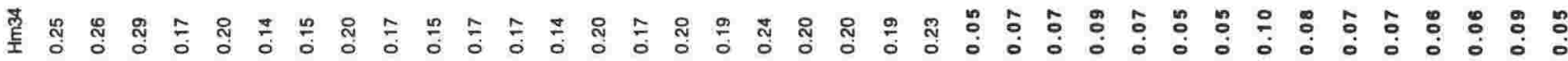

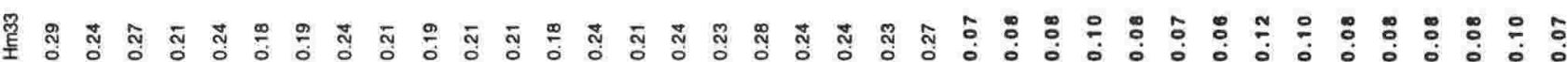

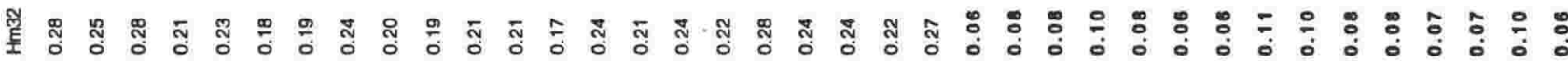

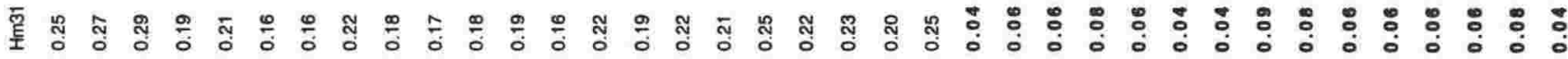

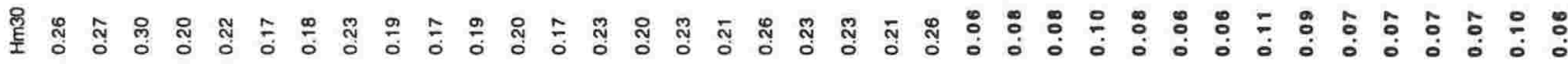
疍 


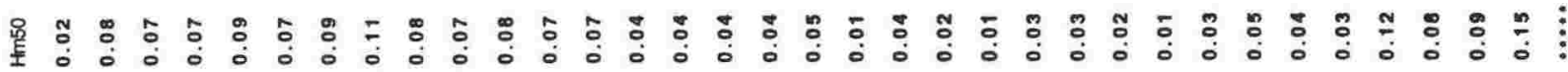
茎 罣

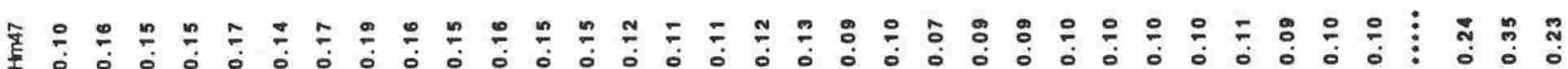

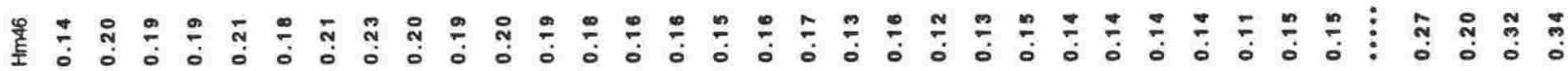

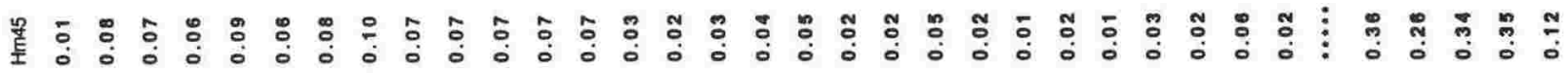

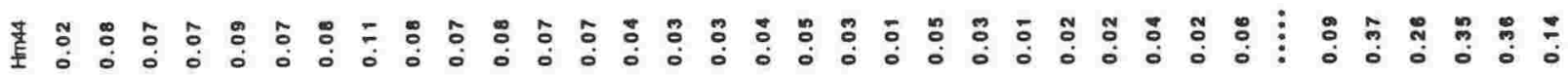
塞

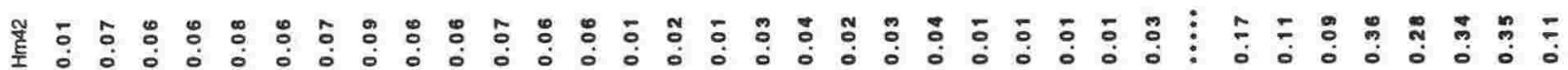

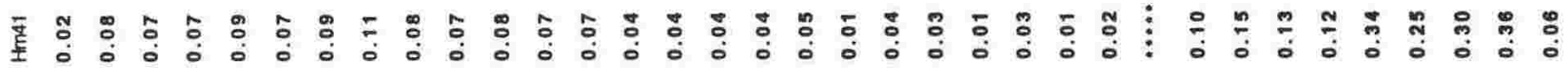

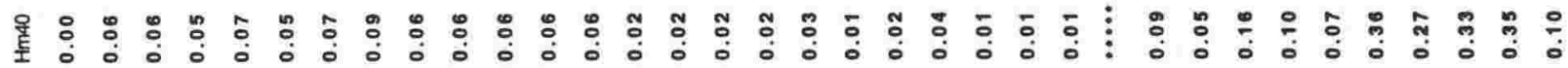

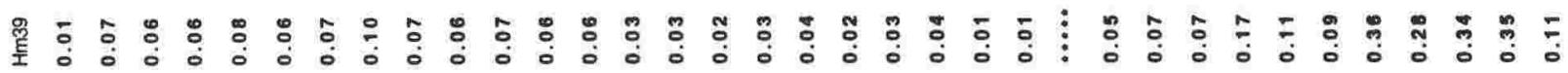

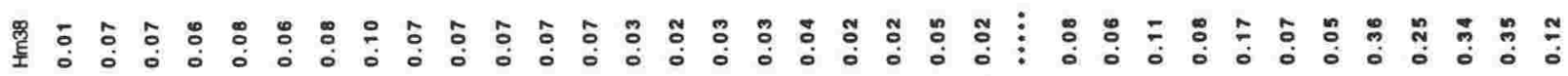

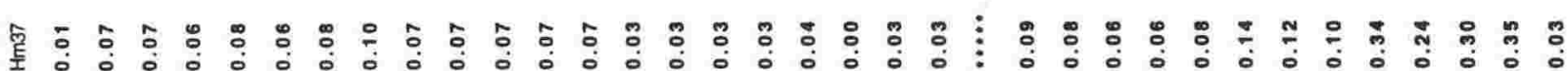
鹿 崖

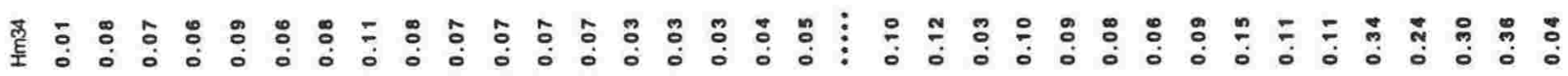

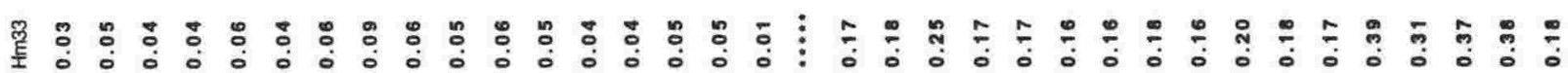
等 号

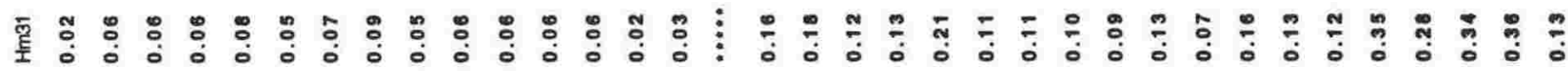

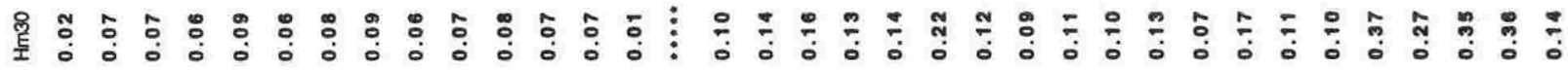
承 


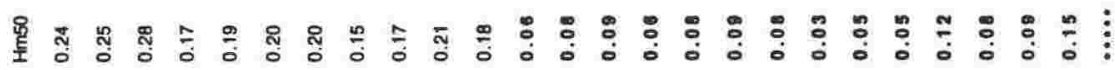

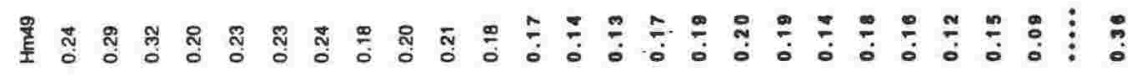

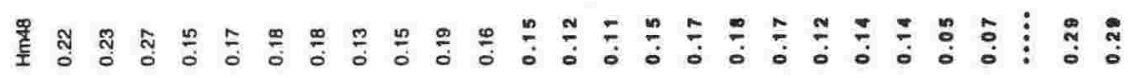

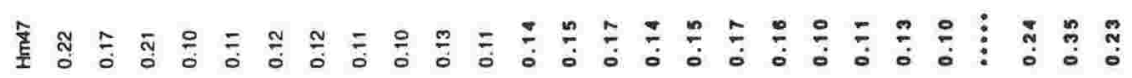

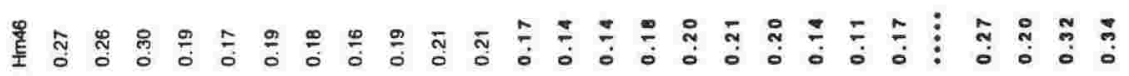

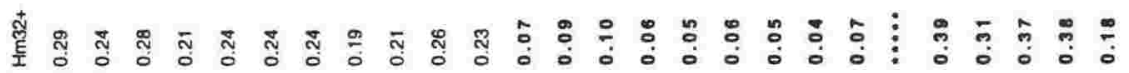

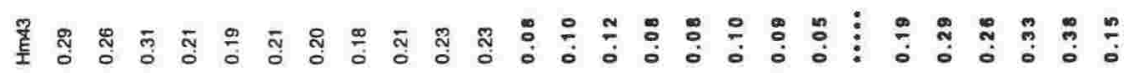

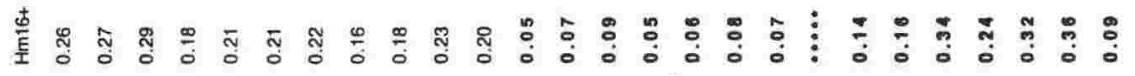

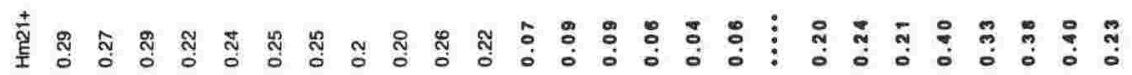

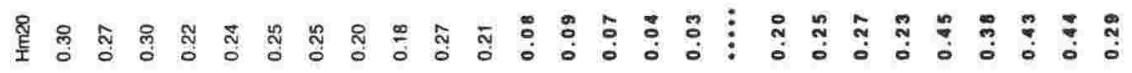

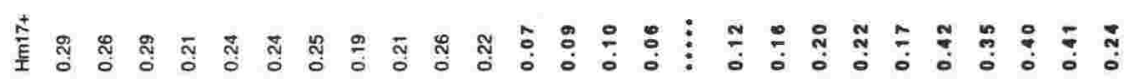

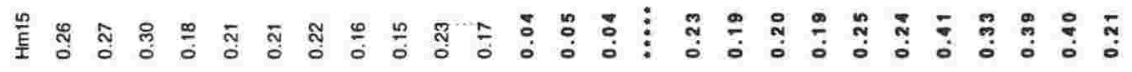

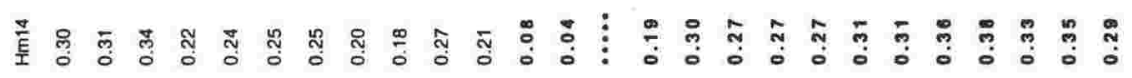

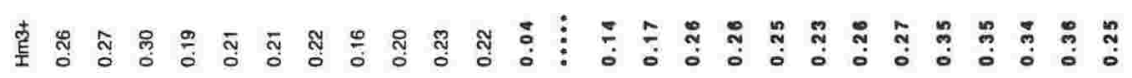

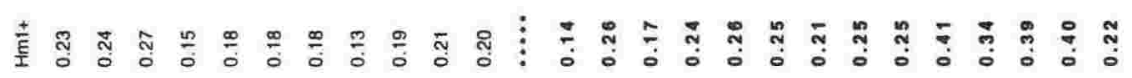

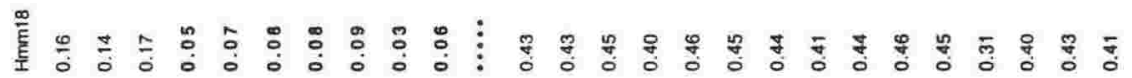

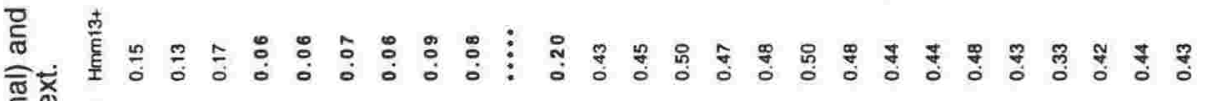

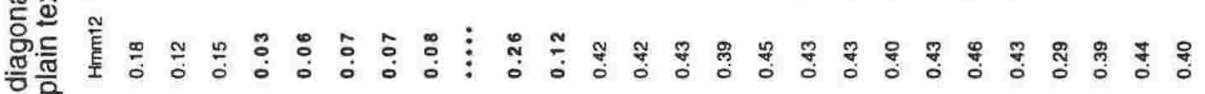

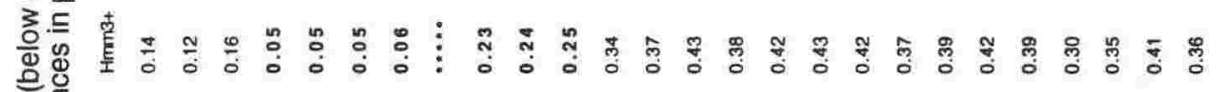

造产

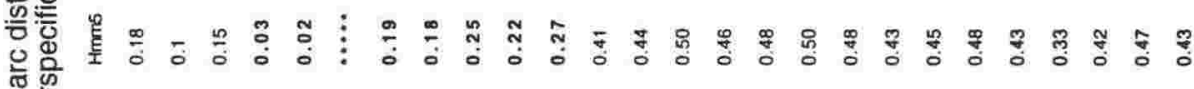

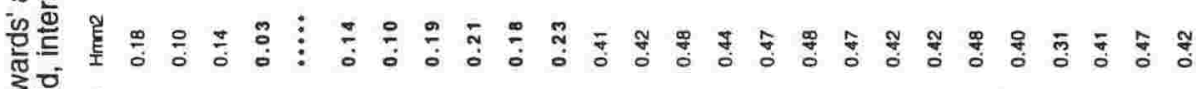

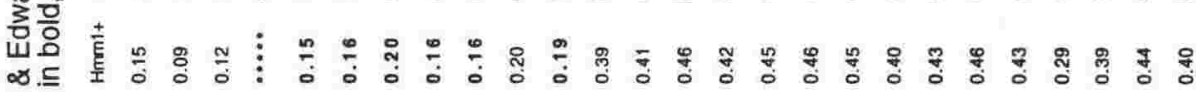

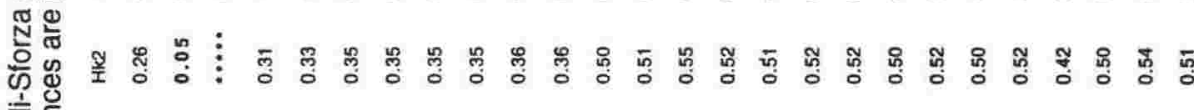

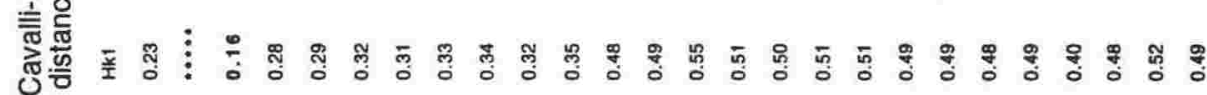

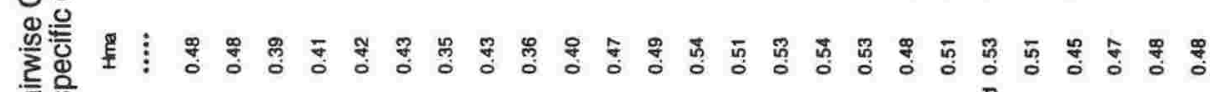

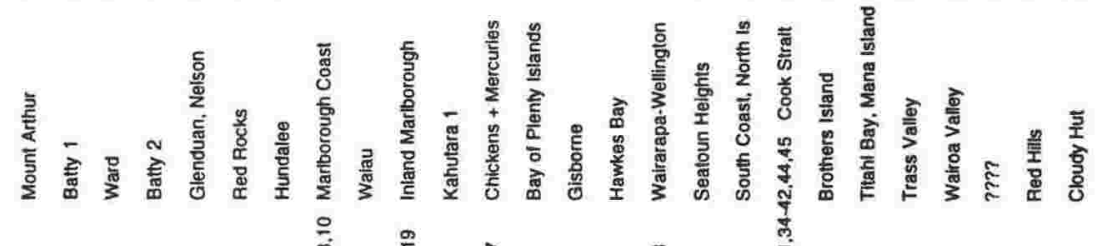

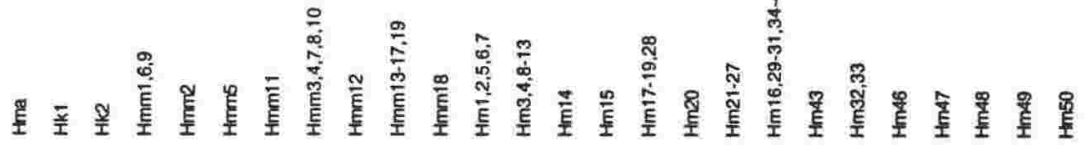


Table 4.5.3.7 Summary of genetic distances (Nei's D) within and between proposed species of the northern group of the $H$. maculatus complex.

\begin{tabular}{lllll}
\hline Species & 1 & 2 & 3 & 4 \\
\hline 1 H. "Mount Arthur" & - & & & \\
2 H. "Kaikouras" & $0.26-0.28$ & 0.02 & & \\
3 H. "Marlborough mini" & $0.12-0.20$ & $0.08-0.17$ & $0.00-0.10$ & \\
4 H. maculatus & $0.13-0.35$ & $\left(0.17^{*}\right)-0.26-0.38$ & $\left(0.07^{*}\right)-0.11-0.35$ & $0.00-0.13-\left(0.24^{* *}\right)$
\end{tabular}

"All pairwise D values $<0.26$ between $H$. maculatus and $H$. "Kaikouras", and $<0.11$ between $H$. maculatus and $H$. "Marlborough mini" involve the single $H$. maculatus from Red Hills.

${ }^{* \star}$ All pairwise $D$ values $>0.13$ within $H$. maculatus involve inland Nelson populations.

Np-1 the Trass and Red Hills populations are fixed for the (c) allele, shared with some northern North Island $H$. maculatus and many southern group populations, but not with $H$. "Mount Arthur", $H$. "Kaikouras", $H$. "Marlborough mini", or other $H$. maculatus populations in the southern part of the species' range. At Pep-3, the Red Hills animal was homozygous for the (b) allele common in other $H$. maculatuscomplex species, rather than the (c) allele which was fixed in all other $H$. maculatus populations. The Sod-2(d) allele was found at a frequency of 0.67 in the Wairoa Valley population. This allele was otherwise absent from $H$. maculatus except for single heterozygotes in the Kaihoka Lakes, Maud, and Chetwodes populations, but was very common in $H$. "Marlborough mini", H. "Kaikouras", some southern group species, and $H$. duvaucelii.

In WPGMA (Figures 4.5.3.10, 4.5.3.11), distance Wagner (Figure 4.5.3.12, 4.5.3.13) (BIOSYS package), and neighbour joining (Figure 4.5.3.14) (MEGA package) phenograms, $H$. "Marlborough mini" and $H$. "Kaikouras" are sister groups (except that the Marlborough Coast populations of $H$. "Marlborough mini" cluster outside $H$. "Kaikouras" in the neighbour-joining tree, and the DISWAG tree for grouped, but not ungrouped populations). $H$. "Mount Arthur" clusters next to this species pair in all phenograms. The mid-point of the distance Wagner and neighbour joining trees falls within $H$. maculatus, which shows greater within-species diversity than the other 3 species.

\subsubsection{MORPHOLOGY}

All 4 species of the northern group of the $H$. maculatus complex have diagnostic morphological characters (Table 4.5.4.1). 


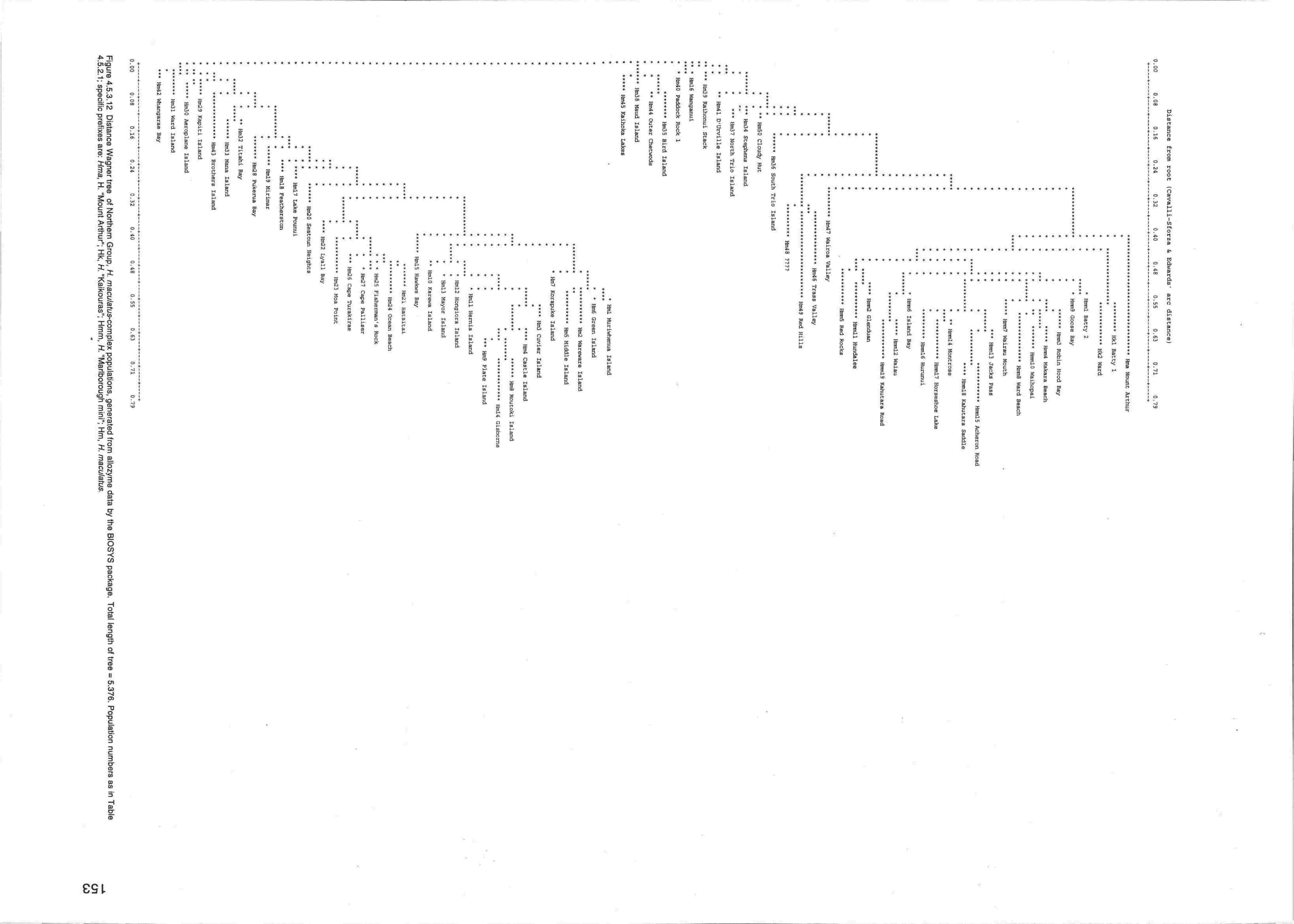




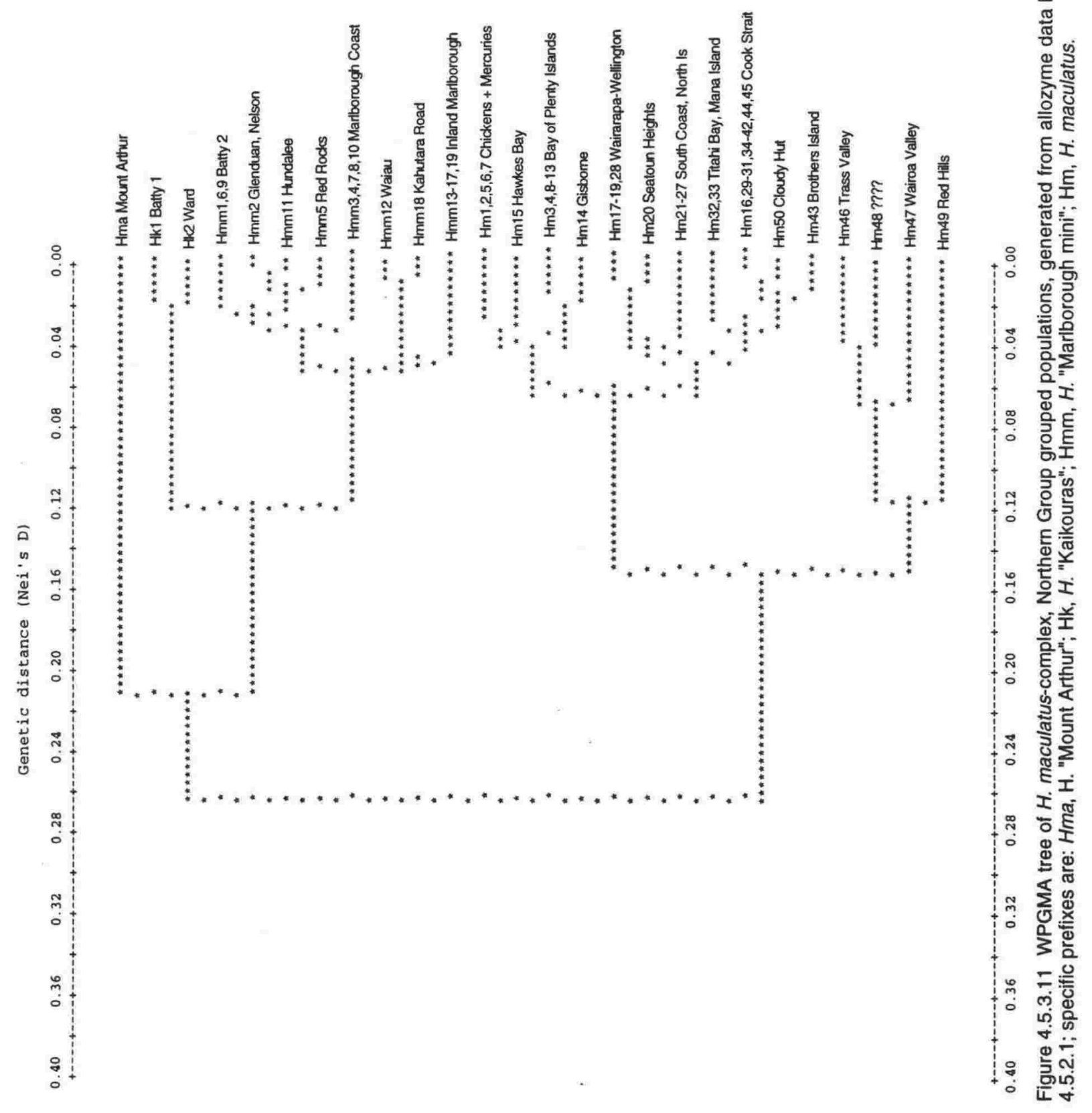




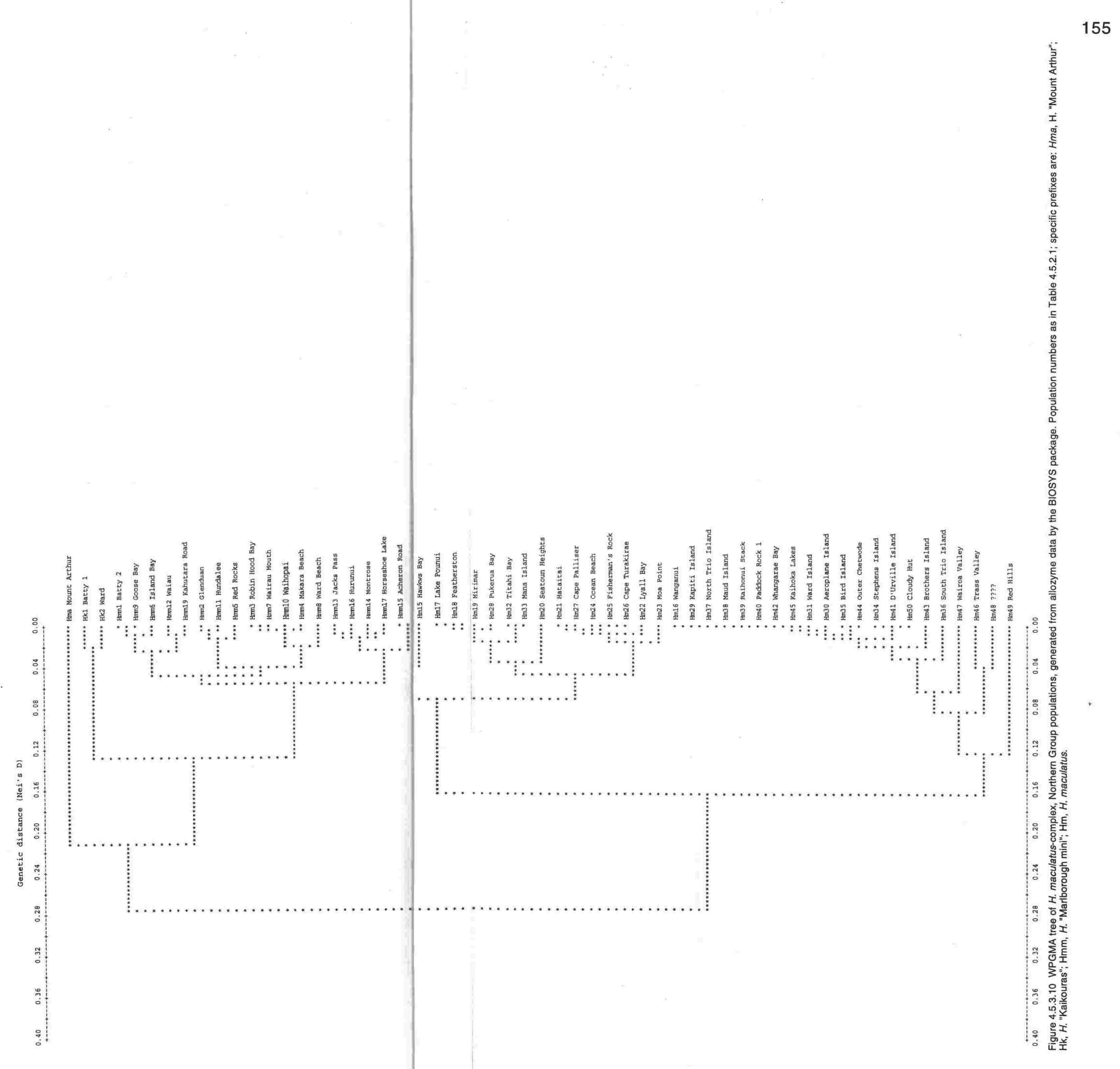


Distance from root (Cavalli-sforza \& Edwards' arc distance)

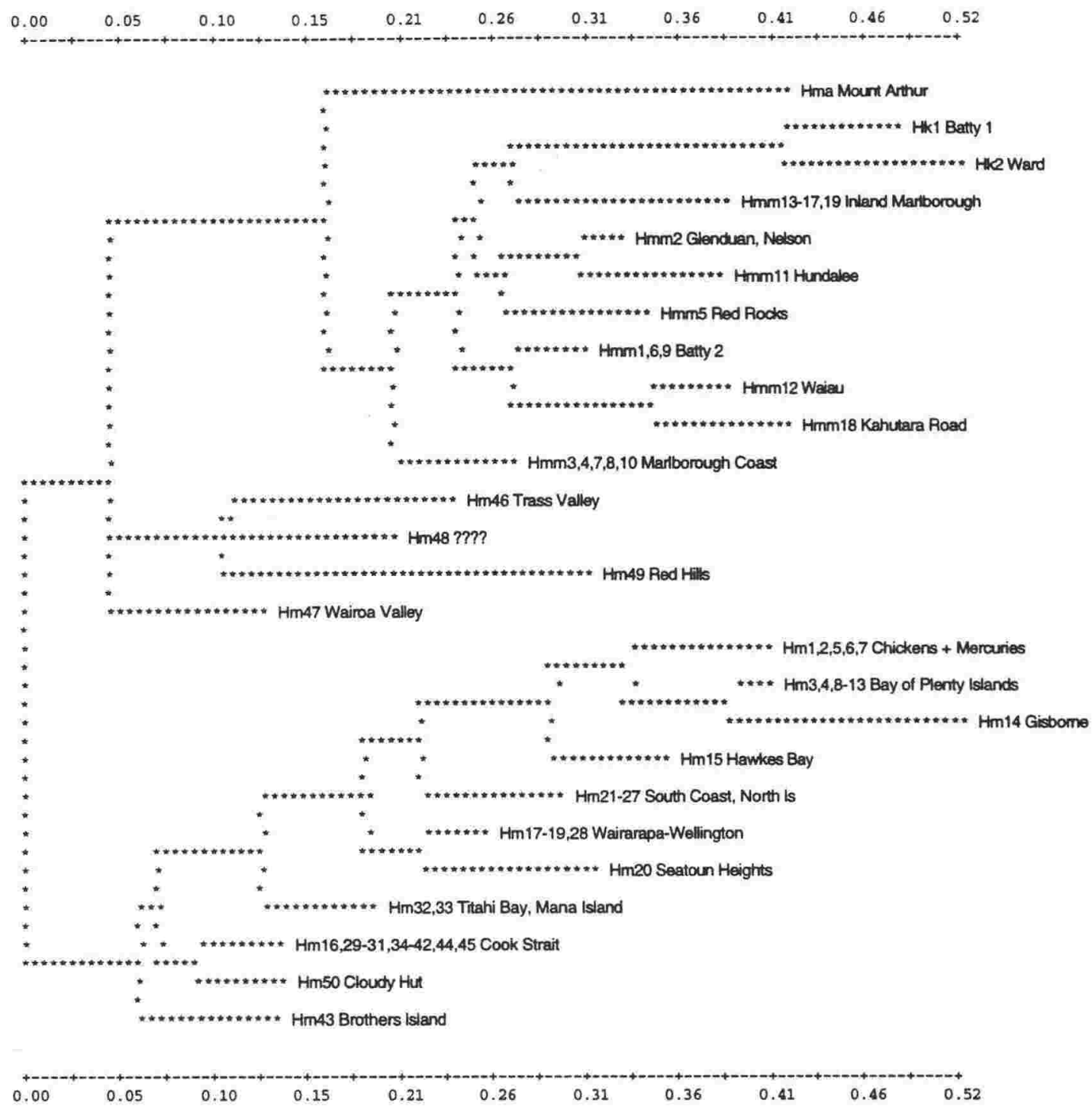

Figure 4.5.3.13 Distance Wagner tree of Northern Group, $H$. maculatus-complex grouped populations, generated from allozyme data by the BIOSYS package. Total length of tree $=3.151$. Population numbers as in Table 4.5.2.1; specific prefixes are: Hma, H. "Mount Arthur"; Hk, $H$. "Kaikouras"; Hmm, H. "Marlborough mini"; Hm, H. maculatus. 


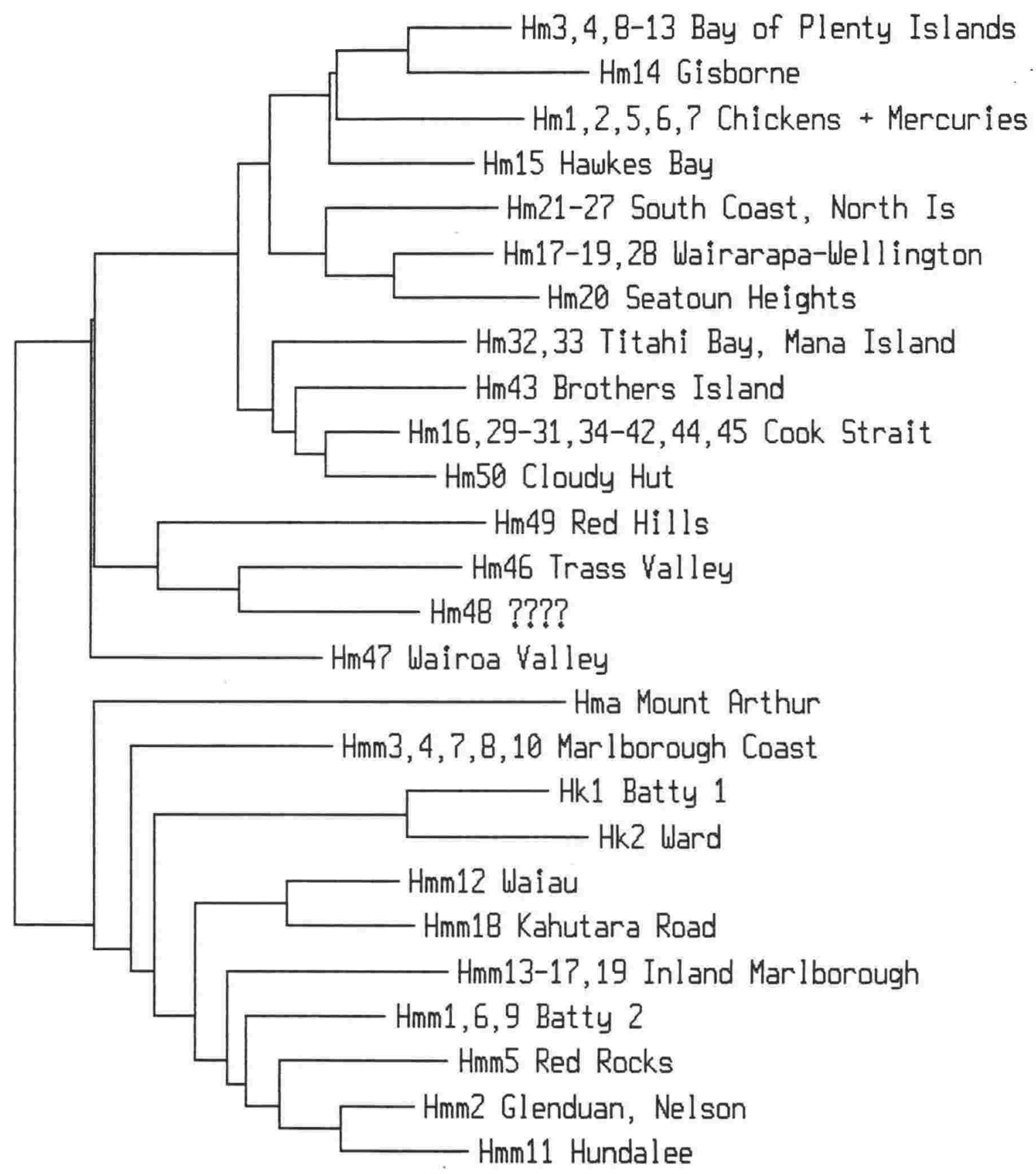

Figure 4.5.3.14 neighbour joining tree of $H$. maculatus-complex, northern group populations, generated from allozyme data by the MEGA package. 
Table 4.5.4.1 Morphological characters of species of the northern group of the $H$. maculatus complex. Diagnostic characters are underlined.

\begin{tabular}{|c|c|c|c|c|}
\hline & H. "Mt Arthur" & H. "Kaikouras" & $\begin{array}{l}\text { H. "Marlborough } \\
\text { mini" }\end{array}$ & H. maculatus \\
\hline Superciliary spines & 3-5 & 0 & 0 & (0)-many \\
\hline Nostril/rostral & narrow contact & excluded & excluded & excluded \\
\hline Mental shape & trapezium & triangle & trapezium & trapezium \\
\hline Upper labials & $\underline{7-10}$ & $10-13$ & $10-12$ & $9-12$ \\
\hline Lower labials & $\overline{5-8}$ & $9-13$ & $10-12-(15)$ & $8-11$ \\
\hline Distal phalange proportion & $\mathrm{c.1} / 4$ & c. $1 / 3$ & c. $1 / 3$ & c. $1 / 3$ \\
\hline Distal phalange shape & tapering & tapering & tapering & narrow \\
\hline Fourth toe lamellae & $11-12$ & $(10)-11-12$ & $\underline{9-10}$ & (11) $-\underline{12-15}$ \\
\hline Preanal pores & 3 rows & 4-7 rows & $2-4$ rows & $3-8$ rows \\
\hline Femoral pores & 1 row & 2 rows & 2 rows & $2-4$ rows \\
\hline Basic colour & grey & brown & brown & brown \\
\hline
\end{tabular}

Morphologically $H$. "Mount Arthur" is closer to $H$. "Southern Alps" than to other members of the northern group, although allozyme electrophoresis indicates a much closer relationship with the latter. The former 2 share the same greyish colour (although $H$. "Mount Arthur" is much darker than $H$. "Southern Alps"), short distal phalanges, and less prominent eye, but differ in the higher lamellae counts and longer, shallower snout of $H$. "Mount Arthur" (see Chapter 3). H. "Mount Arthur" also resembles the southern group of the $H$. maculatus complex in the relationship between the nostril, rostral, anterior nasal, and first labial. In the other species of the northern group, the nasal and first labial are broadly in contact, forming a definite suture, and separating the rostral and nostril, as illustrated by Robb \& Rowlands (1977) for $H$. maculatus. In the southern group and $H$. "Mount Arthur" these 2 scales usually either meet only at their corners, or just fail to meet, allowing slight contact between the rostral and nostril.

Most populations in $H$. "Marlborough mini" are small to very small geckos from Marlborough, north Canterbury, Nelson, and the Wellington Coast. Specimens from Nelson Boulder Bank grow larger than the others, and have a bolder colour pattern, but are otherwise similar, and I include them in $H$. "Marlborough mini".

\subsubsection{DISCUSSION}

The northern group includes $H$. maculatus, $H$. "Marlborough mini", $H$. "Kaikouras", and $H$. "Mount Arthur". The ranges of the former 2 overlap extensively in Nelson and Marlborough and abut near Wellington (Figure 
4.5.2.1), indicating that they interact as biological species, although they have not been found in microsympatry, apparently because of ecotopic separation in the zone of overlap. In the South Island, H. maculatus is found almost entirely in forest, or in previously forested habitats. $H$. "Marlborough mini" is found mainly in open habitats, associated with native plant communities (e.g., Sophora prostrata, Pachystegia spp.) which indicate long-term absence of forest.

H. "Kaikouras" and $H$. "Marlborough mini" were collected in sympatry at Batty (upper Blue Duck catchment, Seaward Kaikouras) confirming their separate species status despite the low genetic distance $(D=0.08)$ between them. The geographic range of $H$. "Kaikouras" is entirely surrounded by that of $H$.

"Marlborough mini". The 2 are similar in both allozyme genotypes and appearance, but distinguished by 2 fixed and one frequency differences in allozymes, and by higher lamellae counts, larger numbers of preanal pores, and a triangular rather than trapezium-shaped mental in $H$. "Kaikouras". $H$. "Kaikouras" has been collected only in the northern Seaward Kaikoura Range north of the northern limit of $H$. "Southern Alps". The 2 populations of $H$. "Kaikouras" sampled, about $65 \mathrm{~km}$ apart, were genetically much closer to each other $(D=0.02)$ than to any of the neighbouring or sympatric populations of $H$. "Marlborough mini" $(D \geq 0.08)$.

The exact phylogenetic position within the northern group of $H$. "Mount Arthur" is not clear from allozyme analyses. In phenetic trees it is the sister group of $H$. "Marlborough mini" plus $H$. "Kaikouras", with $H$. maculatus the sister group of this group of 3 species. Its position in parsimony trees is unstable and no one position is strongly supported (Chapter 5). The morphological features shared with $H$. "Southern Alps" are consistent with a basal position in the northern group, but could also result from convergence in a similar, sub-alpine environment. This population is considered to deserve specific status, despite the limitations of small sample size, because it is the sister species of a clade of at least 2 species. A female from this population was in mid-pregnancy in April, indicating that, like highaltitude populations of $H$. "Otago" (Cree 1994), the Mount Arthur population probably has a 2-year reproductive cycle, unlike the rest of the northern group, which all appeared to have annual cycles.

Apart from a group of $H$. maculatus populations in inland Nelson, the larger genetic distances (Nei's $D>0.1$ ) within species are between geographically widely separated populations, suggesting isolation by distance. The genetically divergent Nelson populations of $H$. maculatus cannot be simply explained by recent 
introgression from any of the adjacent species. Polymorphism and heterozygosity in these populations are not unusually high. The Np-1(c) allele cannot have come from any other northern group species, and several fixed differences argue against introgression from $\mathrm{H}$. "Southern Alps" or $\mathrm{H}$. brunneus. It appears more likely that the high genetic diversity in inland Nelson exists because this is the area of origin of $H$. maculatus, where the species has the longest history of in situ evolution (Chapter 7). Separate species status for this group of populations is not considered justified by the present data, as they do not form a uniform group. There is substantial genetic divergence among them, along with size and habitat differences between them, but no obvious morphological separation from $H$. maculatus as a whole. Recognition of 2 or 3 new species from this group would be premature before further collecting can clarify patterns of geographic variation.

\subsection{The hoplodactylus maculatus complex, Southern Group}

\subsubsection{INTRODUCTION}

In this section I justify the recognition of 4 new species ( $H$. "Otago", $H$. "Cromwell Gorge", $H$. "Danseys Pass", and $H$. "Southern Alps"), and suggest that the 2 most widespread of these, $H$. "Otago" and $H$. "Southern Alps", probably contain further undiagnosed species. A fifth new species in this group was shown in section 3.4 .5 to be conspecific with Cope's (1868) Pentadactylus brunneus.

Possible taxonomic subdivision of South Island $H$. maculatus sensu Robb \& Rowlands 1977 was first suggested by Rowlands (pers. comm.) who considered that variation was mainly between populations, and that captive breeding was much more readily achieved between animals from the same locality than in mixed collections. He singled out Otago Peninsula as an area which had a distinctive population.

Robb (1982, and pers. comm.) considered a specimen from the top of the Old Man Range to represent a new species morphologically distinct from $H$. maculatus.

The discovery of sympatric large and small species of the $H$. maculatus complex in Marlborough then Southland (sections 4.3.1, 4.4.1, \& 4.5.1) was followed by collection of samples of $H$. maculatus in the Mackenzie Basin which were considered to include both "maxi" and "mini" forms. These samples were included in my survey. 


\subsubsection{SAMPLING}

Samples were collected from $273 \mathrm{H}$. maculatus sensu Robb \& Rowlands (1977) from 81 populations referable to this species group; more than from any other group (Figure 4.6.2.1, Table 4.6.2.1). Sampling distribution reflects both actual distribution of the animals (e.g., the absence of samples from areas of intensive agriculture on the Canterbury plains and in Southland) and ease of access (e.g., the clustering of samples of $H$. "Southern Alps" near the major roads through the mountains). After the patchy nature of variation began to become apparent, potential boundary zones (e.g., the Clutha Valley near Alexandra) were sampled more intensively.

Samples were also collected from $34 \mathrm{H}$. duvaucelii from 17 populations (Figure 4.6.2.2, Table 4.6.2.1).

\subsubsection{ALLOZYME RESULTS}

Allozyme variation was detected at 19 loci in the southern group of the $H$. maculatus complex (Table 4.6.3.1). Two Mdh-1(ac) heterozygotes in the Hakataramea Pass population of $H$. "Southern Alps" were the only variants at this locus in this group; all other populations were fixed for $M d h-1(\mathrm{c})$. Other loci variable within this group all had more geographically widespread polymorphisms (Table 4.6.3.1; Figures 4.6.3.1-18).

Levels of polymorphism were higher than in most other groups in this study (Table 4.6.3.2). There was only one instance within this group of high observed polymorphism with lower than expected heterozygosity, indicating possible sympatry of reproductively isolated species (at Omarama, where one animal differed from the other 8 by 3 fixed and one almost fixed differences). Apart from this site, allozymes identified only geographic clusters of populations.

Population differentiation was fine-grained, with many apparently fixed differences (allowing for the limitations on detecting polymorphism imposed by some small sample sizes) between geographically close populations (Figures 4.6.3.1-18). Larger-scale geographic clusters were not defined by diagnostic alleles or fixed differences between the groups as a whole, but by discontinuities in gene flow indicated by a concentration of pairwise fixed differences between individual populations across geographical boundaries. There was little uniformity of populations even on a very local scale, and therefore little opportunity for grouping populations for analysis. 


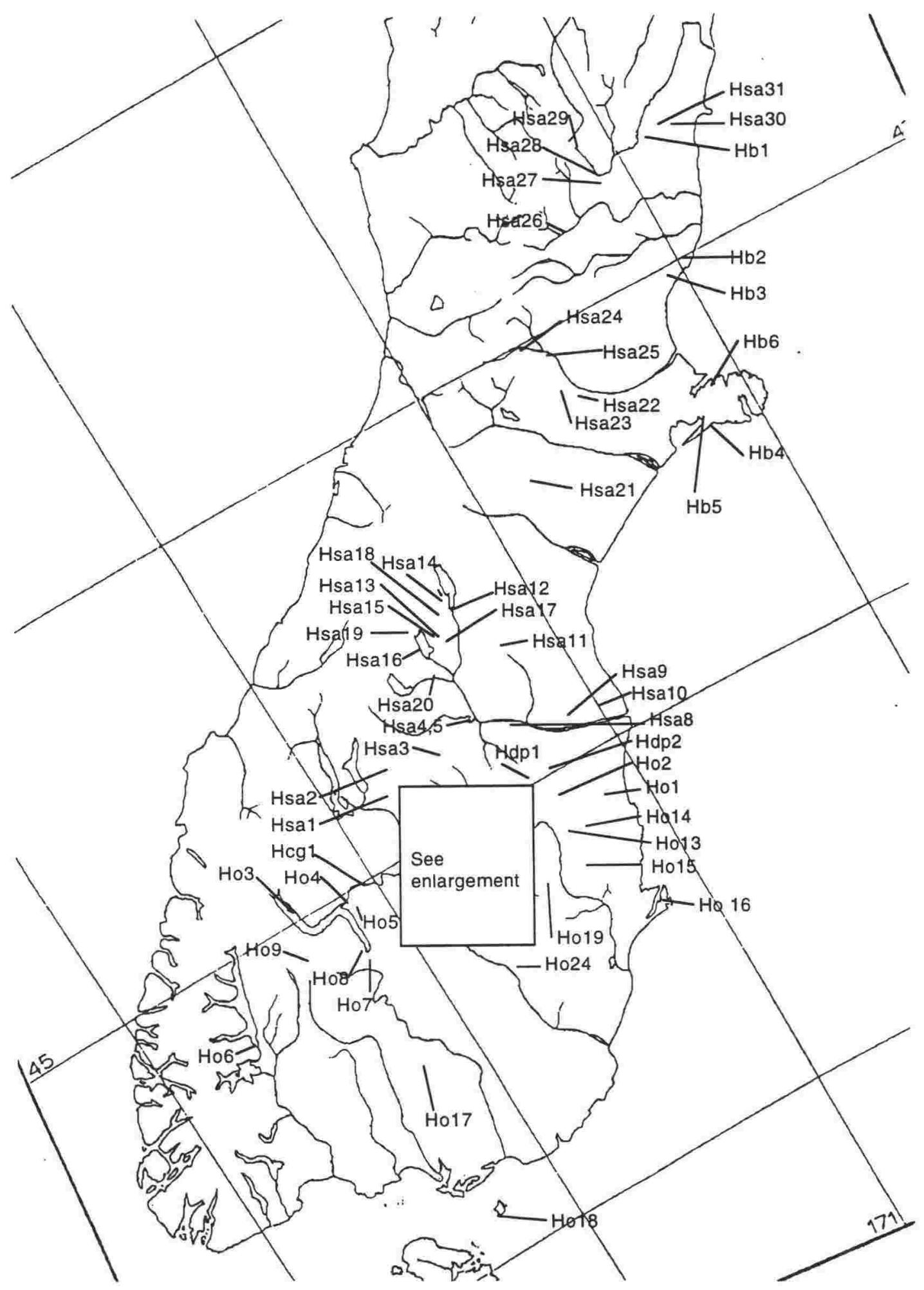

Figure 4.6.2.1 Sampling locations for populations assigned to the southern group of the $H$. maculatus complex. For population key see Table 4.6.2.1. For Central Otago populations see enlargement, next page. 


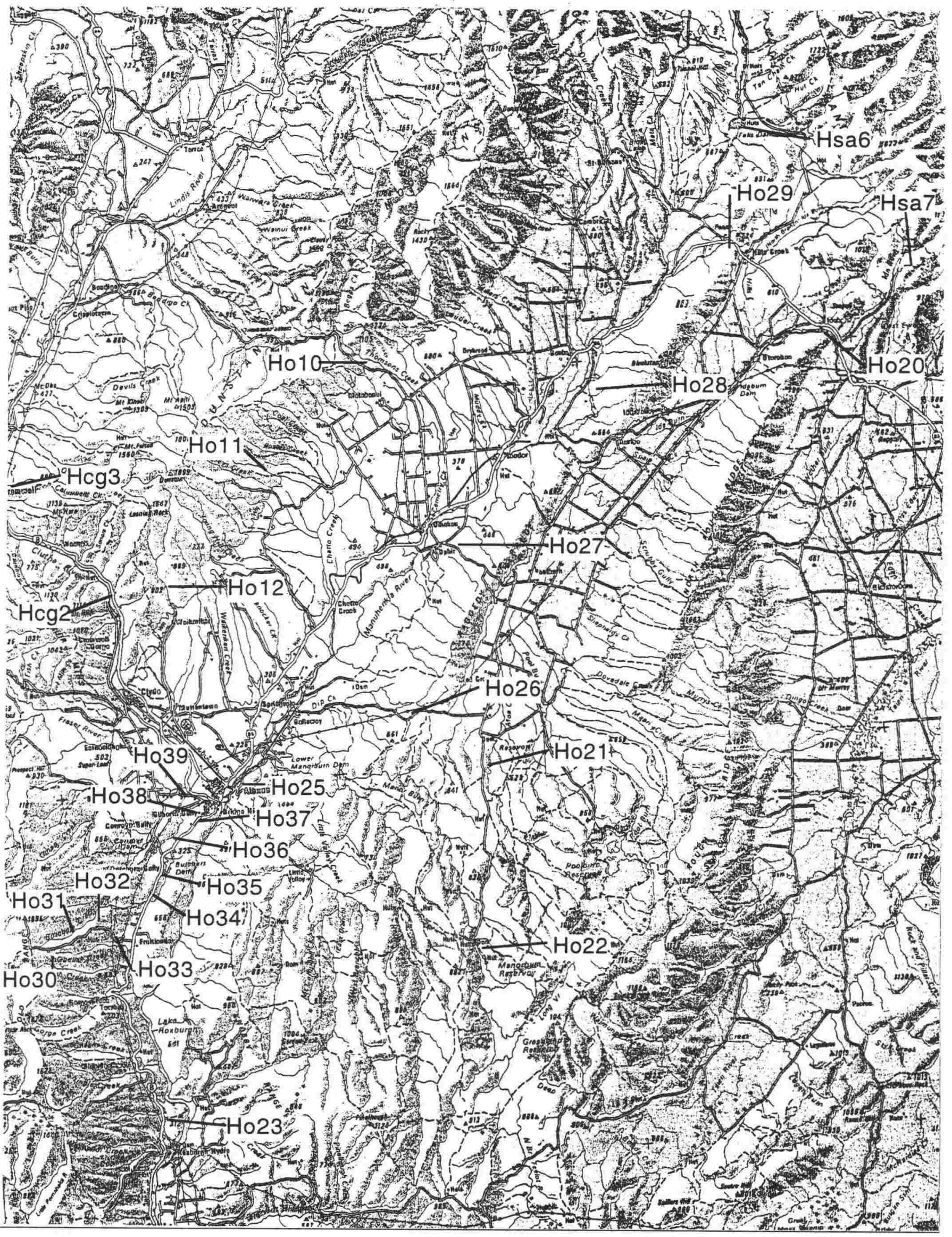

Figure 4.6.2.1 (continued) enlargement, Central Otago area - see previous page. 
Table 4.6.2.1 Localities and population codes for samples of $H$. maculatus complex, southern group species (refer to Figures 4.6.2.1 and 4.6.2.2).

\begin{tabular}{|c|c|c|}
\hline $\begin{array}{l}\text { Population } \\
\text { code }\end{array}$ & Species & Locality \\
\hline Hdp1 & H. "Dansey's Pass" & Kyeburn Diggings \\
\hline Hdp2 & H. "Dansey's Pass" & Dansey's Pass \\
\hline Ho1 & H. "Otago" & Horse Range \\
\hline $\mathrm{Ho} 2$ & H. "Otago" & Mount Pisgah \\
\hline $\mathrm{Ho3}$ & H. "Otago" & Tree Island \\
\hline Ho4 & H. "Otago" & Kelvin Heights \\
\hline Ho5 & H. "Otago" & Remarkables \\
\hline Ho6 & H. "Otago" & Te Anau \\
\hline $\mathrm{Ho} 7$ & H. "Otago" & Nokomai \\
\hline Ho8 & H. "Otago" & Kingston \\
\hline Ho9 & H. "Otago" & Gorge Burn \\
\hline Ho10 & H. "Otago" & Thomson's Saddle, Dunstan Range 1 \\
\hline Ho11 & H. "Otago" & Dunstan Range 2 \\
\hline Ho12 & H. "Otago" & Waikerikeri, Dunstan Range \\
\hline Ho13 & H. "Otago" & Taieri Ridge \\
\hline Ho14 & H. "Otago" & Deepdell \\
\hline Ho15 & H. "Otago" & Billy Ridge \\
\hline Ho16 & H. "Otago" & Harbour Cone, Otago Peninsula \\
\hline Ho17 & H. "Otago" & Hokonui Hills \\
\hline Ho18 & H. "Otago" & Islet, Ruapuke Island, Foveaux Strait \\
\hline Ho19 & H. "Otago" & Glenavon \\
\hline $\mathrm{Ho} 2 \mathrm{O}$ & H. "Otago" & Idaburn \\
\hline Ho21 & H. "Otago" & Manorburn Road \\
\hline $\mathrm{Ho} 22$ & H. "Otago" & Manorburn Dam \\
\hline Ho23 & H. "Otago" & Roxburgh \\
\hline Ho24 & H. "Otago" & Beaumont \\
\hline $\mathrm{Ho25}$ & H. "Otago" & Alexandra lookout \\
\hline Ho26 & H. "Otago" & Galloway \\
\hline Ho27 & H. "Otago" & South Raggedy Range \\
\hline $\mathrm{Ho} 28$ & H. "Otago" & Becks \\
\hline Ho29 & H. "Otago" & North Raggedy Range \\
\hline НозО & H. "Otago" & Old Man Range 4 (top) \\
\hline Ho31 & H. "Otago" & Old Man Range 3 \\
\hline Но32 & H. "Otago" & Old Man Range 2 \\
\hline Ноз3 & H. "Otago" & Old Man Range (bottom) \\
\hline Но34 & H. "Otago" & Butchers Gully \\
\hline Ho35 & H. "Otago" & Butchers Dam \\
\hline Ноз6 & H. "Otago" & Alexandra (3 km E) \\
\hline Ho37 & H. "Otago" & Alexandra (SE) \\
\hline Ho38 & H. "Otago" & Aranui Dam (Alexandra) \\
\hline Ноз9 & H. "Otago" & Alexandra West \\
\hline Hcg1 & H. "Cromwell Gorge" & Nevis Bluff \\
\hline Hcg2 & H. "Cromwell Gorge" & Cromwell Gorge \\
\hline Hcg3 & H. "Cromwell Gorge" & Cromwell, east of River \\
\hline Hsa1 & H. "Southern Alps" & Lindis Pass 1 \\
\hline Hsa2 & H. "Southern Alps" & Lindis Pass 2 \\
\hline Hsa3 & H. "Southern Alps" & Manuherikia River \\
\hline Hsa4 & H. "Southern Alps" & Omarama 1 \\
\hline Hsa5 & H. "Southern Alps" & Omarama 2 \\
\hline Hsa6 & H. "Southern Alps" & Falls Dam \\
\hline Hsa7 & H. "Southern Alps" & Little Mount Ida \\
\hline Hsa8 & H. "Southern Alps" & Kurow \\
\hline Hsa9 & H. "Southern Alps" & Waitaki River \\
\hline Hsa10 & H. "Southern Alps" & Waimate \\
\hline Hsa11 & H. "Southern Alps" & Hakataramea Pass \\
\hline Hsa12 & H. "Southern Alps" & Tekapo \\
\hline
\end{tabular}




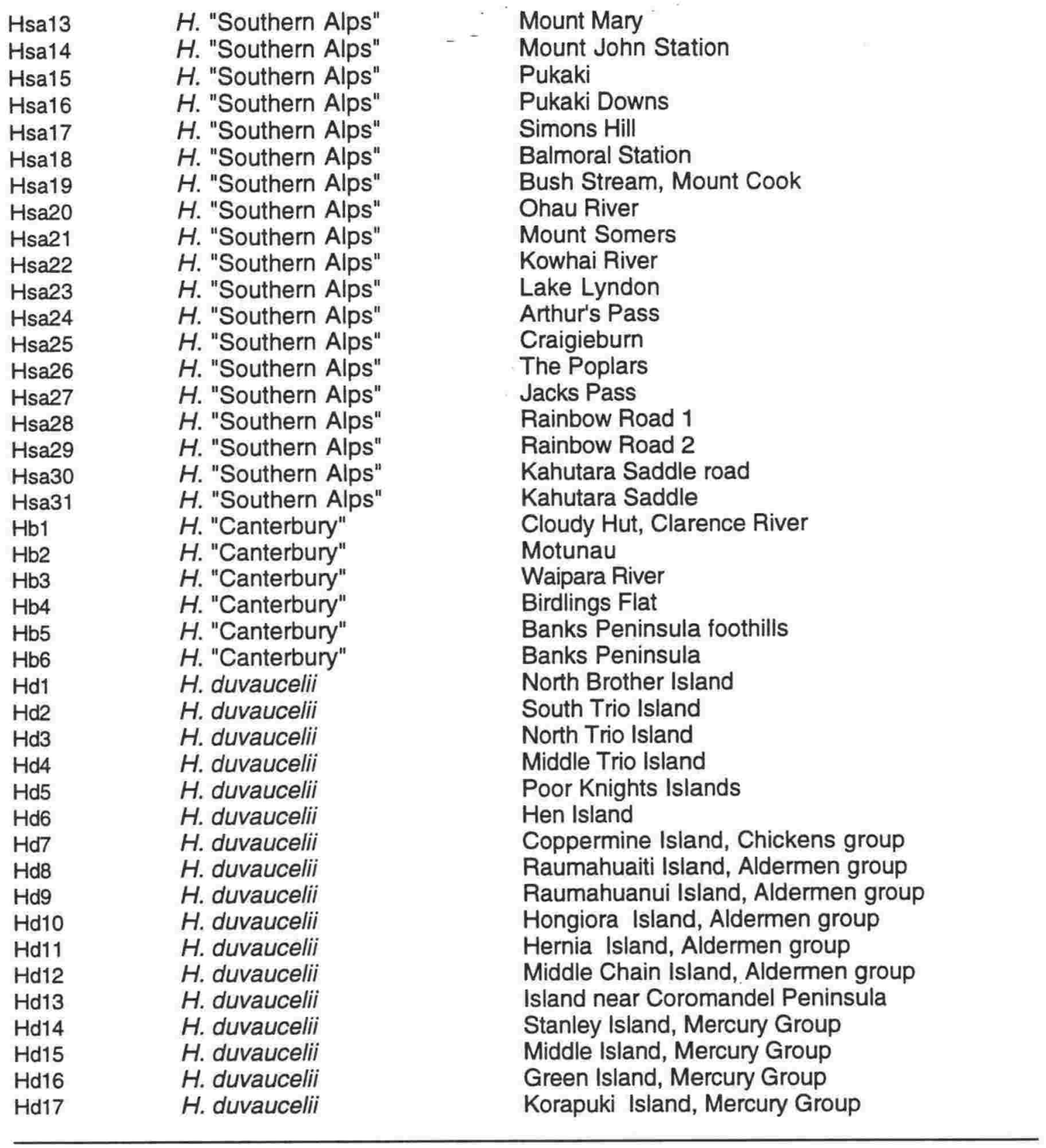




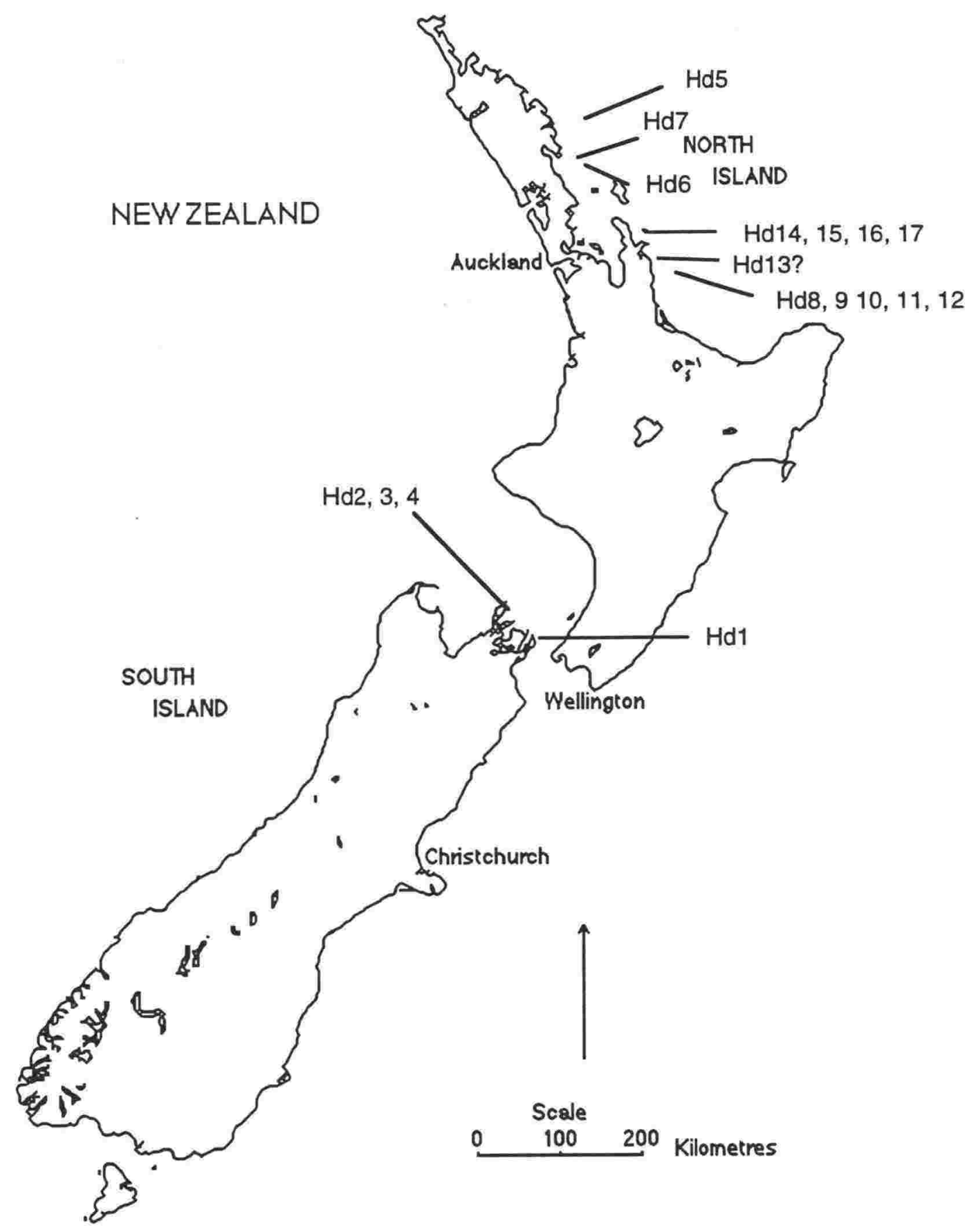

Figure 4.6.2.2 Sampling locations for $H$. duvaucelii populations. For population key see Table 4.6.2.1. 
Table 4.6.3.1 Allelic frequencies in populations of the southern group of the $H$. maculatus complex.

\begin{tabular}{|c|c|c|c|c|c|c|c|c|c|c|c|c|c|c|c|}
\hline Locus & Hdp1 & Hdp2 & Ho1 & $\mathrm{Ho} 2$ & $\mathrm{Ho3}$ & $\mathrm{Ho} 4$ & $\begin{array}{l}\text { Population } \\
\text { Ho5 }\end{array}$ & Ho6 & $\mathrm{Ho} 7$ & $\mathrm{HoB}$ & $\mathrm{Hog}$ & Ho10 & Ho11 & Ho12 & Ho13 \\
\hline (N) & 5 & 4 & 1 & 1 & 5 & 2 & 2 & 1 & 1 & 1 & 5 & 3 & 1 & 2 & 9 \\
\hline$A k-1$ & 1.00 & 1.00 & 1.00 & 1.00 & 1.00 & 1.00 & 1.00 & 1.00 & 1.00 & 1.00 & 1.00 & 1.00 & 1.00 & 1.00 & - \\
\hline b & 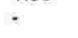 & $\cdot$ & 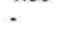 & - & $\cdot$ & - & $\cdot$ & - & $\cdot$ & $\cdot$ & 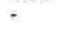 & $\cdot$ & $\cdot$ & - & 1.00 \\
\hline \multicolumn{3}{|l|}{$\begin{array}{l}b \\
G d-2 \\
b\end{array}$} & - & - & 1.00 & 1.00 & 1.00 & 1.00 & 1.00 & 1.00 & 1.00 & 1.00 & 1.00 & 1.00 & - \\
\hline $\mathrm{c}$ & 1.00 & 1.00 & 1.00 & 1.00 & - & - & 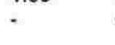 & 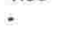 & $\cdot$ & - & $\cdot$ & $\cdot$ & - & $\cdot$ & 1.00 \\
\hline \multicolumn{2}{|l|}{ Got-1 } & . & & 0.50 & 1.00 & 1.00 & 1.00 & . & 1.00 & 1.00 & 1.00 & 1.00 & 1.00 & 0.75 & 0.28 \\
\hline c & 1.00 & 1.00 & 1.00 & 0.50 & $\cdot$ & $\cdot$ & 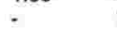 & 1.00 & 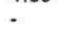 & - & - & $\cdot$ & $\cdot$ & 0.25 & 0.72 \\
\hline Got-2 & 1.00 & 1.00 & 1.00 & 1.00 & 1.00 & 1.00 & 1.00 & 1.00 & 1.00 & 1.00 & 1.00 & 1.00 & 1.00 & 1.00 & 1.00 \\
\hline$\vec{b}$ & - & $\cdot$ & $\cdot$ & - & 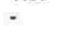 & - & $\cdot$ & $\cdot$ & - & 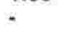 & $\cdot$ & $\cdot$ & $\cdot$ & - & 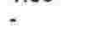 \\
\hline$G p-1$ & & & & & & & & & & & & & & & \\
\hline $\begin{array}{l}\mathrm{a} \\
\mathrm{b}\end{array}$ & $\begin{array}{l}0.10 \\
0.90\end{array}$ & $i .00$ & 1.00 & 0.50 & 1.00 & 1.00 & 1.00 & 1.00 & 1.00 & 1.00 & 0.90 & 0.67 & 1.00 & 1.00 & $\begin{array}{r}0.50 \\
0.50\end{array}$ \\
\hline \multicolumn{11}{|l|}{ Gp-4 } & 0.10 & & - & - & \\
\hline $\begin{array}{l}a \\
c\end{array}$ & 0.80 & 1.00 & - & - & - & - & - & . & - & - & 1.00 & - & - & 1.00 & - \\
\hline c & 0.80 & 1.00 & - & $\therefore \quad$ & 0.10 & - & 0.75 & 1.00 & 1.00 & 1.00 & $\cdot$ & - & . & - & - \\
\hline g & 0.20 & $:$ & 1.00 & 1.00 & : & : & : & $:$ & . & $\cdot$ & - & - & - & - & 0.22 \\
\hline$i$ & - & . &. &. & 0.90 & 1.00 & 0.25 & $\dot{.}$ & : & $\dot{.}$ & : & 1.00 & 1.00 & $:$ & 0.78 \\
\hline \multicolumn{15}{|l|}{ Gpd-1 } & - \\
\hline a & - & - & - in & 100 & 100 & - & . & . & . & . & . & . & - & . & - \\
\hline $\begin{array}{l}c \\
d\end{array}$ & 0.20 & 0.50 & $\begin{array}{l}1.00 \\
-\end{array}$ & 1.00 & 1.00 & 1.00 & 1.00 & 1.00 & 1.00 & 1.00 & 1.00 & 0.67 & 1.00 & - & 1.00 \\
\hline d & 0.80 & 0.50 & $\cdot$ & $\cdot$ & $\cdot$ & $\cdot$ & - & - & $\cdot$ & - & $\cdot$ & 0.33 & $\cdot$ & 1.00 & - \\
\hline \multicolumn{16}{|l|}{ Gpi-1 } \\
\hline $\begin{array}{l}b \\
d\end{array}$ & 1.00 & 1.00 & 1.00 & $\dot{-}$ & 1.00 & 1.00 & 1.00 & 1.00 & 1.00 & 1.00 & 1.00 & 1.00 & 1.00 & 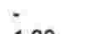 & 0.50 \\
\hline 1 & - & - & . & 1.00 & $\cdot$ &. &. & - & - & $\because$ & 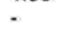 & $\begin{array}{l}1.00 \\
.\end{array}$ & 1.00 & 1.00 & 0.50 \\
\hline \multicolumn{16}{|l|}{$l c d-1$} \\
\hline$a$ & 1.00 & 1.00 & 1.00 & 1.00 & 1.00 & 1.00 & 1.00 & 1.00 & 1.00 & 1.00 & 1.00 & 1.00 & 1.00 & 1.00 & - \\
\hline & $\cdot$ & $\cdot$ & - & - & $\cdot$ & - & - & $\cdot$ & $\cdot$ & $\cdot$ & $\cdot$ & $\cdot$ & 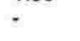 & - & 1.00 \\
\hline $\begin{array}{l}a \\
c\end{array}$ & 1.00 & 1.00 & 1.00 & 1.00 & 1.00 & 1.00 & 100 & 1.00 & $\overline{1}_{100}$ & 1.00 & $i .00$ & 1.00 & 1.00 & 1.00 & ; \\
\hline d & - & - & $\because$ & $\therefore$ & - & . & $\begin{array}{l}1,00 \\
.\end{array}$ & $\begin{array}{l}1.00 \\
-\end{array}$ & $\begin{array}{l}1.00 \\
-\end{array}$ & $\begin{array}{l}1.00 \\
-\end{array}$ & 1.00 & .00 & .00 & $\begin{array}{l}1.00 \\
.\end{array}$ & 1.00 \\
\hline$f$ & - & - & - & - & - & - & . & - & - & - & . & - & . & $:$ & $\dot{-}$ \\
\hline Mdh-1 & & & & & & & & & & & & & & & \\
\hline a & $\cdot$ & - & - & - & - & . & - & - & - & - & - & - & . & - & - \\
\hline c & 1.00 & 1.00 & 1.00 & 1.00 & 1.00 & 1.00 & 1.00 & 1.00 & 1.00 & 1.00 & 1.00 & 1.00 & 1.00 & 1.00 & 1.00 \\
\hline $\begin{array}{c}M e-1 \\
a\end{array}$ & 1.00 & 1.00 & 1.00 & 1.00 & 1.00 & 1.00 & 1.00 & 1.00 & 1.00 & 1.00 & 1.00 & 1.00 & 1.00 & 1.00 & 1.00 \\
\hline $\begin{array}{l}a \\
b\end{array}$ & $-\quad$ & .00 &. & 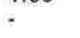 & 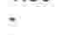 & - &. &. & $\therefore$ & $\therefore$ & $\cdot$ & - & $\cdot$ & - &. \\
\hline $\mathrm{c}$ & - & - & - & - & - & - & - & - & - & . & - & - & - & - & - \\
\hline d & - & $\cdot$ & - & $\cdot$ & - & $\cdot$ & . & - & $\cdot$ & - & $\cdot$ & $\cdot$ & . & - & . \\
\hline Mpi-1 & & & & & & & & & & & & & & & \\
\hline $\begin{array}{l}c \\
d\end{array}$ & : & $\dot{-}$ & 1.00 & 0.50 & 1.00 & 1.00 & 1.00 & 1.00 & 1.00 & 1.00 & 1.00 & 1.00 & $\begin{array}{l}1.00 \\
-\end{array}$ & $\begin{array}{l}1.00 \\
-\end{array}$ & $\begin{array}{l}1.00 \\
-\end{array}$ \\
\hline i & 1.00 & 1.00 & . & 0.50 & : & : & $\therefore$ & : & $\overline{-}$ & 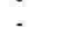 & . & . & . & - & $\dot{-}$ \\
\hline$N p-1$ & & & & & & & & & & & & & & & \\
\hline a & . & . & - & - & - & - & - & . & - & - & - & - & - & - & - \\
\hline b & - & - & - & - & - & - & - & - & - & - & - & - & $=$ & - & - \\
\hline c & 1.00 & 1.00 & 1.00 & 1.00 & 1.00 & 1.00 & 1.00 & 1.00 & 1.00 & 1.00 & 1.00 & 1.00 & 1.00 & 1.00 & 1.00 \\
\hline Pep-3 & . & . & - & - & . & . & . & . & - & . & - & - & - & - & . \\
\hline $\begin{array}{l}a \\
b\end{array}$ & 1.00 & 1.00 & 1.00 & 1.00 & 1.00 & 1.00 & 1.00 & 1.00 & 1.00 & 1.00 & 1.00 & 1.00 & 1.00 & 1.00 & 1.00 \\
\hline Pgd-1 & & & & & & & & & & & & & & & \\
\hline e & 1.00 & 1.00 & 1.00 & 1.00 & 1.00 & 1.00 & 1.00 & 1.00 & 1.00 & 1.00 & 1.00 & 1.00 & 1.00 & 1.00 & - \\
\hline 1 & - & - & - & - & - & - & - & - & - & - & - & 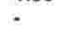 & - & - & 1.00 \\
\hline h & $\cdot$ & $\cdot$ & r & - & $\cdot$ & - & $\cdot$ & - & - & - & $\cdot$ & - & - & - & - \\
\hline Pgm-1 & & & & & & & & & & & & & & & \\
\hline b & 1.00 & 1.00 & 1.00 & 1.00 & 0.80 & 0.50 & 1.00 & 1.00 & - & - & - & 1.00 & 1.00 & 0.50 & 1.00 \\
\hline d & - & - & $\cdot$ & $\cdot$ & 0.20 & 0.50 & - & - & 1.00 & 1.00 & 1.00 & $\cdot$ & - & 0.50 & - \\
\hline Pgm-2 & & & & & & & & & & & & & & & \\
\hline c & $\begin{array}{l}0.90 \\
0.10\end{array}$ & 1.00 & - & - & - & 0.50 & 0.75 & 1.00 & - & 100 & $\begin{array}{l}0.70 \\
0.30\end{array}$ & $\begin{array}{l}0.17 \\
0.83\end{array}$ & $\begin{array}{l}0.50 \\
0.50\end{array}$ & 1.00 & $\begin{array}{l}0.39 \\
0.61\end{array}$ \\
\hline e & 0.10 & $\cdot$ & 1.00 & 1.00 & 1.00 & 0.50 & 0.25 & 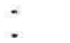 & 1.00 & $\begin{array}{l}1.00 \\
-\end{array}$ & 0.30 & 0.83 & 0.00 & $\therefore$ & $\begin{array}{l}0.61 \\
-\end{array}$ \\
\hline Sod-2 & & 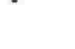 & 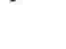 & & & 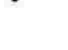 & & & & & & & & & \\
\hline b & - & - & - & - & . & 0.75 & 0.75 & - & 0.50 & 1.00 & - & - & - & - & - \\
\hline c & 0.30 & 0.12 & 0.50 & - & 1.00 & 0.25 & 0.25 & 1.00 & 0.50 & - & 0.60 & 0.83 & - & 0.25 & 0.56 \\
\hline d & 0.70 & 0.88 & 0.50 & 1.00 & - & . & - & - & - & - & 0.40 & 0.17 & 1.00 & 0.75 & 0.44 \\
\hline e & - & - & - & - & - & - & - & - & - & - & $\cdot$ & - & 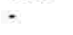 & 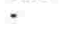 & - \\
\hline
\end{tabular}


Table 4.6.3.1 (continued)

\begin{tabular}{|c|c|c|c|c|c|c|c|c|c|c|c|c|c|c|c|}
\hline Locus & Ho14 & Ho15 & Ho16 & Ho17 & Ho18 & Ho19 & $\begin{array}{l}\text { Population } \\
\text { Ho2O }\end{array}$ & Ho21 & Ho22 & Ho23 & Ho24 & Ho24a" & Ho25 & Ho26 & $\mathrm{Ho} 27$ \\
\hline (N) & 3 & 4 & 7 & 1 & 1 & 2 & 7 & 2 & 2 & 7 & 8 & 1 & 13 & 1 & 2 \\
\hline $\begin{array}{l}A k-1 \\
a\end{array}$ & - & - & - & - & - & - & - & - & - & - & - & - & $=$ & - & \\
\hline b & 1.00 & 1.00 & 1.00 & 1.00 & 1.00 & 1.00 & 1.00 & 1.00 & 1.00 & 1.00 & 1.00 & 1.00 & 1.00 & 1.00 & 1.00 \\
\hline $\begin{array}{c}G d-2 \\
b\end{array}$ & is & $\therefore$ & 50 & in & ino & in & $\therefore$ & ino & i & - ino & i & in & ino & $i n$ & 100 \\
\hline c & 1.00 & 1.00 & 1.00 & 1.00 & 1.00 & 1.00 & 1.00 & 1.00 & 1.00 & 1.00 & 1.00 & 1.00 & 1.00 & 1.00 & 1.00 \\
\hline $\begin{array}{l}\text { Got-1 } \\
\text { a } \\
\text { c }\end{array}$ & in & - in & 0.07 & 1.00 & 1.00 & 1.00 & : & 1.00 & 1.00 & 1.00 & 1.00 & 1.00 & 1.00 & 1.00 & 1.00 \\
\hline Got-2 & 1.00 & & & & $\cdot$ & $\cdot$ & 1.00 & $\cdot$ & $\cdot$ & - & $\cdot$ & - & $\cdot$ & $\cdot$ & $\cdot$ \\
\hline $\begin{array}{l}a \\
b\end{array}$ & 1.00 & 1.00 & 1.00 & 1.00 & 1.00 & 1.00 & 1.00 & - & . & 0.85 & 1.00 & 1.00 & 0.81 & 1.00 & 1.00 \\
\hline$G p-1$ & & & 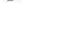 & $\cdot$ & $\cdot$ & $\sim$ & $\cdot$ & 1.00 & 1.00 & 0.15 & $\cdot$ & - & 0.19 & - & $\cdot$ \\
\hline $\begin{array}{l}a \\
b\end{array}$ & $\begin{array}{l}0.83 \\
0.17\end{array}$ & 1.00 & $\begin{array}{l}0.50 \\
0.50\end{array}$ & - & : & 0.25 & 1.00 & 1.00 & 1.00 & 0.79 & 0.81 & 1.00 & 1.00 & 1.00 & 1.00 \\
\hline Gp -4 & & & & 1.00 & 1.00 & 0.75 & $\cdot$ & $\cdot$ & $\cdot$ & 0.21 & 0.19 & $\cdot$ & $\cdot$ & $\cdot$ & $\cdot$ \\
\hline $\begin{array}{l}\mathrm{a} \\
\mathrm{c}\end{array}$ & $\dot{0}_{00}$ & . & . & - & - & - & - & - & - & - & $\because$ & - & - & - & - \\
\hline$i$ & $\begin{array}{l}0.50 \\
0.50\end{array}$ & $:$ & 0.29 & 1.00 & & 0.50 & 0.79 & 1.00 & 0.75 & 0.64 & 0.25 & - & 0.42 & 1.00 & 1.00 \\
\hline$g$ & . & 1.00 & 0.71 & 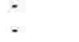 & $:$ & 0.50 & 0.21 & $\vdots$ & $\vdots$ & 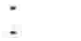 & : & $:$ & 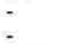 & : & - \\
\hline i & - & $\cdot$ & $\cdot$ & - & - & - & - & - & 0.25 & 0.36 & 0.75 & 1.00 & 0.58 & $:$ & $:$ \\
\hline $\begin{array}{l}G \rho d-1 \\
\mathrm{a}\end{array}$ & - & - & - & - & - & - & - & - & - & - & - & - & & - & \\
\hline c & 1.00 & 1.00 & 1.00 & 1.00 & 1.00 & 1.00 & 1.00 & 1.00 & 1.00 & 1.00 & 1.00 & 1.00 & 1.00 & 1.00 & 1.00 \\
\hline$d$ & $\cdot$ & $\cdot$ & $\cdot$ & $\cdot$ & $\cdot$ & - & $\cdot$ & - & - & $\cdot$ & - & $\cdot$ & - & $\cdot$ & $\cdot$ \\
\hline $\begin{array}{l}\text { Gpi-1 } \\
\mathrm{a}\end{array}$ & . & . & 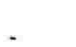 & - & - & - & . & . & . & - & - & - & - & - & \\
\hline $\begin{array}{l}\mathrm{a} \\
\mathrm{d}\end{array}$ & 1.00 & 1.00 & 1.00 & 1.00 & 1.00 & 1.00 & 1.00 & 1.00 & 1.00 & 1.00 & 1.00 & 1.00 & 1.00 & 1.00 & 1.00 \\
\hline$t$ & $\cdot$ & $\cdot$ & - & $\cdot$ & $\cdot$ & $\cdot$ & $\cdot$ & $\cdot$ & $\cdot$ & $\cdot$ & $\cdot$ & - & - & - & $\cdot$ \\
\hline $\begin{array}{l}\mid c d-1 \\
\mathrm{a}-1\end{array}$ & & & & & & & & & & & & & & & \\
\hline $\begin{array}{l}\text { a } \\
\text { b }\end{array}$ & $\begin{array}{l}0.17 \\
0.83\end{array}$ & 1.00 & 1.00 & ${ }^{1.00}$ & 1.00 & 1.00 & 1.00 & 1.00 & 1.00 & 1.00 & 1.00 & 1.00 & 1.00 & 1.00 & 1.00 \\
\hline $1 c d-2$ & & & & & $\cdot$ & $\cdot$ & $\cdot$ & $\cdot$ & $\cdot$ & - & $\cdot$ & $\cdot$ & $\cdot$ & $\cdot$ & $\cdot$ \\
\hline $\begin{array}{l}\mathrm{a} \\
\mathrm{c}\end{array}$ & - & $\therefore$ & . & - & . & - & . & 0.75 & . & - & - & . & - & - & - \\
\hline $\begin{array}{l}c \\
d\end{array}$ & 1.00 & 1.00 & 1.00 & 1.00 & 1.00 & 1.00 & 1.00 & 0.25 & 1.00 & 1.00 & 1.00 & 1.00 & 1.00 & 1.00 & 1.00 \\
\hline $\mathrm{i}$ & : & $:$ & $\vdots$ & $:$ & $:$ & $\vdots$ & $:$ & $:$ & $:$ & $\vdots$ & $\vdots$ & $\vdots$ & : & $\vdots$ & : \\
\hline Mah-1 & & & & & & & & & & & & & & & \\
\hline a & - & - & - & - & . & - & . & - & - & - & - & - & - & - & - \\
\hline c & 1.00 & 1.00 & 1.00 & 1.00 & 1.00 & 1.00 & 1.00 & 1.00 & 1.00 & 1.00 & 1.00 & 1.00 & 1.00 & 1.00 & 1.00 \\
\hline $\begin{array}{l}M e-1 \\
a\end{array}$ & & 1.00 & 1.00 & 1.00 & 1.00 & 1.00 & 1.00 & 1.00 & 1.00 & 1.00 & 1.00 & 1.00 & 1.00 & 1.00 & 1.00 \\
\hline$\vec{b}$ & .00 & . & 8 & 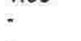 & . & $\therefore$ & 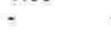 & $\cdot$ & $\cdot$ & $\cdot$ & - &. & &. & .0 \\
\hline c & : & - & - & - & : & : & - & $\cdot$ & - & $\cdot$ & - & - & - & - & - \\
\hline d & - & - & $\cdot$ & $\cdot$ & $\cdot$ & $\cdot$ & $\cdot$ & - & - & $\cdot$ & $\cdot$ & $\cdot$ & - & - & - \\
\hline $\begin{array}{l}\text { Mpi-1 } \\
\text { c }\end{array}$ & 1.00 & 1.00 & 1.00 & 1.00 & 1.00 & 1.00 & 0.86 & 0.25 & - & 0.14 & 0.56 & 1.00 & 0.27 & 1.00 & 1.00 \\
\hline d & . &. & $\cdot$ & 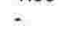 & . &. & - & & - & - & - & & & . & .00 \\
\hline$f$ & $\cdot$ & . & - & $\cdot$ & - & $\cdot$ & 0.14 & 0.75 & 1.00 & 0.86 & 0.44 & - & 0.73 & $\cdot$ & - \\
\hline$N p-1$ & & & & & & & & & & & & & & & \\
\hline $\begin{array}{l}a \\
b\end{array}$ & : & : & $:$ & $:$ & $:$ & $:$ & $:$ & $:$ & $:$ & $\vdots$ & : & : & $\therefore$ & $:$ & $\therefore$ \\
\hline c & 1.00 & 1.00 & 1.00 & 1.00 & 1.00 & 1.00 & 1.00 & 1.00 & 1.00 & 1.00 & 1.00 & 1.00 & 1.00 & 1.00 & 1.00 \\
\hline $\begin{array}{l}\text { Pep-3 } \\
\mathrm{a}\end{array}$ & . & - & 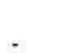 & - & - & - & . & $\cdot$ & . & & & & & & \\
\hline$\vec{b}$ & 1.00 & 1.00 & 1.00 & 1.00 & 1.00 & 1.00 & 1.00 & 1.00 & 1.00 & 1.00 & 1.00 & 1.00 & 1.00 & 1.00 & 1.00 \\
\hline$P g d-1$ & & & & & & & & & & & & & & & \\
\hline e & in & in & $i n$ & $j 00$ & $j 00$ & 1.00 & 1.00 & 1.00 & 1.00 & 1.00 & 1.00 & 1.00 & 1.00 & 1.00 & 1.00 \\
\hline $\begin{array}{l}f \\
h\end{array}$ & 1.00 & 1.00 & 1.00 &.$^{1.00}$ & 1.00 & $:$ & $:$ & $\vdots$ & $\vdots$ & 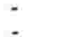 & $:$ & $\vdots$ & $\overline{-}$ & $\vdots$ & $:$ \\
\hline$P g m-1$ & & & & & & & & & & & & & & & \\
\hline b & 1.00 & 1.00 & 1.00 & 1.00 & 1.00 & 1.00 & 1.00 & - & 1.00 & 1.00 & 1.00 & 1.00 & 1.00 & 1.00 & 1.00 \\
\hline d & $\cdot$ & $\cdot$ & $\cdot$ & $\cdot$ & $\cdot$ & $\cdot$ & - & 1.00 & $\cdot$ & $\cdot$ & - & $\cdot$ & $\cdot$ & - & $\cdot$ \\
\hline Pgm-2 & & & & & & & & & & & & & & & \\
\hline $\begin{array}{l}c \\
\text { e }\end{array}$ & 1.00 & 1.00 & 1.00 & .00 & .00 & 1.00 & 1.00 & 1.00 & $i .00$ & $\begin{array}{l}0.71 \\
0.29\end{array}$ & 1.00 & 1.00 & $\begin{array}{l}0.50 \\
0.50\end{array}$ & 1.00 & $\begin{array}{l}0.75 \\
0.25\end{array}$ \\
\hline i &. &. & $\cdot$ & - & - &. & - & - &. & .0 & . & 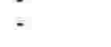 & - & - &. \\
\hline $\begin{array}{l}\text { Sod-2 } \\
\mathrm{b}\end{array}$ & 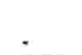 & 7 & 0.29 & * & . & - & - & - & - & 0.64 & 0.12 & - & & - & \\
\hline c & 1.00 & 0.38 & 0.71 & 1.00 & 1.00 & 0.75 & - & 0.75 & 1.00 & 0.21 & 0.44 & 1.00 & 0.54 & . & 0.50 \\
\hline d & $\cdot$ & 0.62 & 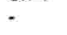 & $\cdot$ & $\cdot$ & 0.25 & 1.00 & 0.25 & - & 0.14 & 0.44 & - & 0.46 & 1.00 & 0.50 \\
\hline e & - &. & - & - & - & - & - & - & - & - & - & - & - & - & - \\
\hline
\end{tabular}


Table 4.6.3.1 (continued)

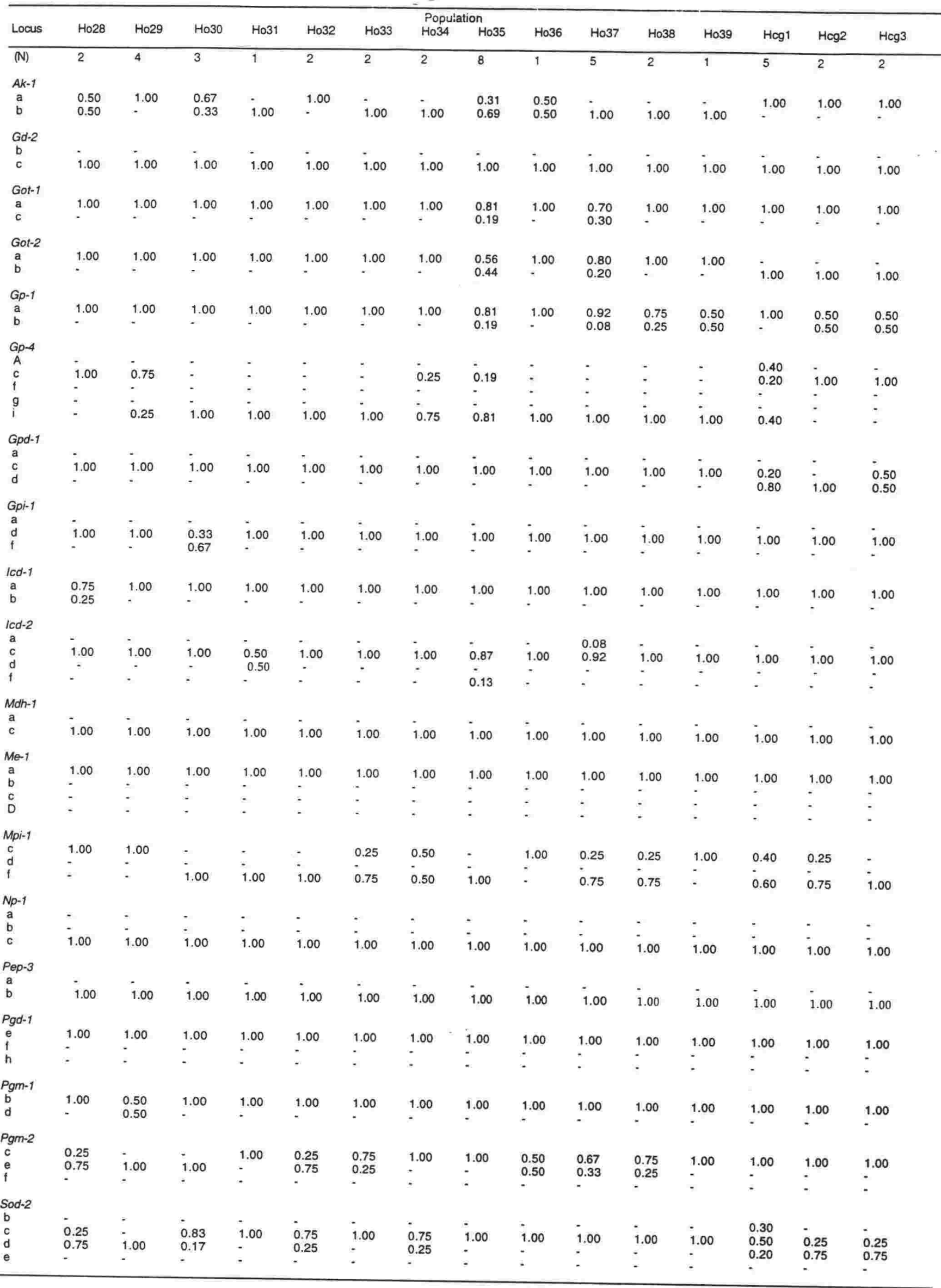


Table 4.6.3.1 (continued)

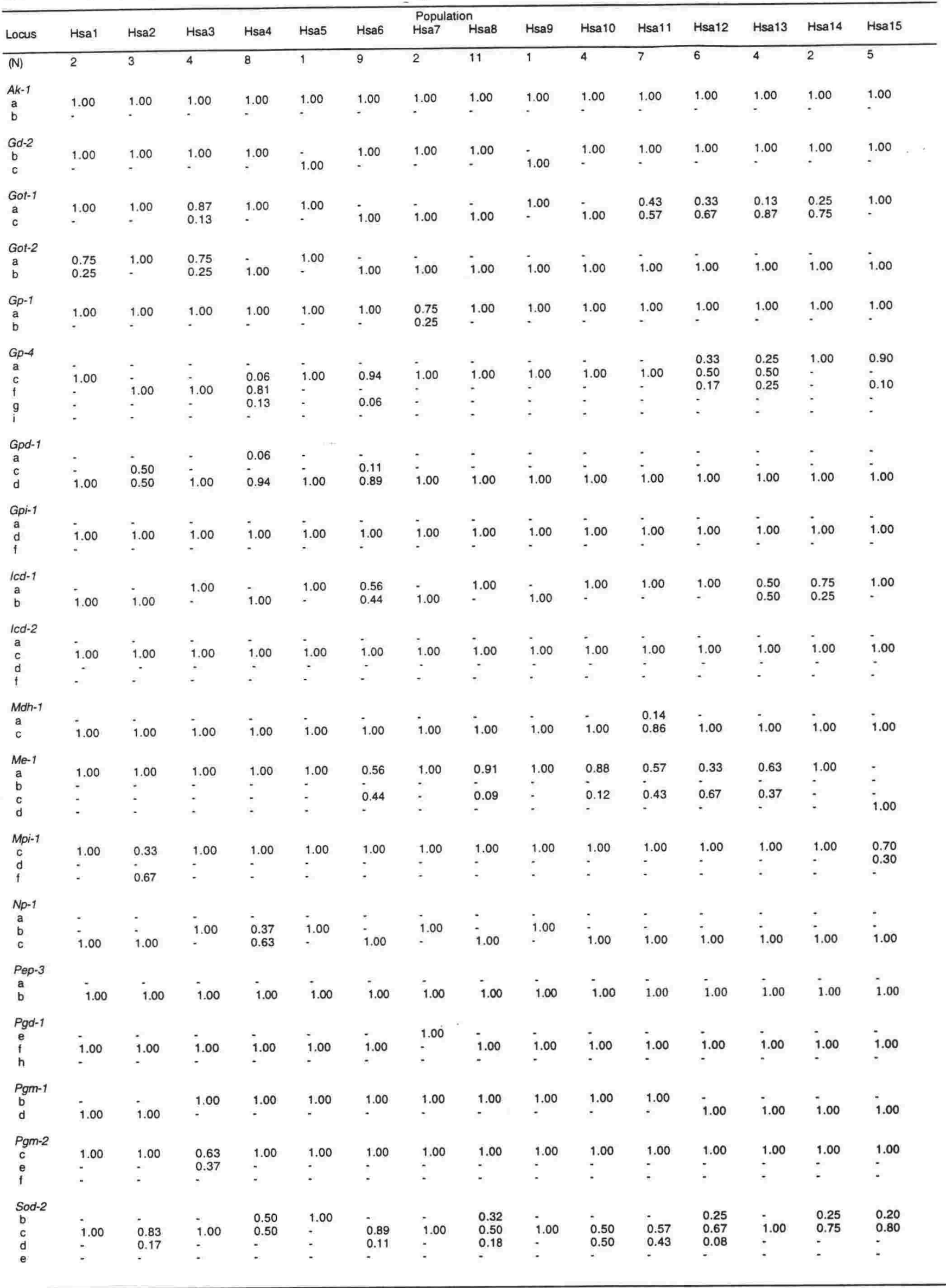


Table 4.6.3.1 (continued)

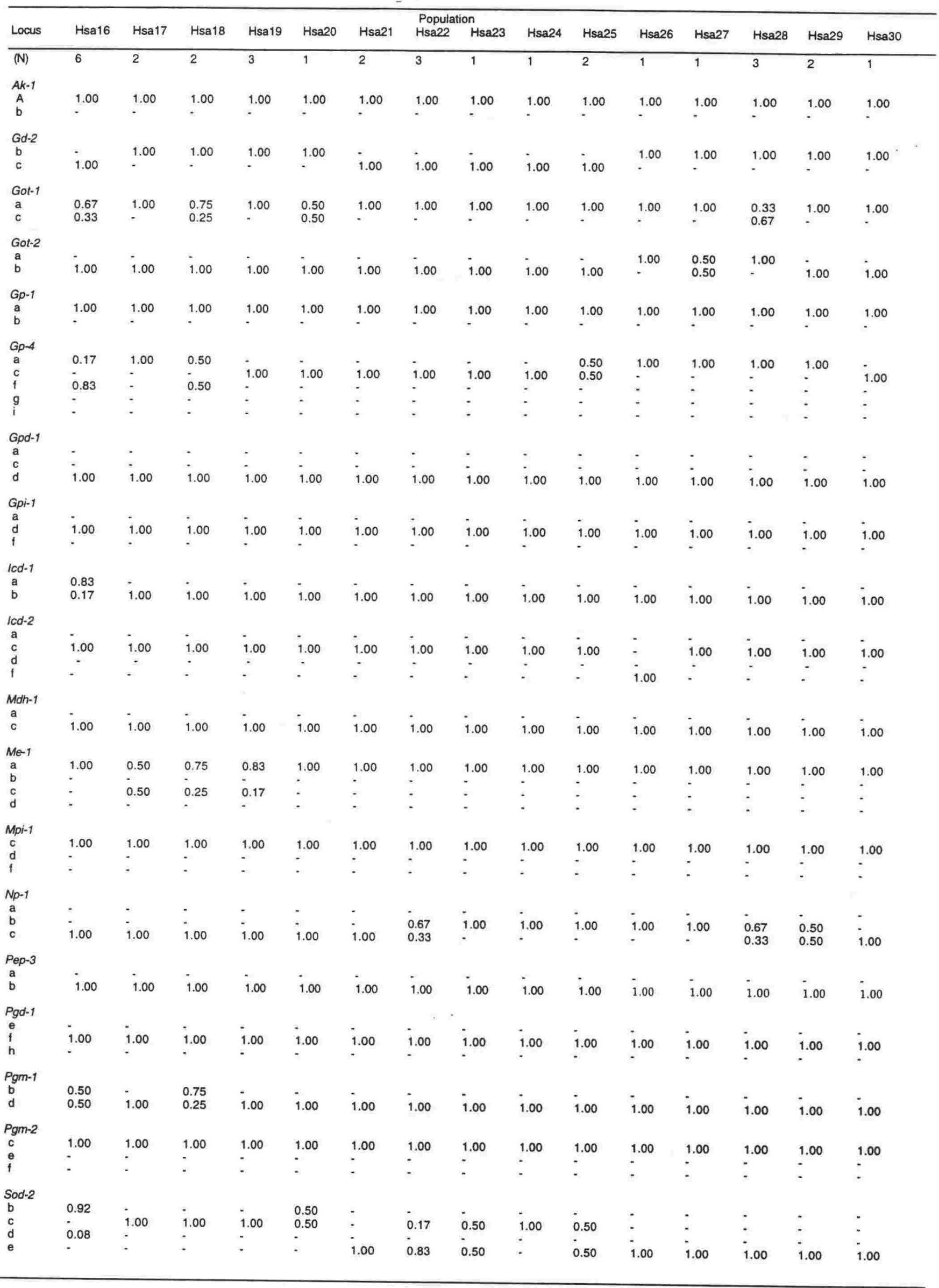


Table 4.6.3.1 (continued)

\begin{tabular}{|c|c|c|c|c|c|c|c|c|c|c|c|c|c|c|c|}
\hline Locus & Hsa31 & Hd1 & Hd2 & $\mathrm{Hd} 3$ & $\mathrm{Hd} 4$ & Hd5 & $\begin{array}{l}\text { Populat } \\
\text { Hd6 }\end{array}$ & $\mathrm{Hd}$ & $\mathrm{H} d 8$ & Hd9 & Hd10 & Hd11 & Hd12 & $\mathrm{Hd} 13$ & Hd14 \\
\hline (N) & 3 & 6 & 2 & 2 & 2 & 2 & 2 & 2 & 1 & 2 & 2 & 2 & 1 & 1 & 1 \\
\hline $\begin{array}{c}A k-1 \\
a\end{array}$ & 1.00 & 1.00 & 1.00 & 1.00 & 1.00 & 1.00 & 1.00 & 1.00 & 1.00 & 1.00 & 1.00 & 1.00 & 1.00 & 1.00 & 1.00 \\
\hline b & $\cdot$ & $\cdot$ & - & $\cdot$ & $\cdot$ & $\cdot$ & $\cdot$ & $\cdot$ & & & & & & & \\
\hline $\begin{array}{c}G d-2 \\
b\end{array}$ & 1.00 & 1.00 & 1.00 & 1.00 & 1.00 & 1.00 & 1.00 & 1.00 & 1.00 & 1,00 & 1.00 & 1.00 & 1.00 & 1.00 & $1.00 \ldots$ \\
\hline c & - & 1.00 & & & & & & & & & & & & & \\
\hline $\begin{array}{l}\text { Got-1 } \\
\text { a }\end{array}$ & 1.00 & 1.00 & 1.00 & 1.00 & 1.00 & 1.00 & 1.00 & 1.00 & 1.00 & 1.00 & 1.00 & 1.00 & 1.00 & 1.00 & 1.00 \\
\hline c & $\cdot$ & $\cdot$ & $\cdot$ & & & - & & & & & & & & & \\
\hline $\begin{array}{l}\text { Got-2 } \\
\text { a }\end{array}$ & 1.00 & - & - & - & - & . & - & - & - & - & $\cdot$ & - & - & - & 1.00 \\
\hline $\mathrm{b}$ &. & 1.00 & 1.00 & 1.00 & 1.00 & 1.00 & 1.00 & 1.00 & 1.00 & 1.00 & 1.00 & 1.00 & 1.00 & 1.00 & 1.00 \\
\hline$G p-1$ & & & & & . & . & - & . & . & - & & & & - & - \\
\hline $\begin{array}{l}\mathrm{a} \\
\mathrm{b}\end{array}$ & 1.00 & 1.00 & 1.00 & 1.00 & 1.00 & 1.00 & 1.00 & 1.00 & 1.00 & 1.00 & 1.00 & 1.00 & 1.00 & 1.00 & 1.00 \\
\hline$G p-4$ & & & & & & & & & & & & & & & \\
\hline $\begin{array}{l}a \\
c\end{array}$ & - & : & 1.00 & 1.00 & 1.00 & 1.00 & 1.00 & 1.00 & 1.00 & 1.00 & 1.00 & 1.00 & 1.00 & 1.00 & . \\
\hline $\begin{array}{l}c \\
f\end{array}$ & 1.00 & 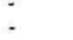 & 1.00 & $\begin{array}{l}1.00 \\
-\end{array}$ & $\begin{array}{l}1.00 \\
-\end{array}$ & 1.00 & 1.00 & 1.00 & - & - & . & 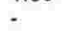 & $\cdot$ & 8 & $\therefore$ \\
\hline $\begin{array}{l}f \\
g\end{array}$ & $\dot{-}$ & . & - & - & . & : & $i$ & 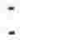 & - & - & - & - & - & . & 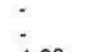 \\
\hline$i$ & . & 1.00 & - & $\cdot$ & - & . & - & $\cdot$ & $\cdot$ & - & - & $\cdot$ & - & $\cdot$ & 1.00 \\
\hline Gpd-1 & & & & & & & & & & & & & & & \\
\hline & & & & & - & - & - & - & 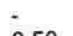 & - 75 & 025 & : & : & - & $\therefore=0$ \\
\hline
\end{tabular}

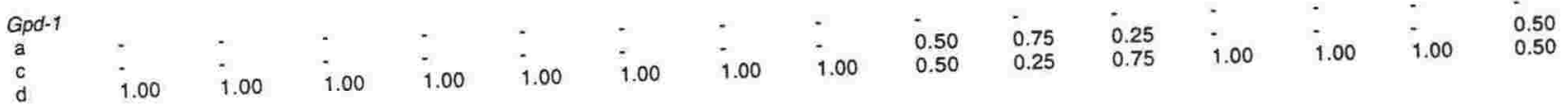

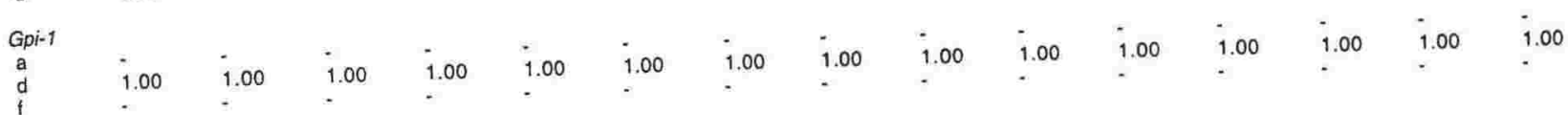

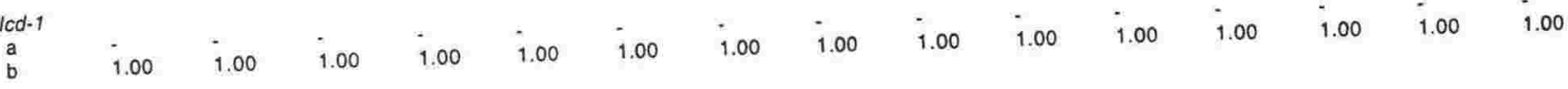

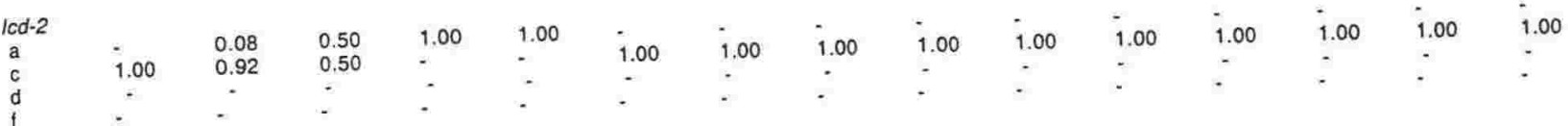

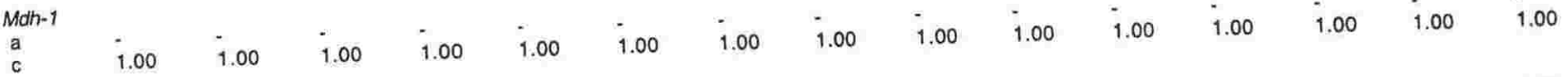

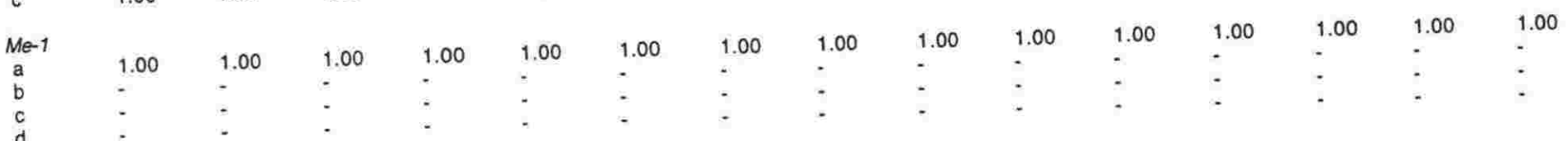

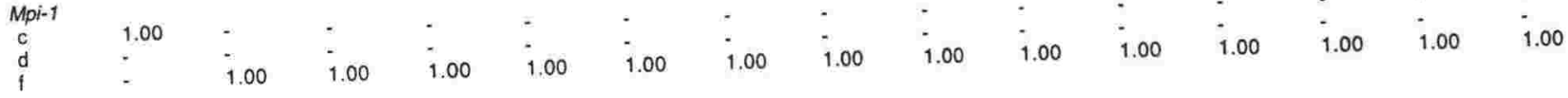

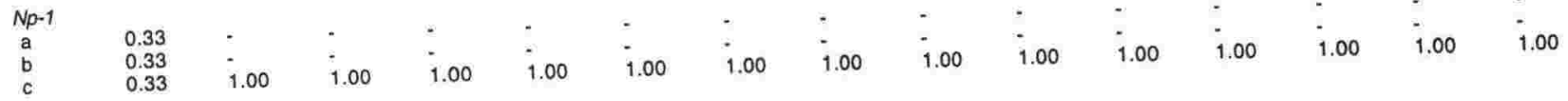

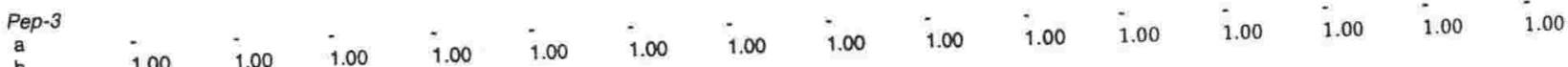

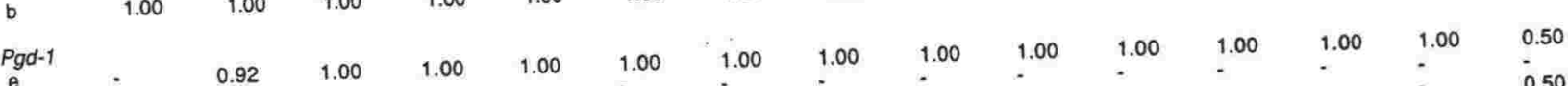

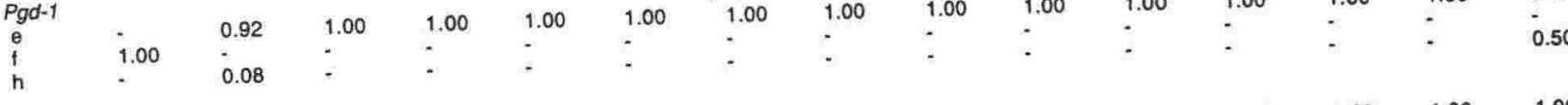

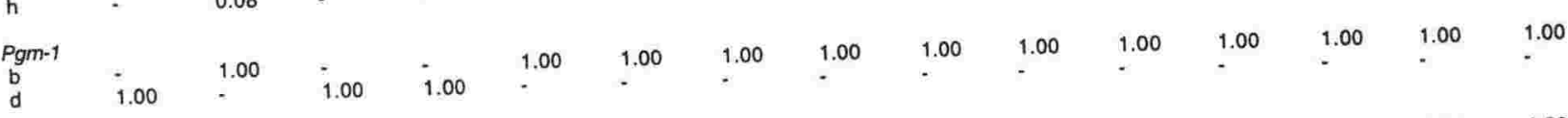

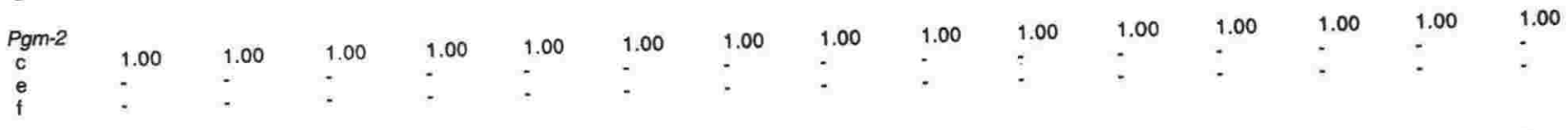

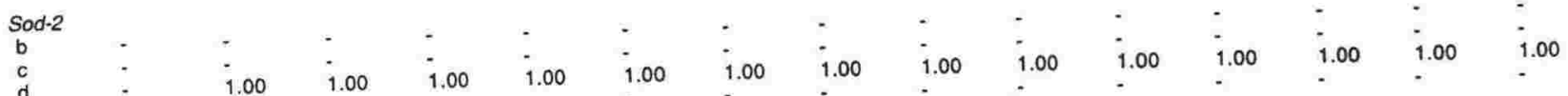
$1.00 \quad 1.00 \quad 1.00 \quad-1.00 \quad-$ 
Table 4.6.3.1 (continued)

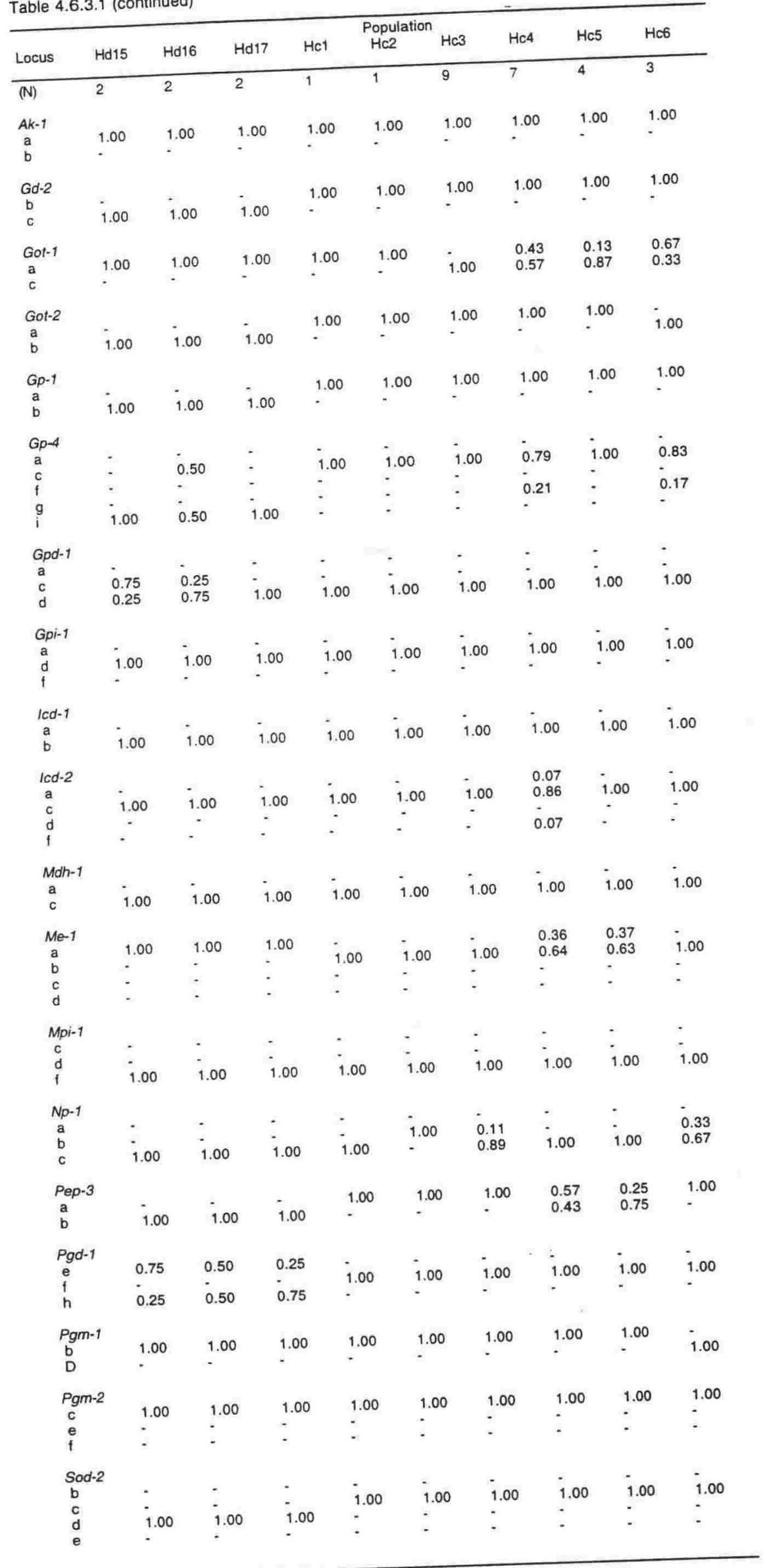




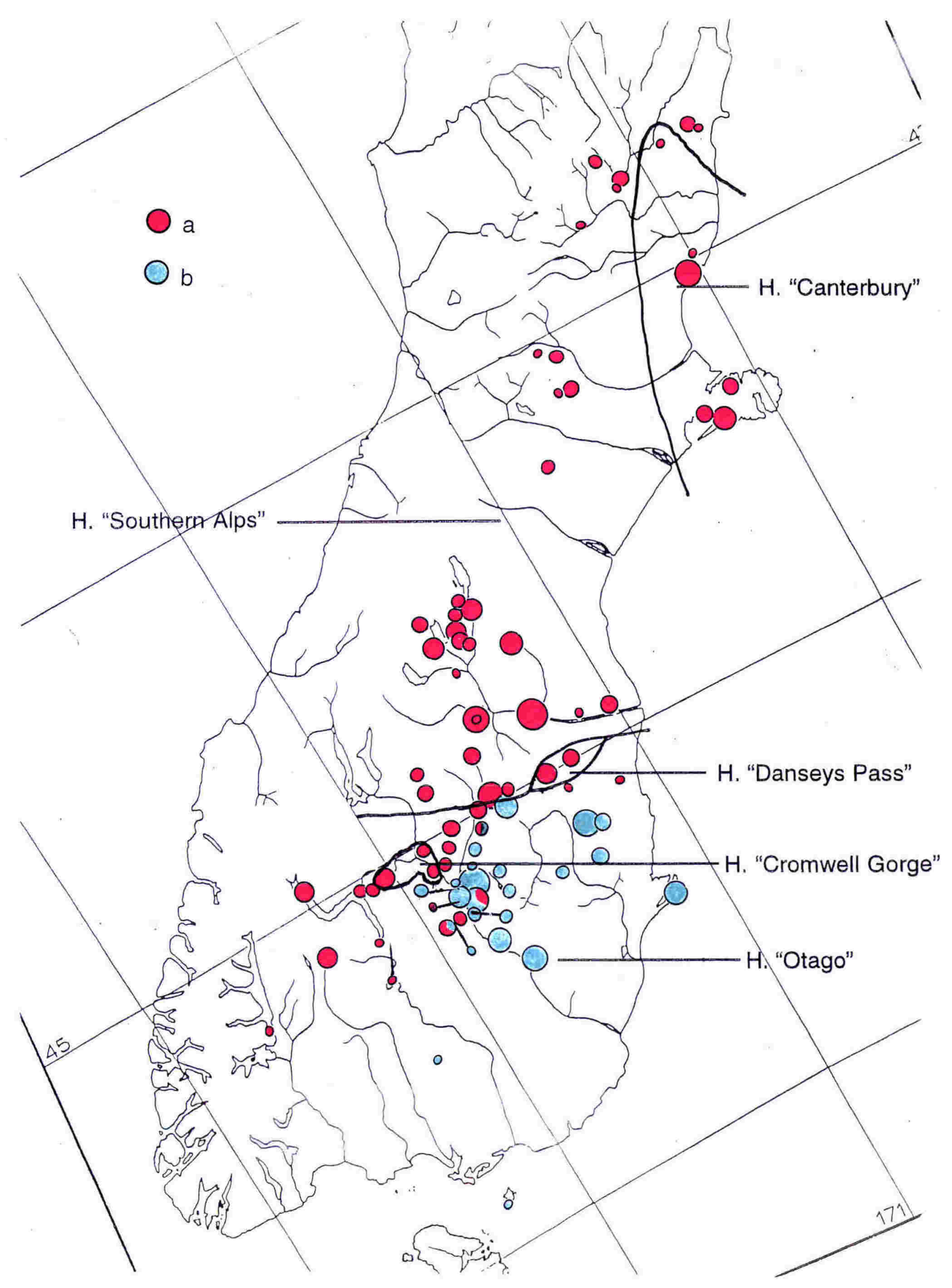

Figure 4.6.3.1 Ak-1 allele distributions among populations of the southern species group of the $\mathrm{H}$. maculatus complex. The total area of the pie chart for each sample is directly proportional to sample size (see Table 4.6.3.1). For the Tara Hills, Omarama sample, the single individual which appeared to be genetically distinct from the rest of the sample is represented as a small circle in the centre of the larger circle representing the remainder of the sample. 


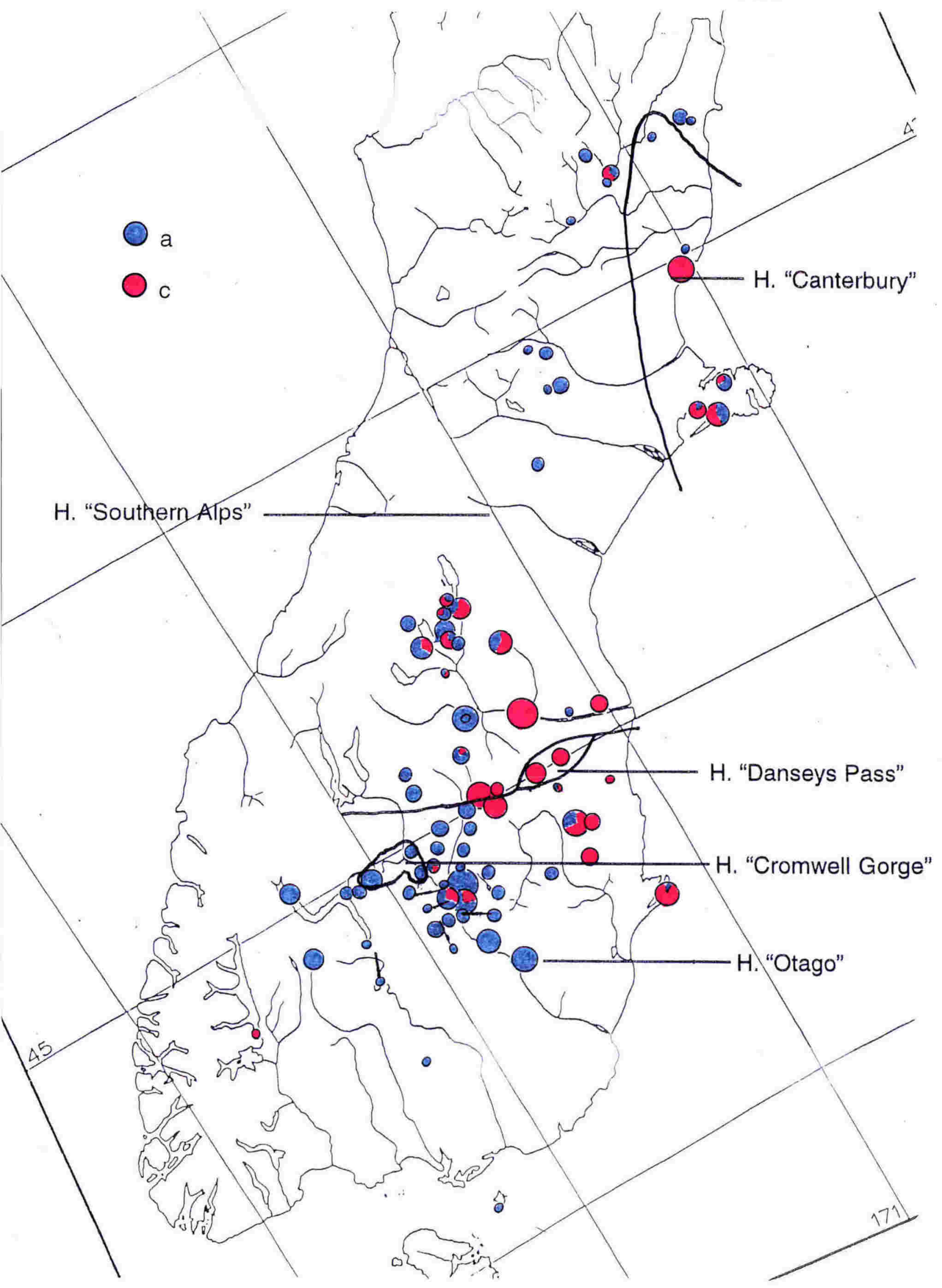

Figure 4.6.3.3 Got-1 allele distributions among populations of the southern species group of the $H$. maculatus complex. The total area of the pie chart for each sample is directly proportional to sample size (see Table 4.6.2.1). For the Tara Hills, Omarama sample, the single individual which appeared to be genetically distinct from the rest of the sample is represented as a small circle in the centre of the larger circle representing the remainder of the sample. 


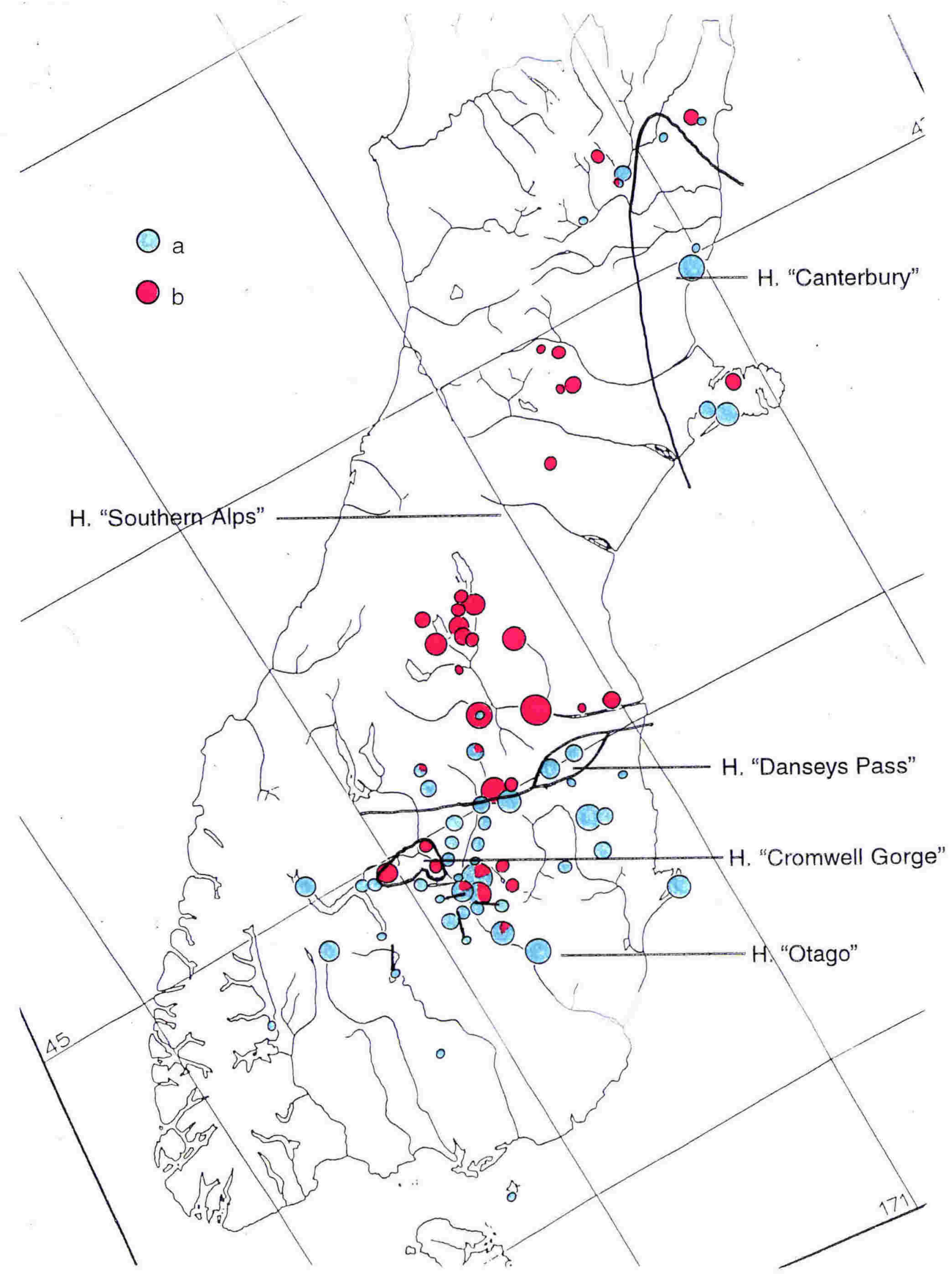

Figure 4.6.3.4 Got-2 allele distributions among populations of the southern species group of the $H$. maculatus complex. The total area of the pie chart for each sample is directly proportional to sample size (see Table 4.6.2.1). For the Tara Hills, Omarama sample, the single individual which appeared to be genetically distinct from the rest of the sample is represented as a small circle in the centre of the larger circle representing the remainder of the sample. 


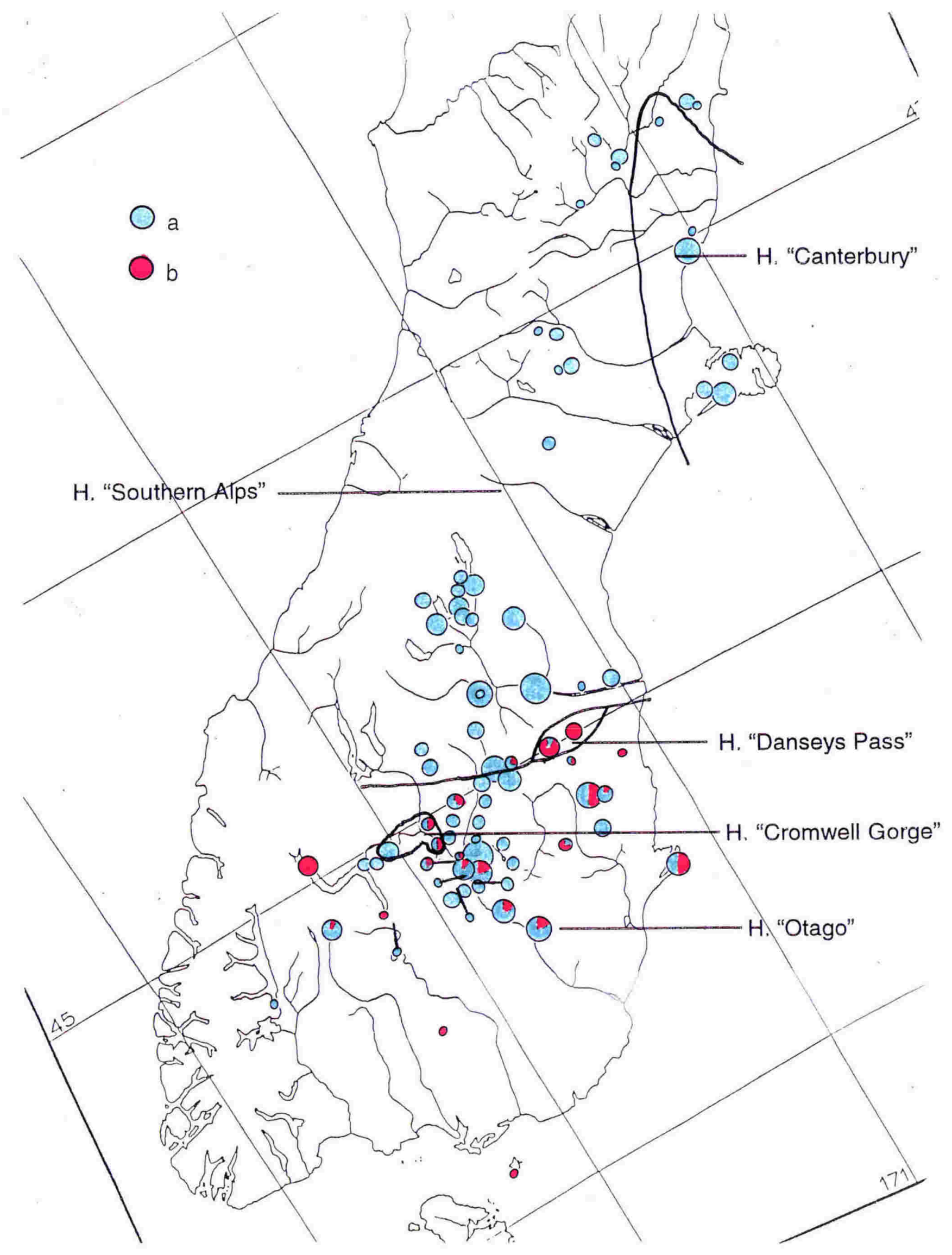

Figure 4.6.3.5 Gp-1 allele distributions among populations of the southern species group of the $H$. maculatus complex. The total area of the pie chart for each sample is directly proportional to sample size (see Table 4.6.2.1). For the Tara Hills, Omarama sample, the single individual which appeared to be genetically distinct from the rest of the sample is represented as a small circle in the centre of the larger circle representing the remainder of the sample. 


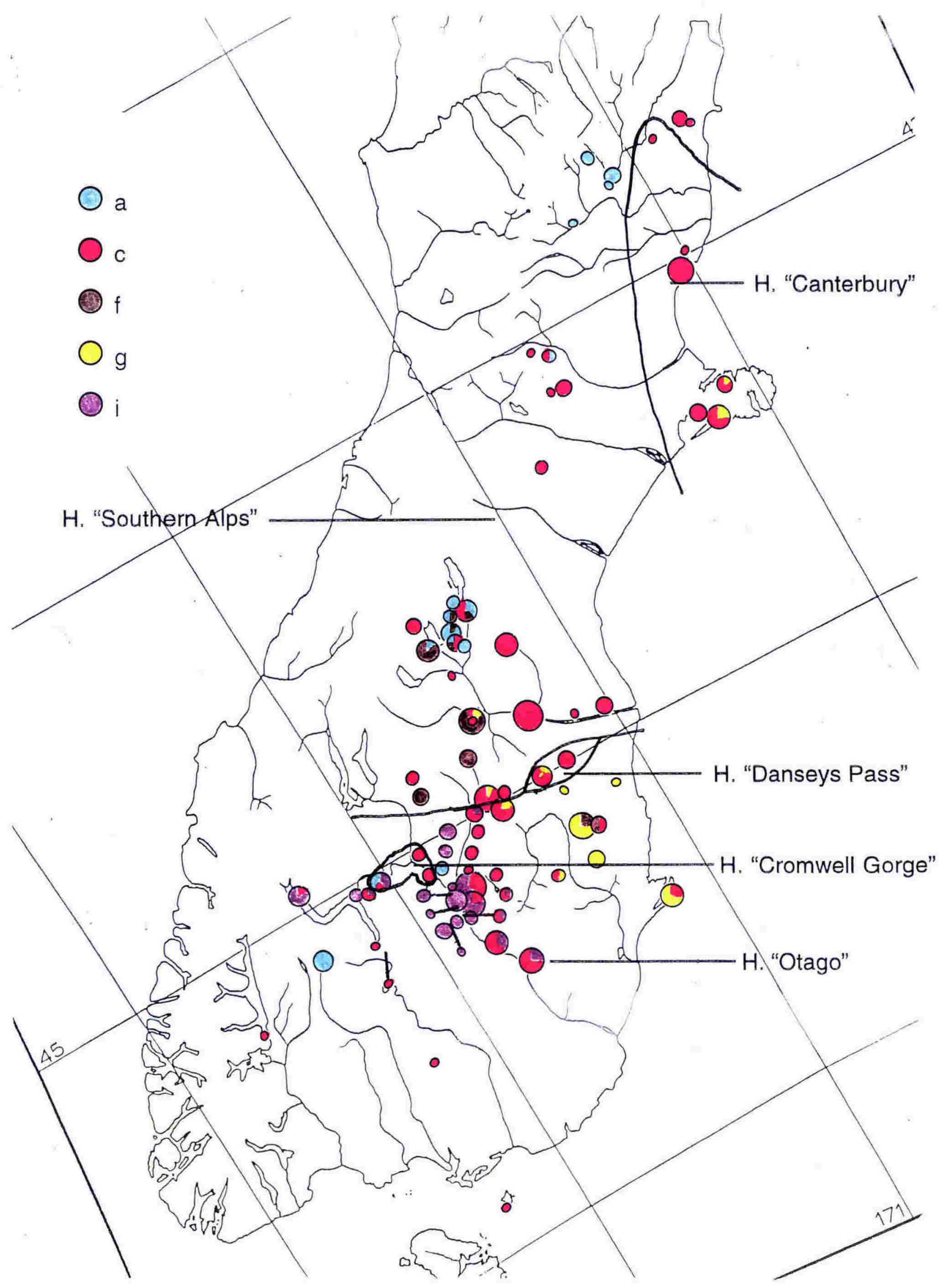

Figure 4.6.3.6 Gp-4 allele distributions among populations of the southern species group of the $H$. maculatus complex. The total area of the pie chart for each sample is directly proportional to sample size (see Table 4.6.2.1). For the Tara Hills, Omarama sample, the single individual which appeared to be genetically distinct from the rest of the sample is represented as a small circle in the centre of the larger circle representing the remainder of the sample. 


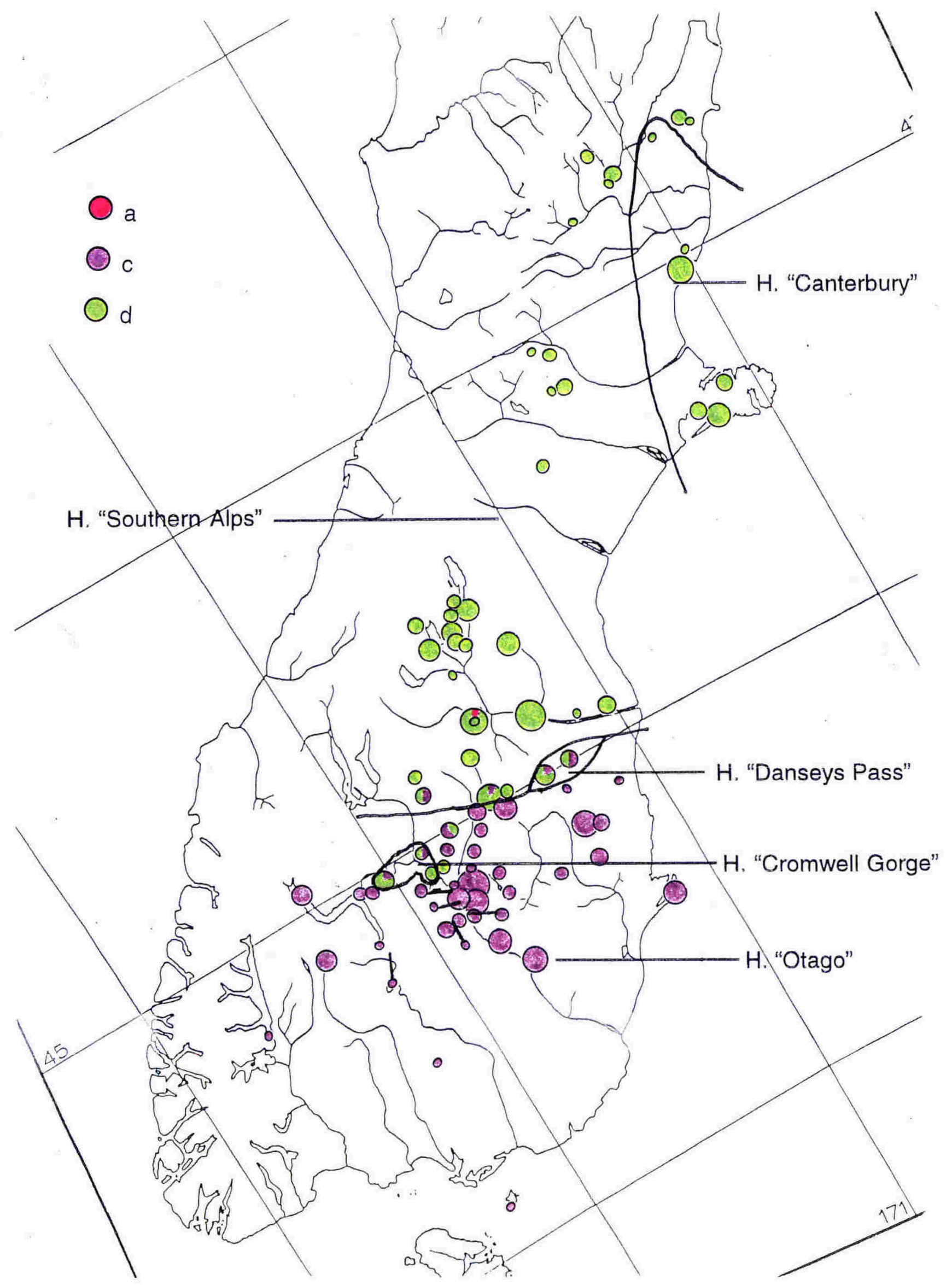

Figure 4.6.3.7 Gpd-1 allele distributions among populations of the southern species group of the $H$. maculatus complex. The total area of the pie chart for each sample is directly proportional to sample size (see Table 4.6.2.1). For the Tara Hills, Omarama sample, the single individual which appeared to be genetically distinct from the rest of the sample is represented as a small circle in the centre of the larger circle representing the remainder of the sample. 


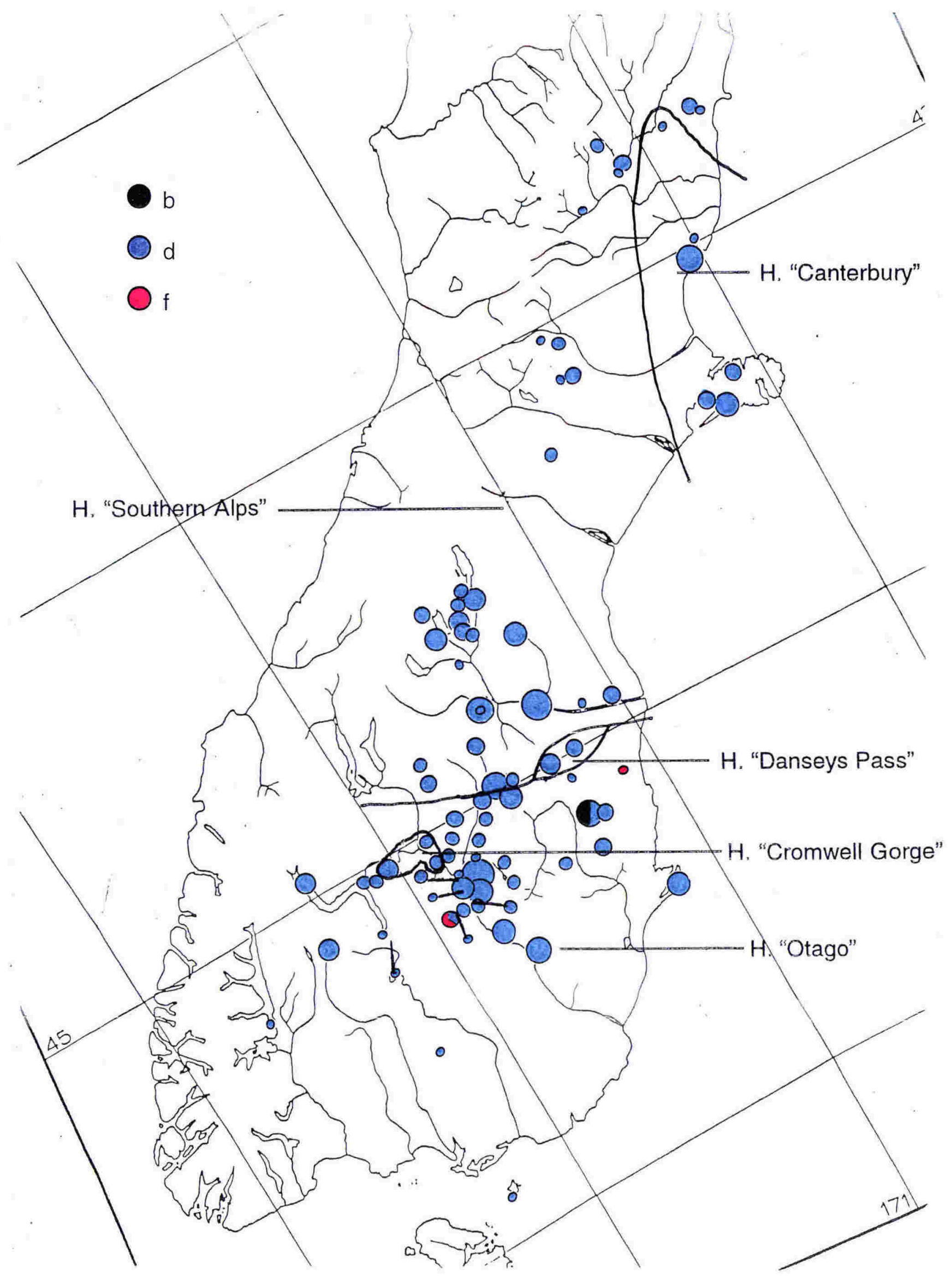

Figure 4.6.3.8 Gpi-1 allele distributions among populations of the southern species group of the $H$. maculatus complex. The total area of the pie chart for each sample is directly proportional to sample size (see Table 4.6.2.1). For the Tara Hills, Omarama sample, the single individual which appeared to be genetically distinct from the rest of the sample is represented as a small circle in the centre of the larger circle representing the remainder of the sample. 


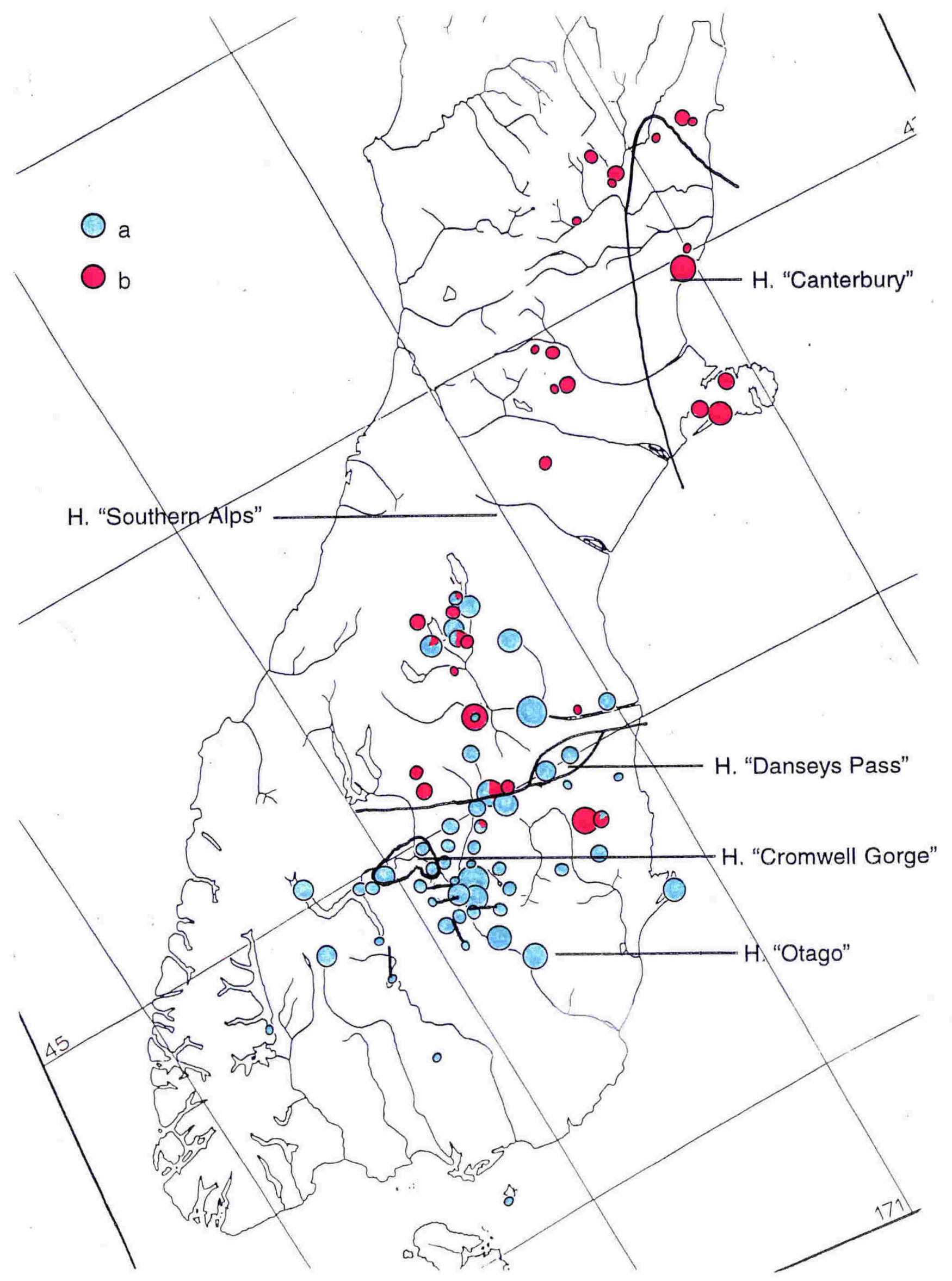

Figure 4.6.3.9 $l c d-1$ allele distributions among populations of the southern species group of the $H$. maculatus complex. The total area of the pie chart for each sample is directly proportional to sample size (see Table 4.6.2.1). For the Tara Hills, Omarama sample, the single individual which appeared to be genetically distinct from the rest of the sample is represented as a small circle in the centre of the larger circle representing the remainder of the sample. 


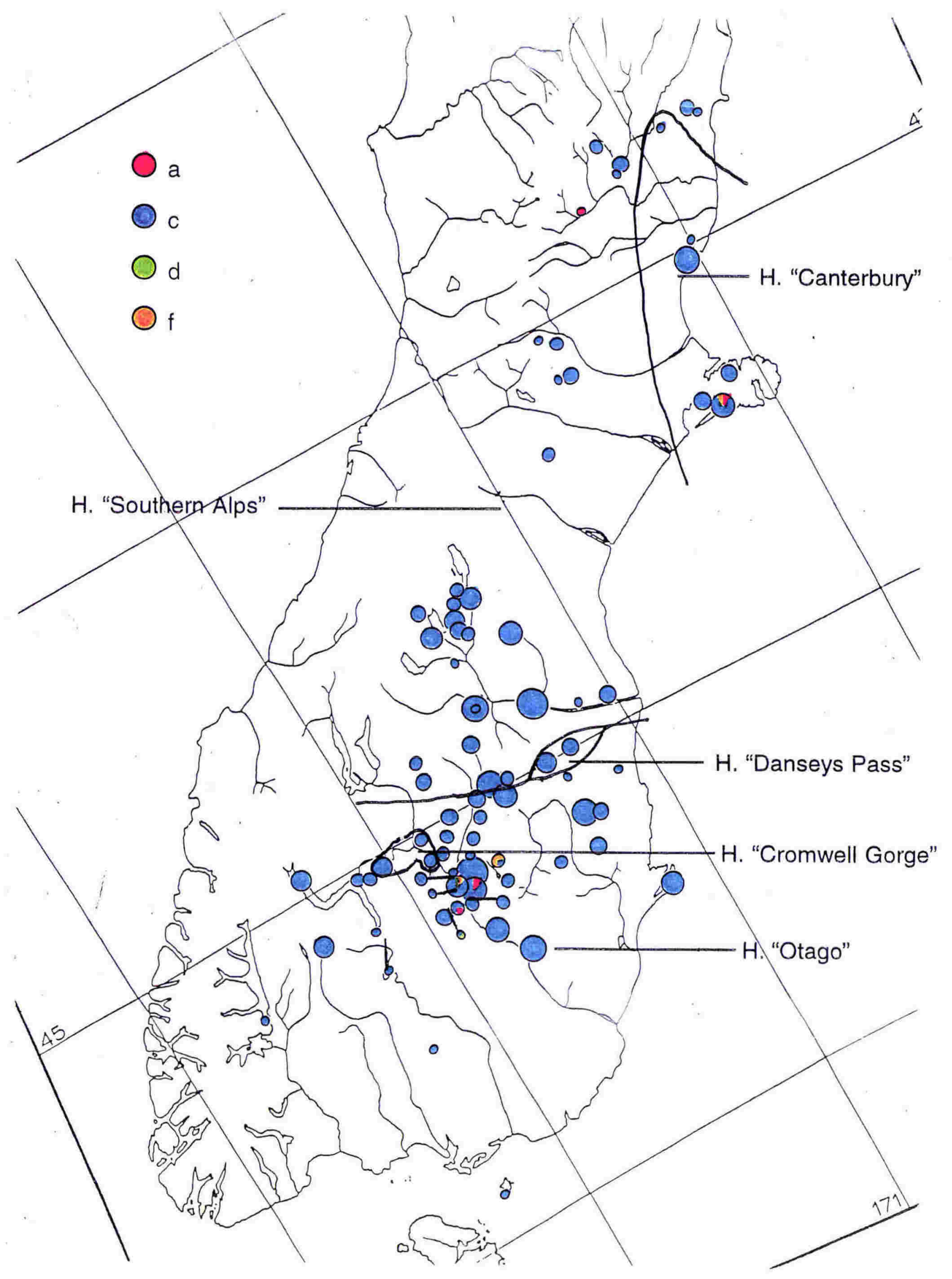

Figure 4.6.3.10 $\mathrm{lcd}$-2 allele distributions among populations of the southern species group of the $H$. maculatus complex. The total area of the pie chart for each sample is directly proportional to sample size (see Table 4.6.2.1). For the Tara Hills, Omarama sample, the single individual which appeared to be genetically distinct from the rest of the sample is represented as a small circle in the centre of the larger circle representing the remainder of the sample. 


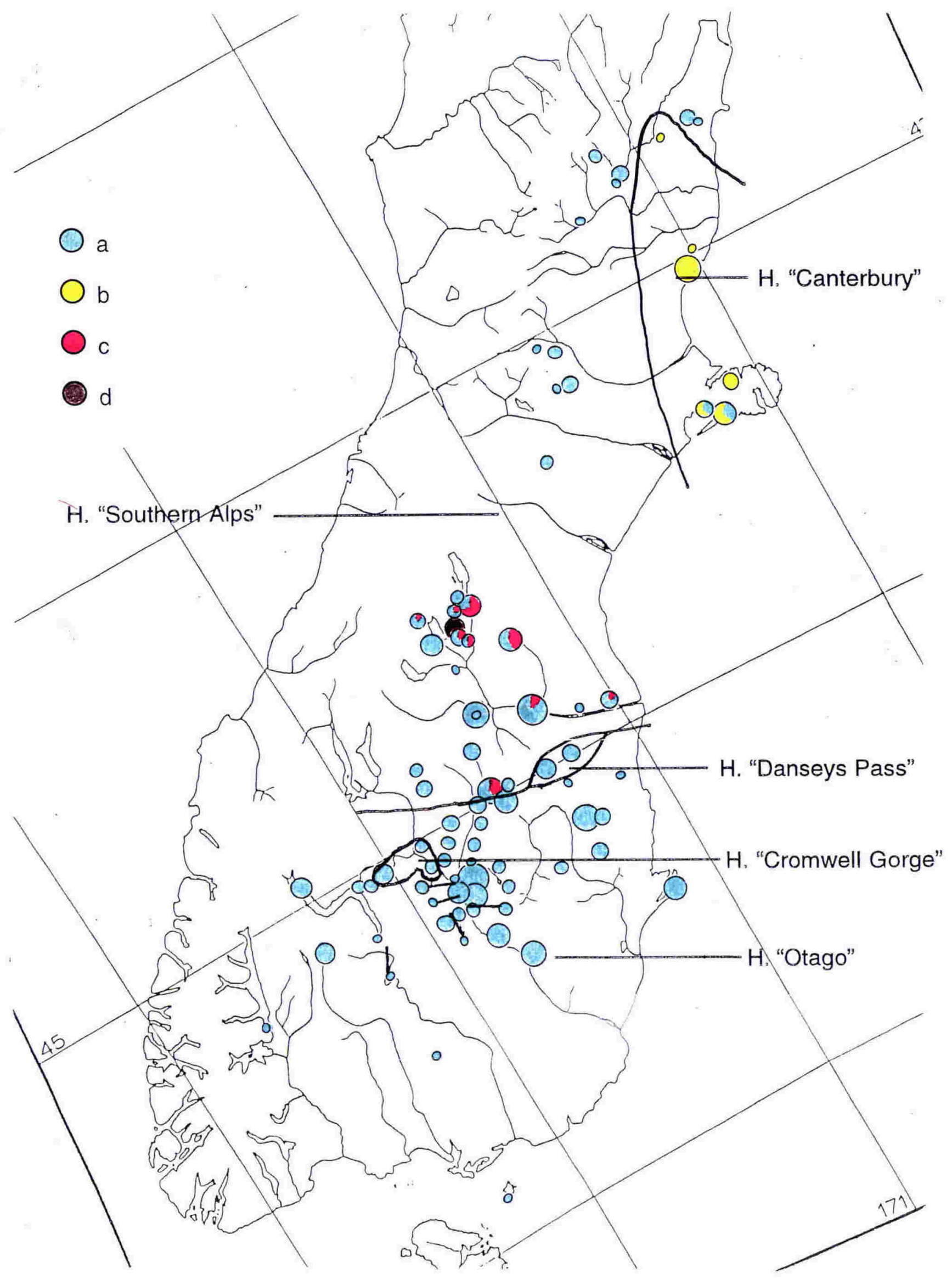

Figure 4.6.3.11 Me-1 allele distributions among populations of the southern species group of the $H$. maculatus complex. The total area of the pie chart for each sample is directly proportional to sample size (see Table 4.6.2.1). For the Tara Hills, Omarama sample, the single individual which appeared to be genetically distinct from the rest of the sample is represented as a small circle in the centre of the larger circle representing the remainder of the sample. 


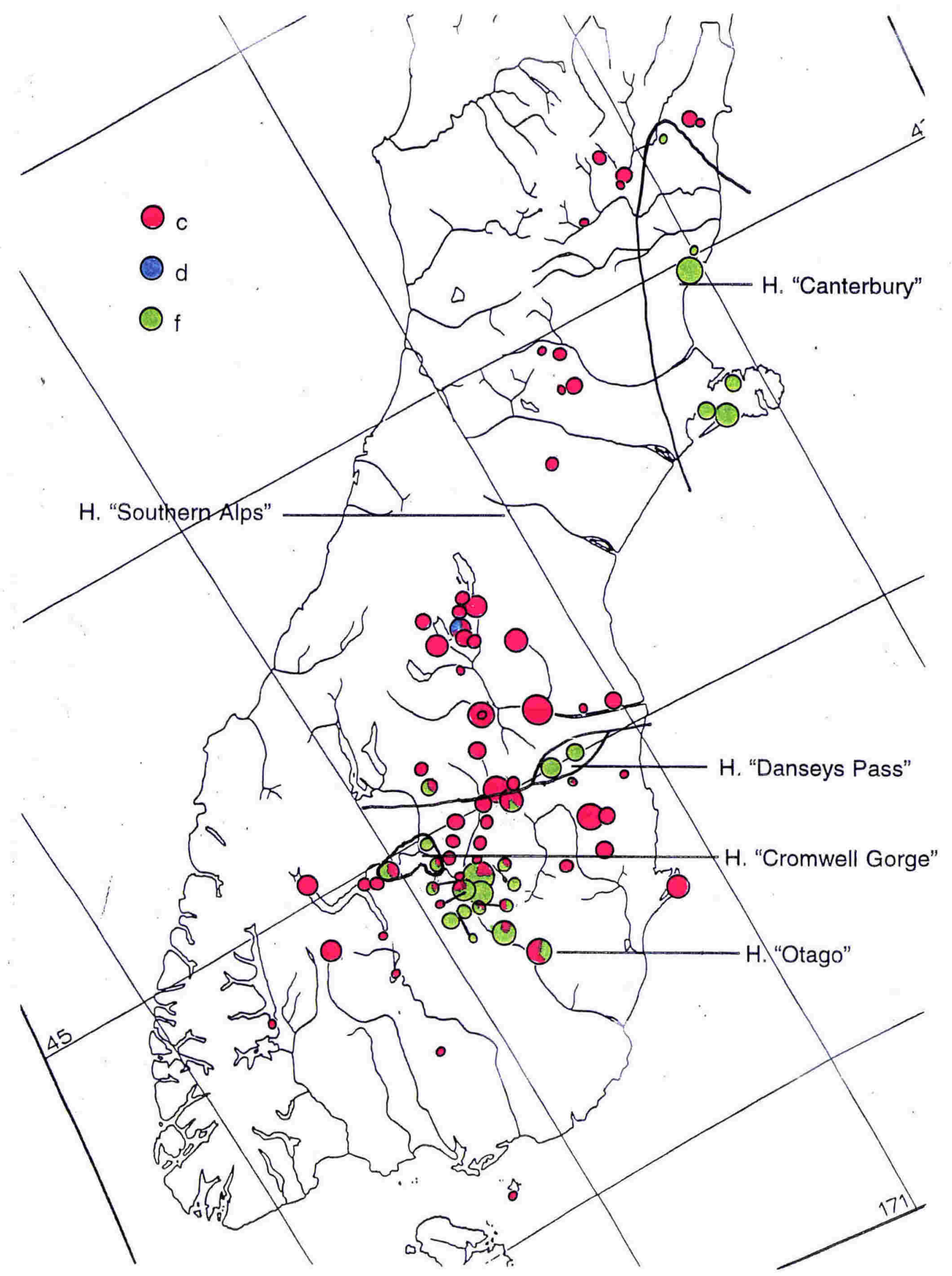

Figure 4.6.3.12 Mpi-1 allele distributions among populations of the southern species group of the $H$. maculatus complex. The total area of the pie chart for each sample is directly proportional to sample size (see Table 4.6.2.1). For the Tara Hills, Omarama sample, the single individual which appeared to be genetically distinct from the rest of the sample is represented as a small circle in the centre of the larger circle representing the remainder of the sample. 


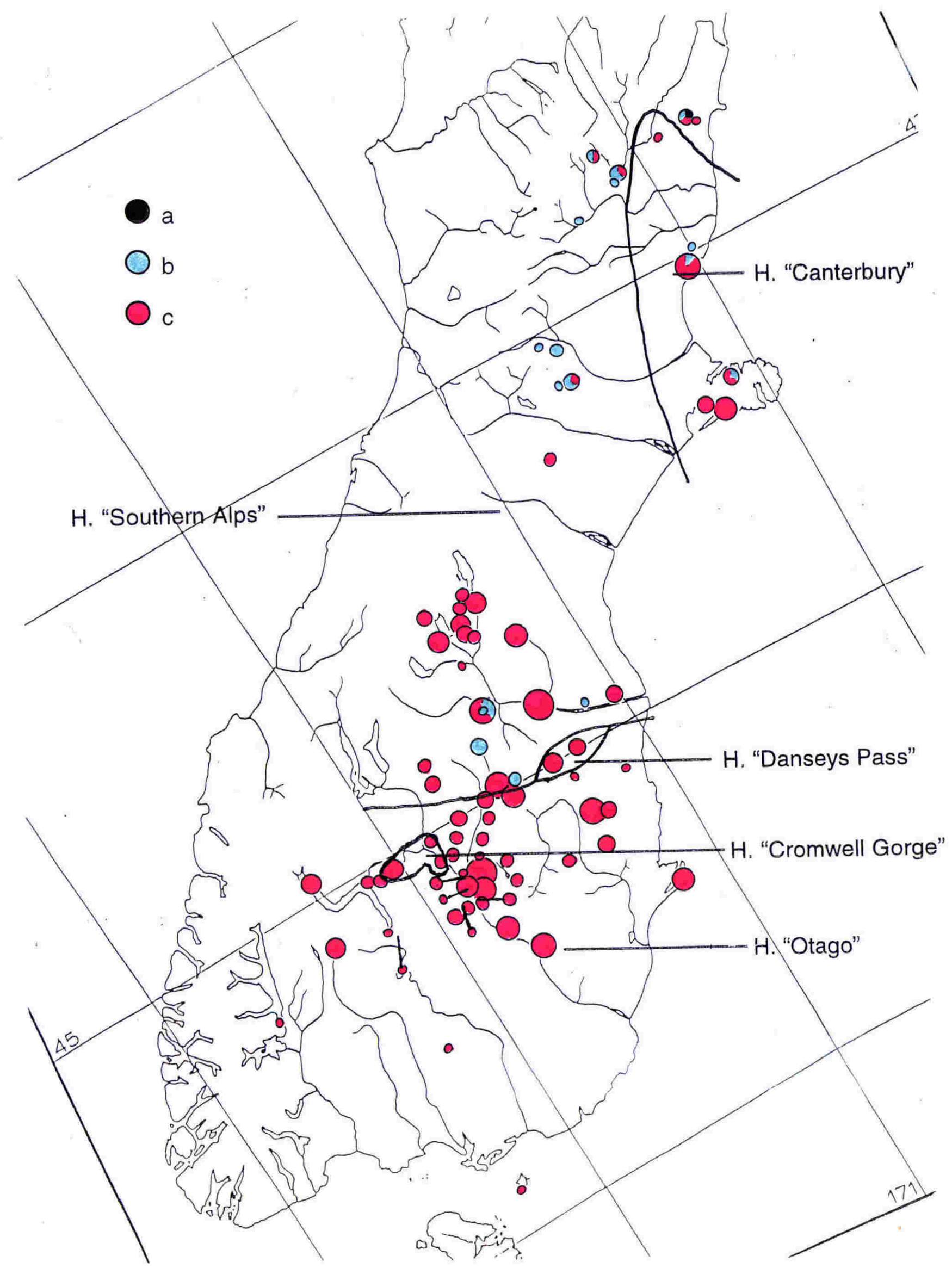

Figure 4.6.3.13 $\mathrm{Np}-1$ allele distributions among populations of the southern species group of the $H$. maculatus complex. The total area of the pie chart for each sample is directly proportional to sample size (see Table 4.6.2.1). For the Tara Hills, Omarama sample, the single individual which appeared to be genetically distinct from the rest of the sample is represented as a small circle in the centre of the larger circle representing the remainder of the sample. 


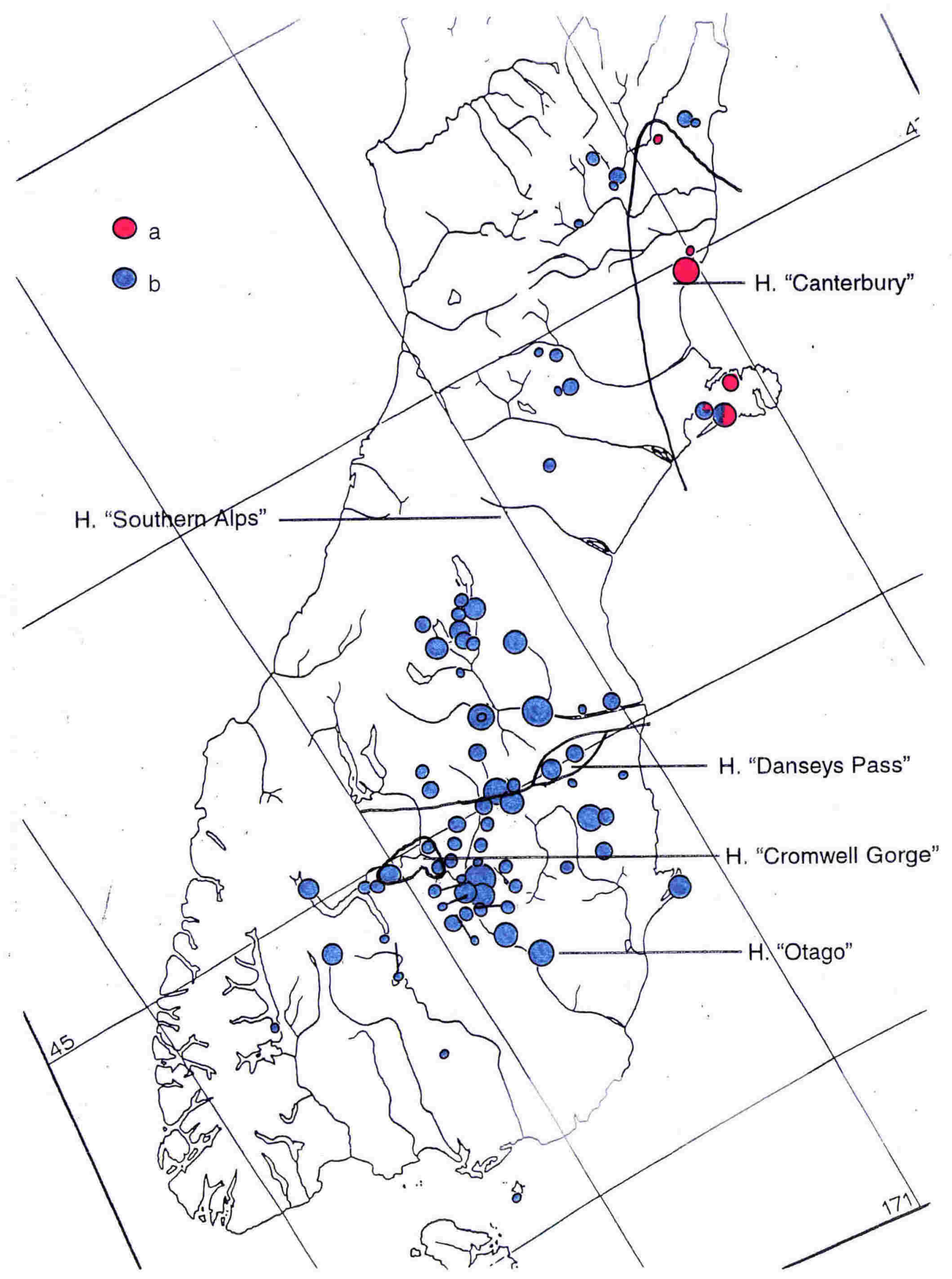

Figure 4.6.3.14 Pep-3 allele distributions among populations of the southern species group of the $H$. maculatus complex. The total area of the pie chart for each sample is directly proportional to sample size (see Table 4.6.2.1). For the Tara Hills, Omarama sample, the single individual which appeared to be genetically distinct from the rest of the sample is represented as a small circle in the centre of the 


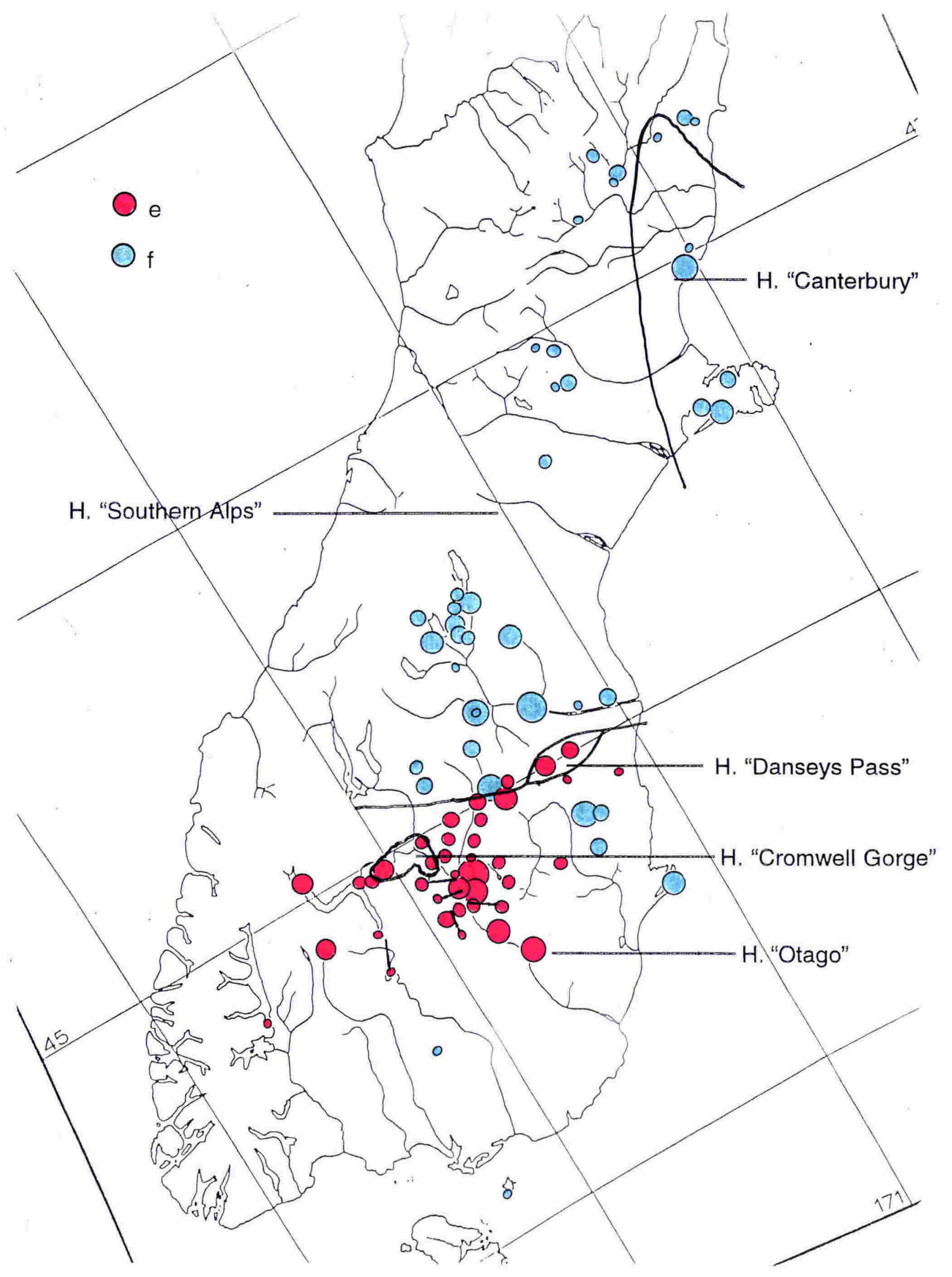

Figure 4.6.3.15 $P g d-1$ allele distributions among populations of the southern species group of the $H$. maculatus complex. The total area of the pie chart for eact sample is directly proportional to sample size (see Table 4.6.2.1). For the Tara Hill Omarama sample, the single individual which appeared to be genetically distinct from the rest of the sample is represented as a small circle in the centre of the larger circle representing the remainder of the sample. 


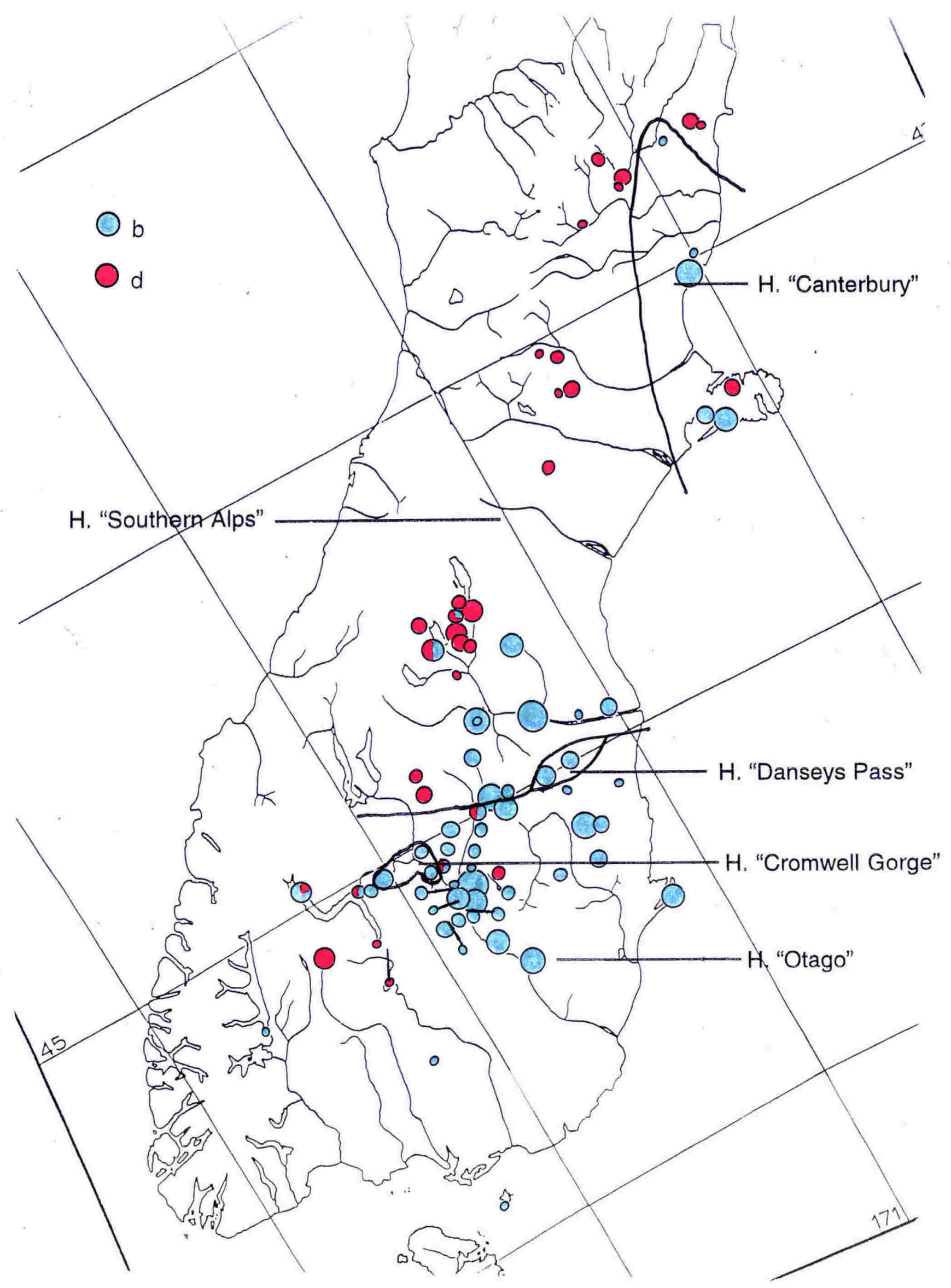

Figure 4.6.3.16 Pgm-1 allele distributions among populations of the southern species group of the $H$. maculatus complex. The total area of the pie chart for each sample is directly proportional to sample size (see Table 4.6.2.1). For the Tara Hills Omarama sample, the single individual which appeared to be genetically distinct from the rest of the sample is represented as a small circle in the centre of the larger circle representing the remainder of the sample. 


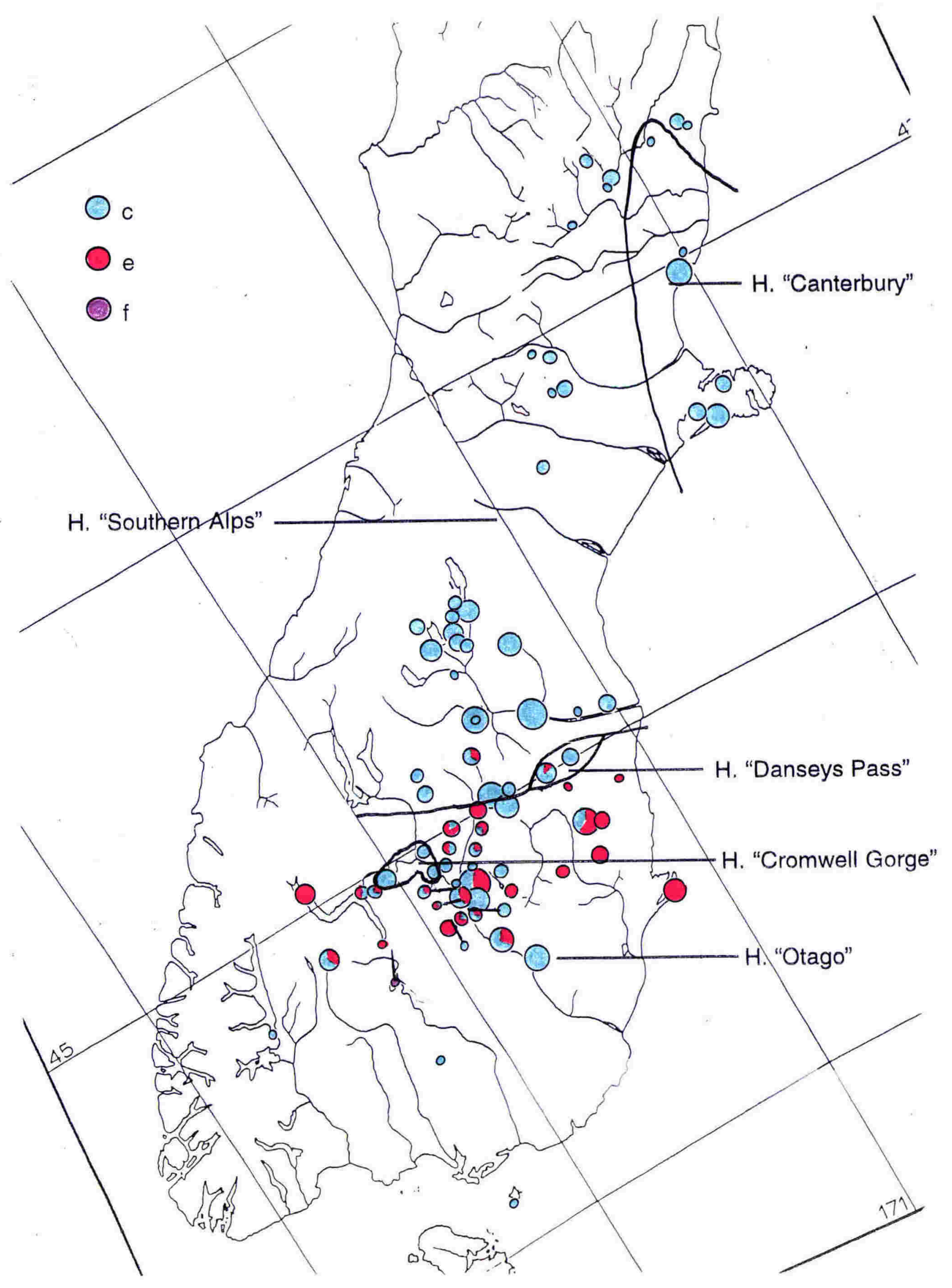

Figure 4.6.3.17 Pgm-2 allele distributions among populations of the southern species group of the $H$. maculatus complex. The total area of the pie chart for each sample is directly proportional to sample size (see Table 4.6.2.1). For the Tara Hills Omarama sample, the single individual which appeared to be genetically distinct from the rest of the sample is represented as a small circle in the centre of the larger circle representing the remainder of the sample. 


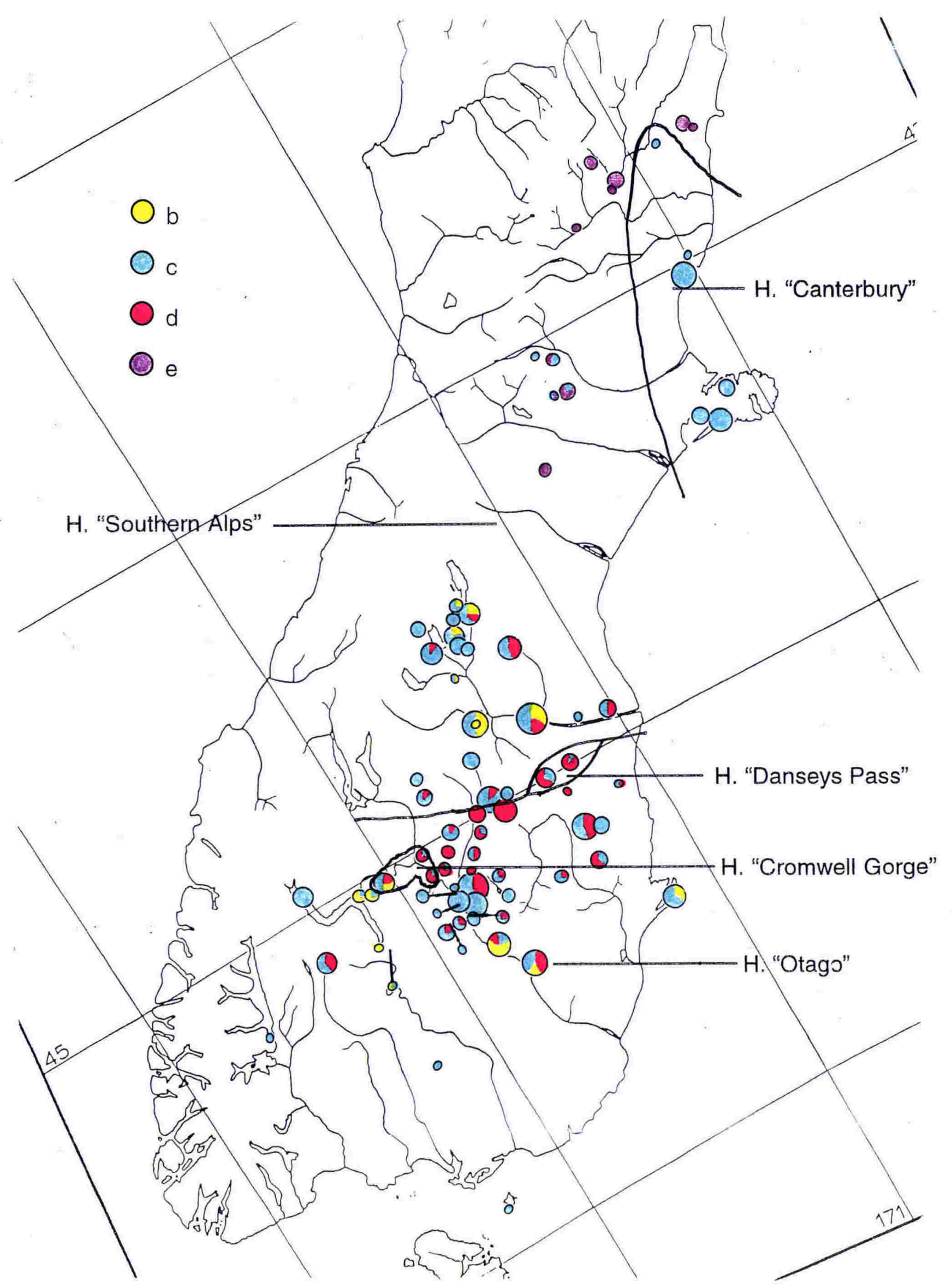

Figure 4.6.3.18 Sod-2 allele distributions among populations of the southern species group of the $H$. maculatus complex. The total area of the pie chart for each sample is directly proportional to sample size (see Table 4.6.2.1). For the Tara Hills, Omarama sample, the single individual which appeared to be genetically distinct from the rest of the sample is represented as a small circle in the centre of the larger circle representing the remainder of the sample. 
Table 4.6.3.2 measures of polymorphism and heterozygosity in populations of the southern group of the $H$. maculatus complex.

\begin{tabular}{|c|c|c|c|c|c|c|}
\hline \multirow{2}{*}{\multicolumn{2}{|c|}{ Population }} & \multirow{3}{*}{$\begin{array}{c}\begin{array}{c}\text { Mean } \\
\text { sample } \\
\text { size per } \\
\text { locus }\end{array} \\
5.0 \\
(0.0)\end{array}$} & \multirow{3}{*}{$\begin{array}{c}\begin{array}{c}\text { Mean no. } \\
\text { of alleles } \\
\text { per locus }\end{array} \\
\begin{array}{l}1.2 \\
(0.1)\end{array}\end{array}$} & \multirow{3}{*}{$\begin{array}{c}\begin{array}{c}\text { Percentage } \\
\text { of loci } \\
\text { polymorphic* }\end{array} \\
18.5\end{array}$} & \multicolumn{2}{|c|}{ Mean heterozygosity } \\
\hline & & & & & \multirow{2}{*}{$\begin{array}{l}\text { direct- } \\
\text { count } \\
0.05 \\
(0.03)\end{array}$} & \multirow{2}{*}{$\begin{array}{l}\begin{array}{l}\text { hdywbg } \\
\text { expected }\end{array} \\
\begin{array}{l}0.06 \\
(0.03)\end{array}\end{array}$} \\
\hline Hdp1 & Kyeburn Diggings & & & & & \\
\hline Hdp2 & Danseys Pass & $\begin{array}{l}4.0 \\
(0.0)\end{array}$ & $\begin{array}{l}1.1 \\
(0.1)\end{array}$ & 7.4 & $\begin{array}{l}0.01 \\
(0.01)\end{array}$ & $\begin{array}{l}0.03 \\
(0.02)\end{array}$ \\
\hline Ho1 & Horse Range & $\begin{array}{l}1.0 \\
(0.0)\end{array}$ & $\begin{array}{l}1.0 \\
(0.0)\end{array}$ & 3.7 & $\begin{array}{l}0.04 \\
(0.04)\end{array}$ & $\begin{array}{l}0.04 \\
(0.04)\end{array}$ \\
\hline $\mathrm{Ho2}$ & Mount Pisgah & $\begin{array}{l}1.0 \\
(0.0)\end{array}$ & $\begin{array}{l}1.1 \\
(0.1)\end{array}$ & 11.1 & $\begin{array}{l}0.11 \\
(0.06)\end{array}$ & $\begin{array}{l}0.11 \\
(0.06)\end{array}$ \\
\hline $\mathrm{Ho3}$ & Tree Island & $\begin{array}{l}5.0 \\
(0.0)\end{array}$ & $\begin{array}{l}1.1 \\
(0.1)\end{array}$ & 7.4 & $\begin{array}{l}0.02 \\
(0.02)\end{array}$ & $\begin{array}{l}0.02 \\
(0.02)\end{array}$ \\
\hline Ho4 & Kelvin Heights & $\begin{array}{l}2.0 \\
(0.0)\end{array}$ & $\begin{array}{l}1.1 \\
(0.1)\end{array}$ & 11.1 & $\begin{array}{l}0.09 \\
(0.05)\end{array}$ & $\begin{array}{l}0.07 \\
(0.04)\end{array}$ \\
\hline Ho5 & Remarkables & $\begin{array}{l}2.0 \\
(0.0)\end{array}$ & $\begin{array}{l}1.1 \\
(0.1)\end{array}$ & 11.1 & $\begin{array}{l}0.06 \\
(0.03)\end{array}$ & $\begin{array}{l}0.06 \\
(0.03)\end{array}$ \\
\hline Ho6 & Te Anau & $\begin{array}{l}1.0 \\
(0.0)\end{array}$ & $\begin{array}{l}1.0 \\
(0.0)\end{array}$ & 0.0 & $\begin{array}{l}0.00 \\
(0.00)\end{array}$ & $\begin{array}{l}0.00 \\
(0.00)\end{array}$ \\
\hline $\mathrm{Ho} 7$ & Nokomai & $\begin{array}{l}1.0 \\
(0.0)\end{array}$ & $\begin{array}{l}1.0 \\
(0.0)\end{array}$ & 3.7 & $\begin{array}{l}0.04 \\
(0.04)\end{array}$ & $\begin{array}{l}0.04 \\
(0.04)\end{array}$ \\
\hline Ho8 & Kingston & $\begin{array}{l}1.0 \\
(0.0)\end{array}$ & $\begin{array}{l}1.0 \\
(0.0)\end{array}$ & 0.0 & $\begin{array}{l}0.00 \\
(0.00)\end{array}$ & $\begin{array}{l}0.00 \\
(0.00)\end{array}$ \\
\hline Ho9 & Gorge Burn & $\begin{array}{l}5.0 \\
(0.0)\end{array}$ & $\begin{array}{l}1.1 \\
(0.1)\end{array}$ & 11.1 & $\begin{array}{l}0.03 \\
(0.02)\end{array}$ & $\begin{array}{l}0.04 \\
(0.03)\end{array}$ \\
\hline Ho10 & Dunstan Range 1 & $\begin{array}{l}3.0 \\
(0.0)\end{array}$ & $\begin{array}{l}1.1 \\
(0.1)\end{array}$ & 14.8 & $\begin{array}{l}0.05 \\
(0.03)\end{array}$ & $\begin{array}{l}0.06 \\
(0.03)\end{array}$ \\
\hline Ho11 & Dunstan Range 2 & $\begin{array}{l}2.0 \\
(0.0)\end{array}$ & $\begin{array}{l}1.0 \\
(0.0)\end{array}$ & 3.7 & $\begin{array}{l}0.04 \\
(0.04)\end{array}$ & $\begin{array}{l}0.03 \\
(0.03)\end{array}$ \\
\hline Ho12 & Waikerikeri & $\begin{array}{l}2.0 \\
(0.0)\end{array}$ & $\begin{array}{l}1.1 \\
(0.1)\end{array}$ & 11.1 & $\begin{array}{l}0.04 \\
(0.03)\end{array}$ & $\begin{array}{l}0.06 \\
(0.04)\end{array}$ \\
\hline $\mathrm{H} 013$ & Taieri Ridge & $\begin{array}{l}9.0 \\
(0.0)\end{array}$ & $\begin{array}{l}1.3 \\
(0.1)\end{array}$ & 22.2 & $\begin{array}{l}0.07 \\
(0.03)\end{array}$ & $\begin{array}{l}0.11 \\
(0.04)\end{array}$ \\
\hline Ho14 & Deepdell & $\begin{array}{l}3.0 \\
(0.0)\end{array}$ & $\begin{array}{l}1.1 \\
(0.1)\end{array}$ & 11.1 & $\begin{array}{l}0.04 \\
(0.02)\end{array}$ & $\begin{array}{l}0.05 \\
(0.03)\end{array}$ \\
\hline Ho15 & Billy Ridge & $\begin{array}{l}4.0 \\
(0.0)\end{array}$ & $\begin{array}{l}1.0 \\
(0.0)\end{array}$ & 3.7 & $\begin{array}{l}0.03 \\
(0.03)\end{array}$ & $\begin{array}{l}0.02 \\
(0.02)\end{array}$ \\
\hline Ho16 & Otago Peninsula & $\begin{array}{l}7.0 \\
(0.0)\end{array}$ & $\begin{array}{l}1.1 \\
(0.1)\end{array}$ & 14.8 & $\begin{array}{l}0.05 \\
(0.03)\end{array}$ & $\begin{array}{l}0.06 \\
(0.03)\end{array}$ \\
\hline Ho17 & Hokonui Hills & $\begin{array}{l}1.0 \\
(0.0)\end{array}$ & $\begin{array}{l}1.0 \\
(0.0)\end{array}$ & 0.0 & $\begin{array}{l}0.00 \\
(0.00)\end{array}$ & $\begin{array}{l}0.00 \\
(0.00)\end{array}$ \\
\hline Ho18 & Foveaux Strait & $\begin{array}{l}1.0 \\
(0.0)\end{array}$ & $\begin{array}{l}1.0 \\
(0.0)\end{array}$ & 0.0 & $\begin{array}{l}0.00 \\
(0.00)\end{array}$ & $\begin{array}{l}0.00 \\
(0.00)\end{array}$ \\
\hline
\end{tabular}


Table 4.6.3.2 (continued)

\begin{tabular}{|c|c|c|c|c|c|c|}
\hline Ho19 & Glenavon & $\begin{array}{l}2.0 \\
(0.0)\end{array}$ & $\begin{array}{l}1.1 \\
(0.1)\end{array}$ & 11.1 & $\begin{array}{l}0.07 \\
(0.04)\end{array}$ & $\begin{array}{l}0.06 \\
(0.04)\end{array}$ \\
\hline $\mathrm{Ho} 2 \mathrm{O}$ & Idaburn & $\begin{array}{l}7.0 \\
(0.0)\end{array}$ & $\begin{array}{l}1.1 \\
(0.1)\end{array}$ & 7.4 & $\begin{array}{l}0.01 \\
(0.01)\end{array}$ & $\begin{array}{l}0.02 \\
(0.02)\end{array}$ \\
\hline $\mathrm{Ho} 21$ & Manorbum Road & $\begin{array}{l}2.0 \\
(0.0)\end{array}$ & $\begin{array}{l}1.1 \\
(0.1)\end{array}$ & 11.1 & $\begin{array}{l}0.06 \\
(0.03)\end{array}$ & $\begin{array}{l}0.06 \\
(0.03)\end{array}$ \\
\hline $\mathrm{Ho} 22$ & Manorbum Dam & $\begin{array}{l}2.0 \\
(0.0)\end{array}$ & $\begin{array}{l}1.0 \\
(0.0)\end{array}$ & 3.7 & $\begin{array}{l}0.02 \\
(0.02)\end{array}$ & $\begin{array}{l}0.02 \\
(0.02)\end{array}$ \\
\hline Ho23 & Roxburgh & $\begin{array}{l}7.0 \\
(0.0)\end{array}$ & $\begin{array}{l}1.3 \\
(0.1)\end{array}$ & 22.2 & $\begin{array}{l}0.08 \\
(0.03)\end{array}$ & $\begin{array}{l}0.09 \\
(0.03)\end{array}$ \\
\hline Ho24 & Beaumont & $\begin{array}{l}8.0 \\
(0.0)\end{array}$ & $\begin{array}{l}1.2 \\
(0.1)\end{array}$ & 14.8 & $\begin{array}{l}0.08 \\
(0.04)\end{array}$ & $\begin{array}{l}0.07 \\
(0.03)\end{array}$ \\
\hline $\mathrm{Ho} 25$ & Alexandra & $\begin{array}{l}13.0 \\
(0.0)\end{array}$ & $\begin{array}{l}1.2 \\
(0.1)\end{array}$ & 18.5 & $\begin{array}{l}0.05 \\
(0.02)\end{array}$ & $\begin{array}{l}0.08 \\
(0.04)\end{array}$ \\
\hline Ho26 & Galloway & $\begin{array}{l}1.0 \\
(0.0)\end{array}$ & $\begin{array}{l}1.0 \\
(0.0)\end{array}$ & 0.0 & $\begin{array}{l}0.00 \\
(0.00)\end{array}$ & $\begin{array}{l}0.00 \\
(0.00)\end{array}$ \\
\hline $\mathrm{Ho} 27$ & South Raggedy Range & $\begin{array}{l}2.0 \\
(0.0)\end{array}$ & $\begin{array}{l}1.1 \\
(0.1)\end{array}$ & 7.4 & $\begin{array}{l}0.06 \\
(0.04)\end{array}$ & $\begin{array}{l}0.04 \\
(0.03)\end{array}$ \\
\hline $\mathrm{Ho28}$ & Becks & $\begin{array}{l}2.0 \\
(0.0)\end{array}$ & $\begin{array}{l}1.1 \\
(0.1)\end{array}$ & 14.8 & $\begin{array}{l}0.09 \\
(0.05)\end{array}$ & $\begin{array}{l}0.08 \\
(0.04)\end{array}$ \\
\hline Ho29 & North Raggedy Range & $\begin{array}{l}4.0 \\
(0.0)\end{array}$ & $\begin{array}{l}1.1 \\
(0.1)\end{array}$ & 7.4 & $\begin{array}{l}0.02 \\
(0.02)\end{array}$ & $\begin{array}{l}0.04 \\
(0.03)\end{array}$ \\
\hline Но30 & Old Man Range 4 (top) & $\begin{array}{l}3.0 \\
(0.0)\end{array}$ & $\begin{array}{l}1.1 \\
(0.1)\end{array}$ & 11.1 & $\begin{array}{l}0.01 \\
(0.01)\end{array}$ & $\begin{array}{l}0.05 \\
(0.03)\end{array}$ \\
\hline Ho31 & Old Man Range 3 & $\begin{array}{l}1.0 \\
(0.0)\end{array}$ & $\begin{array}{l}1.0 \\
(0.0)\end{array}$ & 3.7 & $\begin{array}{l}0.04 \\
(0.04)\end{array}$ & $\begin{array}{l}0.04 \\
(0.04)\end{array}$ \\
\hline Ho32 & Old Man Range 2 & $\begin{array}{l}2.0 \\
(0.0)\end{array}$ & $\begin{array}{l}1.1 \\
(0.1)\end{array}$ & 7.4 & $\begin{array}{l}0.04 \\
(0.03)\end{array}$ & $\begin{array}{l}0.04 \\
(0.03)\end{array}$ \\
\hline Ho33 & Old Man Range (bottom) & $\begin{array}{l}2.0 \\
(0.0)\end{array}$ & $\begin{array}{l}1.1 \\
(0.1)\end{array}$ & 7.4 & $\begin{array}{l}0.04 \\
(0.03)\end{array}$ & $\begin{array}{l}0.04 \\
(0.03)\end{array}$ \\
\hline Ho34 & Butchers Gully & $\begin{array}{l}2.0 \\
(0.0)\end{array}$ & $\begin{array}{l}1.1 \\
(0.1)\end{array}$ & 11.1 & $\begin{array}{l}0.07 \\
(0.04)\end{array}$ & $\begin{array}{l}0.06 \\
(0.04)\end{array}$ \\
\hline Ho35 & Butchers Dam & $\begin{array}{l}8.0 \\
(0.0)\end{array}$ & $\begin{array}{l}1.2 \\
(0.1)\end{array}$ & 22.2 & $\begin{array}{l}0.09 \\
(0.03)\end{array}$ & $\begin{array}{l}0.08 \\
(0.03)\end{array}$ \\
\hline Ho36 & Alexandra (3 km E) & $\begin{array}{l}1.0 \\
(0.0)\end{array}$ & $\begin{array}{l}1.1 \\
(0.1)\end{array}$ & 7.4 & $\begin{array}{l}0.07 \\
(0.05)\end{array}$ & $\begin{array}{l}0.07 \\
(0.05)\end{array}$ \\
\hline Ho37 & Alexandra (SE) & $\begin{array}{l}5.9 \\
(0.1)\end{array}$ & $\begin{array}{l}1.2 \\
(0.1)\end{array}$ & 22.2 & $\begin{array}{l}0.07 \\
(0.03)\end{array}$ & $\begin{array}{l}0.08 \\
(0.03)\end{array}$ \\
\hline Ho38 & Aranui Dam (Alexandra) & $\begin{array}{l}2.0 \\
(0.0)\end{array}$ & $\begin{array}{l}1.1 \\
(0.1)\end{array}$ & 11.1 & $\begin{array}{l}0.06 \\
(0.03)\end{array}$ & $\begin{array}{l}0.06 \\
(0.03)\end{array}$ \\
\hline Но39 & Alexandra West & $\begin{array}{l}1.0 \\
(0.0)\end{array}$ & $\begin{array}{l}1.0 \\
(0.0)\end{array}$ & 3.7 & $\begin{array}{l}0.04 \\
(0.04)\end{array}$ & $\begin{array}{l}0.04 \\
(0.04)\end{array}$ \\
\hline $\mathrm{Hcg} 1$ & Nevis Bluff & $\begin{array}{l}5.0 \\
(0.0)\end{array}$ & $\begin{array}{l}1.3 \\
(0.1)\end{array}$ & 18.5 & $\begin{array}{l}0.07 \\
(0.04)\end{array}$ & $\begin{array}{l}0.10 \\
(0.04)\end{array}$ \\
\hline
\end{tabular}


Table 4.6.3.2 (continued)

\begin{tabular}{|c|c|c|c|c|c|c|}
\hline $\mathrm{Hcg} 2$ & Cromwell Gorge & $\begin{array}{l}2.0 \\
(0.0)\end{array}$ & $\begin{array}{l}1.1 \\
(0.1)\end{array}$ & 11.1 & $\begin{array}{l}0.07 \\
(0.04)\end{array}$ & $\begin{array}{l}0.06 \\
(0.04)\end{array}$ \\
\hline $\mathrm{Hcg} 3$ & Cromwell $\mathrm{E}$ of River & $\begin{array}{l}2.0 \\
(0.0)\end{array}$ & $\begin{array}{l}1.1 \\
(0.1)\end{array}$ & 11.1 & $\begin{array}{l}0.06 \\
(0.04)\end{array}$ & $\begin{array}{l}0.07 \\
(0.04)\end{array}$ \\
\hline Hsa1 & Lindis Pass 1 & $\begin{array}{l}2.0 \\
(0.0)\end{array}$ & $\begin{array}{l}1.0 \\
(0.0)\end{array}$ & 3.7 & $\begin{array}{l}0.02 \\
(0.02)\end{array}$ & $\begin{array}{l}0.02 \\
(0.02)\end{array}$ \\
\hline $\mathrm{Hsa} 2$ & Lindis Pass 2 & $\begin{array}{l}3.0 \\
(0.0)\end{array}$ & $\begin{array}{l}1.1 \\
(0.1)\end{array}$ & 11.1 & $\begin{array}{l}0.03 \\
(0.02)\end{array}$ & $\begin{array}{l}0.05 \\
(0.03)\end{array}$ \\
\hline \multirow[t]{2}{*}{ Hsa3 } & Manuherikia River & $\begin{array}{l}4.0 \\
(0.0)\end{array}$ & $\begin{array}{l}1.1 \\
(0.1)\end{array}$ & 11.1 & $\begin{array}{l}0.02 \\
(0.01)\end{array}$ & $\begin{array}{l}0.05 \\
(0.03)\end{array}$ \\
\hline & Omarama (combined) & $\begin{array}{l}9.0 \\
(0.0)\end{array}$ & $\begin{array}{l}1.3 \\
(0.1)\end{array}$ & 25.9 & $\begin{array}{l}0.04 \\
(0.03)\end{array}$ & $\begin{array}{l}0.08 \\
(0.03)\end{array}$ \\
\hline Hsa4 & Omarama 1 & $\begin{array}{l}8.0 \\
(0.0)\end{array}$ & $\begin{array}{l}1.2 \\
(0.1)\end{array}$ & 14.8 & $\begin{array}{l}0.04 \\
(0.03)\end{array}$ & $\begin{array}{l}0.06 \\
(0.03)\end{array}$ \\
\hline Hsa5 & Omarama 2 & $\begin{array}{l}1.0 \\
(0.0)\end{array}$ & $\begin{array}{l}1.0 \\
(0.0)\end{array}$ & 0.0 & $\begin{array}{l}0.00 \\
(0.00)\end{array}$ & $\begin{array}{l}0.00 \\
(0.00)\end{array}$ \\
\hline Hsa6 & Falls Dam & $\begin{array}{l}9.0 \\
(0.0)\end{array}$ & $\begin{array}{l}1.2 \\
(0.1)\end{array}$ & 18.5 & $\begin{array}{l}0.05 \\
(0.02)\end{array}$ & $\begin{array}{l}0.06 \\
(0.03)\end{array}$ \\
\hline Hsa7 & Little Mount Ida & $\begin{array}{l}2.0 \\
(0.0)\end{array}$ & $\begin{array}{l}1.0 \\
(0.0)\end{array}$ & 3.7 & $\begin{array}{l}0.02 \\
(0.02)\end{array}$ & $\begin{array}{l}0.02 \\
(0.02)\end{array}$ \\
\hline Hsa8 & Kurow & $\begin{array}{l}11.0 \\
(0.0)\end{array}$ & $\begin{array}{l}1.1 \\
(0.1)\end{array}$ & 7.4 & $\begin{array}{l}0.02 \\
(0.02)\end{array}$ & $\begin{array}{l}0.03 \\
(0.02)\end{array}$ \\
\hline Hsa9 & Waitaki River & $\begin{array}{l}1.0 \\
(0.0)\end{array}$ & $\begin{array}{l}1.0 \\
(0.0)\end{array}$ & 0.0 & $\begin{array}{l}0.00 \\
(0.00)\end{array}$ & $\begin{array}{l}0.00 \\
(0.00)\end{array}$ \\
\hline Hsa10 & Waimate & $\begin{array}{l}4.0 \\
(0.0)\end{array}$ & $\begin{array}{l}1.1 \\
(0.1)\end{array}$ & 7.4 & $\begin{array}{l}0.03 \\
(0.02)\end{array}$ & $\begin{array}{l}0.03 \\
(0.02)\end{array}$ \\
\hline Hsa11 & Hakataramea Pass & $\begin{array}{l}7.0 \\
(0.0)\end{array}$ & $\begin{array}{l}1.1 \\
(0.1)\end{array}$ & 14.8 & $\begin{array}{l}0.06 \\
(0.03)\end{array}$ & $\begin{array}{l}0.07 \\
(0.03)\end{array}$ \\
\hline Hsa12 & Tekapo & $\begin{array}{l}6.0 \\
(0.0)\end{array}$ & $\begin{array}{l}1.2 \\
(0.1)\end{array}$ & 14.8 & $\begin{array}{l}0.04 \\
(0.03)\end{array}$ & $\begin{array}{l}0.08 \\
(0.04)\end{array}$ \\
\hline Hsa13 & Mount Mary & $\begin{array}{l}4.0 \\
(0.0)\end{array}$ & $\begin{array}{l}1.2 \\
(0.1)\end{array}$ & 14.8 & $\begin{array}{l}0.02 \\
(0.01)\end{array}$ & $\begin{array}{l}0.08 \\
(0.04)\end{array}$ \\
\hline Hsa14 & Mount John Station & $\begin{array}{l}2.0 \\
(0.0)\end{array}$ & $\begin{array}{l}1.1 \\
(0.1)\end{array}$ & 11.1 & $\begin{array}{l}0.06 \\
(0.03)\end{array}$ & $\begin{array}{l}0.06 \\
(0.03)\end{array}$ \\
\hline Hsa15 & Pukaki & $\begin{array}{l}5.0 \\
(0.0)\end{array}$ & $\begin{array}{l}1.1 \\
(0.1)\end{array}$ & 11.1 & $\begin{array}{l}0.03 \\
(0.02)\end{array}$ & $\begin{array}{l}0.04 \\
(0.02)\end{array}$ \\
\hline Hsa16 & Pukaki Downs & $\begin{array}{l}6.0 \\
(0.0)\end{array}$ & $\begin{array}{l}1.2 \\
(0.1)\end{array}$ & 18.5 & $\begin{array}{l}0.04 \\
(0.03)\end{array}$ & $\begin{array}{l}0.07 \\
(0.03)\end{array}$ \\
\hline Hsa17 & Simons Hill & $\begin{array}{l}2.0 \\
(0.0)\end{array}$ & $\begin{array}{l}1.0 \\
(0.0)\end{array}$ & 3.7 & $\begin{array}{l}0.04 \\
(0.04)\end{array}$ & $\begin{array}{l}0.03 \\
(0.03)\end{array}$ \\
\hline Hsa18 & Balmoral Station & $\begin{array}{l}2.0 \\
(0.0)\end{array}$ & $\begin{array}{l}1.1 \\
(0.1)\end{array}$ & 14.8 & $\begin{array}{l}0.06 \\
(0.03)\end{array}$ & $\begin{array}{l}0.08 \\
(0.04)\end{array}$ \\
\hline Hsa19 & Bush Stream Mount Cook & $\begin{array}{l}3.0 \\
(0.0)\end{array}$ & $\begin{array}{l}1.0 \\
(0.0)\end{array}$ & 3.7 & $\begin{array}{l}0.01 \\
(0.01)\end{array}$ & $\begin{array}{l}0.01 \\
(0.01)\end{array}$ \\
\hline
\end{tabular}


Table 4.6.3.2 (continued)

\begin{tabular}{|c|c|c|c|c|c|c|}
\hline $\mathrm{Hsa2O}$ & Ohau River & $\begin{array}{l}1.0 \\
(0.0)\end{array}$ & $\begin{array}{l}1.1 \\
(0.1)\end{array}$ & 7.4 & $\begin{array}{l}0.07 \\
(0.05)\end{array}$ & $\begin{array}{l}0.07 \\
(0.05)\end{array}$ \\
\hline Hsa21 & Mount Somers & $\begin{array}{l}2.0 \\
(0.0)\end{array}$ & $\begin{array}{l}1.0 \\
(0.0)\end{array}$ & 0.0 & $\begin{array}{l}0.00 \\
(0.00)\end{array}$ & $\begin{array}{l}0.00 \\
(0.00)\end{array}$ \\
\hline Hsa22 & Kowhai River & $\begin{array}{l}3.0 \\
(0.0)\end{array}$ & $\begin{array}{l}1.1 \\
(0.1)\end{array}$ & 7.4 & $\begin{array}{l}0.01 \\
(0.01)\end{array}$ & $\begin{array}{l}0.03 \\
(0.02)\end{array}$ \\
\hline Hsa23 & Lake Lyndon & $\begin{array}{l}1.0 \\
(0.0)\end{array}$ & $\begin{array}{l}1.0 \\
(0.0)\end{array}$ & 3.7 & $\begin{array}{l}0.04 \\
(0.04)\end{array}$ & $\begin{array}{l}0.04 \\
(0.04)\end{array}$ \\
\hline Hsa24 & Arthur's Pass & $\begin{array}{l}1.0 \\
(0.0)\end{array}$ & $\begin{array}{l}1.0 \\
(0.0)\end{array}$ & 0.0 & $\begin{array}{l}0.00 \\
(0.00)\end{array}$ & $\begin{array}{l}0.00 \\
(0.00)\end{array}$ \\
\hline Hsa25 & Craigiebum & $\begin{array}{l}2.0 \\
(0.0)\end{array}$ & $\begin{array}{l}1.1 \\
(0.1)\end{array}$ & 7.4 & $\begin{array}{l}0.00 \\
(0.00)\end{array}$ & $\begin{array}{l}0.05 \\
(0.03)\end{array}$ \\
\hline Hsa26 & The Poplars & $\begin{array}{l}1.0 \\
(0.0)\end{array}$ & $\begin{array}{l}1.0 \\
(0.0)\end{array}$ & 0.0 & $\begin{array}{l}0.00 \\
(0.00)\end{array}$ & $\begin{array}{l}0.00 \\
(0.00)\end{array}$ \\
\hline Hsa27 & Jack's Pass & $\begin{array}{l}1.0 \\
(0.0)\end{array}$ & $\begin{array}{l}1.0 \\
(0.0)\end{array}$ & 3.7 & $\begin{array}{l}0.04 \\
(0.04)\end{array}$ & $\begin{array}{l}0.04 \\
(0.04)\end{array}$ \\
\hline Hsa28 & Rainbow Road 1 & $\begin{array}{l}3.0 \\
(0.0)\end{array}$ & $\begin{array}{l}1.1 \\
(0.1)\end{array}$ & 7.4 & $\begin{array}{l}0.03 \\
(0.03)\end{array}$ & $\begin{array}{l}0.04 \\
(0.03)\end{array}$ \\
\hline Hsa29 & Rainbow Road 2 & $\begin{array}{l}2.0 \\
(0.0)\end{array}$ & $\begin{array}{l}1.0 \\
(0.0)\end{array}$ & 3.7 & $\begin{array}{l}0.00 \\
(0.00)\end{array}$ & $\begin{array}{l}0.03 \\
(0.03)\end{array}$ \\
\hline Hsa30 & Kahutara Saddle road & $\begin{array}{l}1.0 \\
(0.0)\end{array}$ & $\begin{array}{l}1.0 \\
(0.0)\end{array}$ & 0.0 & $\begin{array}{l}0.00 \\
(0.00)\end{array}$ & $\begin{array}{l}0.00 \\
(0.00)\end{array}$ \\
\hline Hsa31 & Kahutara Saddle & $\begin{array}{l}3.0 \\
(0.0)\end{array}$ & $\begin{array}{l}1.1 \\
(0.1)\end{array}$ & 3.7 & $\begin{array}{l}0.00 \\
(0.00)\end{array}$ & $\begin{array}{l}0.03 \\
(0.03)\end{array}$ \\
\hline $\mathrm{Hb} 1$ & Cloudy Hut & $\begin{array}{l}1.0 \\
(0.0)\end{array}$ & $\begin{array}{l}1.0 \\
(0.0)\end{array}$ & 0.0 & $\begin{array}{l}0.00 \\
(0.00)\end{array}$ & $\begin{array}{l}0.00 \\
(0.00)\end{array}$ \\
\hline $\mathrm{Hb} 2$ & Motunau & $\begin{array}{l}1.0 \\
(0.0)\end{array}$ & $\begin{array}{l}1.0 \\
(0.0)\end{array}$ & 0.0 & $\begin{array}{l}0.00 \\
(0.00)\end{array}$ & $\begin{array}{l}0.00 \\
(0.00)\end{array}$ \\
\hline $\mathrm{Hb} 3$ & Waipara River & $\begin{array}{l}9.0 \\
(0.0)\end{array}$ & $\begin{array}{l}1.0 \\
(0.0)\end{array}$ & 3.7 & $\begin{array}{l}0.00 \\
(0.00)\end{array}$ & $\begin{array}{l}0.01 \\
(0.01)\end{array}$ \\
\hline $\mathrm{Hb} 4$ & Birdlings Flat & $\begin{array}{l}7.0 \\
(0.0)\end{array}$ & $\begin{array}{l}1.2 \\
(0.1)\end{array}$ & 18.5 & $\begin{array}{l}0.10 \\
(0.05)\end{array}$ & $\begin{array}{l}0.08 \\
(0.03)\end{array}$ \\
\hline Hb5 & Banks Peninsula foothills & $\begin{array}{l}4.0 \\
(0.0)\end{array}$ & $\begin{array}{l}1.1 \\
(0.1)\end{array}$ & 11.1 & $\begin{array}{l}0.04 \\
(0.03)\end{array}$ & $\begin{array}{l}0.05 \\
(0.03)\end{array}$ \\
\hline $\mathrm{Hb} 6$ & Banks Peninsula & $\begin{array}{l}3.0 \\
(0.0)\end{array}$ & $\begin{array}{l}1.1 \\
(0.1)\end{array}$ & 11.1 & $\begin{array}{l}0.04 \\
(0.03)\end{array}$ & $\begin{array}{l}0.05 \\
(0.03)\end{array}$ \\
\hline Hd1 & North Brother Island & $\begin{array}{l}6.0 \\
(0.0)\end{array}$ & $\begin{array}{l}1.1 \\
(0.1)\end{array}$ & 7.4 & $\begin{array}{l}0.01 \\
(0.01)\end{array}$ & $\begin{array}{l}0.01 \\
(0.01)\end{array}$ \\
\hline $\mathrm{Hd} 2$ & South Trio Island & $\begin{array}{l}2.0 \\
(0.0)\end{array}$ & $\begin{array}{l}1.0 \\
(0.0)\end{array}$ & 3.7 & $\begin{array}{l}0.00 \\
(0.00)\end{array}$ & $\begin{array}{l}0.03 \\
(0.03)\end{array}$ \\
\hline $\mathrm{Hd} 3$ & North Trio Island & $\begin{array}{l}2.0 \\
(0.0)\end{array}$ & $\begin{array}{l}1.0 \\
(0.0)\end{array}$ & 0.0 & $\begin{array}{l}0.00 \\
(0.00)\end{array}$ & $\begin{array}{l}0.00 \\
(0.00)\end{array}$ \\
\hline $\mathrm{Hd} 4$ & Middle Trio Island & $\begin{array}{l}2.0 \\
(0.0)\end{array}$ & $\begin{array}{l}1.0 \\
(0.0)\end{array}$ & 0.0 & $\begin{array}{l}0.00 \\
(0.00)\end{array}$ & $\begin{array}{l}0.00 \\
(0.00)\end{array}$ \\
\hline Hd5 & Poor Knights Islands & 2.0 & 1.0 & 0.0 & 0.00 & 0.00 \\
\hline
\end{tabular}




$\begin{array}{lllllll} & & (0.0) & -(0.0) & & (0.00) & (0.00) \\ \text { Hd6 } & \text { Hen Island } & 2.0 & 1.0 & 0.0 & 0.00 & 0.00 \\ & & (0.0) & (0.0) & & (0.00) & (0.00) \\ \text { Hd7 } & \text { Coppermine Island } & 2.0 & 1.0 & 0.0 & 0.00 & 0.00 \\ & & (0.0) & (0.0) & & (0.00) & (0.00) \\ \text { Hd8 } & \text { Raumahuaiti Island } & 1.0 & 1.0 & 3.7 & 0.04 & 0.04 \\ & & (0.0) & (0.0) & & (0.04) & (0.04) \\ \text { Hd9 } & \text { Raumahuanui Island } & 2.0 & 1.0 & 3.7 & 0.02 & 0.02 \\ & & (0.0) & (0.0) & & (0.02) & (0.02) \\ \text { Hd10 } & \text { Hongiora Island } & 2.0 & 1.0 & 3.7 & 0.02 & 0.02 \\ & & (0.0) & (0.0) & & (0.02) & (0.02) \\ \text { Hd11 } & \text { Hemia Island } & 2.0 & 1.0 & 0.0 & 0.00 & 0.00 \\ & & (0.0) & (0.0) & & (0.00) & (0.00) \\ \text { Hd12 } & \text { Middle Chain Island } & 1.0 & 1.0 & 0.0 & 0.00 & 0.00 \\ & & (0.0) & (0.0) & & (0.00) & (0.00) \\ \text { Hd13 } & \text { near Coromandel Pen. } & 1.0 & 1.0 & 0.0 & 0.00 & 0.00 \\ & & (0.0) & (0.0) & & (0.00) & (0.00) \\ \text { Hd14 } & \text { Stanley Island } & 1.0 & 1.1 & 7.4 & 0.07 & 0.07 \\ & & (0.0) & (0.1) & & (0.05) & (0.05) \\ \text { Hd15 } & \text { Middle Island } & 2.0 & 1.1 & 7.4 & 0.04 & 0.04 \\ & & (0.0) & (0.1) & & (0.03) & (0.03) \\ \text { Hd16 } & \text { Green Island } & 2.0 & 1.1 & 11.1 & 0.06 & 0.07 \\ & & (0.0) & (0.1) & & (0.04) & (0.04) \\ \text { Hd17 } & \text { Korapuki Island } & 2.0 & 1.0 & 3.7 & 0.02 & 0.02 \\ & & (0.0) & (0.0) & & (0.02) & (0.02) \\ & & & & & & \end{array}$

In preliminary clustering analyses, populations from Canterbury and OtagoSouthland always each formed monophyletic groups. The smallest pairwise genetic distance between populations of these groups was $D=0.13$ (between Te Anau $H$. "Otago" and Falls Dam, Kurow, and Waimate $H$. "Southern Alps"), and pairwise genetic distances between geographically close populations across the geographic boundary were higher $(D>0.15$; most $>0.2$ ). These groups were therefore analysed separately to reduce the sizes of phenograms.

Trees generated from allozyme data by WPGMA (Figures 4.6.3.19-20) showed discrete geographical clusters of genetically related populations. However, examination of the distance matrices from which these trees were generated revealed that genetic distances were continuously distributed, with no clear separation of between-species and within-species or 


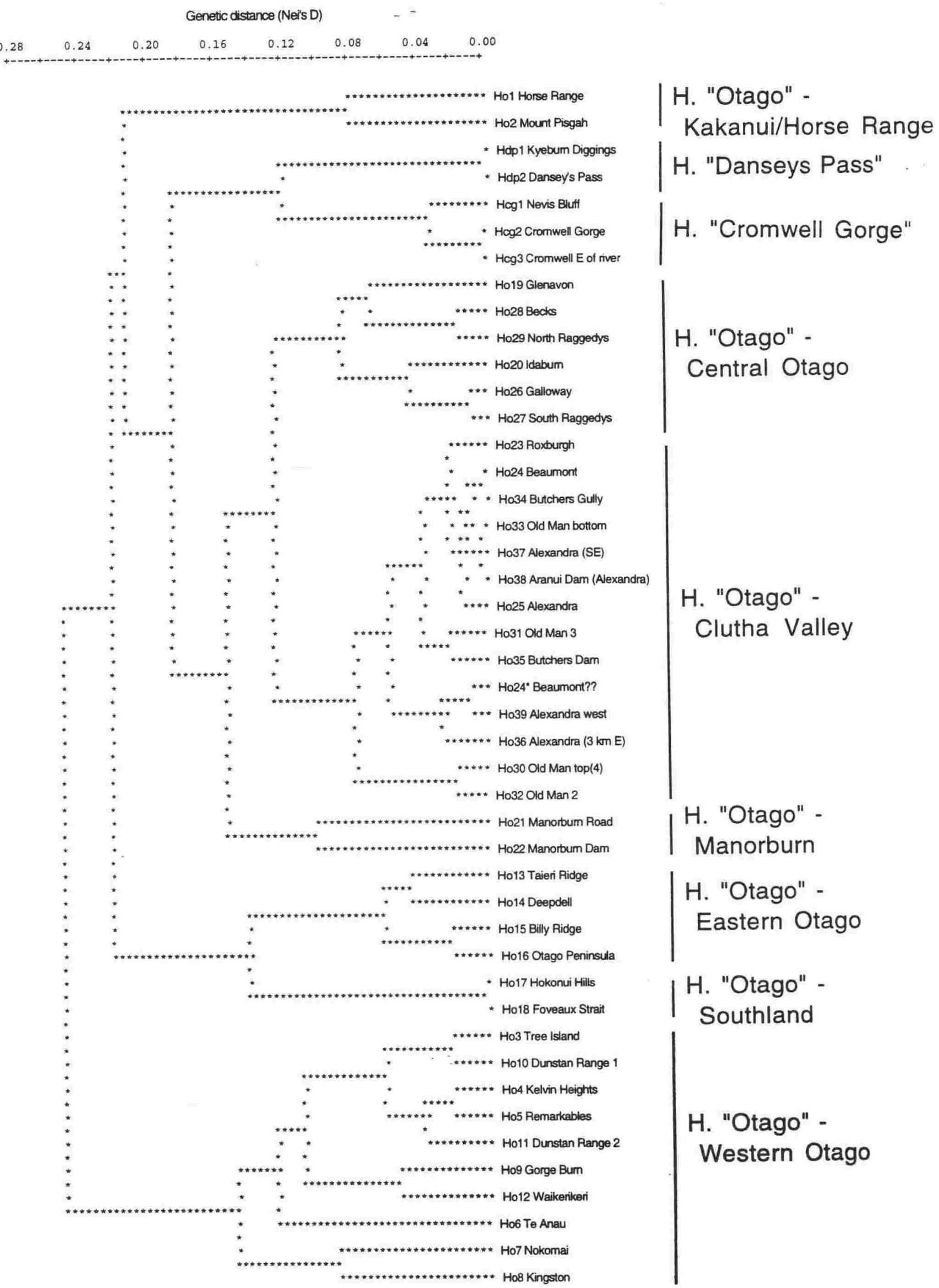

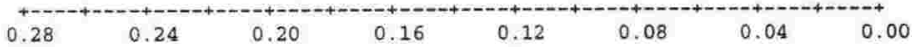

Figure 4.6.3.19 WPGMA tree of Otago-Southland, $H$. maculatus-complex populations, generated from allozyme data by the BIOSYS package. 


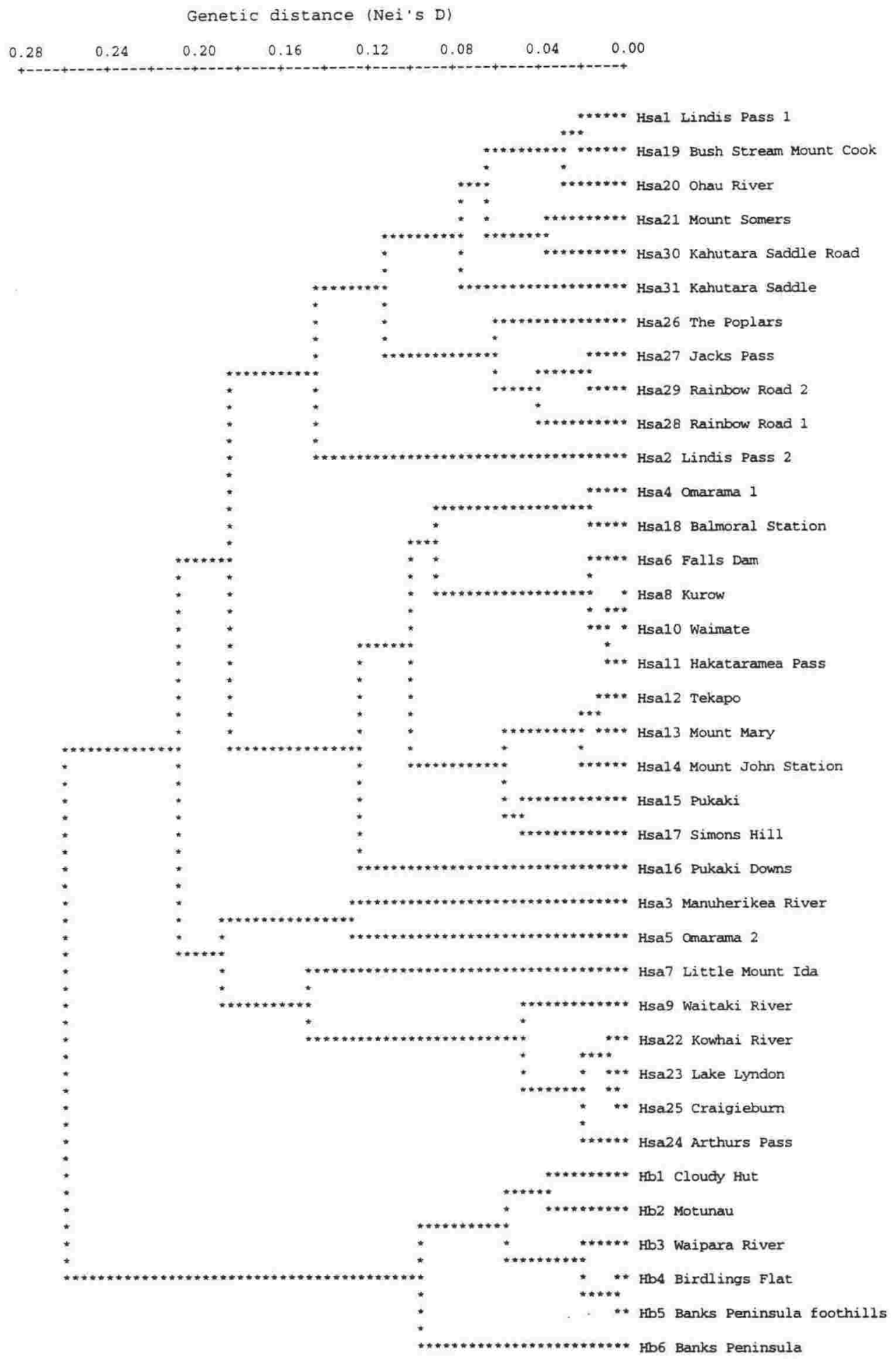

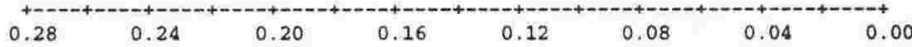

Figure 4.6.3.20 WPGMA tree of Canterbury H. maculatus-complex populations, generated from allozyme data by the BIOSYS package. 
within-group genetic distances (Nei's D, Tables 4.6.3.3 \& 4.6.3.4 for OtagoSouthland and Canterbury populations respectively, summarised in Table 4.6.3.5). In particular, $H$. "Otago" and $H$. "Southern Alps" include some unusually large intraspecific distances (Table 4.6.3.5).

However, distance Wagner (Figures 4.6.3.21-22) (BIOSYS package), and neighbour joining (Figures 4.6.3.23-24) (MEGA package) procedures, generated using Cavalli-Sforza \& Edwards' arc distance (Tables 4.6.3.6 \& 4.6.3.7), showed less clear structure, indicating that the clusters in the WPGMA phenogram may not be monophyletic clades.

In scatterplots of pairwise genetic and geographic distances, discontinuity between within-group and between-group genetic distances was most apparent at about Nei's $D=0.08$, similar to the genetic distance between the most closely related sympatric species in this study (see following sections).

\subsubsection{H. "Danseys Pass"}

The 2 genetically indistinguishable populations of $H$. "Danseys Pass" (9 km apart) differ from all neighbouring populations sampled (20-60 km away) by Nei's $D>0.1$ (Figure 4.6.3.1.1). They are roughly equidistant from the Kakanui/Horse Range and most Central Otago populations of $H$. "Otago" ( $D=0.1-0.2)$, with larger genetic distances to eastern Otago and Manorburn populations of $H$. "Otago" ( $D=0.2-0.3)$.

\subsubsection{H. "Cromwell Gorge"}

$H$. "Cromwell Gorge" is found very close to western populations of $H$. "Otago", with continuous habitat between them. They are always separated by $D>0.1$ (Figure 4.6.3.2.1), regardless of geographic proximity, indicating absence of gene flow. Similarly, pairwise genetic distances of $\mathrm{D} \geq 0.08$ between $H$. "Cromwell Gorge" and Clutha Valley and Central Otago populations of $H$. "Otago", with no effect of geographic proximity within this range of distances (Figure 4.6.3.2.2-3) suggest an absence of gene flow.

\subsubsection{H. "Otago"}

As discussed above (sections 4.6.3, 4.6.3.1, \& 4.6.3.2), the distributions of $H$. "Southern Alps" and $H$. "Danseys Pass" meet that of $H$. "Otago" at abrupt parapatric boundaries, and the range of $H$. "Otago" overlaps that of $H$. "Cromwell Gorge". 
MNN I

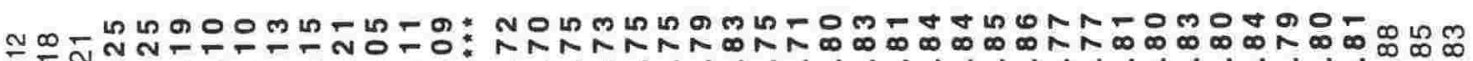

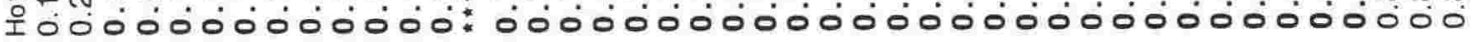
=

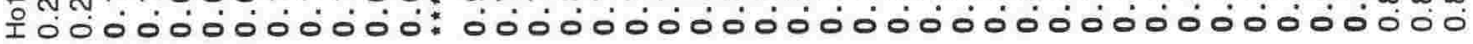
은

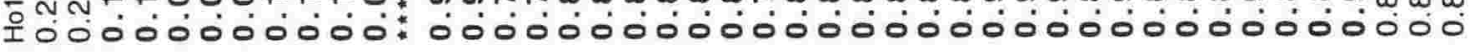

N I m $⿻$ 의

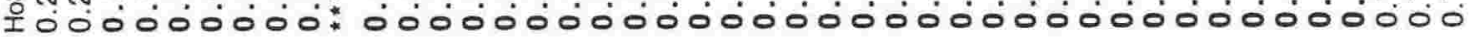

ON ó

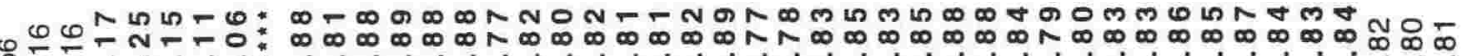

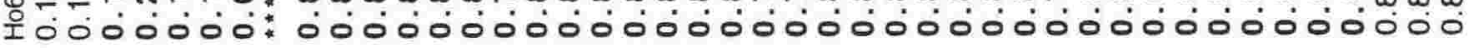

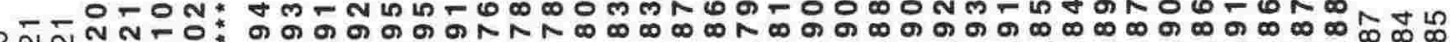
I

운

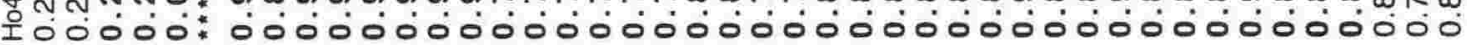

mo* mL I

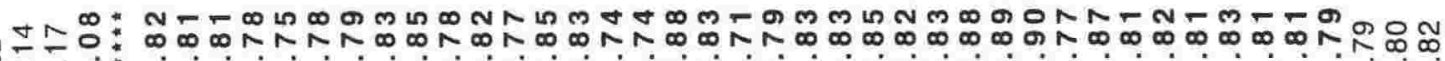

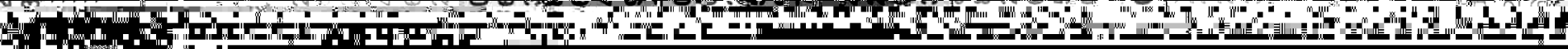
H.

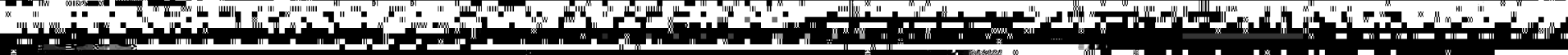
, (1)

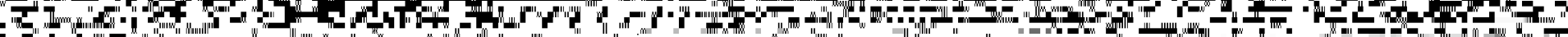

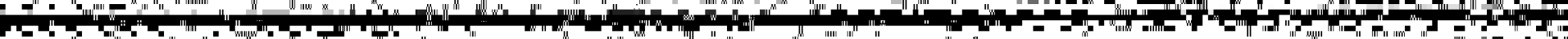

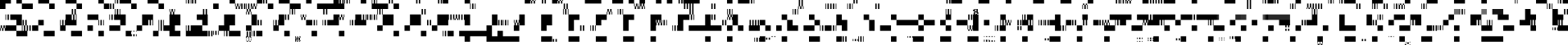

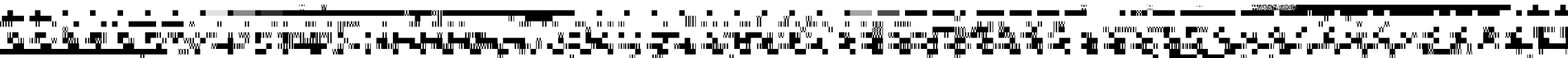
H th r r

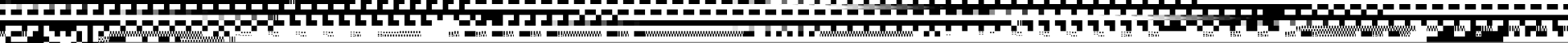


슴유.

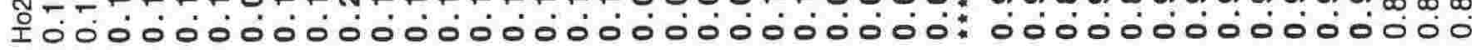
Mำกำ

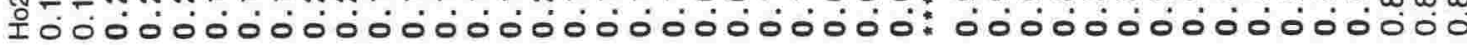

LNA

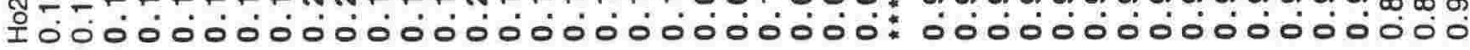
IN

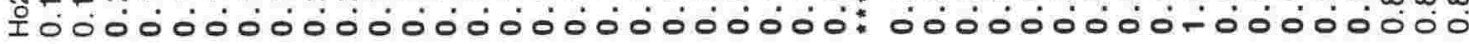

픈ㄷㄴ

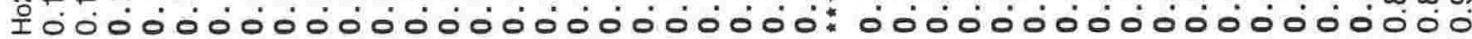
는

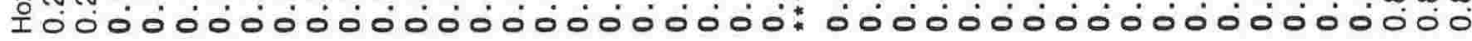
$\bar{N}$ N $N$ m

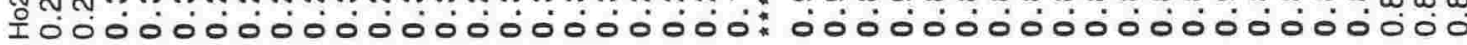
몬ㅁำ Í

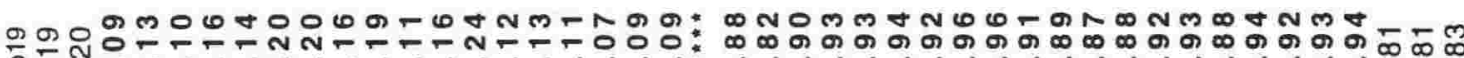
全 m N $\bar{N} N$ m

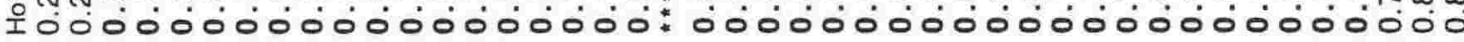
든

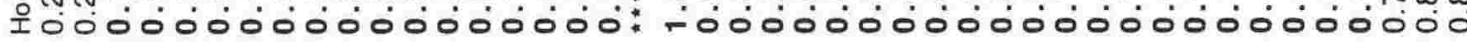

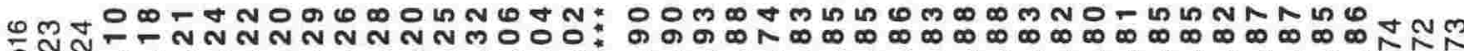

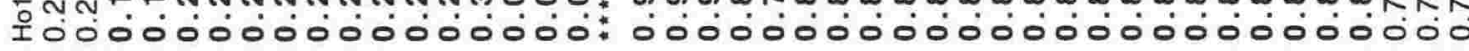
nᄂ

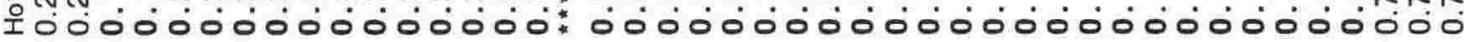

ए

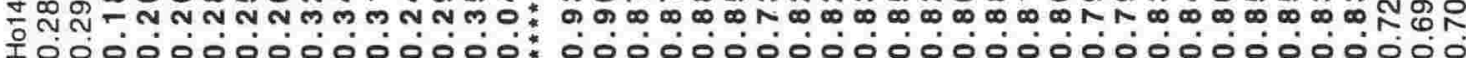

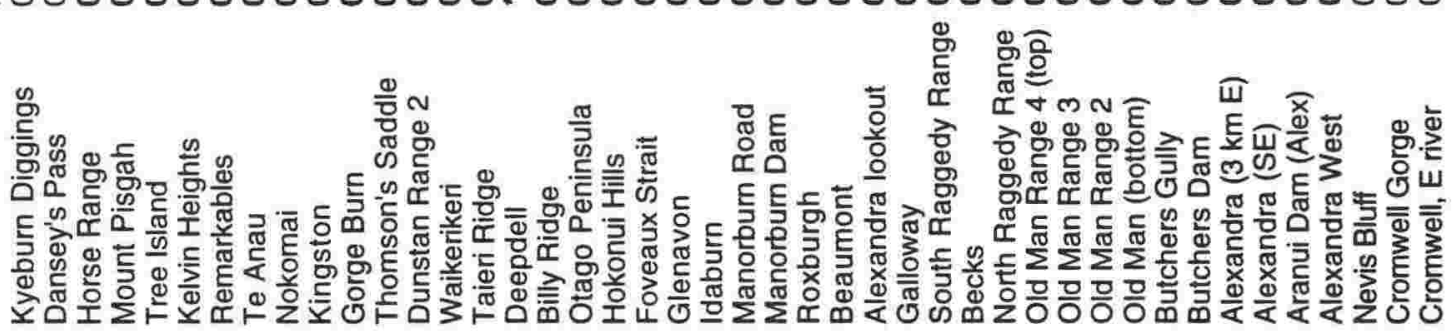

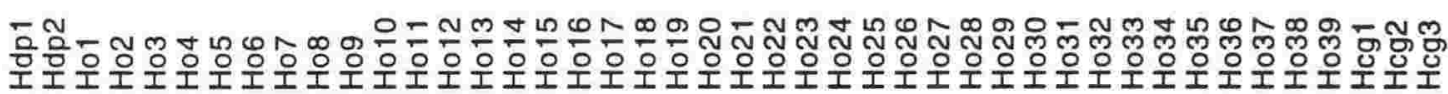




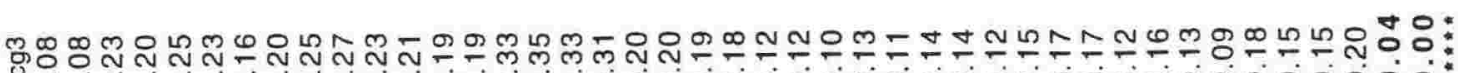
To0000000000000000000000000000000000000000000:

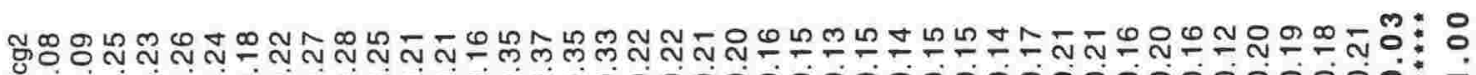

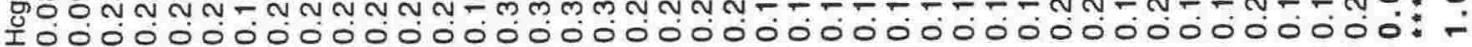

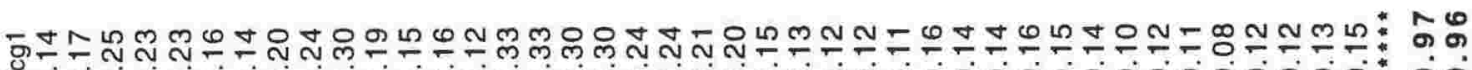

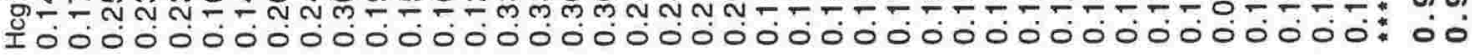

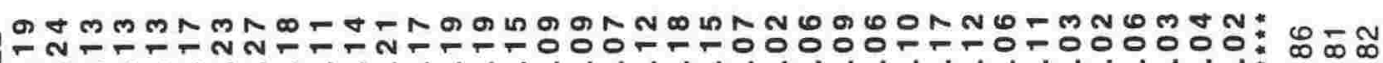

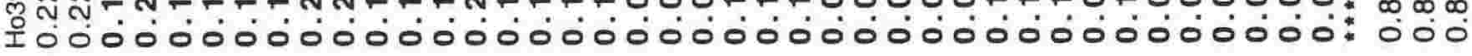
m I ติ I ஜํำ

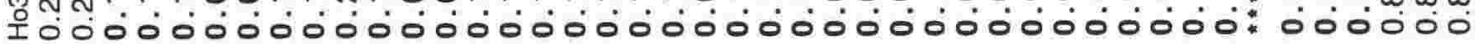
以ㄴ

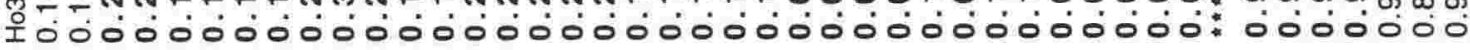

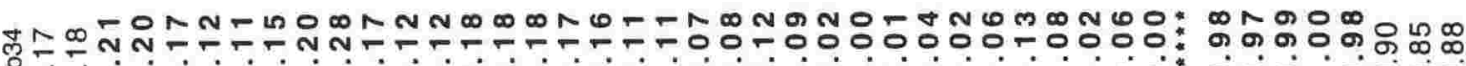

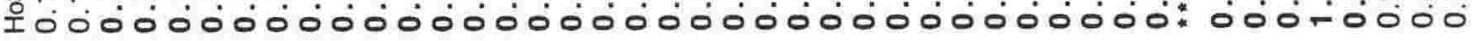

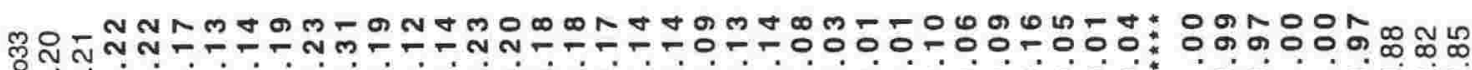

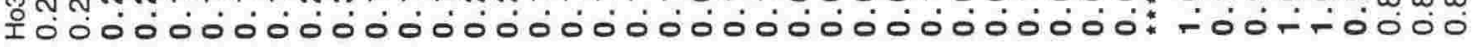

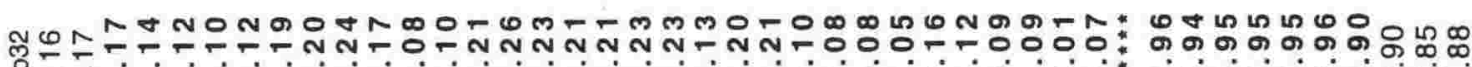

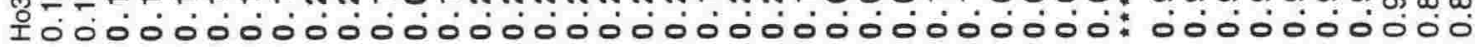

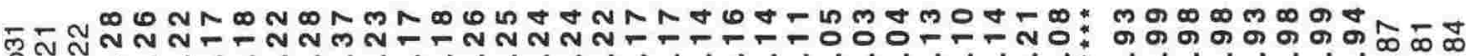

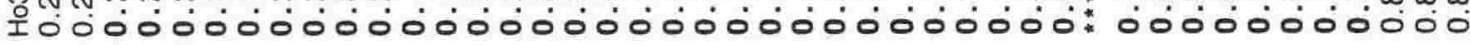

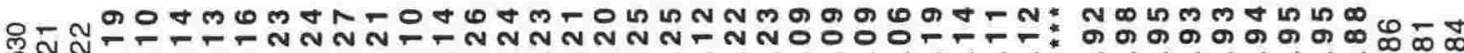

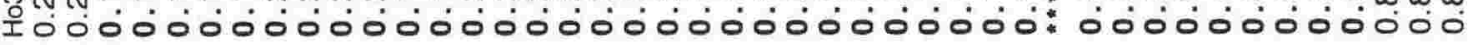

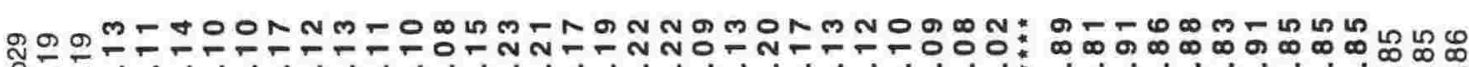

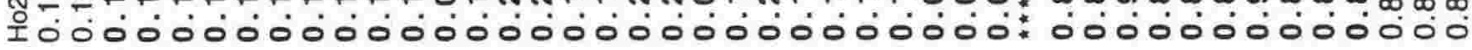

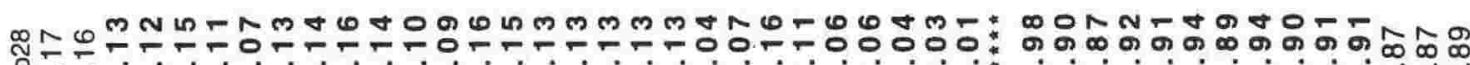

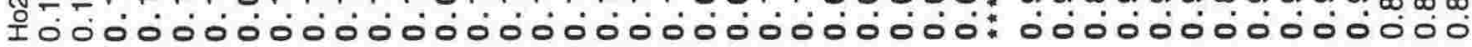

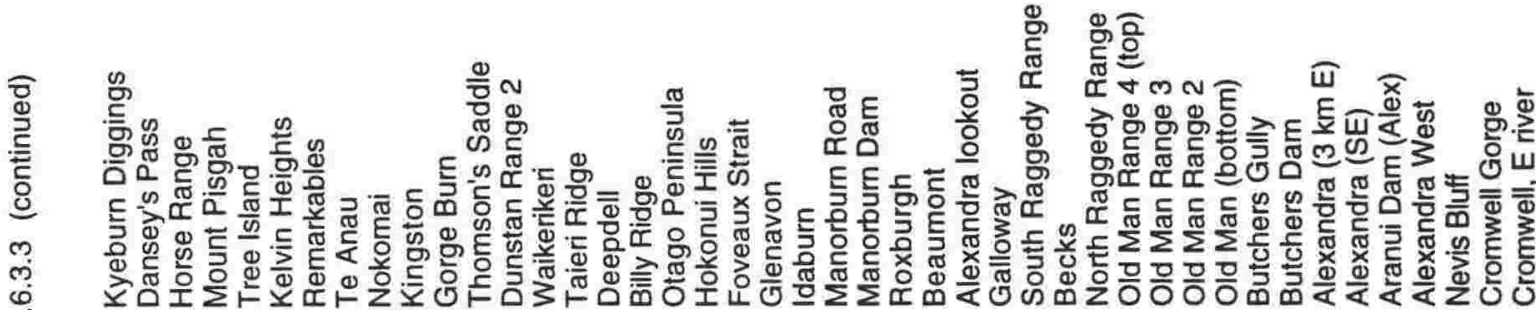
メロエ

은음

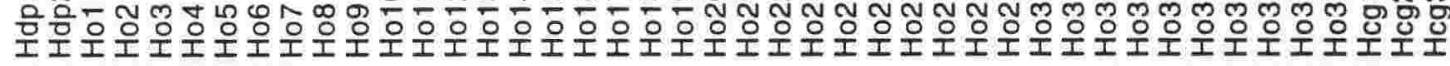




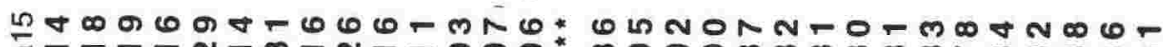

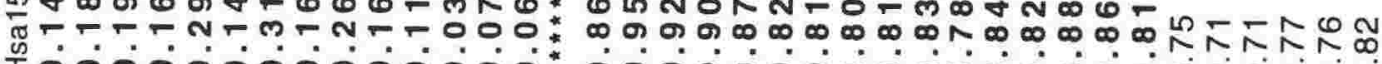

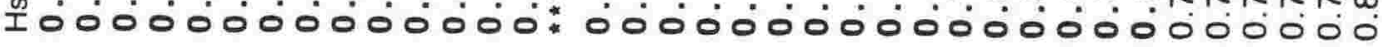

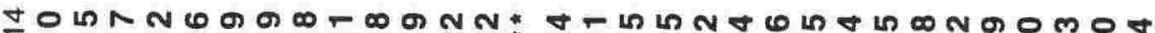

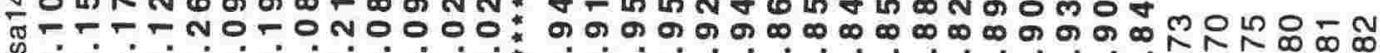

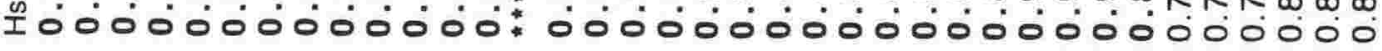

MNMNーR

乐, -

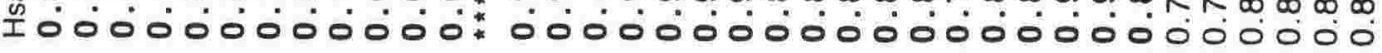

N-NN

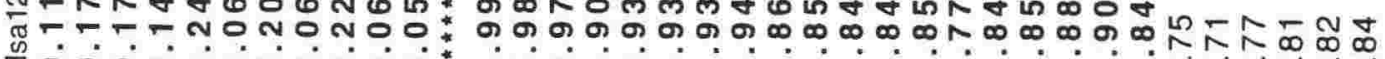

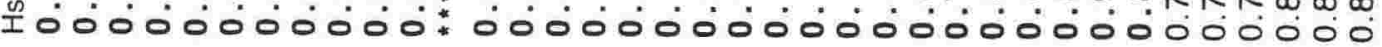

=mmm-

-

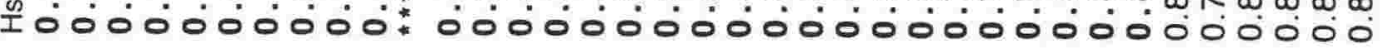
은 $\operatorname{Ln}$ L

ॠบ?

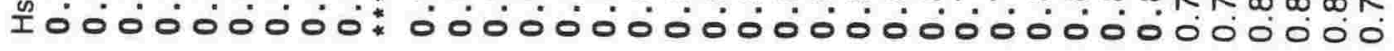

刃ำ

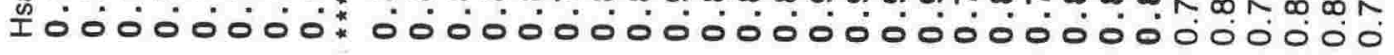

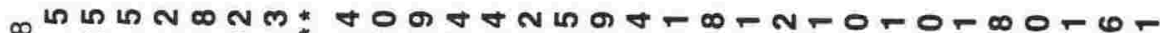

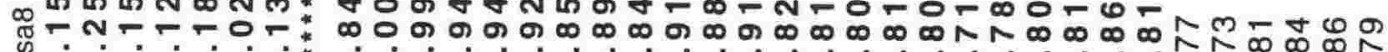

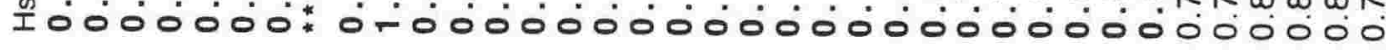

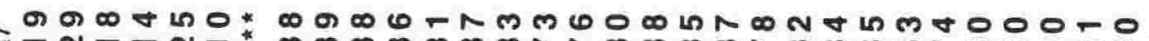

ల్ల

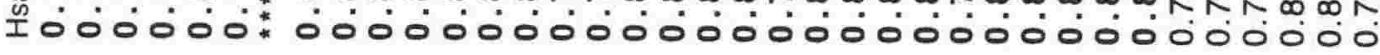

ตN-

שึ I00000* $0000000000000000000000000000^{\circ} 0^{\circ}$

ம

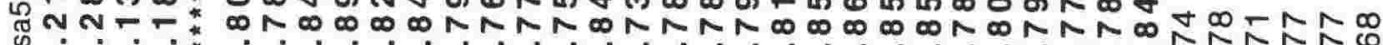

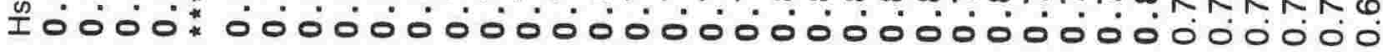

प

雨

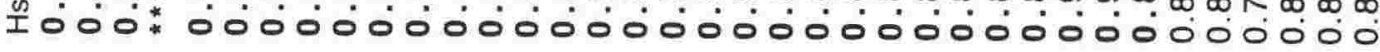

๓ $\omega_{*}^{*}-\infty$ L

雨 I00*0000000000000000000000000000000000

ம*

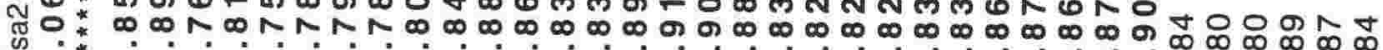

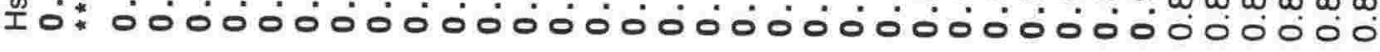

- + in

* \%

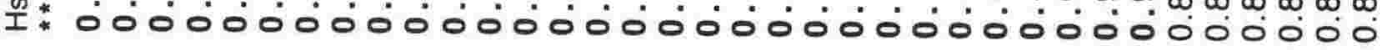

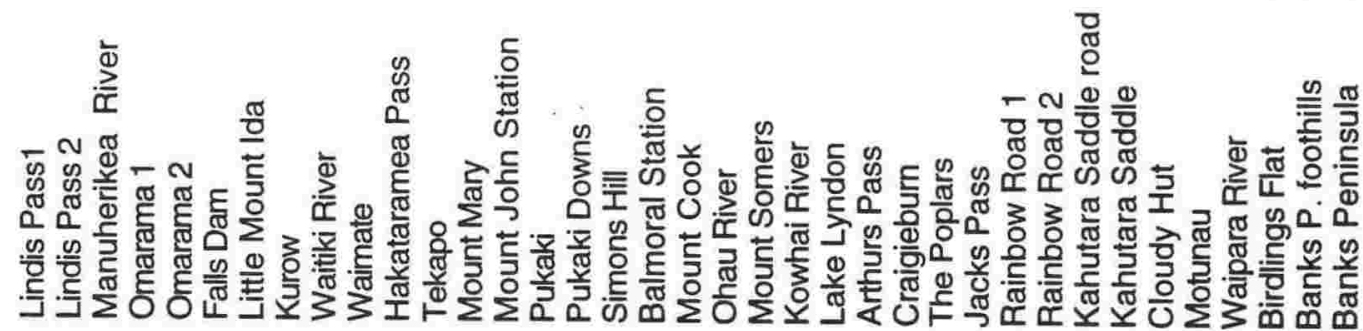

๘ 


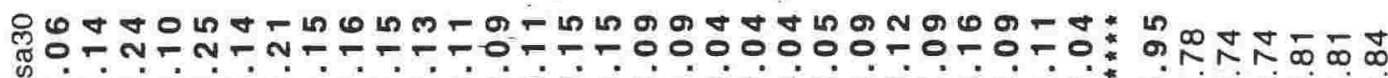

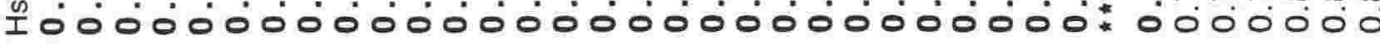

균-

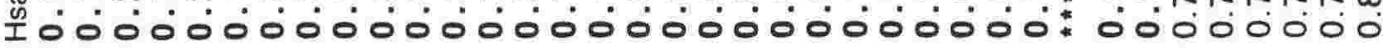

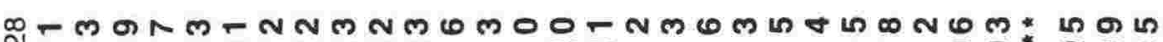

กั- -

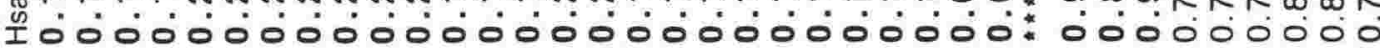

ำก

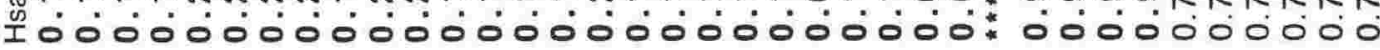

ம ת - - n

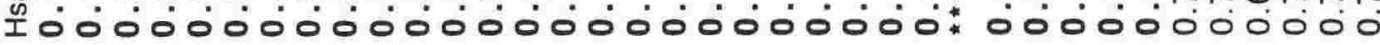

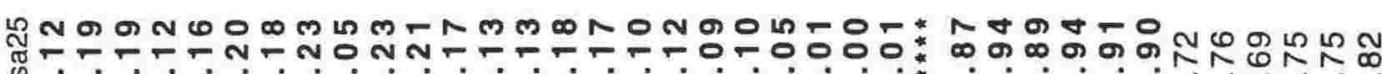

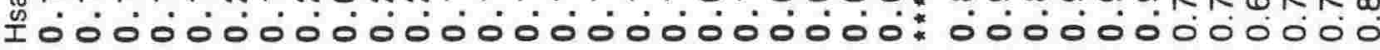

鬲

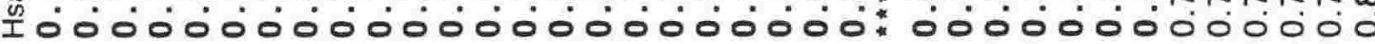

근

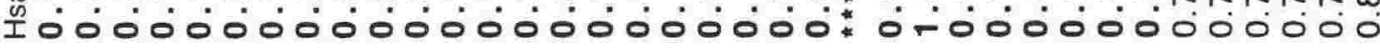

Nom

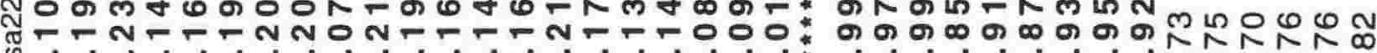

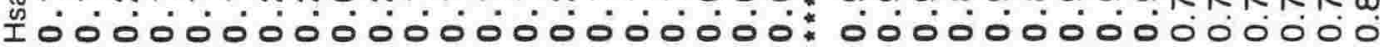

זٓ이

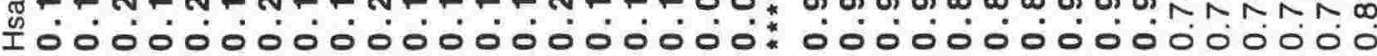

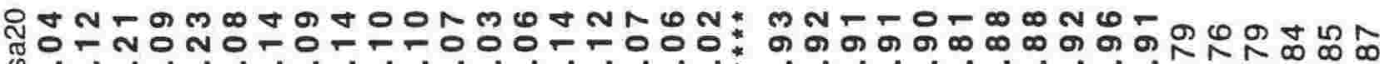

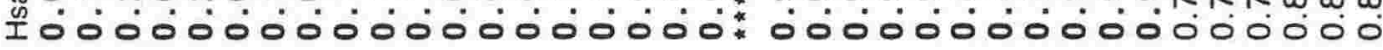

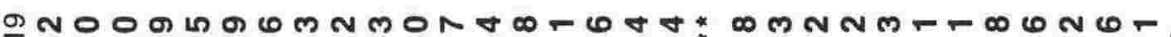

TO

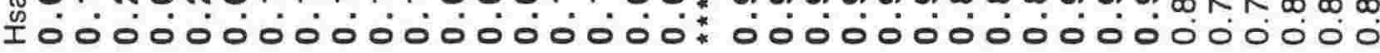

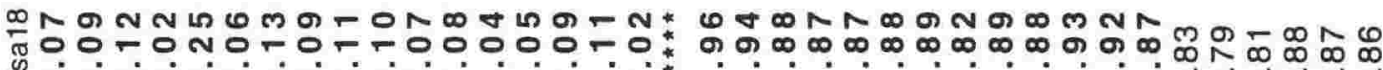

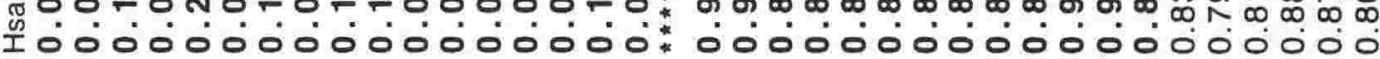

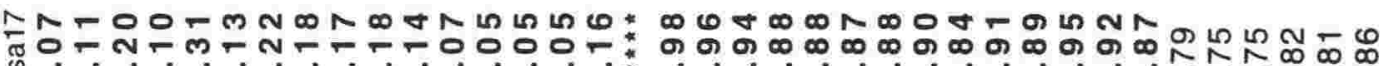

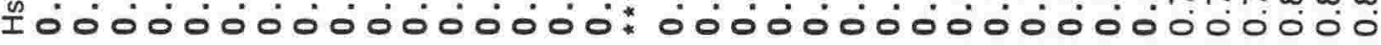

m m

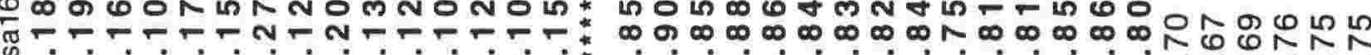

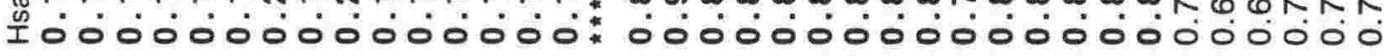

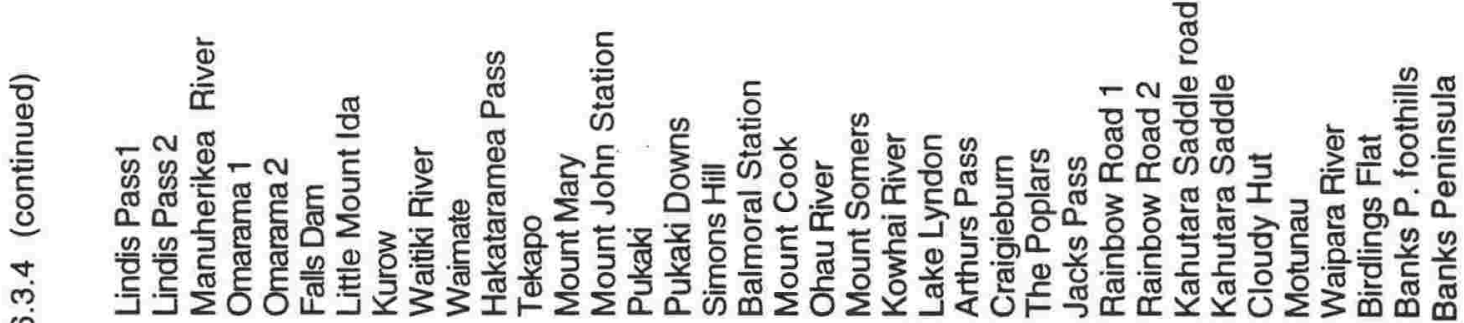




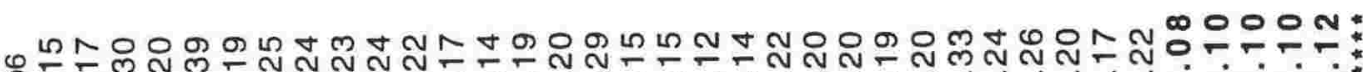

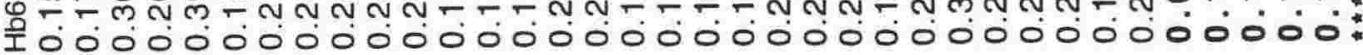

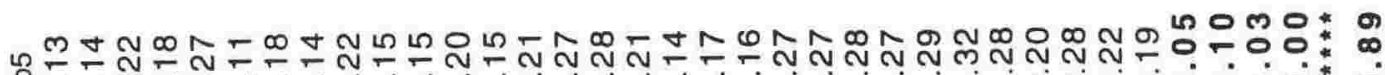

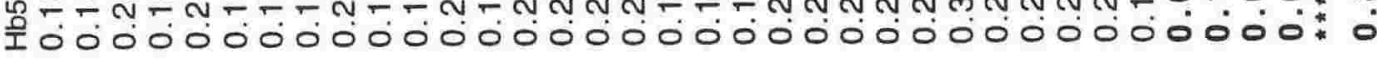

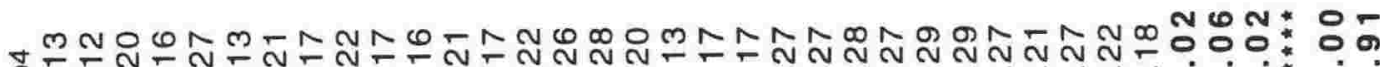

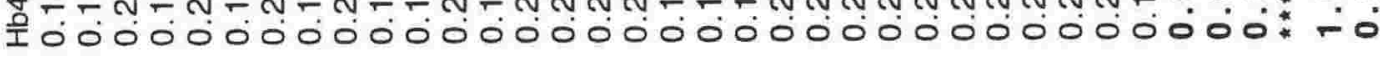

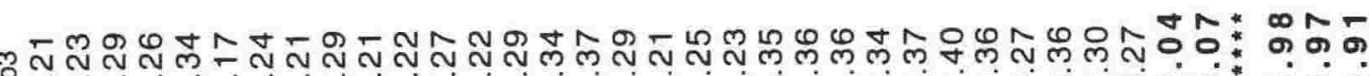
全000000000000000000000000000000000\%

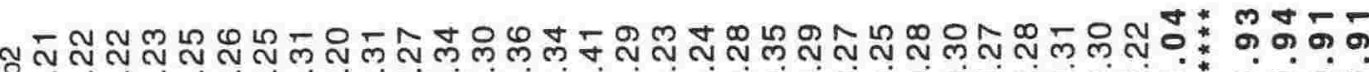
全0000000000000000000000000000000*0000

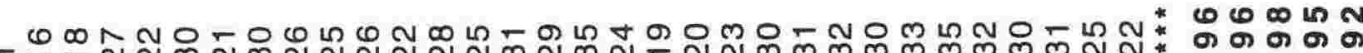

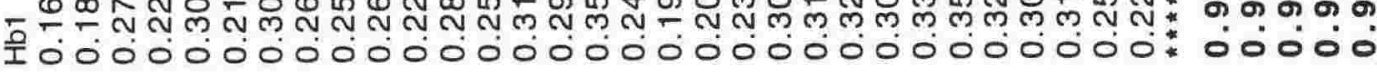
อั I0000000000000000000000000

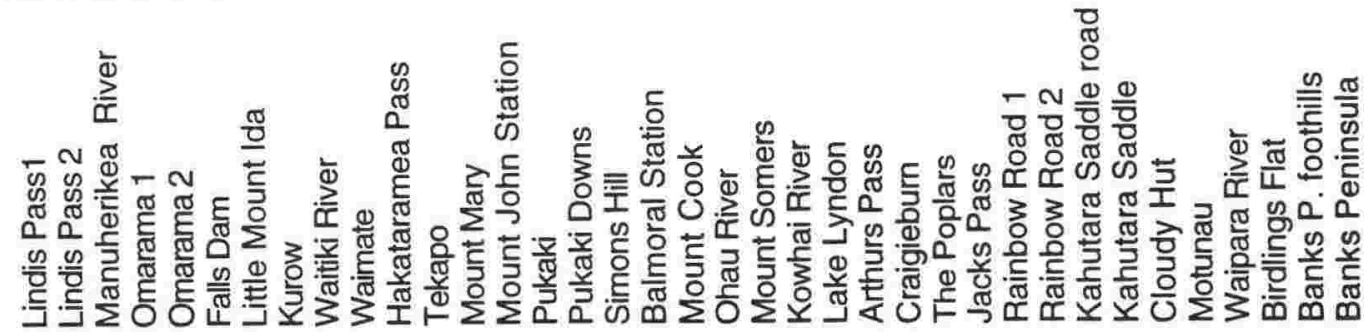
๘

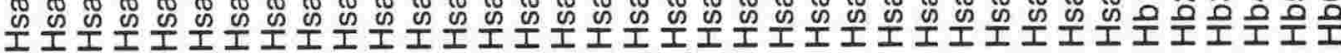


Table 4.6.3.5 Ranges of genetic distances (Nei's D) within and between proposed species of the southern group of the $H$. maculatus complex. Withinspecies values are in bold type.

\begin{tabular}{lllllll}
\hline Species & 1 & 2 & 3 & 4 & 5 & 6 \\
\hline 1 H. "Southern Alps" & $0.00-0.34$ & & & & & \\
2 H. brunneus & $0.11-0.41$ & $0.00-0.12$ & & & & \\
3 H. "Cromwell Gorge" & $0.13-0.49$ & $0.26-0.36$ & $0.00-0.04$ & & & \\
4 H. "Danseys Pass" & $0.21-0.54$ & $0.20-0.45$ & $0.08-0.17$ & 0.00 & & \\
5 H. "Otago" & $0.13-0.65$ & $0.18-0.66$ & $0.08-0.37$ & $0.13-0.30$ & $0.00-0.37$ & \\
6 H. duvaucelii & $0.17-0.52$ & $0.26-0.47$ & $0.05-0.22$ & $0.12-0.22$ & $0.15-0.60$ & $0.00-0.14$ \\
& & & & & &
\end{tabular}

There is geographic structure within $H$. "Otago", with boundaries indicated by size, colour pattern, and allozyme differences. However, these boundaries are less well defined than those between $H$. "Otago" and $H$. "Cromwell Gorge" or $H$. "Danseys Pass", because the geographic distances separating the populations are greater and some of the pairwise genetic distances across the boundaries are lower. The 6 geographic groups within $H$. "Otago" seen on the WPGMA phenogram generated from allozyme data (Figure 4.6.3.19) are:

1) the Kakanui and Horse Ranges

2) Eastern Otago, south of the Shag River and east of the Taieri River

3) Central Otago between the Manuherikia and Taieri Rivers

4) the Manorburn Dam area of Central Otago

5) the Clutha Valley from Alexandra eastwards

6) western Otago, from the Dunstan and Old Man Ranges west

7) eastern Southland and Foveaux Strait.

I examine the validity of these groups below.

The 2 single specimens from the Kakanui and Horse Ranges are separated from each other by about $23 \mathrm{~km}$ and $\mathrm{D}=0.08$ (Figure 4.6.3.3.1), and from all other populations $20-60 \mathrm{~km}$ distant by $\mathrm{D}>0.1$.

Western populations were mostly separated from Central Otago populations by larger genetic distances than those within these groups; however, there was considerable genetic diversity over small geographic distances within both, which increased the spread of both within-group and between-group distances (Figure 4.6.3.3.2, 4.6.3.3.3). All but 2 pairwise between-group genetic distances were $D \geq 0.08$. The 2 exceptions were $D=0.06$ between 


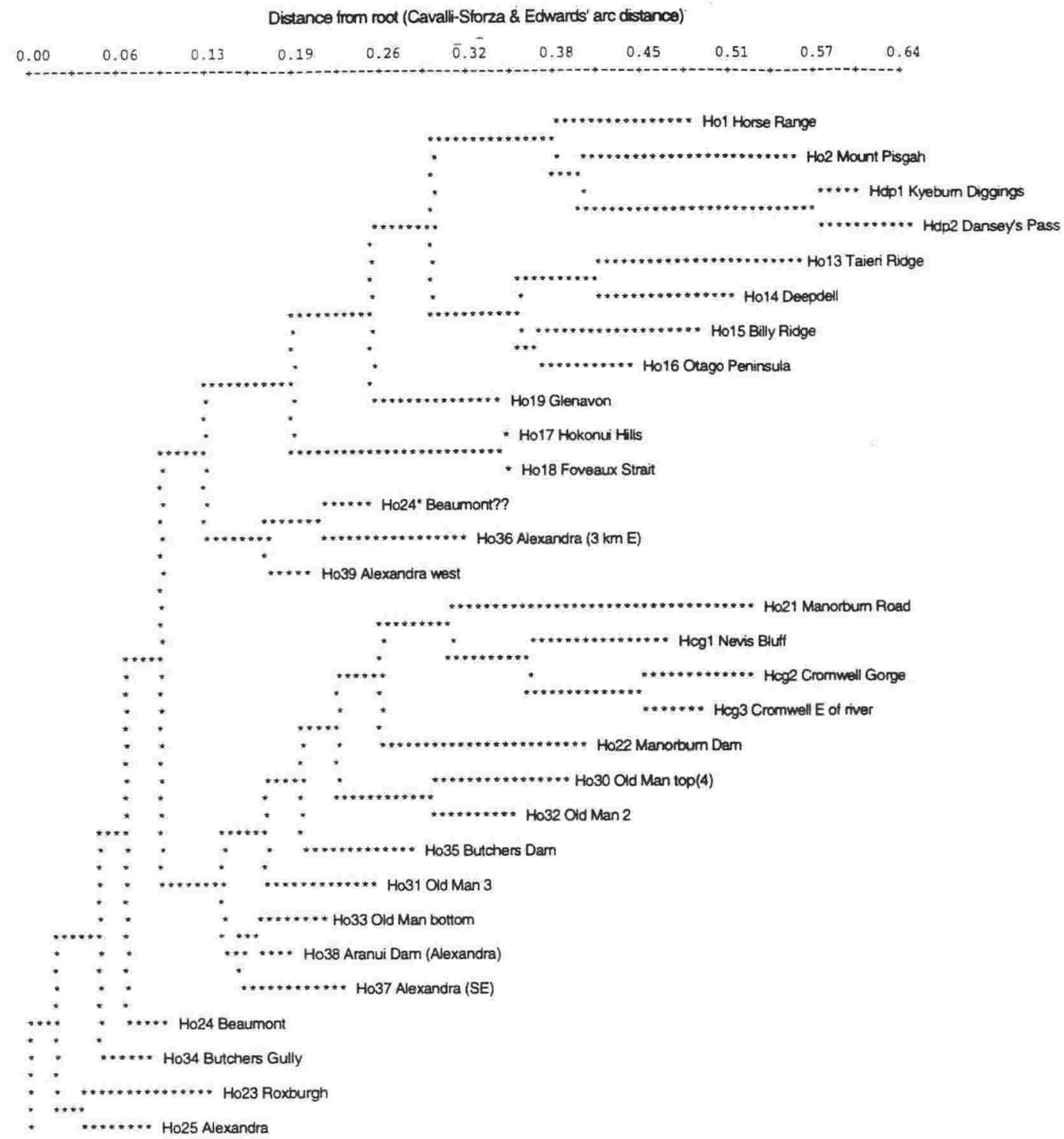

Ho3 Tree island
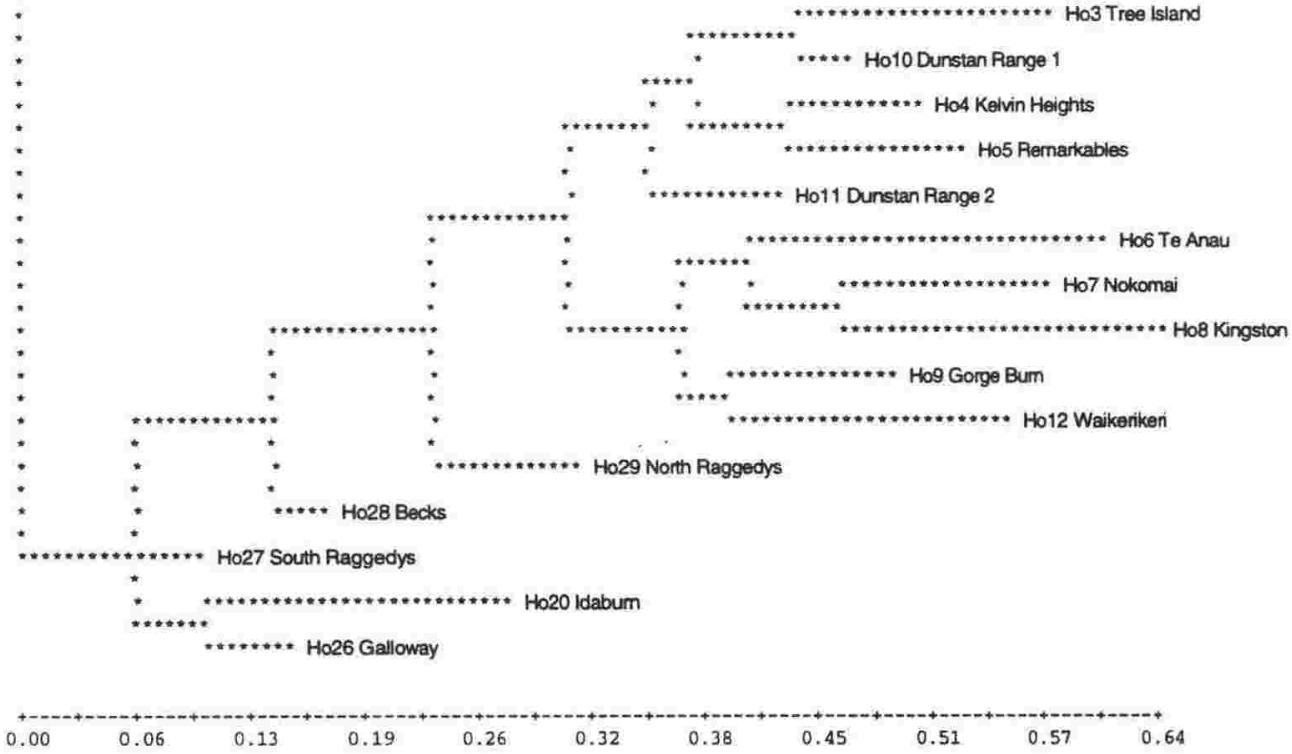

Figure 4.6.3.21 Distance Wagner tree of Otago-Southland, $H$. maculatus-complex populations, generated from allozyme data by the BIOSYS package. Total length of tree $=5.806$. 
Distance from root (Cavalli-Sforza \& Edwards' arc distance)

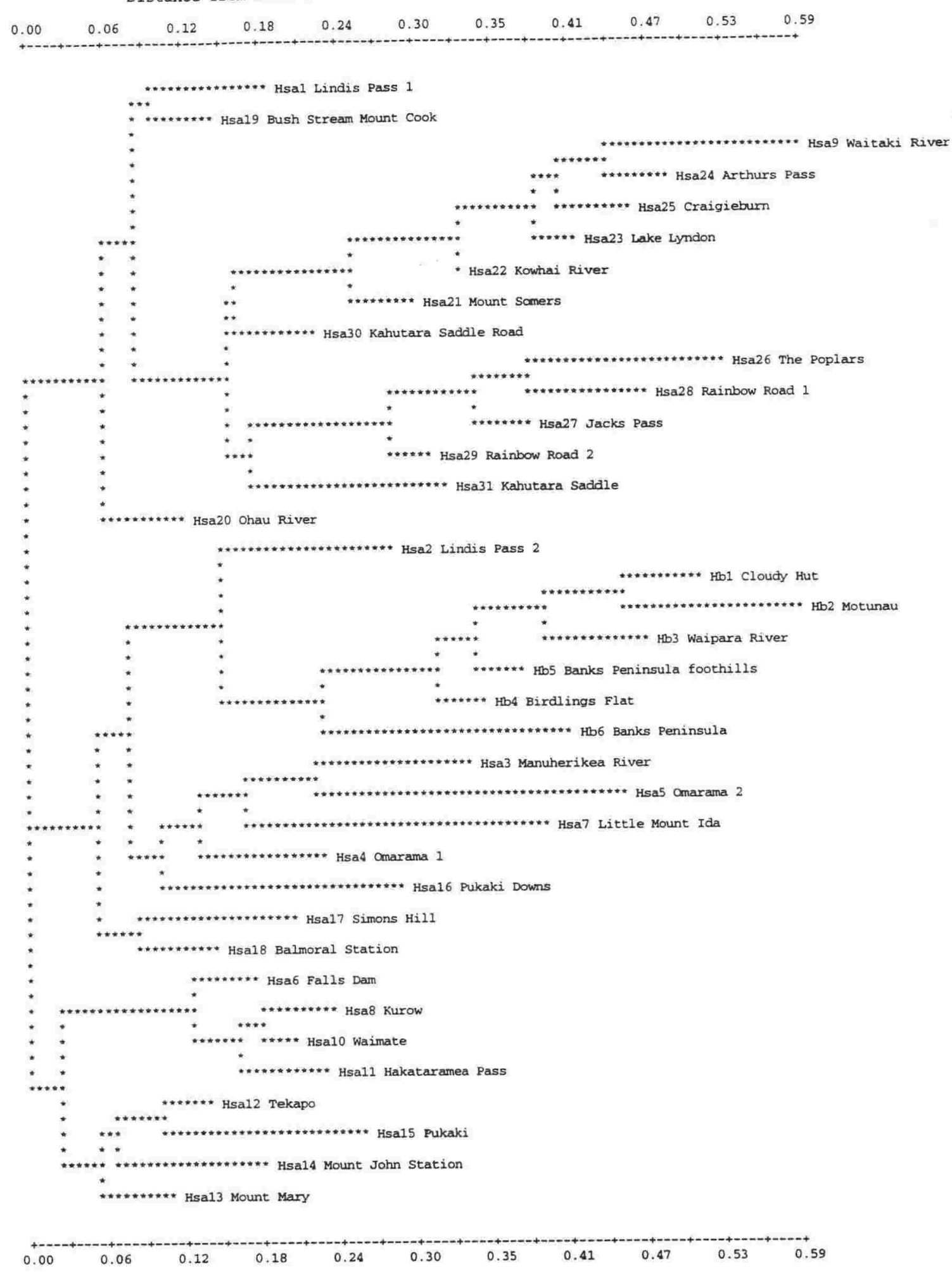

Figure 4.6.3.22 Distance Wagner tree of Canterbury $H$. maculatus-complex populations, generated from allozyme data by the BIOSYS package. Total length of tree $=4.835$. 


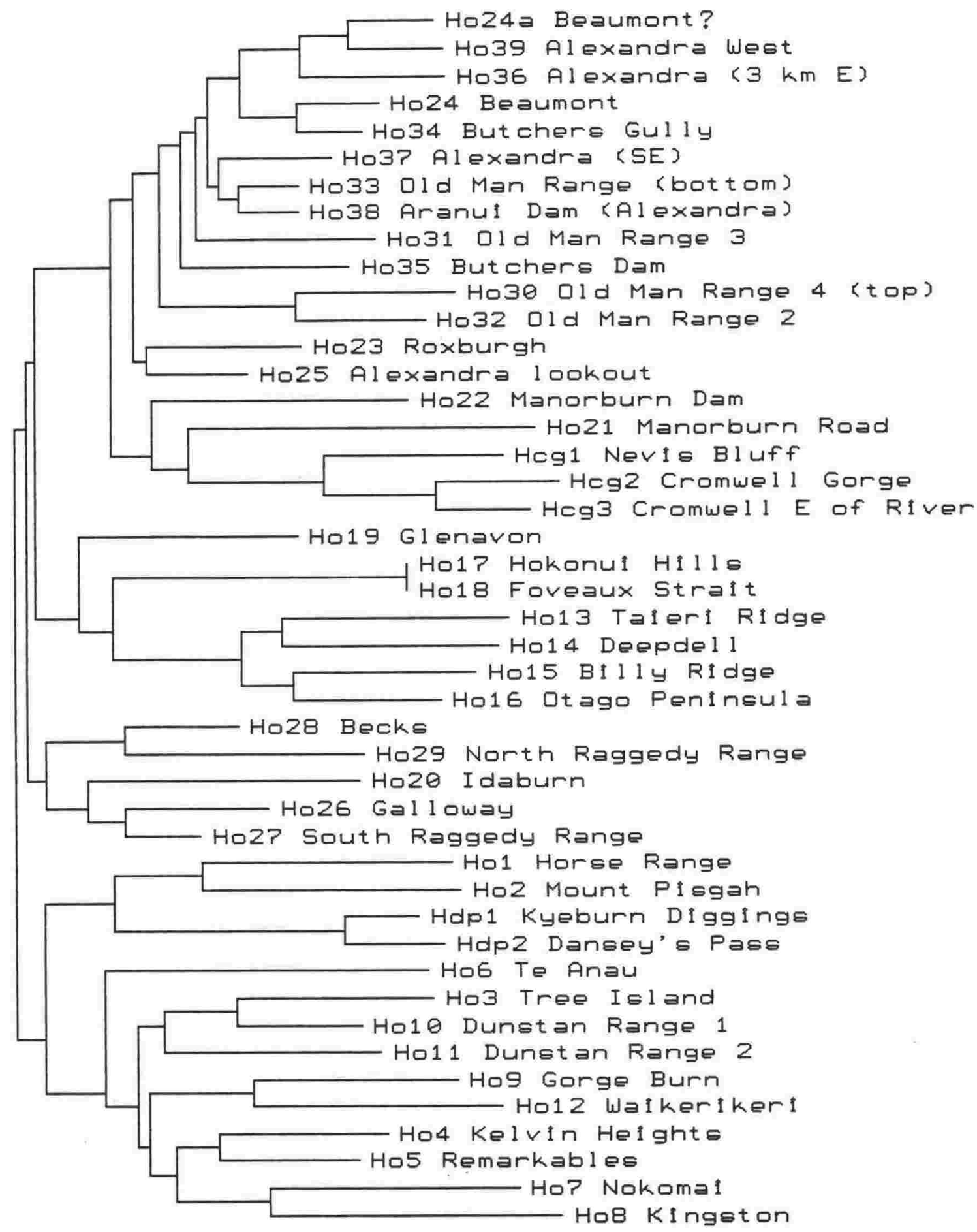

Figure 4.6.3.23 neighbour joining tree of Otago-Southland populations of the southern group of the $H$. maculatus complex, generated by the MEGA package from Cavalli-Sforza \& Edwards' arc distances (27 allozyme loci). Population numbers as in Table 4.6.2.1. 


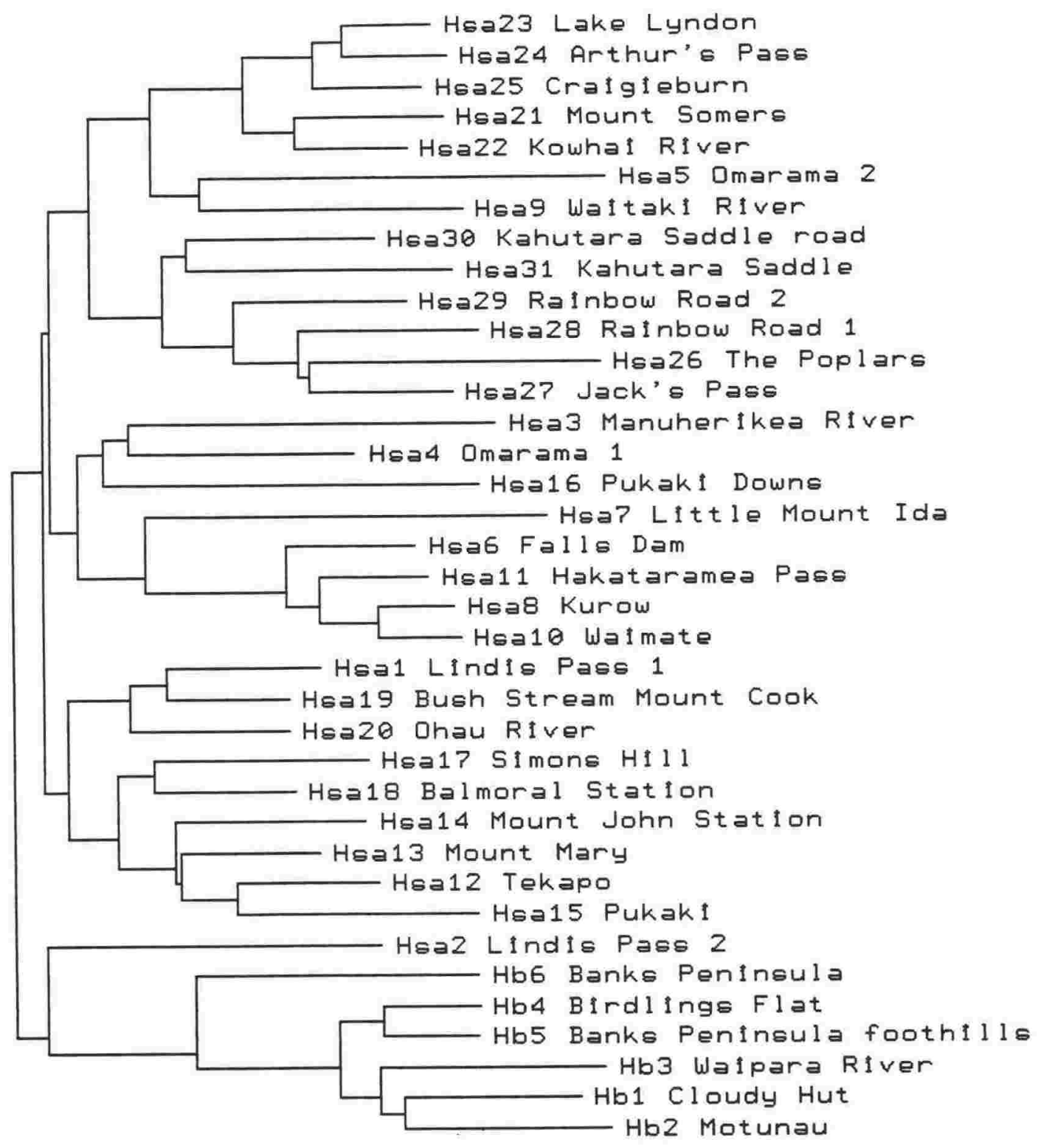

Figure 4.6.3.24 neighbour joining tree of Canterbury populations of the southern group of the $H$. maculatus complex, generated by the MEGA package from Cavalli-Sforza \& Edwards' arc distances (27 allozyme loci). Population numbers as in Table 4.6.2.1. 
뜌ำ

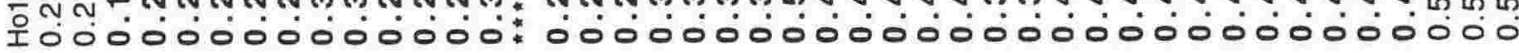

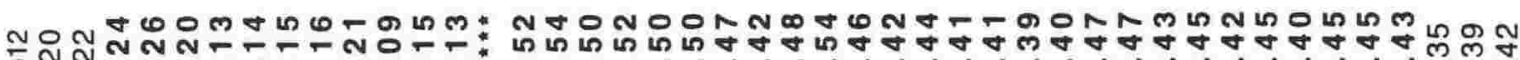

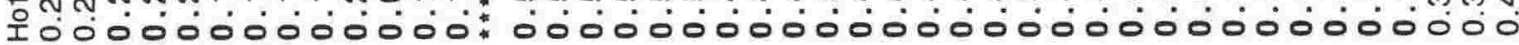

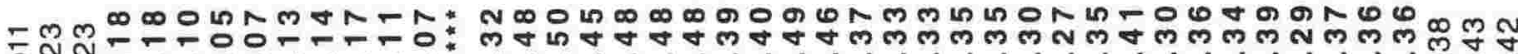

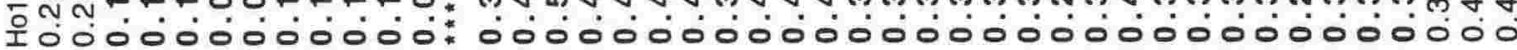

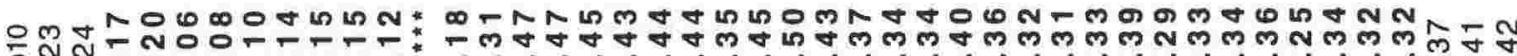

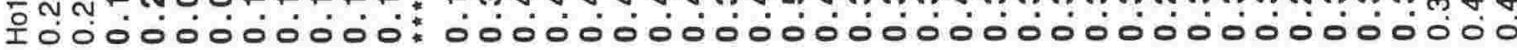

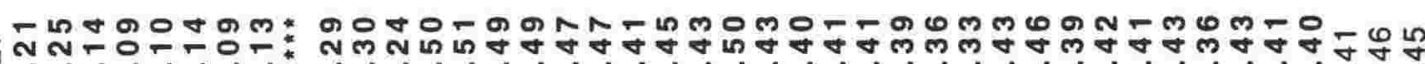

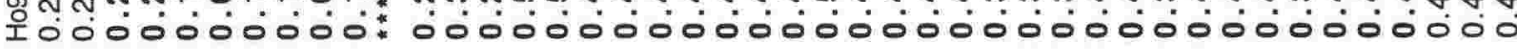
m

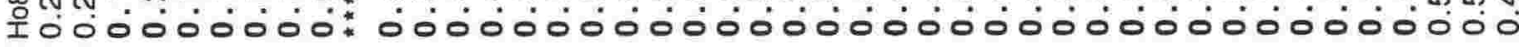

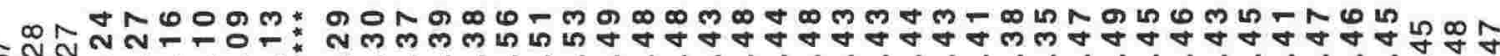

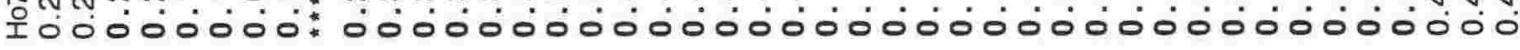

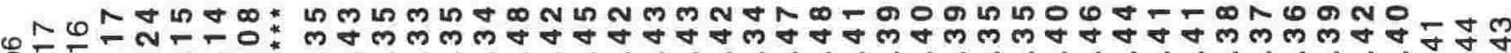

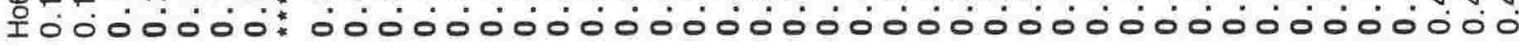

กำ N 웅ㅇㅇ: 0000000000000000000000000000000000000

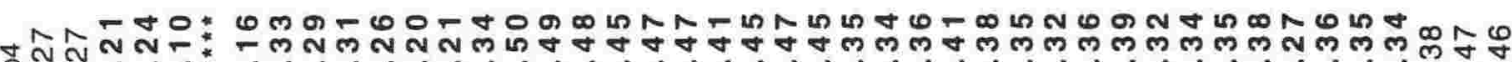

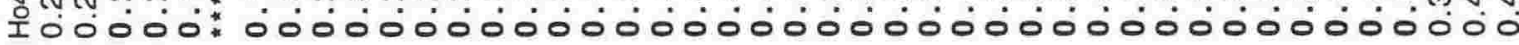

以

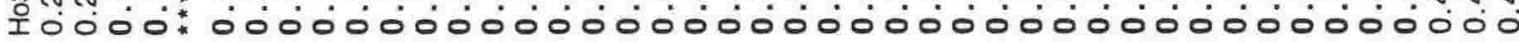

므:*

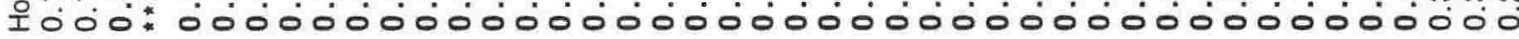

웅." 0000000000000000000000000000000000000000

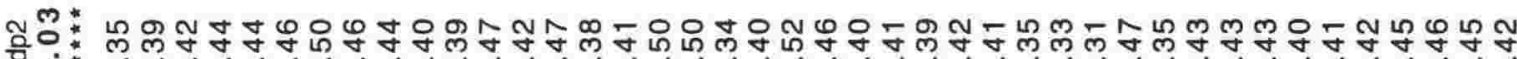

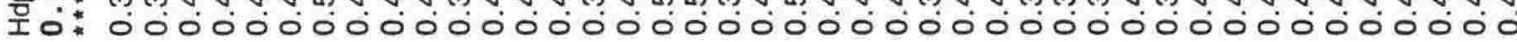

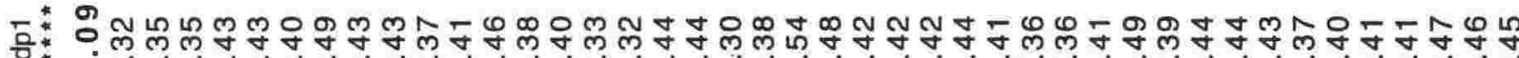

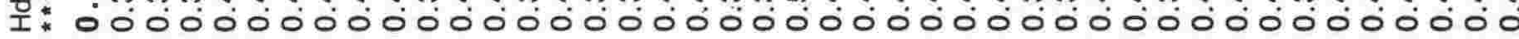

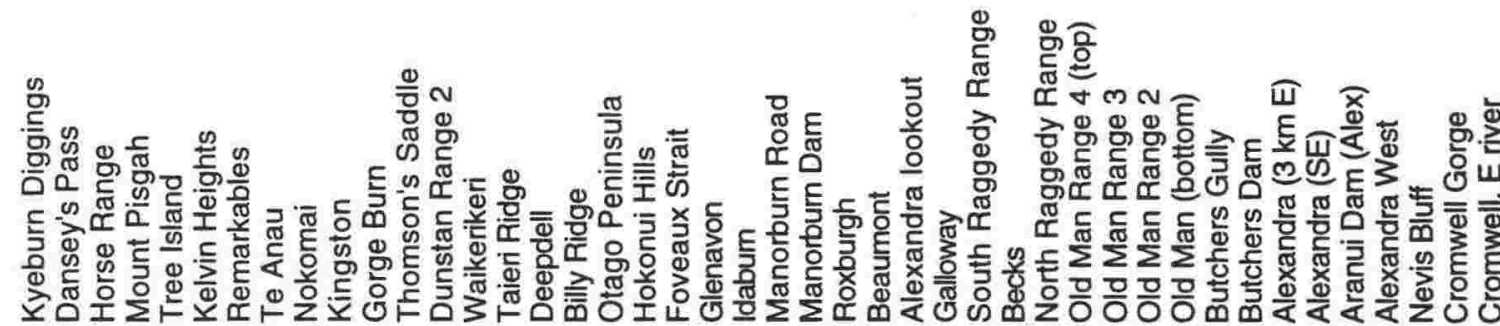

-

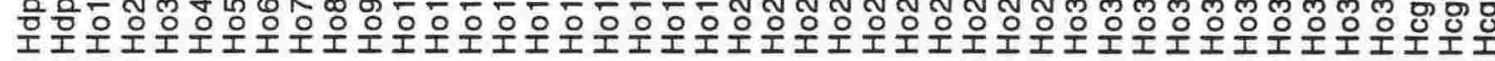




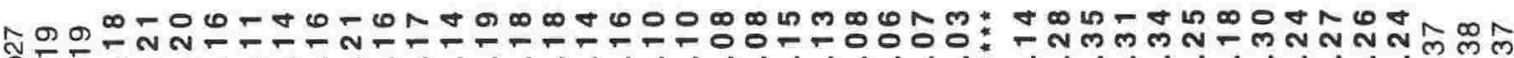

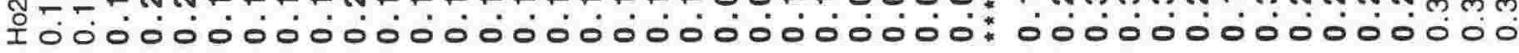
mONOON NoO

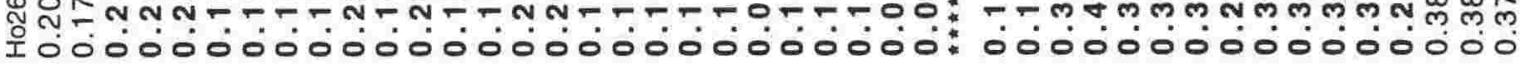
mo 은

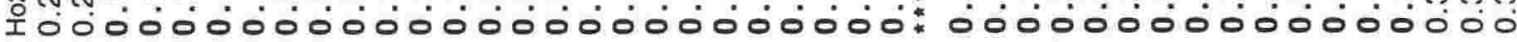

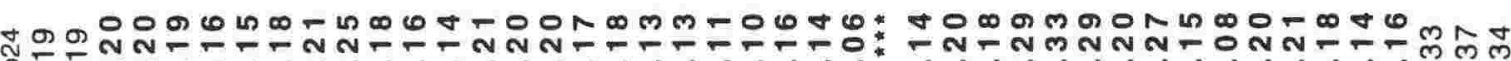

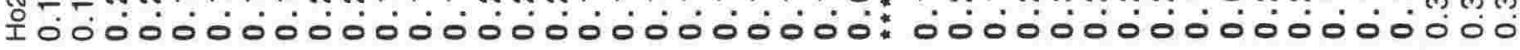
$m \infty n N-N N m-5 m-0 m \omega m-0 m$ n

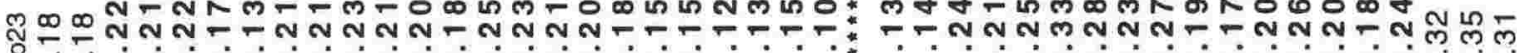

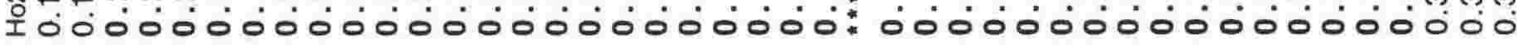
Nָ

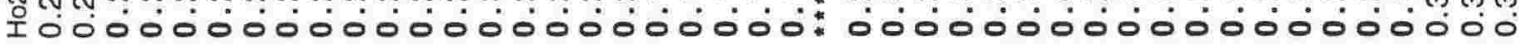
స్ I00000000000000000000"*000000000000000000000

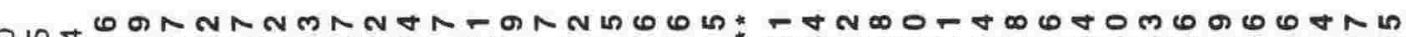
N

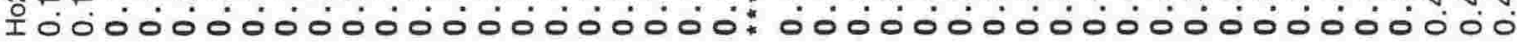
-

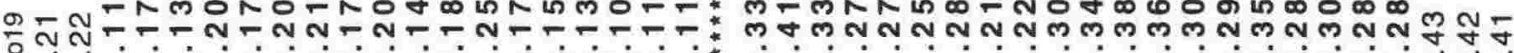

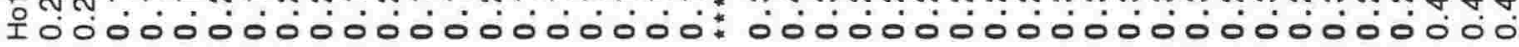

O m N

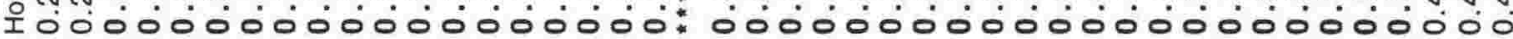
슨유

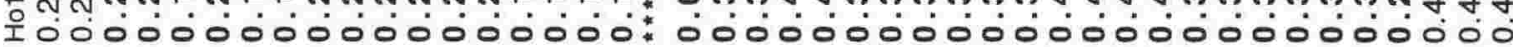
m

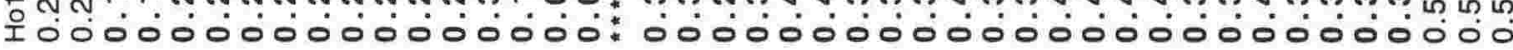

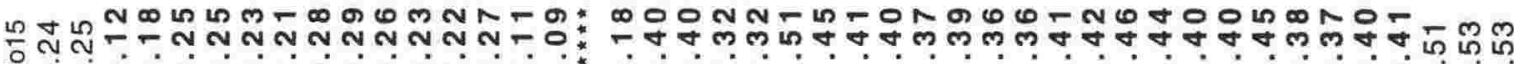

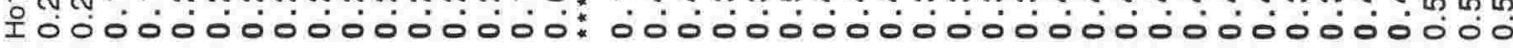

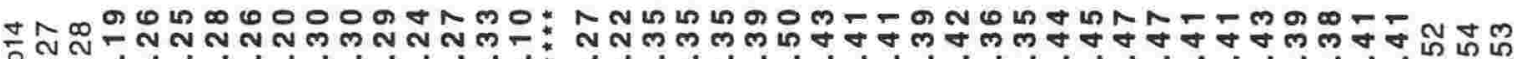
I

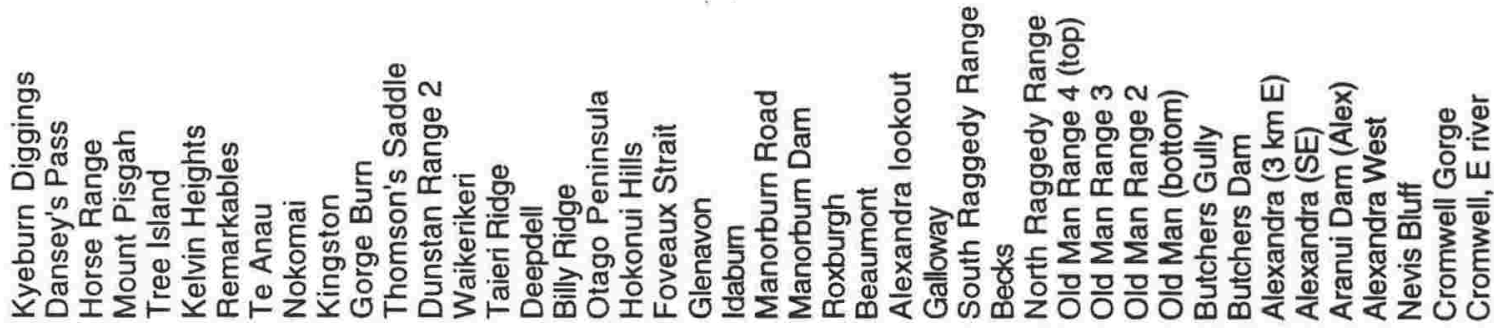

즌믐 곤 


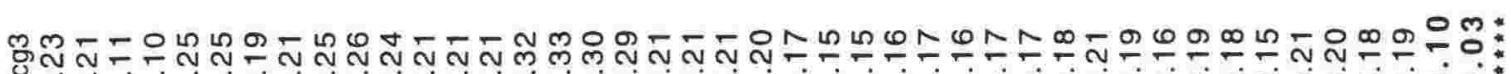
I00000

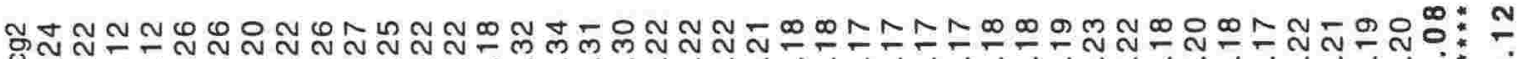

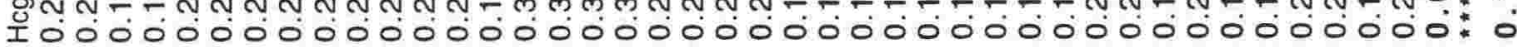

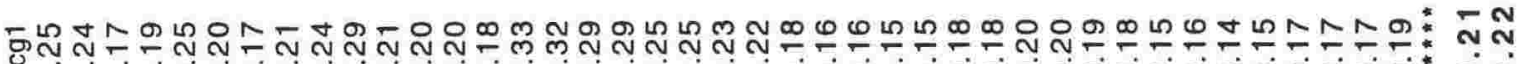

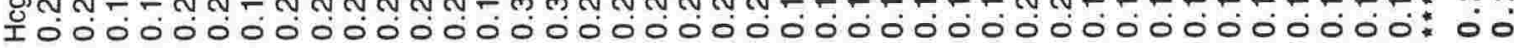

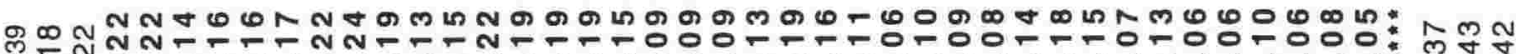

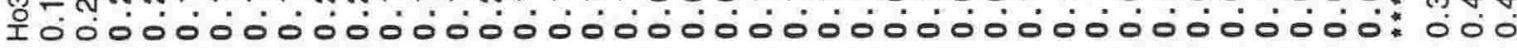

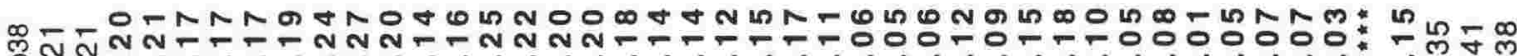

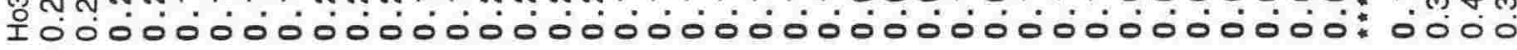
ฟิธลธลกัก

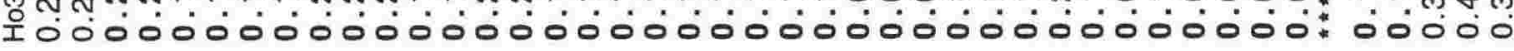

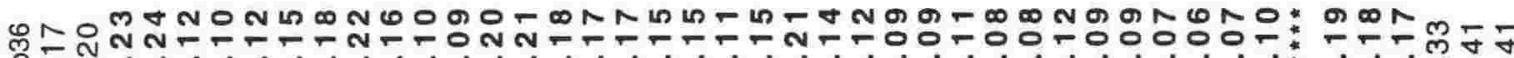
I

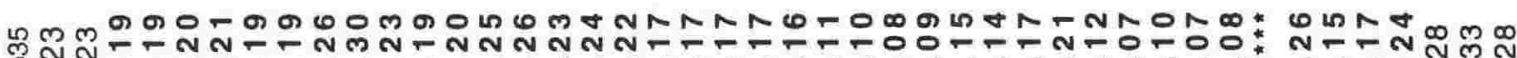

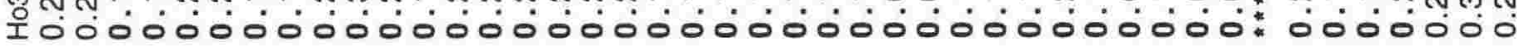

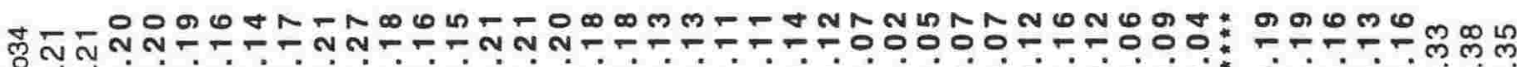

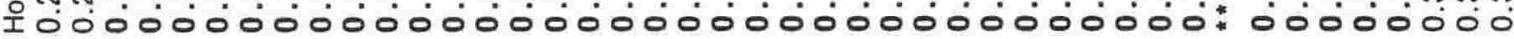

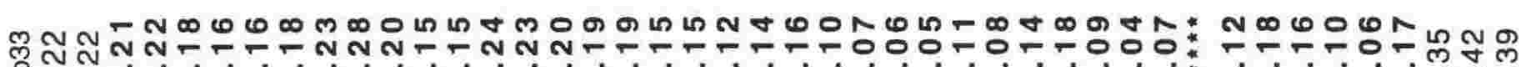

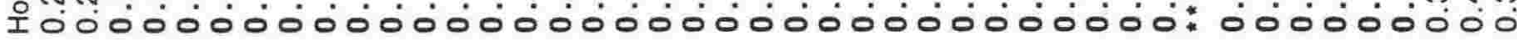
Nָ-

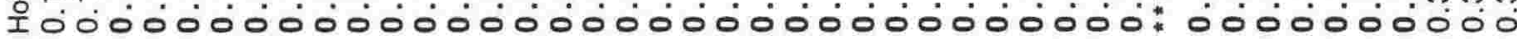
-

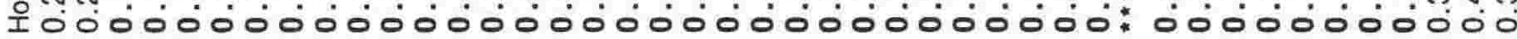

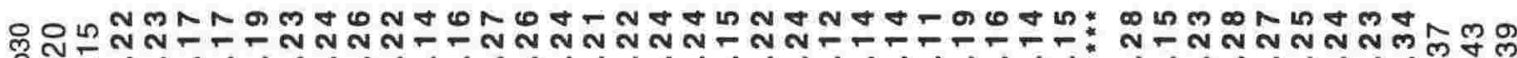

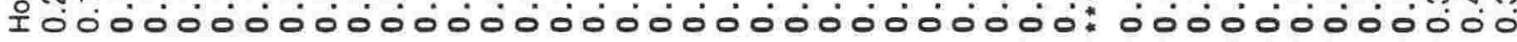

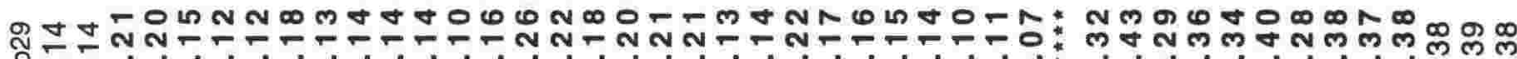

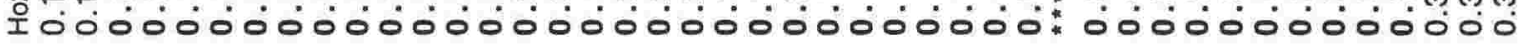

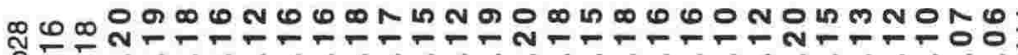

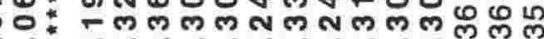

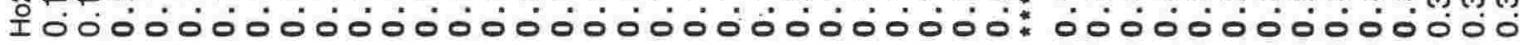

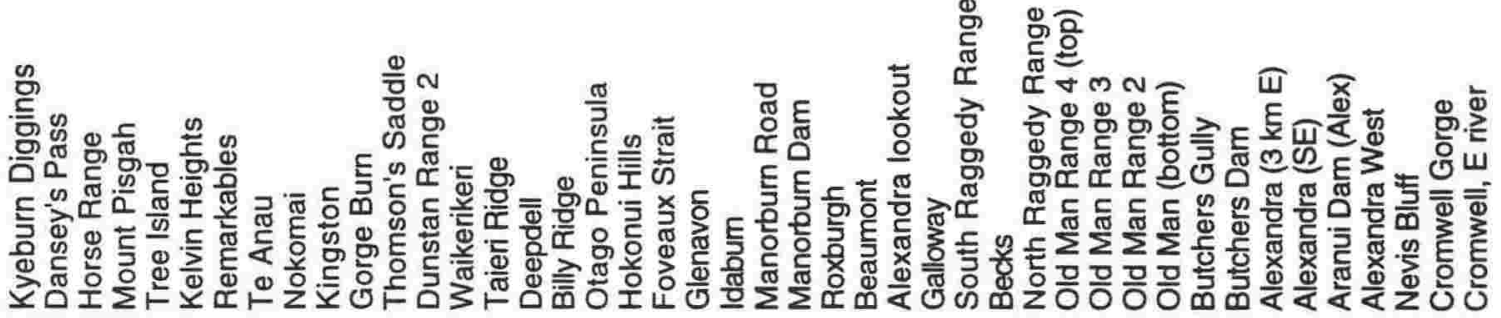

음음

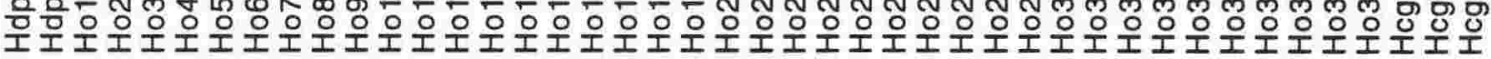




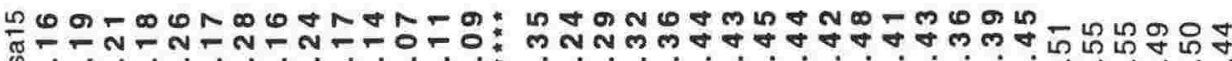

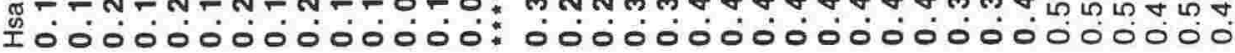

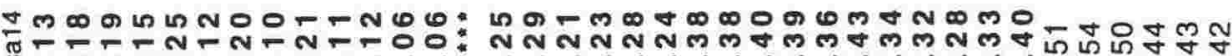

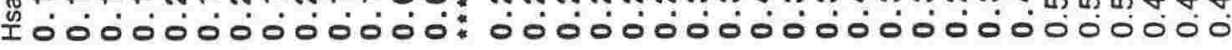

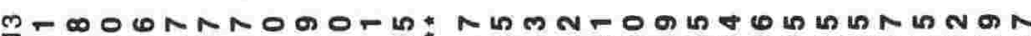

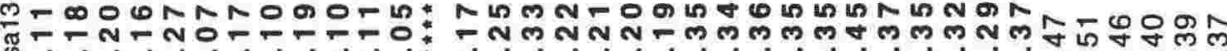

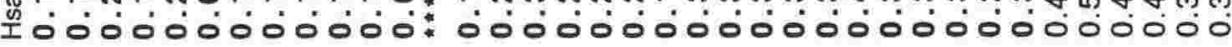

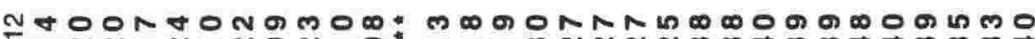

d.

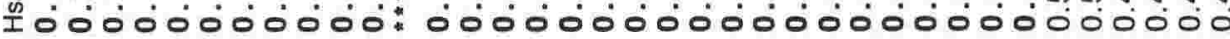

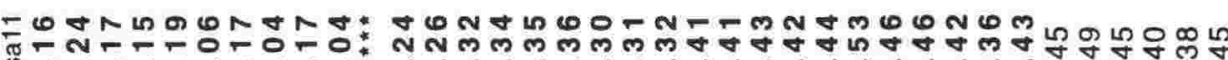

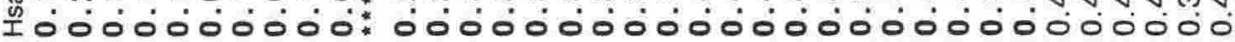

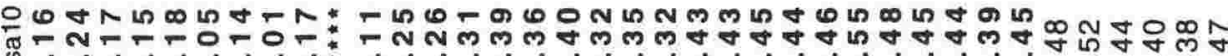

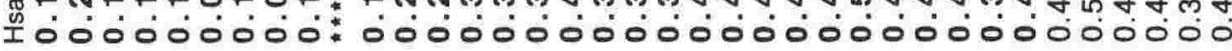

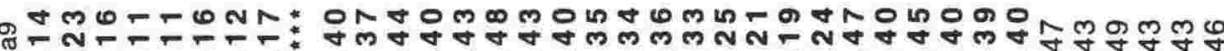

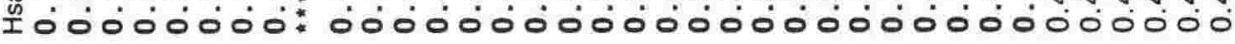

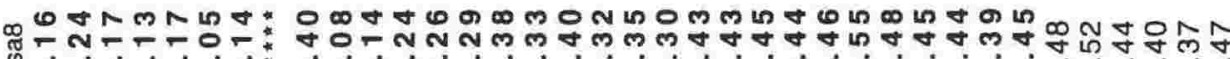

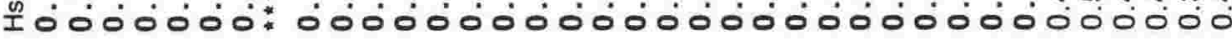
m

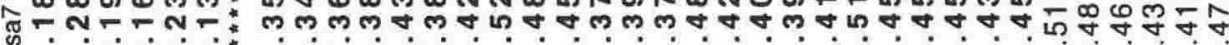

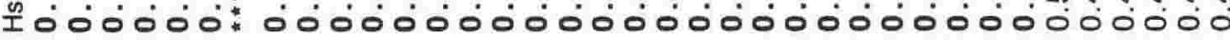

๒ூ I0000.* 0000000000000000000000000000000

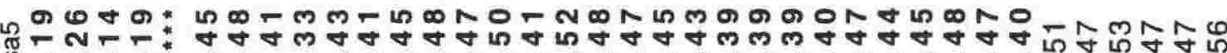

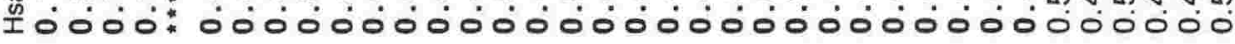

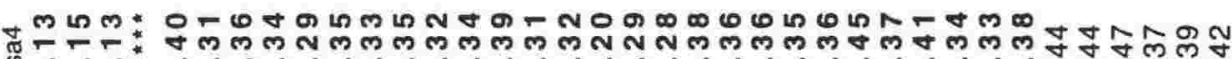
I00** 0000000000000000000000000000

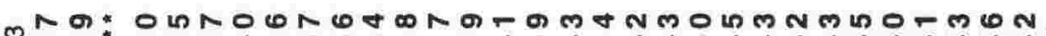

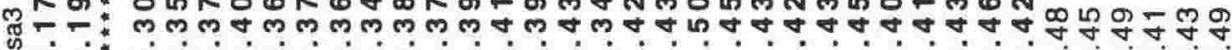

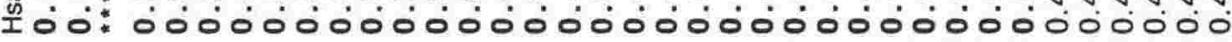

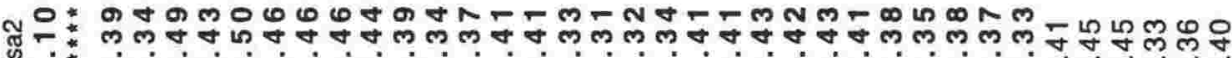
范* 0000000000000000000000000000000000

₹ I* 000000000000000000000000000000000

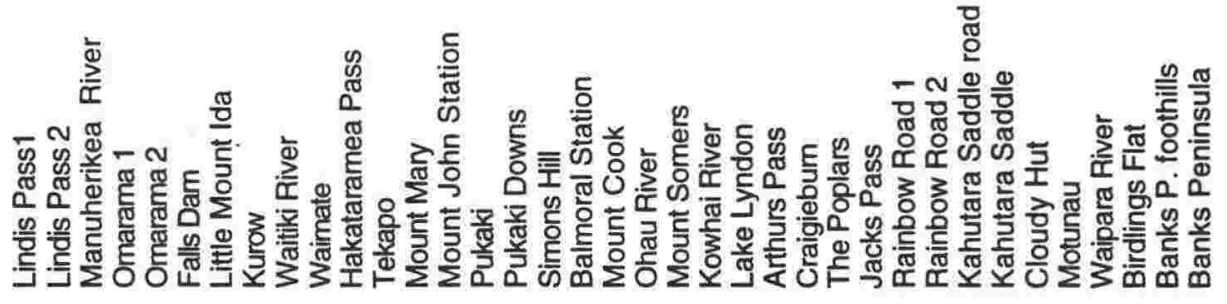

- ㅆம

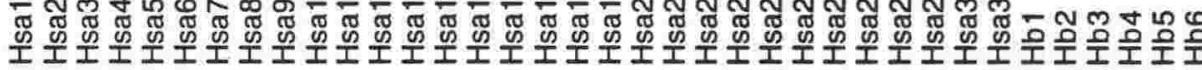


으

गुำ I000000000000000000000000\% 000000 มูก

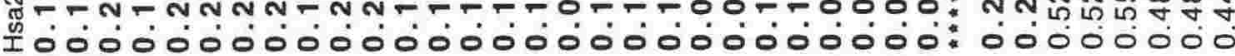
חึ็

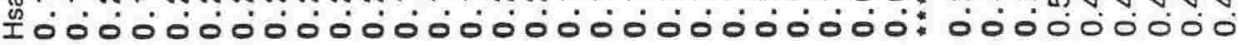
ลิ NNm

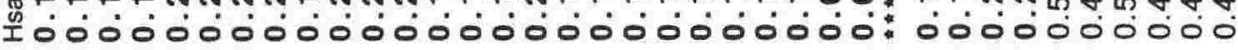

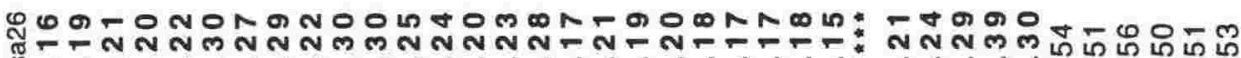

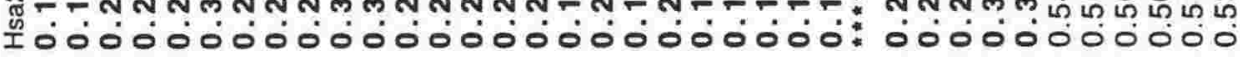
Mี

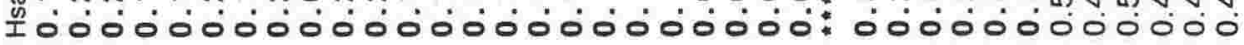

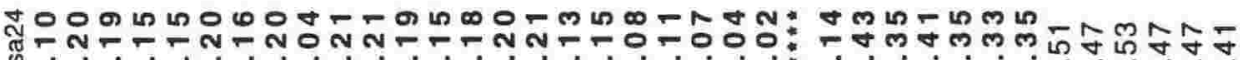

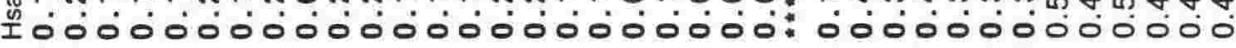

근 Í

กี้

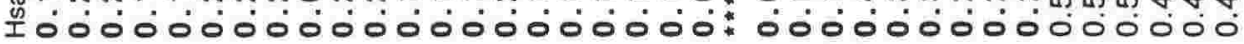

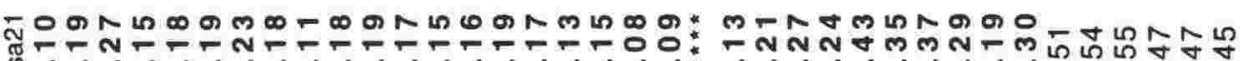
Í

공 Í

MmmOF

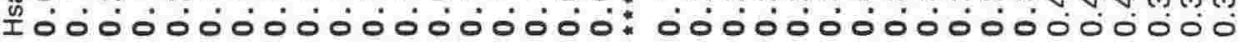

DE

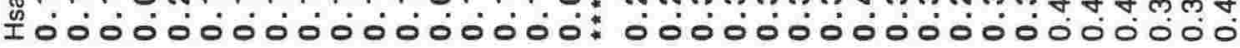

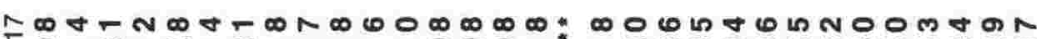

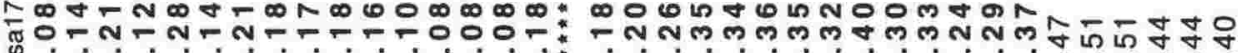
I0000000000000000"00000000000000000000

엠

ก

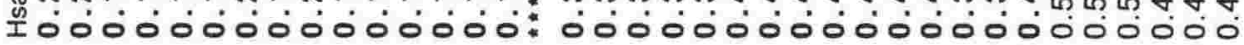

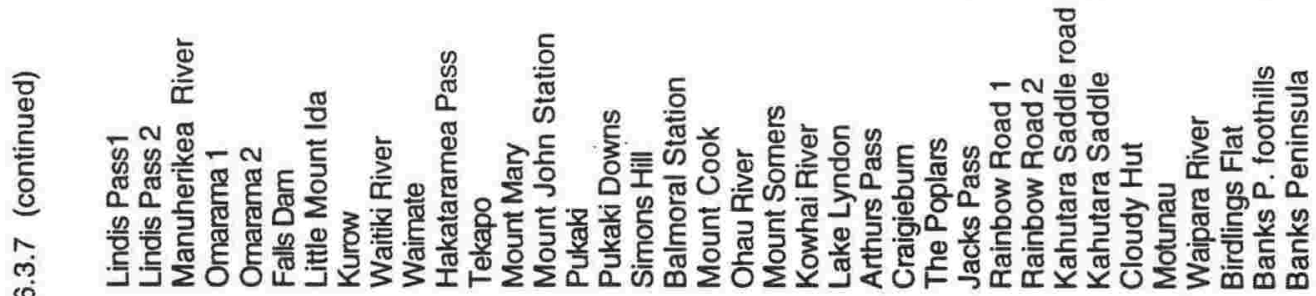

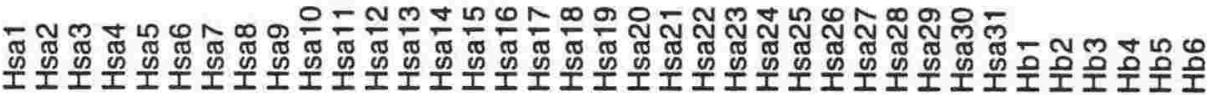




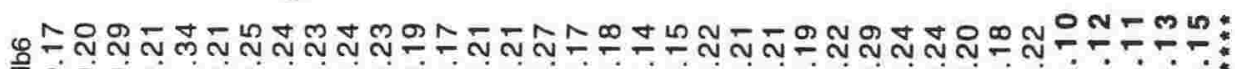
Dojo,

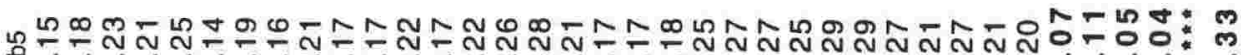

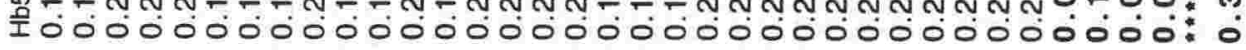

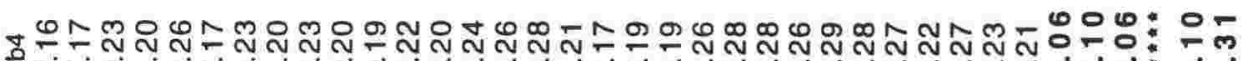

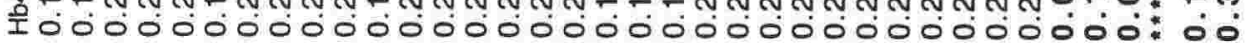

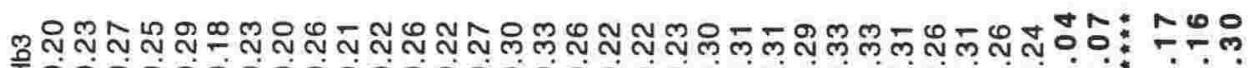
10000000000000000000000000000000 000

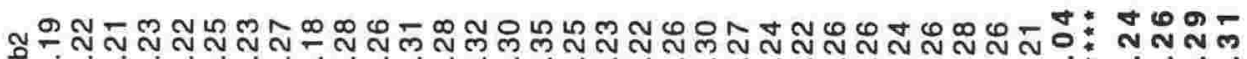

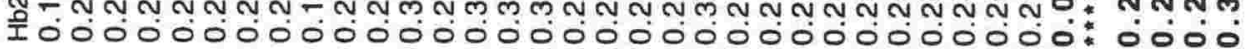
-

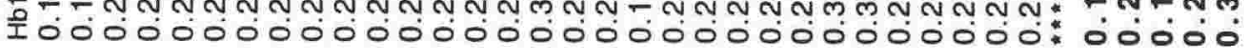

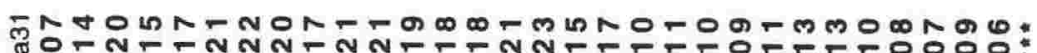
ת

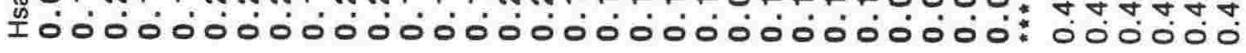

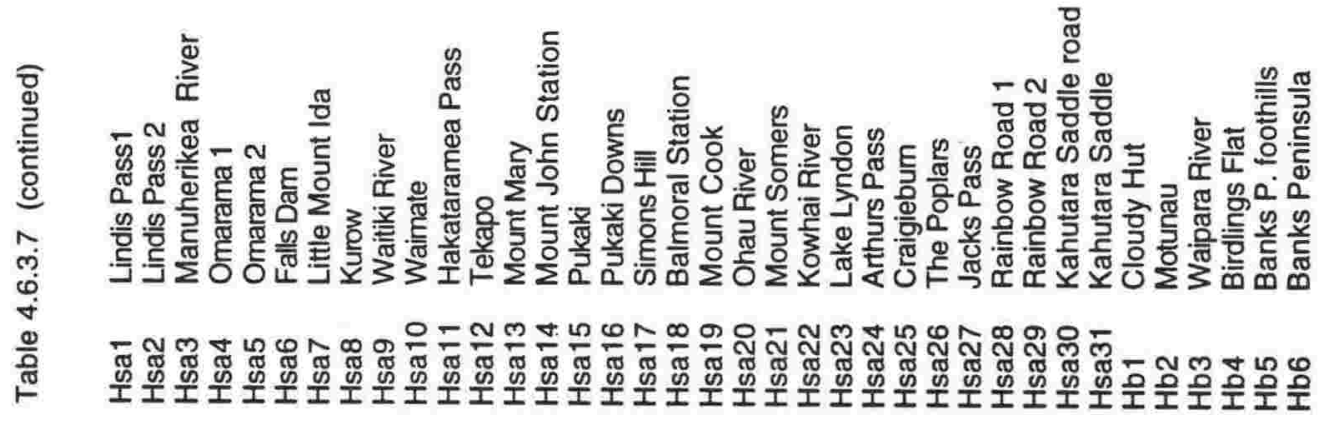




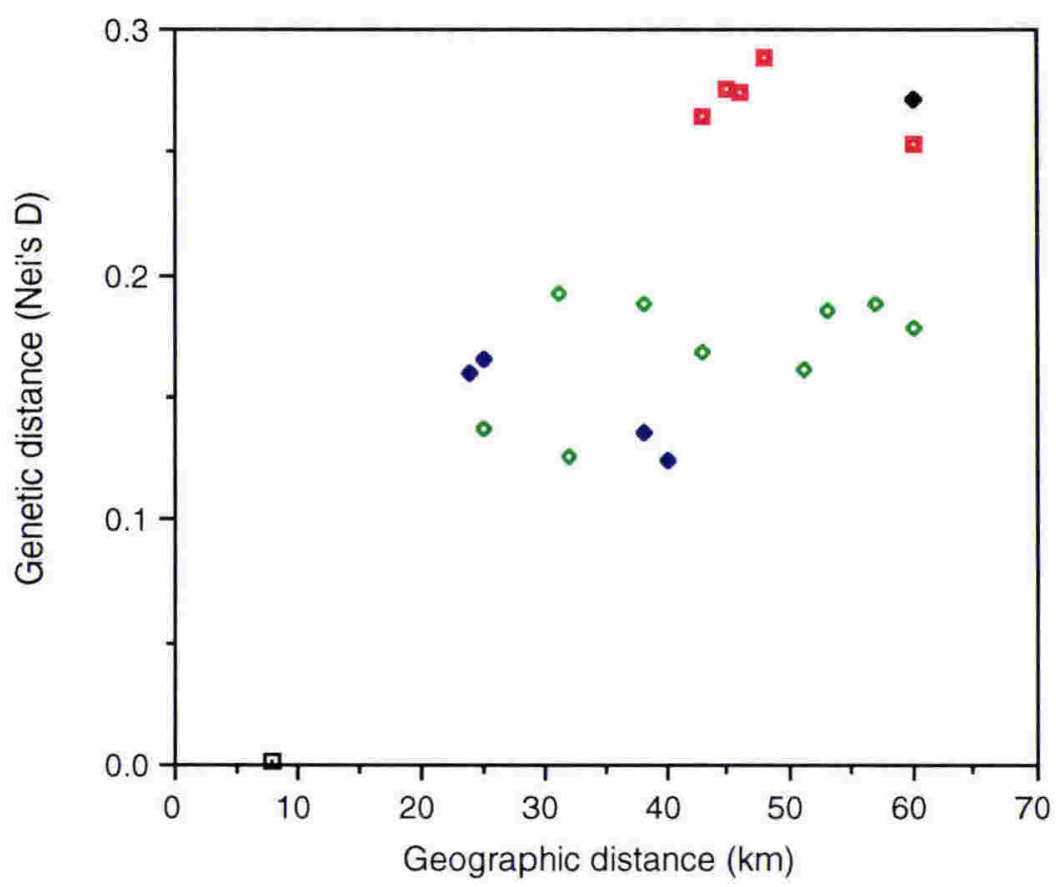

Figure 4.6.3.1.1 Relationship of genetic and geographic distances between pairs of populations within $H$. "Dansey's Pass" (open square), between $H$. "Dansey's Pass" and Kakanui-Horse Range populations of $H$. "Otago" (blue diamonds), between $H$. "Dansey's Pass" and Eastern Otago populations of $H$. "Otago" (red squares), between $H$. "Dansey's Pass" and Manorburn populations of $H$. "Otago" (black diamond), and between $H$. "Dansey's Pass" and Central Otago populations of $H$. "Otago" (green diamonds).

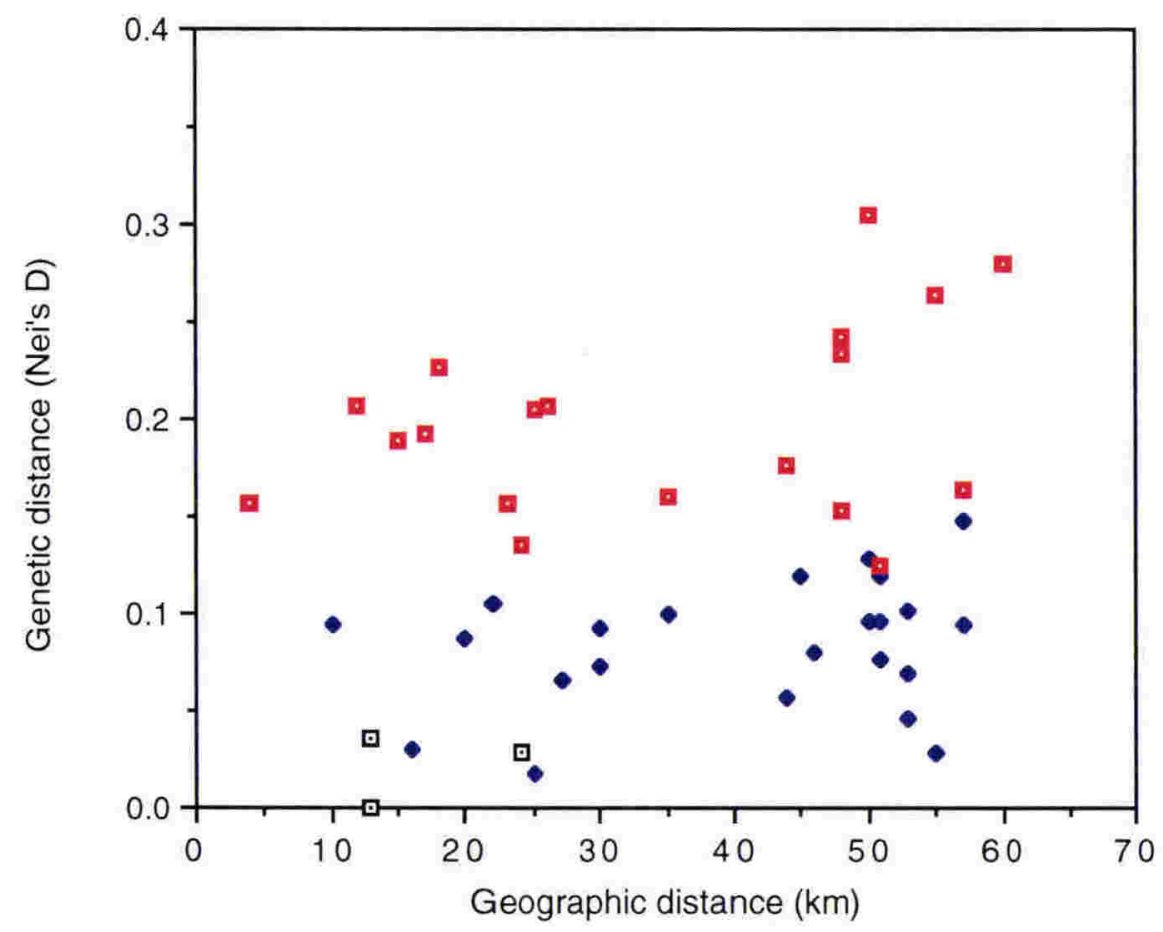

Figure 4.6.3.2.1 Relationship of genetic and geographic distances between pairs of populations within $H$. "Cromwell Gorge" (open squares), within western $H$. "Otago" (blue diamonds), and between $H$. "Cromwell Gorge" and western $H$. "Otago" (red squares). 


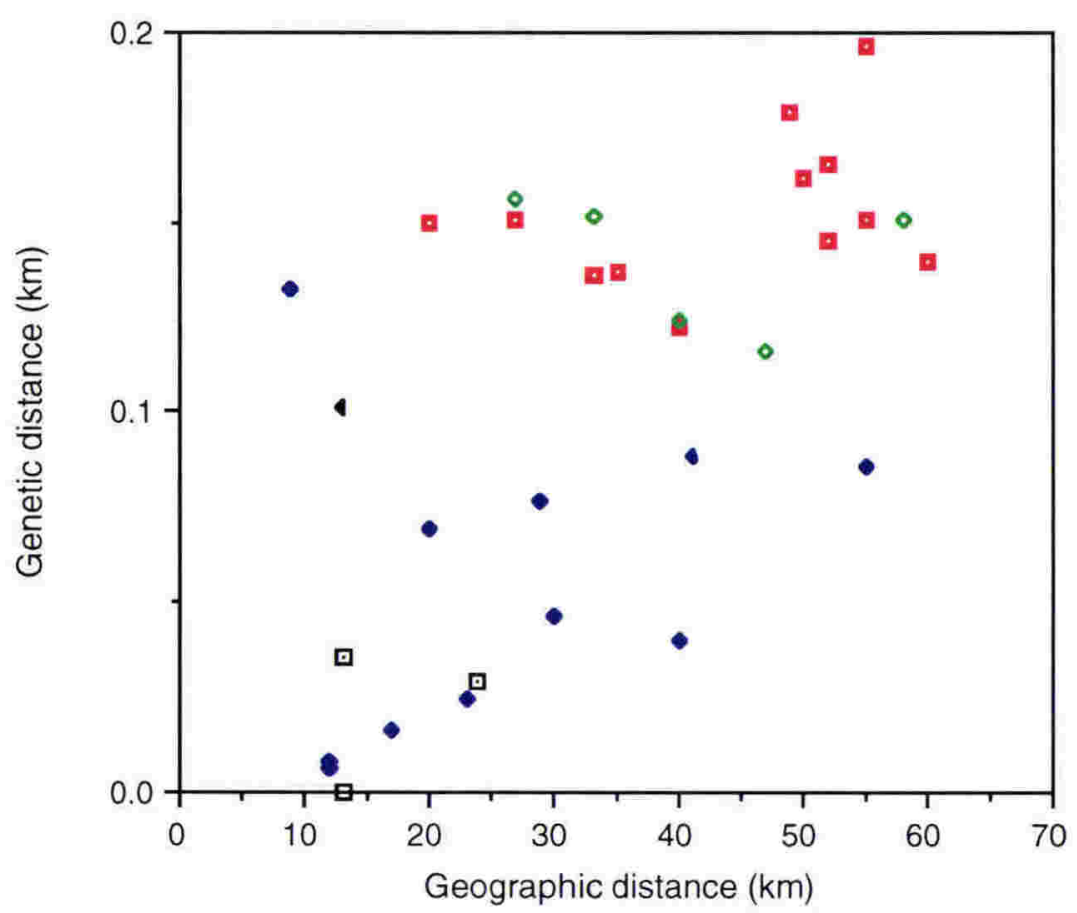

Figure 4.6.3.2.2 Relationship of genetic and geographic distances between pairs of populations within $H$. "Cromwell Gorge" (open squares), within Central Otago $H$. "Otago" (blue diamonds), between $H$. "Cromwell Gorge" and Central Otago H. "Otago" (red squares), within Manorburn $H$. "Otago" (black diamond), and between $H$. "Cromwell Gorge" and Manorburn H. "Otago" (green diamonds).

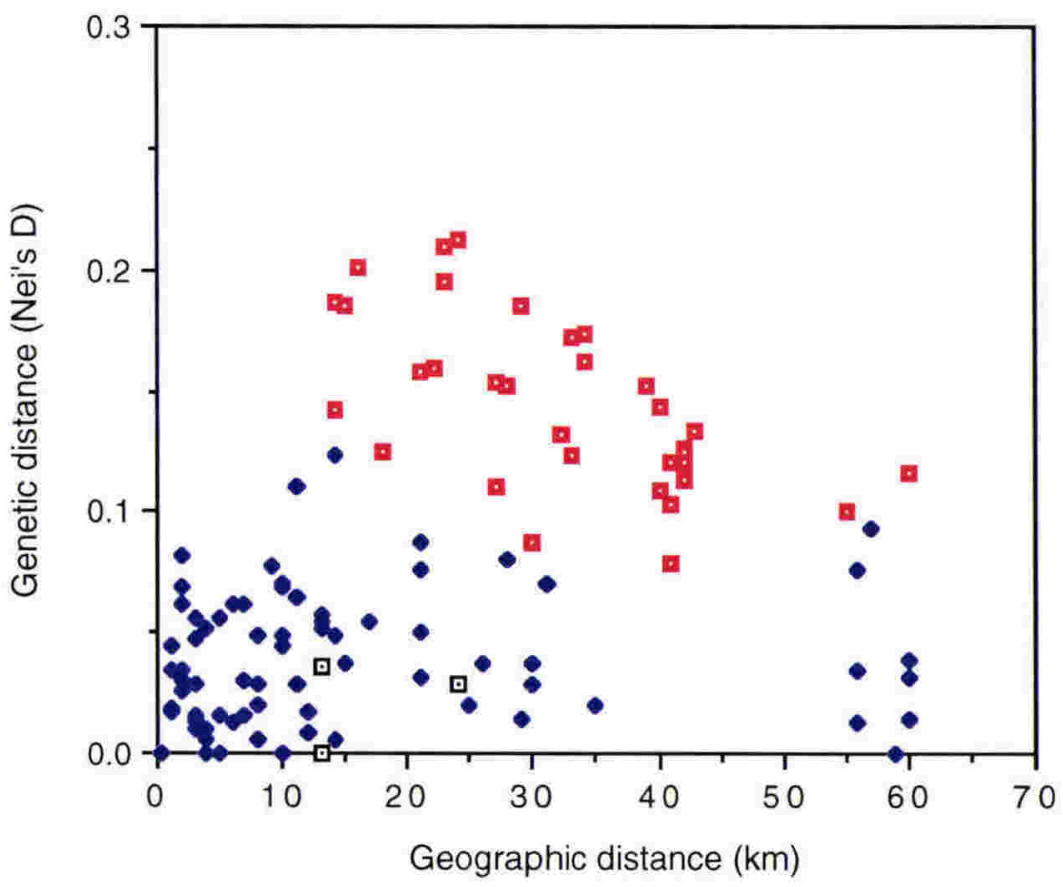

Figure 4.6.3.2.3 Relationship of genetic and geographic distances between pairs of populations within $H$. "Cromwell Gorge" (open squares), within Clutha Valley H. "Otago" (blue diamonds), and between $H$. "Cromwell Gorge" and Clutha Valley $H$. "Otago" (red squares). 


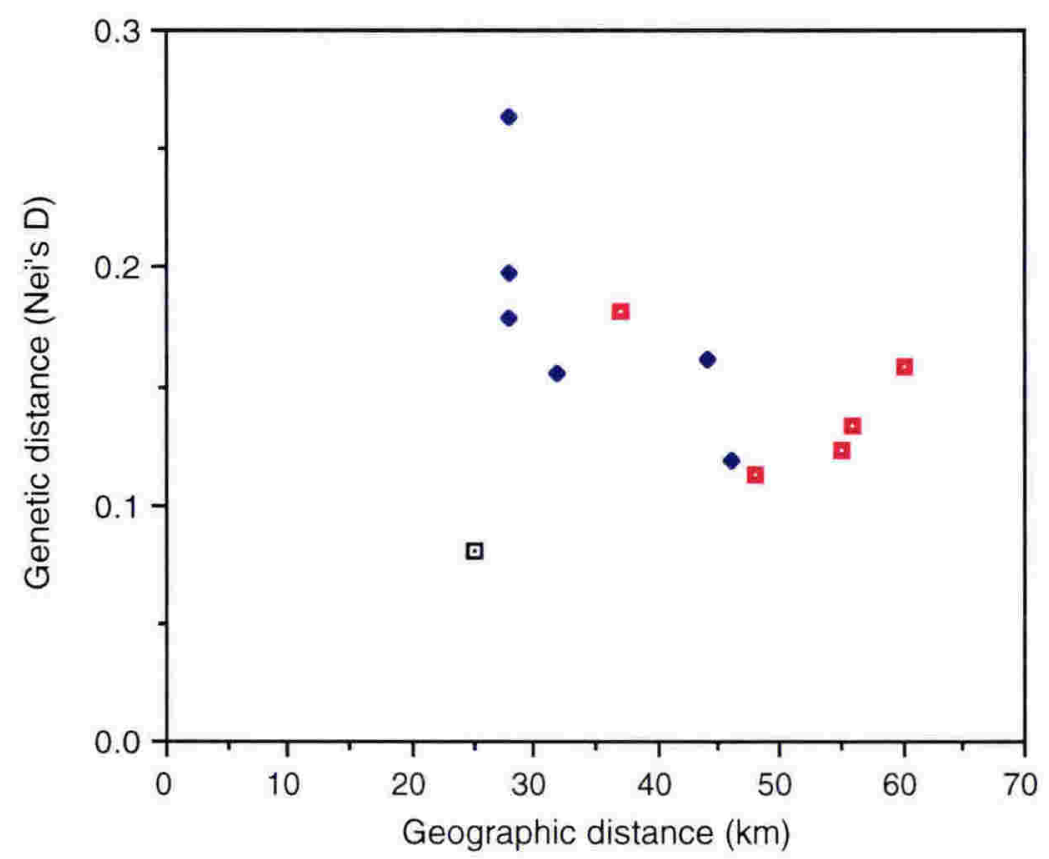

Figure 4.6.3.3.1 Relationship of genetic and geographic distances within H. "Otago", between pairs of populations within Kakanui/Horse Ranges (open square), between Kakanui/Horse Range and eastern Otago (blue diamonds), and between Kakanui/Horse Range and central Otago (red squares).

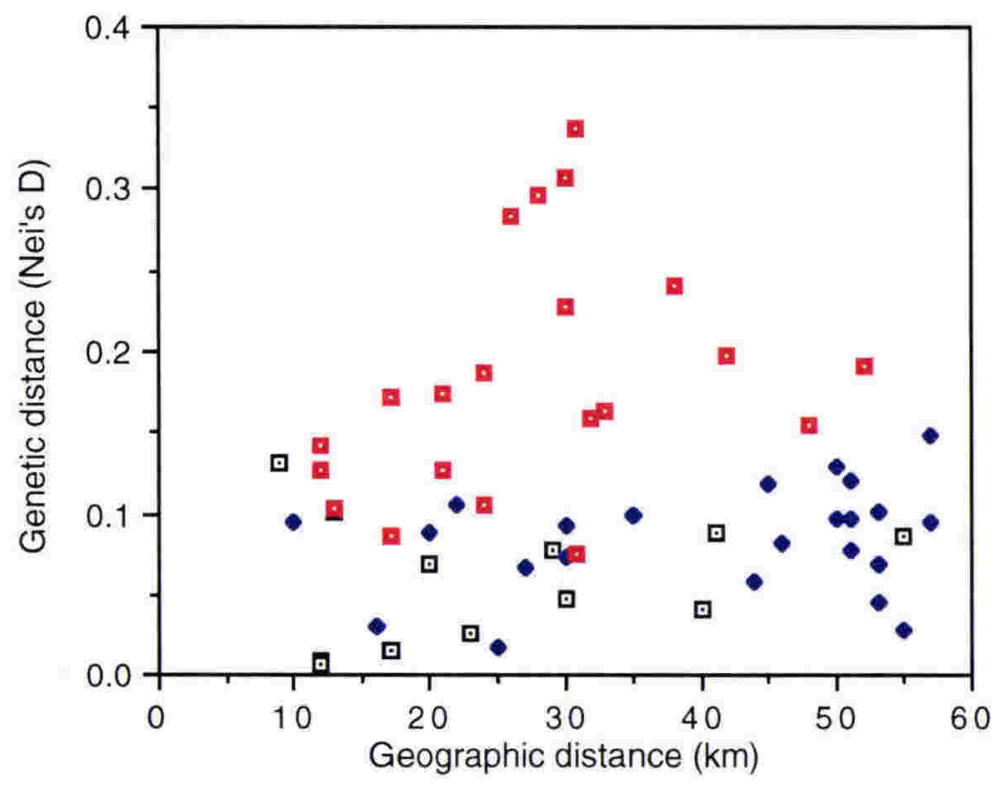

Figure 4.6.3.3.2 Relationship of genetic and geographic distances within H. "Otago", between pairs of populations within central Otago (open squares), between pairs of populations within western Otago (blue diamonds), and between central Otago and western Otago (red squares). 
Alexandra ( $3 \mathrm{~km}$ east) and Dunstan, and $\mathrm{D}=0.07$ between Alexandra ( $3 \mathrm{~km}$ east) and Kelvin Heights.

No abrupt geographic or genetic boundary separates Clutha Valley populations from the main group of Central Otago populations (Figure 4.6.3.3.4), as between-group pairwise distances overlap extensively with within-group distances, despite Clutha Valley populations forming a separate cluster in most analyses (Figures 4.6.3.19, 21, 23). The latter effect is probably partly an artefact of the high sampling intensity over small geographic distances in the Clutha Valley, whereas the rest of Central Otago was sampled more thinly.

The North Raggedys and Idaburn samples, both in the Central Otago group and separated by only $9 \mathrm{~km}$, have a genetic distance of $D=0.13$ between them, but there are lower genetic distances between both these populations and those further south on the Raggedy Range. The valley between these sites has alluvial soil without exposed rocks of any size, and has been heavily modified for agriculture. The genetic structure of this group of populations suggests the presence of gene flow around the head of the valley, about $40 \mathrm{~km}$ to the south.

In the Manorburn area, 2 samples were collected which were genetically very divergent from those from the rest of Central Otago, including the Clutha Valley (Figure 4.6.3.3.5, 4.6.3.3.6). The Manorburn Road and Galloway samples, only $6 \mathrm{~km}$ apart, were separated by $D=0.14$. However, some genetic distances to Clutha Valley populations were lower (e.g., $D=0.05$ between Alexandra lookout (Ho25) and Manorburn Dam (Ho22); Table 4.6.3.3; Figure 4.6.3.3.6).

Eastern populations of $H$. "Otago" were separated from Central Otago populations by $D>0.1$ over a range of geographic distances (Figure 4.6.3.3.7), except for a distance of $D=0.07$ between the Otago Peninsula and Glenavon populations, $55 \mathrm{~km}$ apart.

\subsubsection{H. brunneus and $H$. "Southern Alps"}

Populations north of the schist/greywacke boundary in north Otago are further subdividable into mainly montane ( $H$. "Southern Alps") and mainly lowland ( $H$. brunneus) forms. Though these have not been found syntopically, their ranges abut in the southern Kaikouras. Pairwise genetic 


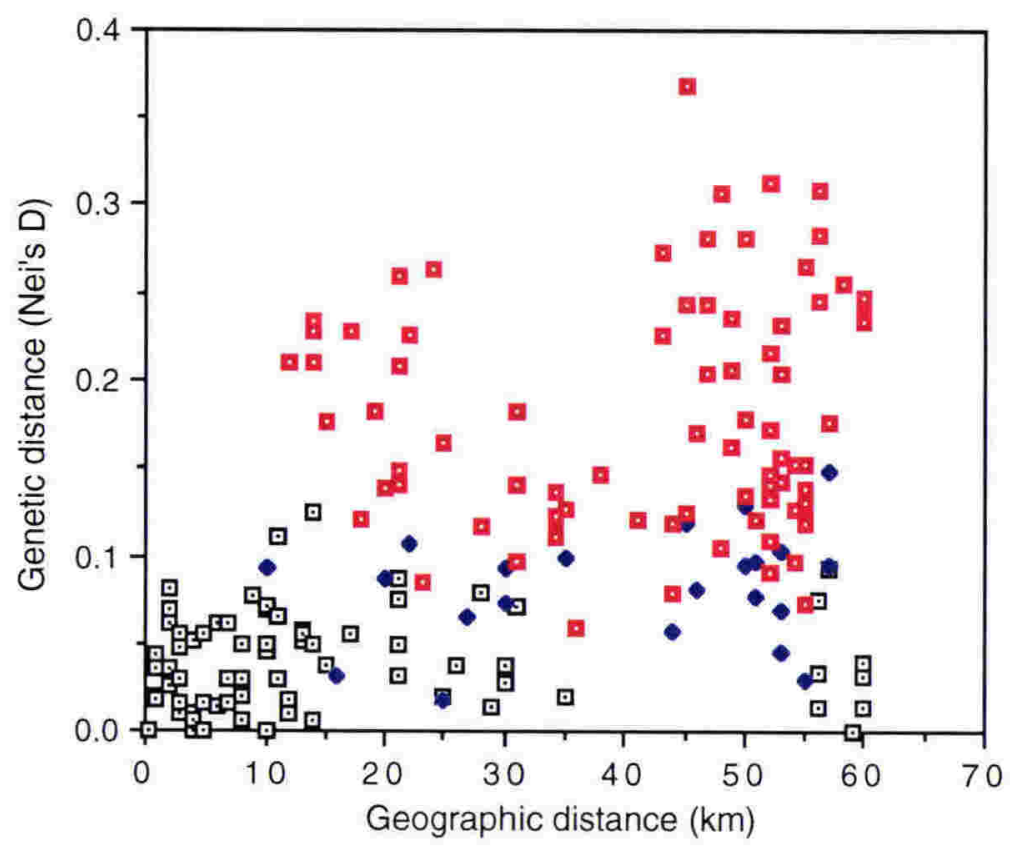

Figure 4.6.3.3.3 Relationship of genetic and geographic distances within $H$. "Otago", between pairs of populations within the Clutha Valley (open squares), between pairs of populations within western Otago (blue diamonds), and between Clutha Valley and western Otago (red squares).

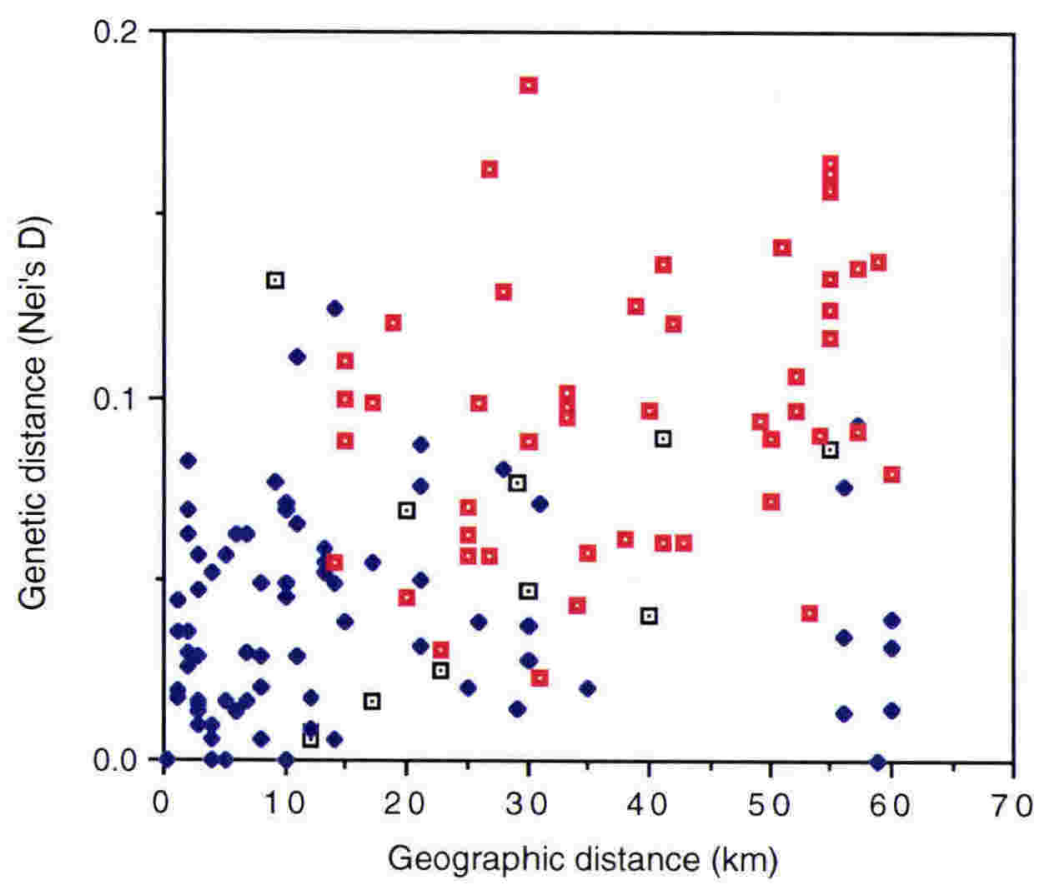

Figure 4.6.3.3.4 Relationship of genetic and geographic distances within $H$. "Otago", between pairs of populations within central Otago (open squares), between pairs of populations within the Clutha Valley (blue diamonds), and between Clutha Valley and central Otago (red squares). 


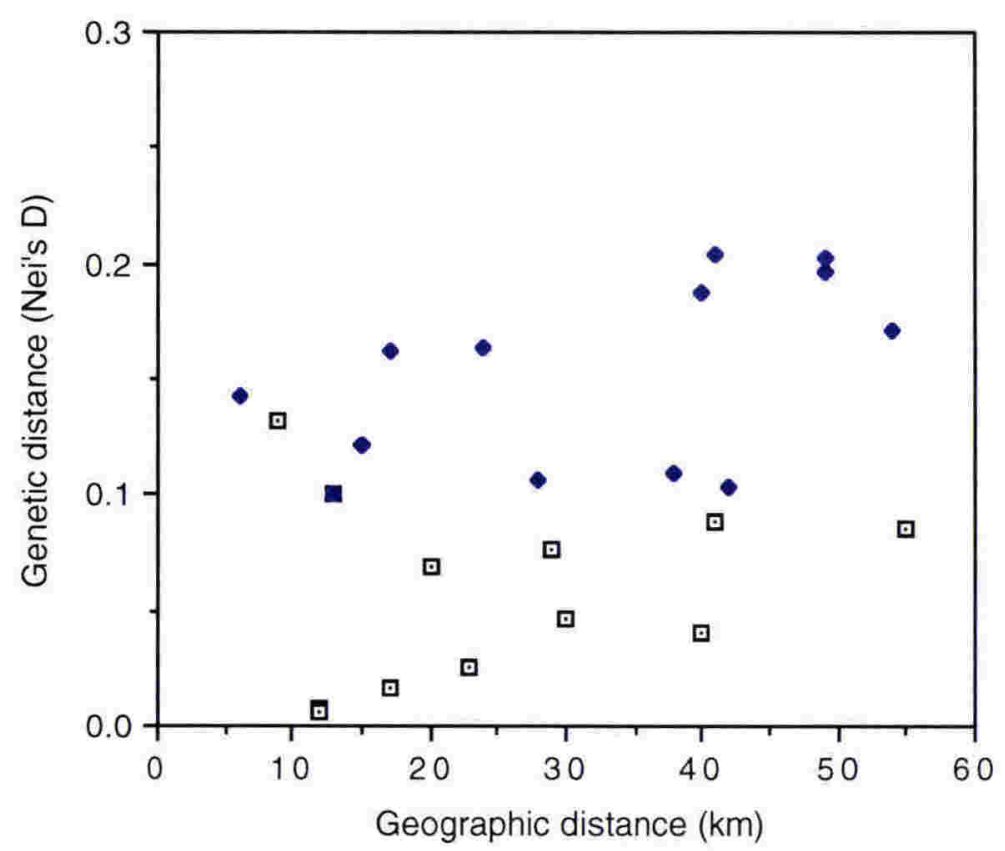

Figure 4.6.3.3.5 Relationship of genetic and geographic distances within $H$. "Otago", between pairs of populations within central Otago (open squares), between pairs of populations within Manorburn (blue square), and between Manorburn and central Otago (blue diamonds).

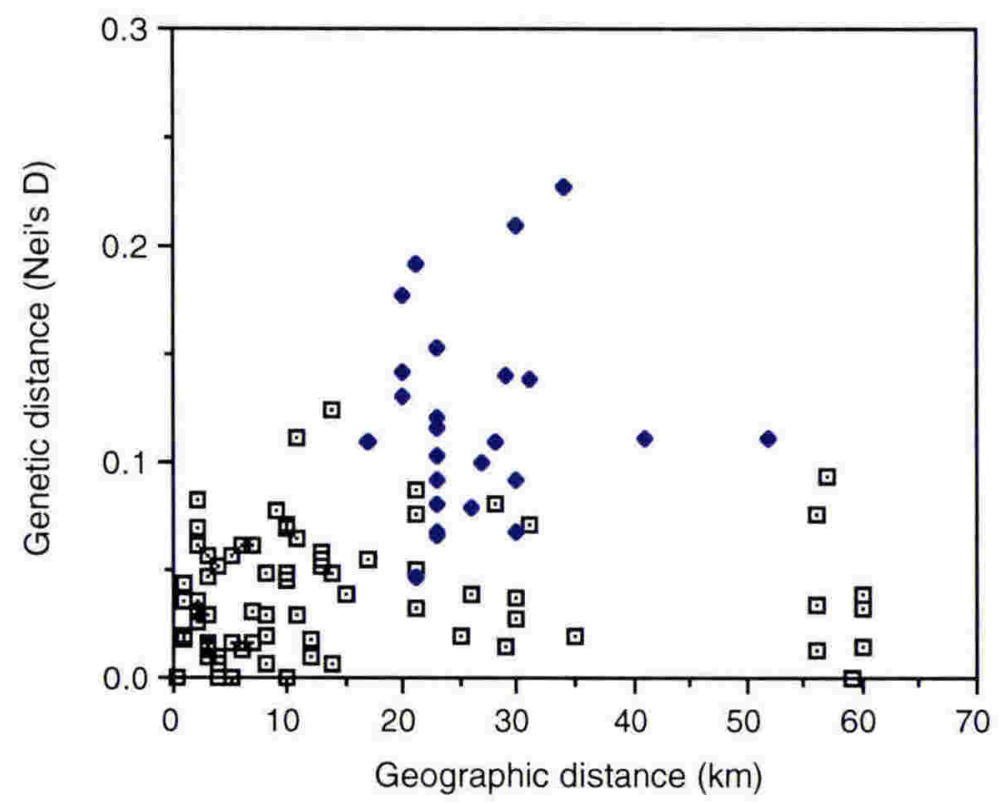

Figure 4.6.3.3.6 Relationship of genetic and geographic distances within H. "Otago", between pairs of populations within the Clutha Valley (open squares), and between Manorburn and the Clutha Valley (blue diamonds). 


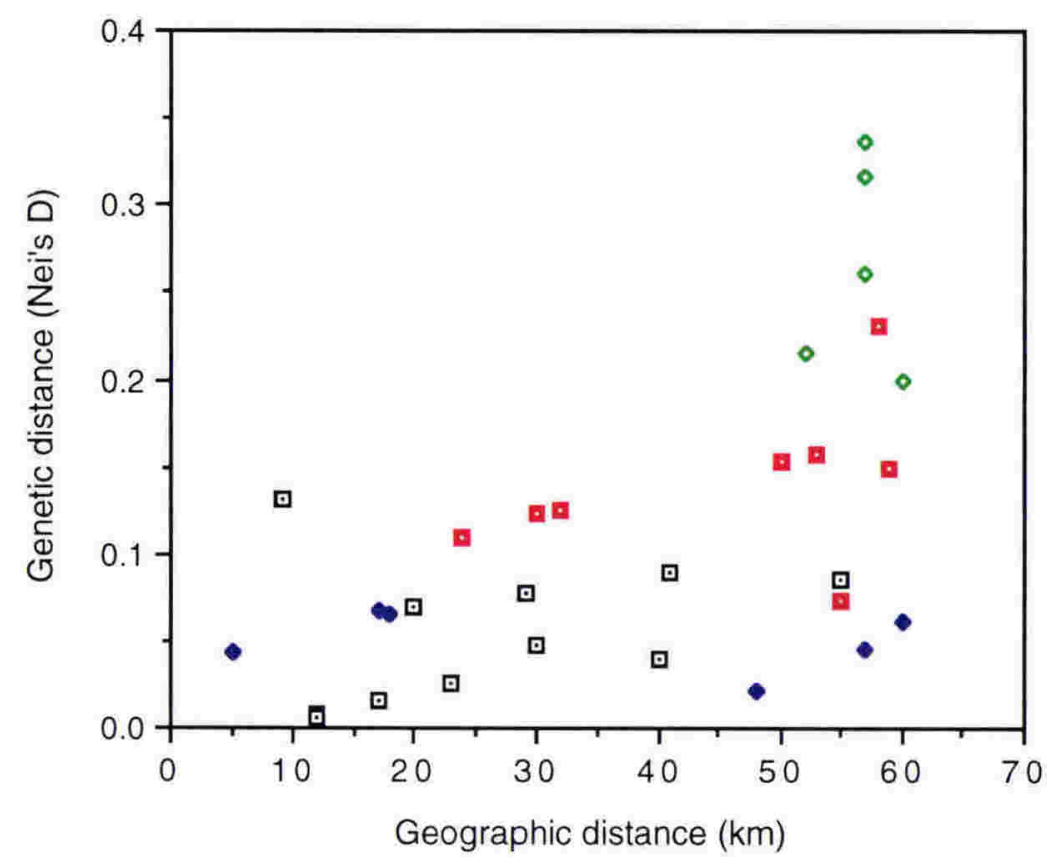

Figure 4.6.3.3.7 Relationship of genetic and geographic distances within $\mathrm{H}$. "Otago", between pairs of populations within central Otago (open squares), between pairs of populations within eastern Otago (blue diamonds), between pairs of populations between eastern and central Otago (red squares), and between pairs of populations between eastern Otago and Manorburn (green diamonds).

distances between populations of the 2 species are consistently higher than pairwise distances between populations separated by the same geographic distance (Figure 4.6.3.4.1) within species.

In the most southern part of the range of $H$. "Southern Alps", there is considerable genetic diversity over small geographic distances (Figure 4.6.3.4.2), but the geographic pattern of this variation was very complex. Both large and small-bodied forms from the McKenzie Basin fall within this group.

\subsubsection{H. duvaucelii}

There was little genetic diversity within $H$. duvaucelii (Tables 4.6.3.1-2, 4.6.3.5.1-2). The most divergent populations were those from the Trios Islands in Cook Strait (Figures 4.6.3.5.1-3), with a second split separating populations from the Brothers Islands in Cook Strait and the Mercury group off Coromandel Peninsula from the remainder of the northern populations. 


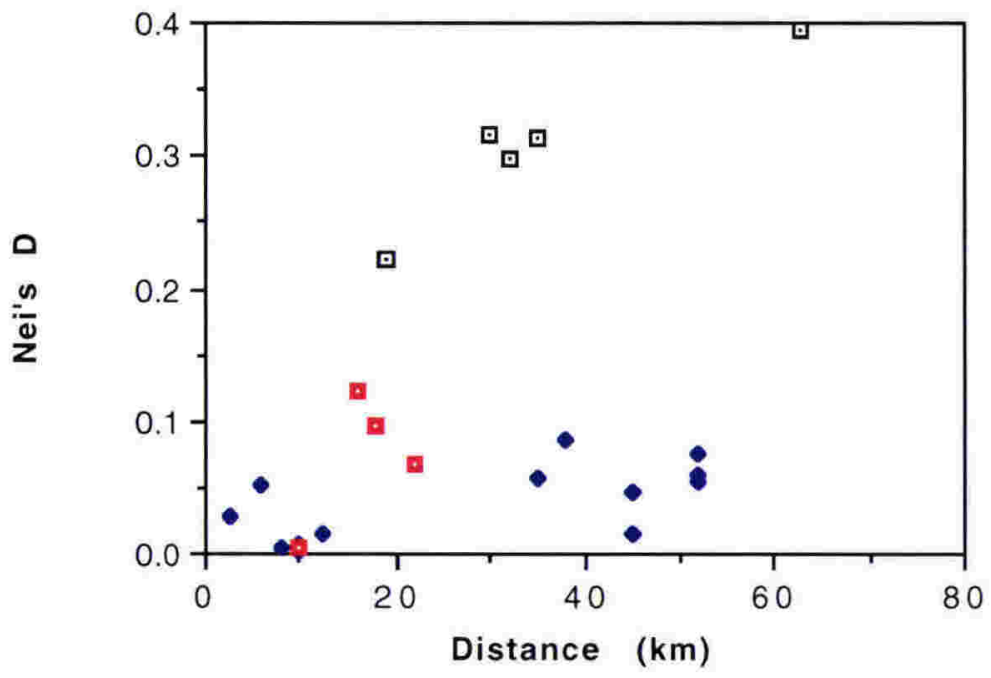

Figure 4.6.3.4.1 Scatter-plot showing relationship between genetic distance and geographic distance between populations within $H$. "Canterbury" (blue diamonds), within $H$. "Southern Alps" in the northern part of its range where it abuts $H$. "Canterbury" (red squares), and between these two species (open squares).

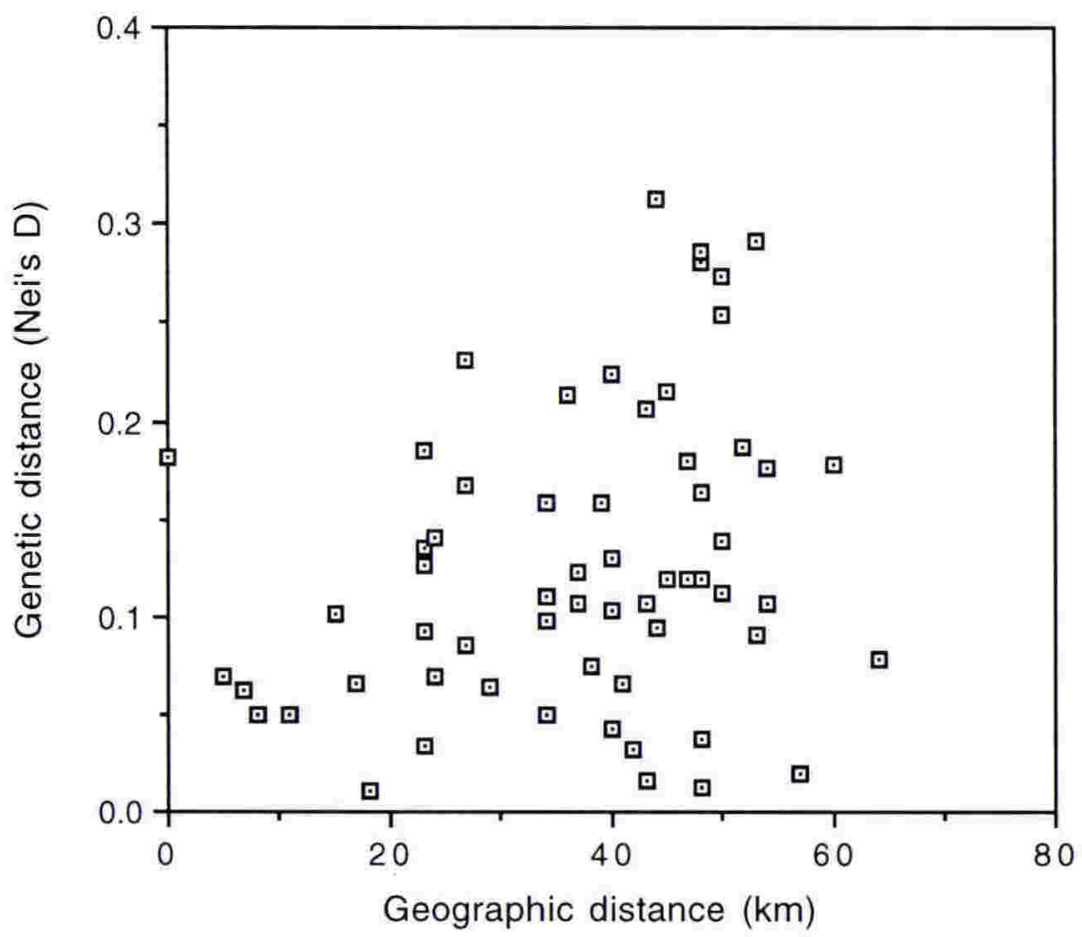

Figure 4.6.3.4.2 Scatter-plot showing relationship between genetic distance and geographic distance between populations within $H$. "Southern Alps". 


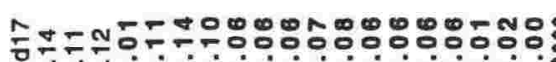

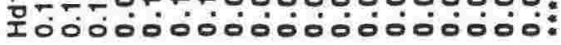

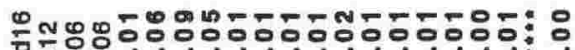

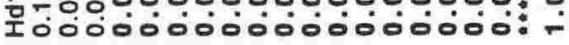

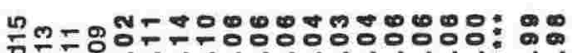

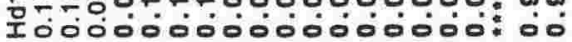

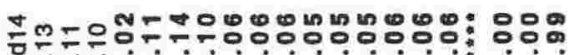

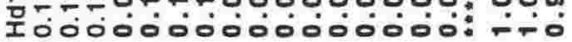

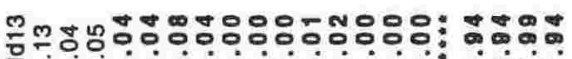

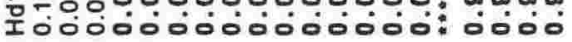

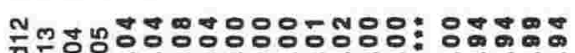
10000000000000 -0000

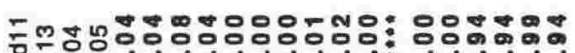
I0000000000001-0000

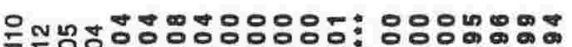

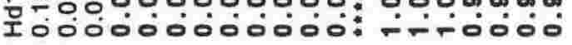

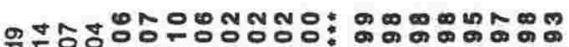
To0000000000 00000000

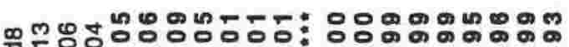

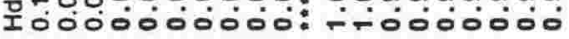

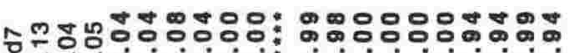

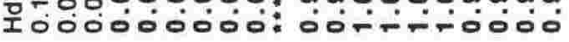

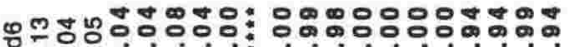
I00000000:-00-7-7000

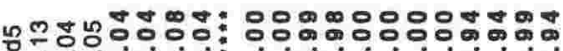
I0000000\%-00-7-0000

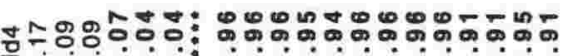
I000000: 0000000000000

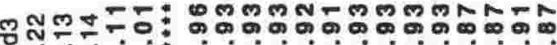
To0000: 0000000000000

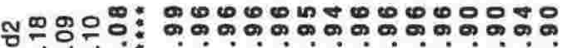
I0000: 0000000000000

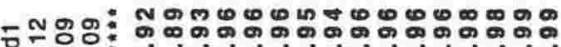

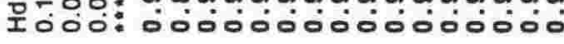

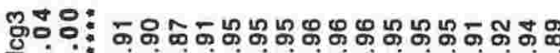

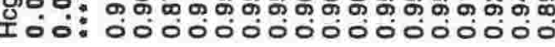
్ㅐㅇㅠ: 유. I0:-00000000000000000

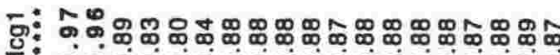
I: 0000000000000000000

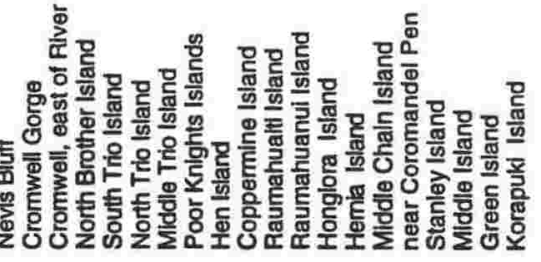

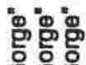

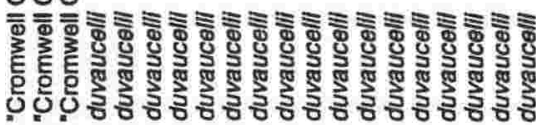

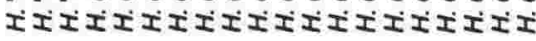

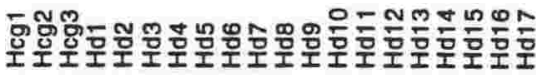

คิ๊

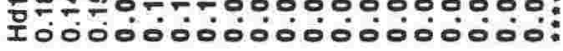

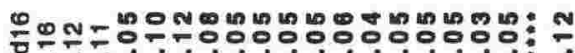

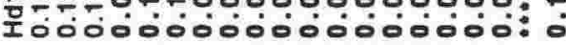

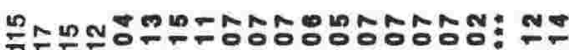

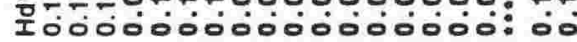

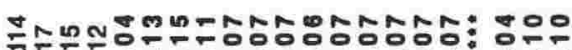

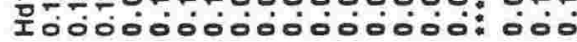

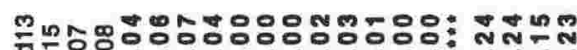

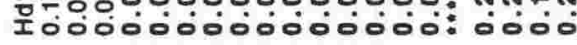

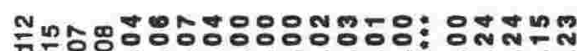
Tó000000000000:0000

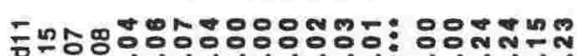

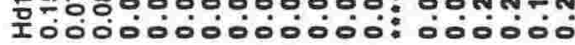

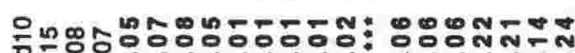

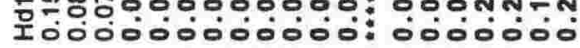

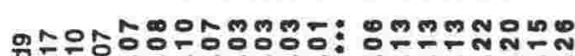

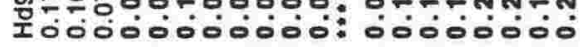

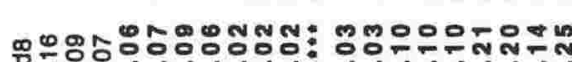

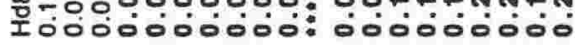

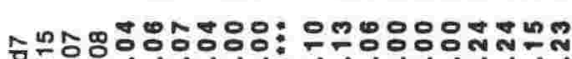

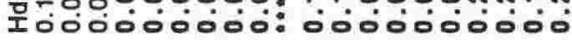

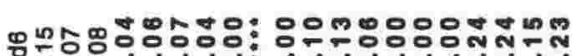

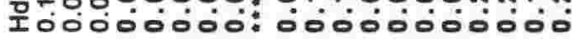

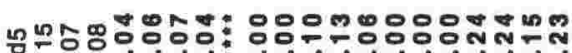
Tó0000 0000000000

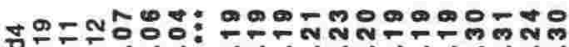

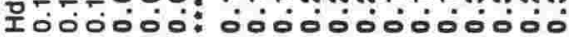

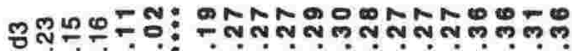
둥oㅇ 00000000000

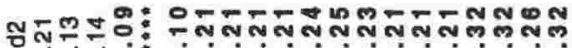

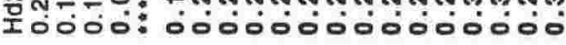
โ ผำำ ㅆำ

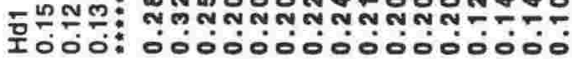

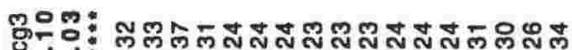

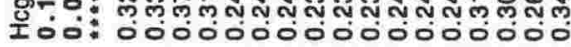

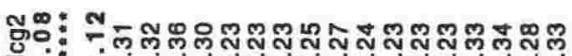

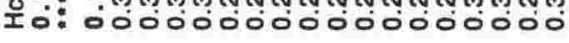

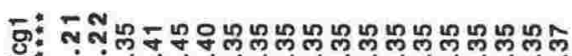
ํ.0000000000000000

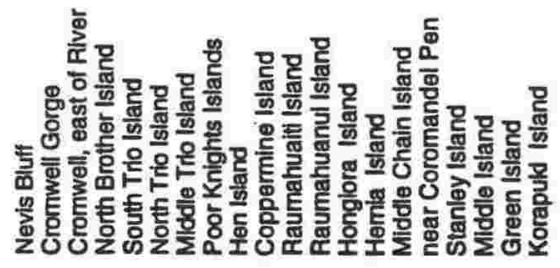

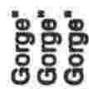

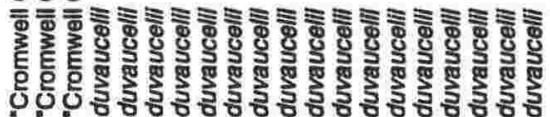

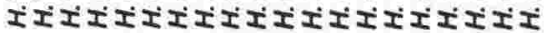

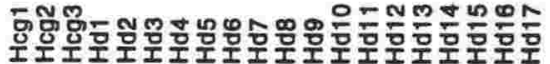




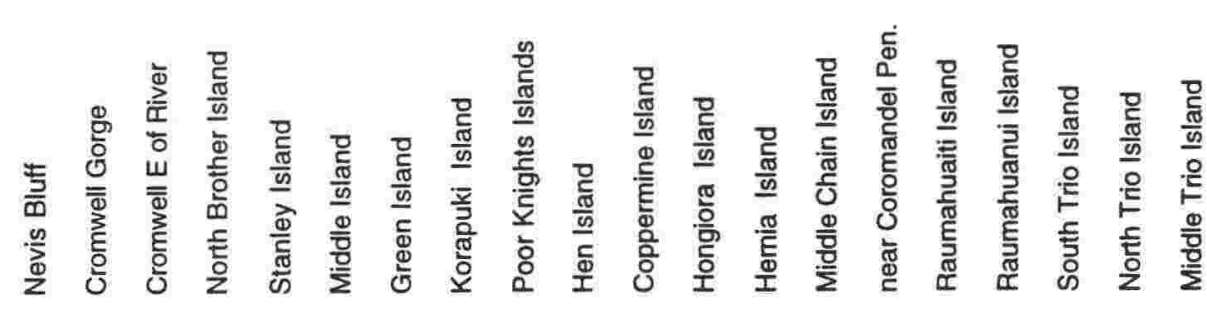

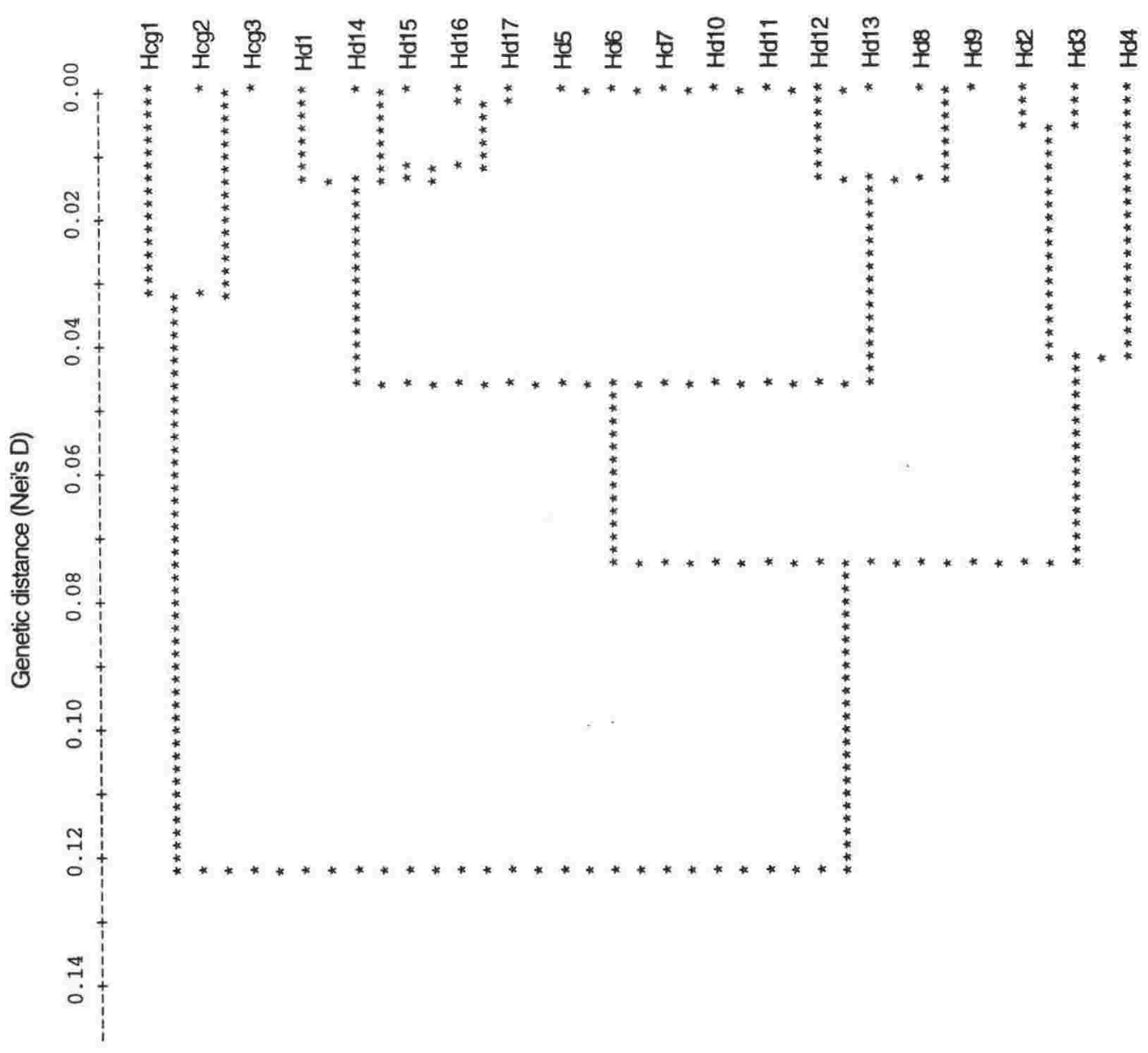



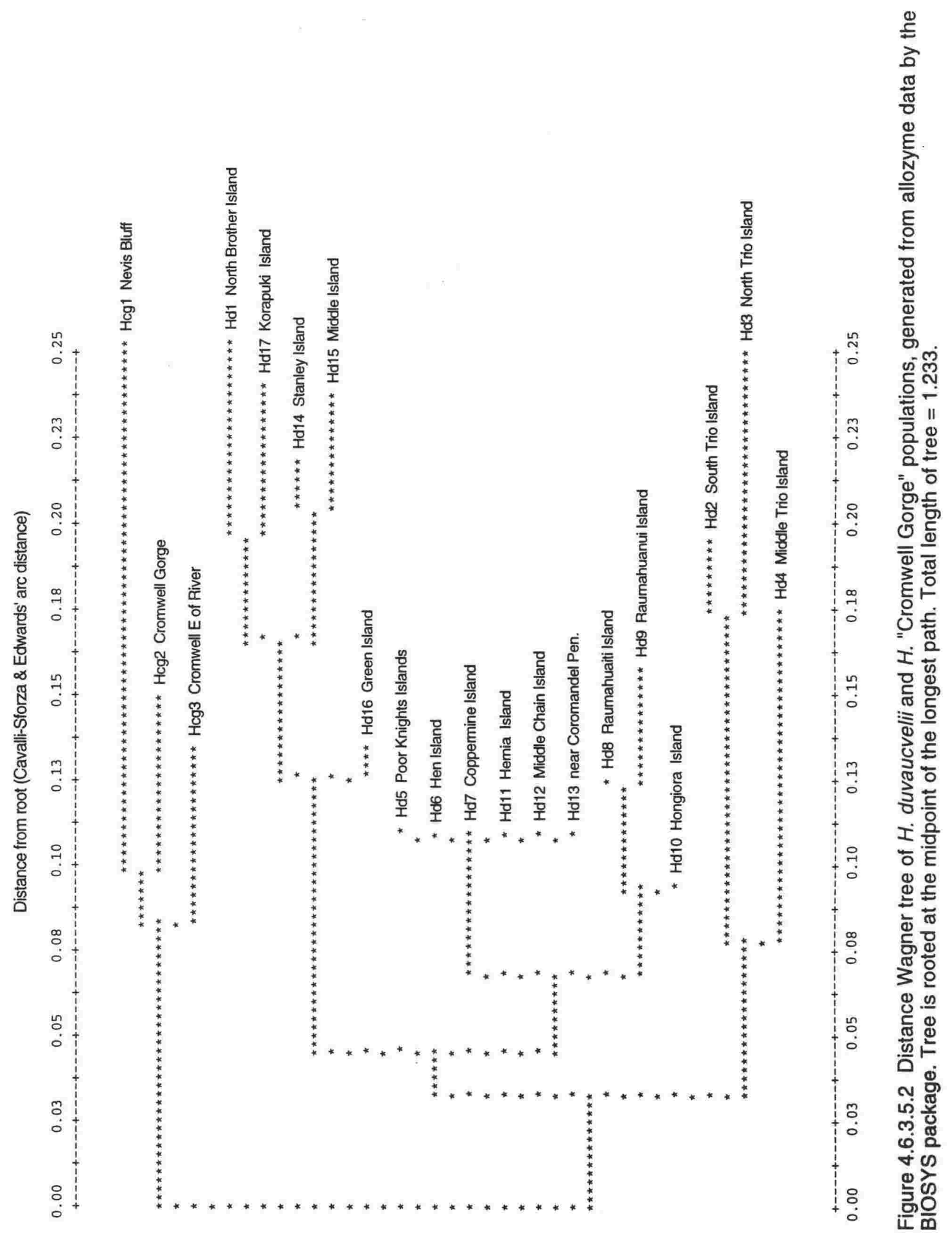


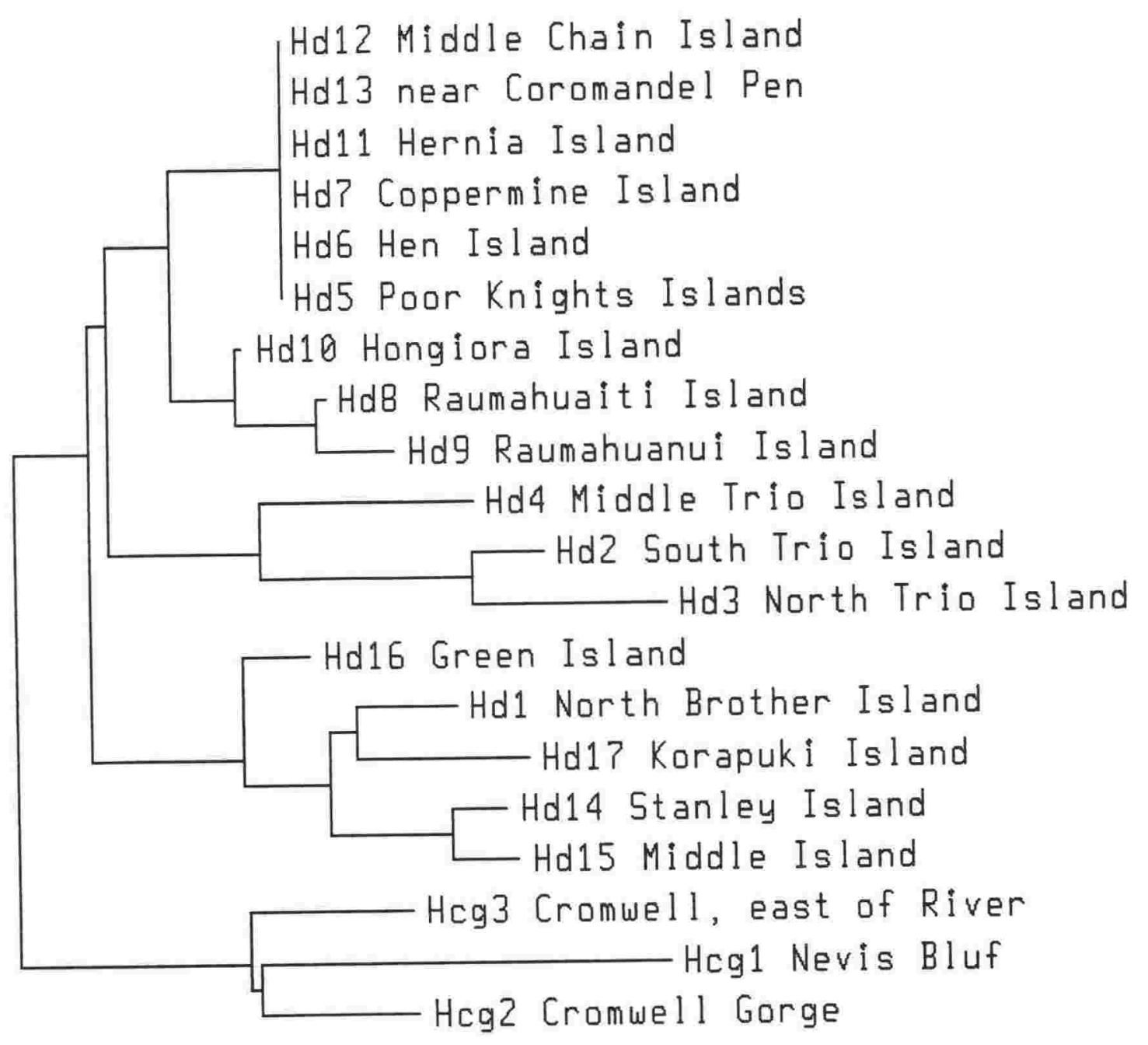

Figure 4.6.3.5.3 Neighbour-joining tree of $H$. duvaucelii and $H$. "Cromwell Gorge" populations from allozyme data (27 loci, Cavalli-Sforza \& Edwards' arc distance). 


\subsubsection{MORPHOLOGY}

Like allozyme variation, morphological variation among populations was complex and fine-grained, and this population-level variation generated "noise" which made diagnostic differences among species or regional groups of populations difficult to detect. For example, within $\mathrm{H}$. "Cromwell Gorge", specimens from Nevis Bluff in the Kawarau Gorge and a museum specimen (NMNZR342) from the Shotover Valley had the nostril broadly in contact with the rostral scale (as does the genetically similar $\mathrm{H}$. duvaucelii, but no other $H$. maculatus complex species), whereas specimens from the Cromwell Gorge had the nostril and rostral separated by contact between the first nasal and first upper labial scales. Within the eastern Otago population group of $H$. "Otago", inland populations had 12-16 (usually 1315) upper labial scales, whereas the genetically similar Otago Peninsula population had 10-12. Most of the size variation in $H$. brunneus is between, rather than within, populations (Freeman 1993). A phylogenetic species concept would use these population-level diagnostic differences to define many new species. Statistical analysis of population-level morphological variation was beyond the scope of this thesis.

The most immediately obvious variable concordant with species and population clusters identified by allozyme analysis is body size, and there were some instances of considerable size differences over small geographic distances between populations across group boundaries (Figure 4.6.4.1). Other morphological characters useful in discriminating individual pairs of proposed species are number of rows of femoral pores, ratio of tail length to snout-vent length, toe shape (degree of dilation of sub-digital pads, relative length of the distal phalanges, and whether the distal phalanges narrow abruptly from the subdigital pad or taper from the end of the pad), eye colour, body colour, presence or absence of speckling on the belly, and tendency to dorso-ventral flattening (Table 4.6.4.1). However, none of these characters are diagnostic autapomorphies at the species level.

\subsubsection{DISCUSSION}

The higher levels of heterozygosity and polymorphism detected in the southern group of the $H$. maculatus complex than in other New Zealand geckos increase the potential for sampling artefacts in small samples. This increases the likelihood of falsely large genetic distances between 


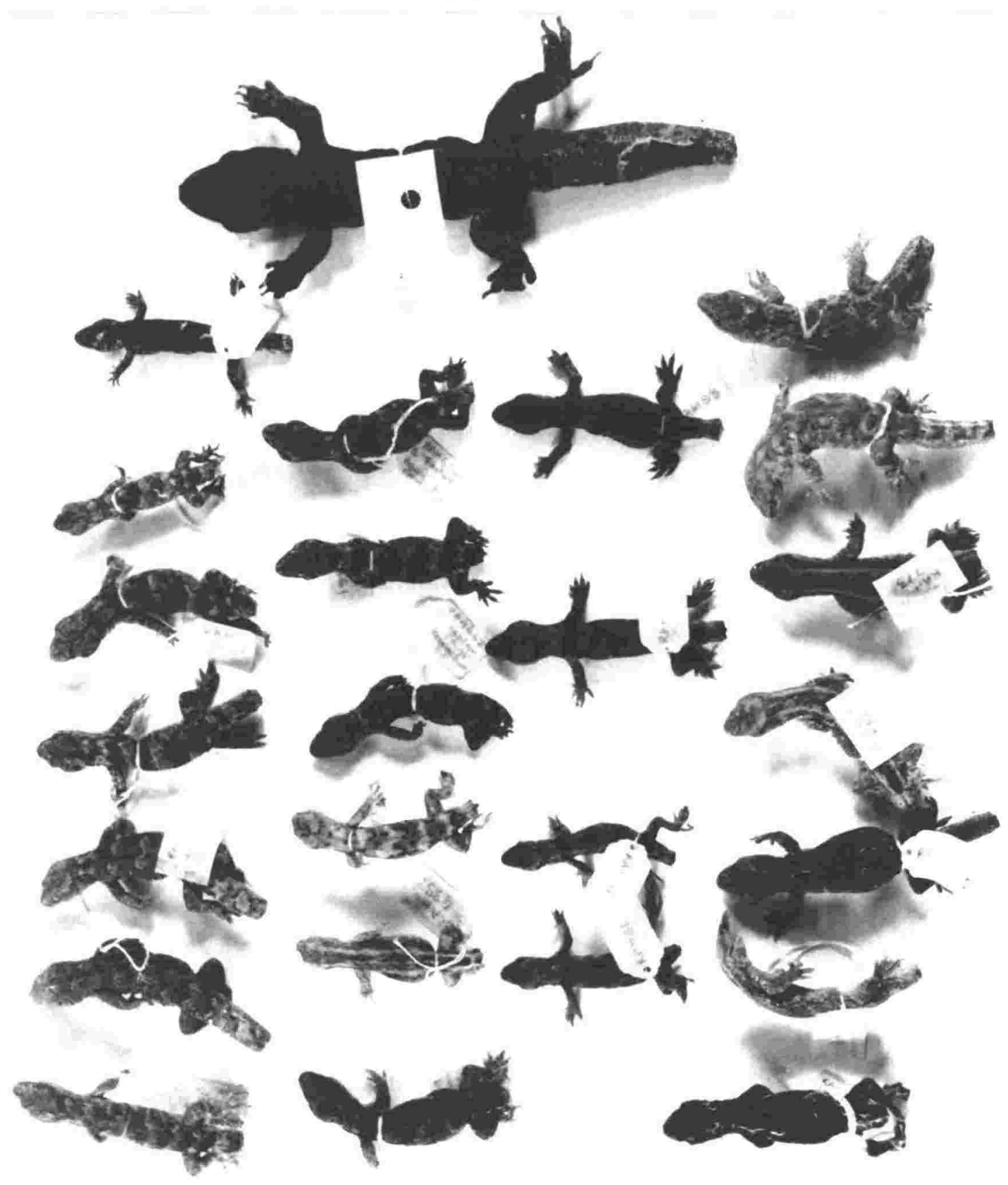

Figure 4.6.4.1 Representative, sexually mature examples of Otago populations of the $H$. maculatus complex. Top, $H$. duvaucelii, FT179; left (top to bottom), $H$. "Cromwell Gorge": RAH61, Nevis Bluff; RAH444, Cromwell Gorge; H. "Otago": RAH457, Dunstan Range 2; RAH438, Waikerikeri; RAH274, Tree Island, Lake Wakatipu; RAH175, Gorge Burn; RAH481, Te Anau; left of centre (top to bottom), H. "Otago": RAH454, Becks; RAH461, south Raggedy Range; RAH462, Manorburn Road; RAH470, Butchers Dam; RAH435, Alexandra west; RAH419, Old Man Range 3; right of centre (top to bottom), $H$. "Otago": RAH53, Idaburn; RAH115, Glenavon; RAH51, Roxburgh; RAH 31, Beaumont; right (top to bottom), $H$. "Danseys Pass": RAH316, Kyeburn Diggings; RAH304, Danseys Pass; $H$. "Otago": RAH273, Horse Range; RAH164, Taieri Ridge; RAH132, Billy Ridge; RAH149, Otago Peninsula, RAH339, Ruapuke islet. 


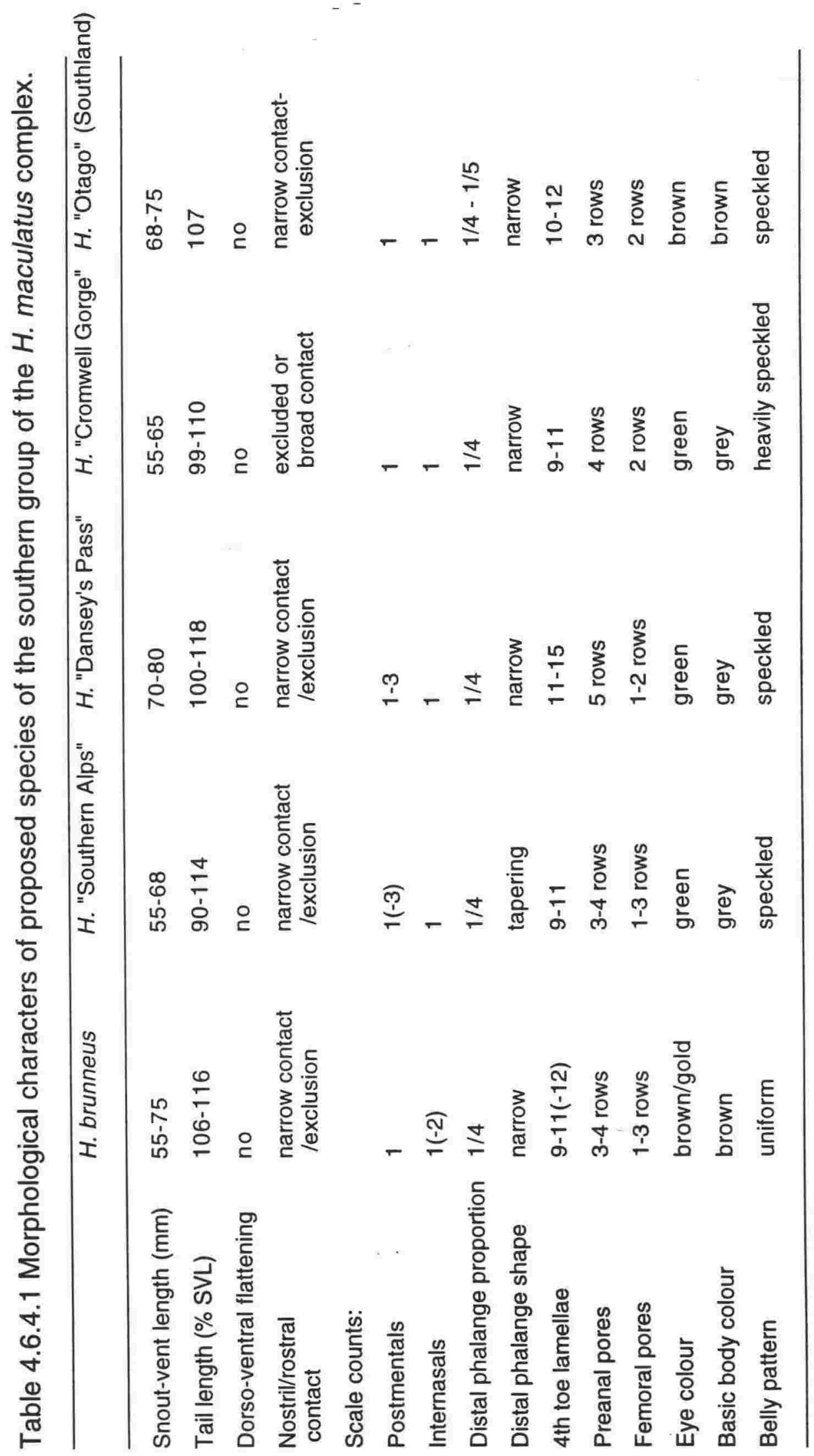




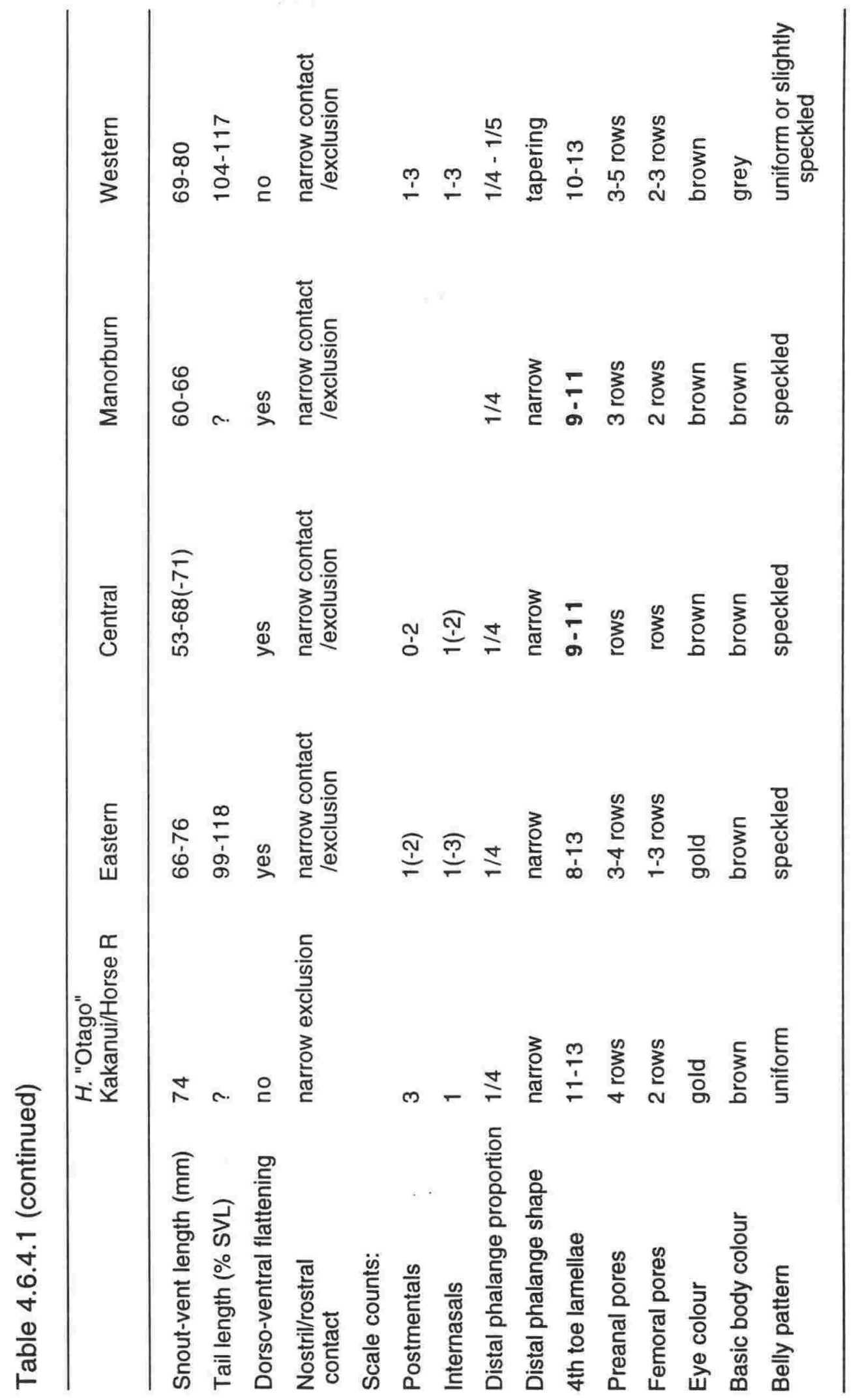


inadequately sampled populations, and of inaccurate recovery of phylogeny (Archie et al. 1989). However, there were 21 samples of $\geq 5$ individuals (= $\geq 10$ alleles) from this group.

As discussed in Section 4.1.2, the value Nei's $D=0.15$, which has been quoted as a value above which pairs of allopatric populations are unlikely to be conspecific (e.g., Thorpe 1983; Highton 1989) must be considered in conjunction with the mix of rates of evolution in the particular loci included in each study (Sarich 1977). For example, fast-evolving esterase loci are often scored in allozyme studies, but could not be scored in this study because of great variability at 2 or more overlapping loci. The exclusion of these fastevolving loci will have "scaled down" the observed rate of evolution.

Genetic distances between the most closely related sympatric taxa in the New Zealand gecko fauna as a whole provide a benchmark at which specific differences are likely in this group, measured by genetic distances generated from this particular set of allozyme loci. Sympatric or adjacent $H$. "Marlborough mini"/H. "Kaikouras", H. pacificus/H. "Matapia", and $H$. granulatus/H. kahutarae are all separated by Nei's $D$ of $0.08-0.11$. Despite its current northern distribution, $H$. duvaucelii falls phylogenetically within the southern group of the $H$. maculatus complex, and is separated from some Otago populations by very low genetic distances (e.g., Nei's $D$ between Cromwell Gorge $H$. "Cromwell Gorge" and several $H$. duvaucelii populations is 0.05 ). As the $40-120 \mathrm{~g} \mathrm{H}$. duvaucelii is very unlikely to be able to interbreed naturally with the 4-5 g H. "Cromwell Gorge", and is clearly on a separate evolutionary trajectory, the 2 must be separate species despite the close similarity of their allozymes.

Georges \& Adams (1996) presented a conservative method for drawing taxonomic conclusions from allozyme data. Populations or groups of populations sharing at least one allele at all assayed loci are sequentially combined for analysis until groups separated by diagnostic fixed differences are obtained. These groups may contain individual pairs of populations separated by fixed differences if they can be linked by mutual sharing of alleles with other, polymorphic populations. By these criteria, $H$. duvaucelii plus the entire $H$. maculatus complex form a single diagnosable taxon. This approach clearly fails to describe adequately taxonomic diversity in this group. 
The problems of interpreting allozyme data for the southern group of the $\mathrm{H}$. maculatus complex do not arise from lack of differences between proposed species (all $D \geq 0.08$, supported by morphological differences), but from large allozyme differences within species. I have taken the conservative approach in this project that geographically adjacent populations should only be separated taxonomically where there is clear evidence for absence of gene flow across a boundary zone. Analysis of further, independent datasets is required when allozyme variation does not identify terminal clades of geographically circumscribed and/or morphologically distinct clusters of populations. Even very large genetic distances between populations need not imply separate species status if, for example, they are at the extremes of a cline of intermediate populations.

The deepest genetic separation in the southern group is between parapatric populations north and south of a line in North Otago closely associated with the geological boundary between schist rocks to the south and greywacke to the north. There are no entirely fixed differences diagnostic of these 2 groups because of the high degree of intragroup heterogeneity, but there is no evidence of gene flow across the boundary. Individual populations separated by small geographic distances across this boundary are morphologically distinct, and have 3 or more fixed allozyme differences between them.

The group of populations north of the schist/greywacke boundary is further subdividable into mainly montane and mainly lowland forms, which differ in colour, head shape, ecology, and physiology (Tocher 1992, 1993). Though these forms have not been found syntopically, their ranges abut in the southern Kaikouras. Pairwise genetic distances between populations of the 2 species are consistently higher than pairwise distances between populations within species separated by the same geographic distance (Figure 4.6.3.4.1). They are therefore considered to be separate species, $H$. "Southern Alps" and $H$. brunneus.

H. "Southern Alps" as I have defined it in this study clearly contains undiagnosed species in the southern part of its range; one specimen from Tara Hills, Omarama, differed at 4 loci from the other 8 specimens in the sample, but was morphologically indistinguishable, and both groups fell within the general cluster of $H$. "Southern Alps" in phenograms (Figures 4.6.3.20, 4.6.3.22, 4.6.3.24). The population at Little Mount Ida differed at 2 
loci from neighbouring populations, and was also distinguished by smaller body size and shorter distal phalanges than its neighbours, but again fell within the general cluster of $H$. "Southern Alps" populations. Many pairs of populations only small distances apart geographically were separated by relatively large genetic distances (Figure 4.6.3.4.2). The apparent paraphyly of $H$. "Southern Alps" with respect to $H$. brunneus probably also reflects the presence of these undiagnosed species.

South of the Schist-Greywacke boundary, the deepest separation is between $H$. "Cromwell Gorge" and the rest. $H$. "Cromwell Gorge", a very small species, is most closely allied with the very large $H$. duvaucelii. Bones resembling those of $H$. duvaucelii, now confined to offshore islands from Cook Strait north, have been found in subfossil deposits throughout New Zealand, including Otago (T. Worthy, Palaeofaunal Surveys, pers. comm.), so the 2 were formerly sympatric or nearly so.

The range of $H$. "Cromwell Gorge" is completely surrounded by that of $H$. "Otago", although the 2 appear to be separated altitudinally and have not to date been found syntopically. Museum specimens indicate that both are probably widespread in the area between Lake Wanaka, the Dunstan Range, and Lake Wakatipu, but no specimens from this area were available for my study. The 2 are very different in size; western populations of $H$. "Otago" weigh 2-3 times more than H. "Cromwell Gorge". Genetic distances between the 2 groups are consistently higher than those within groups, independent of geographic distance between them, indicating absence of gene flow, and therefore separate species status.

Samples from Kyeburn Diggings and Danseys Pass, $15 \mathrm{~km}$ apart, were morphologically and genetically indistinguishable $(D=0.00)$ from each other, and separated by genetic distances of $D>0.13$ and by morphological differences from all neighbouring populations, justifying specific status as $H$. "Danseys Pass".

The remaining populations in Southland and Otago south of the schistgreywacke boundary are considered a single species, $H$. "Otago", in this analysis. However this species is subdivided into 6 geographic clusters with allozyme and morphological differences between them, sometimes across quite abrupt boundaries: (1) the Kakanui and Horse Ranges, (2) eastern Otago east of the Taieri River, (3) Central Otago including the Clutha Valley, 
(4) Manorburn, (5) western Otago, and (6) Southland-Foveaux Strait. Examination of DNA variation would be highly desirable to provide more information on the significance of this separation, but I consider that the present evidence does not quite justify further formal subdivision of this group. The relationship between genetic variation and reported geographic variation in reproductive cycles in $H$. "Otago" (Cree 1994) also requires further investigation.

Both the Manorburn and Kakanui/Horse Range groups are pairs of adjacent but genetically disparate populations; each showed genetic distances between the samples within the group which are almost as large as those to neighbouring groups.

Samples from the Manorburn group (Manorburn Road and Manorburn Dam) consisted of somewhat larger animals than those from neighbouring populations, and were separated from neighbouring populations and each other by large genetic distances over short geographic distances in apparently continuous habitat (Figures 4.6.3.3.5, 4.6.3.3.6). The 2 Manorburn populations differed genetically by $D=0.10$.

Single specimens from Mount Pisgah (Kakanui Mountains) and the Horse Range differed by $D=0.08$. They were most closely allied to eastern populations of $H$. "Otago", but were separated from that group by $\mathrm{D}>0.10$.

In the eastern Otago group, samples from Otago Peninsula and Taieri Ridge, $60 \mathrm{~km}$ apart but both east of the Taieri River, are more similar in allozymes ( $D=0.061$ ) than those from Billy Ridge (eastern Otago group) and Glenavon (Central Otago group) $(D=0.110)$, both near Middlemarch and only $16 \mathrm{~km}$ apart, but on opposite sides of the Taieri. Specimens from east of the river are larger and less speckled than those from Central Otago, and usually have bright yellow, rather than brown or brownish yellow, eyes.

Western and Central Otago populations of $H$. "Otago" meet at the Manuherikia Valley and the eastern slopes of the Old Man Range. Western populations are distinguished by large body size, high scale counts, broadly dilated toe pads, and very short distal phalanges. They have been recorded from near Lake Te Anau, the Eglinton Valley, the Eyre Mountains, Tree Island in Lake Wakatipu, The Remarkables, Kelvin Heights, the Slate Range, the Old Man Range, and the Dunstan Mountains. The specimen from the top 
of the Old Man Range considered by Robb (1982) to represent a new species is morphologically typical of this group. However, all samples from that range which I have analysed are closest to the Central Otago group in both allozymes and morphology, although a few specimens are larger, possibly indicating gene flow from western populations.

Samples from an islet near Ruapuke Island, Foveaux Strait, and the Hokonui Range, Southland, were genetically identical to each other, and roughly equidistant between the eastern and Central Otago population groups. However, there is a large area between these localities and the eastern and Central Otago groups from which I have no samples.

\subsection{THE H. GRANULATUS COMPLEX}

\subsubsection{INTRODUCTION}

H. granulatus (sensu Thomas 1981), H. kahutarae, and H. rakiurae form a close group in phylogenetic analyses of allozyme data (Chapter 6).

H. granulatus has been recognised since 1845 (Chapter 4). McCann (1955) described Heteropholis nebulosus from offshore islands around Stewart Island, but it has had a taxonomically unstable history since then. Robb (1980a) included $H$. nebulosus in Hoplodactylus in her popular monograph, while retaining its separate identity. Thomas (1981) comprehensively examined the set of characters traditionally used in gecko taxonomy, and concluded that there were no significant morphological differences between Hoplodactylus granulatus and McCann's Heteropholis nebulosus. He reduced the latter to synonymy with the former. $H$. granulatus (sensu Thomas 1981 ) is found over most of the country (Pickard \& Towns 1988).

The other 2 related species are far more recent discoveries with limited distributions in inaccessible habitats. Thomas (1981) described $H$. rakiurae from southern Stewart Island, and Whitaker (1984b) described $H$. kahutarae from high altitude rock bluffs in the Kaikoura Ranges. Since its discovery $H$. kahutarae has been regarded as very distinctive, with no obvious close relationship with any other recognised species (Whitaker 1984b, Bauer 1990).

Both Thomas (1982) and Robb (1982) referred to the existence of a possible undescribed species of Hoplodactylus known from a single preserved specimen from the Open Bay Islands, off the West Coast of the South Island 
(Robb referred to it only as from the West Coast). There is still only the one, poorly preserved specimen available, but further individuals have recently been photographed.

My phylogenetic analysis of allozyme results (Chapter 5 and below), consistently placed $H$. kahutarae within $H$. granulatus sensu Thomas (1981), and revealed substantial genetic divergence among populations of $H$. granulatus. Taxonomic reassessment of this variation, and of the status of $H$. nebulosus and $H$. kahutarae, was therefore necessary. $H$. rakiurae, although closely related, always fell just outside the cluster of $H$. granulatus populations plus $H$. kahutarae (see Chapter 5), so was not included in this analysis.

\subsubsection{SAMPLE COLLECTION}

Allozyme results were obtained from 27 specimens from 12 populations of the $H$. granulatus complex. $H$. granulatus proved extremely difficult to collect because of its cryptic, arboreal, nocturnal lifestyle. Despite considerable search effort, most of the samples whose localities are mapped in Figure 4.7.2.1 were obtained via amateur collectors, sample sizes are very small, some samples have no locality data, and geographic coverage is very patchy.

\subsubsection{ALLOZYME RESULTS}

Electrophoresis (27 loci) revealed variation at 15 loci in the $H$. granulatus complex, including $H$. nebulosus and $H$. kahutarae (Table 4.7.3.1). Polymorphic loci were as close to Hardy-Weinberg equilibrium within populations as could be expected given the mostly very small sample sizes; there was no evidence for cryptic species within samples (Tables 4.7.3.1 \& 2).

The single specimen of $H$. nebulosus (from Zero Rock) had fixed differences from all $H$. granulatus at $I c d-1$ and $I c d-2$. There were at least 4 fixed differences between this individual and any single $H$. granulatus population. The geographically closest population from which electrophoretic samples were available, from Ngahere ( $\mathrm{Hg} 9$ ), was separated from $\mathrm{H}$. nebulosus by $\mathrm{D}$ $=0.24$ (Table 4.7.3.3) (6 fixed differences), but the genetically most similar $H$. granulatus samples were from Reefton and Trass Valley $(D=0.17 ; 4$ fixed and 2-3 frequency differences). 


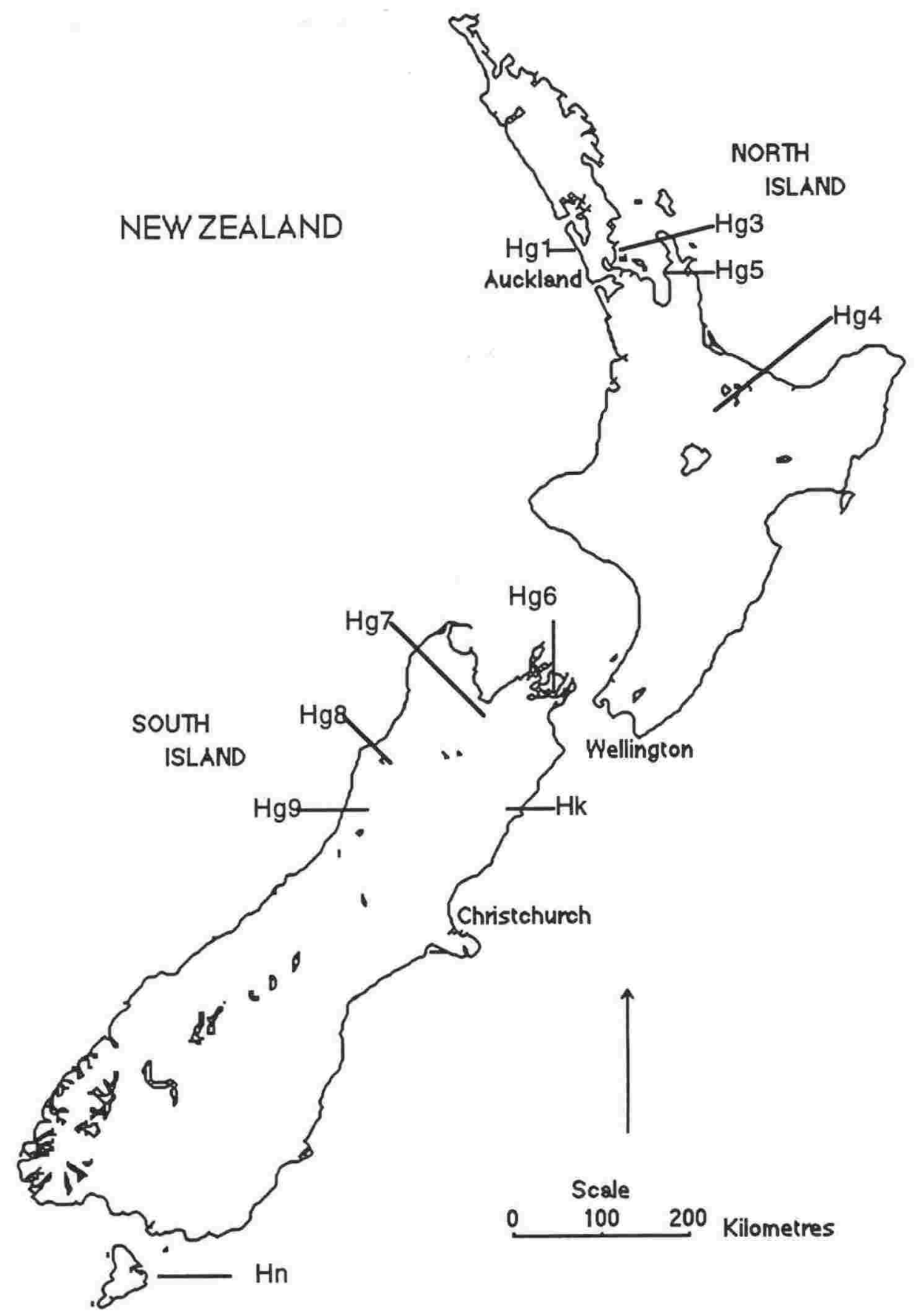

Figure 4.7.2.1 Collecting localities for samples of the $\mathrm{H}$. granulatus complex.

Hg1 H. granulatus

Hg2 H. granulatus

Hg3 H. granulatus

$\mathrm{Hg} 4 \quad H$. granulatus

Hg5 H. granulatus

Hg6 H. granulatus
Bethels Beach

?1 (captive collection)

Northcross

Rotorua

Coromandel?

Maud Island
$\mathrm{Hg} 7 \mathrm{H}$. granulatus Trass Valley, Nelson

Hg8 H. granulatus

Hg9 H. granulatus

Hg10 H. granulatus

Hn $H$. nebulosus

Hk H. kahutarae
Reefton

Ngahere

?2 (captive collection)

Zero Rock 
Table 4.7.3.1 Allele frequencies at variable allozyme loci in populations of the $H$. granulatus complex.

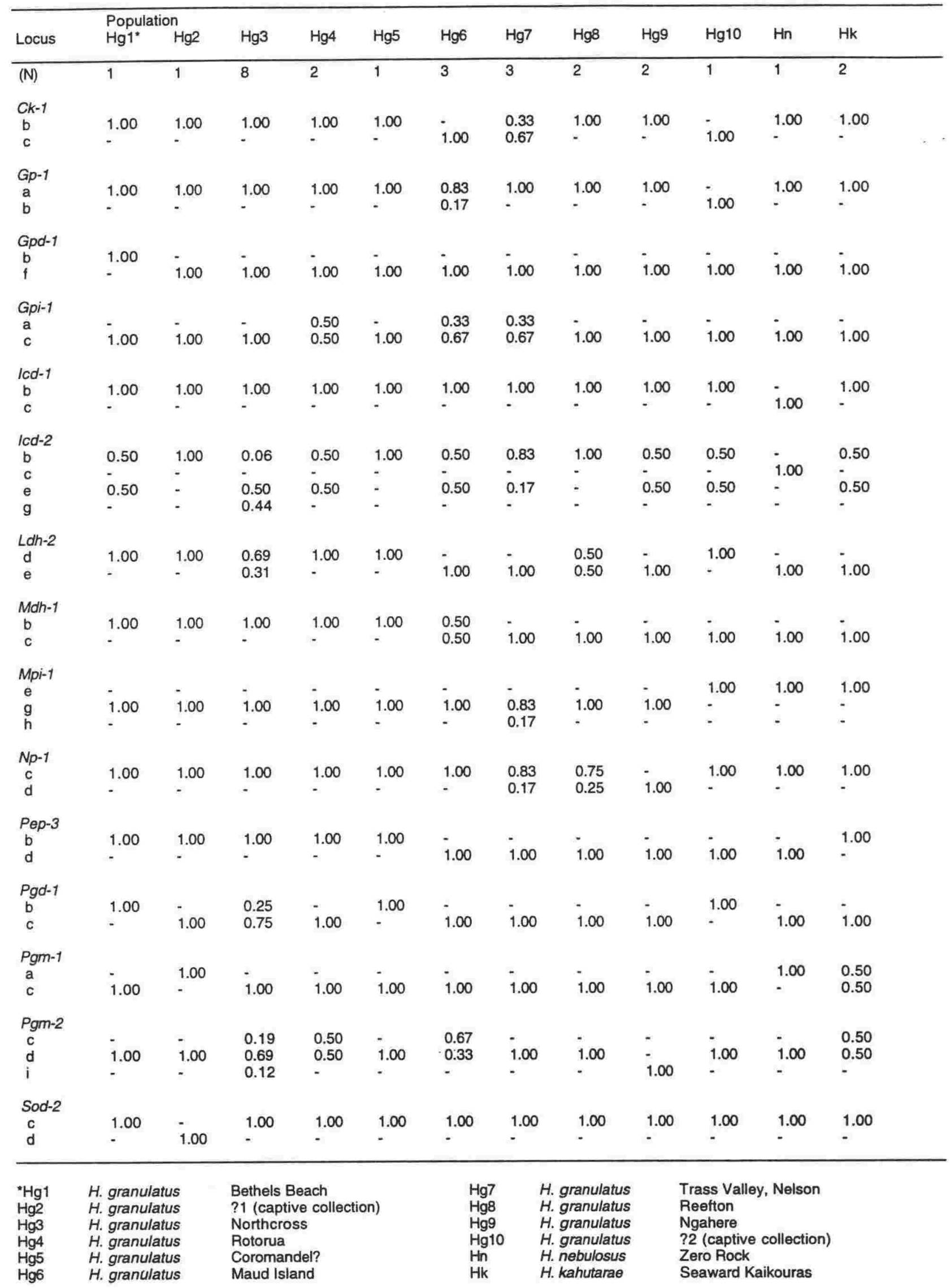


Table 4.7.3.2 Measures of polymorphism and heterozygosity in populations of the $H$. granulatus complex.

\begin{tabular}{|c|c|c|c|c|c|}
\hline \multirow[b]{2}{*}{ Population } & \multirow{2}{*}{$\begin{array}{l}\text { Mean } \\
\text { sample } \\
\text { size per } \\
\text { locus }\end{array}$} & \multirow{2}{*}{$\begin{array}{l}\text { Mean no. } \\
\text { of alleles } \\
\text { per locus }\end{array}$} & \multirow{2}{*}{$\begin{array}{l}\text { Percentage } \\
\text { of loci } \\
\text { polymorphic* }\end{array}$} & \multicolumn{2}{|c|}{ Mean heterozygosity } \\
\hline & & & & $\begin{array}{l}\text { direct- } \\
\text { count }\end{array}$ & $\begin{array}{l}\text { hdywbg } \\
\text { expected** }\end{array}$ \\
\hline Hg1 Bethel's Beach & $\begin{array}{l}1.0 \\
(0.0)\end{array}$ & $\begin{array}{l}1.0 \\
(0.0)\end{array}$ & 3.7 & $\begin{array}{l}0.04 \\
(0.04)\end{array}$ & $\begin{array}{l}0.04 \\
(0.04)\end{array}$ \\
\hline Hg2 Loc?1 & $\begin{array}{l}1.0 \\
(0.0)\end{array}$ & $\begin{array}{l}1.0 \\
(0.0)\end{array}$ & 0.0 & $\begin{array}{l}0.00 \\
(0.00)\end{array}$ & $\begin{array}{l}0.00 \\
(0.00)\end{array}$ \\
\hline Hg3 Northcross (Auckland) & $\begin{array}{l}8.0 \\
(0.0)\end{array}$ & $\begin{array}{l}1.2 \\
(0.1)\end{array}$ & 14.8 & $\begin{array}{l}0.07 \\
(0.03)\end{array}$ & $\begin{array}{l}0.07 \\
(0.04)\end{array}$ \\
\hline Hg4 Rotorua & $\begin{array}{l}2.0 \\
(0.0)\end{array}$ & $\begin{array}{l}1.1 \\
(0.1)\end{array}$ & 11.1 & $\begin{array}{l}0.00 \\
(0.00)\end{array}$ & $\begin{array}{l}0.07 \\
(0.04)\end{array}$ \\
\hline Hg5 Coromandel? & $\begin{array}{l}1.0 \\
(0.0)\end{array}$ & $\begin{array}{l}1.0 \\
(0.0)\end{array}$ & 0.0 & $\begin{array}{l}0.00 \\
(0.00)\end{array}$ & $\begin{array}{l}0.00 \\
(0.00)\end{array}$ \\
\hline Hg6 Maud Island & $\begin{array}{l}3.0 \\
(0.0)\end{array}$ & $\begin{array}{l}1.2 \\
(0.1)\end{array}$ & 18.5 & $\begin{array}{l}0.06 \\
(0.03)\end{array}$ & $\begin{array}{l}0.10 \\
(0.04)\end{array}$ \\
\hline Hg7 Trass Valley & $\begin{array}{l}3.0 \\
(0.0)\end{array}$ & $\begin{array}{l}1.2 \\
(0.1)\end{array}$ & 18.5 & $\begin{array}{l}0.04 \\
(0.02)\end{array}$ & $\begin{array}{l}0.08 \\
(0.03)\end{array}$ \\
\hline Hg8 Reefton & $\begin{array}{l}2.0 \\
(0.0)\end{array}$ & $\begin{array}{l}1.1 \\
(0.1)\end{array}$ & 7.4 & $\begin{array}{l}0.06 \\
(0.04)\end{array}$ & $\begin{array}{l}0.04 \\
(0.03)\end{array}$ \\
\hline Hg9 Ngahere & $\begin{array}{l}2.0 \\
(0.0)\end{array}$ & $\begin{array}{l}1.0 \\
(0.0)\end{array}$ & 3.7 & $\begin{array}{l}0.00 \\
(0.00)\end{array}$ & $\begin{array}{l}0.03 \\
(0.03)\end{array}$ \\
\hline Hg10 Loc?2 & $\begin{array}{l}1.0 \\
(0.0)\end{array}$ & $\begin{array}{l}1.0 \\
(0.0)\end{array}$ & 3.7 & $\begin{array}{l}0.04 \\
(0.04)\end{array}$ & $\begin{array}{l}0.04 \\
(0.04)\end{array}$ \\
\hline Hn H. nebulosus & $\begin{array}{l}1.0 \\
(0.0)\end{array}$ & $\begin{array}{l}1.0 \\
(0.0)\end{array}$ & 0.0 & $\begin{array}{l}0.00 \\
(0.00)\end{array}$ & $\begin{array}{l}0.00 \\
(0.00)\end{array}$ \\
\hline Hk H. kahutarae & $\begin{array}{l}2.0 \\
(0.0)\end{array}$ & $\begin{array}{l}1.1 \\
(0.1)\end{array}$ & 11.1 & $\begin{array}{l}0.07 \\
(0.05)\end{array}$ & $\begin{array}{l}0.07 \\
(0.04)\end{array}$ \\
\hline
\end{tabular}

H. kahutarae has previously been regarded as so morphologically distinct that its affinities were unknown, and its species status is unquestioned. However, it shared all its alleles with $H$. granulatus, although not with any single population. $H$. kahutarae falls within $H$. granulatus sensu Thomas (1981) in phenograms derived from allozyme data (Figures 4.7.3.1-3). It differed from the Reefton and Trass Valley samples of $H$. granulatus by $D=0.11$, and from both $H$. nebulosus and the Northcross (Auckland) sample of $H$. granulatus by $D=$ 0.12 (Table 4.7.3.3). 
ำ ำ

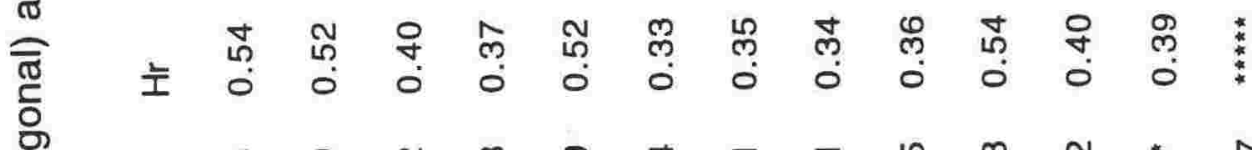

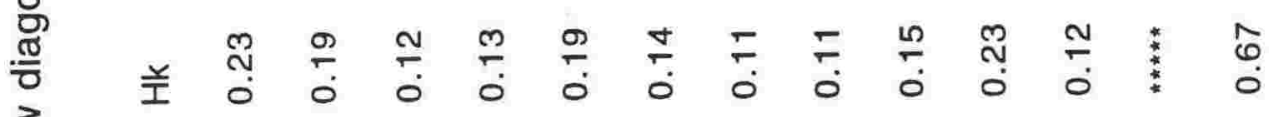
亭

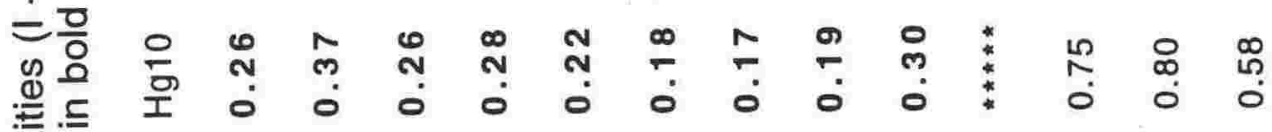
喜岏 先䇂

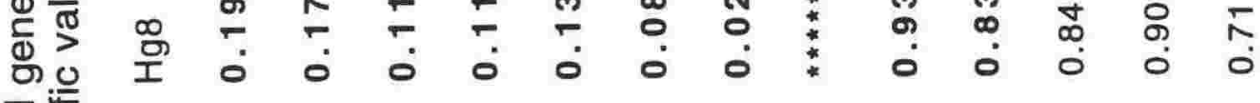
穷毞

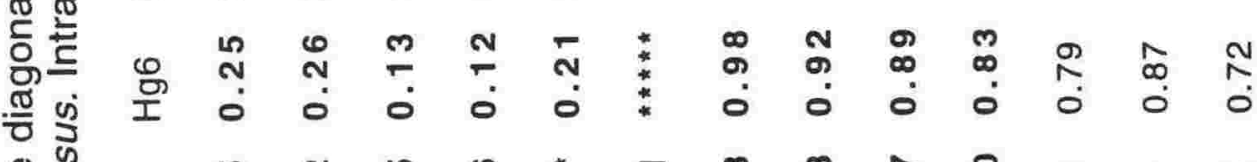

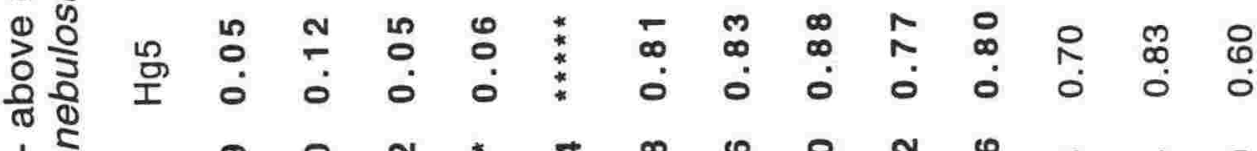

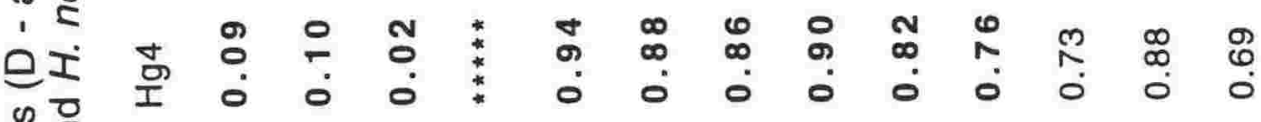

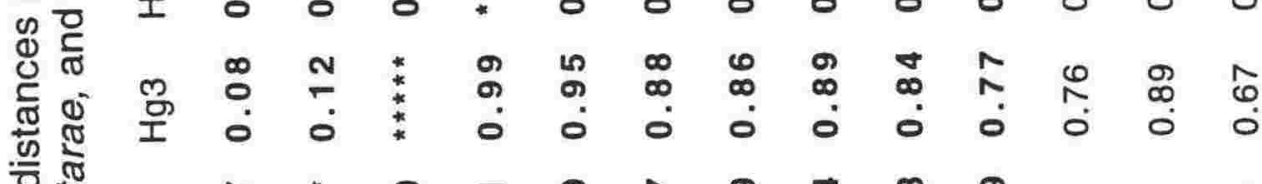
忨

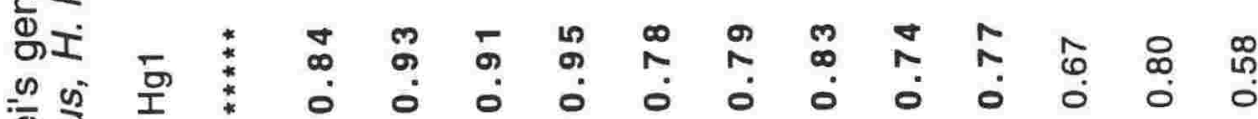
$\sum \frac{0}{\infty}$ 约 즌 元 $I$

ल ठ ตุ่ ก

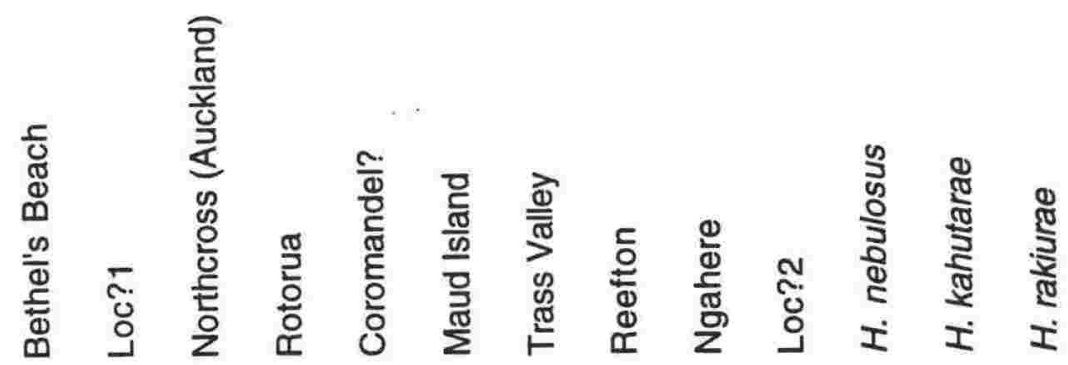
高言高

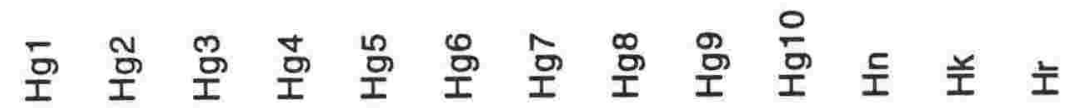




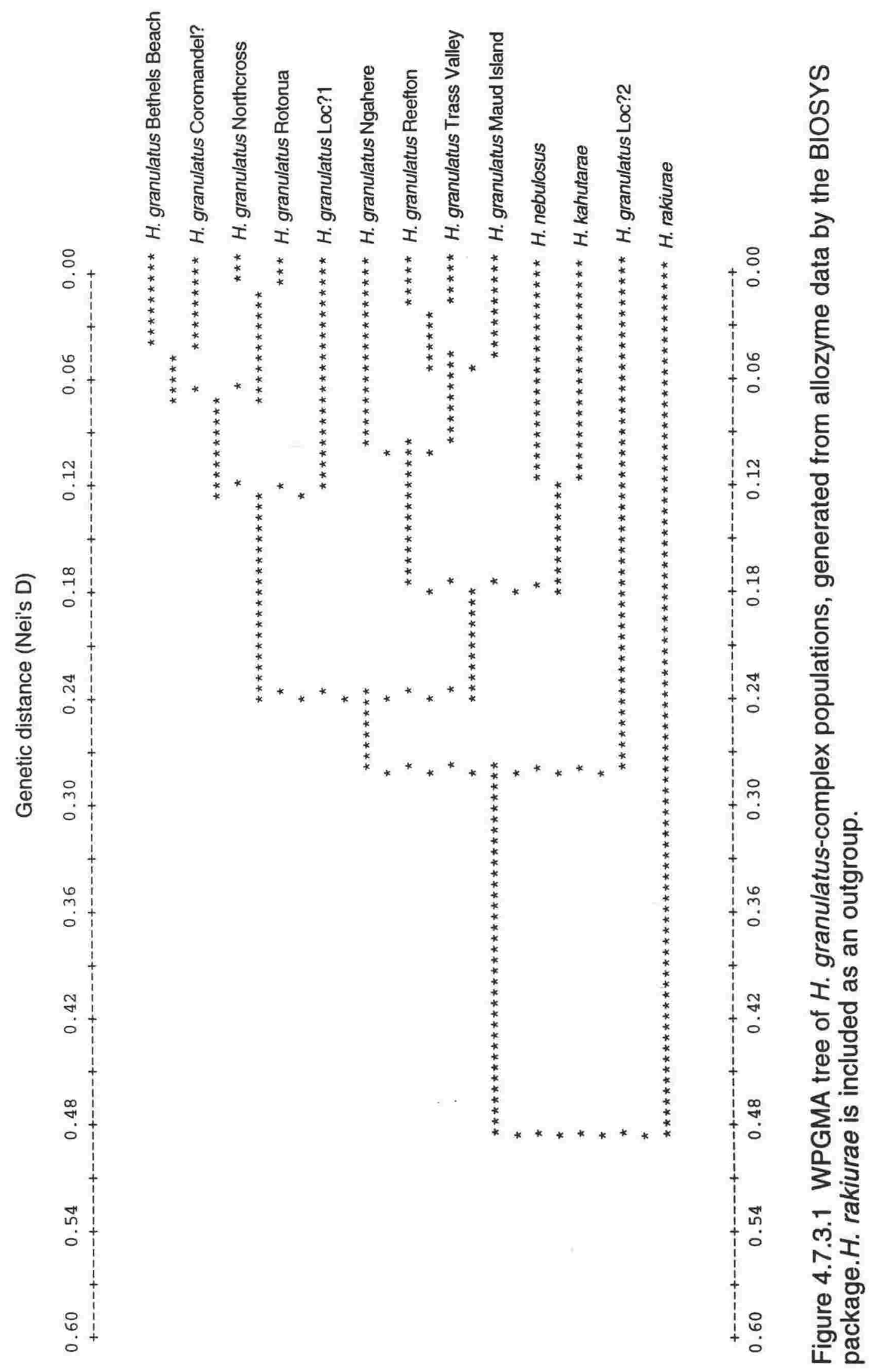




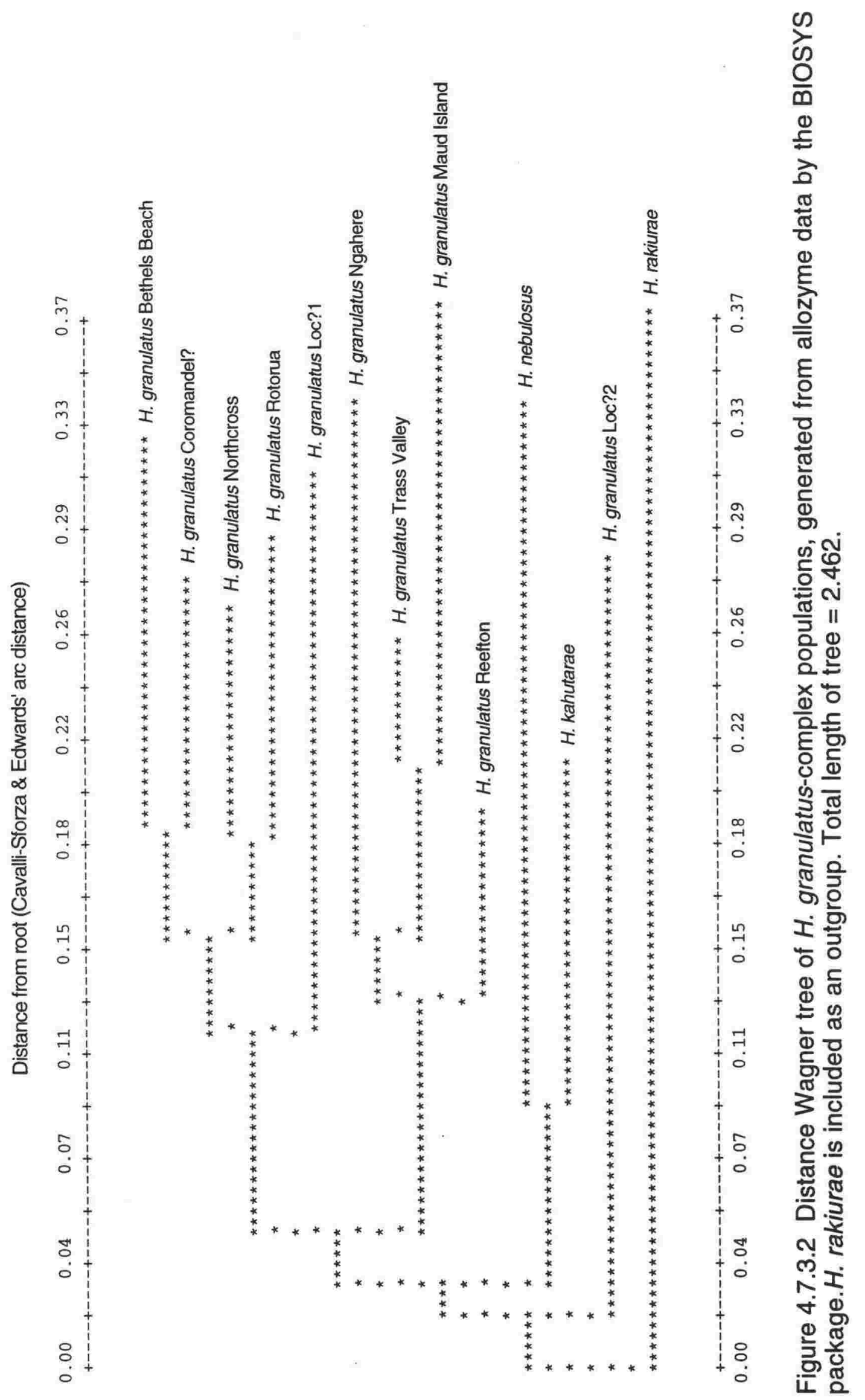




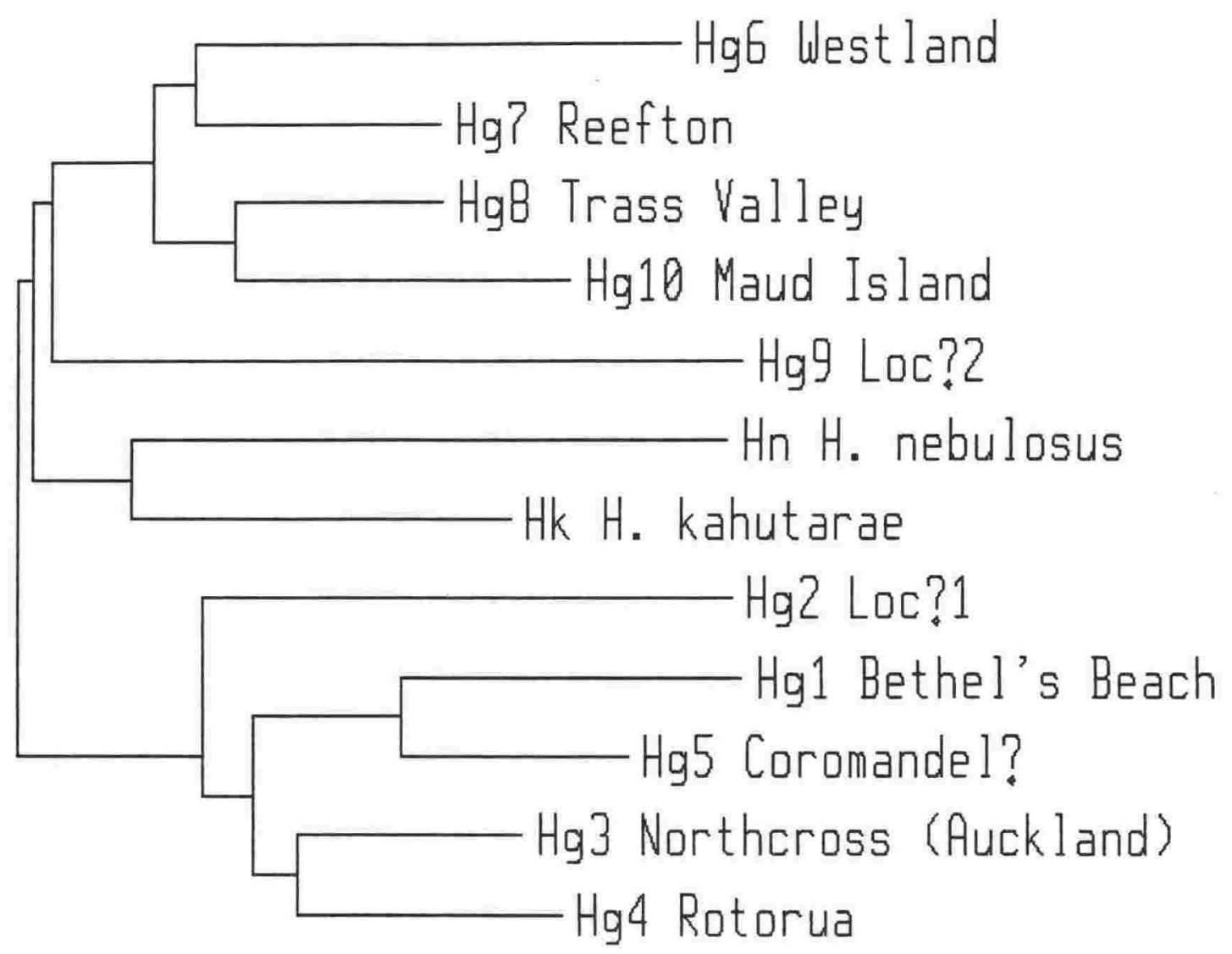

Figure 4.7.3.3 Neighbour-joining tree of $H$. granulatus-complex populations, generated from Cavalli-Sforza \& Edwards' (1967) distances (27 allozyme loci) using the MEGA programme. 
Some genetic distances between populations of $H$. granulatus were higher than the lowest distances between $H$. granulatus and either $H$. nebulosus or $H$. kahutarae, for all 3 genetic distance measures used (Tables 4.7.3.3. \& 4.7.3.4). Within $H$. granulatus, Pep-3 showed a fixed difference, and Mdh-1 an almost fixed difference between North and South Island populations, but no samples were obtained from the southern half of the North Island so a clinal connection between the 2 groups is possible. In the South Island, allozyme variation appeared to be clinal; the Maud Island and Ngahere populations had fixed differences at 3 loci $(C k-1, N p-1, P g m-2)$, but the Trass and/or Reefton populations are polymorphic at $\mathrm{Ck}-1$ and $\mathrm{Np}-1$.

The most genetically divergent specimen of $H$. granulatus was an animal (CD990 - Hg10 in Table 4.7.3.1) that had died in captivity at Mount Bruce National Wildlife Centre. It was of unknown provenance, but believed to be from the North Island. Morphologically, it was indistinguishable from other North Island specimens. My attempts to trace its origin have failed because of poor record-keeping at Mount Bruce at the time. This specimen differed from all other North Island $H$. granulatus at 5 of 27 loci (Ck-1, Gp-1, Mdh-1, Mpi-1, Pep-3), but at 4 of these loci shared a South Island allele, and at the fifth (Mpi-1) was homozygous for an allele otherwise seen only in $H$. nebulosus and $H$. kahutarae. At Pgd-1, the specimen was homozygous for an allele otherwise seen only in the North island, and at $L d h-2$, it had an allele common in the North Island, but only seen in the South Island in 2 heterozygotes from Reefton. It had no unique alleles.

\subsubsection{MORPHOLOGY AND ECOLOGY}

The genetic diversity in $H$. granulatus is not reflected in morphological or ecological diversity. There is a polymorphism in the presence of distinct scale rings on the tail marking the position of autotomy planes. Many North Island specimens lack these rings, as does the type of $H$. granulatus (Bauer 1990), although the lack of these rings is not diagnostic of $H$. granulatus, contrary to the claim of Bauer (1990), as all South Island and some North Island specimens examined had them. In the South Island, populations show no diagnostic morphological differences, but differ in the bolder, brighter, less speckled colour pattern of the Westland populations, and in the shape of the tail, which is slender and cylindrical in Westland populations, and thicker and somewhat square in cross-section in the Nelson-Marlborough populations (which appear to be morphologically indistinguishable from those in the North Island). 
$\frac{3}{9}$

$\stackrel{\oplus}{\varrho}$

क

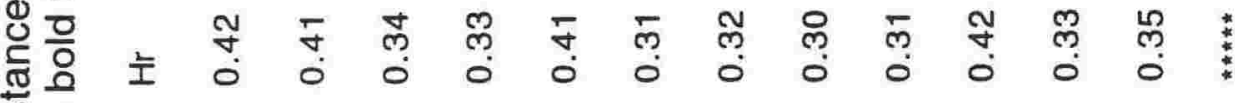

号.

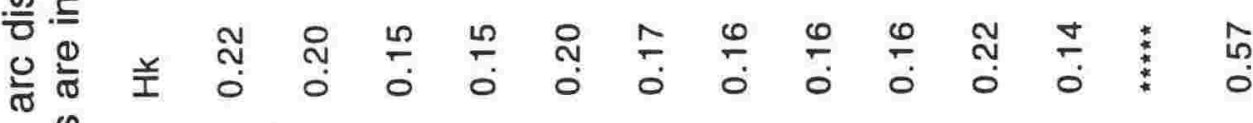

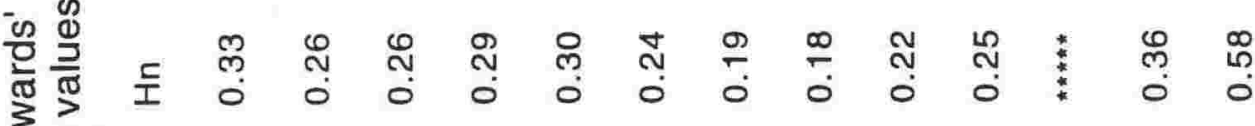

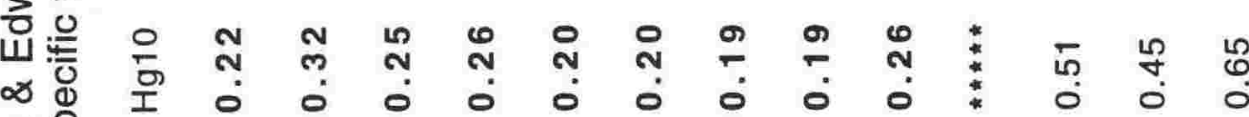

กี้ สี้ 0 ก

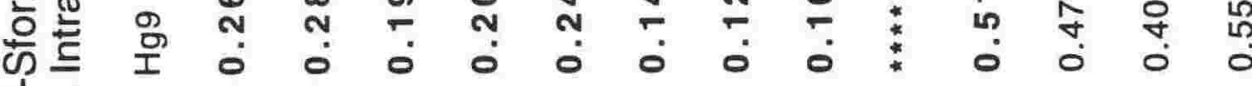

㲾永

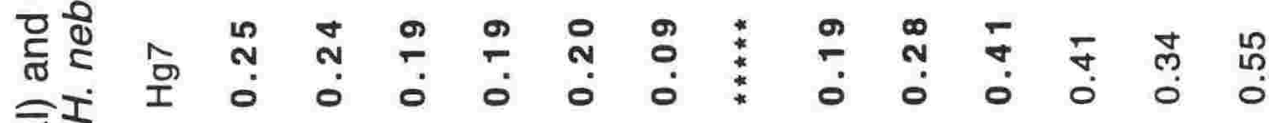

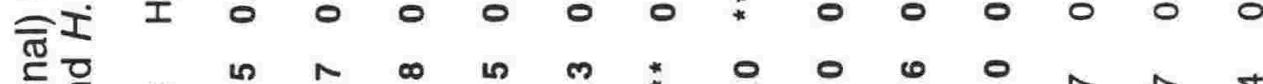

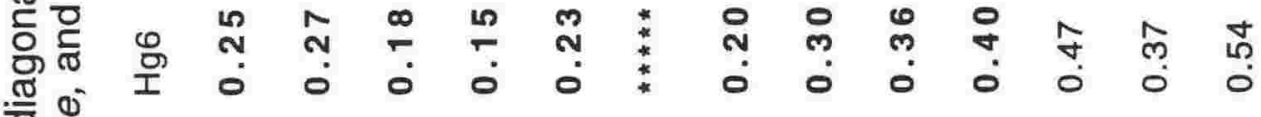

泀

山

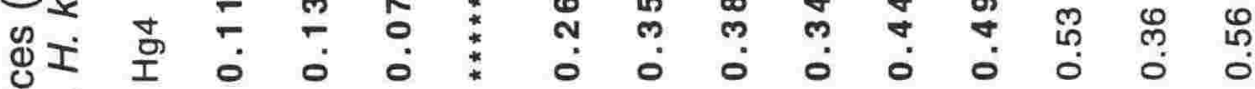

到

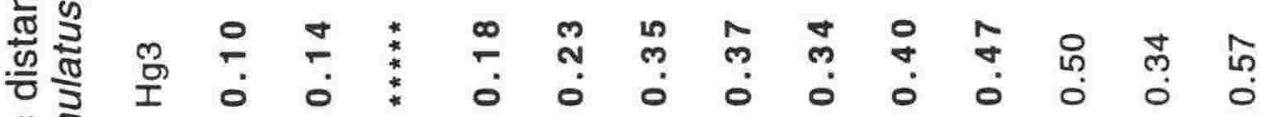

을

ฮั

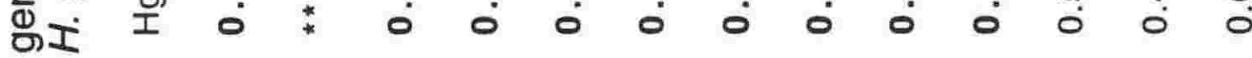

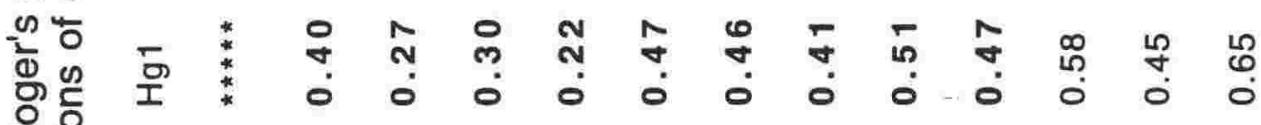
뚜은

$\frac{\pi}{5}$

즌 흥

สํ

$\sum$

$\forall$

ต ซ

กิ

๑ 등

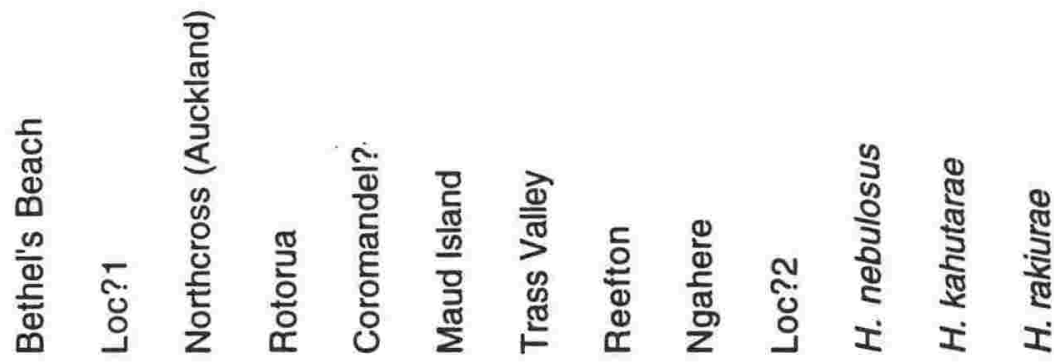

응 음 $\frac{\pi}{0}$

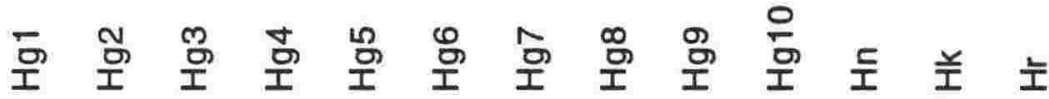


Based on very limited evidence, reproductive cycles probably vary within $H$. granulatus; a female from Reefton collected while pregnant gave birth in November (R Morris pers. comm.), whereas $H$. granulatus, including Nelson specimens, typically give birth in February-April (pers. obs.; B. Goertz, Reptile Research Centre, pers. comm.). Three Westland females held in captivity with North Island males for more than 10 years never bred, whereas North Island females in the same cage gave birth every year (pers. obs.).

Because Thomas's (1981) treatment was so thorough, and few additional specimens have become available, the traditional morphological characters (such as scale counts) which he analysed and found no difference between $H$. granulatus and $H$. nebulosus in will not be discussed further.

Morphologically, $H$. nebulosus differs from $H$. granulatus in such largely unquantifiable characters as colour and bluntness of the snout. The belly, rather than being heavily mottled with grey and white as in $H$. granulatus, is dull pink with only scattered dark spots. The dorsal pattern is basically the same as in other populations (though with more of a tendency to longitudinal stripes on the neck and forepart of the body), but the pattern is less prominent, and the background colouring brighter and more uniform, usually in dull pinks and/or greens. The white blotchy markings around the mouth which are prominent in $\mathrm{H}$. granulatus, are absent in $\mathrm{H}$. nebulosus, and the eye-ear stripe, which is also prominent and white in $H$. granulatus, is light buff in $H$. nebulosus. The snout is much shorter, deeper, and more blunt in $H$. nebulosus (shorter than eye-ear measurement) than in $\mathrm{H}$. granulatus (longer than eye-ear measurement), and lacks the concavity seen in profile in typical H. granulatus (Chapter 3). The difference in snout shape was not discussed by Thomas (1981); from the small number of specimens available this character appears to be diagnostic.

In northern New Zealand, $H$. granulatus is present on the mainland and Waiheke, Great and Little Barrier Islands, the largest, most heavily forested islands in the Hauraki Gulf, but is absent from many smaller islands, some of which have been very little modified, indicating that it has probably never been found on these. In the Marlborough Sounds, $H$. granulatus is present in forest on islands within the shelter of the sounds such as Maud Island, but absent from exposed, petrel-burrowed islands such as the Brothers, Stephens Island, and the Trios. H. nebulosus, on the other hand, is known only from small, exposed, heavily petrel-burrowed, mammal-free offshore islands. Because Stewart Island itself lies directly between some of these 
islands, it is assumed that $H$. nebulosus was formerly found there, and is now extinct as a result of the presence of introduced mammalian predators. It has also apparently disappeared from Kundy Island, the type locality, since the introduction of weka (Gallirallus australis), a flightless, terrestrial rail. This suggests that $H$. nebulosus must spend a considerable part of its time on the ground. $H$. granulatus, in contrast, is strongly arboreal, and has survived the presence of the same predators in large areas of the North and South Islands.

The Open Bay Island specimen (Figure 3.4.1.1) and photographs of other specimens taken on the island appear externally very similar to $H$. nebulosus, but the preserved specimen, a mature male, is considerably smaller than (roughly half the mass of) mature $\mathrm{H}$. nebulosus or $\mathrm{H}$. granulatus, and faint longitudinal stripes are visible. No other member of the $H$. granulatus complex has longitudinal stripes the full length of the body, although some, particularly some $H$. nebulosus, have them on the neck and shoulder region. The specimen photographed more recently had markings similar to $H$. nebulosus.

A small juvenile specimen from subalpine scrub in Fiordland (Figure 3.4.1.2), the only specimen available from this area, has markings somewhat suggestive of $H$. rakiurae, and low lamellae counts and single conical cloacal spurs, which are not consistent with $H$. granulatus. This specimen almost certainly represents a new species.

\subsubsection{DISCUSSION}

\subsubsection{H. nebulosus}

The allozyme results of this study confirm the conclusions of Thomas (1982) and Robb (1982) that $H$. nebulosus is closely related to $H$. granulatus, and that McCann's original placement among the green geckos was inappropriate (see Chapter 5).

The magnitude of the allozyme difference between the Zero Rock sample and the others justifies specific recognition for $H$. nebulosus, regardless of its morphological similarity to $H$. granulatus, and despite the absence of allozyme samples from $H$. granulatus material from the south of the South Island. $H$. nebulosus is more similar to $H$. kahutarae ( $D=0.12$ ) than to any population of $H$. granulatus $(D \geq 0.17$ ). The genetic distance between $H$. nebulosus and Bethels Beach $H$. granulatus is the same as that between $H$. nebulosus and $H$. rakiurae $(D=0.40)$, undoubtedly 
a specific difference. In phenograms $H$. nebulosus is the sister group of $H$. kahutarae, and these 2 fall within $H$. granulatus. The small amount of ecological information available on the Stewart island region populations supports their taxonomic separation from $H$. granulatus.

\subsubsection{H. granulatus}

The large genetic distances among populations within $H$. granulatus, geographic variation in reproductive cycles, the lack of progeny from Westland females held in captivity with North Island males, and branching patterns in trees generated from allozyme data all indicate the possibility of specific distinction at least between North and South Island populations. However, the groups generated by analysis of allozyme genotypes and suggested by morphological and reproductive information are not congruent; in appearance and reproductive cycles Nelson-Marlborough populations resemble North Island populations rather than the more genetically similar Westland ones.

The $440 \mathrm{~km}$ gap between the Maud Island and Rotorua samples means that clinal variation with isolation by distance is also a strong possibility from the observed pattern of allozyme variation. If populations in this gap are genetically intermediate, their inclusion in a phenetic analysis would have the effect of reducing the separation between the North and South Island groups, and this in turn could mean that $H$. granulatus could form a monophyletic group, with $H$. nebulosus and $H$. kahutarae falling outside it. Samples of $H$. granulatus were too small and geographically well-separated for the shape of the clines to be assessed; sharply stepped clines with concordant patterns for several characters would imply speciation as in the $H$. maculatus complex, whereas continuous clines would not. Subdivision of $H$. granulatus using the information available from this study would therefore be premature.

The allozyme profile of CD990, fixed for South Island alleles at most variable loci, but for North Island alleles at some, suggests that a southern North Island origin is most likely for this specimen. Collection of southern North Island specimens would clarify this, but proved impossible during this study despite considerable effort. 


\subsubsection{H. kahutarae}

Is $H$. kahutarae derived within $H$. granulatus? It certainly appears to be derived within $H$. granulatus sensu Thomas (1981) (i.e., including $H$. nebulosus), as it falls within this group in all trees (Figures 4.7.3.1 - 4.7.3.3). However, in all trees it appears as the sister species of $H$. nebulosus, which $I$ consider specifically distinct from $H$. granulatus. This pair often clusters within $H$. granulatus; in the WPGMA tree (Figure 4.7.3.1) H. kahutarae plus $H$. nebulosus fall between the North Island and South Island populations of H. granulatus. CD990 in particular falls outside $H$. kahutarae plus $H$. nebulosus in all trees. However, this may be an artefact of the absence of adequate samples from the southern North Island; larger, more evenly distributed samples may be genetically intermediate between existing samples, and reduce the observed genetic distances within $\mathrm{H}$. granulatus, as discussed above.

However, regardless of the exact branching pattern within this complex, there is strong evidence for $H$. kahutarae having been derived within $H$. granulatus plus $H$. nebulosus, or from a recent common ancestor of these 2 . This origin, combined with the strong morphological similarity of $H$. nebulosus and all $H$. granulatus populations, indicates that the highly distinct morphology (Whitaker 1982; Bauer 1990) of $H$. kahutarae must have arisen recently by accelerated morphological evolution, perhaps in response to its unusual, extremely high-altitude habitat.

\subsubsection{Other possible cryptic species}

The Fiordland and Open Bay Islands specimens probably represent one or more further undescribed species; further collecting in these areas is necessary.

\subsection{NAULTINUS}

\subsubsection{INTRODUCTION}

There has been dispute for many years about the appropriate taxonomic level at which to recognise the morphological diversity of the New Zealand green geckos. Many species were described in the nineteenth century, but this century all green geckos were usually referred to Naultinus elegans until McCann (1955) resurrected the generic name Heteropholis Fischer, 1882 to include all the South Island species ( 3 new species plus 2 resurrected from informal synonymy with $N$. elegans). Robb \& Hitchmough (1980) resurrected $N$. grayii from synonymy with $N$. elegans on the basis of diagnostic 
morphological differences and demographic differences including 5 months' separation of birth seasons.

Meads' (1982) captive hybridisation experiments showed full interfertility of all Naultinus and Heteropholis species in captivity. F1, F2, backcross, and multispecies hybrids were produced with no loss of fertility. He used these results, interpreted using a strict isolation species concept, to suggest reduction of all known taxa of New Zealand green geckos (including some morphologically distinct populations with no formal taxonomic status) to subspecies of Naultinus elegans. Gill (1986) used this subspecific nomenclature in his popular field guide.

However, Bauer (1990) argued on the basis of differences in external morphology, spatial separation, and temporal separation (presumably in reproductive events) that species-level recognition was justified, and pointed out that, as far as can be determined from their highly fragmented remnant distributions, Naultinus spp. show abrupt parapatric boundaries between the recognised species. He formalised the often-suggested synonymy of Heteropholis with Naultinus. His approach is consistent with an evolutionary species concept.

In this thesis I also use an evolutionary species concept. I consider that specific recognition is justified if 2 taxa maintain differences across a parapatric boundary or hybrid zone which is very narrow relative to the geographic distributions of the taxa, even if there is evidence for limited gene flow across this boundary.

Most former descriptions of Naultinus species fail to represent the geographic variation present within the species. For example, the redescription of $N$. stellatus by McCann (1955) reflects the fact that his neotype is from Lake Rotoiti, and therefore has the features (which I suggest below may be derived from introgression from $N$. rudis) common in that population but not in populations to the north. The original type of the species, from Mount Arthur (Hutton 1872), probably differed in many characters from the neotype.

Bauer's (1990, appendix 3) character-state matrix makes no allowance for polymorphism or geographic variation within species in such characters as rostral-nostril contact. The keys of Towns (1985) and Bauer (1990) are 
inconsistent with each other in some details, and both fail to provide correct identifications for many specimens. For example, for $N$. stellatus, Towns' couplet 7 states "body scales of equal size but conical or tuberculate" whereas Bauer's couplet 1 states "scalation of dorsal body surface heterogeneous". In Towns' couplet 5 the correct choice for this species is "scales not enlarged" whereas in Bauer's couplet 3 the correct choice is "head and nape only with heterogeneous conical scales". Both keys also fail because some characters do not differ as clearly as the keys imply or are not consistent. For example, the distinction between granular and conical or tuberculate scales, used in both keys to separate $N$. gemmeus and $N$. stellatus (granular) from N. tuberculatus and N. poecilochlorus (conical or tuberculate) is unclear to me after examining a range of specimens. Similarly, the couplet "supraciliary scales granular" or "supraciliary scales conical" which (alone in Bauer's key, and in combination with colour descriptions in Towns') separates $N$. gemmeus from N. stellatus, results in misclassification of most Nelson Lakes animals and some other specimens of $N$. stellatus, which have strongly conical supraciliary scales.

Agricultural development has destroyed green gecko habitat in many areas, exterminating many populations, and therefore making interpretation of geographic variation more difficult. For example, on the Canterbury Plains north of Banks Peninsula there is a gap between the distributions of $N$. gemmeus and N. rudis (Pickard \& Towns 1988), which is very likely to be an artefact of habitat destruction. Bull \& Whitaker (1975) reported populations of $N$. gemmeus and $N$. rudis about $30 \mathrm{~km}$ apart near Hanmer, but they were referring Lewis Pass populations, now identified as $N$. tuberculatus, to $N$. gemmeus.

In the following sections I compare allozyme data for populations of all recognised species of Naultinus, re-examine external morphological variation in the genus, and discuss them in the light of distribution data for the various forms.

\subsubsection{SAMPLING}

Samples for electrophoresis were collected from 42 individual Naultinus from 24 populations (Table 4.8.2.1).Sample distribution is uneven (Figure 4.8.2.1; Table 4.8.2.1), and many of the specimens used for allozyme electrophoresis came from captive collections and therefore had only general, rather than specific locality data. However, because field collection 
Table 4.8.2.1 Collecting localities for samples of Naultinus spp. (see Figure 4.8.2.1).

$\begin{array}{lll}\text { Ng1 } & \text { N. grayii } & \text { Kaitaia } \\ \text { Ng2 } & \text { N. grayii } & \text { Mangonui } \\ \text { Ng3 } & \text { N. grayii } & \text { South Kaimaumau swamp } \\ \text { Ng4 } & \text { N. grayii } & \text { North Kaimaumau swamp } \\ \text { Ng5 } & \text { N. grayii } & \text { Cape Reinga } \\ \text { Ngxe } & \text { N. grayii x elegans } & \text { captive collection } \\ \text { Nee1 } & \text { N. e. elegans } & \text { Huntly } \\ \text { Nee2 } & \text { N. e. elegans } & \text { Auckland } \\ \text { Nee3 } & \text { N. e. elegans } & \text { Glenfield } \\ \text { Nep1 } & \text { N. e. punctatus } & \text { Paremata } \\ \text { Nep2 } & \text { N. e. punctatus } & \text { Mount Bruce } \\ \text { Nep3 } & \text { N. e. punctatus } & \text { Palmerston North } \\ \text { Nep4 } & \text { N. e. punctatus } & \text { Wellington } \\ \text { Nm1 } & \text { N. manukanus } & \text { Stephens Island } \\ \text { Nm2 } & \text { N. manukanus } & \text { Marlborough Sounds } \\ \text { Nmx? } & \text { N. manukanus } x & \text { captive collection } \\ \text { Nr } & \text { N. rudis } & \text { captive collection } \\ \text { Ns1 } & \text { N. stellatus } & \text { Nelson Lakes } \\ \text { Ns2 } & \text { N. stellatus } & \text { Sandy Bay } \\ \text { Ns3 } & \text { N. stellatus } & \text { Maitai Valley } \\ \text { Ns4 } & \text { N. stellatus } & \text { Adele Island } \\ \text { Ns5 } & \text { N. stellatus } & \text { Golden Bay } \\ \text { Nsxe } & \text { N. stellatus } x \text { elegans } & \text { captive collection } \\ \text { Nt } & \text { N. tuberculatus } & \text { Lewis Pass } \\ \text { Nge1 } & \text { N. gemmeus } & \text { Hakataramea Pass } \\ \text { Nge2 } & \text { N. gemmeus } & \text { Banks Peninsula } \\ \text { Nge3 } & \text { N. gemmeus } & \text { Otago Peninsula } \\ & & \end{array}$

of green geckos is extremely difficult and time consuming, and all species are protected, better samples are unlikely to become available in the foreseeable future. General localities are considered correct for these samples because the collectors who supplied them had kept animals from different locations separate, and both fine-scale morphological features unlikely to be obvious to the collectors, and allozyme profiles were consistent with the collectors' information.

Three known, captive-bred hybrid individuals were also surveyed for allozyme genotypes (Table 4.8.2.1), but were not included in any phylogenetic analysis.

\subsubsection{ALLOZYMES}

Of the 27 allozyme loci scored for all populations, only 6 were polymorphic (Table 4.8.3.1). Three loci were not taxonomically informative: Got-2 varied only between the Hakataramea Pass population of $N$. gemmeus (homozygous Got-2a) and the rest (homozygous Got-2c). For Sod-2 the only variation was a single heterozygote in the Sandy Bay population of $N$. 


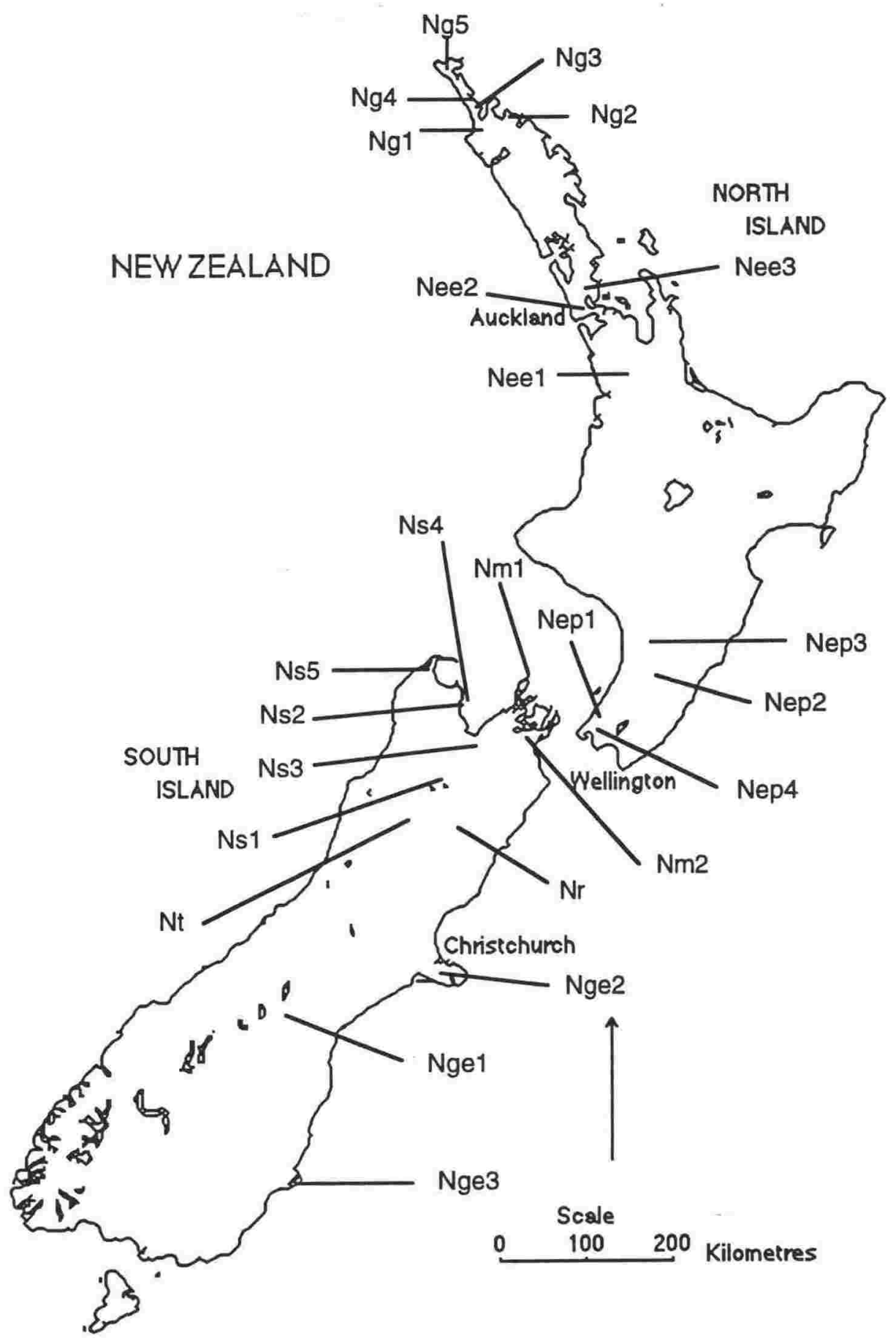

Figure 4.8.2.1 Collecting localities for samples of Naultinus. See Table 4.8.2.1 for key. 


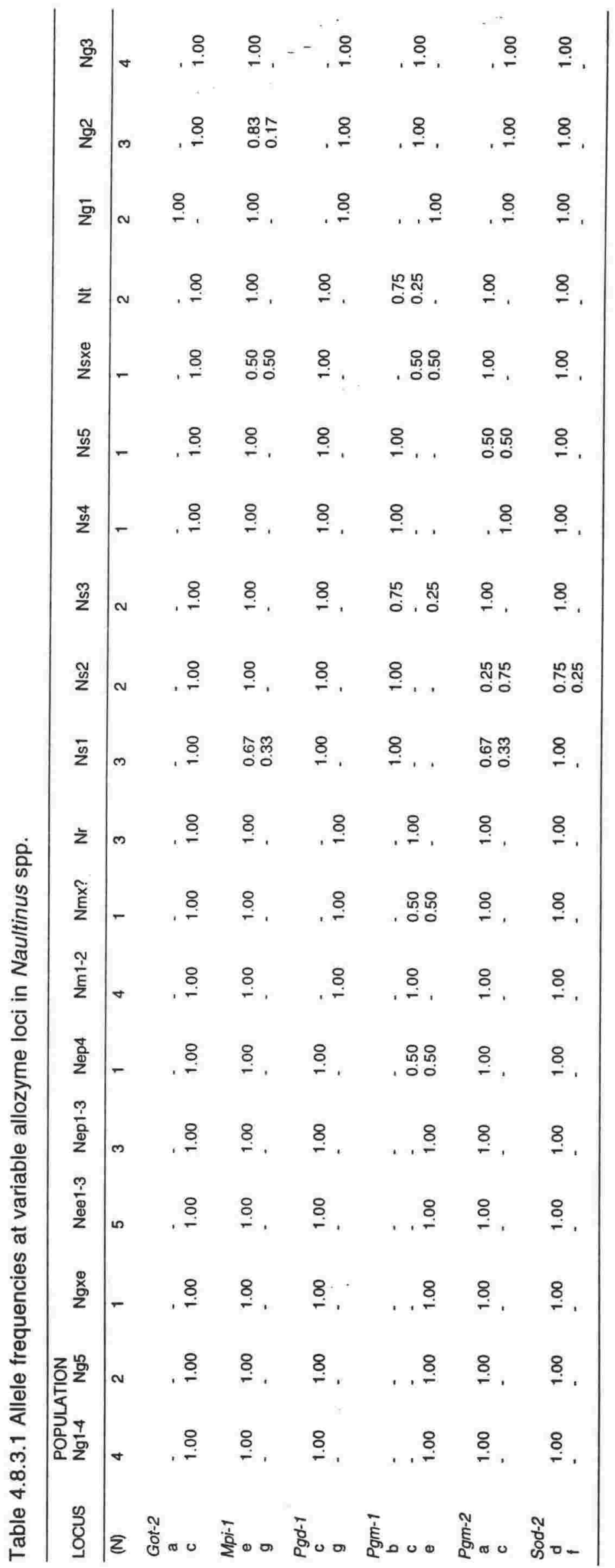


stellatus. Mpi-1 (Figure 4.8.3.1) had a polymorphism for an uncommon allele (Mpi-1(g)) shared by Banks Peninsula N. gemmeus and Nelson Lakes N. stellatus.
ost polymorphic loci showed strong geographic structure in their shism (Figures 4.8.3.2-4). Pgd-1 (Figure 4.8.3.2) showed a fixed $\Xi$ between species from the eastern South Island (N. gemmeus, $N$. manukanus - Pgd-1 $(\mathrm{g})$ ) and those from the north-western South tuberculatus, N. stellatus) and the North Island (N. elegans, N. $\gamma^{d-1(c))}$.

'Figure 4.8.3.3), N. stellatus was polymorphic for alleles which showed a fixed difference between N. gemmeus (Pgm-2(c)) and the genus (Pgm-2(a)).

Pgm-1 showed the most complex polymorphism (Figure 4.8.3.4). $N$. manukanus, $N$. rudis, and the Banks Peninsula and Otago Peninsula populations of $N$. gemmeus were fixed for Pgm-1(c), which was also found in N. tuberculatus and N. e. punctatus (single heterozygotes in each). Pgm-1(e) was found in all other North Island specimens sampled, was fixed in Hakataramea Pass N. gemmeus, and was found in a single heterozygous Maitai Valley N. stellatus. Pgm-1(a) was found in all other N. stellatus and N. tuberculatus.

As far as could be detected from the small samples available, levels of polymorphism were very low (Table 4.8.3.2). There was no evidence of genetic structuring within samples; observed heterozygosity was close to Hardy-Weinberg expectation in all populations (sample sizes were too small to permit tests for significance).

Matrices of genetic distance (Nei's D and I - Table 4.8.3.3, Cavalli-Sforza \& Edwards' arc distance and Rogers' D - Table 4.8.3.4) showed small genetic distances (Nei's $D=0.00-0.04$ ) within species, except for the Hakataramea Pass population of $N$. gemmeus, which differed from the other 2 populations of this species by $D=0.08$. Between-species distances ranged from 0.00 (among all North Island species, and between $N$. manukanus and $N$. rudis) to 0.15 (between N. tuberculatus and Hakataramea Pass N. gemmeus). 


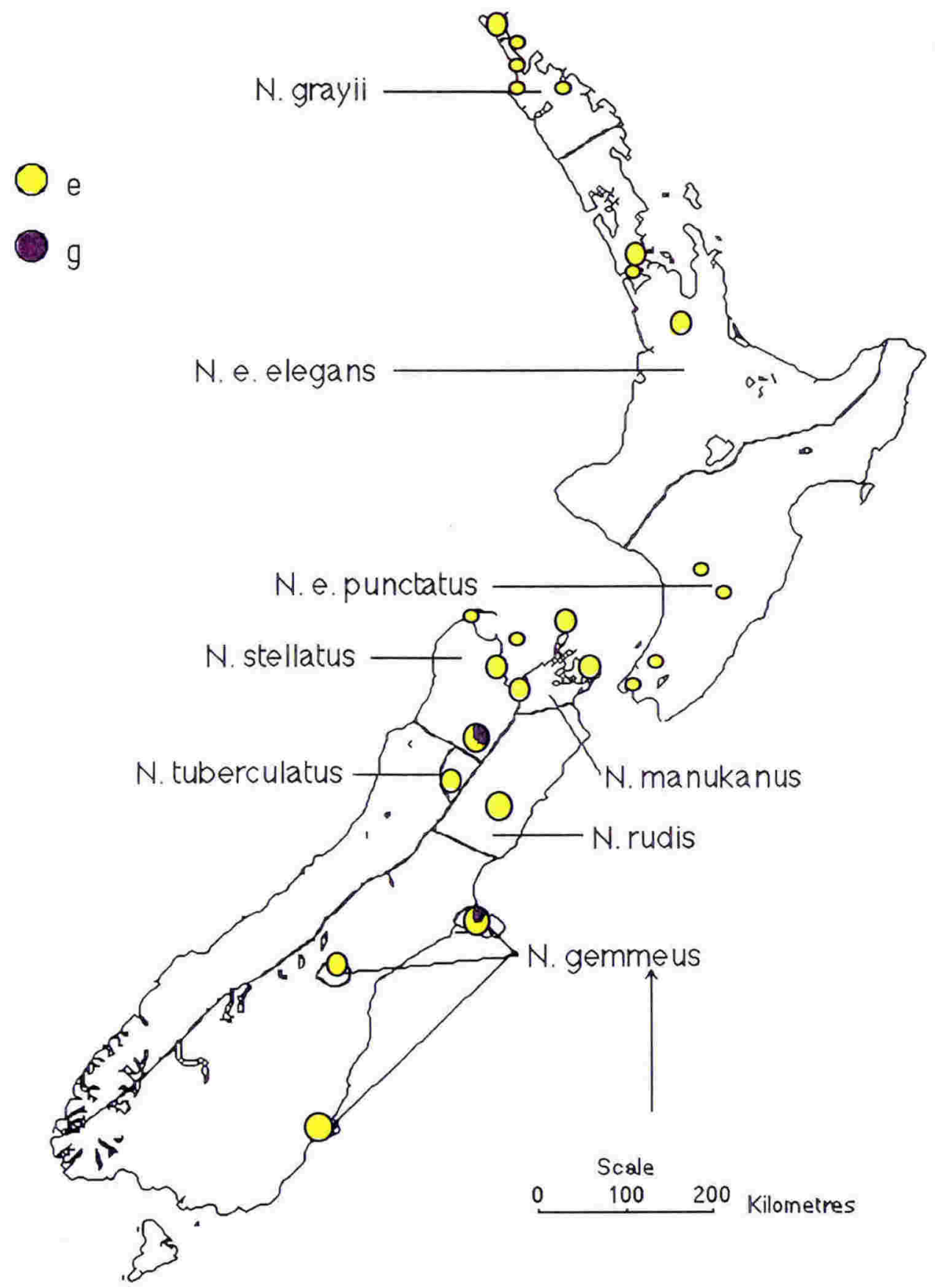

Figure 4.8.3.1 Distributions of alleles of Mpi-1 among populations of Naultinus spp., mapped onto the currently recognised species' distributions. 


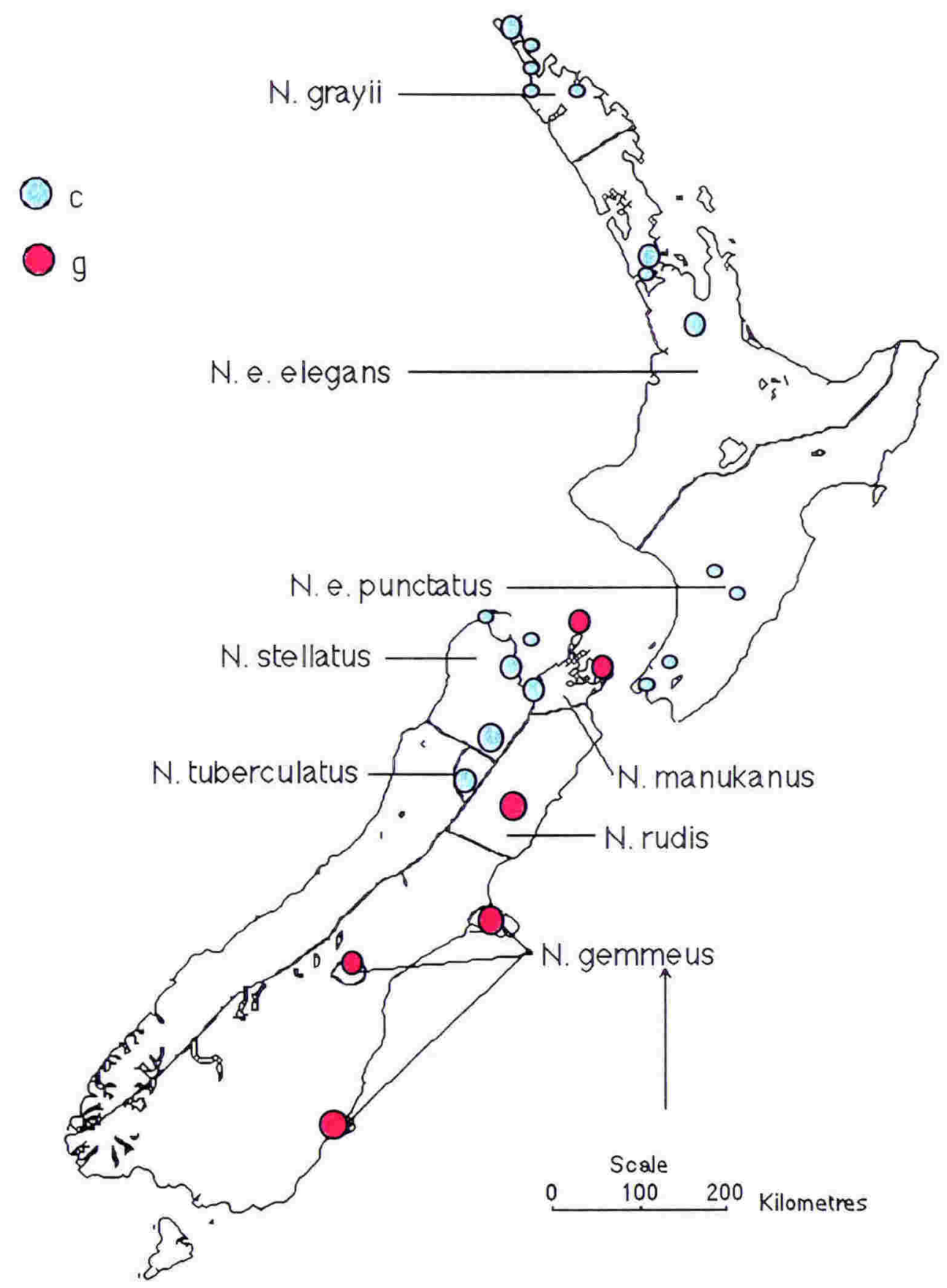

Figure 4.8.3.2 Distributions of alleles of Pgd-1 among populations of Naultinus spp., mapped onto the currently recognised species' distributions. 


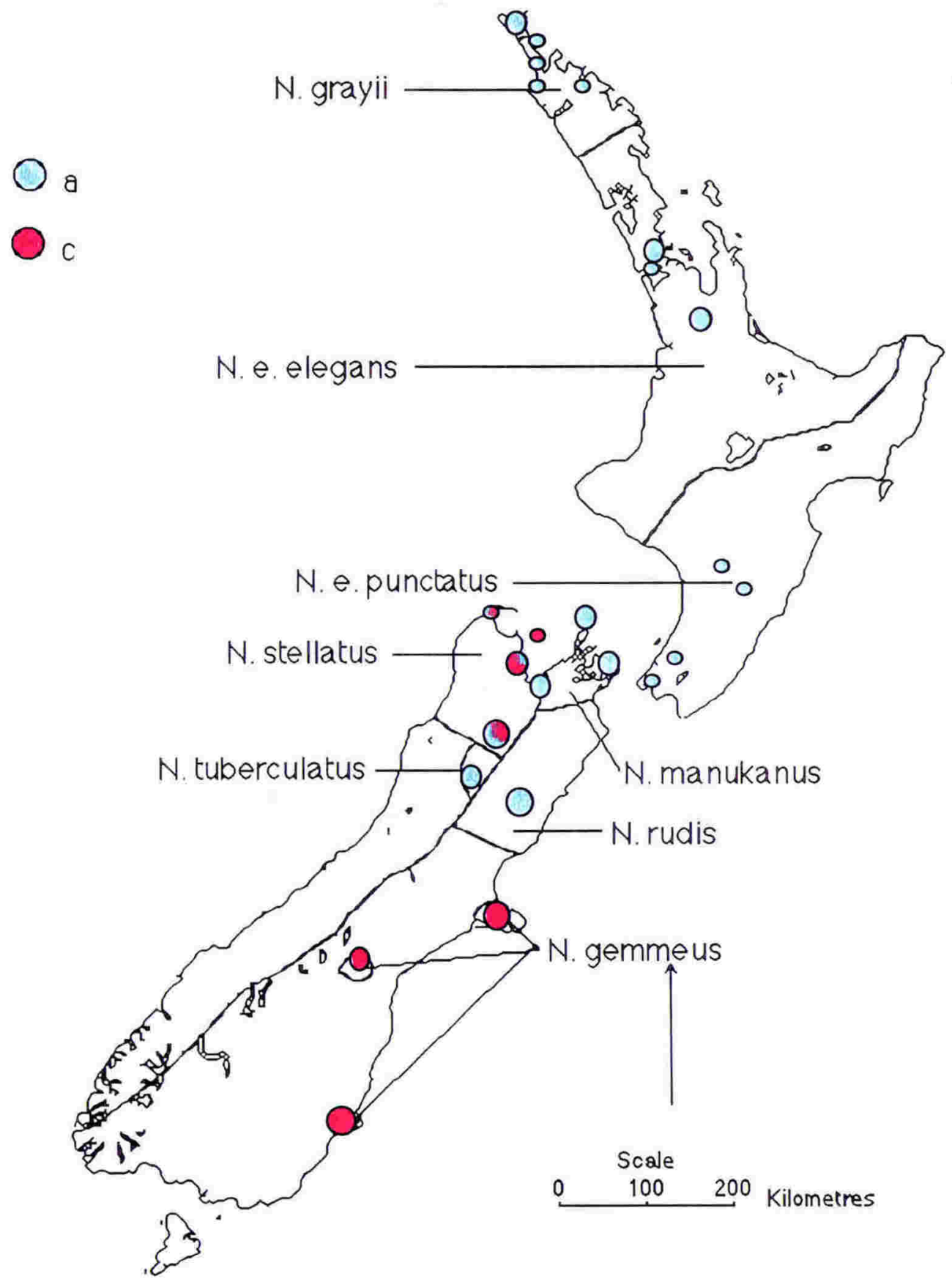

Figure 4.8.3.3 Distributions of alleles of Pgm-2 among populations of Naultinus spp., mapped onto the currently recognised species' distributions. 


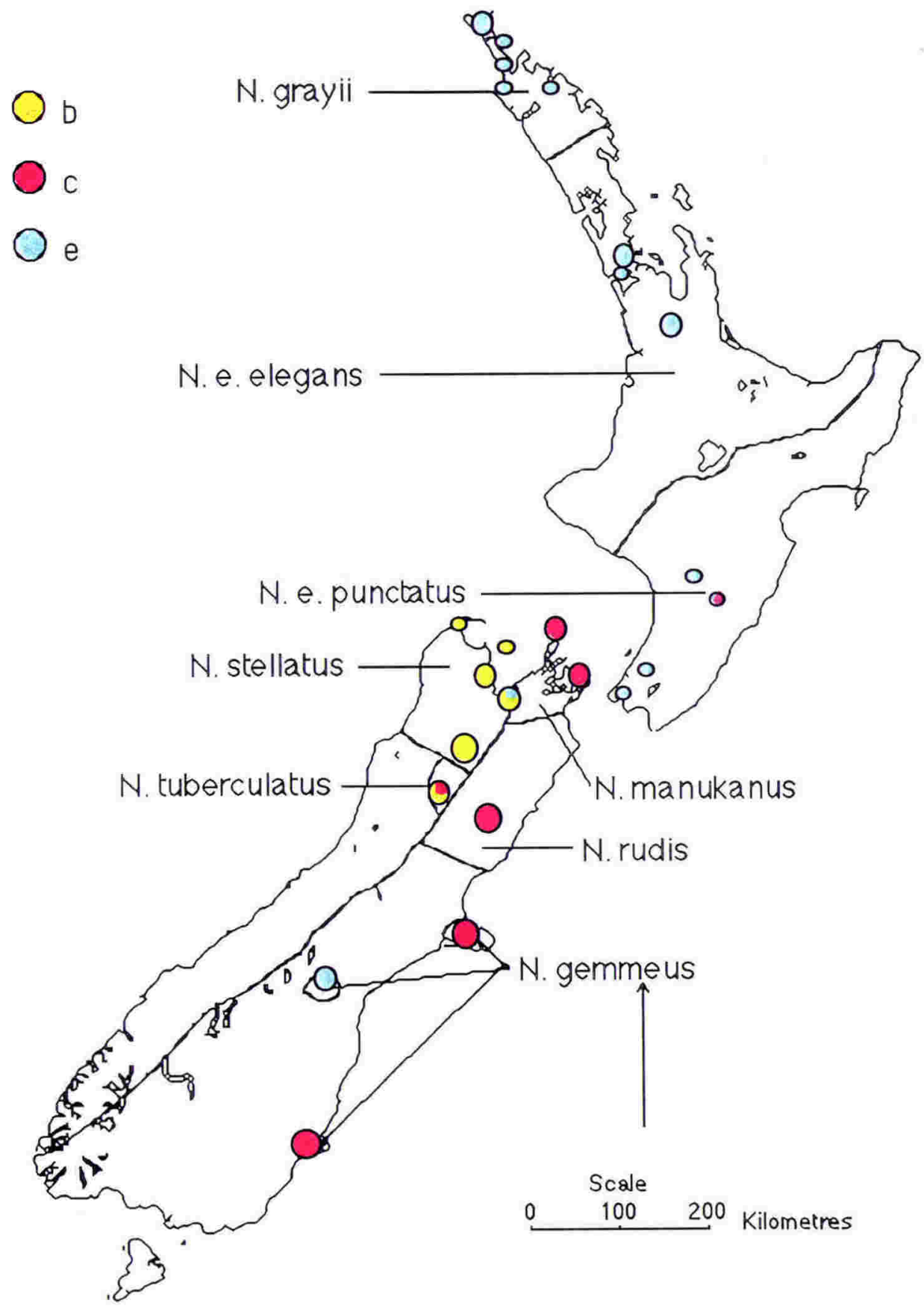

Figure 4.8.3.4 Distributions of alleles of Pgm-1 among populations of Naultinus spp., mapped onto the currently recognised species' distributions. 
Table 4.8.3.2 Measures of polymorphism and heterozygosity for grouped samples of Naultinus spp (standard errors in parentheses).

\begin{tabular}{|c|c|c|c|c|c|}
\hline \multirow[b]{2}{*}{ Population } & \multirow{2}{*}{$\begin{array}{l}\text { Mean } \\
\text { sample } \\
\text { size per } \\
\text { locus }\end{array}$} & \multirow[b]{2}{*}{$\begin{array}{l}\text { Mean no. } \\
\text { of alleles } \\
\text { per locus }\end{array}$} & \multirow{2}{*}{$\begin{array}{l}\text { Percentage } \\
\text { of loci } \\
\text { polymorphic* }\end{array}$} & \multicolumn{2}{|c|}{ Mean heterozygosity } \\
\hline & & & & $\begin{array}{l}\text { direct- } \\
\text { count }\end{array}$ & $\begin{array}{l}\text { hdywbg } \\
\text { expected** }\end{array}$ \\
\hline N. grayii & $\begin{array}{l}4.0 \\
(0.0)\end{array}$ & $\begin{array}{l}1.0 \\
(0.0)\end{array}$ & 0.0 & $\begin{array}{l}0.00 \\
(0.00)\end{array}$ & $\begin{array}{l}0.00 \\
(0.00)\end{array}$ \\
\hline N. grayii (North Cape) & $\begin{array}{l}2.0 \\
(0.0)\end{array}$ & $\begin{array}{l}1.0 \\
(0.0)\end{array}$ & 0.0 & $\begin{array}{l}0.00 \\
(0.00)\end{array}$ & $\begin{array}{l}0.00 \\
(0.00)\end{array}$ \\
\hline N. e. elegans & $\begin{array}{l}5.0 \\
(0.0)\end{array}$ & $\begin{array}{l}1.0 \\
(0.0)\end{array}$ & 0.0 & $\begin{array}{l}0.00 \\
(0.00)\end{array}$ & $\begin{array}{l}0.00 \\
(0.00)\end{array}$ \\
\hline N. e. punctatus & $\begin{array}{l}4.0 \\
(0.0)\end{array}$ & $\begin{array}{l}1.0 \\
(0.0)\end{array}$ & 3.7 & $\begin{array}{l}0.01 \\
(0.01)\end{array}$ & $\begin{array}{l}0.01 \\
(0.01)\end{array}$ \\
\hline N. manukanus & $\begin{array}{l}4.0 \\
(0.0)\end{array}$ & $\begin{array}{l}1.0 \\
(0.0)\end{array}$ & 0.0 & $\begin{array}{l}0.00 \\
(0.00)\end{array}$ & $\begin{array}{l}0.00 \\
(0.00)\end{array}$ \\
\hline N. rudis & $\begin{array}{l}3.0 \\
(0.0)\end{array}$ & $\begin{array}{l}1.0 \\
(0.0)\end{array}$ & 0.0 & $\begin{array}{l}0.00 \\
(0.00)\end{array}$ & $\begin{array}{l}0.00 \\
(0.00)\end{array}$ \\
\hline N. stellatus (Nelson Lakes) & $\begin{array}{l}3.0 \\
(0.0)\end{array}$ & $\begin{array}{l}1.1 \\
(0.1)\end{array}$ & 7.4 & $\begin{array}{l}0.00 \\
(0.00)\end{array}$ & $\begin{array}{l}0.04 \\
(0.03)\end{array}$ \\
\hline N. stellatus (Sandy Bay) & $\begin{array}{l}2.0 \\
(0.0)\end{array}$ & $\begin{array}{l}1.1 \\
(0.1)\end{array}$ & 7.4 & $\begin{array}{l}0.04 \\
(0.03)\end{array}$ & $\begin{array}{l}0.04 \\
(0.03)\end{array}$ \\
\hline N. stellatus (Maitai Valley) & $\begin{array}{l}2.0 \\
(0.0)\end{array}$ & $\begin{array}{l}1.0 \\
(0.0)\end{array}$ & 3.7 & $\begin{array}{l}0.02 \\
(0.02)\end{array}$ & $\begin{array}{l}0.02 \\
(0.02)\end{array}$ \\
\hline N. stellatus (Adele Island) & $\begin{array}{l}1.0 \\
(0.0)\end{array}$ & $\begin{array}{l}1.0 \\
(0.0)\end{array}$ & 0.0 & $\begin{array}{l}0.00 \\
(0.00)\end{array}$ & $\begin{array}{l}0.00 \\
(0.00)\end{array}$ \\
\hline N. stellatus (Golden Bay) & $\begin{array}{l}1.0 \\
(0.0)\end{array}$ & $\begin{array}{l}1.0 \\
(0.0)\end{array}$ & 3.7 & $\begin{array}{l}0.04 \\
(0.04)\end{array}$ & $\begin{array}{l}0.04 \\
(0.04)\end{array}$ \\
\hline N. tuberculatus & $\begin{array}{l}2.0 \\
(0.0)\end{array}$ & $\begin{array}{l}1.0 \\
(0.0)\end{array}$ & 3.7 & $\begin{array}{l}0.02 \\
(0.02)\end{array}$ & $\begin{array}{l}0.02 \\
(0.02)\end{array}$ \\
\hline N. gemmeus (Hakataramea) & $\begin{array}{l}2.0 \\
(0.0)\end{array}$ & $\begin{array}{l}1.0 \\
(0.0)\end{array}$ & 0.0 & $\begin{array}{l}0.00 \\
(0.00)\end{array}$ & $\begin{array}{l}0.00 \\
(0.00)\end{array}$ \\
\hline N. gemmeus (Banks Pen.) & $\begin{array}{l}3.0 \\
(0.0)\end{array}$ & $\begin{array}{l}1.0 \\
(0.0)\end{array}$ & 3.7 & $\begin{array}{l}0.01 \\
(0.01)\end{array}$ & $\begin{array}{l}0.01 \\
(0.01)\end{array}$ \\
\hline N. gemmeus (Otago Pen.) & $\begin{array}{l}4.0 \\
(0.0)\end{array}$ & $\begin{array}{l}1.0 \\
(0.0)\end{array}$ & 0.0 & $\begin{array}{l}0.00 \\
(0.00)\end{array}$ & $\begin{array}{l}0.00 \\
(0.00)\end{array}$ \\
\hline
\end{tabular}


离

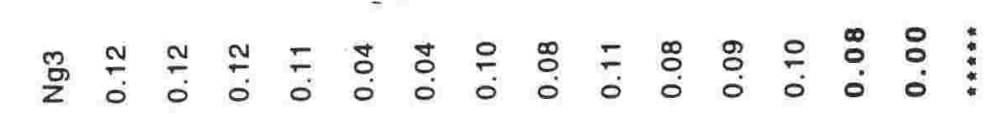

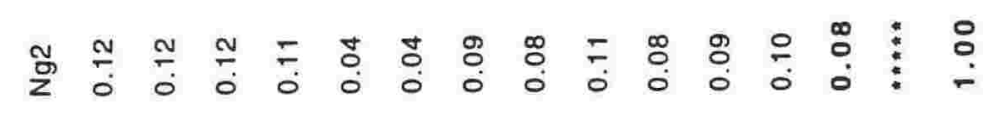

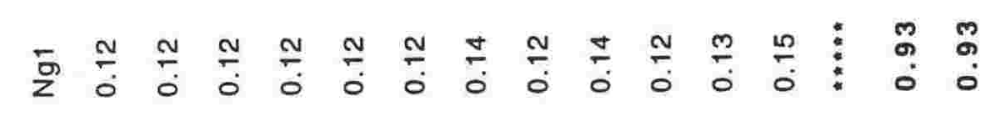

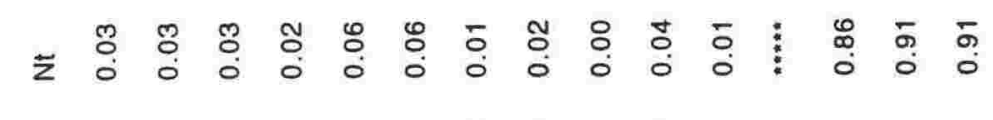

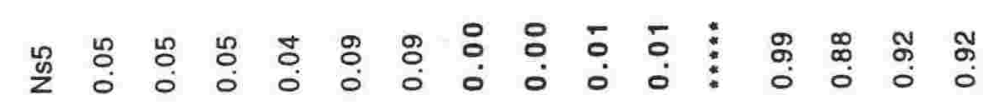

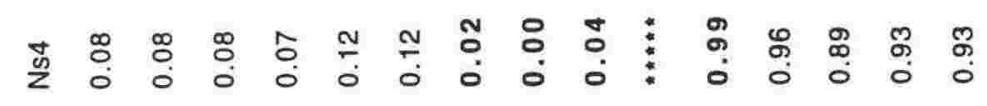

๓

๙

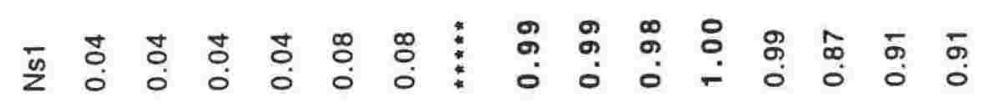

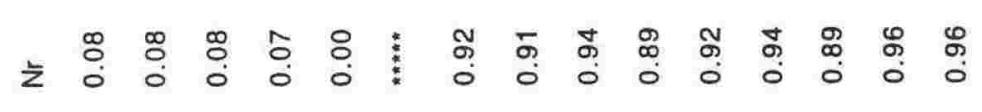

冡

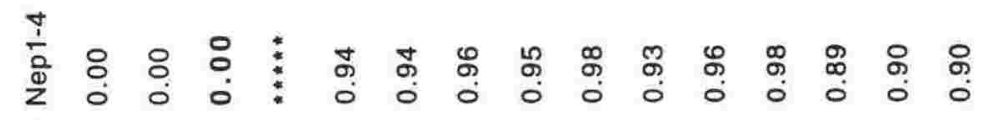

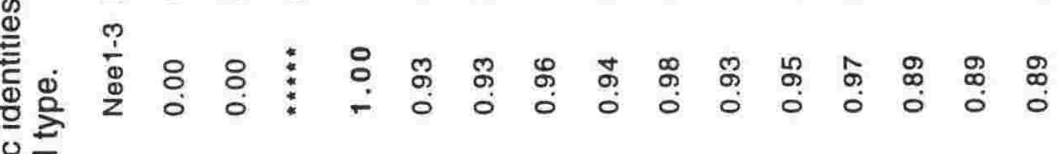

焉思

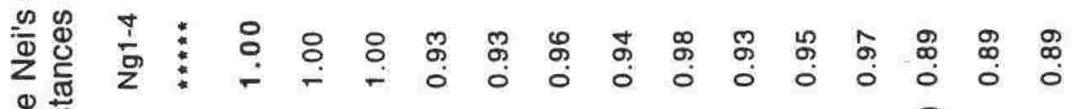

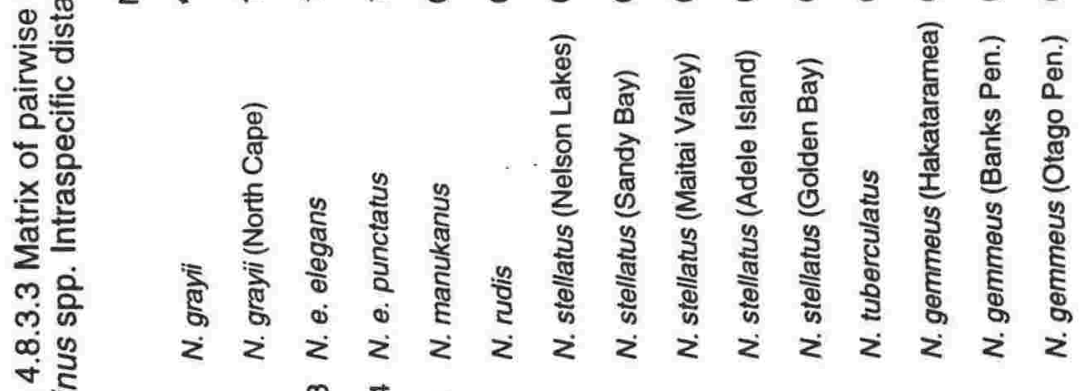

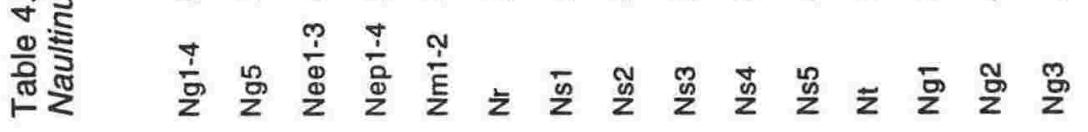




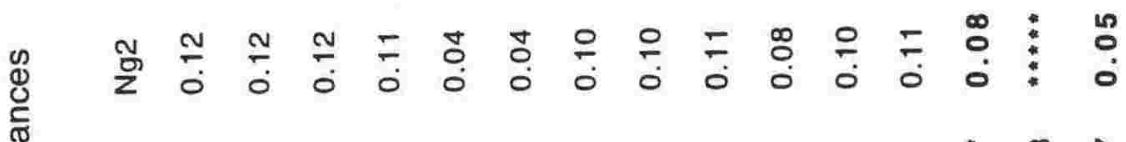

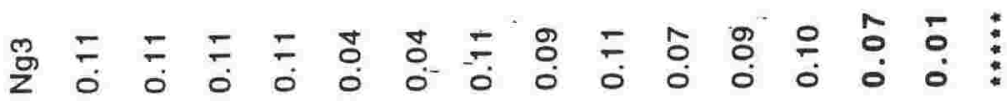

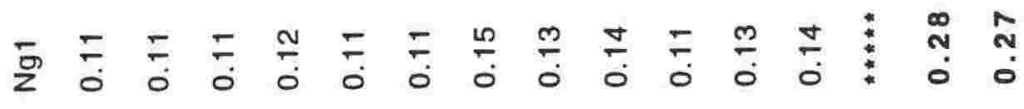

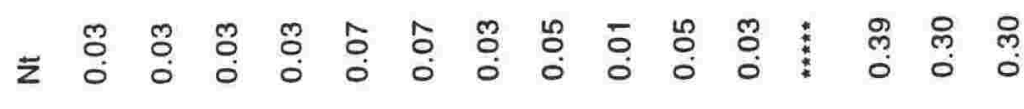

ลัก

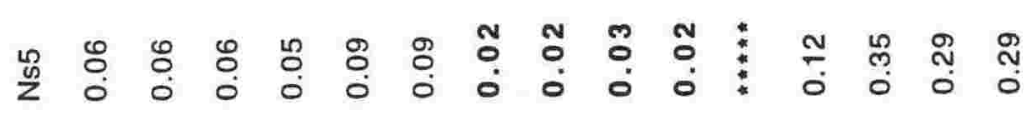

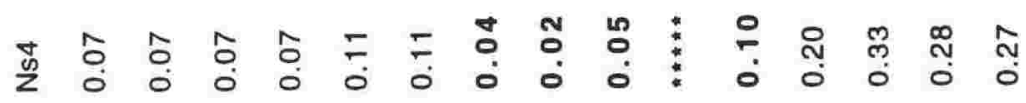
은 స్త్

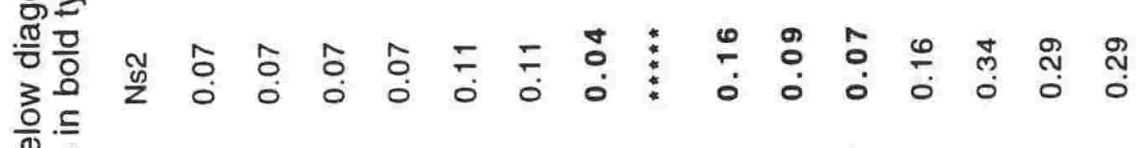

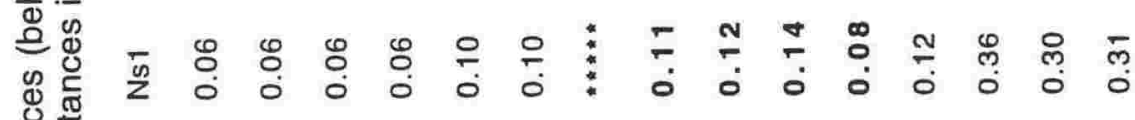
悹 $\frac{\pi}{0}$

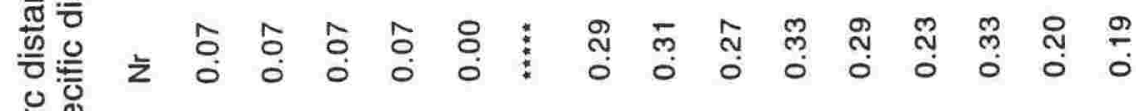

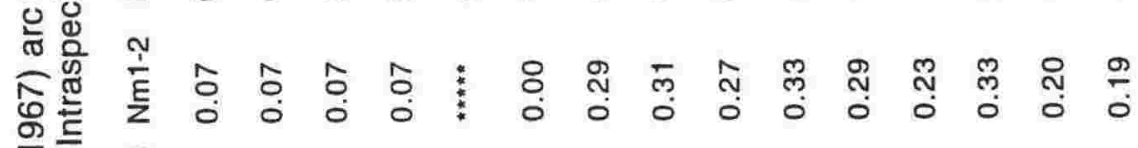

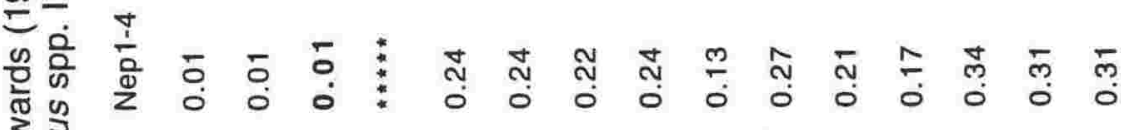
㟧

め 흥

员员

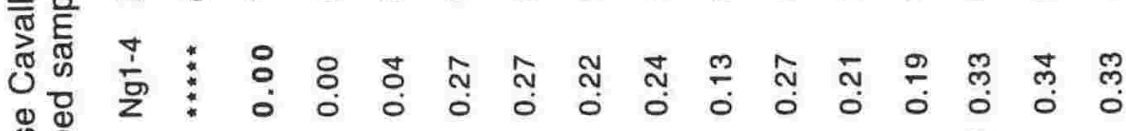

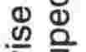

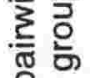
응 둥

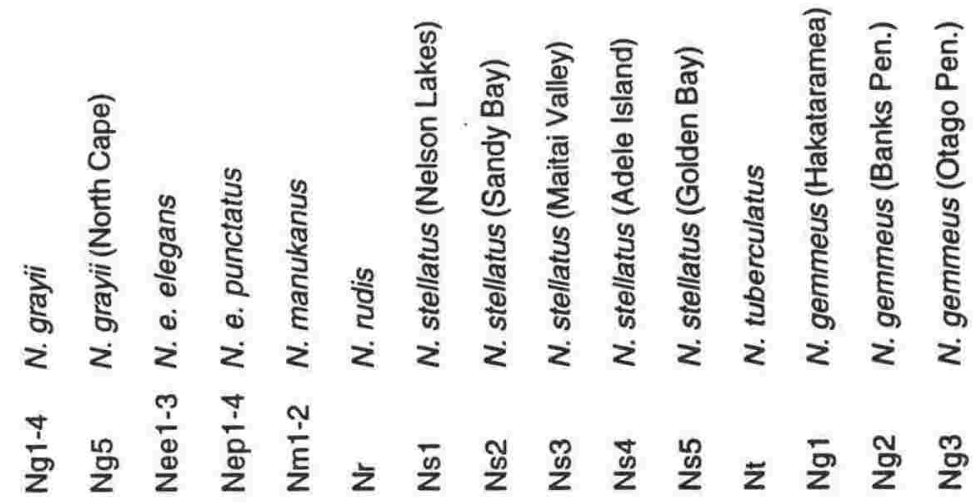


WPGMA (Figure 4.8.3.5), distance Wagner (Figure 4.8.3.6), and neighbour joining (Figure 4.8.3.7) phenograms consistently identified several groups, but differed somewhat in the relationships suggested among these groups. Groups common to all 3 trees were:

- all North Island samples

- $\quad$. tuberculatus plus $N$. stellatus (except that this group is paraphyletic to the North Island group in the distance Wagner phenogram)

- North Island species plus N. tuberculatus and N. stellatus

- $\quad$ N. manukanus plus $N$. rudis

- Otago Peninsula and Banks Peninsula N. gemmeus

- N. manukanus, $N$. rudis, and N. gemmeus (except that Hakataramea Pass N. gemmeus is the sister group of all the other taxa in the WPGMA phenogram).

The Hakataramea Pass sample clustered first with the other populations of $N$. gemmeus only in the distance Wagner phenogram. It was the sister group of $N$. manukanus, $N$. rudis, and Otago Peninsula and Banks Peninsula $N$. gemmeus in the neighbour-joining tree, and the sister group of all the other taxa in the WPGMA tree.

$N$. tuberculatus fell within N. stellatus in the WPGMA and neighbour-joining trees, but was the sister group of $N$. stellatus plus the North Island species in the distance Wagner tree.

The 3 captive-bred hybrids all had allozyme genotypes consistent with their recorded parentage (Table 4.8.2.1).

\subsubsection{MORPHOLOGY}

As Bauer (1990) pointed out, most character differences among Naultinus species involve visible external morphology. Dorsal colour pattern (Figure 4.8.4.1), ventral colour (Figure 4.8.4.2), foot-pad colour (Figure 4.8.4.3), mouth colour (Figure 4.8.4.4), tongue colour (Figure 4.8.4.5), secondary coloration in sexually mature males (Figure 4.8.4.6), presence or absence of contact between the nostrils and rostral scale (Figure 4.8.4.7), and presence or absence of greatly enlarged scales on the dorsum (Figure 4.8.4.8) all vary geographically, mainly among species, but also sometimes among populations within species of green geckos. Some character states occur in geographically contiguous clusters of species/populations (e.g., mottled brown belly colouring, Figure 4.8.4.2), but others show disjunct distributions 


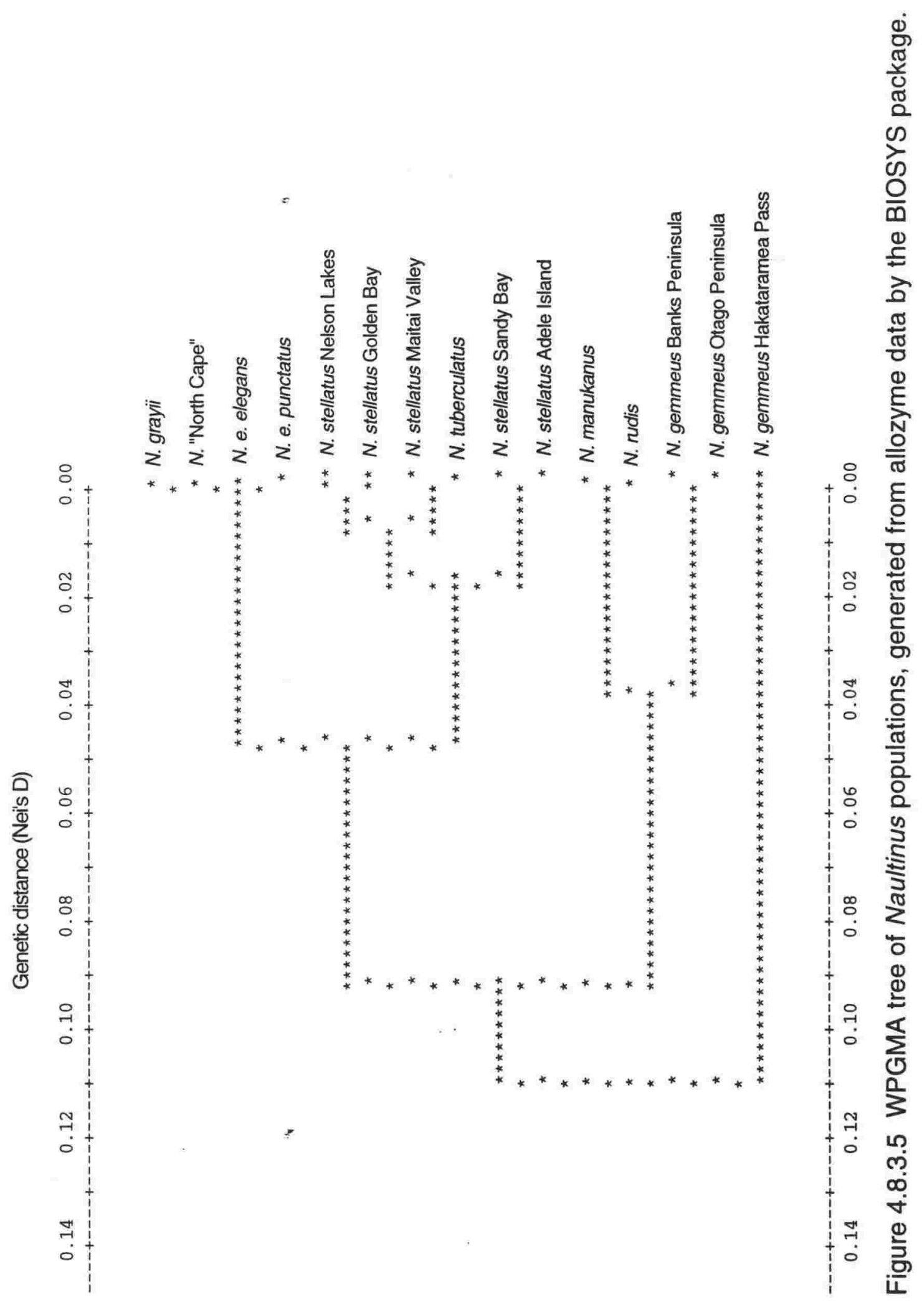




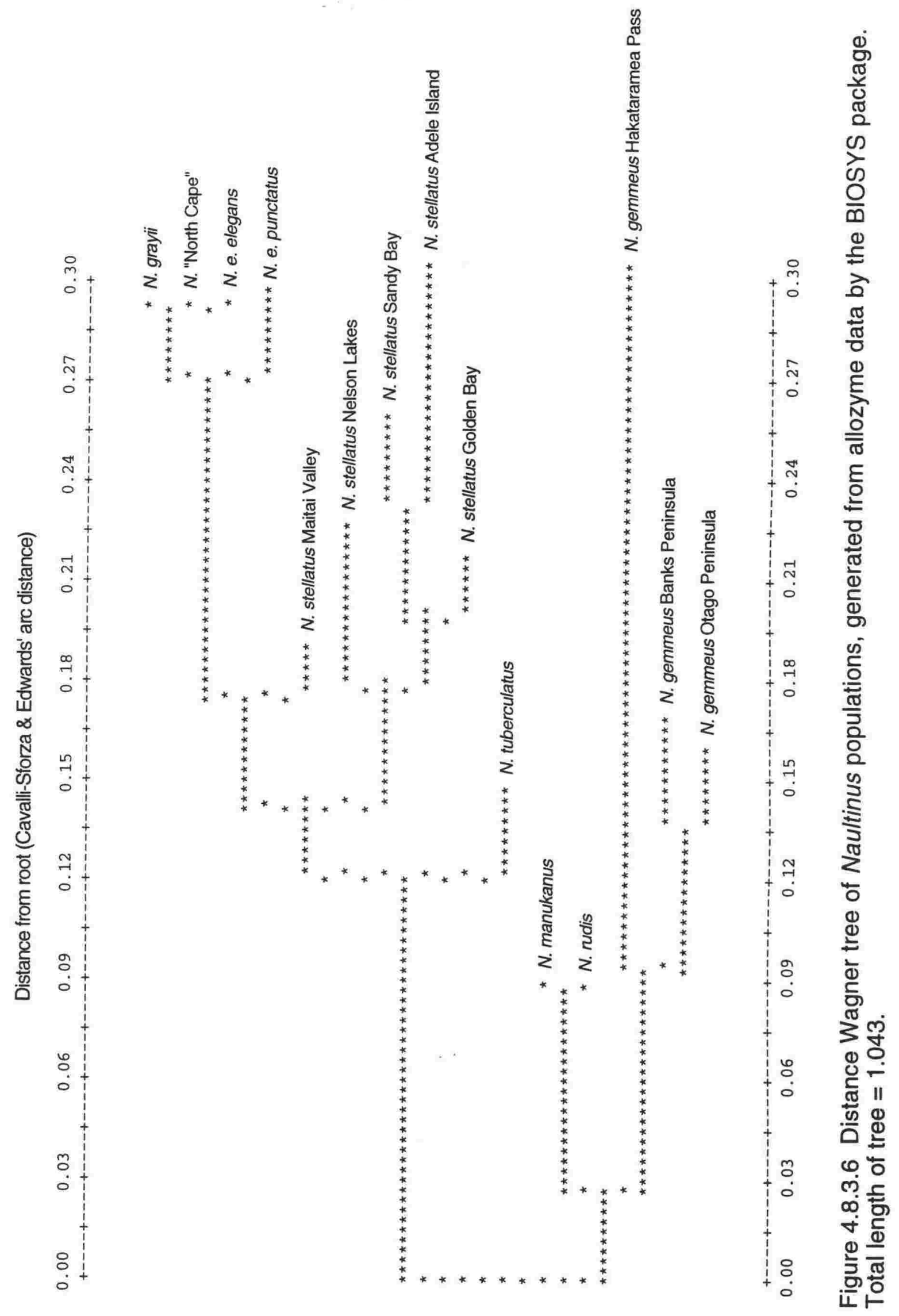




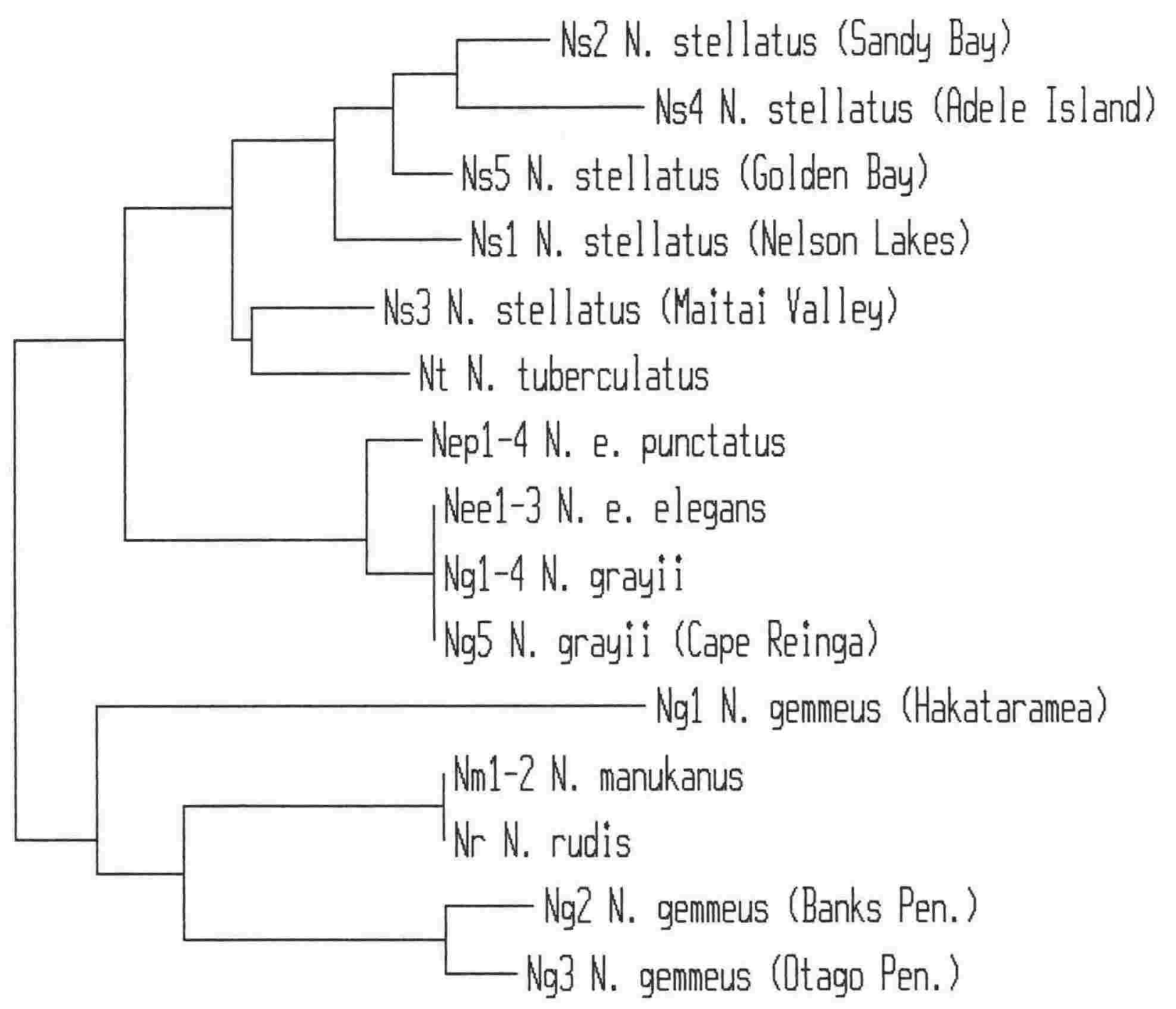

Figure 4.8.3.7 Neighbour-joining tree of Naultinus populations, generated from Cavalli-Sforza \& Edwards' (1967) distances (27 allozyme loci) using the MEGA programme. 
NEW ZEALAND

DA Large circular blotches

D Broad transverse stripes

Complexpaltem of small spots and narrowstripes

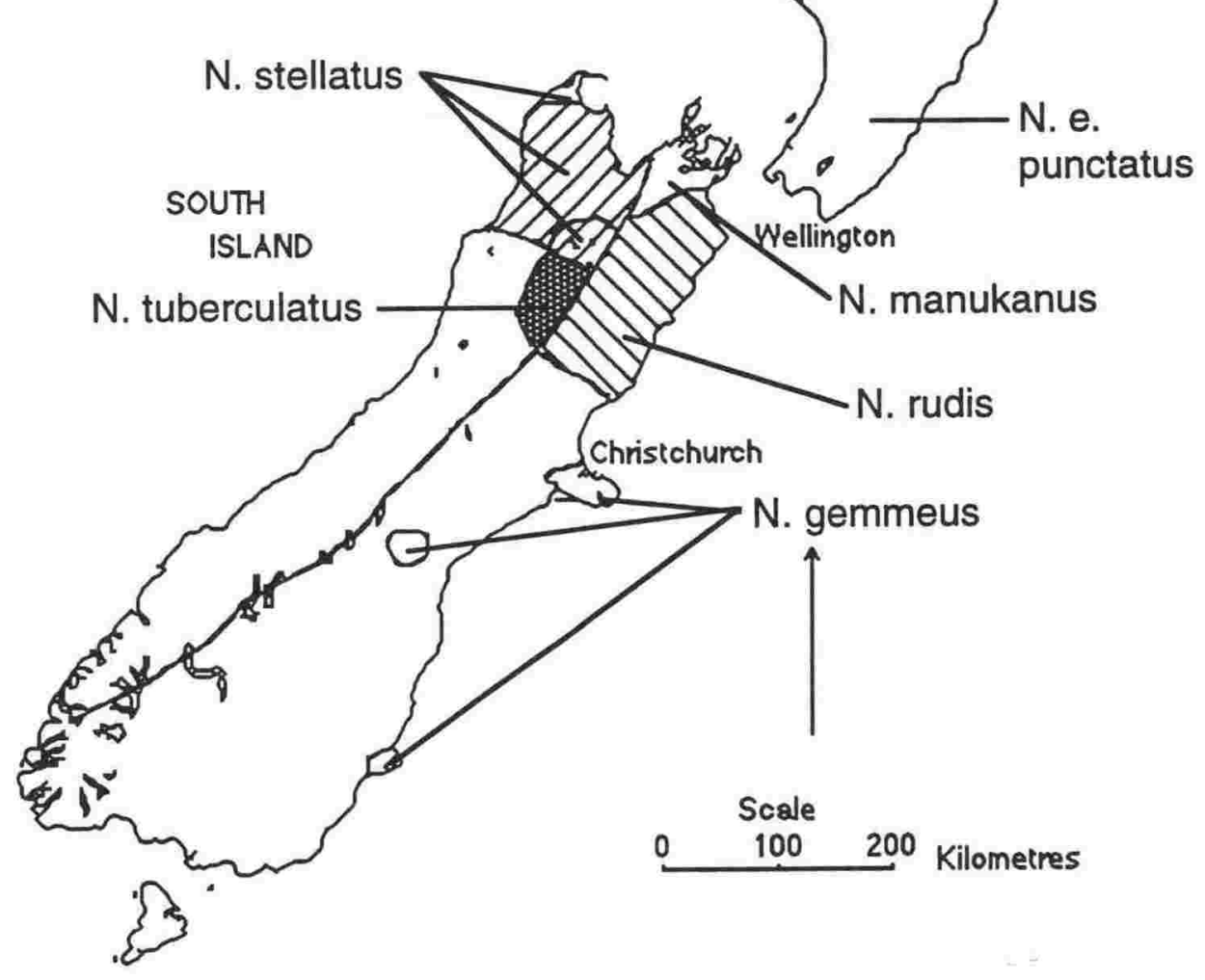

Figure 4.8.4.1 Geographic variation in dorsal colour pattern of Naultinus spp., mapped onto the currently recognised species' distributions. 


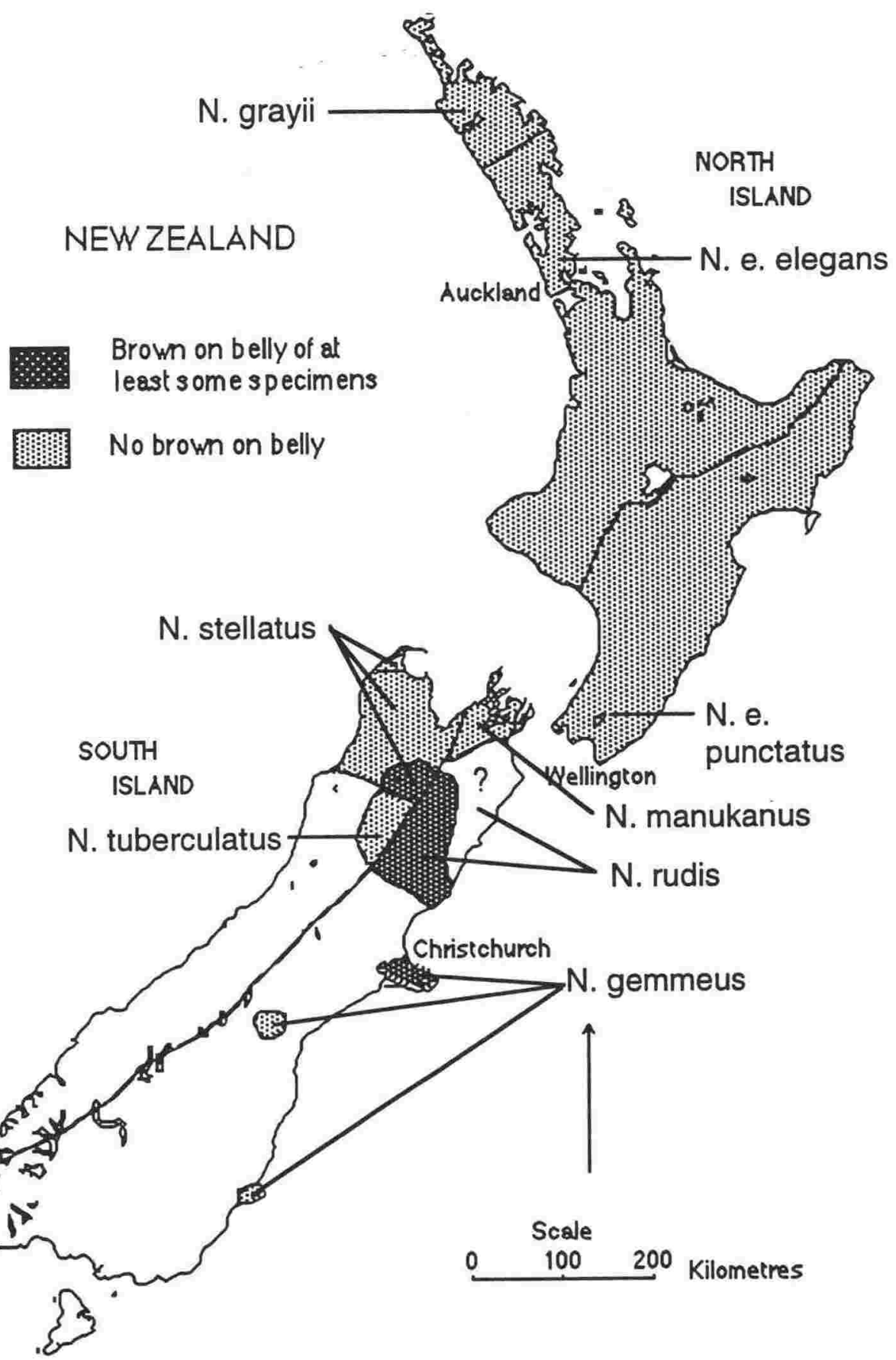

Figure 4.8.4.2 Geographic variation in ventral colour pattern of Naultinus spp., mapped onto the currently recognised species' distributions. 


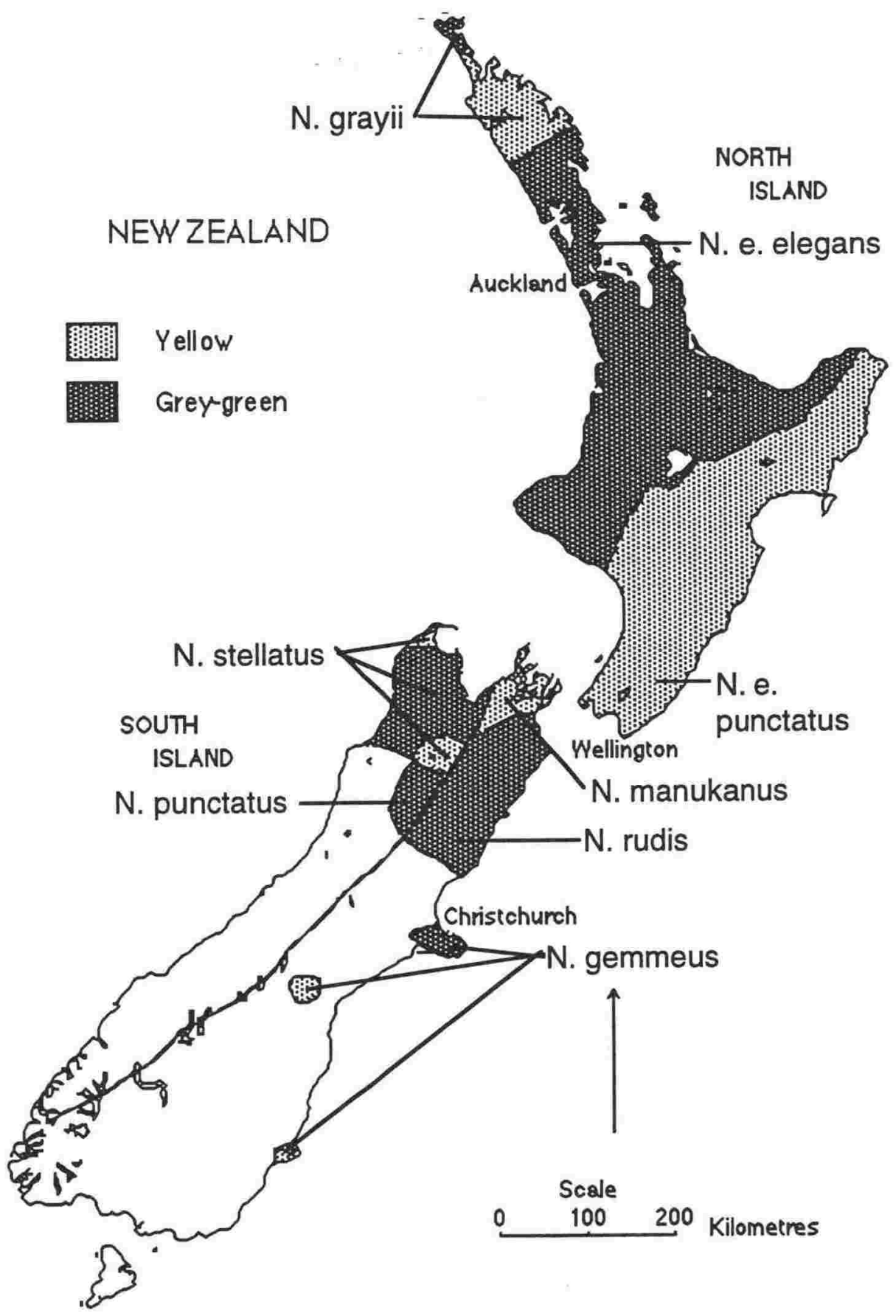

Figure 4.8.4.3 Geographic variation in foot-pad colour of Naultinus spp., mapped onto the currently recognised species' distributions. 


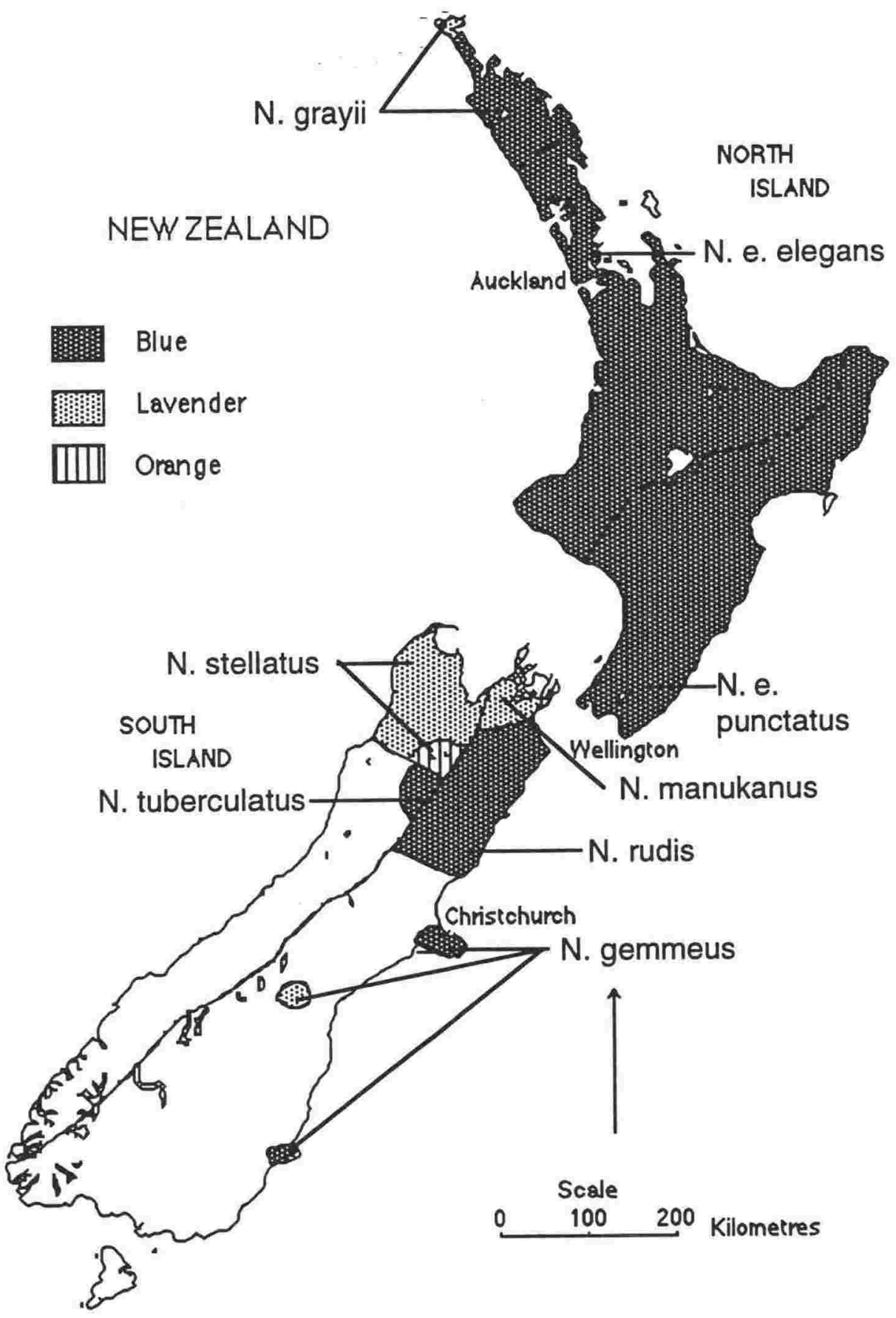

Figure 4.8.4.4 Geographic variation in mouth colour of Naultinus spp., mapped onto the currently recognised species' distributions. 


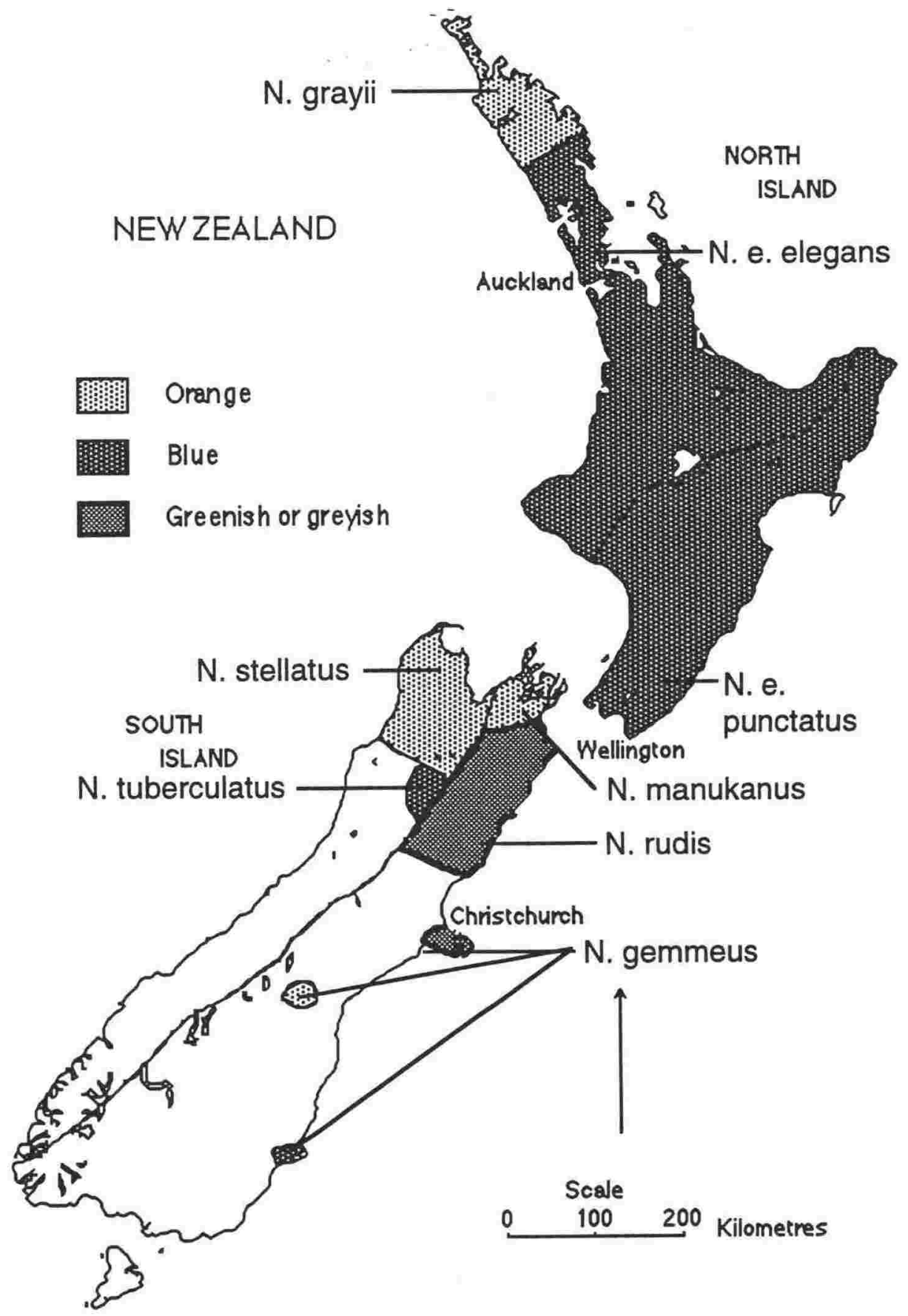

Figure 4.8.4.5 Geographic variation in tongue colour of Naultinus spp., mapped onto the currently recognised species' distributions. 
N. grayii

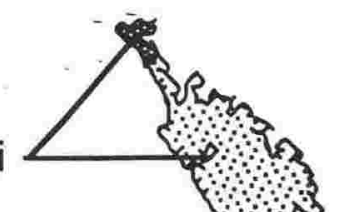

… None

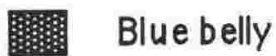

Blue flank

Da Brown dorsum

MV Dark green dorsum

Auckland

एव N. e. elegans

N. stellatus

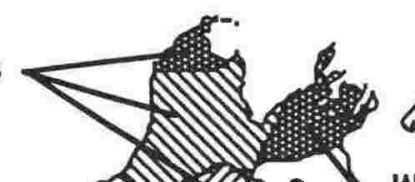

N.e.

punctatus

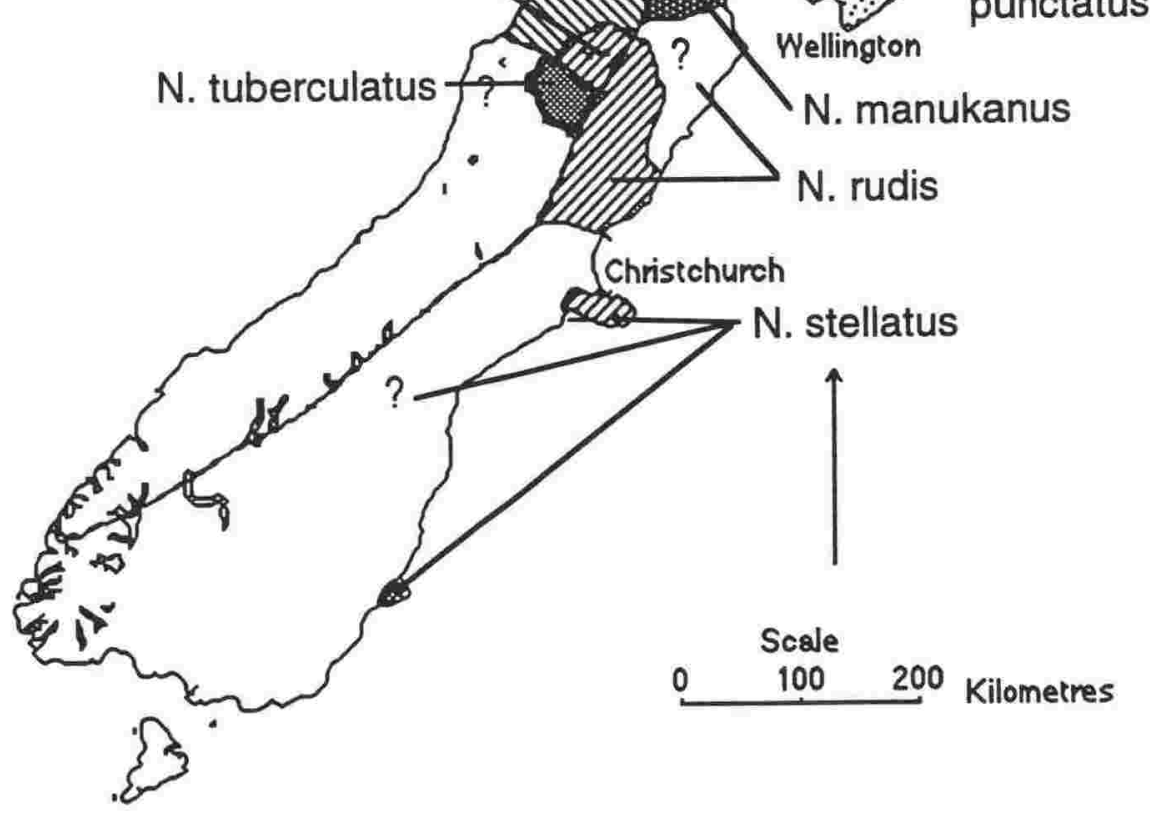

Figure 4.8.4.6 Geographic variation in secondary colouration in sexually mature males of Naultinus spp., mapped onto the currently recognised species' distributions. 
NEW ZEALAND

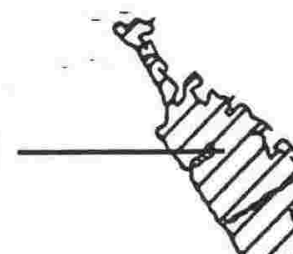

DX Excludedfrom nostril

DV Touchingnostril

NORTH

ISLAND

N. e. elegans पू $\mathrm{N}$

Auckland

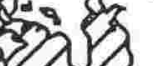

N. tuberculatus

N. stellatus

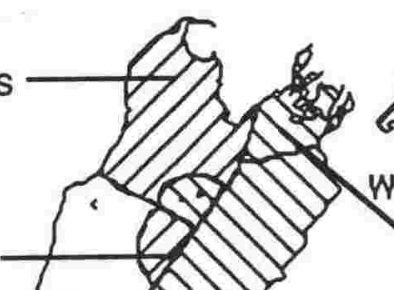

N. rudis

Chrisichurch

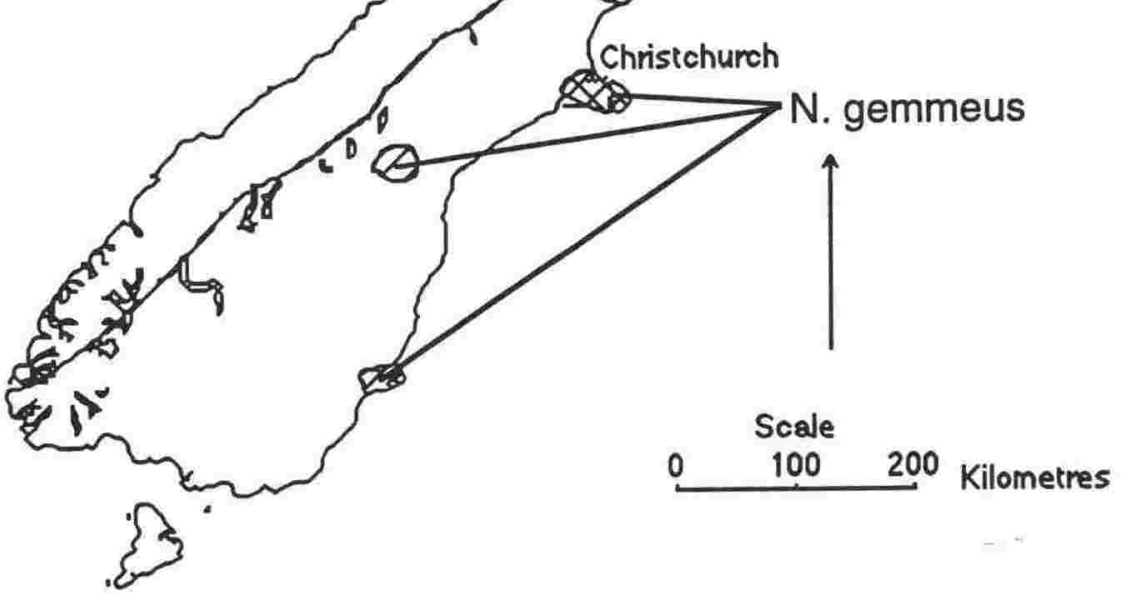

है

Figure 4.8.4.7 Geographic variation in presence or absence of contact between the nostrils and rostral scale of Naultinus spp., mapped onto the currently recognised species' distributions. 


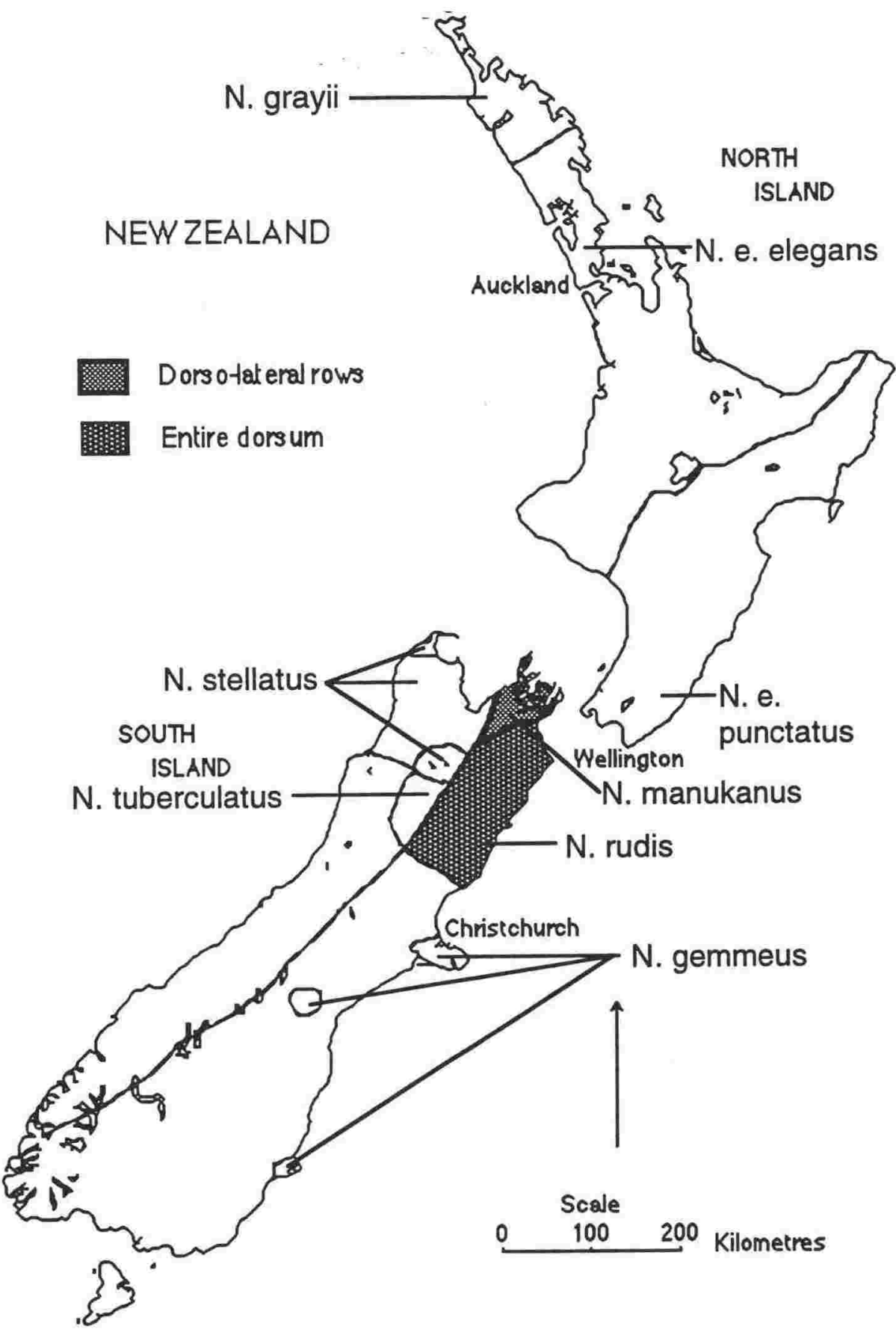

Figure 4.8.4.8 Geographic variation in presence of greatly enlarged scales on the dorsum of Naultinus spp., mapped onto the currently recognised species' distributions. 
Table 4.8.4.1. Morphological characters of north-western South Island Naultinus populations examined in this study.

\begin{tabular}{|c|c|c|c|c|c|}
\hline $\begin{array}{l}\text { Species/ } \\
\text { population }\end{array}$ & $\begin{array}{l}\text { Rostral/ } \\
\text { nostril }\end{array}$ & $\begin{array}{l}\text { Snout } \\
\text { scales }\end{array}$ & & $\begin{array}{l}\text { Supracilian } \\
\text { scales }\end{array}$ & $\begin{array}{l}\text { Body } \\
\text { scales }\end{array}$ \\
\hline $\begin{array}{l}\text { N. stellatus } \\
\text { Golden Bay } \\
\text { Sandy Bay } \\
\text { Maitai Valley }\end{array}$ & $\begin{array}{l}\text { Excluded } \\
\text { Excluded } \\
\text { Excluded }\end{array}$ & \multicolumn{2}{|c|}{$\begin{array}{l}\text { Small, rounded } \\
\text { Small, rounded } \\
\text { Flat, imbricate } \\
\text { or small, rounded }\end{array}$} & $\begin{array}{l}\text { Conical } \\
\text { Rounded } \\
\text { Rounded }\end{array}$ & $\begin{array}{l}\text { Tuberculate } \\
\text { Tuberculate } \\
\text { Tuberculate }\end{array}$ \\
\hline $\begin{array}{l}\text { Blue Glen } \\
\text { Nelson Lakes }\end{array}$ & $\begin{array}{l}\text { Excluded } \\
\text { Touch or } \\
\text { excluded }\end{array}$ & \multicolumn{2}{|c|}{$\begin{array}{l}\text { Medium, rounded } \\
\text { Large, irregular }\end{array}$} & $\begin{array}{l}\text { Rounded } \\
\text { Conical }\end{array}$ & $\begin{array}{l}\text { Tuberculate } \\
\text { Tuberculate }\end{array}$ \\
\hline N. tuberculatus & Excluded & \multicolumn{2}{|c|}{ Large, irregular } & Conical & Tuberculate \\
\hline $\begin{array}{l}\text { Species/ } \\
\text { population }\end{array}$ & $\begin{array}{l}\text { Snout } \\
\text {-vent (mm) }\end{array}$ & $\begin{array}{l}\text { Tongue } \\
\text { colour }\end{array}$ & $\begin{array}{l}\text { Foot-pad } \\
\text { colour }\end{array}$ & \multicolumn{2}{|c|}{$\begin{array}{c}\text { Male } 2^{\circ} \\
\text { colour }\end{array}$} \\
\hline $\begin{array}{l}\text { N. stellatus } \\
\text { Golden Bay } \\
\text { Sandy Bay } \\
\text { Maitai Valley } \\
\text { Blue Glen } \\
\text { Nelson Lakes } \\
\text { N. tuberculatus }\end{array}$ & $\begin{array}{l}45-55 \\
50-60 \\
65-70 \\
65 \\
70-80 \\
65-85\end{array}$ & $\begin{array}{l}\text { Orange } \\
\text { Orange } \\
\text { Orange } \\
\text { Orange } \\
\text { Orange } \\
\text { Black }\end{array}$ & $\begin{array}{l}\text { Yellow } \\
\text { Grey } \\
\text { Grey } \\
\text { Yellow } \\
\text { Grey }\end{array}$ & \multicolumn{2}{|c|}{$\begin{array}{l}\text { Blue belly } \\
\text { Darker green dorsum } \\
\text { Darker green dorsum } \\
\text { ? } \\
\text { Brown dorsum } \\
\text { None? }\end{array}$} \\
\hline
\end{tabular}

(e.g., lavender mouth colour, Figure 4.8.4.4, foot colour, Figure 4.8.4.3, blue belly in mature males Figure 4.8.4.6), suggesting a complex history of geographic spread and gene flow. The significance of this diversity is discussed with respect to particular species boundaries below.

$N$. stellatus shows more population-level morphological variation than other species. Morphological characters of this species, and the genetically similar and geographically adjacent $N$. tuberculatus, are summarised in Table 4.8.4.1; mouth and tongue colour, dorsal colour pattern, belly colour, and absence of sexual colour dimorphism all diagnostically separate these 2 species.

\subsubsection{DISCUSSION}

Allozyme electrophoresis revealed relatively little allozyme differentiation among the recognised species of Naultinus, comparable with that within the $H$. pacificus complex, and considerably less than that within either the $H$. 
maculatus or $H$. granulatus complexes. This genetic similarity is consistent with the results of Meads' (1982) captive hybridisation experiments which showed full interfertility of all Naultinus species in captivity. The total absence of sympatry between any Naultinus species pair is also entirely consistent with these results, although sympatric species pairs in the $H$. maculatus and $H$. pacificus complexes differ by genetic distances comparable to the larger between-species differences among Naultinus species.

This shallow genetic divergence within Naultinus implies very much more recent speciation than that within any other genus of New Zealand endemic lizards (this thesis; Daugherty et al. 1990a). The North Island species, which are morphologically well-differentiated but genetically identical at the loci I examined (see below), are an extreme example.

Many morphological characters are concordant with the species boundaries. This is not surprising, as the species were recognised on the basis of some of these same characters. However, other characters do not agree as well with the accepted species boundaries, reflecting geographic variation within recognised species (particularly N. gemmeus; mouth colour, Figure 4.8.4.4, foot colour, Figure 4.8.4.3, belly colour, Figure 4.8.4.2, sexual dimorphism, Figure 4.8.4.6; also N. stellatus, Table 4.8.4.1), and in some instances indicating past introgression across well-supported species boundaries (e.g., Nelson Lakes N. stellatus, see below).

The presence of 3 different forms of sexual colour dimorphism (blue belly, blue flank, brown or dark green dorsum, Figure 5.8.4.6) in different taxa suggests a strong visual component in courtship (although it is also possible that the secondary male colours might be significant only in the context of inter-male conflict). Although interspecific hybrids are easily obtained in captivity, mating tends to be assortative when there is mate choice (Robb 1980a). I have observed a single pair of $N$. grayii courting in a cage among about $60 \mathrm{~N}$. elegans, which were ignoring them and vice versa. This suggests a mechanism by which speciation might proceed at much lower levels of genetic divergence, in comparison to the nocturnal, non-dichromic, presumably more pheromone-mediated Hoplodactylus species. Similarly, birds, which have strongly visual mate recognition systems, are well known to frequently have little or no measurable allozyme divergence among closely related species (Patton \& Avise 1986). 


\subsubsection{N. gemmeus}

The Banks Peninsula population of $N$. gemmeus shows fixed allozyme differences from its closest neighbours, $N$. rudis at Pgm-2 and $N$. tuberculatus at Pgd-1 and Pgm-2 (Table 5.8.3.1). Morphologically, Banks Peninsula $N$. gemmeus differ from $N$. rudis in tongue colour, enlarged scales

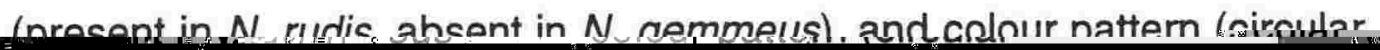

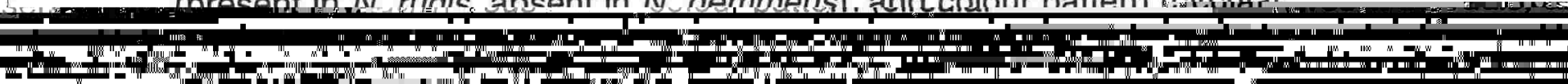


rostral, but is otherwise similar, though less colourfully patterned than most Otago Peninsula specimens. I could not locate the Green Island specimens, but saw photographs. The animals appeared very similar to the Hakataramea Pass specimens, with the same orange and lavender mouth pigment, and orange colouring around the nostrils and corners of the mouth. In view of this widespread range, and the discrepancies between patterns of morphological and allozyme differentiation in the widely separated samples available, I consider that further formal separation of populations of $N$. gemmeus would be premature at this stage. Since the allozyme divergence between the Hakataramea population and the 2 coastal populations is one of the deepest in the genus, further taxonomic separation may be warranted when more information becomes available.

\subsubsection{Separation of eastern and western species pairs in the northern South Island}

The best-supported taxonomic boundary in Naultinus is that separating the populations on the eastern (N. manukanus and $N$. rudis) and western $(N$. stellatus and $N$. tuberculatus) sides of the northern third of the South Island. This boundary zone extends about $150 \mathrm{~km}$ from the coast east of Nelson city ( $N$. stellatus to the west meeting $N$. manukanus to the east) in an almost straight line south-south-west to end between Hanmer ( $N$. rudis in the east) and Lewis Pass (N. tuberculatus in the west) at the southern limit of the range of $N$. rudis. It is well supported by allozymes - Pgd-1 shows a fixed and $P g m-1$ an almost fixed difference across it, and Pgm-2(c) is common in the west but absent in the east.

The single morphological feature which is completely concordant with this boundary throughout its length is the presence of greatly enlarged scales on the dorsum of populations to the east ( $N$. rudis and $N$. manukanus), and their absence to the west ( $N$. stellatus and N. tuberculatus). Bauer's (1990) character-state matrix (his appendix 3 ) lists all Naultinus spp. except $N$. rudis and $N$. manukanus as having the nostril not in contact with the rostral, in contradiction of McCann's descriptions, in which only N. tuberculatus and the North Island species have this condition. However, I have found this character to be more variable within and between other species (Figure 4.8.4.7).

Populations of $N$. stellatus and $N$. manukanus have been found only a few kilometres apart in the northern South Island (Bull \& Whitaker 1975; Pickard \& Towns 1988). They are readily distinguished; $N$. stellatus has a blotched 
pattern on an olive-green background and no enlarged scales, whereas $N$. manukanus is usually brighter green with smaller more discrete markings, and has distinct dorso-lateral rows of greatly enlarged scales. Bull \& Whitaker (1975) reported hybridisation at the zone of contact, but only one putative wild hybrid was examined (A. H. Whitaker, pers. comm.), so the extent of hybridisation is unknown.

Compared with the more northern populations of $N$. stellatus, the Nelson Lakes population differs in several characters which are present in many specimens from this population but are not completely fixed: larger size, brown rather than green dorsal background colour in adult males, mottled brown and cream belly, large irregular snout scales, and rostral scale in contact with the nostril. All of these characters could be explained by introgression from the neighbouring population of $N$. rudis (or vice versa). However, this does not mean that there are no barriers to gene flow across the boundary between these species; allozyme differences at 2 loci, mouth and tongue colour, foot-pad colour, the presence of enlarged dorsal scales, and details of the dorsal colour pattern all support the validity of the boundary at this point.

\subsubsection{N. stellatus and N. tuberculatus}

The nomenclatural confusion involving $N$. tuberculatus, $N$. stellatus, and $N$. poecilochlorus is discussed in Chapter 3 . In this thesis I regard populations from Lewis Pass and the West Coast south of the Buller Gorge as belonging to $N$. tuberculatus, and those north of the Buller Gorge, including the Golden Bay-Cape Farewell area, as belonging to $N$. stellatus.

The justification for separate species status for $N$. tuberculatus is questionable. Allozyme frequency differences, but no fixed differences, separate $N$. stellatus from $N$. tuberculatus from Lewis Pass, although small sample sizes mean that these frequency differences may be sampling artefacts. One $N$. tuberculatus was heterozygous for an allele (Pgm-1(c)) not seen in any N. stellatus. Both Pgm-2(a) and Pgm-2(c) were common in N. stellatus, but only Pgm-2(a) was found in N. tuberculatus. At Mpi-1 and Sod-2 the most common allele seen in N. stellatus was the only one seen in N. tuberculatus.

Although diagnostic morphological differences between $N$. tuberculatus and $N$. stellatus as a whole are fewer because of the substantial variation within 
$N$. stellatus, the geographically closest population of $N$. stellatus (Nelson Lakes) differs from N. tuberculatus (Lewis Pass) in dorsal colour pattern, ventral colour, mouth and tongue colour, presence of sexual dimorphism, and foot-pad colour (Table 4.8.4.1).

The possibility of limited gene flow between $N$. tuberculatus and $N$. stellatus is suggested by the appearance of a specimen collected in about 1970 at Shenandoah and held in captivity. This animal combined the mouth colour of $N$. stellatus with elements of the colour pattern of $N$. tuberculatus (B. W. Thomas pers. comm.). However, typical $N$. stellatus and $N$. tuberculatus have been found less than $30 \mathrm{~km}$ apart (Robb 1980b), and much of the suitable habitat in this "gap" has not been searched. The morphological differences between the 2 taxa over a short geographic distance justify their continued recognition at species level in the absence of further information to the contrary. Populations of $N$. stellatus separated by similar distances may show statistical, but not diagnostic differences.

\subsubsection{N. rudis and N. manukanus}

I found no allozyme differences between $N$. manukanus and $N$. rudis.

Thomas (1982) described strongly clinal variation in size, colour pattern, and distribution of enlarged scales from north to south within N. manukanus, and found the most southern populations of this species are almost indistinguishable in these characters from $N$. rudis. He therefore suggested that the 2 species be synonymised. Robb (1980a) recorded differences in foot-pad, mouth, and tongue colour between the 2 species. Perhaps most significantly, the 2 differ in the form of sexual colour dimorphism, males of $N$. manukanus having a light blue belly, and many males of $N$. rudis a brown dorsum. Distributions of these characters near the putative boundary should be investigated further before the validity of $N$. manukanus as a separate species can be judged.

\subsubsection{Separation between North and South Island populations}

North Island species of Naultinus have no diagnostic alleles. They are most similar in allozyme genotype to $N$. stellatus (Figures 4.8.3.5-7), but differ from it by an almost fixed difference at Pgm-1 and a major frequency difference at $P g m-2$, and from N. manukanus by a fixed difference at Pgd-1, and an almost fixed difference at Pgm-1. 
There is also a fixed difference across Cook Strait in scale texture; North Island specimens have much smaller, more uniform-sized, less conical scales then any South Island specimens, although the Stephens Island and particularly the Cape Farewell populations are closer than other South Island populations to the North Island condition.

There have been repeated comments in the literature that $N$. manukanus from Stephens Island are morphologically almost indistinguishable from $N$. elegans from the Wellington area (N. e. punctatus) (e.g., Bull \& Whitaker 1975; Thomas 1982); McCann (1955) included the Stephens Island population in $N$. elegans. In fact the 2 differ in body size, mouth and tongue colour (Figures 4.8.4.4, 4.8.4.5), form of sexual dimorphism (Figure 4.8.4.6), rostral-nostril contact (Figure 4.8.4.7), and the presence of enlarged scales on the head and pelvic region in Stephens Island N. manukanus (Figure 4.8.4.8).

Bull \& Whitaker (1975) and Thomas (1982) also stated the north-western populations of $N$. stellatus (formerly referred to $N$. tuberculatus) to be morphologically similar to the North Island species. Pairwise comparisons of these populations with $N$. grayii and the $2 N$. elegans subspecies reveal multiple morphological differences in each instance (body size, snout scale shape, mouth and tongue colour (Figures 4.8.4.4, 4.8.4.5), form of sexual dimorphism (Figure 4.8.4.6), foot colour (Figure 4.8.4.3)). However, atypical $N$. grayii populations from the extreme north of the North Island differ diagnostically from Cape Farewell populations only in foot-pad colour (Figures 4.8.4.1-8).

\subsubsection{Species boundaries in the North Island}

The 2 recognised Naultinus species ( $N$. grayii and $N$. elegans) in the North Island differ by 5 months in their birth season, and morphologically in snout shape, earhole size, tongue colour, and several scale features, and $N$. grayii has additional diagnostic differences from each of the $N$. elegans subspecies (Robb \& Hitchmough 1980). N. elegans has 2 well-differentiated subspecies (N. e. elegans and N. e. punctatus), which differ diagnostically in size, form of secondary male colouring, foot-pad colour, cloacal spur colour, and form of markings (Robb \& Hitchmough 1980). Populations from the North Cape area represent an undescribed taxon distinct from the neighbouring $N$. grayii in body size, mouth colour, form of male secondary sex colouring, foot colour, and in lacking the flattened snout scales which are 
a diagnostic character for adult $N$. grayii. The population from the North Cape area was not recognised as distinct by Robb \& Hitchmough (1980); the specimen from this area examined by Robb \& Hitchmough (1980, fig. 7) was assumed to be a subadult.

In contrast to this morphological diversity, no allozyme variation was found in North Island Naultinus except for a single N. e. punctatus from Mount Bruce which was heterozygous for Pgm-1(e), the common North Island allele, and Pgm-1(c) the allele found in N. manukanus, N. rudis, and N. gemmeus.

There is evidence of introgressive hybridisation at both the northern and southern boundaries of the range of N. grayii. A specimen seen by $P$. Anderson (Department of Conservation, pers. comm.) at Opua, Bay of Islands, in the southern part of the range of $N$. grayii, showed reddish-purple rather than orange-red tongue colour, which perhaps indicates introgression from N. e. elegans. RAH253, from the northern end of Kaimaumau Swamp, while in all other respects typical of $N$. grayii, had a pale lilac mouth lining as in the North Cape population, again probably indicating introgression.

Specimens from further south in this swamp area are typical $N$. grayii in all respects.

Robb \& Hitchmough (1980) suggested that the presence of some unusually large individuals in the otherwise typical $N$. e. elegans population near Turangi might indicate introgression from $N$. e. punctatus. This remains the only evidence for introgression between these subspecies, despite the fact that the 2 taxa have now been found very close together at several points along a boundary that spans the widest part of the North Island (DoC mapping scheme).

Therefore, although there is evidence from a few specimens for the possibility of hybridisation at each of the geographic boundaries between North Island Naultinus taxa, any hybrid zones must be very narrow relative to the overall distributions of the taxa. This suggests that, by the species criteria followed in this study, species status is justified for all 4 taxa. However, in the absence of supporting genetic evidence I am not proposing any formal taxonomic change. More variable genetic systems such as DNA sequences from very variable regions of the genome, or microsatellite DNA should be used to assess gene flow across these boundaries. 


\subsection{Allozyme Confirmation OF, THE IDENTITY OF \\ MAUD ISLAND HOPLODACTYLUS STEPHENSI AND \\ MANA ISLAND H. CHRYSOSIRETICUS}

\subsubsection{INTRODUCTION}

The taxonomic status and therefore the conservation priority of allopatric populations is often problematical (e.g., Frost \& Hillis 1990). Allozyme electrophoresis has been used to detect cryptic allopatric species among New Zealand frogs (Leiopelma spp., Bell et al. in press) and tuatara (Sphenodon spp., Daugherty et al. 1990b) on islands in Cook Strait. Allozyme electrophoresis was therefore appropriate for assessing genetic divergence between Cook Strait island populations of the very rare Hoplodactylus stephensi and between island and mainland populations of H. chrysosireticus.

H. chrysosireticus was known from Mana Island (Daniel et al. 1972) before the species was formally described (Robb 1980a, b), but none was seen on the island between 1972 and 1991. In February 1991 a Department of Conservation survey team rediscovered the species on the island. In total several hundred animals were seen; they were found in large numbers in some flax (Phormium tenax) bushes, but were patchily distributed.

$H$. stephensi was long considered a Stephens Island endemic, both before and after its formal description (Robb 1980a, b). In May 1989, Brian Bell (pers. comm.) photographed a striped brown gecko on Maud Island. The gecko was tentatively identified as $H$. stephensi from these photographs. In March 1991 a survey led by A H Whitaker located several more specimens.

\subsubsection{SAMPLES}

Two individuals from each of the newly discovered/rediscovered populations of $H$. chrysosireticus and $H$. stephensi were collected for electrophoretic analysis. Two Stephens Island specimens of $H$. stephensi, and 3 mainland Taranaki specimens (ex captivity) of $H$. chrysosireticus had already been collected for this study.

\subsubsection{RESULTS}

Despite the superficial similarity of their longitudinally striped colour patterns, $H$. chrysosireticus and $H$. stephensi are not closely related. There were fixed 

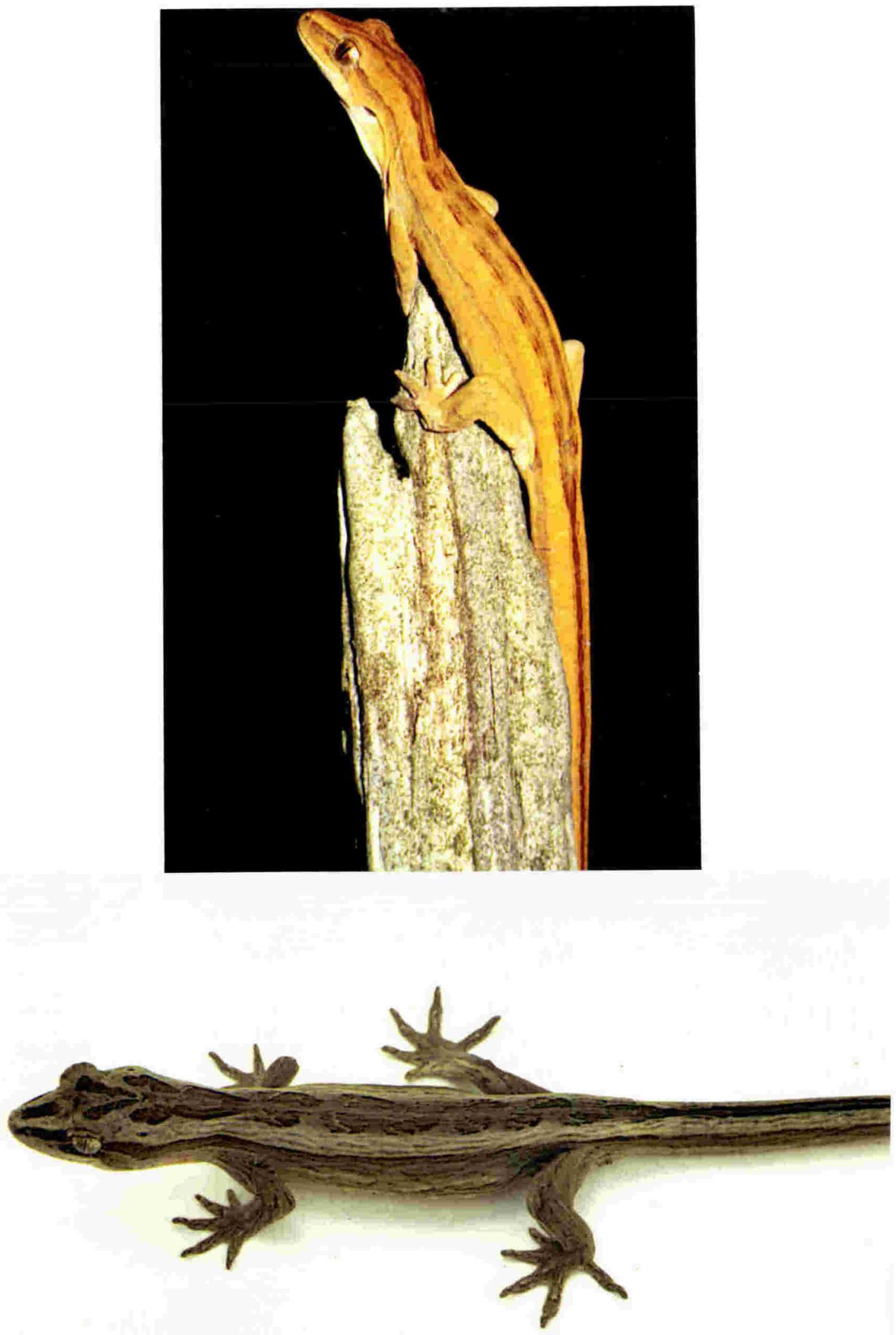

Figure 4.9.3.1 H. stephensi from Stephens Island (above, photographed by A. Cree) and Maud Island (below), showing differences in head markings. 
differences between the 2 species at 18 of the 27 allozyme loci scored in this study (Chapter 5).

In contrast, there were no fixed differences and only one apparent frequency difference between the $2 \mathrm{H}$. stephensi populations. Both Stephens Island specimens were Mpi-1(eg) heterozygotes, and both Maud Island specimens were Mpi-1(e) homozygotes, but this frequency difference could easily be a sampling artefact, given the small samples $(n=2)$ from each population. However, the Maud Island specimens were smaller than mature adult specimens from Stephens Island 70 and $72 \mathrm{~mm}$ snout-vent, vs 79 and 80 $\mathrm{mm}$ snout-vent), and had pronounced markings on their heads, reminiscent of $H$. rakiurae, which are absent from animals in the Stephens Island population (Figure 4.9.3.1).

There was one fixed difference $(G p-4=$ albumin) between Mana Island ( $G p$ 4 (c)) and mainland Taranaki (Gp-4 (i)) H. chrysosireticus. The Mana Island specimens were larger ( 66 and $71 \mathrm{~mm}$ snout-vent vs about $60 \mathrm{~mm}$ snoutvent) than the Taranaki ones, but otherwise morphologically indistinguishable.

\subsubsection{Discussion}

The differences in allozymes, size, and markings between the conspecific populations of $H$. stephensi and $H$. chrysosireticus are no greater than those between allopatric populations in other Hoplodactylus species, and do not justify taxonomic separation. However, conservation programmes should aim to preserve the geographic structure of genetic diversity (Vrijenhoek et al. 1985). The populations within each species are not identical, and should be managed as separate units. Any management must avoid any possibility of mixing animals originating from different populations.

The history of discovery of these populations indicates that both species are extremely cryptic in habits. $H$. stephensi was recognised as a potentially separate species on Stephens Island by the 1960s (Sharell 1966) and was apparently then regarded as common, but for the last 20 years has been apparently uncommon and difficult to locate predictably (e.g., Newman 1980). There has been a long history of biological research on Maud Island, including numerous visits by herpetologists, but $H$. stephensi was discovered there only in 1989 and is still difficult to locate. Both islands are free of introduced mammalian predators (except for occasional brief 
colonisations of stoats on Maud), yet on both either $H$. stephensi is very uncommon, or its primary habitat has not been found. It has not been found in sympatry with any introduced mammal, but its low population density or extremely cryptic habits even on mammal-free Maud and Stephens Islands suggest that there must be other causes for its apparent rarity apart from mammalian predators.

H. chrysosireticus, in contrast, until recently co-existed with introduced mammals throughout its range. It was discovered in Taranaki (an intensively settled area) only in the 1960s, and it was found to be common in flax, probably its primary habitat, only in the late 1970s (Wilkinson 1981); until then it had been found only as a rare occupant of household gardens. On Mana Island this species went undetected between 1972 and 1991. It may have been genuinely rarer during this period because of the impact of very high mouse population densities. However, a substantial portion of the rediscovered population must have co-existed with mice, because the lag of less than 2 years between mouse eradication in 1989 and the rediscovery of the $H$. chrysosireticus population was insufficient for major population growth in an animal with maximum productivity of 2 offspring per adult female per year and minimum age at maturity of probably 3-4 years.

It is therefore likely that there are undetected extant populations of both species, which may be at low densities in the presence of introduced predators. Any translocations should be planned with this possibility in mind and follow intensive surveying to detect existing native populations. The low reproductive rate of geckos (maximum 2 offspring per adult female per year; minimum generation time 4 years in many Hoplodactylus species) means that recovery of populations to detectable levels after removal of predators may be slow. Our current poor knowledge of $H$. stephensi means that a confident conclusion that the species is absent in any particular location may remain impossible.

Mana Island is the only island locality for $H$. chrysosireticus. The population on Matapia Island, off Ninety Mile Beach, recorded by Pickard \& Towns (1988), is in fact a new species, very closely allied to, but sympatric with, $H$. pacificus (section 5.2). It differs by 14 fixed differences (some at different loci) from both $H$. chrysosireticus and $H$. stephensi (Chapter 5). 


\subsection{SUMMARY}

The patterns of variation observed within each of the major species groups/complexes in the New Zealand gecko fauna are very different.

The $H$. pacificus complex and Naultinus have similar, low levels of allozyme divergence within them. Naultinus has only allopatric or parapatric taxa, often with concordant morphological and allozyme differences across narrow boundaries. The $H$. pacificus complex has morphologically and genetically divergent species on isolated offshore islands, but also has sympatric but very closely related species on a tiny offshore island which has been isolated for only about 10000 years.

The 2 major groups of the $H$. maculatus complex have much more genetic diversity, but the biogeographical pattern of this variation varies between them. In the southern group the taxa are all parapatric (except for apparent sympatry at the Omarama site), whereas the northern group has sympatry or extensive overlap of distributions among 3 of its 4 species.

The $H$. granulatus complex has both high levels of genetic divergence within species $(H$. granulatus itself), and morphologically highly distinct species with little allozyme divergence ( $H$. kahutarae).

These different patterns of divergence within species complexes mean that simplistic application of distance values alone cannot resolve species status, as has been found by other authors, e.g., Frost \& Hillis's (1990) criticism of Highton's (1989) analysis of Plethodon salamander speciation.

As outlined in section 4.1.2, I have used an evolutionary/concordance species concept for allopatric or parapatric taxa, i.e., taxa are considered separate species if there is concordant evidence from 2 or more genetically unlinked markers that they are on separate evolutionary trajectories, as indicated by sympatry, contact at a parapatric boundary or narrow hybrid zone, degree of genetic divergence from sister taxa, or phylogenetic relationships to other clades of species.

Using these criteria I propose 11 new species, resurrect 2 species from synonymy, and recommend areas for further investigation where allozyme results are suggestive but not conclusive. These species and the evidence supporting their recognition are as follows. 


\section{Within the $\boldsymbol{H}$. pacificus complex:}

- $\quad$ H. "Matapia", because it is sympatric with H. pacificus on Matapia Island, with 2 fixed differences separating them, although allozyme separation from allopatric populations of $H$. pacificus is minimal;

- $\quad \boldsymbol{H}$. "Three Kings" and $\boldsymbol{H}$. "Poor Knights", because phylogenetic analysis indicates that they are the sister group of $H$. pacificus plus $H$. "Matapia", and the genetic and morphological differences between $H$. "Three Kings" and $H$. "Poor Knights" are greater than those between $H$. pacificus and $H$. "Matapia", and between other sympatric species pairs.

H. "Southern mini", because of the substantial genetic separation $(D=$ 0.38-0.40) and diagnostic morphological differences from its sister species, H. chrysosireticus.

\section{Within the northern group of the $\boldsymbol{H}$. maculatus complex:}

- $\quad H$. "Marlborough mini", because its range extensively overlaps on a broad scale, and abuts on a local scale, that of $H$. maculatus; these two species form separate groups in dendrograms, and (with one exception) are separated by pairwise differences of $D=0.11-0.35$;

- $\quad H$. "Kaikouras", because its range falls entirely within that of $H$. "Marlborough mini" and is overlapped by that of $H$. maculatus, and it is sympatric with $H$. "Marlborough mini" at one site, with 2 fixed differences between them;

- $\quad$ H. "Mount Arthur", because neighbour-joining analysis places it as the sister taxon of the clade including the other 3 species of the northern group, and it is separated from all other populations by $D \geq 0.12$, which is larger than the genetic distance between sympatric $H$. "Kaikouras" and $H$. "Marlborough mini".

\section{Within the southern group of the $\boldsymbol{H}$. maculatus complex:}

- $\quad H$. "Southern Alps", because its range abuts those of $H$. brunneus, $H$. "Otago", and $H$. "Danseys Pass", with genetic distances of $D \geq 0.11$, concordant morphological differences, and no evidence of gene flow across these boundaries;

- $\quad H$. brunneus (resurrected from synonymy) because it is broadly parapatric with, but altitudinally separated from, $H$. "Southern Alps", with no evidence of gene flow between them as evidenced by genetic divergence; 
H. "Danseys Pass", because its range abuts those of $H$. "Southern Alps" and $H$. "Otago", with genetic distances of $D \geq 0.13$, concordant morphological differences, and no evidence of gene flow across these boundaries;

- $\quad \boldsymbol{H}$. "Cromwell Gorge", because its range is completely overlapped by that of $H$. "Otago", with allozyme divergence between closely parapatric populations;

- $\quad H_{\text {. }}$ "Otago", because its range overlaps that of $H$. "Cromwell Gorge" with genetic distances of $D \geq 0.11$ and no evidence of gene flow between them, and abuts those of $H$. "Southern Alps" and $H$. "Danseys Pass", with genetic distances of $D \geq 0.13$, concordant morphological differences, and no evidence of gene flow between them;

- $\quad$ further subdivision of both $H$. "Southern Alps" and $H$. "Otago" may be warranted, but requires more data.

\section{Within the $H$. granulatus complex:}

- $\quad H$. nebulosus (resurrected from synonymy) is specifically distinct from $H$. granulatus, on the basis of substantial genetic separation $(D=0.17$ 0.40 ), and differences in head shape, colour, and ecology; and because it is genetically closer to $H$. kahutarae $(D=0.12)$ than to $H$. granulatus;

- $H$. kahutarae is genetically close to $H$. nebulosus $(D=0.12)$ and some populations of $H$. granulatus ( $D \geq 0.11$ ), despite its very distinct morphology; - $\quad$ on the basis of morphology of museum specimens, there appears to be at least one undescribed species in the Fiordland-Open Bay Islands area, for which samples for genetic analysis were unavailable.

\section{Within Naultinus:}

- $\quad$ allozyme divergence supports the existence of barriers to gene flow between $N$. gemmeus and $N$. rudis; $N$. gemmeus and $N$. tuberculatus; $N$. rudis+manukanus and $N$. stellatus+tuberculatus; and between North Island and South Island taxa;

- there are no allozyme differences among any North Island species, or between $N$. rudis and $N$. manukanus, and only frequency differences between $N$. stellatus and $N$. tuberculatus;

- $\quad$ compared to genetic distances among some other species of Naultinus, there is substantial genetic divergence between populations within N. gemmeus;

- no formal taxonomic changes are suggested for Naultinus (except for the corrections to nomenclature of Nelson-West Coast populations 
presented in Chapter 3), but additional work using more rapidly-evolving genetic systems is recommended. 


\section{Chapter 5: PHYLOGENY}

\subsection{INTRODUCTION}

In this chapter I

- evaluate the higher-level phylogenetics of the Diplodactylinae to provide a context in which to examine the phylogeny of the New Zealand geckos

- reassess Bauer's (1990) cladistic analysis of morphological data from the New Zealand species

- perform cladistic and phenetic analyses of my allozyme data from New Zealand geckos

- compare the phylogenies generated from morphological and allozyme information, and form a consensus conclusion

- examine discrepancies between this consensus and the existing classification of New Zealand geckos

- suggest possible generic rearrangements, and

- present a personal view of the likely evolutionary history of geckos in New Zealand.

There is general agreement that the New Zealand genera are part of a monophyletic subfamily, the Diplodactylinae, of the family Gekkonidae (King 1987a, b; Kluge 1987; Greer 1989; Bauer 1990). This subfamily is distinguished by parchment-like rather than calcareous eggshells (except in viviparous species), lack of calcareous endolymphatic sacs, and having the pupil either slit-like or with more than 3 scallops on each edge. However, these features are believed to be plesiomorphic, and the only known synapomorphy is the larger than usual number of scleral ossicles (Kluge 1987; Greer 1989).

The Diplodactylinae occur in Australia, New Caledonia and New Zealand. As outlined in the introduction to this thesis, Kluge $(1967 a, b)$ divided the subfamily into 2 tribes, and placed the New Zealand genera in the Tribe Carphodactylini, along with the New Caledonian Eurydactylodes, 
Rhacodactylus, and Bavayia and the northern Australian

Pseudothecadactylus, Carphodactylus, Phyllurus, and Nephrurus. The other Australian genera formed the Tribe Diplodactylini.

Kluge (1967a, b) considered the New Zealand geckos to be a "natural group" most closely related to the New Caledonian genera, which in tum are most closely related to Pseudothecadactylus. He believed that speciation had occurred during southward dispersal from the northern Australia/New Guinea region, and that the New Zealand genera were the most primitive in the subfamily. His work predated widespread acceptance of continental drift as a possible underlying factor in biogeographical distributions, and his hypothesised time-scale for evolution of the major Gekkonid groups was based on his understanding of geological events. He believed that the Diplodactylinae arose during the Upper Cretaceous. This is also the period during which New Zealand is now believed to have separated from Gondwanaland. It is now therefore possible to propose an alternative hypothesis that, rather than dispersing to New Zealand over water, the geckos form part of the Gondwanan element of our fauna. Greer (1989) presented Kluge's (1967a, b) view in an informal summary tree (Figure 5.1.1).

King (1987a, b) described the karyotypes of most diplodactyline genera and species, although New Zealand species were poorly represented in his work. The karyotype data were consistent with the existence of 2 major phylogenetic groups within the subfamily, largely, but not entirely, agreeing with Kluge's tribes Diplodactylini and Carphodactylini (Figure 5.1.2). The Australian genus Oedura, placed by Kluge in the Diplodactylini, contained groups of species with 2 different karyotypes, one of which was identical to the highly derived form found in most Carphodactylini. The other Oedura group had a karyotype intermediate between that considered primitive in the subfamily and the derived carphodactyline state. Strophurus, another genus of Diplodactylini, also had karyotypes intermediate between the 2 main groups. An important conclusion of King's (1987b) work was that, since the derived carphodactyline karyotype is present in Australia, New Caledonia, and New Zealand, evolution within the tribe must have been well advanced before these landmasses separated.

Bauer (1990) considered the narrow-toed, padless Australian genera Carphodactylus, Phyllurus, and Nephrurus to be ancestral, and the padded 

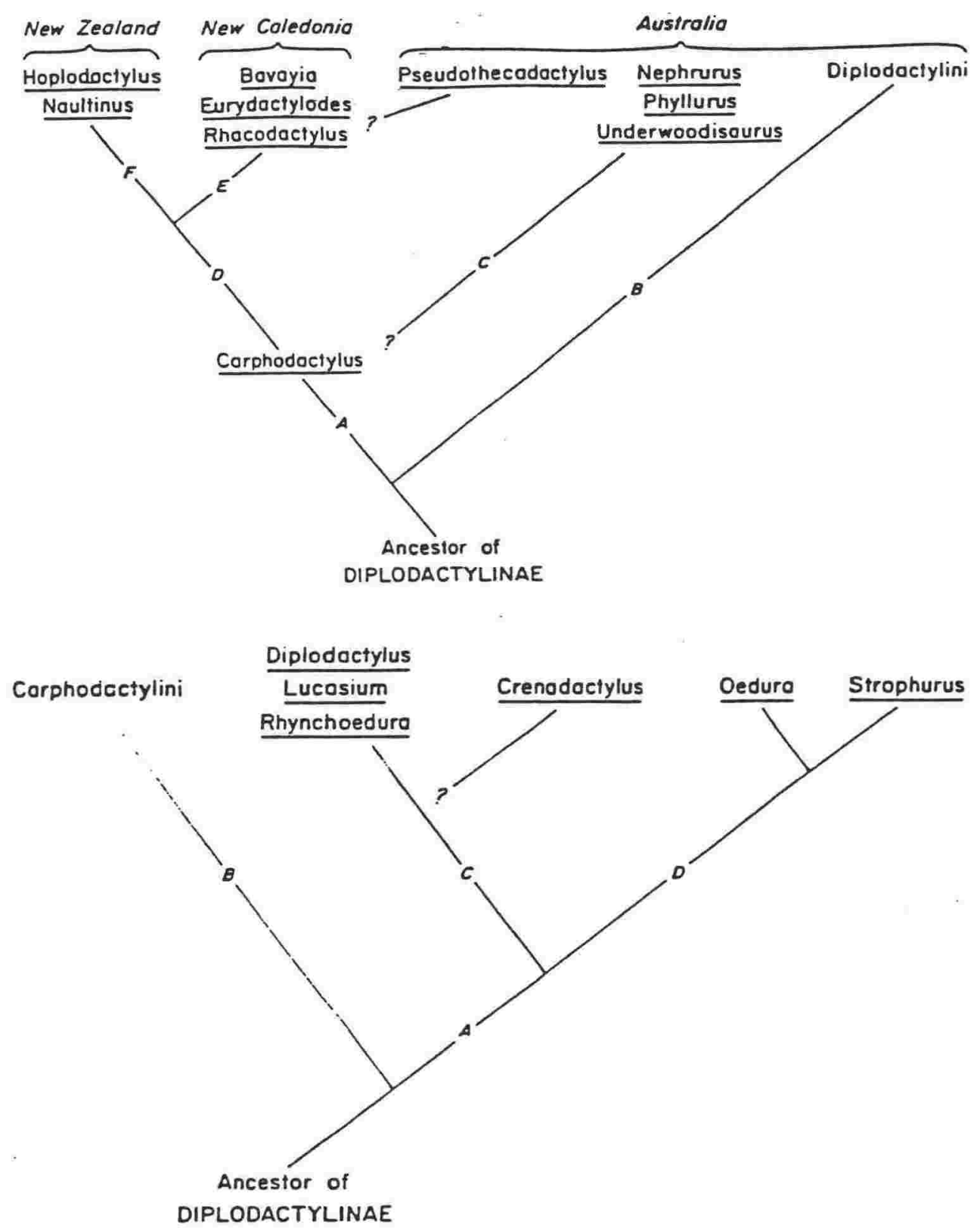

Figure 5.1.1 (figure and caption reproduced from Greer 1989, figs 34 \& 35) "Schematic diagram of the probable relationships in the subfamily Diplodactylinae.

(top) genera within the tribe Carphodactylini [Diplodactylini as outgroup] (largely after Kluge 1967b). The diagnostic characters signalled by the letters are as follows: A - preanal pores in a large patch; B - premaxillary bones fused; terminal digital pads; C - spiny scales: carrot-shaped tail, and preanal pores absent; D - premaxillary bones fused; E - basal digital pad; terminal tail pad; F - ovoviviparous reproduction.

(bottom) genera within the tribe Diplodactylini [Carphodactylini as outgroup] (largely from personal observations). The diagnostic characters signalled by the letters are as follows: A - terminal digital pads; B - preanal pores in large patch; C - external ear opening relatively small; overall size small; D - basal digital pads; lateral cloacal bones lost." 


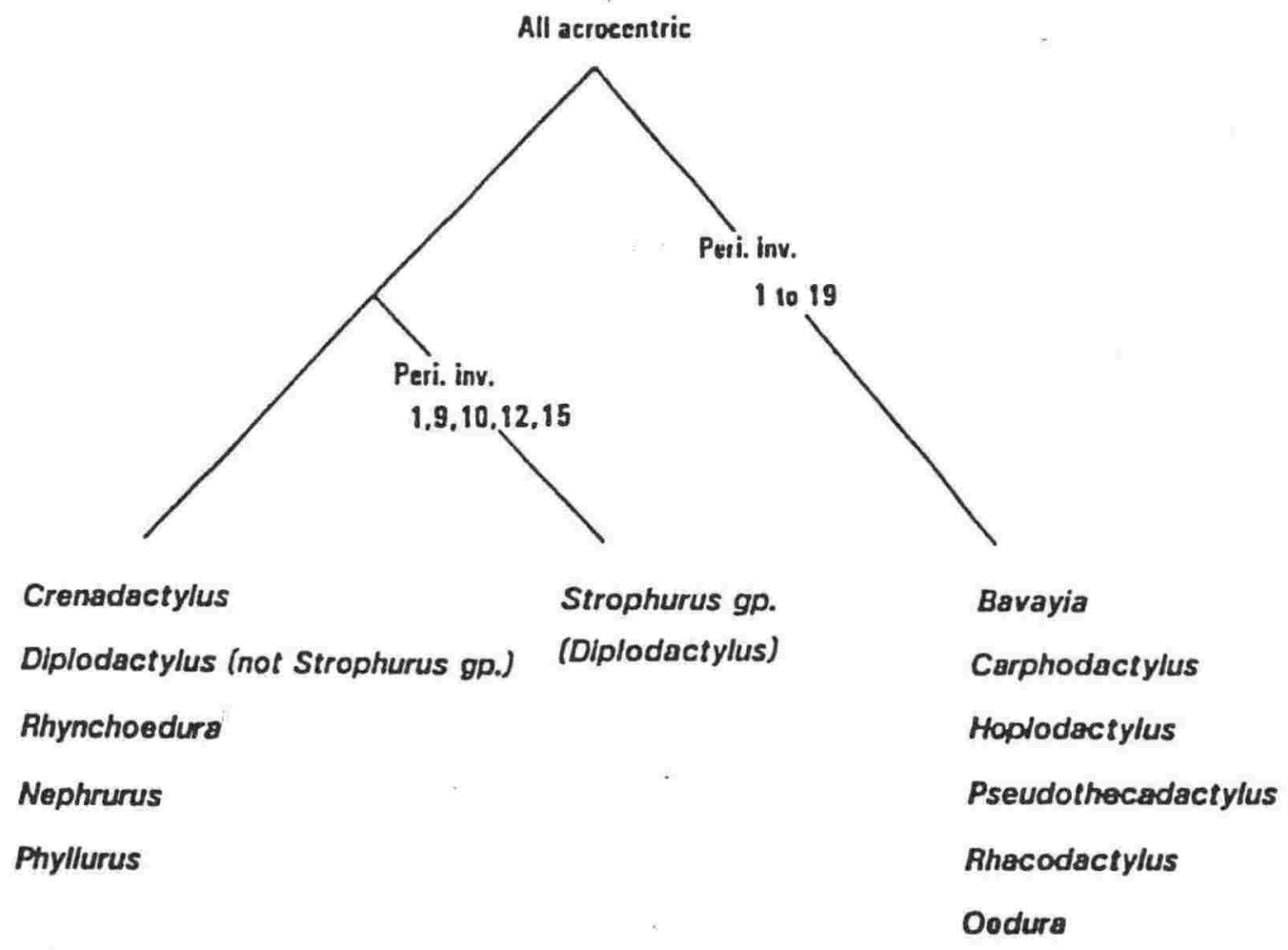

Figure 5.1.2 (figure and caption reproduced from King 1987b, fig. 8) "Phylogeny showing the 3 major groups present in the Diplodactylinae distinguished by the presence of shared derived chromosomal rearrangements. Minor differences are not included. It should be noted that the inclusion of Nephrurus and Phyllurus (Carphodactylini) with Crenadactylus, Diplodactylus, and Rhynchoedura (Diplodactylini) does not mean that these genera are in the wrong tribes. It simply means that these genera all share the ancestral $2 n=38$ karyomorph. However, the inclusion of Oedura (Diplodactylini) with the Carphodactylini does have such an implication and is based on the possession of 19 shared derived rearrangements. Note that the $D$. strophorus group of the genus Diplodactylus remains as an independent entity." NB Diplodactylus (Strophorus) elderi, which has morphological characters associating it with the Strophorus group (Kluge 1967b; Russel \& Rosenberg 1981), has pericentric inversions of chromosomes 17 and 18 only; in Oedura lesueurii and $O$ rhombifer, only chromosome pairs 3,6, and 13-18 are biarmed, rather than all chromosomes as in the rest of Oedura. 


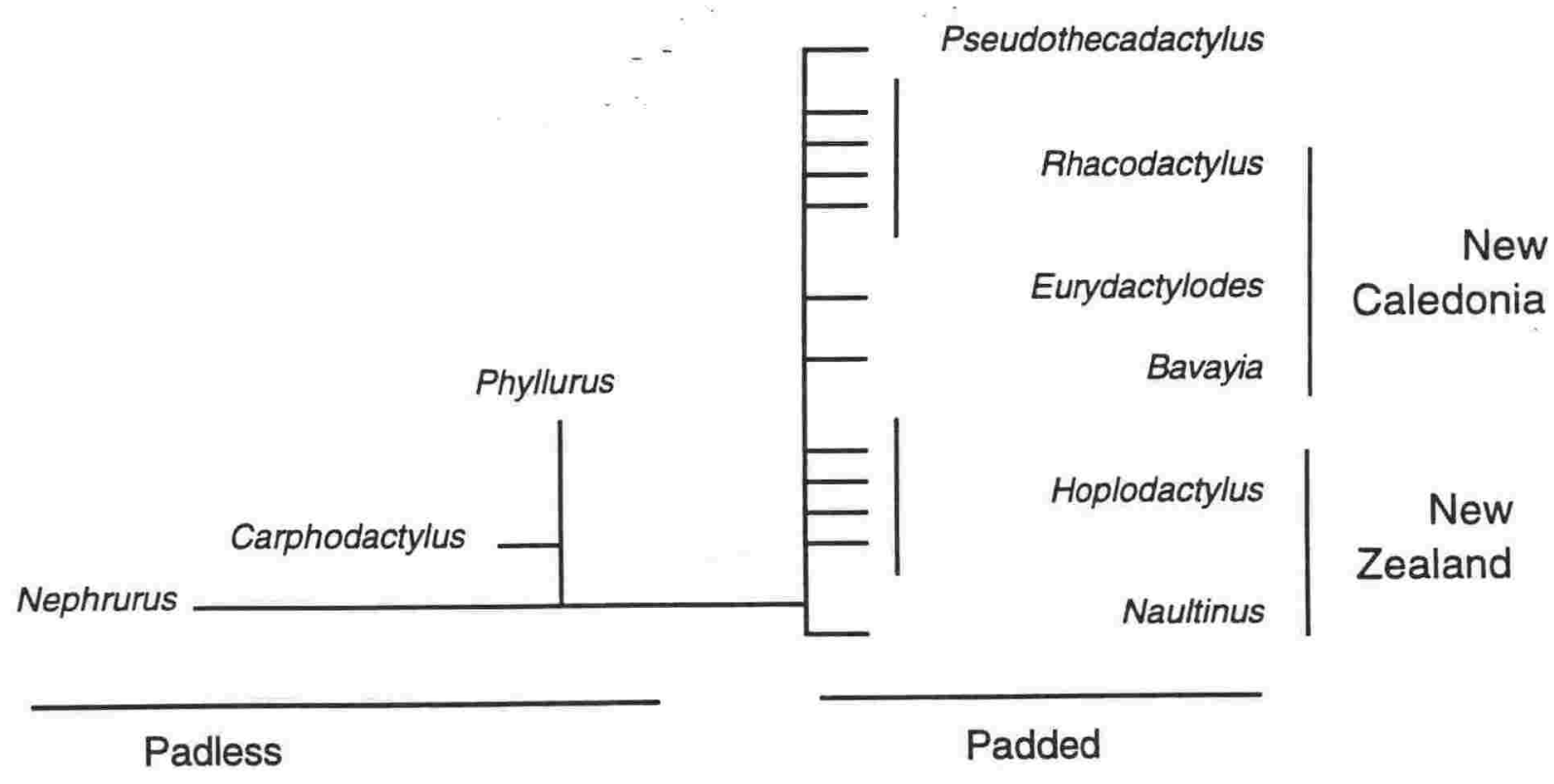

Figure 5.1.3 (Redrawn from Bauer 1990, fig. 16) Consensus cladogram of carphodactyline genera. The subgenus $P$ seudothecadactylus is included within Rhacodactylus.

New Zealand and New Caledonian genera and Pseudothecadactylus to be a monophyletic derived group, rooted between Naultinus and Hoplodactylus (Figure 5.1.3).

\subsection{Phylogenetics of the Diplodactylinae Based on} MORPHOLOGICAL ANALYSIS

If Greer's (1989) summary trees for the Carphodactylini and Diplodactylini are combined and examined, many character state reversals and parallel changes are obvious (Figure 5.1.1). Fused premaxillary bones diagnose both the Diplodactylini (character B) and the padded Carphodactylini (New Zealand and New Caledonian species plus Pseudothecadactylus - character D). The Carphodactylini are diagnosed at $A$ as having preanal pores in a large patch, but the first split within this group leads to a group with preanal pores absent (character $\mathrm{C}$ ). Basal digital pads are presented as occurring in the New Caledonian genera and Pseudothecadactylus (character E) and in Oedura and Strophurus (character D of the Diplodactylini). However, basal pads also occur in the New Zealand genera (Bauer 1990), so this condition should be at position $D$, not $E$ of the carphodactyline tree. A simple rearrangement of the combined tree (Figure 5.2.1) produces a tree which is 


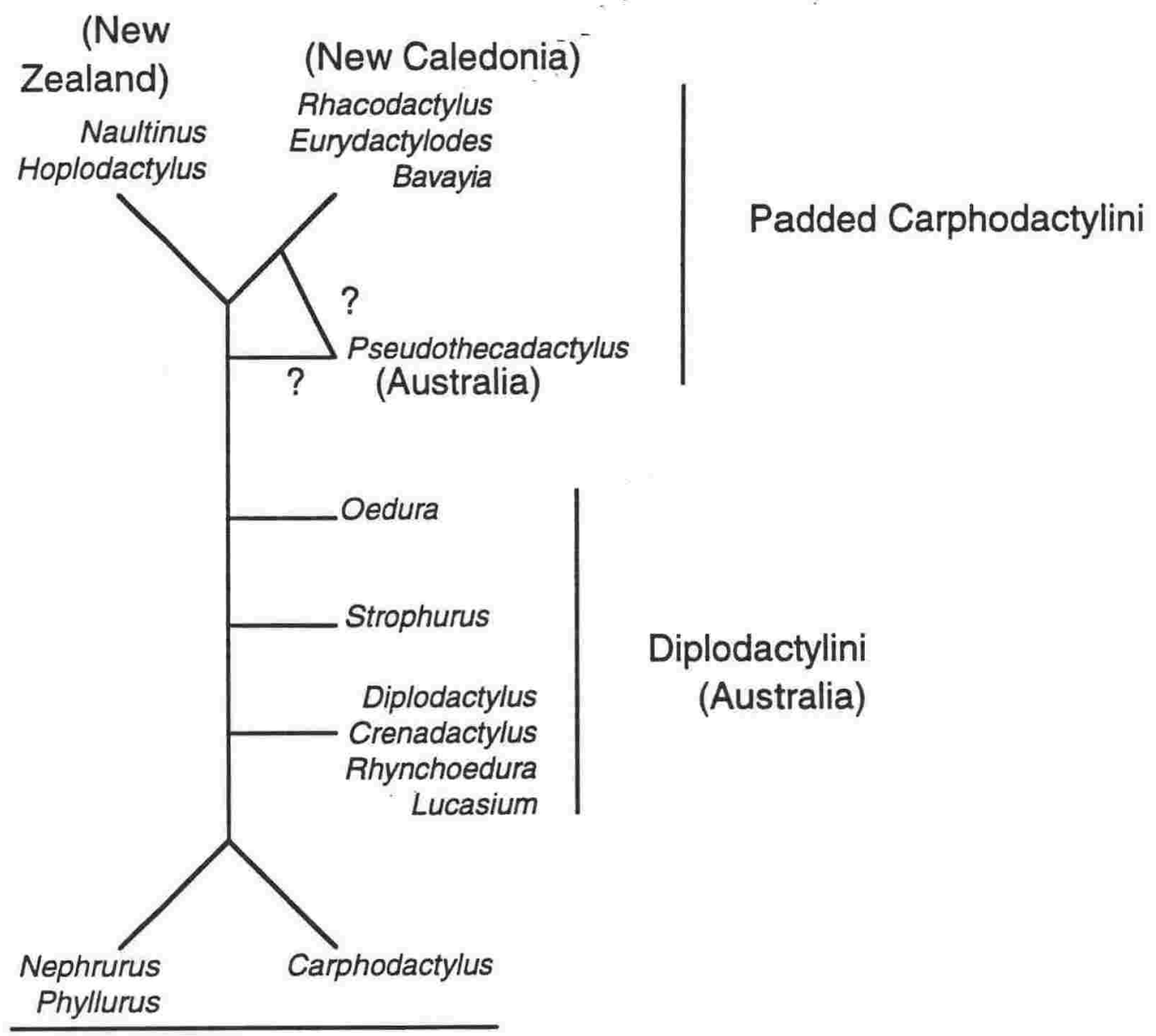

Padless Carphodactylini

(Australia)

Figure 5.2.1 Informal rearrangement of figure 5.1.1, prepared by hand, with the 2 tribes combined in a single tree, and branching order rearranged to remove obvious character state reversals.

far more parsimonious for the small set of characters presented by Greer (1989).

The position of Oedura near the padded Carphodactylini, and particularly near Pseudothecadactylus, is also suggested by direct comparison of the generic descriptions of Cogger (1975), which differ only in the absence of enlarged subdigital apical plates and presence of terminal subcaudal scansors in Pseudothecadactylus. In turn, Oedura has several morphological characters in common with Strophurus, and Strophurus is sufficiently similar to Diplodactylus to have been regarded as a subgenus of it for most of its history. 
Bauer's (1990) carphodactyline cladogram had no edge lengths presented, but my re-analysis of Bauer's data matrix using the same parsimony analysis package (PAUP) showed that the edge between the padded and padless lineages was very long compared to the edges within the 2 lineages, particularly the padded lineage (Figure 5.2.2). (I use edge rather than branch in this situation because of the possibility that the root of the tree falls within the edge between the lineages, meaning that the edge is possibly composed of 2 branches). These long edges mean that most characters vary between the 2 lineages, rather than within either lineage, so very few characters are available to determine the position of the root of the padded lineage. Any homoplasy in these characters will have an exaggerated effect on the position of this root. Very high levels of homoplasy are indeed found between these 2 major groups within the Carphodactylini (Table 5.2.1). This analysis attributes more than a third of the patristic distance (30 of 78 steps) between Pseudothecadactylus australis and Carphodactylus laevis to homoplasy. The possibility that homoplasy between Pseudothecadactylus and the New Caledonian genera in relatively few characters has falsely linked these groups therefore cannot be excluded using this dataset.

The root of the padded Carphodactyline group which Bauer (1990) placed between Naultinus and the rest of the group has no significant bootstrap support (Figure 5.2.2). Although the most informative trees are usually obtained by including the maximum possible amount of data (both taxa and characters) in a cladistic analysis (Kluge 1989), in these particular circumstances it is informative to investigate the effects of possible homoplasy in the outgroups by selectively removing some taxa. If some of the padless Carphodactyline genera, the more distant outgroups to the padded group, are removed from Bauer's dataset, the position of the root of the padded lineage changes. Removing Phyllurus alone or Carphodactylus alone has no effect. Removing only Nephrurus moves the root to between the New Zealand and New Caledonian genera (Figure 5.2.3). Removing both Nephrurus and Phyllurus moves the root to between Pseudothecadactylus and the rest of the padded taxa (Figure 5.2.4), and generates bootstrap support of $52 \%$ for a grouping of Carphodactylus and Pseudothecadactylus (Figure 5.2.5), implying that the New Caledonian and New Zealand genera may form a monophyletic group. While none of these positions has any greater claim than the others to be considered "correct", this instability confirms that the position of the root is poorly supported, and should be regarded as uncertain. 

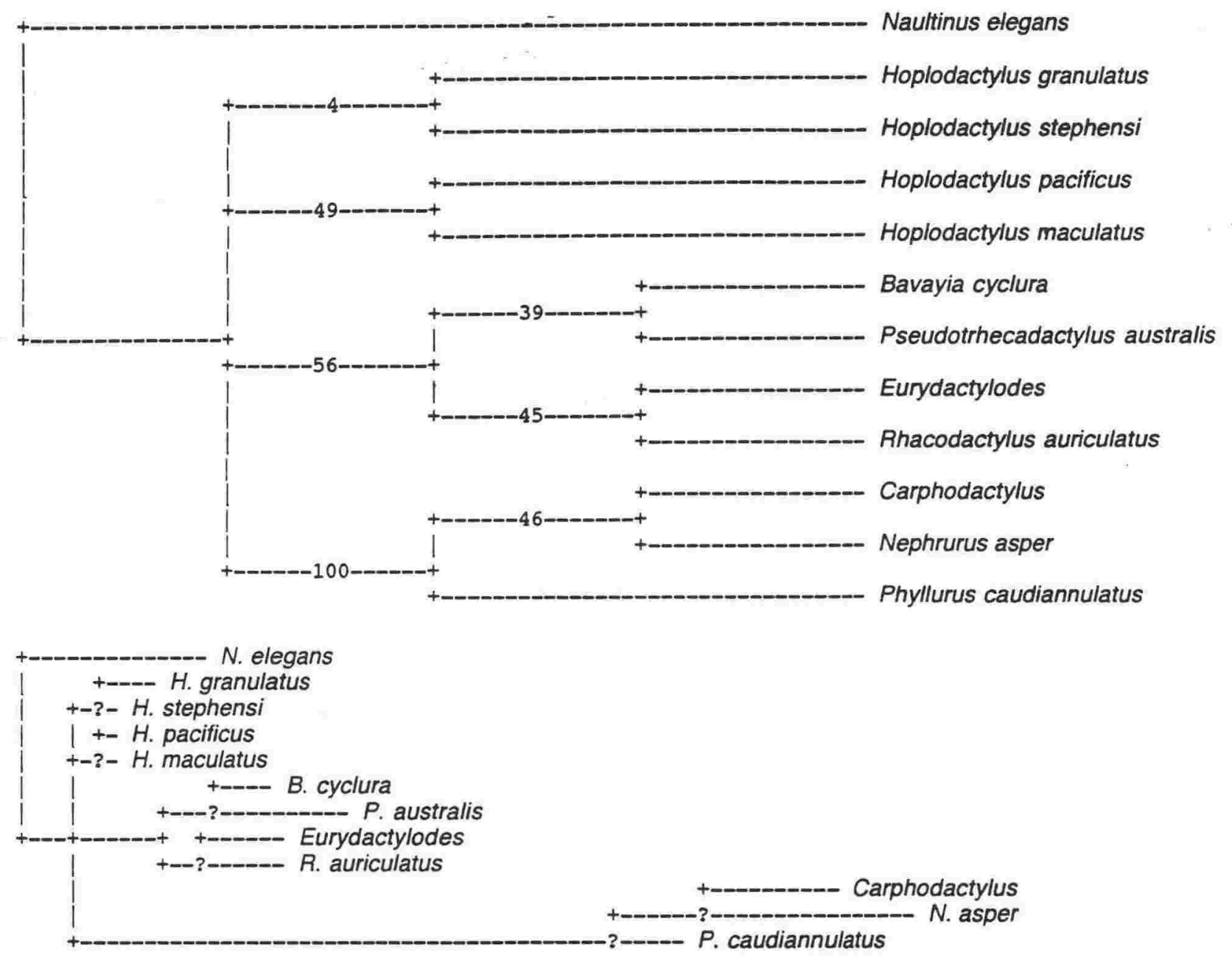

Figure 5.2.2 My bootstrap 50\% majority-rule consensus cladogram and phylogram (plus other groups compatible with this tree) using Bauer's (1990) morphological dataset, (branch and bound search). Tree length $=149$; Consistency index $(\mathrm{Cl})=0.644 ; \mathrm{Cl}$ excluding uninformative characters $=0.566$; Retention index $(\mathrm{RI})=0.624$; Rescaled consistency index $(\mathrm{RC})=0.402 ; \mathrm{f}$ value $=$ $452 ; \mathrm{f}$-ratio $=0.2172$ (multistate unordered and stepmatrix characters excluded from f-value calculations).

Table 5.2.1 Pairwise homoplasy matrix for cladogram in Figure 5.2.2. Values in this matrix are the differences between pairwise numbers of character state differences between taxa and assigned branch lengths on the phylogram. The degree by which assigned branch length exceeds the number of character differences measures the amount of homoplasy between the 2 populations.

\begin{tabular}{|c|c|c|c|c|c|c|c|c|c|c|c|}
\hline & 1 & 2 & 3 & 4 & 5 & 6 & 7 & 8 & 9 & 10 & 11 \\
\hline N. elegans & - & & & & & & & & & & \\
\hline H. granulatus & 10 & - & & & & & & & & & \\
\hline H. stephensi & 4 & 0 & - & & & & & & & & \\
\hline H. pacificus & 0 & 0 & 2 & _ & & & & & & & \\
\hline H. maculatus & 2 & 0 & 2 & 0 & - & & & & & & \\
\hline B. cyclura & 2 & 0 & 2 & 4 & 4 & - & & & & & \\
\hline Eurydactylodes & 4 & 2 & 2 & 2 & 2. & 2 & - & & & & \\
\hline R. auriculatus & 0 & 0 & 0 & 2 & 0 & 0 & 0 & - & & & \\
\hline$P$. australis & 8 & 4 & 2 & 4 & 4 & 0 & 0 & 6 & - & & \\
\hline Carphodactylus & 26 & 10 & 10 & 10 & 12 & 20 & 24 & 20 & 30 & - & \\
\hline N. asper & 26 & 10 & 10 & 10. & 10 & 20 & 22 & 18 & 26 & 0 & - \\
\hline P. caudiannulatus & 16 & 0 & 0 & 0 & 2 & 8 & 12 & 8 & 16 & 0 & 0 \\
\hline
\end{tabular}




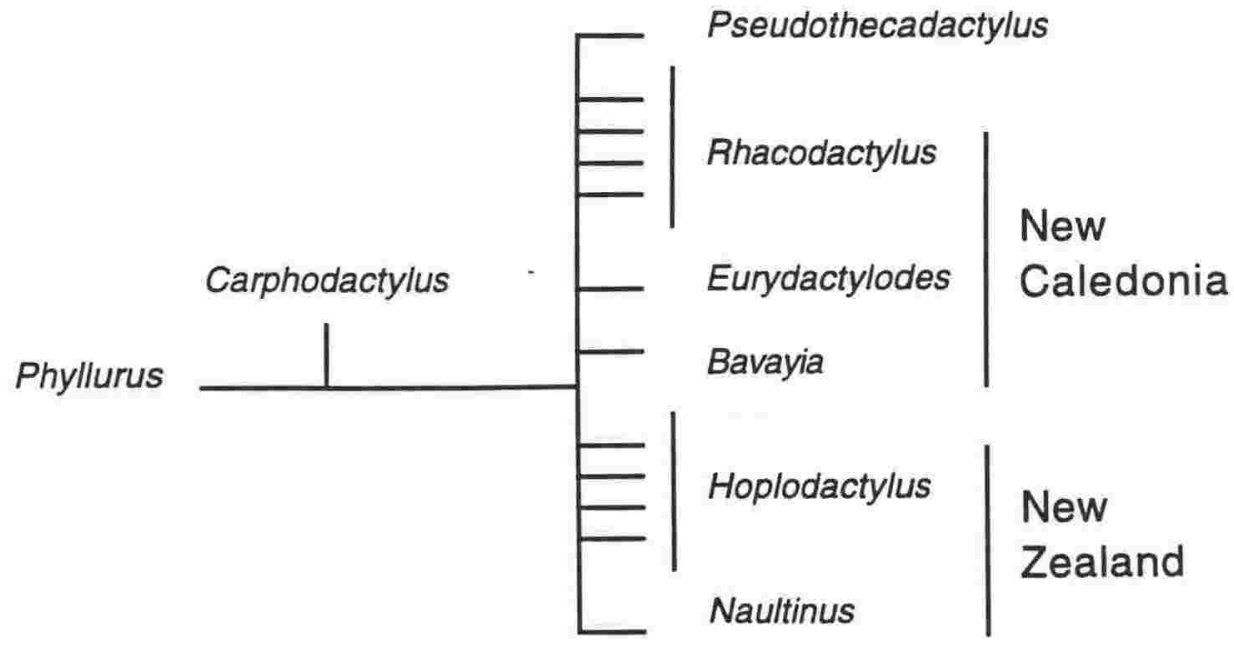

Figure 5.2.3 Maximum parsimony cladogram from Bauer's (1990) morphological dataset, omitting Nephrurus spp. (branch and bound search).

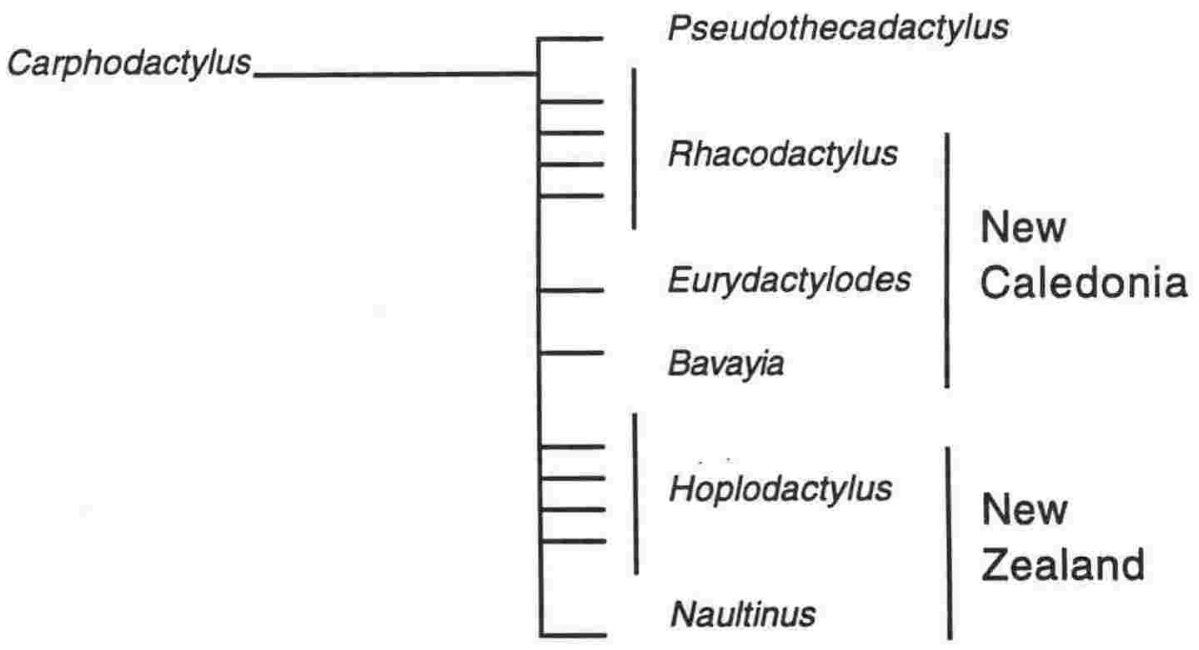

Figure 5.2.4 Maximum parsimony cladogram from Bauer's (1990) morphological dataset, omitting Nephrurus and Phyllurus spp. (branch and bound search). 

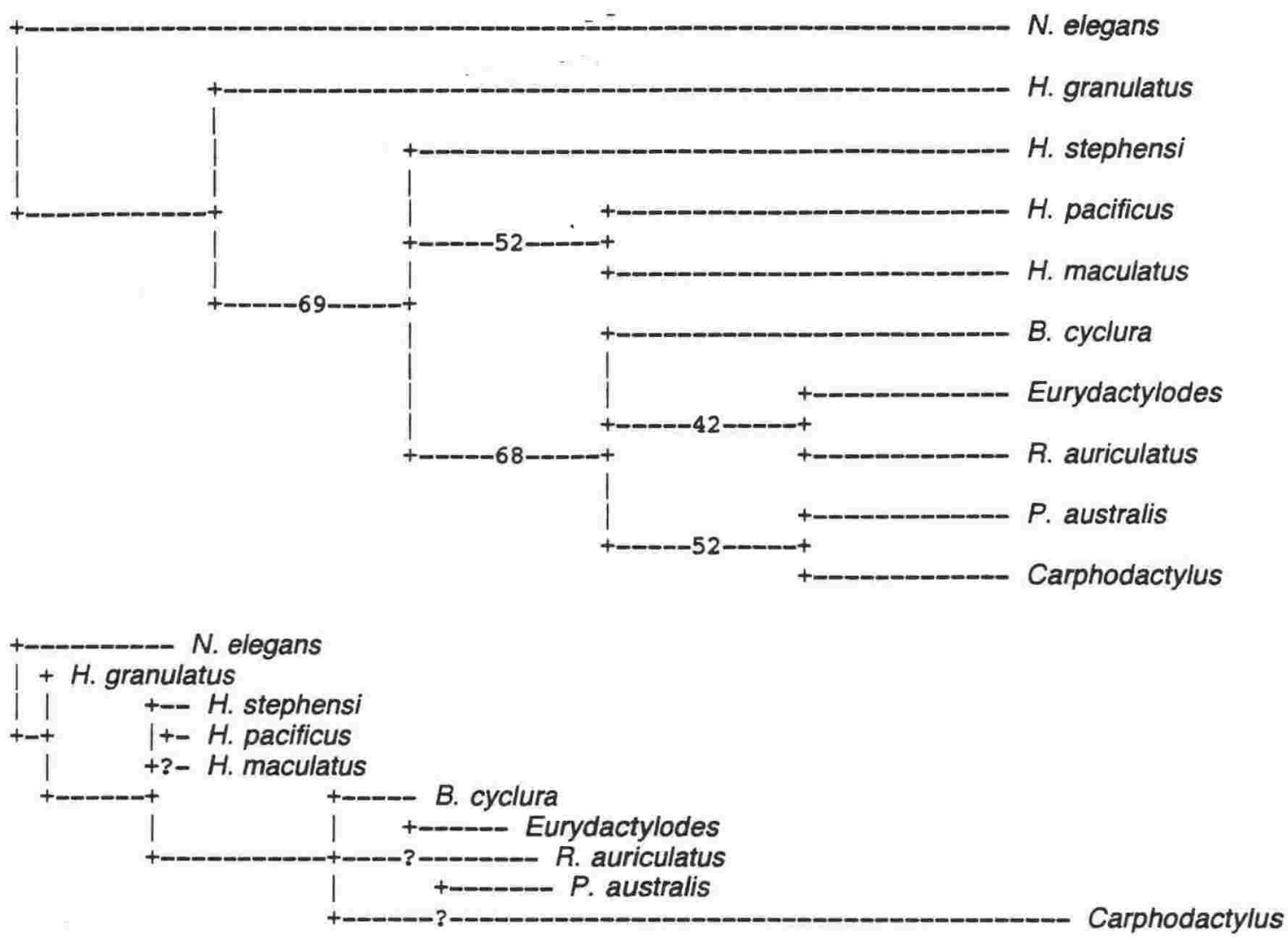

Figure 5.2.5 My bootstrap 50\% majority-rule consensus cladogram and phylogram (plus other groups compatible with this tree) using Bauer's (1990) morphological dataset, omitting Nephrurus and Phyllurus spp. (branch and bound search). Tree length $=108$; Consistency index $(\mathrm{Cl})=0.704 ; \mathrm{Cl}$ excluding uninformative characters $=0.508$; Retention index $(\mathrm{RI})=0.508$; Rescaled consistency index $(R C)=0.357$; $f$ value $=204$; f-ratio $=0.2004$; (multistate unordered and stepmatrix characters excluded from f-value calculations).

In view of the doubt cast on the monophyly of the Carphodactylini by King's cytogenetic analysis and by my informal rearrangement of Greer's tree (Figure 5.2.1), I conducted a parsimony (PAUP) analysis in which the genera placed by Kluge $(1967 a, b)$ in the Diplodactylini were added to Bauer's data matrix for the Carphodactylini. I did not examine specimens of Diplodactylini to assess character states myself; I gathered as much data as possible from the literature, including Bauer's (1990) statements of character states in Diplodactylini which he discussed as outgroups for many characters, and primary descriptions of species and genera of Diplodactylini (King \& Gow 1983; Kluge 1967b; Russel 1979; Russel \& Rosenberg 1981). Data on some characters were unavailable; the dataset was therefore less complete for the Diplodactylini than the Carphodactylini. Because I examined no Australian or 
New Caledonian taxa myself, I made no changes to Bauer's data matrix, although I disagree with his assessment of some character states for New Zealand species (see Section 5.3).

The size of the combined dataset meant that only heuristic searches were possible because of computer time constraints. However, bootstrapped searches were carried out on various subsets of the data. All these searches placed the Diplodactylini in a compact group on the long edge between the padded and padless Carphodactyline lineages (Figure 5.2.6). The compactness of the Diplodactylini in this tree is probably partly an artefact; the characters most likely to be missing from the dataset are the most variable ones, which will have had the effect of reducing edge lengths for this group relative to the Carphodactylini, for which the dataset is complete. However, the short edge lengths also reflect the morphological conservatism of this group, which I hypothesise resulted in its being distinguished as a tribe in the first place. There is no reason to suspect that the position of the Diplodactylini on the tree has been caused by any similar bias in the dataset.

Figure 5.2.6 agrees very well with the informal rearrangement of Greer's (1989) tree (Figure 5.2.1). Both are much more consistent with King's (1987a, b) cytogenetic data than is the existing, 2-tribe classification (Figure 5.1.1). When the cytogenetic groups are mapped onto the morphological tree (Figure 5.2.7), only the position of Carphodactylus is not reconcilable between the 2 datasets, with the cytogenetic data placing it with the padded lineage, and the morphological data in the padless lineage, separated from the padded lineage by the Diplodactylini. I thus conclude that neither tribe is monophyletic, a paraphyletic Diplodactylini having given rise independently to the padded and padless lineages of the Carphodactylini.

All the searches of the extended dataset placed the root of the padded lineage between the New Zealand and New Caledonian genera (Figure 5.2.6), and within the New Zealand fauna, placed the root between the broadtoed \& narrow-toed Hoplodactylus.

Bauer et al. (1997) found that allozyme results from Rhacodactylus and Pseudothecadactylus spp. conflicted strongly with Bauer's (1990) hypothesis from analysis of morphological variation that Pseudothecadactylus fell within Rhacodactylus, and should therefore be synonymised with it. This further casts doubt on the correctness of the topology of Bauer's (1990) tree. 


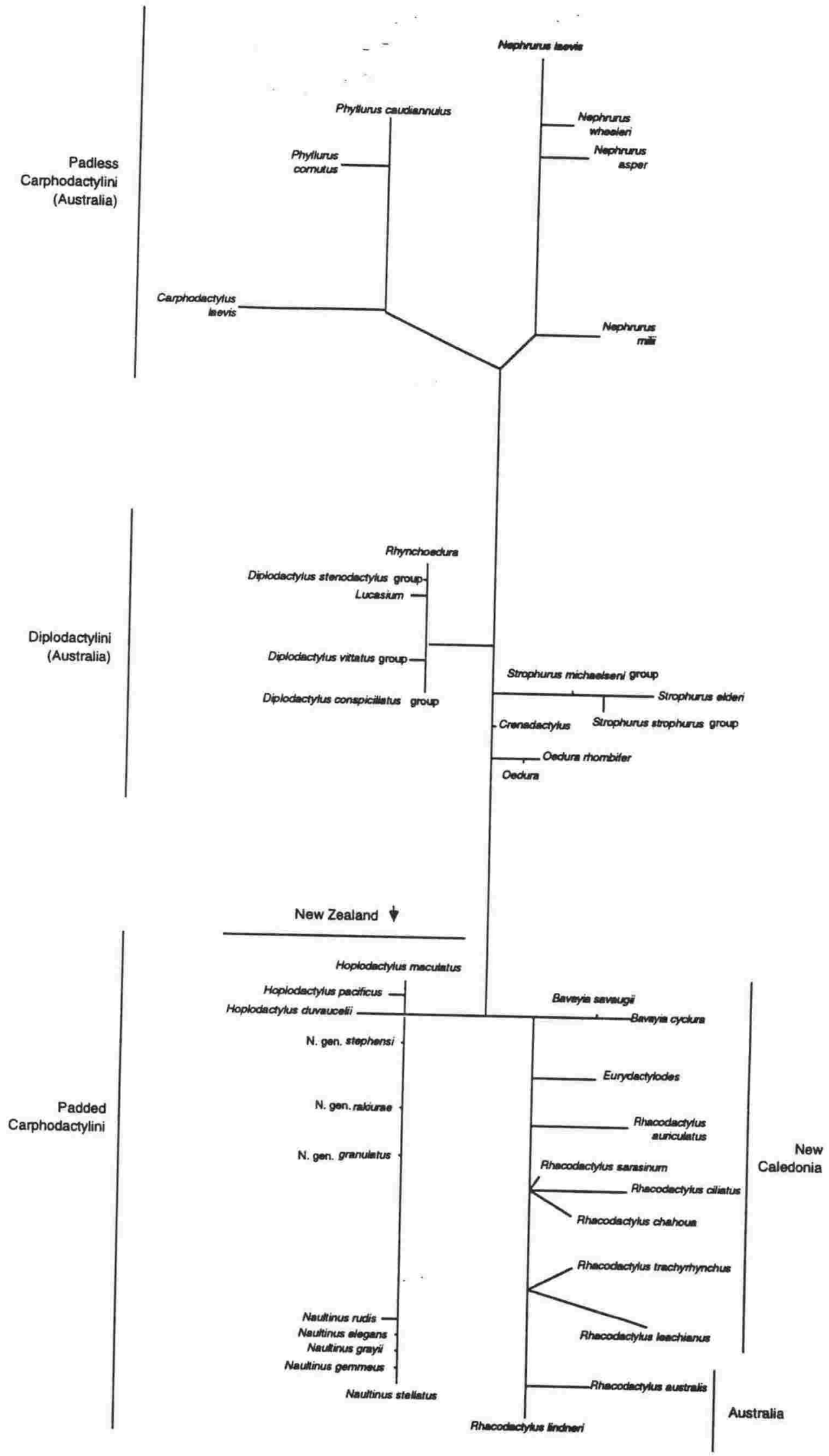

Figure 5.2.6 Maximum parsimony cladogram from Bauer's (1990) morphological dataset, with taxa from the Diplodactylini added (branch and
bound search). 


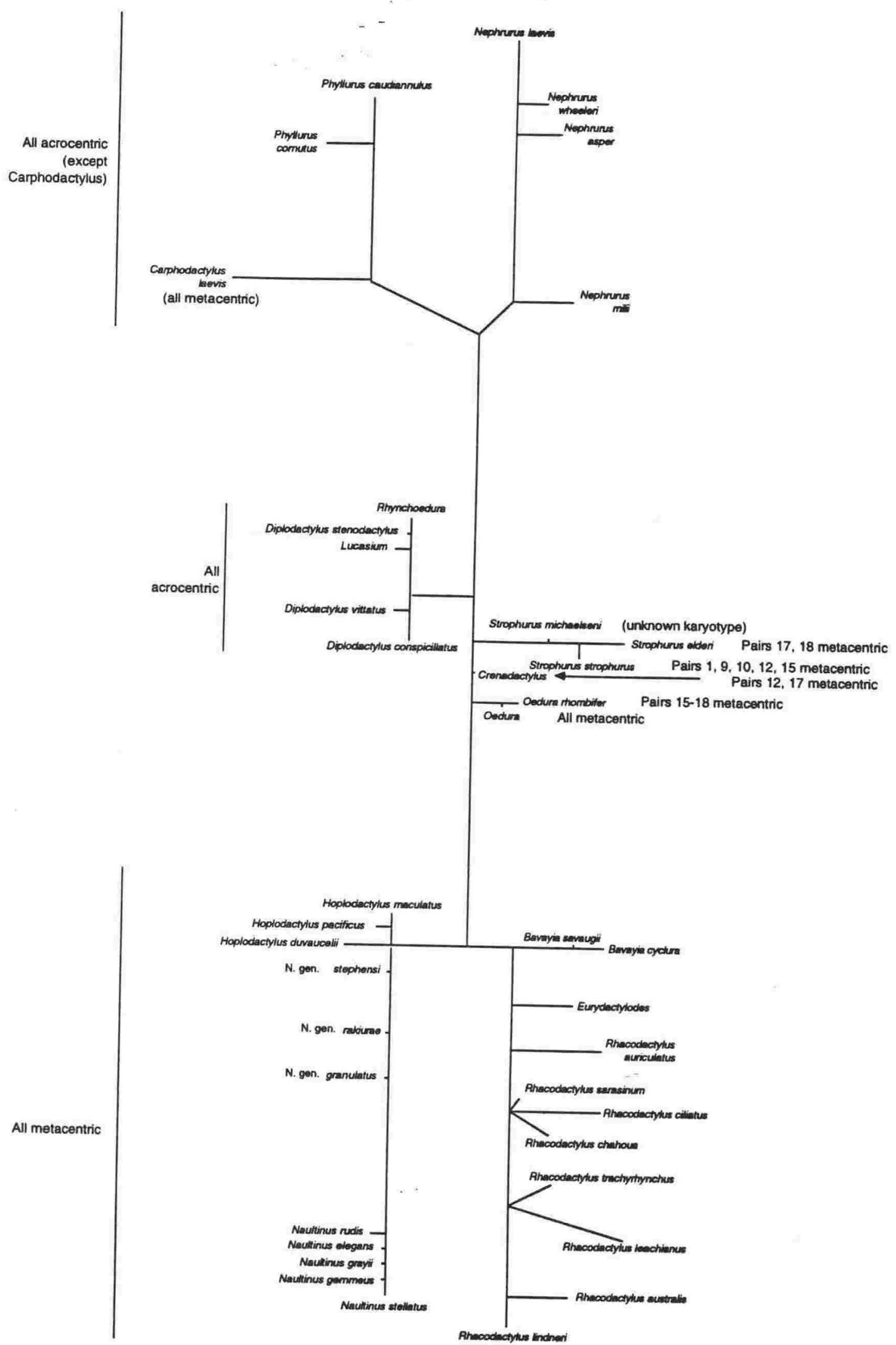

Figure 5.2.7 Karyotype data from King (1987b) mapped onto the maximum parsimony cladogram from Figure 5.2.6. 


\subsection{A RE-ANALYSIS OF PHYLOGENETIC RELATIONSHIPS AMONG NEW}

\section{ZEALAND GECKOS FROM MORPHOLOGY}

I found either discrepancies between Bauer's (1990) text and data matrix, or disagreement between my observations and his for 10 out of the 25 characters which varied among New Zealand species. I did not examine skeletal material (characters 1-59), and could only assess external characters. These disagreements are outlined below. This must call into question the accuracy of his entire dataset; I had no opportunity to assess character states for non-New Zealand species.

- H. granulatus is listed in Bauer's (1990) text as sharing the condition found in Naultinus of autotomy planes restricted to the first 5 to 12 postpygal vertebrae, rather than present throughout the tail as in other Hoplodactylus, but in the data matrix (character 28) it is listed as sharing the typical Hoplodactylus condition. Judging by the presence of the scale annuli which are associated with these planes (character 103), I have found this condition to vary within $H$. granulatus, with South Island populations having the typical Hoplodactylus condition, and North Island populations being polymorphic for the 2 conditions. Since characters 28 and 103 probably reflect a single underlying character, the validity of including both is very questionable.

- Bright green pigment fading to bright pink in preservative (character 60) is seen in $\mathrm{H}$. rakiurae as well as Naultinus rather than being restricted to Naultinus as claimed by Bauer (1990).

- There is a contradiction between Bauer's (1990) text and character matrix for the presence of pigment in the mouth and tongue (characters 63 and 64). Naultinus, $H$. granulatus, and $H$. kahutarae are correctly listed in both places as possessing this pigment, and $H$. rakiurae, not mentioned in the text, is correctly listed in the character matrix as possessing pigment, but $H$. stephensi, listed in the text as possessing pigment, is listed in the matrix as lacking it on the tongue. However, the tongue of this species is bright red (Robb 1980a, plate 12), as in several Naultinus species. Most Hoplodactylus species have pigment on the tongue, but not in the remainder of the mouth lining; however, they are all regarded by Bauer (1990) as lacking both. 
- I could detect no difference in peritoneal pigment (character 66) between those species Bauer regarded as having brown pigment and those he regarded as having black pigment. All appeared black to me.

- H. stephensi is listed as having broadly dilated digital pads (character 73). Although the pads of this species are slightly broader than those of other narrow-toed Hoplodactylus species or any Naultinus, they are very much narrower then the narrowest of broad-toed Hoplodactylus toes, and I consider that they would be better scored as having narrow, rather than broadly dilated pads.

- $H$. kahutarae and $H$. rakiurae are confused in the discussion of character states of apical plates (character 75 ); $H$. rakiurae, not $H$. kahutarae, has them on all digits, and $H$. kahutarae, not $H$. rakiurae, has none on any digit. The characters are applied correctly in the individual species accounts.

- All South Island Naultinus spp. were scored by Bauer as having heterogeneous dorsal scales (character 78), whereas I consider that only $N$. rudis and $N$. manukanus have strongly heterogeneous dorsal scales; in the other species, the scales are more or less uniformly large.

- Bauer's (1990) character 79 (nostril contacts/doesn't contact rostral), while of little phylogenetic importance, varies geographically within some species of Naultinus (see Chapter 4); the application of a single character state for these species is therefore misleading.

- H. pacificus is listed as having cloacal spurs (character 95) of the same blunt, conical type as $H$. maculatus, $H$. chrysosireticus, and $H$. duvaucelii, rather than a fan of pointed spurs as in narrow-toed Hoplodactylus and Naultinus, yet the difference in spur form between $H$. pacificus and $H$. maculatus is diagnostic, and has been used as such ever since the 2 were redescribed by Robb \& Rowlands (1977).

- Character 102 (abrupt narrowing of the tail at the pygal/post-pygal boundary) is another character for which Bauer's (1990) text and data matrix are not in agreement. The text states that the tail narrows abruptly in some $H$. chrysosireticus, $H$. maculatus, $H$. pacificus, $H$. stephensi, and $H$. delcourti, but only $H$. maculatus and $H$. pacificus are listed in the data 
matrix as having this character. I also observed this condition in $H$. duvaucelii. Visibility of this character is dependent upon the condition of the animal; very fat specimens do not show it because fat is accumulated in the tail, meaning that it sometimes even broadens rather than narrows at this point.

- Bauer (1990) lists Naultinus and $H$. rakiurae as lacking distinct scale rows associated with autotomy septa (character 103) in his character state discussion, but does not list $H$. granulatus in this section. However, this absence is listed in the diagnosis for $H$. granulatus but not for $H$. rakiurae. In the character state matrix, all Hoplodactylus are listed as having these scale rows. I have found these scale rows to be absent in Naultinus and some northern populations of $H$. granulatus, but present in other populations of $H$. granulatus and in $H$. rakiurae, as well as in all other Hoplodactylus spp.

I therefore re-analysed (using PAUP) a representative subset of Bauer's data with corrections as described above (Table 5.3.1). However, the corrections to the New Zealand data made no difference to the topology of the tree (Figure 5.3.1). The tree, like all others in this thesis, is unrooted, and the superficial appearance of Naultinus spp. as outgroups is an artefact of data entry order: the significant structure is indicated by bootstrap support for groups of taxa. A bootstrapped heuristic search showed strong support for monophyletic Naultinus (82\%), monophyletic Bavayia (86\%), and monophyletic Pseudothecadactylus (in Bauer's nomenclature Rhacodactylus lindneri plus $R$. australis, $85 \%$ ), but otherwise did not resolve relationships among the padded Carphodactyline species. A split between the padless Carphodactylini and the rest was also strongly supported (98\%). The Diplodactylini were not strongly supported as a separate monophyletic group.

The New Zealand taxa from this corrected dataset were subjected to a bootstrapped branch and bound search (100 replications), with and without the presence of Bavayia cyclura and Rhacodactylus australis as potential outgroups (Figures 5.3.2, 5.3.3). 


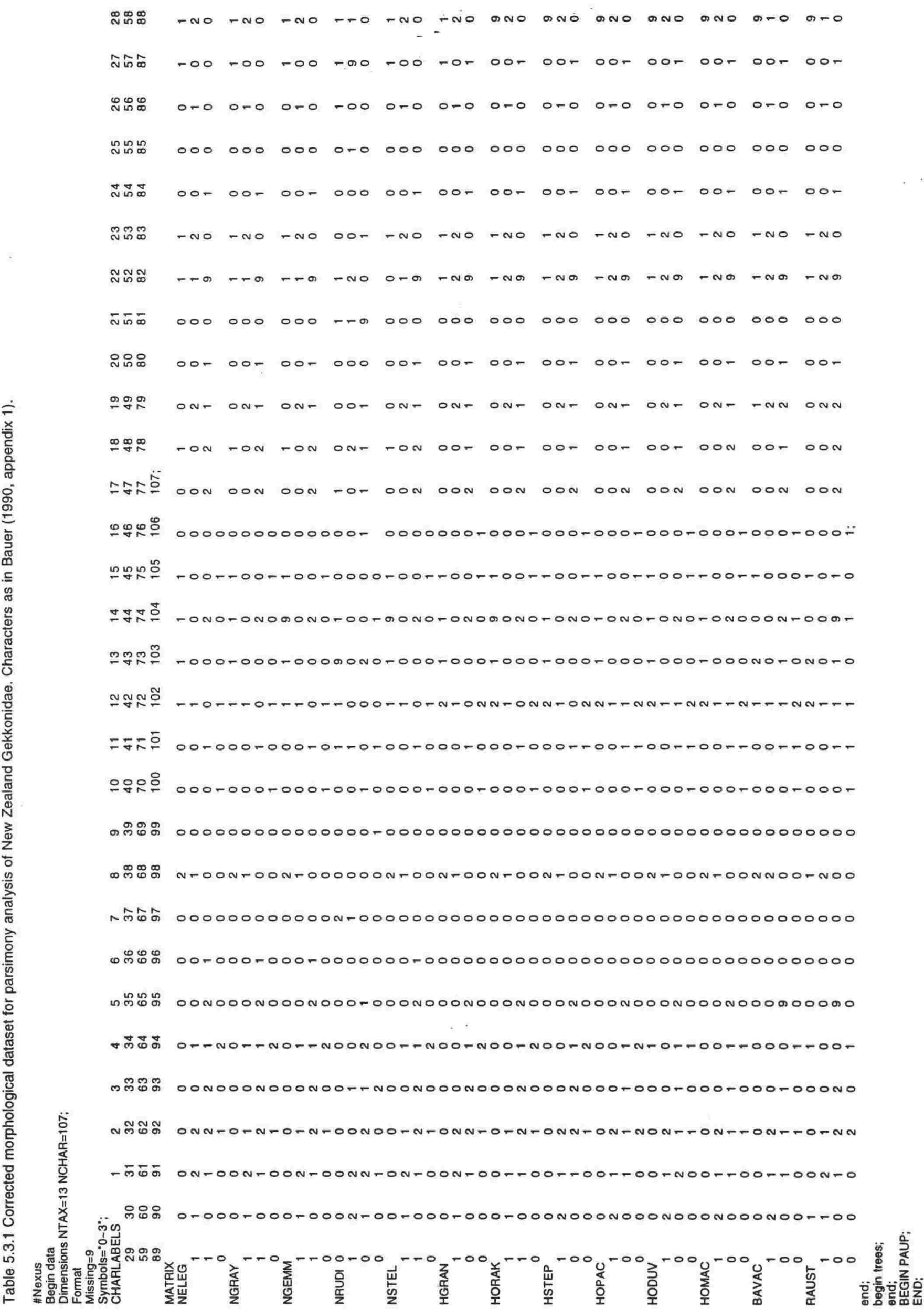




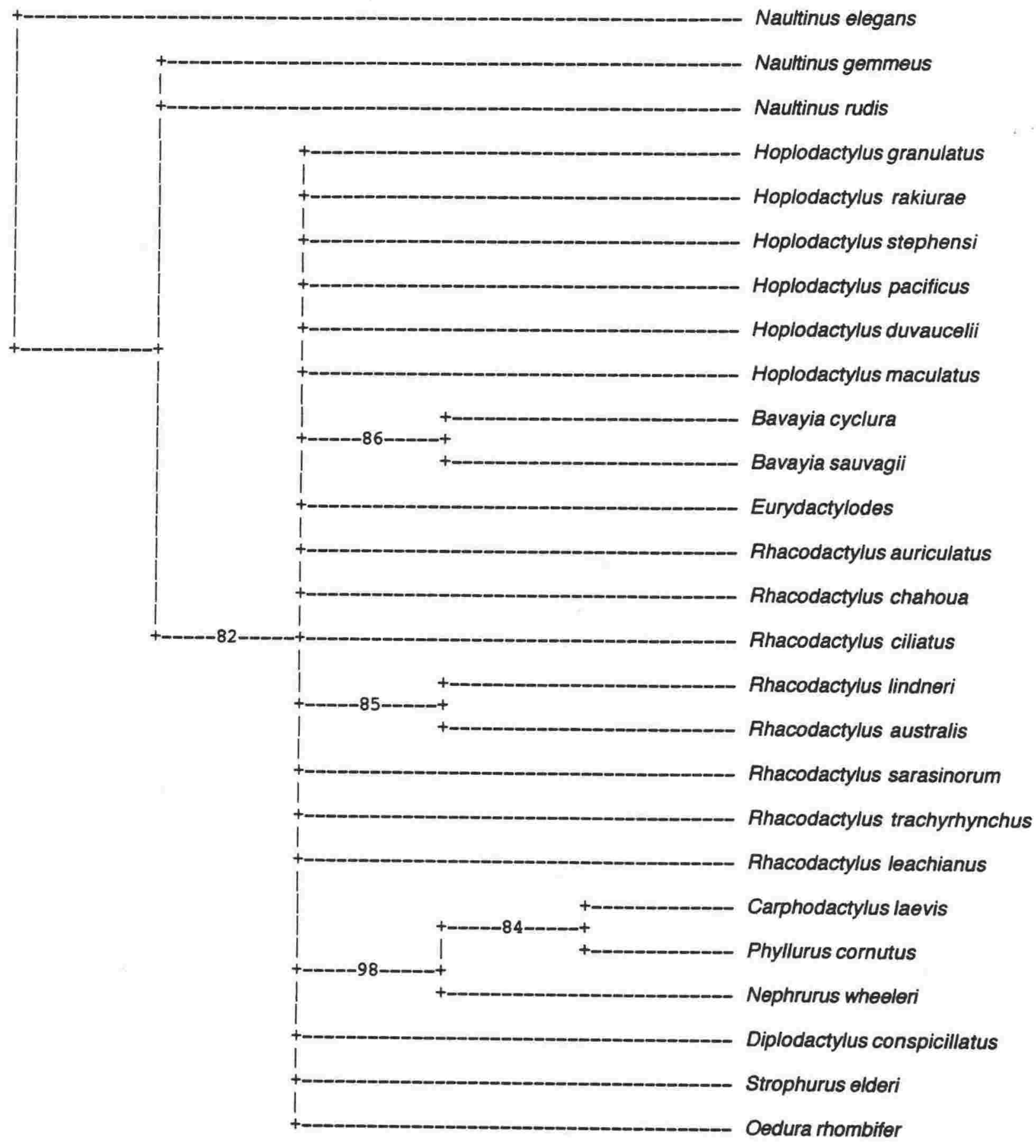

Figure 5.3.1 Bootstrap 80\% majority-rule consensus tree (PAUP package; bootstrapped heuristic search, 100 replications) from a representative subsample of Bauer's (1990) morphological dataset, with corrections to character states for New Zealand species, and with representative Diplodactylini added. 

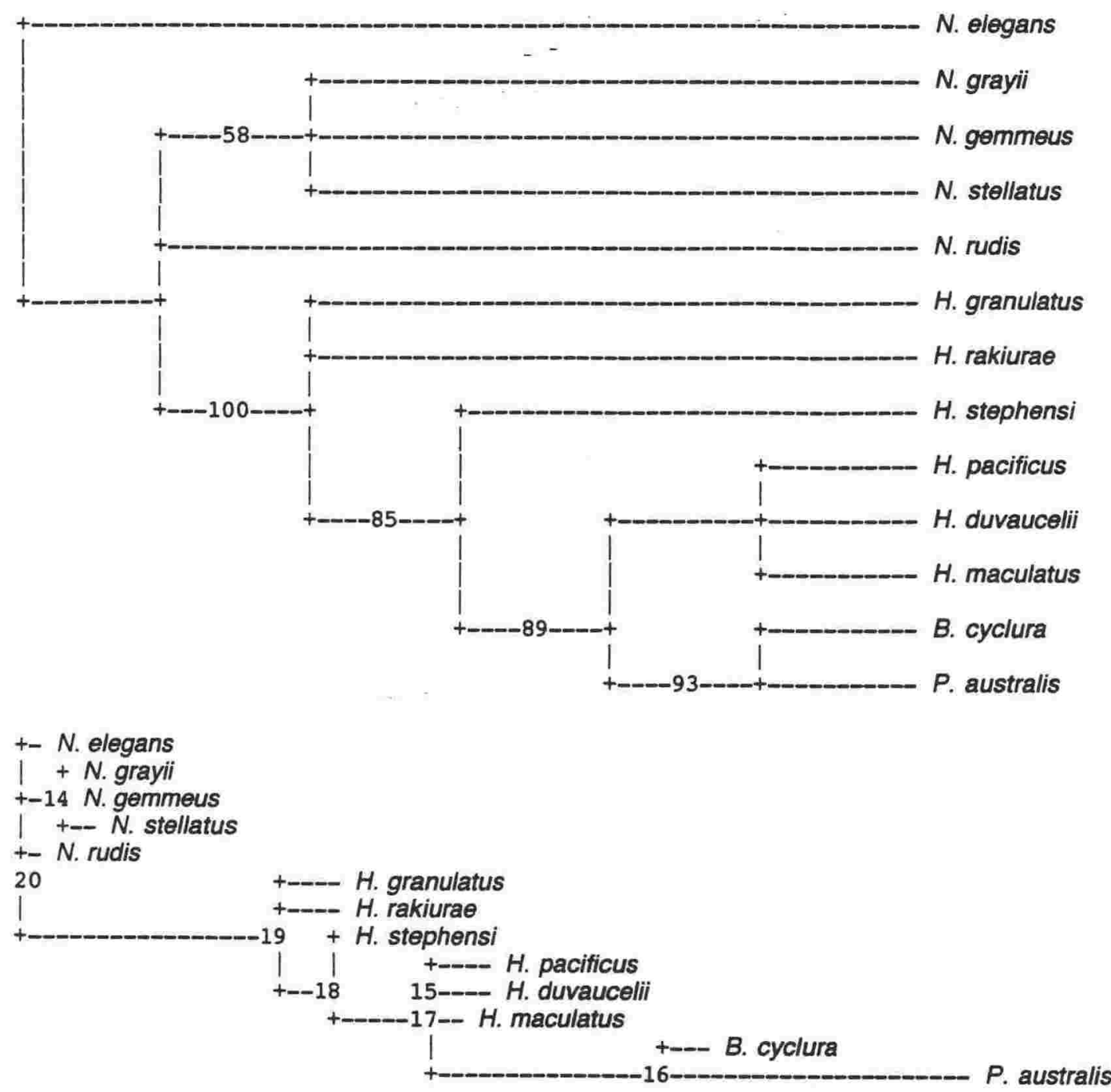

Figure 5.3.2 Bootstrap 50\% majority-rule consensus cladogram and phylogram (plus other groups compatible with this tree) for New Zealand geckos plus Rhacodactylus australis and Bavayia cyclura from morphological characters. Tree length $=54$; Consistency index $(\mathrm{Cl})=0.685$; $\mathrm{Cl}$ excluding uninformative characters $=0.638$; Retention index $(\mathrm{RI})=0.813$; Rescaled consistency index $(R C)=0.557 ; f$ value $=166 ;$ fratio $=0.1526$.

In both trees there was very strong support for groupings of: all Naultinus (100\%); Naultinus plus $H$. granulatus and $H$. rakiurae (85 and $91 \%$ ), and this group plus $H$. stephensi ( 89 and $99 \%$ ). In the tree with only New Zealand taxa, there was also very strong support (99\%) for a broad-toed group of Hoplodactylus ( $H$. pacificus, $H$. maculatus, and $H$. duvaucelii), and $61 \%$ support for a grouping of $H$. maculatus and $H$. duvaucelii to the exclusion of H. pacificus. However, when Bavayia cyclura and Rhacodactylus australis were included in the analysis, support for a broad-toed group of Hoplodactylus was only $11 \%$. 

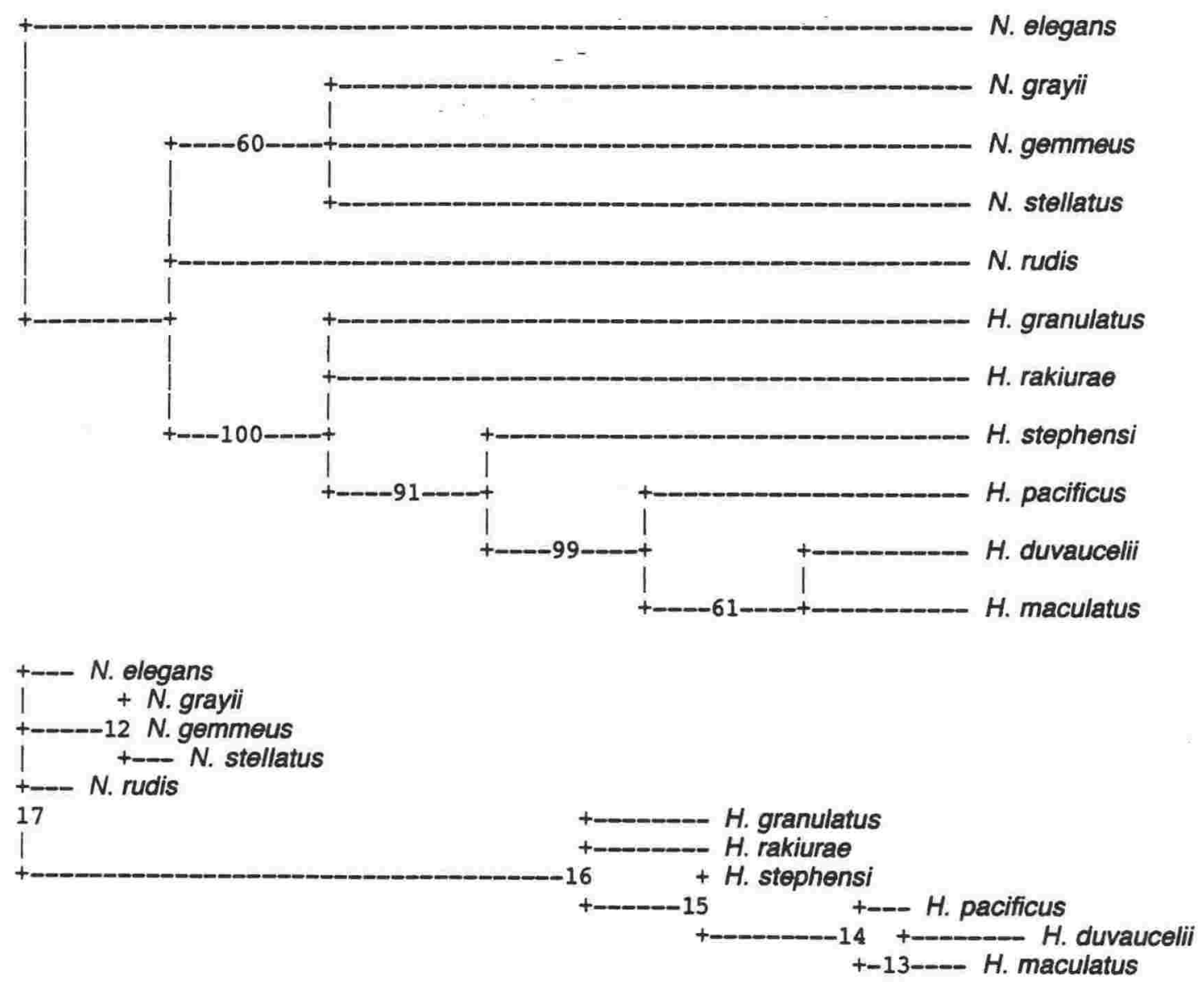

Figure 5.3.3 Bootstrap 50\% majority-rule consensus cladogram and phylogram (plus other groups compatible with this tree) for New Zealand geckos only, from morphological characters. Tree length $=30$; Consistency index $(\mathrm{Cl})=0.800 ; \mathrm{Cl}$ excluding uninformative characters $=0.760$; Retention index $(\mathrm{Rl})=0.906$; Rescaled consistency index $(R C)=0.725 ; f$ value $=50 ; f$ ratio $=0.0856$.

Bavayia cyclura and Rhacodactylus australis were grouped together with $93 \%$ bootstrap support. The tree is consistent with the New Zealand taxa forming a monophyletic group, with the root probably falling between the broad-toed and narrow-toed sections of Hoplodactylus, or possibly within the broad-toed group, and with Hoplodactylus being paraphyletic with respect to Naultinus. However, the outgroup status of Bavayia and Rhacodactylus is unconfirmed. The root could be elsewhere on the tree, which would make Hoplodactylus paraphyletic with respect to Bavayia and Rhacodactylus, as suggested by Bauer (1990). 


\subsection{Phylogenetic Relationships Within the NeW ZeAland Gecko FAUNA FROM ALLOZYME DATA}

An attempt was made to survey representative Carphodactylini from outside New Zealand (Table 5.4.1) for allozyme characters. However, only liver was available for the non-New Zealand species, so loci scored from muscle samples could not be surveyed. Some loci scored in New Zealand species from liver did not give scorable results from the non-New Zealand species. Homology of loci between New Zealand and non-New Zealand species was not clear for some stains which showed multiple loci on gels. For these reasons too few loci could be scored to include potential outgroups in this analysis. However, the difficulties encountered in extending allozyme screening systems which worked well for all New Zealand species to related New Caledonian and Australian genera suggest that the New Zealand species are probably all more closely related to each other than to any species outside New Zealand. That is, the hypothesis that Hoplodactylus is paraphyletic with respect to the New Caledonian genera is not supported.

Allozyme genotype data from all samples were pooled without weighting within each species recognised in this study (Chapters $3 \& 4$ ) to provide a dataset for phylogenetic analysis of New Zealand species. Pooled allele frequencies are presented in Table 5.4.2.

WPGMA (Figure 5.4.1) and distance Wagner (Figure 5.4.2) trees were generated from these pooled data using the BIOSYS-1 package. A matrix of Cavalli-Sforza \& Edwards' (1967) arc distances was also generated using

Table 5.4.1 Australian Diplodactylini and New Caledonian Carphodactylini used for allozyme analysis.

Diplodactylini:

Diplodactylus conspicillatus

Strophurus ciliaris

Nephrurus laevissimus

Nephrurus wheeleri

Nephrurus milii

Oedura leseurii

Oedura tryoni

Carphodactylini:

Bavayia cyclura

Bavayia sauvagii

Rhacodactylus leachianus 
Table 5.4.2 Allozyme allele frequencies in species of New Zealand Gekkonidae, pooled without weighting over all populations of each species. Species key:

$\begin{array}{lllll}\mathrm{Ng} & \mathrm{N} . \text { grayii } & \mathrm{Ng}(\mathrm{hp}) & \mathrm{N} . \text { gemmeus } \\ \mathrm{Ng}(\mathrm{nc}) & \mathrm{N} . \text { grayii } & \text { (North Cape) } & \mathrm{Ng} & \mathrm{N} \text {. gemmeus } \\ \mathrm{Nee} & \mathrm{N} . \text { e. elegans } & \mathrm{Hs} & \mathrm{H} \text {. stephensi } \\ \mathrm{Nep} & \mathrm{N} . \text { e. punctatus } & \mathrm{Hg} & \mathrm{H} \text {. granulatus } \\ \mathrm{Nm} & \mathrm{N} . \text { manukanus } & \mathrm{Hn} & \mathrm{H} \text {. nebulosus } \\ \mathrm{Nr} & \mathrm{N} \text {. rudis } & \mathrm{Hk} & \mathrm{H} \text {. kahutarae } \\ \mathrm{Ns} & \mathrm{N} \text {. stellatus } & \mathrm{Hr} & \mathrm{H} \text {. rakiurae } \\ \mathrm{Nt} & \mathrm{N} \text {. tuberculatus } & \mathrm{Htk} & \mathrm{H} \text {. "Three Kings" }\end{array}$

\begin{tabular}{lllllllllllllllllll}
\hline \multicolumn{3}{c}{ Lecus } & $\mathrm{Ng}$ & $\mathrm{Ng}(\mathrm{nc})$ & $\mathrm{Nee}$ & $\mathrm{Nep}$ & $\mathrm{Nm}$ & $\mathrm{Nr}$ & $\mathrm{Ns}$ & $\mathrm{Nt}$ & $\mathrm{Ng}(\mathrm{hp})$ & $\mathrm{Ng}$ & $\mathrm{Hs}$ & $\mathrm{Hg}$ & $\mathrm{Hn}$ & $\mathrm{Hk}$ & $\mathrm{Hr}$ & $\mathrm{Htk}$ \\
\hline (n) & 4 & 2 & 5 & 4 & 4 & 3 & 9 & 2 & 2 & 7 & 4 & 24 & 1 & 2 & 9 & 3 &
\end{tabular}

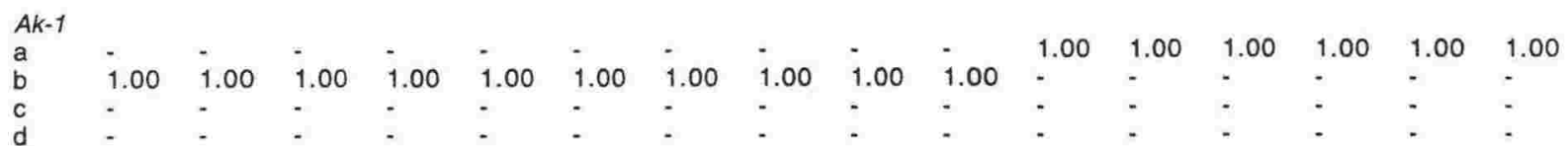

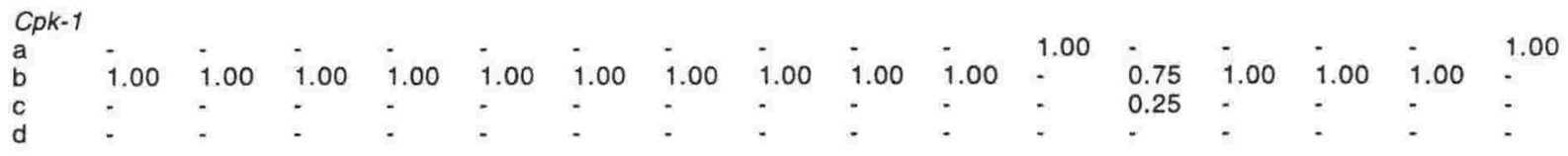

Gd-2

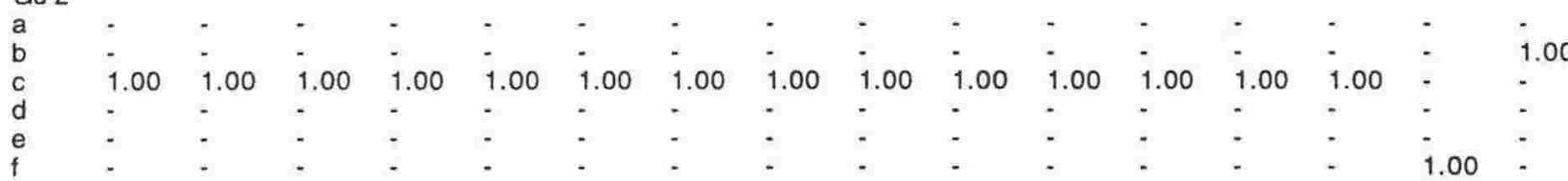

$\begin{array}{lllllllllllllllll}\text { Got-1 } & 1.00 & 1.00 & 1.00 & 1.00 & 1.00 & 1.00 & 1.00 & 1.00 & 1.00 & 1.00 & 1.00 & 1.00 & 1.00 & 1.00 & 1.00 & 1.00\end{array}$

Got-2

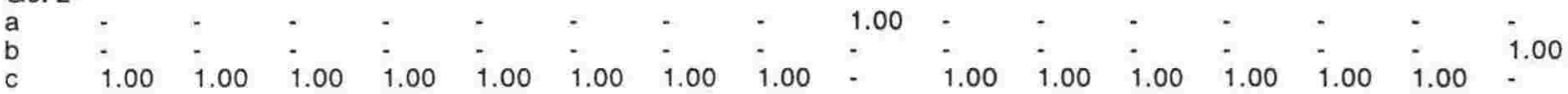

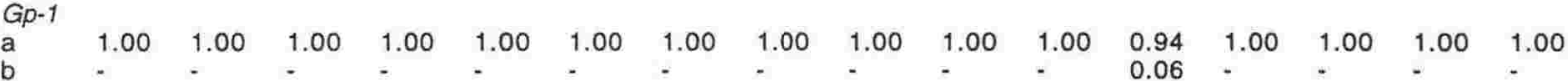

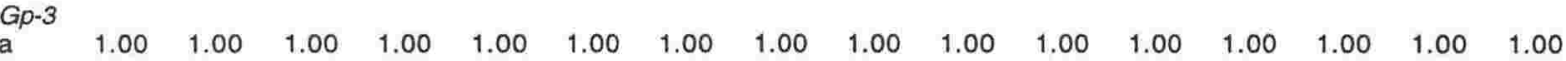

$G p-4$

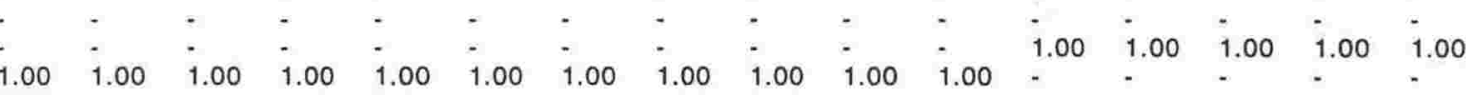

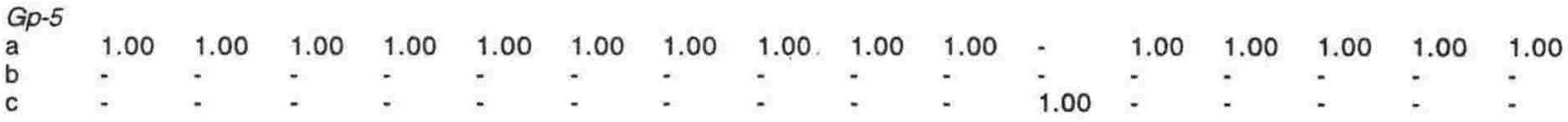

Gpd-1

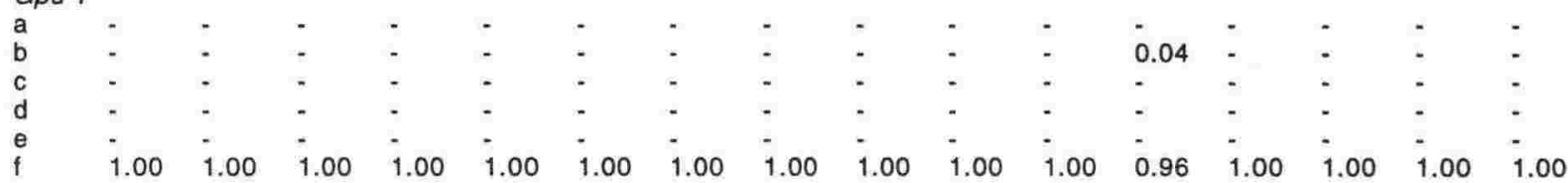

Gpi-1

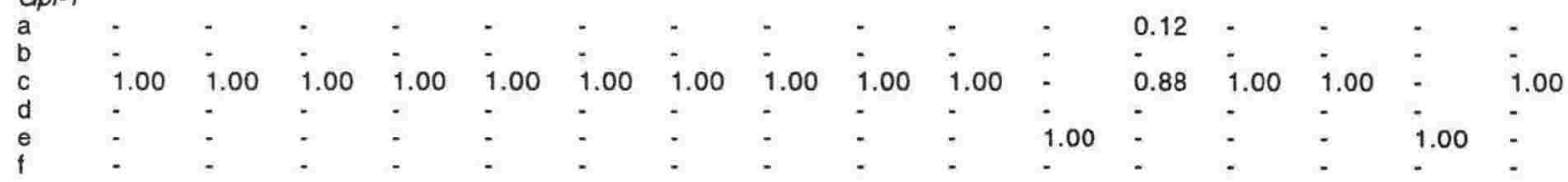


Table 5.4.2 (continued)

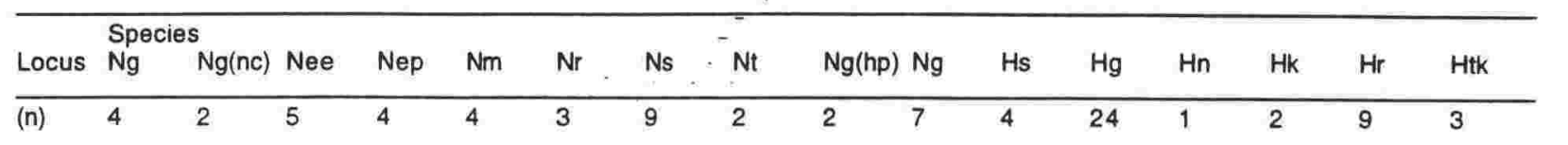
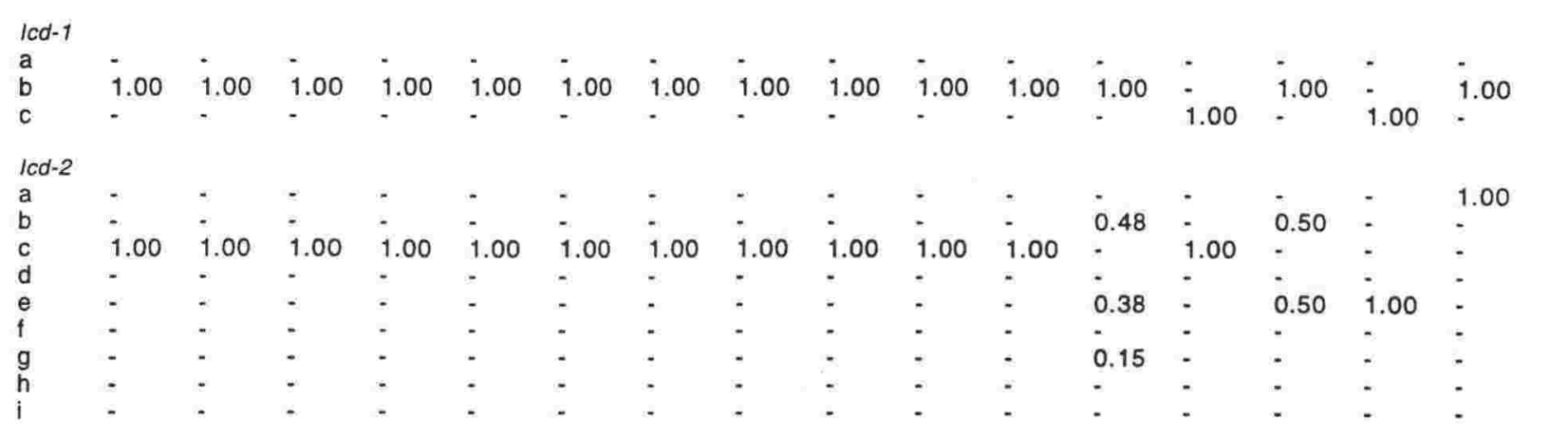

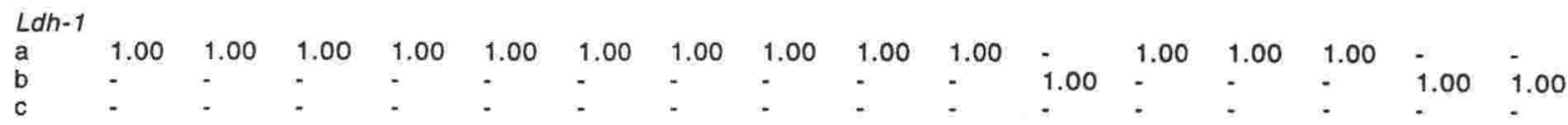
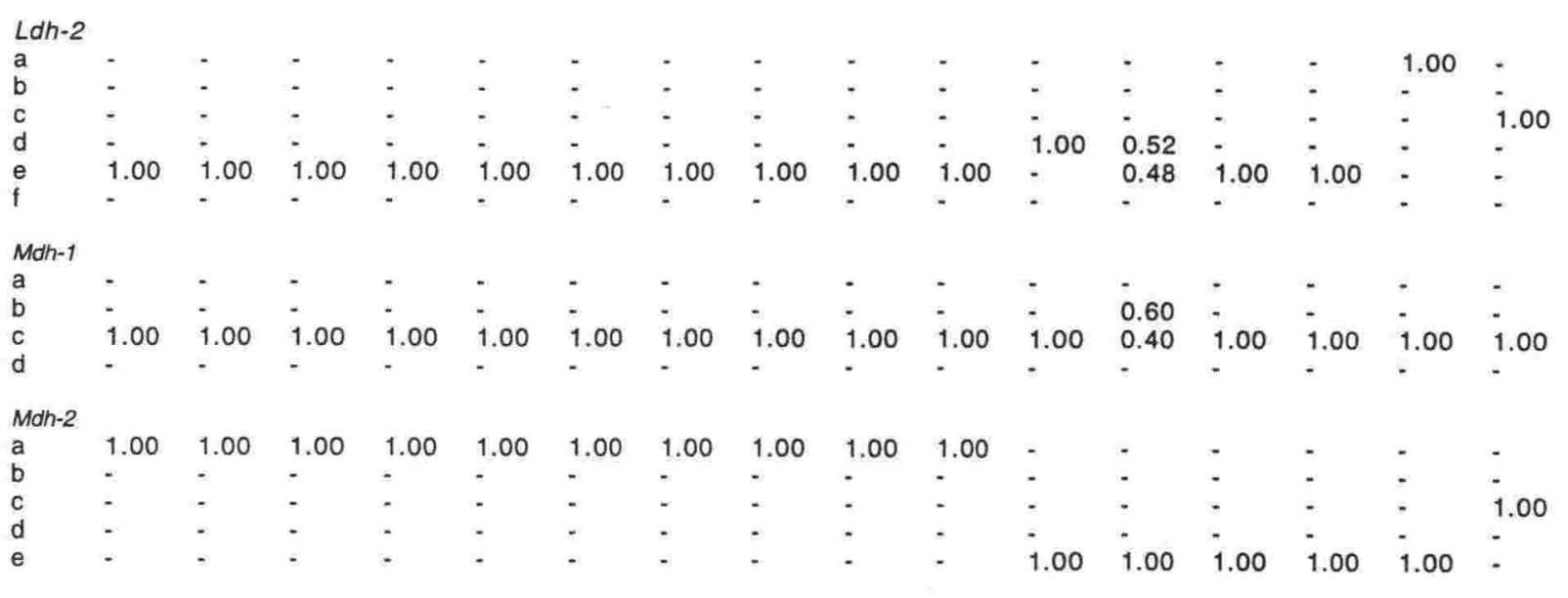

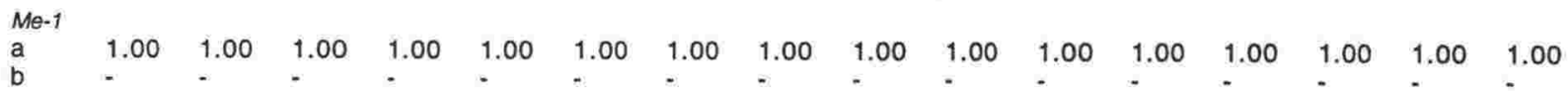
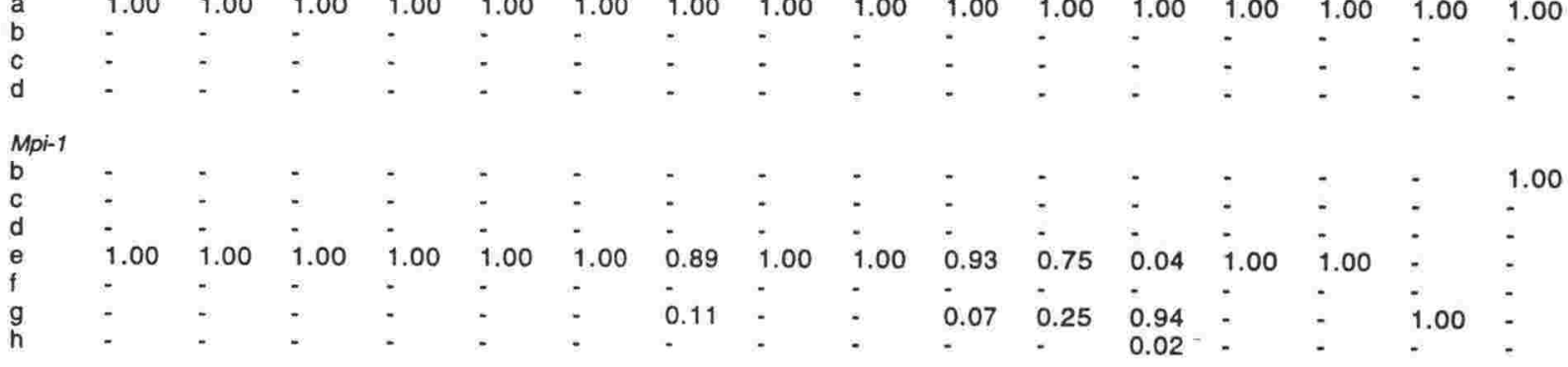

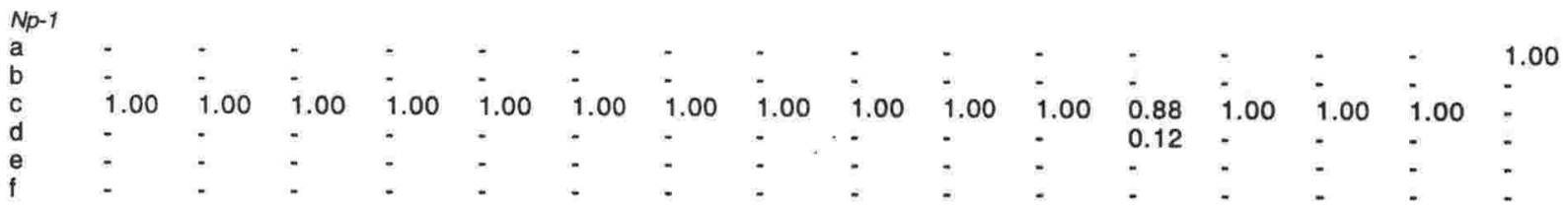

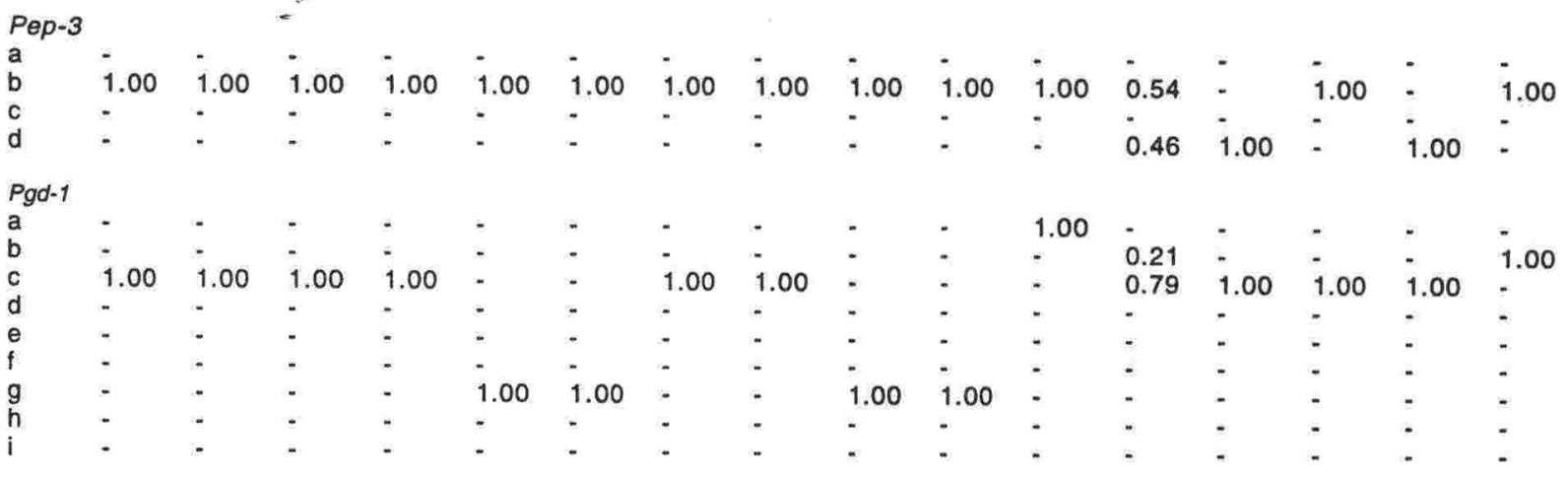


Table 5.4 .2 (continued)

\begin{tabular}{|c|c|c|c|c|c|c|c|c|c|c|c|c|c|c|c|c|}
\hline Locus & $\begin{array}{l}\text { Specie } \\
\mathrm{Ng}\end{array}$ & $\mathrm{Ng}(\mathrm{nc})$ & $\mathrm{Nee}$ & Nep & $\mathrm{Nm}$ & $\mathrm{Nr}$. & Ns . & $\mathrm{Nt}$ & $\mathrm{Ng}(\mathrm{hp})$ & $\mathrm{Ng}$ & Hs & $\mathrm{Hg}$ & $\mathrm{Hn}$ & $\mathrm{Hk}$ & $\mathrm{Hr}$ & Htk \\
\hline (n) & 4 & 2 & 5 & 4 & 4 & 3 & 9 & 2 & 2 & 7 & 4 & 24 & 1 & 2 & 9 & 3 \\
\hline \multicolumn{17}{|c|}{ Pgm-1 } \\
\hline a & - & - & $\cdot$ & - & - & - & - & - & - & - & - & 0.04 & 1.00 & 0.50 & $\cdot$ & $\cdot$ \\
\hline b & $\cdot$ & - & - & - & - & - & 0.94 & 0.75 & - & - & - & - & - & $i$ & $\cdot$ & $\cdot$ \\
\hline c & - & - & - & 0.12 & 1.00 & 1.00 & - & 0.25 & - & 1.00 & 1.00 & 0.96 & * & 0.50 & 1.00 & - \\
\hline d & - & - & - & - & - & - & - & - & - & - & - & $\cdot$ & - & - & - & 1.00 \\
\hline e & 1.00 & 1.00 & 1.00 & 0.88 & - & - & 0.06 & - & 1.00 & $\cdot$ & - & - & $\cdot$ & - & $\cdot$ & - \\
\hline \multicolumn{17}{|l|}{ Pgm-2 } \\
\hline a & 1.00 & 1.00 & 1.00 & 1.00 & 1.00 & 1.00 & 0.56 & 1.00 & - & - & - & - & - & $\cdot$ & $\cdot$ & - \\
\hline b & - & - & $\cdot$ & - & - & - & - & - & $=$ & - & - & - & - & $=$ & - & $\cdot$ \\
\hline c & - & - & - & - & - & - & 0.44 & - & 1.00 & 1.00 & 1.00 & 0.19 & - & 0.50 & 0.83 & $\cdot$ \\
\hline d & - & - & - & - & - & - & - & - & - & $=$ & - & 0.69 & 1.00 & 0.50 & - & 1.00 \\
\hline e & - & - & - & - & - & - & - & - & - & - & - & $=$ & - & - & - & $\cdot$ \\
\hline$f$ & - & - & - & - & - & - & - & - & - & - & - & - & - & - & - & - \\
\hline $\mathrm{g}$ & - & - & - & - & - & - & - & - & - & - & - & - & - & - & 0.17 & - \\
\hline h & - & - & - & - & - & - & - & - & - & - & - & - & - & - & - & - \\
\hline $\mathrm{i}$ & - & - & - & - & - & - & - & - & - & - & $\cdot$ & 0.12 & - & - & $\cdot$ & - \\
\hline \multicolumn{17}{|l|}{$S d h-2$} \\
\hline a & 1.00 & 1.00 & 1.00 & 1.00 & 1.00 & 1.00 & 1.00 & 1.00 & 1.00 & 1.00 & 1.00 & 1.00 & 1.00 & 1.00 & 1.00 & 1.00 \\
\hline \multicolumn{17}{|l|}{ Sod-2 } \\
\hline a & - & - & - & - & - & - & - & - & - & - & - & - & - & - & 1.00 & - \\
\hline b & - & $\cdot$ & - & - & - & - & - & - & - & - & - & - & - & - & - & - \\
\hline c & - & - & - & - & - & - & - & - & - & - & - & 0.96 & 1.00 & 1.00 & - & 0.83 \\
\hline d & 1.00 & 1.00 & 1.00 & 1.00 & 1.00 & 1.00 & 0.94 & 1.00 & 1.00 & 1.00 & 1.00 & 0.04 & - & - & - & 0.17 \\
\hline e & - & - & - & - & - & - & - & $\cdot$ & - & - & - & $\cdot$ & - & - & - & - \\
\hline$f$ & - & - & - & - & - & - & 0.06 & - & - & - & - & - & - & - & - & - \\
\hline \multicolumn{17}{|l|}{ Sod-3 } \\
\hline a & 1.00 & 1.00 & 1.00 & 1.00 & 1.00 & 1.00 & 1.00 & 1.00 & 1.00 & 1.00 & ; & 1.00 & 1.00 & 1.00 & 1.00 & 1.00 \\
\hline b & - & - & - & - & - & $\cdot$ & $\cdot$ & - & - & $\cdot$ & 1.00 & $\cdot$ & - & $\cdot$ & $\cdot$ & $=$ \\
\hline c & - & - & - & - & - & - & - & - & - & - & $\cdot$ & - & - & - & $\cdot$ & $\cdot$ \\
\hline
\end{tabular}

Table 5.4 .2 (continued)

Species key:

$\begin{array}{llll}\text { Hpk } & \text { H. "Poor Knights" } & \text { Hsa } & \text { H. "Southern Alps" } \\ \text { Hmat } & \text { H. "Matapia" } & \text { Hd } & \text { H. duvaucelii } \\ \text { Hp } & \text { H. pacificus } & \text { Hca } & \text { H. "Canterbury" } \\ \text { Hc } & \text { H. chrysosireticus } & \text { Hma } & \text { H. "Mount Arthur" } \\ \text { Hsm } & \text { H. "Southern mini" } & \text { Hkai } & \text { H. "Kaikouras" } \\ \text { Hdp } & \text { H. "Dansey's Pass" } & \text { Hmm } & \text { H. "Marlborough mini" } \\ \text { Ho } & \text { H. "Otago" } & \text { Hm } & \text { H. maculatus } \\ \text { Hcg } & \text { H. "Cromwell Gorge" } & & \end{array}$

$\mathrm{Hcg} H$. "Cromwell Gorge"

\begin{tabular}{|c|c|c|c|c|c|c|c|c|c|c|c|c|c|c|c|}
\hline Locus & $\begin{array}{l}\text { Specie } \\
\text { Hpk }\end{array}$ & Hmat & $\mathrm{Hp}$ & $\mathrm{Hc}$ & Hsm & $\mathrm{Hdp}$ & Ho & $\mathrm{Hcg}$ & Hsa & $\mathrm{Hd}$ & $\mathrm{Hca}$ & Hma & Hkai & $\mathrm{Hmm}$ & $\mathrm{Hm}$ \\
\hline (n) & 8 & 3 & 39 & 6 & 13 & 9 & 127 & 9 & 103 & 34 & 25 & 2 & 9 & 55 & 153 \\
\hline \multicolumn{16}{|l|}{ Ak-1 } \\
\hline a & 1.00 & 1.00 & 1.00 & 1.00 & 1.00 & 1.00 & 0.29 & 1.00 & 1.00 & 1.00 & 1.00 & 1.00 & 1.00 & 1.00 & 1.00 \\
\hline b & - & - & - & - & - & - & 0.71 & - & - & - & - & $\cdot$ & - & $=$ & - \\
\hline c & - & - & - & - & - & - & - & - & - & - & - & - & - & - & - \\
\hline d & - & - & - & - & - & - & - & - & - & - & $\cdot$ & - & - & - & - \\
\hline
\end{tabular}
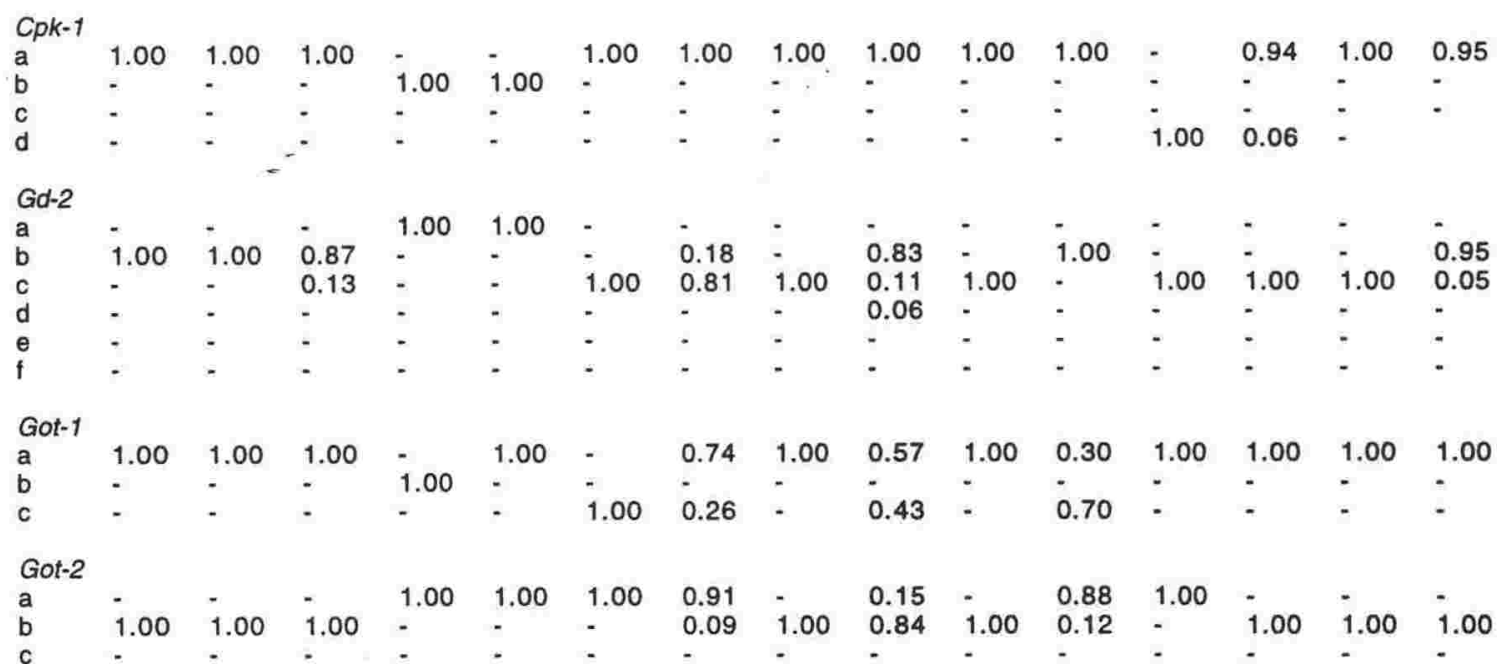
Table 5.4.2 (continued)

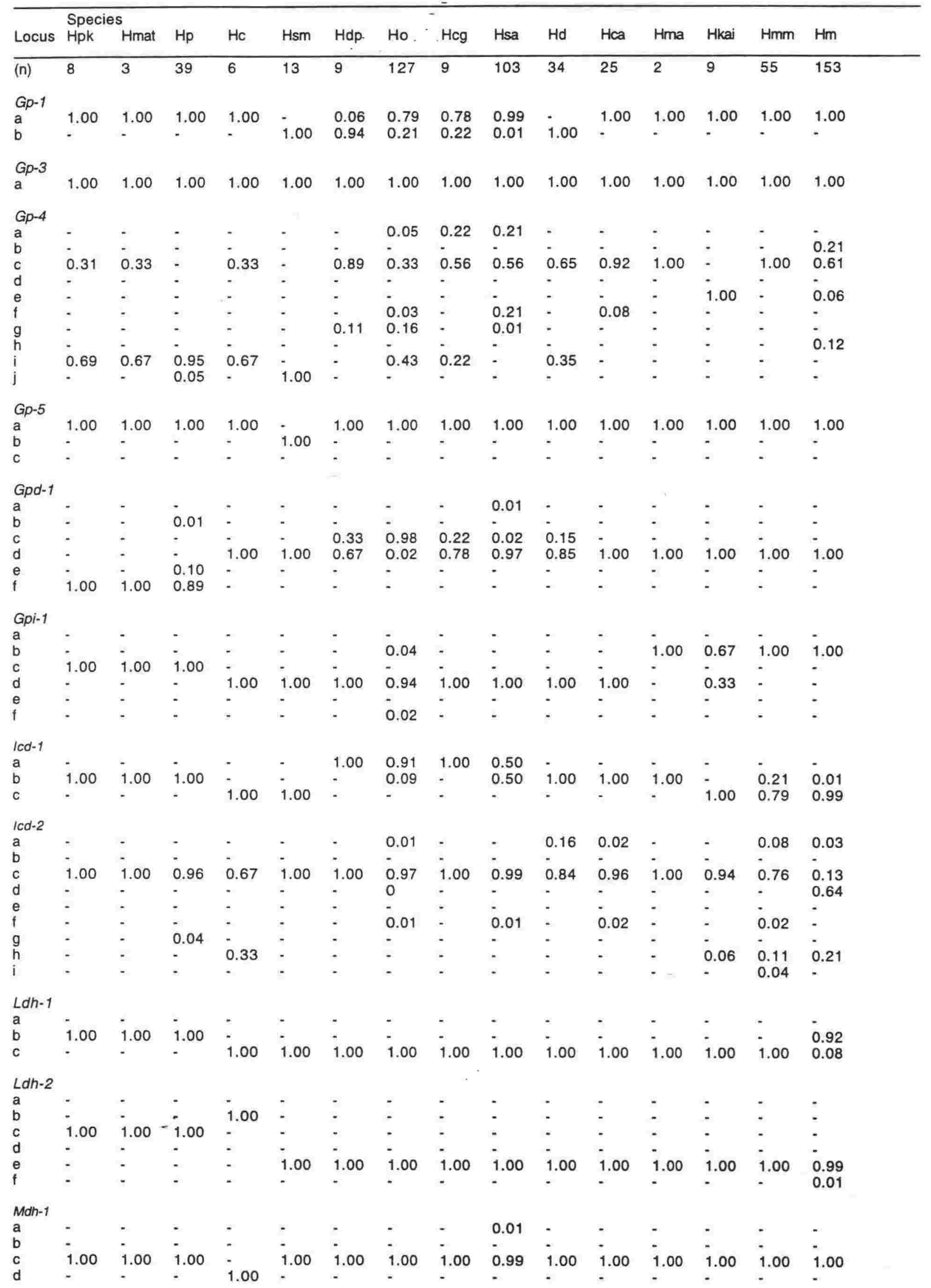


Table 5.4 .2 (continued)

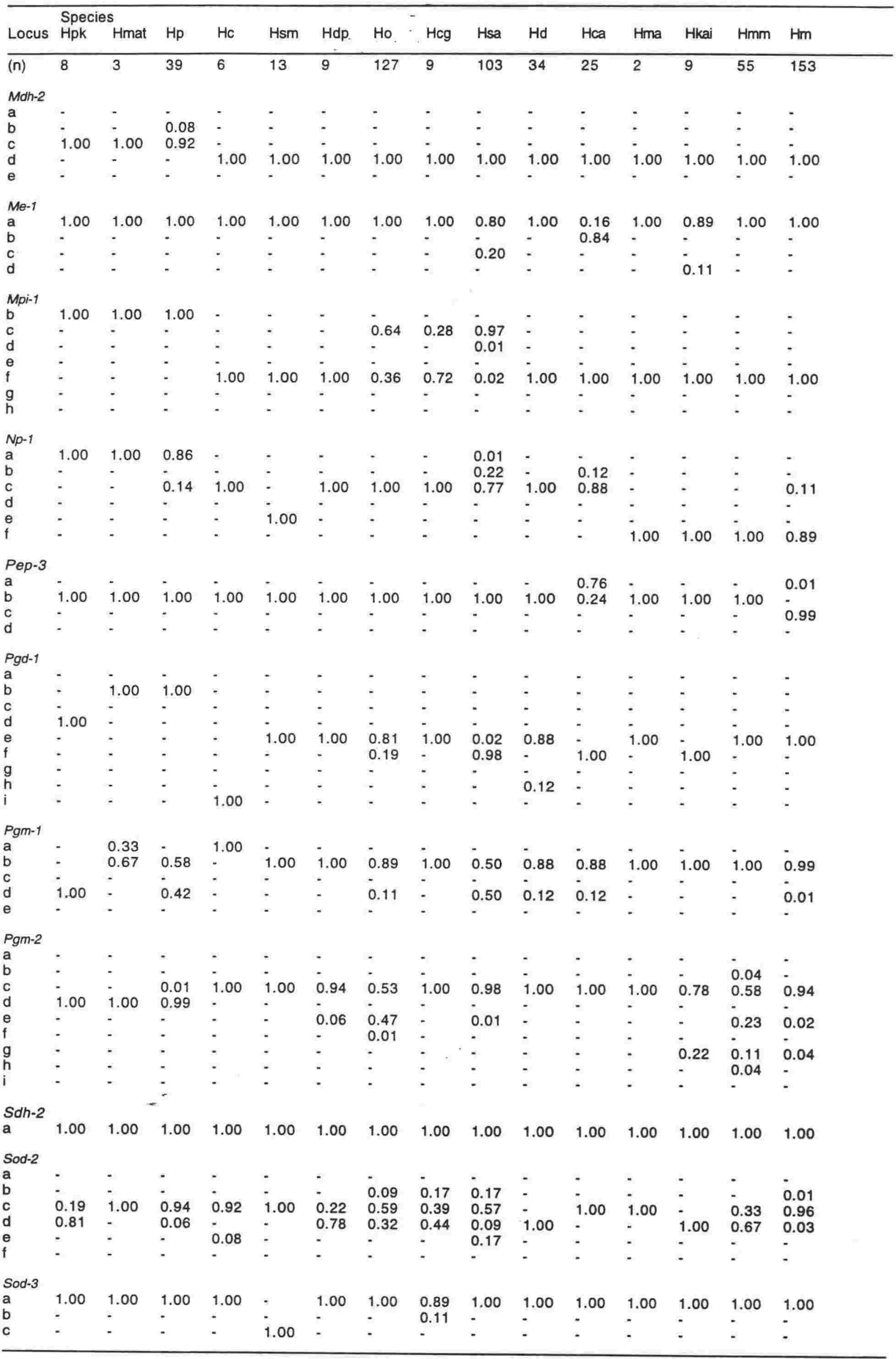


Genetic distance (Nei's D)
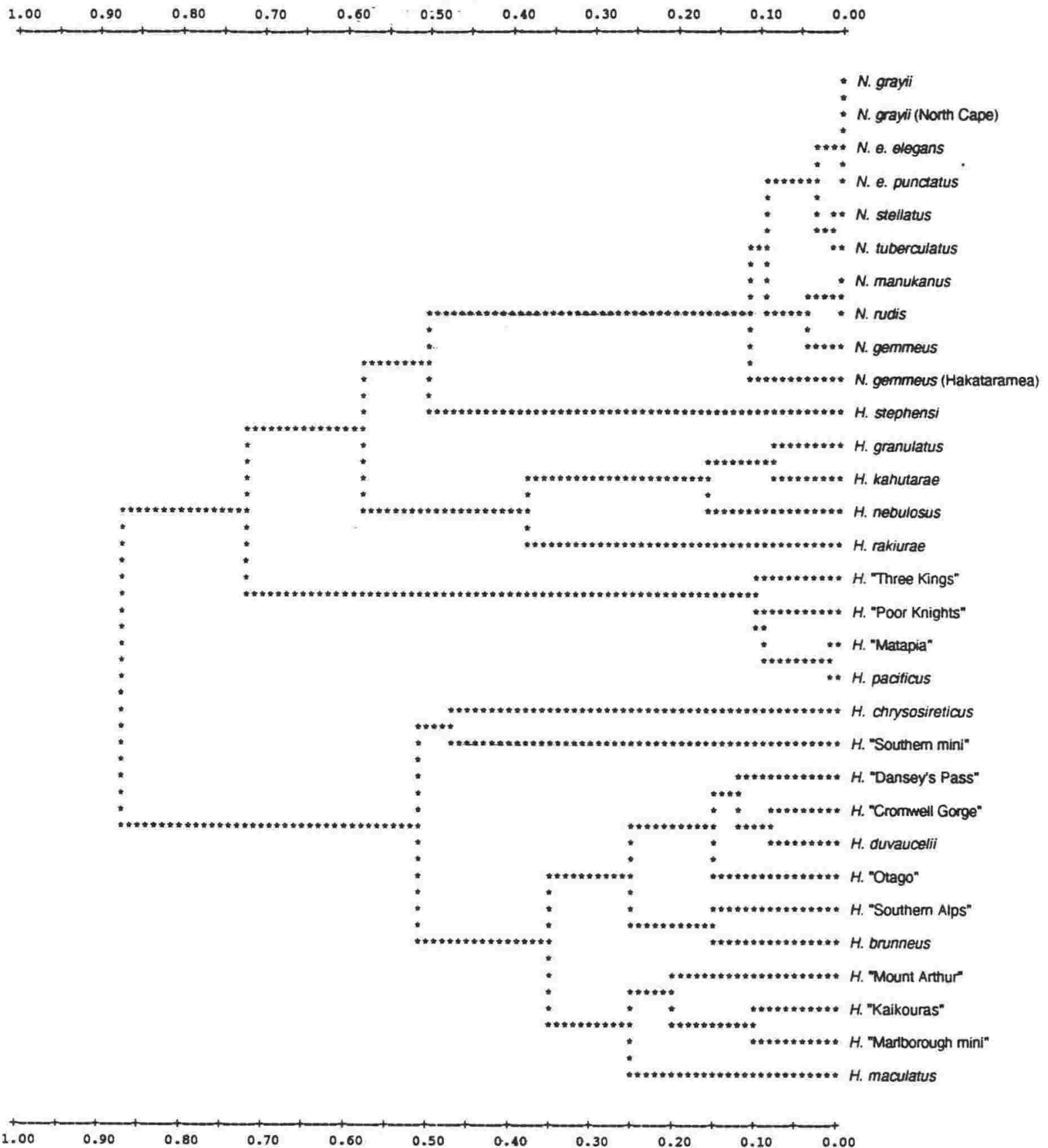

Figure 5.4.1 WPGMA phenogram (BIOSYS package) for New Zealand geckos, generated from allozyme data (27 loci, Nei's D) pooled within species. 
Distance from root

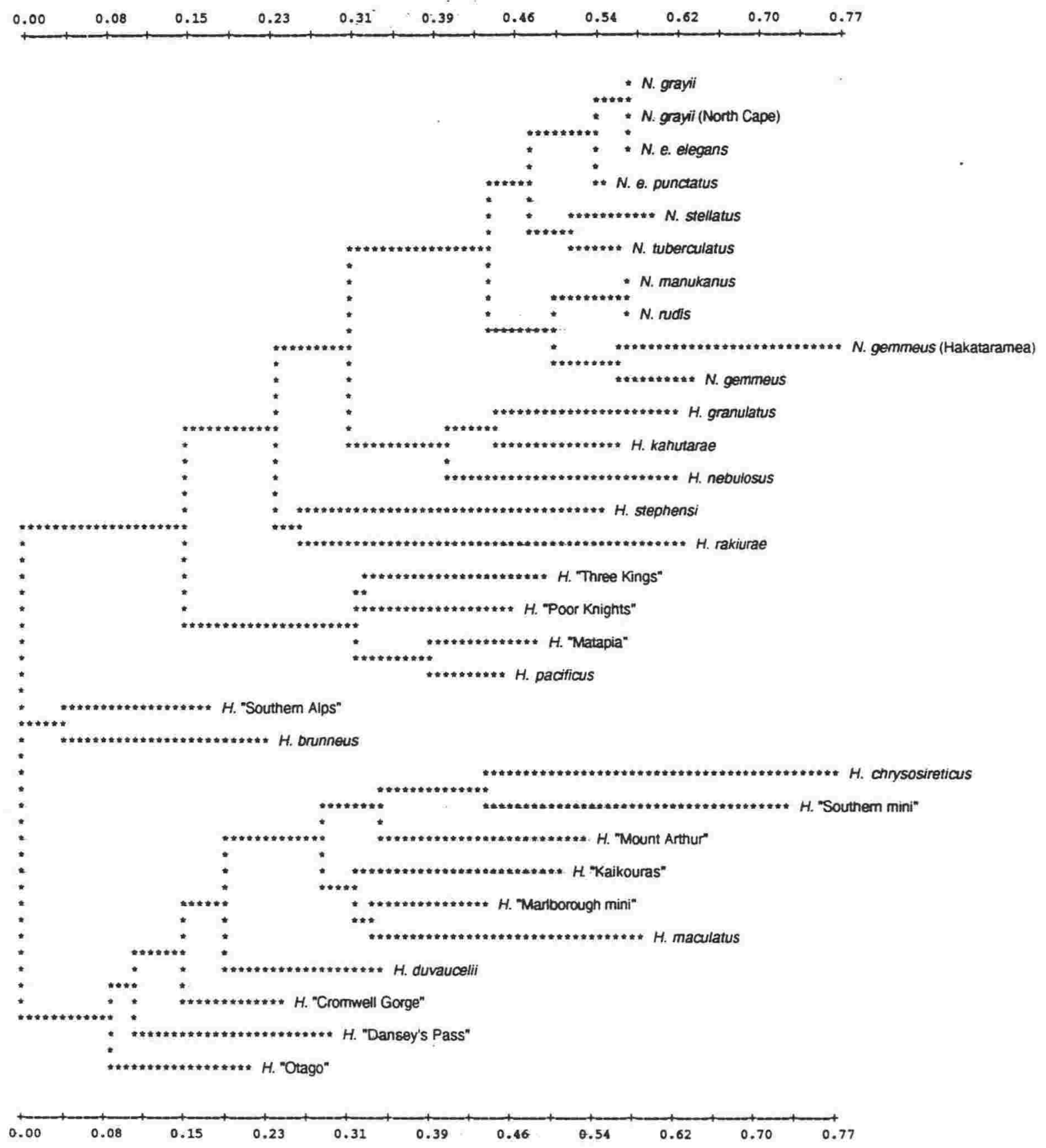

Figure 5.4.2 Distance Wagner phylogram (BIOSYS package) for New Zealand geckos, generated from allozyme data (27 loci, Cavalli-Sforza \& Edwards' arc distance) pooled within species. Total length of tree $=6.021$. 
BIOSYS, then used to produce a neighbour joining Tree (Figure 5.4.3) using the MEGA package.

A summary of all alleles present in each species at each variable locus was generated from Table 5.4.2 to produce a character state matrix for parsimony analysis using PAUP 3.0 (Table 5.4.3). To reduce the numbers of taxa in this dataset, Naultinus and the $H$. pacificus group were each collapsed into a single taxon, as both are clearly monophyletic groups with low levels of divergence among species within the groups relative to the distances to their closest sistergroups. Because of limitations of the numbers of taxa which could be run on PAUP in a reasonable time, bootstrapped branch and bound searches were performed only on various subsets of this datafile (Figure 5.4.4-8). Even heuristic searches using the whole dataset caused the computer to run out of available memory and crash, because very large numbers of equally short trees were found.

Trees generated by WPGMA, neighbour joining, and maximum parsimony (Figure 5.4.1-8) from my allozyme data are in general agreement for the New Zealand taxa. In particular, the WPGMA and neighbour joining trees are very similar, differing only in branching order within closely related species groups. The position of the root of these trees is unknown, which means that no groups can be conclusively regarded as monophyletic. However, the following groupings are supported by most tree-building methods.

The primary split is between the following 2 groups.

- Narrow-toed Hoplodactylus (i.e., H. stephensi, H. rakiurae, $H$. kahutarae, $H$. nebulosus, and $H$. granulatus) plus Naultinus. This group is recovered by all phenetic methods and in all parsimony trees, with 63$98 \%$ support in the latter.

- Broad-toed Hoplodactylus (i.e. the $H$. maculatus and $H$. pacificus complexes). Both phenetic methods place the $H$. pacificus complex closer to the narrow-toed group than to the $H$. maculatus complex. However, bootstrap values in parsimony analysis give stronger support (63-98\%) to a broad-toed group including the $H$. maculatus and $H$. pacificus complexes.

Within the narrow-toed group there are 2 or 3 groups. 


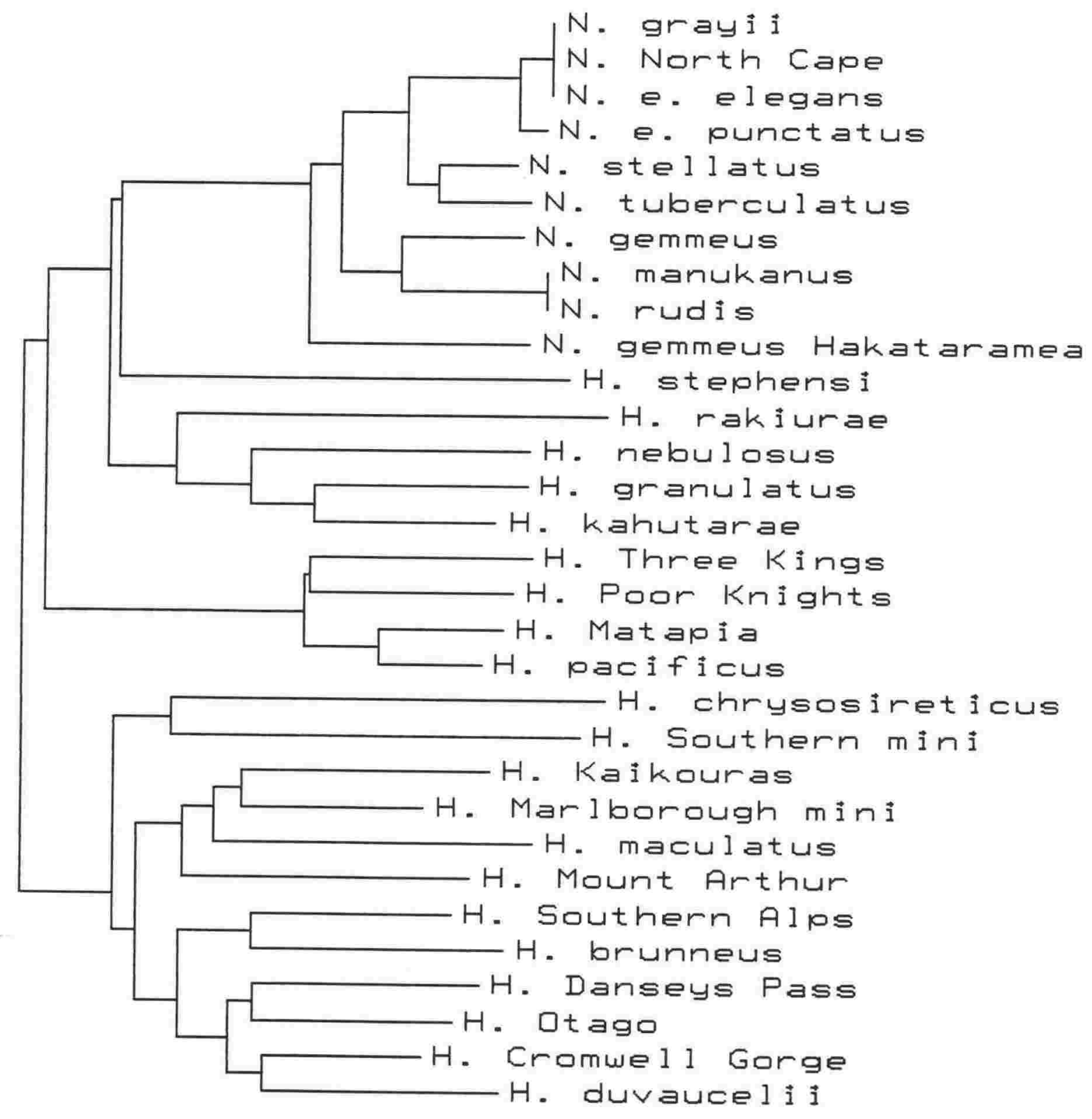

Figure 5.4.3 neighbour joining phylogram (MEGA package) for New Zealand geckos, generated from allozyme data (27 loci, Cavalli-Sforza \& Edwards' arc distance) pooled within species. 


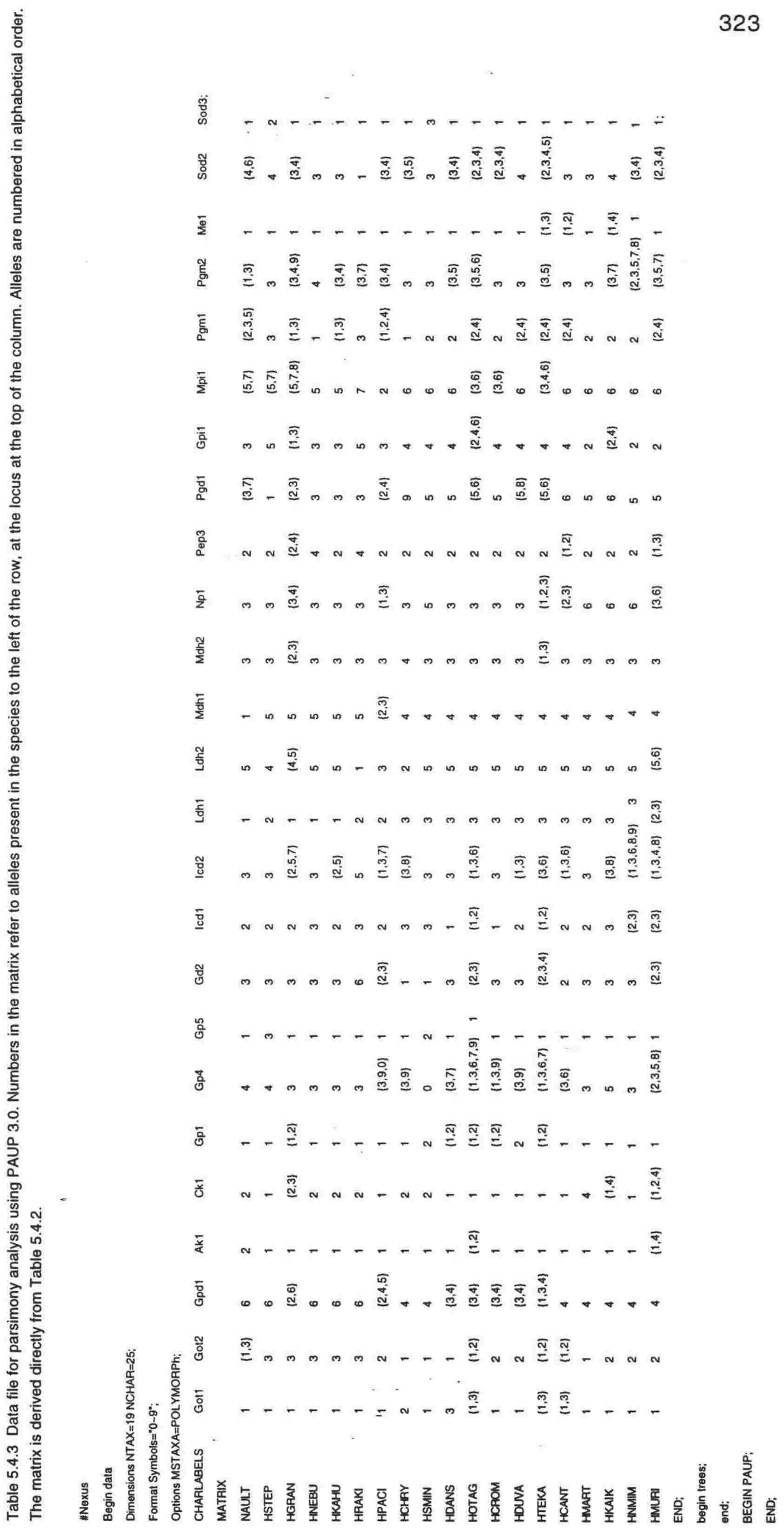




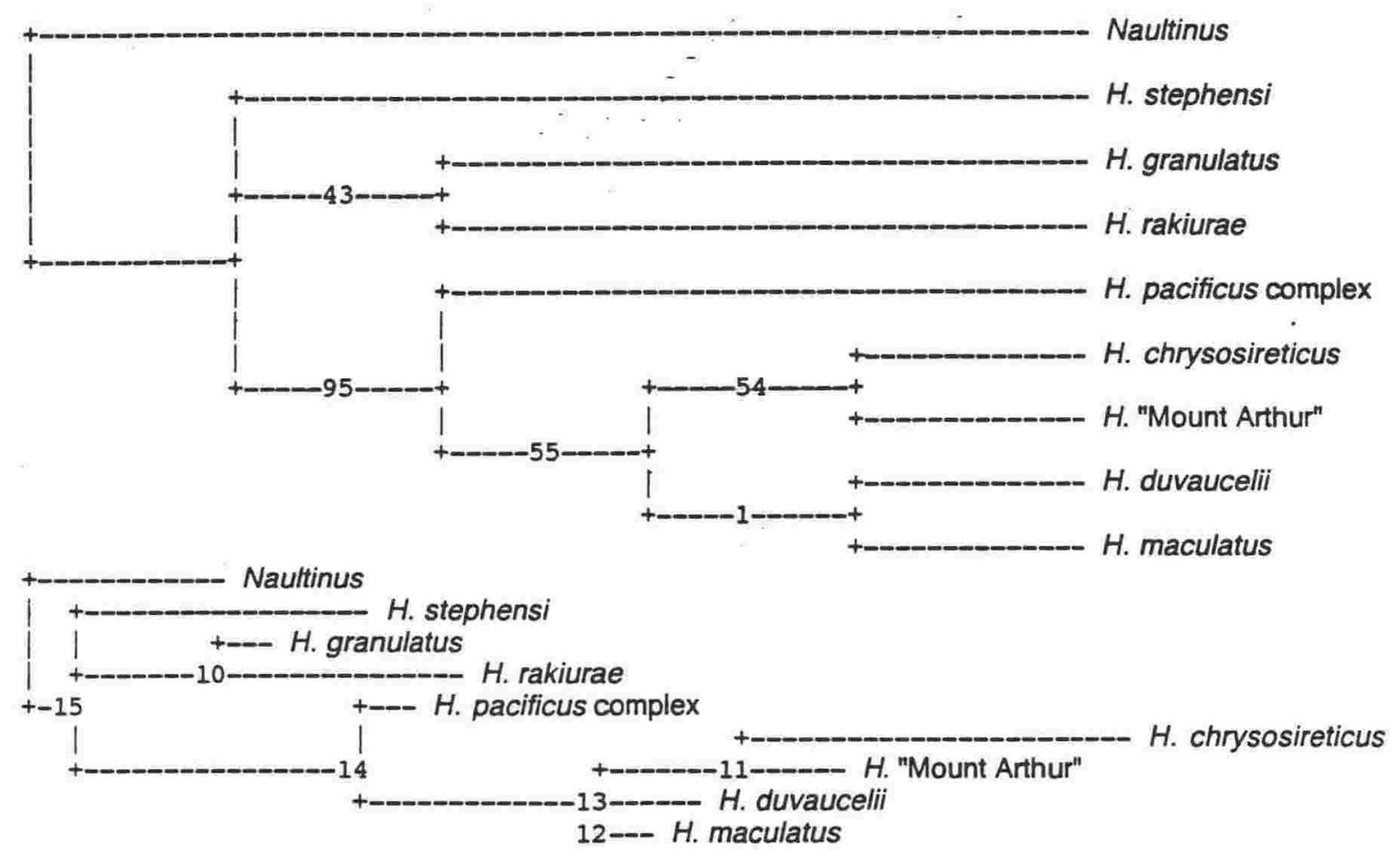

Figure 5.4.4 Maximum Parsimony cladogram and phylogram (PAUP package) for representative New Zealand gecko species, generated from allozyme data (25 variable loci) pooled within species. Bootstrap values are presented on branches of the cladogram. Tree length $=116$; Consistency index $(\mathrm{Cl})=0.681 ; \mathrm{Cl}$ excluding uninformative characters $=0.661$; Retention index $(\mathrm{RI})=0.351$; Rescaled consistency index $(R C)=0.239 ; f$ value $=5 ; \mathrm{f}$-ratio $=0.1163$ (multistate unordered and stepmatrix characters excluded from $\mathrm{f}$-value calculations)

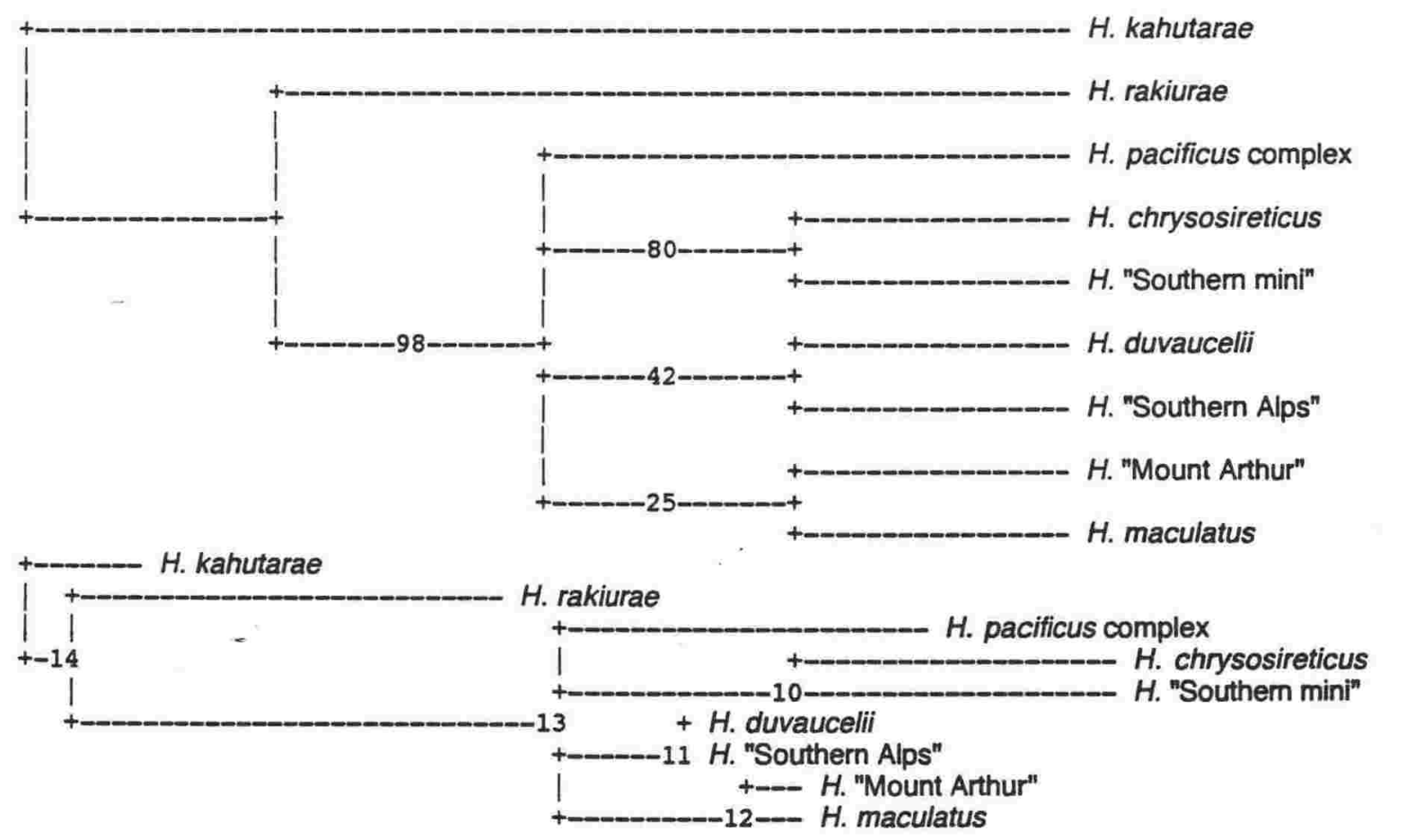

Figure 5.4.5 Maximum Parsimony cladograms and phylograms (PAUP package) for representative New Zealand gecko species, generated from allozyme data (25 variable loci) pooled within species. 


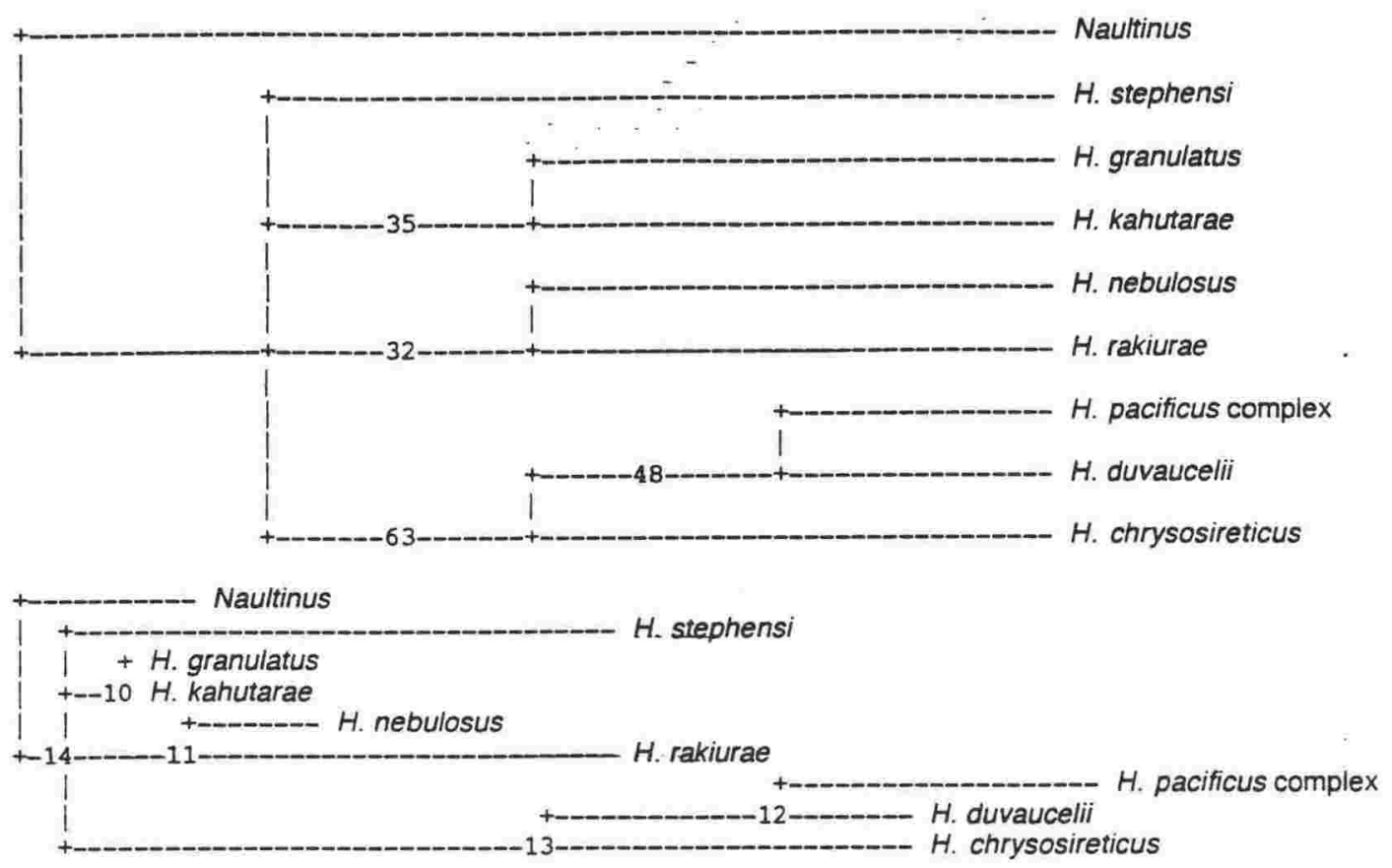

Figure 5.4.6 Maximum Parsimony cladograms and phylograms (PAUP package) for representative New Zealand gecko species including all narrow-toed Hoplodactylus, generated from allozyme data (25 variable loci) pooled within species. Bootstrap values are presented on branches of the cladogram. Tree length $=101$; Consistency index $(\mathrm{Cl})=0.653$; $\mathrm{Cl}$ excluding uninformative characters $=0.611$; Retention index $(\mathrm{RI})=0.239$; Rescaled consistency index $(R C)=0.156 ; f$ value $=7 ; f$-ratio $=0.1014 ;$ (multistate unordered and stepmatrix characters excluded from f-value calculations).

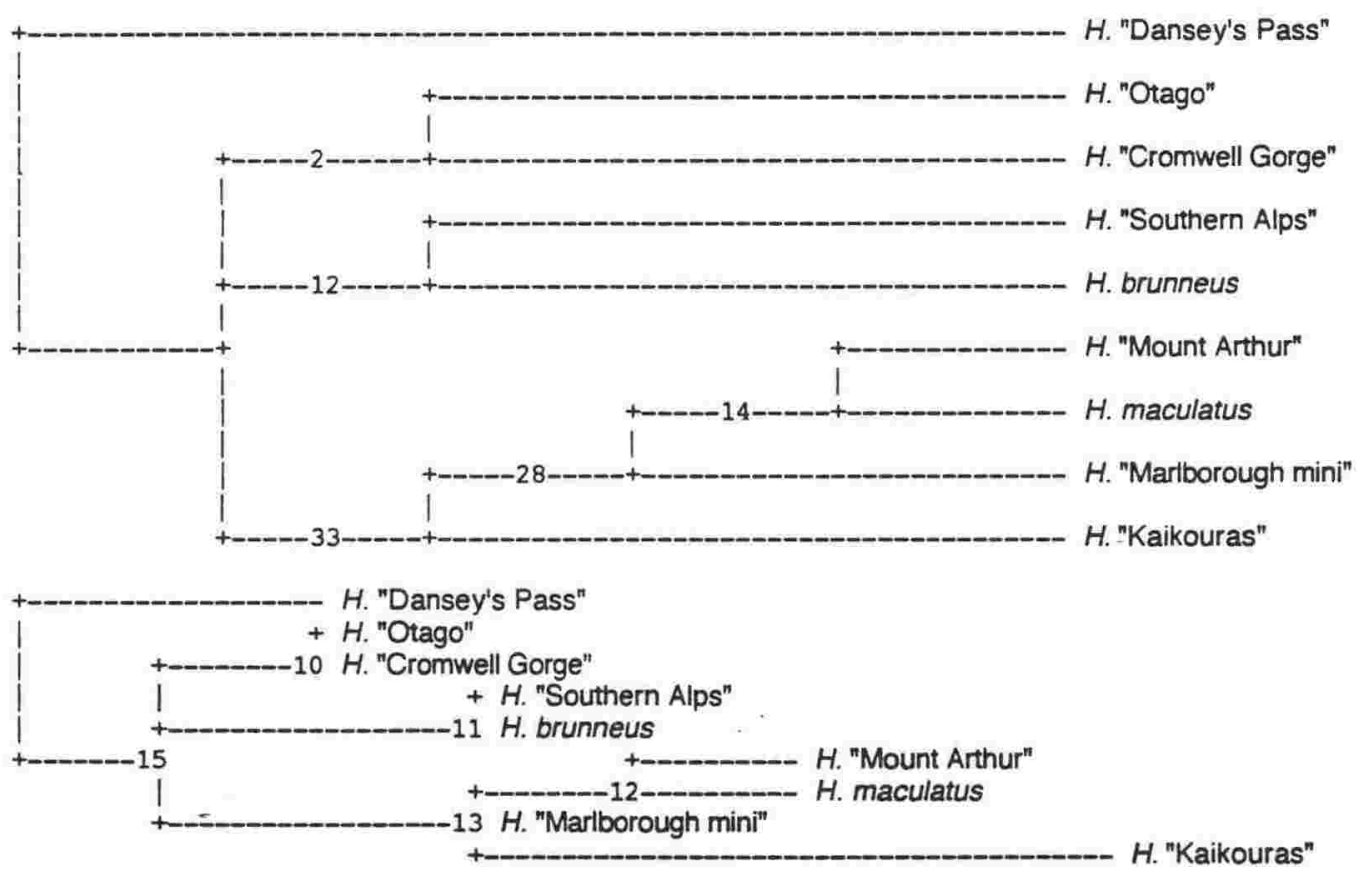

Figure 5.4.7 Maximum Parsimony cladograms and phylograms (PAUP package) for species of the $H$. maculatus complex, generated from allozyme data (25 variable loci) pooled within species. Bootstrap values are presented on branches of the cladogram. Tree length $=119$; Consistency index $(\mathrm{Cl})=0.437 ; \mathrm{Cl}$ excluding uninformative characters $=0.396$; Retention index $(R I)=0.056$; Rescaled consistency index $(R C)=$ $0.025 ; f$ value $=23 ; f$-ratio $=1.0000$ (multistate unordered and stepmatrix characters excluded from f-value calculations) 


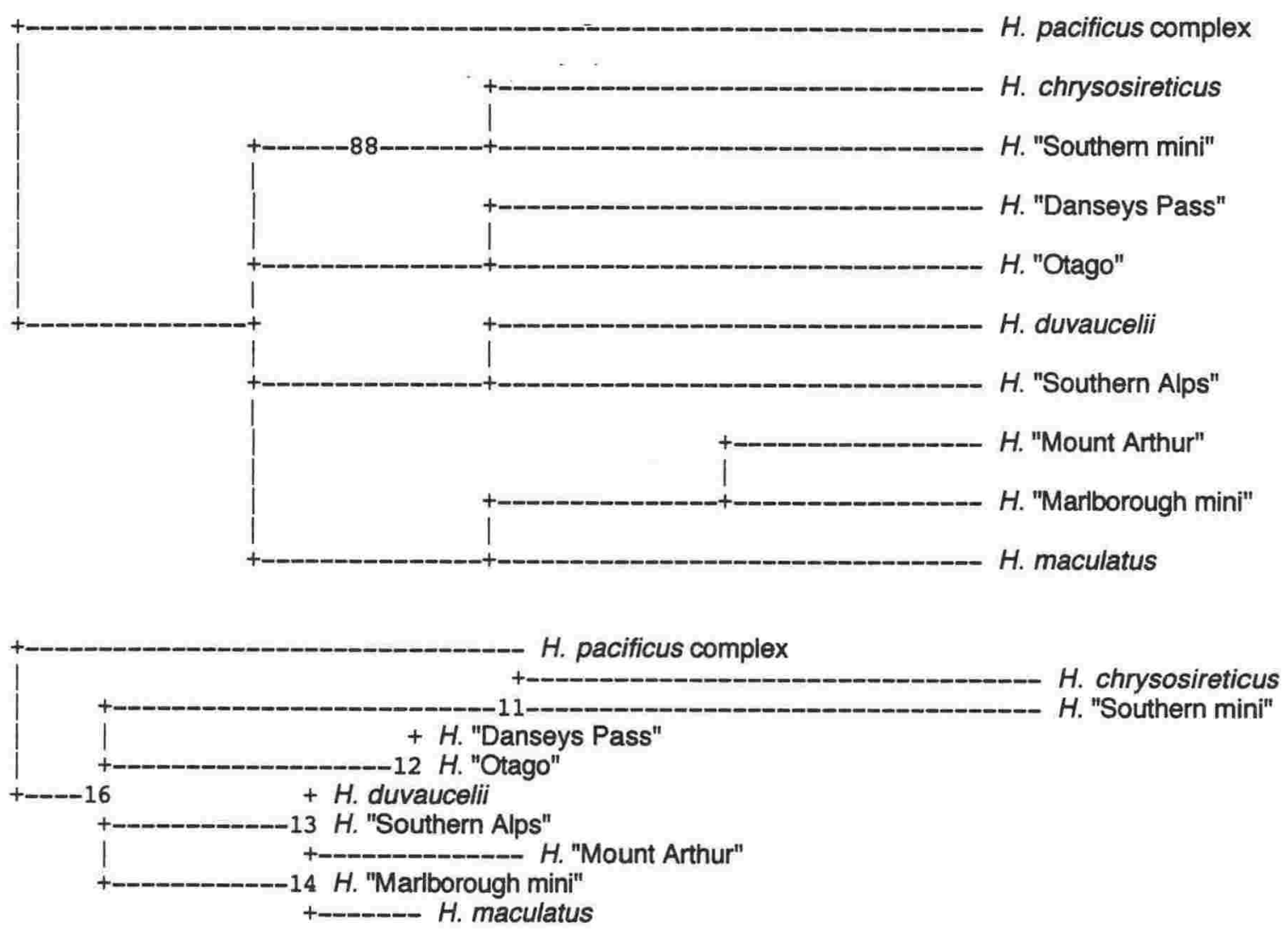

Figure 5.4.8 Maximum parsimony cladograms and phylograms (PAUP package) for members of the $H$. maculatus complex, generated from allozyme data (25 variable loci) pooled within species.

- Naultinus is always present as a monophyletic group in phenetic analyses. This strong support is generated by the high similarity among Naultinus spp. rather than a particularly long edge separating Naultinus from the rest. Naultinus species were pooled for cladistic analysis.

- The $\boldsymbol{H}$. granulatus complex (i.e., $H$. rakiurae, $H$. kahutarae, $H$. nebulosus, and $H$. granulatus). This group appeared in WPGMA and neighbour-joining analyses, but in the distance Wagner tree, $H$. rakiurae was the sister group of $H$. stephensi. In cladistic trees, this group had little or no bootstrap support, but the trees were not incompatible with the existence of the group.

- H. stephensi. The position of this species with respect to Naultinus and the $H$. granulatus complex differs between tree-building methods, and is best regarded as unresolved. In WPGMA and neighbour-joining trees, $H$ stephensi and Naultinus are sister taxa, but the edge combining this pair 
to the exclusion of others is short. In the distance Wagner tree, $H$. rakiurae was the sister group of $H$. stephensi. In parsimony trees, the relationship among $\mathrm{H}$ stephensi, Naultinus, and the other narrow-toed Hoplodactylus species is unresolved.

Within the broad-toed group there were 2 major complexes.

- The $\boldsymbol{H}$. pacificus complex (i.e., $H$. pacificus, $H$. "Poor Knights", $H$. "Three Kings", and $H$. "Matapia"). This group appears in all phenetic trees; it always falls between the narrow-toed group discussed above and the $H$. maculatus complex. The strong support for this clade results from the combination of a long edge leading to the clade and much shorter edges within the clade. The species of this group were pooled for cladistic analysis.

- The $\boldsymbol{H}$. maculatus complex (i.e., $H$. maculatus, $H$. "Mount Arthur", $H$. "Marlborough mini", H. "Kaikouras", H. "Dansey's Pass", H. "Cromwell Gorge", H. "Otago", H. "Southern Alps", H. brunneus, H. duvaucelii, $H$. "Southern mini", and $H$. chrysosireticus.). This group is always present in phenetic analyses, but was more weakly supported, and sometimes appeared paraphyletic to the $H$. pacificus group in bootstrapped PAUP analyses. Because this group is by far the largest in my dataset, and includes far deeper branches than any of the other major groups, the branch separating this clade as a whole from the other major groups is quite short, which leads to poor support.

Within the $H$. maculatus complex are 3 species groups.

- $\quad$. chrysosireticus plus $H$. "Southern mini". This pairing was present in all phenetic and parsimony analyses, and had high bootstrap support $(80-88 \%$ - Figures $5.4 .5,5.4 .8)$ in the latter, although the edge uniting them is very short compared to the substantial distance (Nei's $D=$ $0.380-0.398$ ) between them. Phenetic analyses suggest that this pair is the sister group of the balance of the $H$. maculatus complex; parsimony analyses fail to resolve this relationship.

- The "northern group" of the $H$. maculatus complex ( $H$. maculatus, $H$. "Kaikouras", H. "Marlborough mini", and $H$. "Mount Arthur"). This group was present in both phenetic analyses. It was also found consistently 
(Figures 5.4.5, 5.4.7, 5.4.8) in PAUP analyses, but with very weak bootstrap support.

- The "southern group" of the H. maculatus complex (H. brunneus, $H$. "Southern Alps", H. "Danseys Pass", H. "Otago", and H. "Cromwell Gorge") plus $\boldsymbol{H}$. duvaucelii. This group was present in both phenetic analyses. It was also found (Figures 5.4.5, 5.4.7) in most PAUP analyses, but with weak bootstrap support. In one parsimony analysis including only members of the $H$. maculatus complex (Figure 5.4.8) no monophyletic southern group was recovered, although 2 pairs of species within this group were associated.

The southern group of the $H$. maculatus complex is further subdivided into 2 geographic clusters.

- The Canterbury species ( $H$. brunneus and $H$. "Southern Alps"). This pairing is present in all trees in which both are included, although bootstrap support is low (12\%, Figure 5.4.7).

- The Otago-Southland species (H. "Danseys Pass", H. "Otago", and $\boldsymbol{H}$. "Cromwell Gorge") plus $\boldsymbol{H}$. duvaucelii. This group is present in WPGMA and neighbour-joining phenograms, but is paraphyletic to $H$. chrysosireticus, $H$. "Southern mini", and the northern group of the $H$. maculatus complex in the distance Wagner tree. In cladograms including 2 or more species of this group, the group was either very poorly supported (2\%, Figure 5.4.7) or not recovered (Figure 5.4.8).

\subsection{Congruence BetWEen MORPHOLOGICALLY-BASED AND}

\section{ALLOZYME-BASED PHYLOGENETIC HYPOTHESES}

Cladograms from morphological (Figure 5.3.3) and allozyme (Figure 5.4.4) data from New Zealand geckos are largely consistent for the taxa present in both. Branching patterns are identical except for the position of $H$. stephensi, which in the morphologically-derived tree is the sister group of the rest of the narrow-toed Hoplodactylus plus Naultinus, whereas in the allozyme-based tree these 3 groups form a trichotomy. Branch lengths leading to Naultinus are larger in the morphological than the allozyme-based trees, perhaps reflecting accelerated morphological evolution in Naultinus associated with the change from nocturnal to diurnal activity. 
The 2 datasets were combined to produce a single dataset for those New Zealand species for which morphological data are available (Table 5.5.1, Figure 5.5.1). A bootstrapped branch and bound search (100 replications) of this dataset produced a consensus tree identical in topology to the one for the same taxa from the morphological data alone, and with only small changes in bootstrap support for most groups.

The congruence between the trees derived from the 2 independent datasets, and the high bootstrap values on the tree from the combined dataset, give very high confidence in this tree accurately representing phylogenetic relationships among these New Zealand gecko species. The major unresolved issue is the position of the root of the tree, and therefore whether the New Zealand species form a monophyletic group or are paraphyletic with respect to the New Caledonian genera. However, on circumstantial evidence, I prefer the hypothesis that the New Zealand geckos are a monophyletic group, with the root probably falling between the broad-toed and narrow-toed Hoplodactylus.

\subsection{Generic CLASSIFICATION}

Criteria for recognition of genera vary greatly between major taxonomic groups, but genera are usually simply the next easily recognisable taxonomic group above the species level (Ross 1974). Recently in herpetology, speciose genera have been split into smaller, more narrowly diagnosed genera of a few species each. For example, Leiolopisma, formerly a huge, catch-all genus, was divided by Greer (1974) into 22 genera in 3 major groups. Even after this major revision, Leiolopisma remained a large genus of Australian, New Zealand, and Pacific and Indian Ocean Island species. Hutchinson et al. (1990) removed all the remaining Australian species from Leiolopisma, distributing them among 4 new genera and one resurrected genus, and suggested that Leiolopisma should be restricted to the type-species, $L$. telfairii, and an extinct relative. Sadlier (1986) recognised several new and reinstated genera in New Caledonia, leaving only 4 New Caledonian species in Leiolopisma. Hardy (1977) removed some New Zealand species to Cyclodina, and Patterson \& Daugherty (1995) removed the remaining New Zealand species to Oligosoma.

My allozyme results indicate major genetic divisions within the New Zealand gecko fauna, but not, as the traditional taxonomy implies, primarily between 


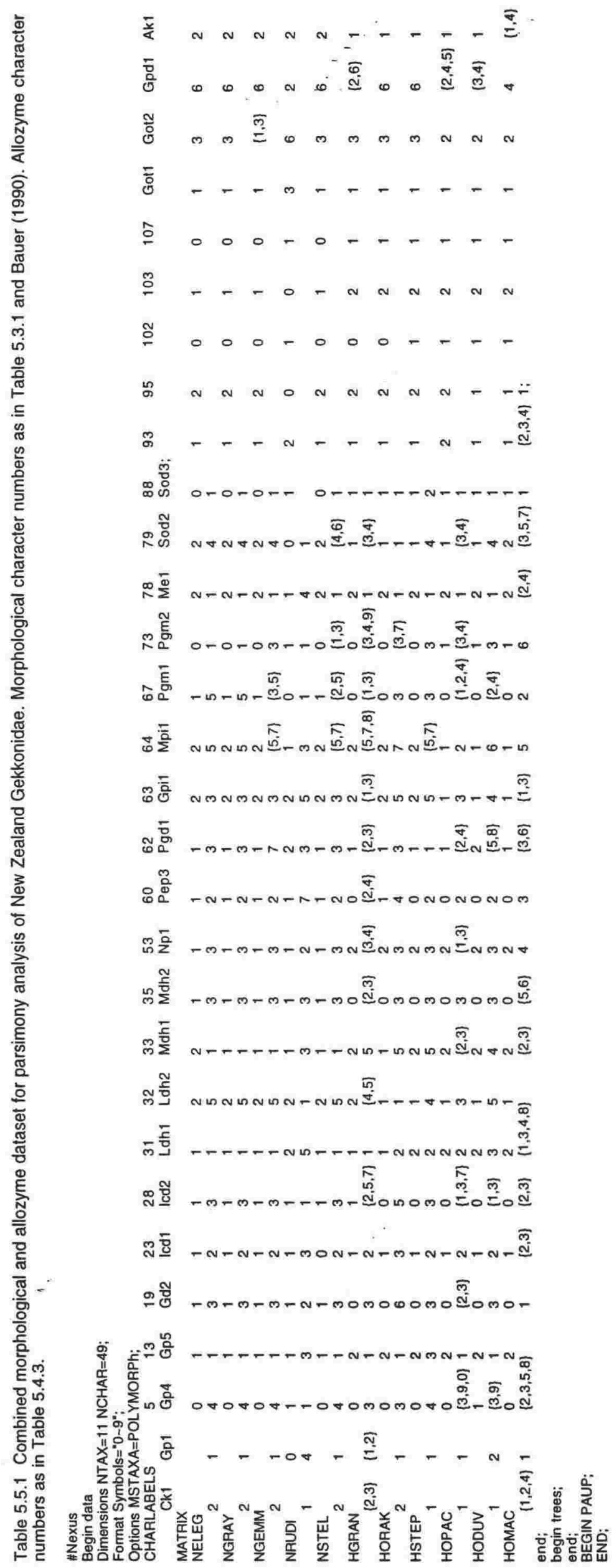



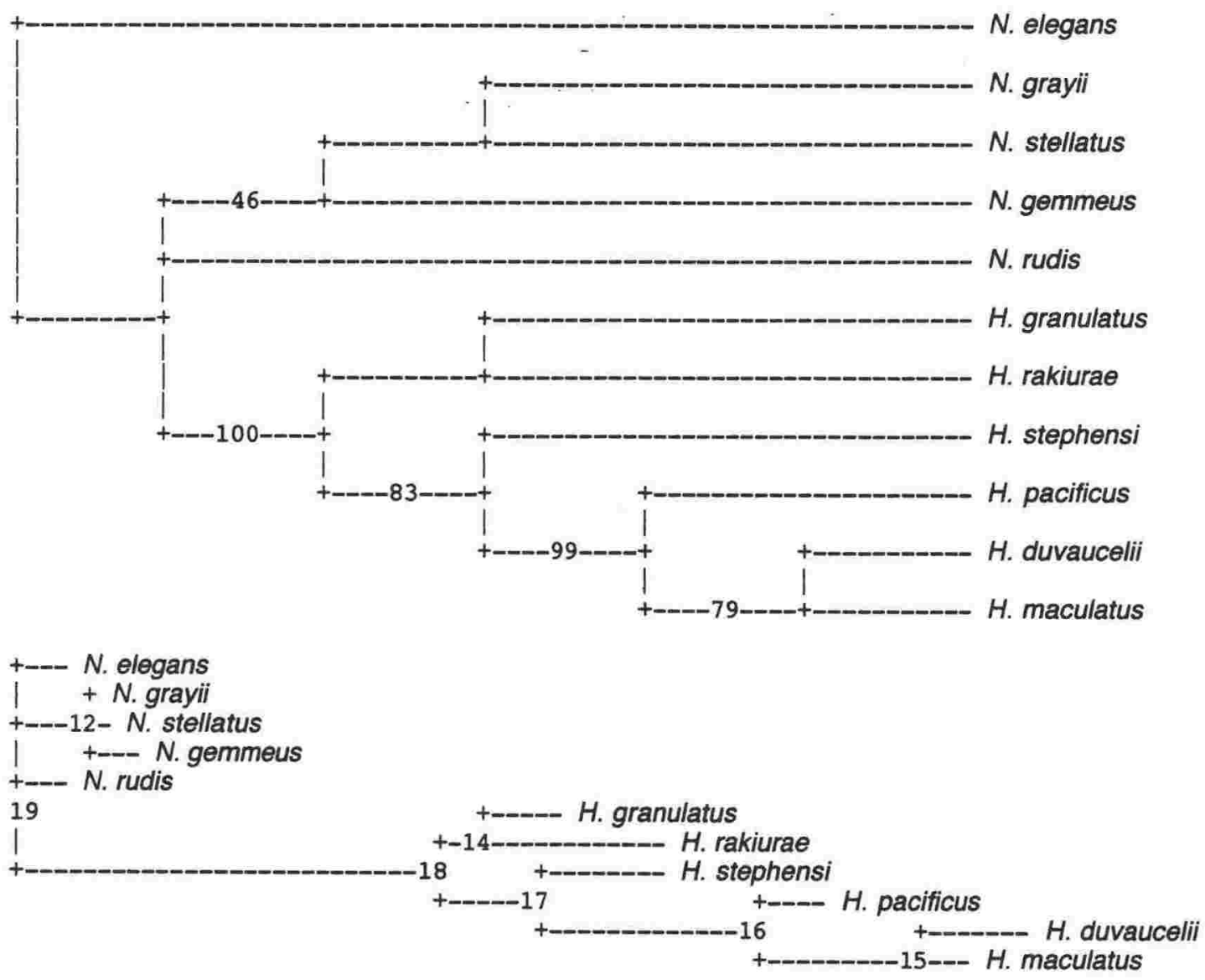

Figure 5.5.1 Bootstrap 50\% majority-rule consensus cladogram and phylogram (plus other groups compatible with this tree) from branch and bound search of combined morphological and allozyme data for New Zealand geckos. Tree length $=136$; Consistency index $(\mathrm{Cl})=0.713 ; \mathrm{Cl}$ excluding uninformative characters $=0.688$; Retention index $(\mathrm{RI})=0.680$; Rescaled consistency index $(\mathrm{RC})=0.485 ; \mathrm{f}$ value $=52$; $\mathrm{f}$-ratio $=0.0836$ (multistate unordered and stepmatrix characters excluded from $\mathrm{f}$-value calculations)

Hoplodactylus and Naultinus. Instead, Naultinus is just one of 4 distinct groups: the others are the $H$. maculatus complex (including $H$. duvaucelii and $H$. chrysosireticus), the $H$. pacificus complex, and the $H$. granulatus complex (including $H$. rakiurae, $H$. nebulosus, and $H$. kahutarae). The phylogenetic position of $H$. stephensi with respect to Naultinus and the $H$. granulatus complex is uncertain.

Generic reassignment is premature in the absence of a strongly supported position for the root of this tree. However, if the root is confirmed to fall between the broad-toed and narrow-toed groups of Hoplodactylus, 3 main possibilities exist for generic classification of the New Zealand geckos. 
- reduce all to a single genus

- recognise 4-5 monophyletic genera:

- Naultinus

- the $H$. granulatus complex

- H. stephensi if it proves on further examination to be the sister group of Naultinus plus the $H$. granulatus complex.

- the H. pacificus complex

- the $H$. maculatus complex

- recognise 3 monophyletic or paraphyletic genera, recognising that clarification of the phylogenetic position of $H$. stephensi is required:

- Naultinus

- the $H$. granulatus complex plus $H$. stephensi

- the $H$. pacificus complex plus the $H$. maculatus complex

I favour the third of these alternatives. The first does not adequately describe the substantial genetic and morphological diversity within the New Zealand gecko fauna. The second separates at the generic level taxa $(H$. stephensi, $H$. pacificus complex, $H$. maculatus complex) that are so similar that before 1977 (Robb \& Rowlands 1977) they were all regarded as conspecific except for the giant $H$. duvaucelii.

The 4-5 groups defined by allozymes are supported by several morphological characters, some of which were not included in Bauer's (1990) analysis.

The broad-toed $H$. pacificus and $H$. maculatus complexes have translucent belly scales (except for large adult $H$. duvaucelii), either uniformly pale or with scattered single darker scales; small, rounded dorsal scales; moderately to broadly dilated digital pads with curved or chevron-shaped distal lamellae; and flesh coloured mouth lining, with pigment absent or confined to the tongue tip. The narrow-toed Naultinus and $H$. granulatus complex species and $H$. stephensi have opaque belly scales, mottled or streaked with dark and light-coloured patches (except for some Naultinus specimens); medium to large conical dorsal scales; feebly dilated digital pads with straight or almost straight transverse lamellae; and blue or black and/or yellow, orange or deep pink pigment in the mouth. 
Within the broad-toed group, H. maculatus complex species have one or 2 blunt cloacal spurs pointing outwards. Members of the $H$. pacificus complex have 2-5 pointed spurs pointing up and back, a condition which they share with the narrow-toed group. Although my allozyme trees place the $H$. pacificus complex in an intermediate position between the $H$. maculatus complex and the narrow-toed group, all informative morphological characters except the form of the cloacal spurs ally this complex with the $H$. maculatus complex. The $H$. pacificus complex also shares its $P e p-1$ and $P e p-2$ isozyme banding patterns (not included in the formal analysis - see Chapter 2) with many the $H$. maculatus complex species. Overall, the $H$. pacificus complex appears closer to the $H$. maculatus complex.

Ecologically and morphologically, the traditional division between the brown and green geckos (Hoplodactylus and Naultinus respectively) is strongly supported (Bauer 1990). Naultinus is distinguished from all New Zealand brown geckos, including the narrow-toed species to which it is most closely related, by each of the following characters: largely cartilaginous pectoral girdle; brightly coloured skin pigment; pupil vertical with smooth margins; diurnal; external ear opening minute; digits with continuous series of scansorial pads; webbing between toes absent; tail elongate and prehensile (Bauer 1990).

Divergence between Naultinus and its closest relatives within Hoplodactylus is several times greater than that within Naultinus, but considerably less than that within Hoplodactylus. Intergeneric hybrids between Naultinus and $H$. granulatus are known (M. Keane, pers. comm.), and confirmed by my own electrophoretic results from one of the hybrids (unpublished data). However, Naultinus is so distinctive morphologically and ecologically, I consider continued generic separation of these groups justified.

\subsection{A PERSONAL VIEW OF DiPLOdACTYLINE GECKO EVOLUTION}

I consider it most likely that the New Zealand and New Caledonian carphodactyline gecko faunas are each monophyletic, are each others' sister groups, and are vicariant in origin. Within Australia, the successive outgroups to these eastern Tasman groups are probably Pseudothecadactylus, the bulk of Oedura (with all-telocentric karyotypes), the Oedura species with mixed karyotypes, Strophurus, and the remaining Diplodactylini and the padless Carphodactylini (Figure 5.7.1). 


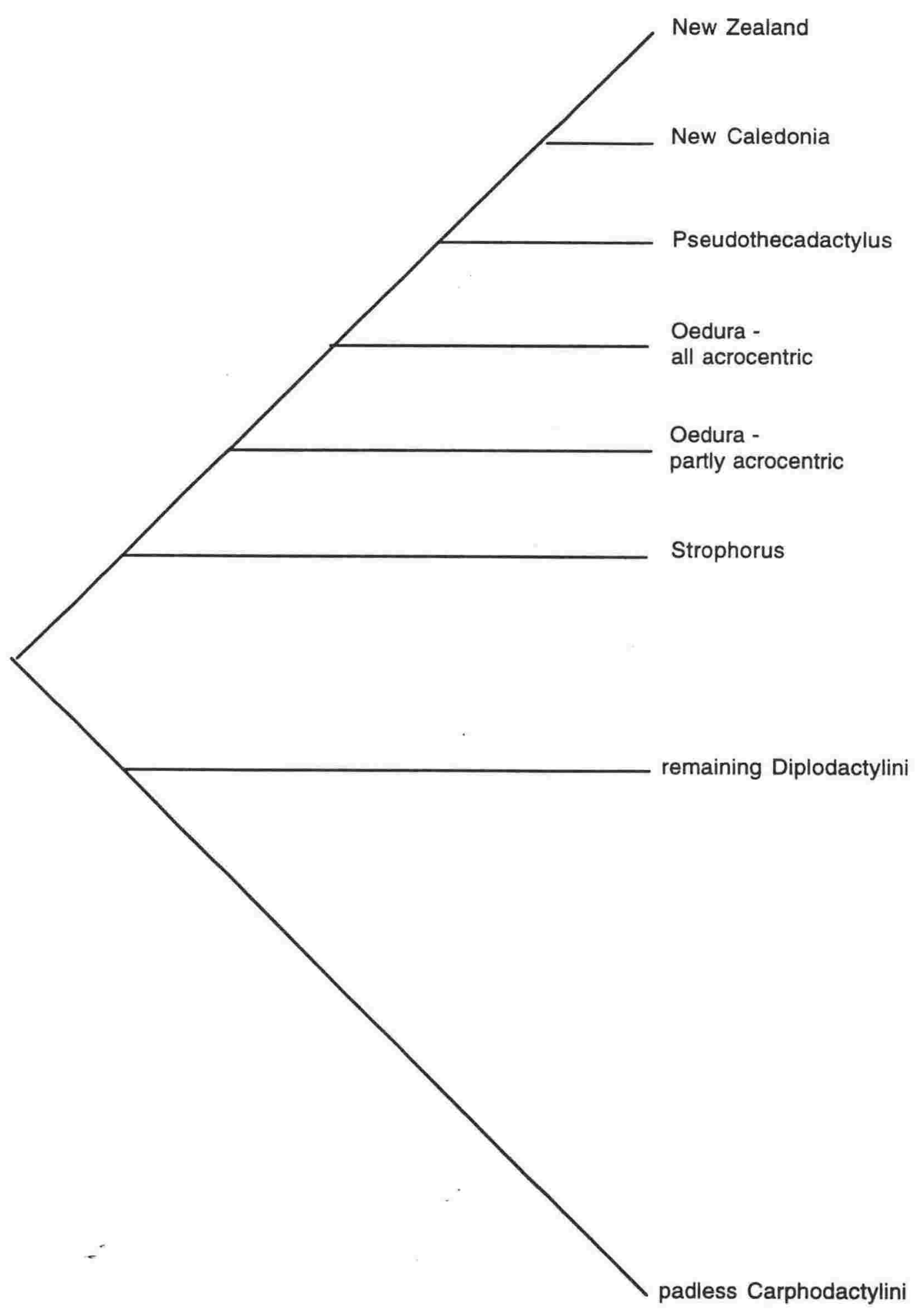

Figure 5.7.1 My hypothesis of outgroup relationships of the New Zealand geckos. 
Within the New Zealand fauna, I consider that the broad-toed and narrowtoed groups (the latter including Naultinus) are each likely to be monophyletic, i.e., the root of the New Zealand portion of the tree is between these groups (Figure 5.7.2).

Within the broad-toed group, there is an early split between the primarily southern $H$. maculatus complex and the entirely northern $H$. pacificus complex. This split may be related to the Pliocene sea strait through what is now the southern North Island. Speciation in the $H$. pacificus complex appears to be much more recent and less diverse than in the $H$. maculatus complex. Dispersal into the North Island by representatives (probably $\mathrm{H}$. chrysosireticus first, and $H$. maculatus, $H$. duvaucelii and to a very limited extent $H$. "Marlborough mini" more recently) of the $H$. maculatus complex has led to widespread sympatry between the first 3 and members of the $H$. pacificus complex.

In the $H$. maculatus complex, the basal group is the species pair of $H$. chrysosireticus and $H$. "southern mini". Both have restricted distributions (although further discoveries are not unlikely), H. chrysosireticus entirely north of Cook Strait, and $H$. "southern mini" entirely southern. Their habitats are very different, $H$. chrysosireticus inhabiting lowland flax bushes, and $H$. "southern mini" subalpine rock screes. The genetic divergence between them is deep.

The balance of the $H$. maculatus complex is divided into 2 groups, centred in the southern and northern South Island. I hypothesise that separation between these groups occurred during transcurrent movement of the Alpine fault. $H$. duvaucelii has spread north from the range of its southern relatives more recently.

Within the northern group, $H$. "Mount Arthur" is probably the sister group of the other 3 species, and $H$. maculatus is probably the sister group of the pair $H$. "Marlborough mini" plus $H$. "Kaikouras".

Within the southern group there is a primary split between $H$. brunneus plus $H$. "Southern Alps" and the remainder of the group. Below this level, phylogenetic relationships in this group are not well resolved. 


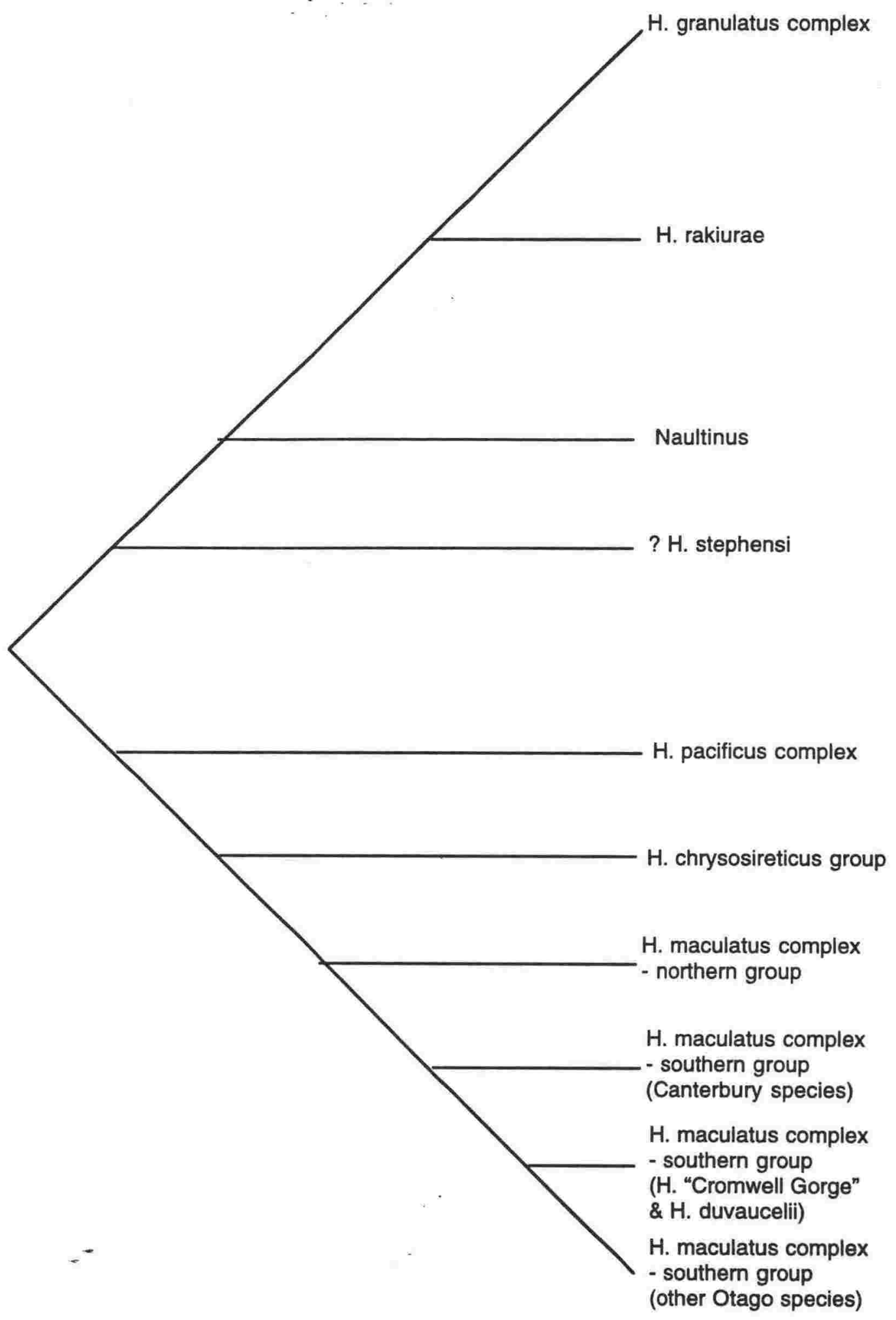

Figure 5.7.1 My hypothesis of evolutionary relationships within the New Zealand gecko fauna. 
In the narrow-toed group, the position of $H$. stephensi is unresolved, but it is either the sister group of the rest of this group (including Naultinus), or diverged from either Naultinus or the $\mathrm{H}$. granulatus complex very soon after these 2 groups diverged. Within both Naultinus and the $H$. granulatus complex the bulk of diversity is in the south, and the basal groups are entirely southern in distribution. The most basal species of the $H$. granulatus complex is $H$. rakiurae. The phylogenetic relationships of the other three species of this group ( $H$. granulatus, $H$. kahutarae, and $H$. nebulosus) are unresolved.

\subsection{CONCLUSIONS}

Phylogenetic analyses of morphological and allozyme characters from New Zealand geckos produce very similar trees. This well-supported phylogeny for New Zealand geckos places Naultinus as a monophyletic group within a paraphyletic Hoplodactylus. Hoplodactylus itself shows a clear separation into broad-toed and narrow-toed groups, the latter being either a close sister group of Naultinus or paraphyletic to it. The most likely position of the root of this tree is between the narrow-toed and broad-toed groups, but this requires confirmation. If it is confirmed, I suggest that these groups be raised to genera.

Reanalysis of Bauer's (1990) phylogenetic analysis of morphological characters in the Carphodactylini suggests that the relationships he proposed among the padded Carphodactylini (New Zealand and New Caledonian taxa and Australian Pseudothecadactylus) are not well supported, and the New Zealand genera may form a monophyletic group.

Reanalysis of phylogenetic patterns within the Diplodactylinae as a whole suggests that the tribes Diplodactylini and Carphodactylini are not monophyletic groups, but represent morphologically more conservative and derived groups respectively in at least 2 clades. 


\section{Chapter 6: EVOLUTIONARY, BIOGEOGRAPHICAL, AND CONSERVATION IMPLICATIONS}

\subsection{INTRODUCTION}

In this thesis I have increased the number of identified extant species in the New Zealand gecko fauna from 16 to 29, with a strong likelihood of further new species being distinguished in future. The traditional view of phylogenetic relationships among the species of the 2 New Zealand genera has also been challenged. The evolutionary and biogeographical implications of these findings are briefly discussed in this chapter.

\subsection{POOR CORRELATION OF RATES OF CHANGE IN ALLOZYMES AND MORPHOLOGY}

Many Hoplodactylus species are so poorly differentiated morphologically that traditional taxonomic techniques have failed to discriminate them. For example:

- All known Hoplodactylus spp. except $H$. duvaucelii and $H$. granulatus were subsumed under the name $H$. pacificus from 1955 (McCann 1955) until 1977 (Robb \& Rowlands 1977).

- Robb \& Rowlands (1977) included H. stephensi in their revised concept of $H$. pacificus, even after separating the latter from $H$. maculatus. Bauer (1990, p.125) suggested that $H$. stephensi was more likely to represent "a recent offshoot from pacificus-maculatus stock" than a relict of a formerly more widespread species as suggested by Towns et al. (1985). My results, in contrast, show very substantial allozyme divergence between $H$. stephensi and all broad-toed Hoplodactylus species, and place $H$. stephensi much closer to Naultinus and other narrow toed Hoplodactylus species. Although several morphological characters support the latter relationship (see Chapter 5), the close similarity of $H$. pacificus and $H$. stephensi in other morphological characters and in general appearance indicate strong morphological conservatism in both species.

- Similarly, the close visual similarity between all members of the $H$. pacificus and $H$. maculatus complexes, which has resulted in their history of taxonomic confusion, contrasts with my results showing strong, 
well-structured genetic differentiation between and within these complexes.

- $H$. nebulosus was regarded as conspecific with $H$. granulatus by Thomas (1981), who found no diagnostic morphological characters separating them. The allozyme results, in contrast, show them to be separated by Nei's $D=0.17-0.40$, distances usually associated with species-level separation.

In contrast to the above examples of morphological similarity between genetically distinct taxa, several of the morphologically most distinctive New Zealand geckos are genetically similar to previously unsuspected sister groups. The following are all examples of morphological differentiation not reflected in genetic differentiation:

- Naultinus probably falls within Hoplodactylus.

- The morphologically distinct species of Naultinus are all very similar to each other in allozyme genetics.

- H. kahutarae falls within $H$. granulatus sensu Thomas (1981) $(=H$. granulatus plus $H$. nebulosus) in all trees, and in many trees falls within H. granulatus.

- $H$. duvaucelii falls within the $H$. maculatus complex in all analyses, and is separated by a small genetic distance (Nei's $D=0.05$ ) from the most closely related population of this complex.

- $\quad H$. "Matapia" falls within H. pacificus in phylogenetic analyses, yet is distinctive in size, colour, and what little is known of its ecology, and is sympatric with morphologically and ecologically more typical $H$. pacificus.

Phylograms of New Zealand gecko species generated from morphological and allozyme characters (Figure 6.2.1) agree closely in branching pattern, but differ in relative lengths of the various branches, most particularly in the much longer branch between Naultinus and Hoplodactylus in the morphological tree. Branch lengths within Naultinus also approach those within Hoplodactylus in the phylogram from morphological characters, whereas in phenograms from allozyme characters (Chapter 5) branch 


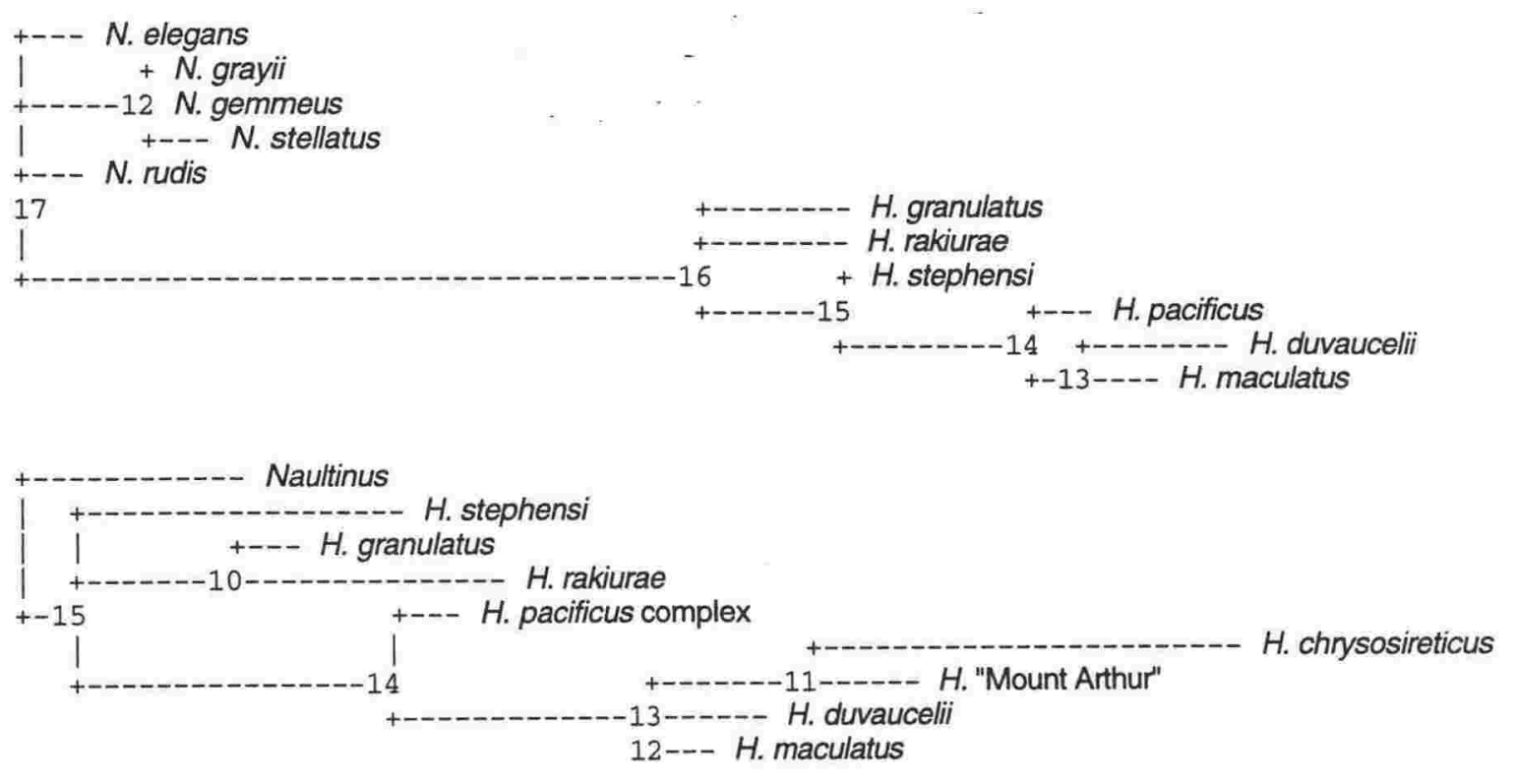

Figure 6.2.1 Maximum parsimony phylograms for New Zealand geckos generated from morphological (above, from Figure 5.4.3) and allozyme (below, from Figure 5.5.4) characters.

lengths within Naultinus are very much shorter than those within Hoplodactylus. This indicates a shift in the rate of either allozyme or morphological evolution between Naultinus and Hoplodactylus; acceleration in morphological evolution in Naultinus is most likely.

In Naultinus and $H$. kahutarae, the distinctive morphology is associated with unusual (for a gecko) ecology. H. kahutarae occupies vertical rock faces in the alpine zone. Naultinus spp. are diurnal, arboreal canopy dwellers, far more exposed to visual predators and more able to use visual cues in intraspecific social behaviour than any of their nocturnal relatives. $H$. duvaucelii is unusual only in its large size, which has allometric effects on osteological development and scale counts. These effects are carried even further in the even larger $H$. delcourti, which is otherwise a very typical member of the $H$. maculatus complex. $H$. "Matapia" could be interpreted as an example of character displacement following a double colonisation of a small island from a single ancestral stock.

The overall picture is one of occasional, rapid morphological change associated with rare niche shifts, against a background of morphological conservatism which fails to reflect the underlying genetic evolution and speciation. 


\subsection{SPECIATION MECHANISMS}

My results, particularly in the southern group of the $H$. maculatus complex (Section 4.6), show allele distributions which for some loci strongly support species boundaries, and indicate that introgression is absent, and at other loci broadly overlap the same species boundaries in geographically congruent shared polymorphisms. The overall pattern of the allozyme variation is a stepped or fragmented cline, which continues across species boundaries. There is a marked absence of diagnostic derived allozyme characters in well-defined species. In my view, these allele distributions suggest parapatric speciation, with the shared polymorphisms being older than the speciation event. This hypothesis requires additional testing using more sensitive genetic techniques to allow assessment of the distributions of more markers across these boundaries, and some additional collection close to these boundaries to assess reproductive isolation.

On Matapia island, the sympatry of 2 morphologically distinct and reproductively isolated species, both of which fall within $H$. pacificus in trees suggest a double colonisation of the island, followed by morphological divergence. Which of the 2 species on the island has been there longer is not clear from the allozyme results. Alternatively this island may preserve a relic of a formerly more widespread polymorphism. If speciation has happened in situ on Matapia Island, it must be recent, as this island is situated in shallow water, and would have been part of the mainland during the last Pleistocene glacial period.

Although I do not advocate the recognition species concept as either universally applicable or a viable species concept for taxonomists (see section 4.1.2), divergence in specific mate recognition systems must be integral in many speciation events. The sensory modes involved in these specific mate recognition systems may strongly affect the degree of correlation between biochemical measures of genetic divergence and speciation. Very speciose groups of genetically closely related species capable of producing viable hybrids between many combinations of parental species are particularly well-documented in groups such as birds, which have visual and auditory courtship displays. Perhaps taxa with mainly visual or auditory mate recognition systems are more able to diverge rapidly than those dependent on chemical senses. The latter might be considered to be more closely linked to the underlying genetic differences measured by such 
techniques as allozyme electrophoresis. This will be discussed further in connection with Naultinus, a genus in which sexual dimorphism in colour has arisen de novo in association with a shift from nocturnal to diurnal activity.

\subsection{PARALLEL MORPHOLOGICAL EVOLUTION CORRELATED WITH HABITAT, AND DIFFERENTIATION OF PARAPATRIC POPULATIONS WITHIN SPECIES}

A possible earlier stage of parapatric divergence is seen in coastal populations in the $H$. maculatus complex. In this complex there is a strong correlation between habitat and body size. All boulder beach populations have small to very small animals, often at high density. These populations, of 3 species, all appear to have been independently derived, in $\mathrm{H}$. maculatus and $H$. brunneus (Table 6.5.1) from adjacent, larger populations from inland, forested or formerly forested habitats where animals weigh at least twice as much as coastal animals of the same age. $H$. "Marlborough mini" also has coastal populations of small animals, but in that species the inland populations are also small, living in open habitats and often sympatric with larger species of the complex.

Freeman (1993) discussed the size difference between Birdlings Flat (coastal) and Banks Peninsula (inland) H. brunneus. He proposed shortterm ecological impact of introduced predators removing larger older animals as an explanation for this difference. However, Anastasiadis \& Whitaker (1987) found that some small, coastal $H$. maculatus are very longlived. I also consider that the minimal intrapopulation size variation, the magnitude of the between-population difference, and its presence at all ages, are inconsistent with Freeman's (1993) explanation. In H. maculatus,

Table 6.5.1 Independently derived groups of coastal populations of small $H$. maculatus-complex geckos. (See population-level phenograms in Chapter $5)$.

H. maculatus

1) Whangarae Bay

2) Cape Palliser, Fisherman's Rock, Cape Turakirae, Baring Head

3) Titahi Bay

4) Pukerua Bay

H. brunneus

Birdlings Flat 
inland specimens do not become sexually mature until they are larger than the maximum size reached in coastal populations. P. Rundlett (School of Biological Sciences, Victoria University of Wellington, pers. comm.) has tested for an effect on size distributions of $H$. maculatus of the presence or absence of mammalian predators, and found no significant effect. Selective removal of large old specimens is not consistent with these observations, and the consistent effect at all ages argues for a longer-term, selective response.

Allozymes provide no support for species-level divergence between these parapatric "ecotypes"; often there is no detectable difference between them, and at most, frequency differences at polymorphic loci. These examples of parallel evolution coupled with some instances of strong differentiation between adjacent apparently conspecific populations not separated by geographic barriers beg further investigation.

\subsection{BIOGEOGRAPHY}

\subsubsection{THE SOUTH ISLAND AS A CENTRE FOR GECKO EVOLUTION}

In the $H$. maculatus complex, 11 of the 12 species are present in the South Island $(H$. duvaucelii is currently confined to offshore islands, probably because of the impact of introduced predators, but is present in recent subfossil deposits in several parts of the South Island). Only $H$. chrysosireticus is confined to the North Island. All 4 North Island species in the $H$. maculatus complex ( $H$. maculatus, $H$. duvaucelii, $H$. chrysosireticus, and $H$. "Marlborough mini") are more closely related to South Island taxa than to each other (Chapter 6 ), and all except $H$. chrysosireticus have extant or subfossil populations on the South Island mainland. All 4 show relatively little genetic differentiation among populations on the North Island and its offshore islands.

Similarly, in the H. granulatus complex, the greatest diversity is in the south, with $H$. granulatus the only species extending to the North Island.

In Naultinus, the North Island species show no allozyme differentiation, and are closely related to $N$. stellatus from north-west Nelson, whereas the South Island species show greater allozyme and morphological differentiation. However, the maximum depth of genetic divergence among Naultinus is much lower than that among species in the $H$. maculatus and $H$. granulatus complexes. 
The $H$. pacificus complex is the only species group confined to North Island, and it has undergone a very modest radiation in the far north.

There is therefore strong circumstantial evidence for most evolution and speciation having happened in the South Island, and for all North Island species except for the $H$. pacificus complex being geologically recent colonists with South Island ancestors.

This phylogeographic pattern is congruent with that proposed for brown kiwis (Apteryx australis) by Baker et al. (1995). In that species also the greatest genetic diversity is in the South Island, and the North Island appears to have been colonised more recently.

\subsubsection{SOUTH ISLAND POPULATIONS OF THE $H$. MACULATUS COMPLEX}

The 2 major groups of the $H$. maculatus complex, the "northern" and "southern" groups have their main centres of diversity in Nelson/Marlborough and Otago respectively. A possible explanation for this distribution pattern is that the 2 groups were formed following separation along the Alpine Fault as this moved formerly continuous land areas apart. Most of the movement along the Alpine Fault is hypothesised to have taken place in the last 10 million years (Kamp 1987). The northern and southern groups of the $H$. maculatus complex cluster at a genetic distance of Nei's $D=$ c. 0.4. If allozyme divergence is clock-like, this implies a crude divergence rate of Nei's $D=1$ corresponding to 25 million years. This rate is not greatly different to that of Nei's $D=1$ corresponding to 22 million years which can be derived from Wilson et al.'s (1974) correlation of immunological, allozyme, and DNA hybridisation distances, particularly given the exclusion of very fast-evolving allozyme loci such as esterases from my dataset.

The close association between the boundaries between rock types (schist/greywacke) and gecko species ( $H$. "Southern Alps"/H. "Otago") in northern Otago suggests a direct causal association. However, the species divergence is shallower than the northern/southern group divergence which I suggest might have followed movement of the Alpine Fault from about 10 million years ago. The rock boundary is considerably older than this; Otago schist has been exposed for about 100 million years (Korsch \& Wellman 1988). Therefore speciation must have occurred along a pre-existing ecotone, making parapatric speciation a possibility. 
Mountain building along the Alpine Fault in the South Island began more recently than transcurrent movement; the presence of schist fragments indicates that Alpine Schist was first exposed only about 2 million years ago (Korsch \& Wellman 1988). If the mountains are about 3 million years old, genetic separation of alpine taxa from their lowland sister groups would therefore be expected to be no greater than about $D=0.12$, based on the above time correlation. The most strictly alpine gecko known is $H$. kahutarae, which is separated from the most similar population of $H$. granulatus by $\mathrm{D}=$ 0.11. $H$. "Kaikouras" and $H$. "Marlborough mini", another species pair from the same area, have similar genetic distances between them. Parapatric populations of $H$. brunneus and $H$. "Southern Alps" in north Canterbury show deeper genetic separation (Figure 4.6.3.4.1). However, both show phylogenetic links to south Canterbury, where unresolved cryptic species appear to be present in $H$. "Southern Alps" (Chapter 4), so their speciation may have preceded mountain building.

In the South Island, geographically close species are closely related, and some have considerable differentiation among populations within species over short geographic distances. If South Island gecko populations were restricted to a few coastal refugia during Pleistocene glaciations, populations should be genetically uniform over broad areas, as the island data indicate no post-Pleistocene differentiation. The allozyme data therefore indicate that $H$. maculatus-complex geckos were widely distributed throughout the Pleistocene. This is not surprising, as many species still occupy subalpine habitats, and these habitats would have been more widespread and continuous during glaciations.

\subsubsection{SOUTH ISLAND POPULATIONS OF NAULTINUS}

The absence of sexual dimorphism in the most southern populations of $N$. gemmeus is almost certainly ancestral, as sexual colour dimorphism is absent in all other Carphodactylini. Most other characters of these populations are shared with other, disjunct populations, particularly in the North Island and the most northern parts of the South Island (Figures 4.7.4.18). This distribution of ancestral characters can be explained by Bull \& Whitaker's (1975) suggestion that the derived morphological characters of $N$. rudis and the more southern populations of $N$. stellatus evolved during southwards dispersal from ancestral populations in the north-eastern and north-western corners respectively of the South Island. Bull \& Whitaker 
(1975) hypothesised that the 2 groups diverged independently as they spread southward from coastal refugia following the retreat of Pleistocene glaciers. Their hypothesis, while highly speculative, remains the one which appears to best fit the observations.

Each north-south cline appears to have been strengthened by the addition through introgression of further derived characters to the already most derived populations at the southern end of the cline (e.g., mottled brown belly colour; brown secondary male colouring). Introgression could have been either from the adjacent cline, and from $N$. gemmeus when contact was made with this species as $N$. rudis expanded south. Reinforcement of specific mate recognition systems may have followed as a secondary development, but the contact zones are so poorly known that this remains speculation.

\subsubsection{ISLAND POPULATIONS AND RATES OF EVOLUTION}

It has been widely assumed in the past that island populations of lizards are often established by rafting vagrants. Geckos such as $\mathrm{H}$. maculatus are biologically well suited to over-water dispersal by rafting on vegetation or driftwood. They live in driftwood and littoral vegetation, forage in the intertidal zone, and are therefore tolerant of high salinity, and can fast for weeks without ill effects. They have post-partum mating, therefore females are effectively permanently pregnant, are very long-lived, with overlapping generations, and very sedentary, all of which would assist the establishment of a new population by a single dispersing female. $H$. maculatus from coastal populations could therefore potentially establish new populations by raft dispersal of a single adult female.

However, if this were true, strong random founder effects on allele frequencies at polymorphic loci would be expected. There would be general shifts in allele frequencies at polymorphic loci (unless subject to very strong selection), a few rare alleles would become common, but most would be lost, resulting in reduced polymorphism and heterozygosity. The overall effect would be greater genetic distances between island populations than those on the same landmass. My results do not support this. Relationships among H. maculatus populations in the Cook Strait area show no effect attributable to isolation of any sort imposed by waterways (Chapter 4). For example, 
Ldh-1 allele frequencies in $H$. maculatus show a cline across Cook Strait, including populations on both main islands and intermediate offshore islands.

This does not mean that these geckos are incapable of establishing populations by rafting. Once a population is large, rare immigration will be undetectable. The evidence of conserved polymorphisms suggests that these populations have always been large, being vicariant in origin Therefore little evidence on rafting is available, but very small stacks, where populations might be expected to occasionally go extinct and be reestablished by dispersal, could be investigated further for this evidence.

The Three Kings and Poor Knights Island groups are the only islands occupied by New Zealand geckos which are believed not to have been connected to the mainland during Pleistocene glacial periods of low sea level, and also the only islands with genetically distinct local endemics. Each of these islands has a depauperate gecko fauna. The Three Kings group has only $H$. "Three Kings", a proposed new species endemic to this island group, and related to $H$. pacificus. The Poor Knights group has $H$. "Poor Knights", another proposed local endemic of the $H$. pacificus complex, and $H$. duvaucelii. $H$. "Three Kings" and $H$. "Poor Knights" differ from each other and from mainland $H$. pacificus by $D=0.09-0.19$ (Chapter 4), but the Poor Knights population of $H$. duvaucelii is indistinguishable genetically and morphologically from populations on other island groups in the Hauraki Gulf. These genetic distances suggest that the $H$. pacificus complex species have been evolving in situ for about 2-4 million years, whereas $H$. duvaucelii is a recent immigrant which must have dispersed to the Poor Knights group over water, perhaps, given the absence of genetic divergence, since the Pleistocene.

Dates of post-Pleistocene separation of the North and South Islands of New Zealand, and of many offshore islands from the mainland, are well established. There has been no detectable differentiation in allozymes or morphology of populations separated by these water barriers relative to populations a similar distance apart on the same landmass. In $H$. maculatus genetic distance/geographic distance relationships are not affected by the presence or absence of water barriers between the populations (Figure 6.6.4.1); island populations and those on the North Island mainland have similar relationships between these 2 distance measures, whereas South 


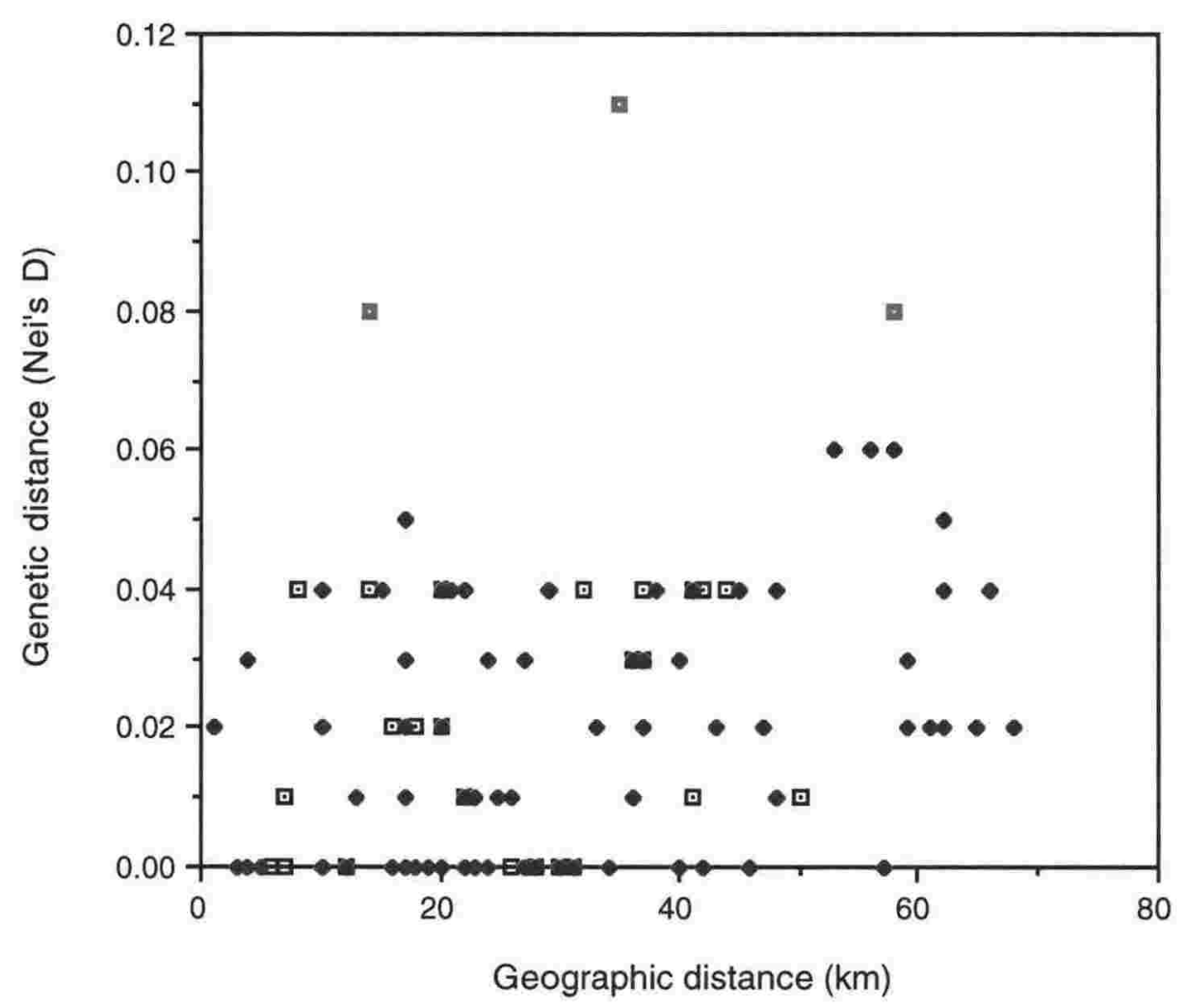

Figure 6.6.4.1 Pairwise geographic and genetic distances between $H$. maculatus populations on the North Island mainland (uncoloured squares), the South Island mainland (red squares), and between populations separated by a seawater barrier in the Cook Strait area (blue diamonds).

Island mainland populations show greater genetic diversity over similar geographic distances. Populations of $H$. pacificus and $H$. duvaucelii show a similar lack of genetic diversity on Pleistocene landbridge islands. In both $H$. maculatus and $H$. duvaucelii, the Trios islands populations show somewhat higher than average genetic distances to adjacent populations (Tables 4.5.3.3 and 4.6.3.1). The Trios are very small islands, where population bottlenecks and consequent allele frequency shifts are more likely than on larger islands.

This observation of no allozyme divergence attributable to seawater barriers provides a zero point for assessment of rates of divergence; a faster-evolving molecule such as the D loop of Mt-DNA should allow calibration of this divergence, aiding interpretation of the histories of mainland lineages. 


\subsection{CONSERVATION}

This study has increased the number of identified extant species of Hoplodactylus from 8 to 21 . Identification of further species is very likely in future, both because more informative techniques (such as DNA sequencing) may clarify species boundaries in variable species such as $H$. "Otago" and $H$. "Southern Alps", which were not satisfactorily resolved using allozymes, and because new discoveries are likely, particularly in remote areas not covered by my survey.

H. maculatus in particular has changed from being a single species distributed almost throughout the country and abundant in many areas, to a complex of 10 species, each of limited distribution. None of these known species appears to be in any immediate danger of extinction; all are at least locally abundant, but some are vulnerable because of their limited range (e.g., H. "Mount Arthur", H. "Cromwell Gorge", H. "Danseys Pass", H. "Kaikouras", H. "Southern mini"). Truly rare species are likely to have escaped detection by the relatively coarse scale of sampling for this study over much of the country. $H$. maculatus itself is now reduced to a locally abundant but very patchily distributed species comparable in conservation status to H. pacificus.

$H$. "Matapia", a member of the $H$. pacificus complex, is the rarest species identified in this study; it is confined to about 1 ha of habitat on a single small island.

The substantial geographic variation detected in many species also merits conservation; no single population can be representative of all but the most locally distributed species, and detectably different populations should be managed separately, as conservation programmes should aim to preserve the geographic structure of genetic diversity (Vrijenhoek et al. 1985). 


\section{Chapter 7: CONCLUSIONS}

\subsection{TAXONOMY}

In this species I recognise 21 species of New Zealand brown geckos (Hoplodactylus), and 7 species of green gecko (Naultinus), bringing the total New Zealand gecko fauna to 28 .

- The identity of Naultinus tuberculatus was clarified by examination of the type specimens. $N$. poecilochlorus should be reduced to synonymy with $N$. tuberculatus. North-west Nelson populations formerly misidentified as $N$. tuberculatus should be included in $N$. stellatus.

My allozyme results, supported by morphological analysis, allow the following taxonomic conclusions to be drawn.

- H. nebulosus should be resurrected from synonymy with $H$. granulatus.

- Hoplodactylus pacificus should be redefined to exclude the Poor Knights and Three Kings populations, which should each be recognised as new species.

- A new species, very closely related to but sympatric with $H$. pacificus, should be recognised from Matapia Island.

- Populations previously assigned to $H$. maculatus sensu Robb \& Rowlands (1977) should be divided among 10 species, in 3 groups.

- One of the $H$. maculatus-complex species, $H$. "Southern mini", is most closely related to $H$. chrysosireticus.

- Another of the H. maculatus-complex species, from lowland Canterbury, is conspecific with Cope's (1868) Pentadactylus brunneus.

- The southern species group of the $H$. maculatus complex includes $H$. duvaucelii, $H$. brunneus, $H$. "Southern Alps", $H$. "Danseys Pass", $H$. "Cromwell Gorge", and $H$. "Otago". All these species are parapatric except for $H$. "Cromwell Gorge", the distribution of which lies within that of $H$. "Otago", and $H$. duvaucelii, which is extinct on the mainland. 
- The northern species group of the $H$. maculatus complex includes $H$. maculatus itself, (more narrowly defined to include only most North Island and some Nelson and Marlborough populations), $H$. "Marlborough mini", $H$. "Kaikouras", and $H$. "Mount Arthur". All except the last, which has a very restricted distribution, are either broadly or narrowly sympatric.

I provide a key for the identification of all known New Zealand species .

\subsection{Phylogenetics}

The position of the root of the phylogeny of New Zealand geckos is hypothesised to fall between the narrow-toed and broad-toed groups of Hoplodactylus; however this position is unconfirmed; some of the groups below could be paraphyletic if the root is elsewhere.

I conclude that there is strong support for the following phylogenetic groups.

- narrow-toed Hoplodactylus plus Naultinus.

- Naultinus

- H. granulatus, H. nebulosus, H. kahutarae, and H. rakiurae

- broad-toed Hoplodactylus

- the H. pacificus complex

- the $H$. maculatus complex plus $H$. duvaucelii and $H$. chrysosireticus

- H. chrysosireticus plus $H$. "Southern mini"

- the "northern group" of the $H$. maculatus complex ( $H$. maculatus, H. "Kaikouras", H. "Marlborough mini", and H. "Mount Arthur")

- the "southern group" of the $H$. maculatus complex $(H$. brunneus, $H$. "Southern Alps", $H$. "Danseys Pass" $H$. "Otago", and $H$. "Cromwell Gorge") plus $H$. duvaucelii.

Phylogenetic analysis of morphological characters from the literature suggests that 
- the New Zealand geckos may form a monophyletic group

- the New Caledonian Carphodactylini may also be monophyletic

- the New Zealand and New Caledonian groups may be sister groups

- the Diplodactylini may be a paraphyletic, morphologically conservative group from which the padded and padless groups of the Carphodactylini have been independently derived, meaning that neither tribe is monophyletic.

\subsection{EVOLUTION}

Genetic divergence within the New Zealand gecko fauna is deep, approaching saturation of allozyme variation between the most divergent species. This indicates a long history of evolution in New Zealand.

Some genetically widely divergent species are so morphologically similar that traditional taxonomies had difficulty distinguishing them (e.g., $\mathrm{H}$. stephensi, all species of the $H$. pacificus complex, and all species of the $H$. maculatus complex except $H$. duvaucelii subsumed under $H$. pacificus by McCann 1955). Conversely, some morphologically highly distinct species have genetically close, previously unsuspected sister group relationships. Rates of change in allozymes and morphology therefore appear poorly correlated.

In the southern group of the $\mathrm{H}$. maculatus complex, the overall pattern of allozyme variation is a stepped or fragmented cline, which continues across species boundaries. There is a marked absence of diagnostic derived allozyme characters in well-defined species. These allele distributions suggest parapatric speciation, with the shared polymorphisms being older than the speciation event. 
There are at least 5 independent cases of coastal populations of very small H. maculatus-complex geckos with larger inland sister-groups, suggesting strong directional selection on body size.

\subsection{BIOGEOGRAPHY}

There is strong circumstantial evidence for most evolution and speciation having happened in the South Island, and for all North Island species except for the $H$. pacificus complex being geologically recent colonists with South Island ancestors.

- In the $H$. maculatus complex, 11 of the 12 species are (or have recently been) present in the South Island.

- Only H. chrysosireticus is confined to the North Island.

- All 4 North Island species in the $H$. maculatus complex ( $H$. maculatus, $H$. duvaucelii, $H$. chrysosireticus, and $H$. "Marlborough mini") are more closely related to South Island taxa than to each other.

- All 4 show relatively little genetic differentiation among populations on the North Island and its offshore islands.

- In the H. granulatus complex, the greatest diversity is in the south, with $H$. granulatus the only species extending to the North Island.

- In Naultinus, the North Island species show no allozyme differentiation, and are closely related to $N$. stellatus from north-west Nelson, whereas the South Island species show greater allozyme and morphological differentiation.

- The H. pacificus complex is the only species group confined to North Island, and it has undergone a very modest radiation in the far north.

The 2 major groups of the $H$. maculatus complex, the "northern" and "southern" groups have their main centres of diversity in Nelson/Marlborough and Otago respectively. A possible explanation for this distribution pattern is that the 2 groups were formed following separation along the Alpine Fault as this moved formerly continuous land areas apart. 
The close association between the boundaries between rock types (schist/greywacke) and gecko species ( $H$. "Southern Alps"/H. "Otago") in northern Otago suggests a direct causal association. Speciation must have occurred along a pre-existing ecotone, making parapatric speciation a possibility.

The evidence of conserved polymorphisms suggests that most island populations of geckos have always been large, being vicariant in origin. Most show no detectable genetic divergence compared to mainland populations separated by equivalent geographic distances.

The Three Kings and Poor Knights Island groups are the only islands occupied by New Zealand geckos which are believed not to have been connected to the mainland during Pleistocene glacial periods of low sea level, and also the only islands with genetically distinct local endemics.

\subsection{Conservation}

Although none of the new species identified in this thesis appears to be critically endangered at present, several (e.g., $H$. "Mount Arthur", $H$. "Cromwell Gorge", H. "Danseys Pass", H. "Kaikouras", H. "Southern mini", and $H$. "Matapia") are vulnerable because of their very restricted known distributions.

The more widespread species have significant geographic variation (potentially including further cryptic species), which conservation management should aim to conserve.

$H$. maculatus, formerly regarded as very widespread and abundant, is now shown to have a more restricted and patchy distribution. 


\section{ACKNOWLEDGEMENTS}

I am very grateful to all the following:

Charles Daugherty for supervision of this thesis, and for access to gecko samples from the CD and FT collections.

The Department of Conservation for permits to collect protected species, and to collect from protected areas.

Landowners who allowed me to collect on their land.

The Royal New Zealand Forest and Bird Protection Society and the Internal Grants Committee of Victoria University of Wellington for financial support.

Tony Whitaker, Geoff Patterson, lan Southey, Graeme Elliott, Kath Walker, Graeme Loh, Peter Anderson, Greg Sherley, Allan Tennyson, Ben Bell, Alison Cree, Robyn Baker, Bruce Thomas, Bernard Goertz, Richard Stocker, Patsy Garrett, Ruth Ainsworth, Janet Butler, Mandy Tocher, Tony Jewell, Conrad Hepers, Rod Morris, Grant Harper, Mike Thorsen, and Mike Kean for providing specimens

Tony Whitaker and Bruce Thomas for valuable advice and discussions, and access to their literature collections.

Graeme Elliott, Graham Wallis, and lan Southey for their company and assistance during field trips.

Ruth Ainsworth, Chris Thorn, Debbie French, Sue Keall, and Anne Conwell for help and support well beyond the call of duty in the laboratory.

Barry Sneddon for allowing me to use his outline maps of New Zealand.

Brett Robertson and Steven Trewick for photography. Graeme Loh and Alison Cree for permission to use their photographs.

Charles Daugherty, Ruth Ainsworth, Mary Morgan-Richards, Mike Beardsell, Geoff Patterson, Mark Hutchinson, and Graham Wallis for constructive comments on various drafts of this thesis. 


\section{REFERENCES}

Allendorf, F. W.; Mitchell, N.; Ryman, N.; Stahl, G. 1977: Isozyme loci in brown trout (Salmo trutta L.): detection and interpretation from population data. Hereditas 86: 179-190.

Anastasiadis, J. M.; Whitaker, A. H. 1987: Longevity of free-living Hoplodactylus maculatus (Reptilia: Gekkonidae). New Zealand journal of ecology 10: 141-142.

Archie, J. W.; Simon, C.; Martin, A. 1989: Small sample size does decrease the stability of dendrograms calculated from allozyme-frequency data. Evolution 43(3): 678683.

Avise, J. C.; Ball, R. M. jr. 1990: Principles of genealogical concordance in species concepts and biological taxonomy. Oxford surveys in evolutionary biology 7: 47-68.

Baker, A. J.; Daugherty, C. H.; Colbourne, R.; McLennan, J. L. 1995: Flightless brown kiwis of New Zealand possess extremely subdivided population structure and cryptic species like small mammals. Proceedings of the National Academy of Science, USA 92: 8254-8258.

Bauer, A. M. 1986: Systematics, biogeography and evolutionary morphology of the Carphodactylini (Reptilia: Gekkonidae). Unpublished PhD thesis, University of California, Berkeley. 869 p.

Bauer, A. M. 1990: Phylogenetic systematics and biogeography of the Carphodactylini (Reptilia: Gekkonidae). Bonner zoologische Monographien, Nr. 30. Bonn, Zoologisches Forschungsinstitut und Museum Alexander Koenig. 218 p.

Bauer, A. M.; Good, D. A.; Sadlier, R. A. 1997: Allozyme evidence for the phylogeny of the giant New Caledonian geckos (Squamata:

Diplodactylinae: Rhacodactylus), with comments on intraspecific variation. Abstract of paper presented at the 7th Society for Research on Amphibians and Reptiles in New Zealand conference, Kaikoura, New Zealand, 31 January - 2 February 1997.

Bell, B. D.; Daugherty, C. H.; Hay, J. M. submitted: Leiopelma pakeka, n. sp. (Anura: Leiopelmatidae), a cryptic species of frog from Maud Island, New Zealand, and a reassessment of the conservation status of Leiopelma hamiltoni from Stephens Island. Journal of the Royal Society of New Zealand, submitted.

Benson, J. A. 1976: The circadian rhythm of locomotor activity in the lizard Hoplodactylus pacificus, and its possible taxonomic use. Tane 22: 119128.

Boulenger, G. A. 1885a: Catalogue of the lizards in the British Museum (Natural History). Volume 1. Taylor and Francis, London, 436 p.

Boulenger, G. A. 1885b: Remarks on the distribution of the Lacertilia. Annals \& magazine of natural history (Series 5) 16 (92): 77-85. 
Bull, P. C.; Whitaker, A. H. 1975: The amphibians, reptiles, birds and mammals. pp 231-236 in Kuschel, G. (ed.): Biogeography and ecology in New Zealand. Junk, The Hague. 689 p.

Buller, W. L. 1871: A list of the lizards inhabiting New Zealand, with descriptions. Transactions of the New Zealand Institute 3: 4-11, 56.

Buth, D. G. 1984: The application of electrophoretic data in systematic studies. Annual review of ecology and systematics 15: 501-522.

Cavalli-Sforza, L. L.; Edwards, A. W. F. 1967: Phylogenetic analysis: models and estimation procedures. Evolution 21: 550-570.

Chrapliwy, P. S.; Smith, H. M.; Grant, C. 1961: The systematic status of the Gekkonid lizard genera Gehyra, Peropus, Hoplodactylus, and Naultinus. Herpetologica 17(1): 5-12.

Clayton, J. W.; Tretiak, D. N. 1972: Amine-citrate buffers for $\mathrm{pH}$ control in starch gel electrophoresis. Journal of the Fisheries Research Board of Canada 29: 1169-1172.

Cogger, H. G. 1975: Reptiles and amphibians of Australia. A. H. and A. W. Reed, Sydney. $584 \mathrm{p}$.

Cope, E. D. 1868: Observations on reptiles of the old world. Proceedings of the Academy of Natural Sciences of Philadelphia 20: 316-323.

Cree, A. 1994: Low annual reproductive output in female reptiles from New Zealand. New Zealand journal of zoology 21 (4): 351-372.

Daniel, M. J.; Whitaker, A. H.; Meads, M. J. 1972: Visit to Mana Island. Unpublished report, Ecology Division, New Zealand Department of Scientific and Industrial Research. 2 p.

Daugherty, C. H.; Patterson, G. B.; Thorn, C. J.; French, D. C. 1990a: Differentiation of the members of the New Zealand Leiolopisma nigriplantare species complex (Lacertilia: Scincidae). Herpetological monographs 4: 61-76.

Daugherty, C. H.; Cree, A.; Hay, J. M.; Thompson, M. B. 1990b: Neglected taxonomy and continuing extinctions of tuatara (Sphenodon). Nature 347 (6289): 177-179.

Dobzhansky, T. 1937: Genetics and the origin of species. Columbia University Press, New York. 364 p.

Donoghue, M J. 1985: A critique of the biological species concept and recommendations for a phylogenetic alternative. The bryologist 88: 172-181.

Echelle, A. A. 1990: In defence of the phylogenetic species concept and the ontological status of hybridogenetic taxa. Herpetologica 46: 109-113. 
Evetts, P. M. 1978: Thermal biology of two Otago lizards Leiolopisma nigriplantare (Peters) and Hoplodactylus pacificus (Gray). Unpublished BSc honours thesis, University of Otago. $104 \mathrm{p}$.

Evetts, P. M.; Grimmond, N. M. 1982: The effects of temperature on oxygen consumption in Leiolopisma nigriplantare maccani and Hoplodactylus maculatus. pp. 409-415 in: Newman D. G. ed.: New Zealand herpetology. Proceedings of a symposium held at the Victoria University of Wellington 29-31 January 1980. New Zealand Wildlife Service occasional publication No. 2. 495 p.

Fischer, J. G. 1882: Herpetologische bemerkungen vorzugsweise über Stücke der Sammlung des Naturhistorischen Museums in Bremen. Abhandlungen herausgegeben von naturwissenschaftlichen Verline zu Bremen 7: 226-238, pl 16.

Freeman, A. 1993: Size differences between populations of Hoplodactylus maculatus in Canterbury, New Zealand. Herpetofauna 23 (1): 9-15.

Frost, D. R.; Hillis, D. M. 1990: Species in concept and practice: herpetological applications. Herpetologica 46: 114-121.

Georges, A.: Adams, M. 1996: Electrophoretic delineation of species boundaries within the short-necked freshwater turtles of Australia (Testudines, Chelidae). Zoological journal of the Linnean Society 118(3): 241-260.

Gill B. J. 1986: Collins handguide to the frogs and reptiles of New Zealand. Collins, Auckland. $112 \mathrm{p}$.

Girard, C. 1857: Descriptions of some new reptiles, collected by the United States Exploring Expedition, under the command of Capt. Charles Wilkes, U. S. N. Part 4: Including the species of saurians, exotic to North America. Proceedings of the Academy of Natural Sciences of Philadelphia (1857): 195-199.

Gorman, G. C.; Renzi, J. R. 1979: Genetic distance and heterozygosity estimates in electrophoretic studies: effects of sample size. Copeia 1979: 242-249.

Gray, J. E. 1842a: Descriptions of some new species of reptiles, chiefly from the British Museum collection. pp 57-59 in "The zoological miscellany", privately published by the author, London, $86 \mathrm{p}$.

Gray, J. E. 1842b: Descriptions of two hitherto unrecorded species of reptiles from New Zealand; presented to the British Museum by Dr Dieffenbach. p 72 in "The zoological miscellany", privately published by the author, London, $86 \mathrm{p}$.

Gray, J. E. 1845: Catalogue of the specimens of lizards in the collection of the British Museum. Newman, London. 289 p.

Greer, A. E. 1974: The generic relationships of the scincid lizard genus Leiolopisma and its relatives. Australian journal of zoology, supplementary series, No. 31, 67 p. 
Greer, A. E. 1989: The biology and evolution of Australian lizards. Surrey Beatty \& Sons, Chipping Norton. 264 p.

Grehan, J. R.; Ainsworth, R. 1985: Orthogenesis and evolution. Systematic zoology 34: 174-192.

Grimmond, N. M.; Evetts, P. M. 1981: The effects of temperature on heat exchange and oxygen consumption in two sympatric New Zealand lizards. pp 257-259 in: Szelenyi, Z.; Szekely, M. (eds): Advances in physiological sciences, Volume 32, Contributions to thermal physiology. Satellite symposium of the 28th International Congress of Physiological Sciences, Pecs, Hungary, 1980. Akademiai Kiado, Budapest.

Hard, G. C. 1954: Notes on reptilia of islands visited by Field Club, 1953-1954. Tane 6: 143-146.

Hardy, G. S. 1977: The New Zealand Scincidae (Reptilia: Lacertilia); a taxonomic and zoogeographic study. New Zealand journal of zoology 4: 221-325.

Harris, H.; Hopkinson, D. A. 1976: Handbook of enzyme electrophoresis in human genetics. North-Holland, Amsterdam.

Highton, R. 1989: Biochemical evolution in the slimy salamanders of the Plethodon glutinosus complex in the eastern United States. Part 1. Geographic protein variation. University of Illinois biological monograph 57: 1-78.

Hillis, D. M. 1984: Misuse and modification of Nei's genetic distance. Systematic zoology 33(2): 238-240.

Hillis, D. M. 1988: Systematics of the Rana pipiens complex: puzzle and paradigm. Annual review of ecology and systematics 19: 39-63.

Hutchinson, M. A.; Donnellan, S. C.; Baverstock, P. B.; Kreig, M.; Simms, S.; Burgin, S. 1990: Immunological relationships and generic revision of the Australian lizards assigned to the genus Leiolopisma (Scincidae: Lygosominae). Australian journal of zoology 38: 535-554.

Hutton, F. W. 1872: Notes on the lizards of New Zealand, with descriptions of two new species. Transactions of the New Zealand Institute 4: 167-172.

Kamp, P. 1987: Age and origin of the New Zealand Orocline in relation to Alpine Fault movement. Journal of the Geological Society of London 144: 641-652.

Kim, J.; Rohlf, F. J.; Sokal, R. R. 1993: The accuracy of phylogenetic estimation using the neighbour-joining method. Evolution 47(2): 471-486.

King, M. 1987a: Origin of the Gekkonidae: chromosomal and albumin evolution suggests Gondwanaland. Search 18(5): 252-254.

King, M. 1987b: Chromosomal evolution in the Diplodactylinae (Gekkonidae: Reptilia). I. Evolutionary relationships and patterns of change. Australian journal of zoology 35: 507-531. 
King, M.; Gow, G. 1983: A new species of Oedura (Gekkonidae: Reptilia) from the Alligator Rivers region of northern Australia. Copeia 1983 (2): 445-449.

Kluge, A. G. 1965: The systematic status of certain Australian lizards of the family Gekkonidae. Australian zoologist 13(2): 121-125.

Kluge, A. G. 1967a: Higher taxonomic categories of gekkonid lizards and their evolution. Bulletin of the American Museum of Natural History 135 (1): 1-60 + 5 plates.

Kluge, A. G. 1967b: Systematics, phylogeny and zoogeography of the lizard genus Diplodactylus Gray (Gekkonidae). Australian journal of zoology 15 (5): 1007-1108.

Kluge, A. G. 1987: A concern for evidence and a phylogenetic hypothesis of relationships among Epicrates (Boidae, Serpentes). Systematic zoology 38: 7-25.

Kluge, A. G. 1989: Cladistic relationships in the Gekkonoidea (Squamata, Sauria). Miscellaneous publications, Museum of Zoology, University of Michigan, No. 173.54 p.

Kluge, A. G. 1991: Checklist of gekkonid lizards. Smithsonian herpetological information service No. 85.35 p.

Korsch, R. J.; Wellman, H. W. 1988: The geological evolution of New Zealand and the New Zealand region. In: Nairn, A. E. M.; Stehli, F. G.; Uyeda, S. (eds) The ocean basins and margins 7B: 411-482. Plenum Publishing Corp., New York.

Kumar, S.; Tamura, K.; Nei, M. 1993: MEGA: molecular evolutionary genetics analysis, version 1.01. The Pennsylvania State University, University Park, PA 16802.

MacAvoy, E. S. 1976: The physiology of lizards from arid regions in Central Otago. Unpublished PhD thesis, University of Otago. $391 \mathrm{p}$.

Mayr, E. 1942: Systematics and the origin of species. Columbia University Press, New York.

Mayr, E. 1963: Animal species and evolution. Harvard University Press, Cambridge, MA.

Mayr, E. 1969: Principles of systematic zoology. McGraw-Hill, New York.

McCallum, J. 1985: Unpublished report on the birds and lizards of the Three Kings Islands to the Secretary for Internal Affairs. 30 January 1985.

Unpubtished report to Secretary for Internal Affairs, Wellington. $3 \mathrm{p}$. [Abstract in Whitaker \& Thomas 1989].

McCallum, J.; Brook, F.; Francis, M. 1986: Unpublished report to the Secretary for Internal Affairs on the birds and lizards of the Three Kings Islands, February 1986. Unpublished report to Secretary of Internal Affairs. 10 p. [Abstract in Whitaker \& Thomas 1989]. 
McCann, C. 1955: The lizards of New Zealand. Gekkonidae and Scincidae. Dominion Museum bulletin No. 17: 1-127.

Mclvor, I. R. 1972: Ecology of a population of Hoplodactylus pacificus on Quail Island in the Lyttelton Harbour. Unpublished MSc thesis, University of Canterbury. $100 \mathrm{p}$.

Meads M. J. 1982: A proposed revision of the Naultinus/Heteropholis species complex. pp. 321-325 in: Newman D. G. ed.: New Zealand herpetology. Proceedings of a symposium held at the Victoria University of Wellington 29-31 January 1980. New Zealand Wildlife Service occasional publication No. 2. 495 p.

Morris, R. W. 1965: Temperature history and metabolic level of a New Zealand gecko, Hoplodactylus pacificus (Gray). Copeia 1965 (2): 248-249.

Murphy, R. W. 1993: The phylogenetic analysis of allozyme data: invalidity of coding alleles by presence/absence and recommended procedures. Biochemical systematics and ecology 21: 25-38.

Murphy, R. W.; Crabtree, C. B. 1985: Evolutionary aspects of isozyme patterns, number of loci, and tissue-specific gene expression in the prairie rattlesnake, Crotalus viridis viridis. Herpetologica 41: 451-470.

Myers, G. S. 1961: The New Zealand lizard names Naultinus and Hoplodactylus. Herpetologica 17(3): 169-172.

Nei, M. 1978: Estimation of average heterozygosity and genetic distance from a small number of individuals. Genetics 89: 583-590.

Nei, M. 1991: Relative efficiencies of different tree-making methods for molecular data. pp 90-128 in: Miyamoto, M. M.; Cracraft, J. L. (eds): Recent advances in phylogenetic studies of DNA sequences. Oxford University Press, Oxford, UK.

Nelson, G. J.; Platnick, N. I. 1981: Systematics and biogeography, cladistics and vicariance. Columbia University Press, New York.

Newman, D. G. 1980: Stephens Island 3-5 November 1980. Unpublished report, Wildlife Service, New Zealand Department of Internal Affairs. 3 p.

Paterson, H. E. H. 1985: The recognition concept of species. Pp 21-29 in: Vrba, E. S. (ed.): Species and speciation. Transvaal Museum monograph, Pretoria, vol. 4.

Patterson, G. B.; Daugherty, C. H. 1990: Four new species and one new subspecies of skink, genus Leiolopisma from New Zealand. Journal of the Royal Society of New Zealand 20: 65-84.

Patterson, G. B.; Daugherty, C. H. 1994: Leiolopisma stenotis, n. sp. (Reptilia: Lacertilia: Scincidae) from Stewart Island, New Zealand. Journal of the Royal Society of New Zealand 24: 125-132. 
Patterson, G. B.; Daugherty, C. H. 1995: Reinstatement of the genus Oligosoma (Reptilia: Lacertilia: Scincidae). Journal of the Royal Society of New Zealand 25: 327-331.

Patton, J. C.; Avise, J. C. 1986: Evolutionary genetics of birds IV Rates of protein divergence in waterfowl (Anatidae). Genetica 68: 129-143.

Pickard C. R. \& Towns, D. R. 1988: Atlas of the amphibians and reptiles of New Zealand. Conservation Sciences publication No. 1. 59 p.

Pollock, M.; MacAvoy, E. S. 1973: The morphology and metabolism of skeletal muscle in hibernating lizards. Proceedings of the Otago Medical School 51(2): 39-40.

Pollock, M.; MacAvoy, E. S. 1978: Morphological and metabolic changes in muscles of hibernating lizards. Copeia 1978 (3): 412-416.

Ridgeway, G. J.; Sherburne, S. W.; Lewis, R. D. 1970: Polymorphisms in the esterases of Atlantic herring. Transactions of the American Fisheries Society 99: 147-151.

Robb, J. 1980a: New Zealand amphibians and reptiles in colour. Collins, Auckland. $128 \mathrm{p}$.

Robb, J. 1980b: Three new species of gekkonid lizards, genera Hoplodactylus Fitzinger and Heteropholis Fischer, from New Zealand. National Museum of New Zealand records 1 (19): 305-310.

Robb, J. 1982: Taxonomy of the New Zealand geckos. pp. 317-320 in: Newman D. G. ed.: New Zealand herpetology. Proceedings of a symposium held at the Victoria University of Wellington 29-31 January 1980. New Zealand Wildlife Service occasional publication No. 2. 495 p.

Robb, J.; Hitchmough, R. A. 1980: Review of the genus Naultinus Gray (Reptilia: Gekkonidae). Records of the Auckland Institute and Museum 16: 189-200.

Robb, J.; Rowlands, R. P. V. 1977: Reinstatement of Hoplodactylus maculatus (Boulenger) with redescription of $H$. pacificus (Gray) (Reptilia: Squamata: Gekkonidae). Records of the Auckland Institute and Museum 14: 133-142.

Robinson, M. 1985: Sexual cycles of New Zealand lizards, with particular reference to the gecko Hoplodactylus maculatus (Boulenger). Unpublished MSc honours thesis, Victoria University of Wellington. $115 \mathrm{p}$.

Rogers, J. S. 1972: Measures of genetic similarity and genetic distance. University of Texas publication 7213: 145-153.

Rosen, D. E. 1978: Vicariant patterns and historical explanation in biogeography. Systematic zoology 27: 159-188.

Rosen, D. E. 1979: Fishes from the uplands and intermontane basin of Guatemala: revisionary studies and comparative geography. Bulletin of the American Museum of Natural History 162: 267-376. 
Ross, H. H. 1974: Biological systematics. Addison-Wesley, Reading, Massachusetts. $345 \mathrm{p}$.

Rowlands, R. P. V. 1976: Mustard marking in Hauraki Gulf geckos (Hoplodactylus sp.). Moko October 1976: 3.

Russel, A. P. 1979: Parallelism and integrated design in the foot structure of Gekkonine and Diplodactyline geckos. Copeia 1979(1): 1-21.

Russel, A. P;. Rosenberg, H. I. 1981: Subgeneric classification in the gekkonid genus Diplodactylus. Herpetologica 37: 86-92.

Sadlier, R. A. 1986: A review of the scincid lizards of New Caledonia. Records of the Australian Museum 39: 1-66.

Saitou, N.; Imanashi, T. 1989: Relative efficiencies of the Fitch-Margoliash, maximum parsimony, maximum likelihood, minimum evolution and neighbour-joining methods of phylogenetic tree reconstruction in obtaining the correct tree. Molecular biology and evolution 6: 514-525.

Saitou, N.; Nei, M. 1987: The neighbour-joining method: a new method for reconstructing phylogenetic trees. Molecular biology and evolution 4 : 406-425.

Sarich, V. M. 1977: Rates, sample sizes, and the neutrality hypothesis for electrophoresis in evolutionary studies. Nature 265: 24-28.

Selander, R. K.; Smith, M. H.; Yang, S. Y.; Johnson, W. E.; Gentry, J. B. 1971: Biochemical polymorphism and systematics in the genus Peromyscus. 1. Variation in the old-field mouse (Peromyscus polionotus). Studies in genetics VI. University of Texas publication 7103: 49-90.

Sharell, R. 1966: The tuatara, lizards and frogs of New Zealand. Collins, London. 94 p.

Shelton, E. 1978: The effect of three light regimes on the locomotor activity in the gecko Hoplodactylus maculatus and the skink Leiolopisma nigriplantare maccani. Unpublished Psychology 2 project, University of Otago, Dunedin.

Smith, M. A. 1933: Remarks on some old world geckoes. Records of the Indian Museum 35(1): 9-19.

Swofford, D. L. 1989: PAUP: Phylogenetic Analysis Using Parsimony. Version 3.0g. Illinois Natural History Survey, Champaign, IL.

Swofford, D. L.; Berlocher, S. H. 1987: Inferring evolutionary trees from gene frequency data under the principle of maximum parsimony. Systematic zoology 36(3): 293325.

Swofford, D. L.; Selander, R. B. 1981: BIOSYS-1: a FORTRAN program for the comprehensive analysis of electrophoretic data in population genetics and systematics. Journal of heredity $72: 281-283$ 
Templeton, A. R. 1989: The meaning of species and speciation: a genetic perspective. Pp 3-27 in: Otte, D.; Endler, J. A. (eds) Speciation and its consequences. Sinauer, Sunderland, Massachusetts.

Thomas, B. W. 1981: Hoplodactylus rakiurae n. sp. (Reptilia: Gekkonidae) from Stewart Island, New Zealand, and comments on the taxonomic status of Heteropholis nebulosus McCann. New Zealand journal of zoology 8: 33-47.

Thomas, B. W. 1982: New Zealand Gekkonidae: evolutionary trends and taxa. pp. 333-339 in: Newman D. G. ed.: New Zealand herpetology. Proceedings of a symposium held at the Victoria University of Wellington 29-31 January 1980. New Zealand Wildlife Service occasional publication No. 2. 495 p.

Thorpe, J. P. 1982: The molecular clock hypothesis: biochemical evolution, genetic differentiation, and systematics. Annual review of ecology and systematics 13: 139-168.

Thorpe, J. P. 1983: Enzyme variation, genetic distance, and evolutionary divergence in relation to levels of taxonomic separation. pp 131-152 in: Oxford, G. S.; Rollinson, D. (eds) Protein polymorphism: adaptive and taxonomic significance. Academic Press, London.

Tocher, M. D. 1992: Paradoxical preferred body temperatures of two allopatric Hoplodactylus maculatus (Reptilia: Gekkonidae) populations from New Zealand. New Zealand natural sciences 19: 53-60.

Tocher, M. D. 1993: Critical minimum body temperatures of Hoplodactylus maculatus (Reptilia: Gekkonidae) from New Zealand. New Zealand natural sciences 20: 41-45.

Towns, D. R. 1985: A field guide to the lizards of New Zealand. New Zealand Wildlife Service occasional publication No. 7. 28 p.

Towns, D. R.; Daugherty, C. H.; Newman, D. G. 1985: An overview of the ecological biogeography of the New Zealand lizards (Gekkonidae, Scincidae). pp 107115 in: Grigg, G.; Shine, R.; Ehmann, H. (eds) Biology of Australasian frogs and reptiles. Surrey Beatty, Chipping Norton, Australia. 527 p.

Vrijenhoek, R. C.; Douglas, M. E.; Meffe, G. K. 1985: Conservation genetics of endangered fish populations in Arizona. Science 229: 400-402.

Waddington, D. C. 1970: New Zealand geckos: family Gekkonidae. Part 1: Hoplodactylus pacificus. New Zealand Herpetological Society newsletter 8: 13.

Whitaker, A. H. 1968: The lizards of the Poor Knights Islands, New Zealand. New Zealand journal of science 11 (4): 623-651.

Whitaker, A. H. 1982: Interim results from a study of Hoplodactylus maculatus (Boulenger) at Turakirae Head, Wellington. pp. 363-374 in: Newman D. G. ed.: New Zealand herpetology. Proceedings of a symposium held at the Victoria 
University of Wellington 29-31 January 1980. New Zealand Wildlife Service occasional publication No. 2. 495 p.

Whitaker, A. H. 1984a: A survey of the lizards of the Lindis Pass area, Otago, 9-19 April 1984. Unpublished report, New Zealand Wildlife Service, Wellington. $24 \mathrm{p}$ + figures.

Whitaker, A. H. 1984b: Hoplodactylus kahutarae n. sp. (Reptilia: Gekkonidae) from the Seaward Kaikoura Range, Marlborough, New Zealand. New Zealand journal of zoology 11: 259-270.

Whitaker, A. H. 1985: A survey of the lizards of the Mount Ida area, Otago, 12-22 March 1985. Otago 'giant' skink survey No. 2. Unpublished report, New Zealand Wildlife Service, Wellington. $36 \mathrm{p}$.

Whitaker, A. H. 1986: A survey of the lizards of the Queenstown area, Otago, 3-12 March 1986. Otago 'giant' skink survey No. 4. Unpublished report, New Zealand Wildlife Service, Wellington. $26 \mathrm{p}$.

Whitaker, A. H. 1987: A survey of the lizards of the Wanaka area, Otago, 1-10 December 1986. Otago 'giant' skink survey No. 5. Unpublished report, New Zealand Wildlife Service, Wellington. $32 \mathrm{p}$.

Whitaker, A. H.; Thomas, B. W. 1989: New Zealand lizards: an annotated bibliography. Lower Hutt, DSIR Ecology Division. 215 p.

Wiley, E. O. 1978: The evolutionary species concept reconsidered. Systematic zoology 28: 88-92.

Wiley, E. O. 1980: Is the evolutionary species fiction? - A consideration of classes, individuals, and historical entities. Systematic zoology 29: 76-80.

Wiley, E. O. 1981: Phylogenetics: the theory and practice of phylogenetic systematics. John Wiley, New York.

Wilkinson, W. D. 1981: The status of the Taranaki gecko Hoplodactylus chrysosireticus in the New Zealand herpetofauna. Herpetofauna (Aust.) 13(1): 18-20.

Wilson, A. C.; Maxson, L. R.; Sarich, V. M. 1974: Two types of molecular evolution. Evidence from studies of interspecific hybridisation. Proceedings of the National Academy of Science, USA 71(7): 2843-2847.

Wright, S. 1978: Evolution and the genetics of populations. Vol. 4 Variability within and among natural populations. The University of Chicago Press, Chicago. 


\section{Appendix 1: Allozyme stain and buffer recipes used in this study}

BUFFERS:

\begin{tabular}{|c|c|c|}
\hline System & Gel buffer & Electrode buffer \\
\hline $\mathrm{AC}^{1}$ & $\begin{array}{l}\mathrm{pH} 6.0 \\
0.841 \mathrm{~g} \text { citric acid / } 2 \mathrm{I} \\
\text { adjust } \mathrm{pH} \text { with } \mathrm{N} \text { - } \\
\text { (3-aminopropyl)-morpholine }\end{array}$ & $\begin{array}{l}\text { Ph } 6.1 \\
16.8 \mathrm{~g} \text { citric acid / } 2 \text { I } \\
\text { adjust pH with } \mathrm{N} \text { - } \\
\text { (3-aminopropyl)-morpholine }\end{array}$ \\
\hline $\mathrm{PH}^{2}$ & $\begin{array}{l}\text { pH } 6.7 \\
12.5 \mathrm{ml} \text { electrode buffer } \\
+17.5 \mathrm{mg} \text { NADP } \\
\text { per } 250 \mathrm{ml}\end{array}$ & $\begin{array}{l}\mathrm{pH} 6.7 \\
37.56 \mathrm{~g} \mathrm{KH}_{2} \mathrm{PO}_{4}+ \\
4.96 \mathrm{~g} \mathrm{NaOH} / 2 \mathrm{I} \\
\text { adjust } \mathrm{pH} \text { with conc. } \mathrm{HCl}\end{array}$ \\
\hline $\mathrm{TC}-1^{1}$ & $\begin{array}{l}\mathrm{pH} 6.7 \\
1.94 \mathrm{~g} \text { TRIS }+ \\
1.26 \mathrm{~g} \text { citric acid } / 2 \mathrm{I} \\
\text { adjust } \mathrm{pH} \text { with } \mathrm{NaOH}\end{array}$ & $\begin{array}{l}\mathrm{pH} 6.3 \\
54 \mathrm{~g} \text { TRIS + } \\
36.14 \mathrm{~g} \text { citric acid / } 2 \text { I } \\
\text { adjust pH with } \mathrm{NaOH}\end{array}$ \\
\hline $\mathrm{PK}^{2}$ & $\begin{array}{l}\mathrm{pH} 8.7 \\
18.40 \mathrm{~g} \text { TRIS }+ \\
2.10 \mathrm{~g} \text { citric acid / } 21\end{array}$ & $\begin{array}{l}\mathrm{pH} 8.2 \\
37.10 \mathrm{~g} \mathrm{boric} \mathrm{acid}+ \\
4.80 \mathrm{~g} \mathrm{NaOH} / 2 \mathrm{I}\end{array}$ \\
\hline $\mathrm{RW}^{1}$ & $\begin{array}{l}\text { pH } 8.5 \\
7.27 \mathrm{~g} \text { TRIS }+ \\
2.10 \mathrm{~g} \text { citric acid }+ \\
20 \mathrm{ml} \text { electrode buffer } / 2 \mathrm{I}\end{array}$ & $\begin{array}{l}\text { pH } 8.1 \\
37.1 \mathrm{~g} \text { boric acid + } \\
5.04 \mathrm{~g} \mathrm{LiOH} / 2 \mathrm{I}\end{array}$ \\
\hline
\end{tabular}

1 Allendorf et al. 1977

2Selander et al. 1971

STAIN BUFFERS:

।

$24.2 \mathrm{~g}$ TRIS/l, $\mathrm{pH} 8$

II $\quad 100 \mathrm{ml}$ stain buffer I : $500 \mathrm{ml}$ distilled water

III Ridgeway gel buffer

STAINS: -

$\begin{array}{ll}\text { AK } & \text { Adenylate kinase } \\ 50 \mathrm{ml} & \text { stain buffer II } \\ 100 \mathrm{mg} & \text { glucose } \\ 50 \mathrm{mg} & \text { ADP } \\ 250 \mathrm{u} / 16 \mathrm{mg} \text { hexokinase } \\ 100 \mathrm{u} \text { G6PDH } \\ 5 \mathrm{mg} \text { NADP } \\ 10 \mathrm{mg} & \text { NBT }\end{array}$




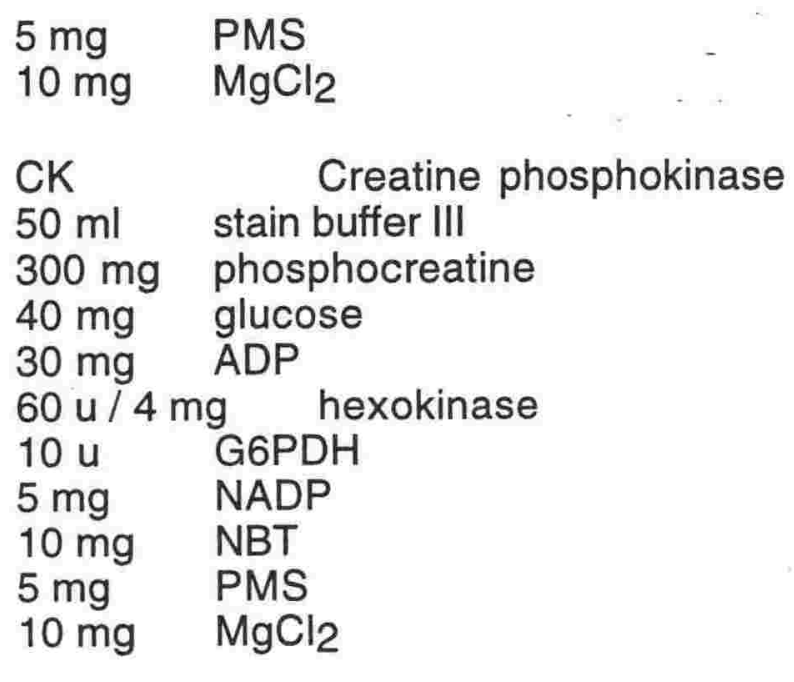

GD Glucose-6-phosphate dehydrogenase

$50 \mathrm{ml}$ stain buffer II

$200 \mathrm{mg}$ glucose-6-phosphate

$5 \mathrm{mg}$ NADP

$10 \mathrm{mg} \quad$ NBT

$5 \mathrm{mg} \quad \mathrm{PMS}$

$10 \mathrm{mg} \quad \mathrm{MgCl}_{2}$

GOT Glutamate-oxalacetate transaminase

$50 \mathrm{ml}$ stain buffer I

$200 \mathrm{mg}$ L-aspartic acid

$100 \mathrm{mg}$ a-ketoglutaric acid

$1 \mathrm{mg}$ pyroxidal-5-phosphate

$300 \mathrm{mg}$ fast blue BB salt

alternative recipe:

$20 \mathrm{ml}$ distilled water

$20 \mathrm{ml}$ stain buffer I

$50 \mathrm{mg}$ ketoglutarate

$100 \mathrm{mg}$ L-aspartate

(add above ingredients together, then adjust $\mathrm{pH}$ back to $8.00-8.10$ )

$60 \mathrm{mg}$ fast garnet $\mathrm{CBC}$

(incubate gel for 20 minutes before adding fast garnet)

GP General protein

$50 \mathrm{ml} \quad 1 \%$ amido black or coomassie brilliant blue in destain (leave 20 minutes, then destain repeatedly until bands visible)

GPD Glycerol-3-phosphate dehydrogenase

$40 \mathrm{ml}$ stain buffer II

$1.3 \mathrm{~g}$ glycerophosphate

$400 \mathrm{mg}$ pyruvic acid

$20 \mathrm{mg} \quad \mathrm{NAD}$

$5 \mathrm{mg} \quad$ MTT

$5 \mathrm{mg} \quad$ PMS

alternative recipe:

$50 \mathrm{ml}$ stain buffer III 
$1 \mathrm{~g} \quad$ glycerophosphate

$5 \mathrm{mg} \quad \mathrm{NAD}$

$10 \mathrm{mg} \quad$ NBT

$5 \mathrm{mg} \quad \mathrm{PMS}$

GPI Glucose phosphate isomerase

$50 \mathrm{ml}$ stain buffer III

$25 \mathrm{mg}$ fructose-6-phosphate

$10 \mathrm{u}$ G6PDH

$5 \mathrm{mg} \quad \mathrm{NADP}$

$10 \mathrm{mg} \quad$ NBT

$5 \mathrm{mg} \quad$ PMS

$10 \mathrm{mg} \quad \mathrm{MgCl}_{2}$

ICD Isocitrate dehydrogenase

$50 \mathrm{ml}$ stain buffer II

$200 \mathrm{mg}$ DL isocitric acid

$5 \mathrm{mg} \quad \mathrm{NADP}$

$10 \mathrm{mg} \quad$ NBT

$5 \mathrm{mg} \quad \mathrm{PMS}$

$10 \mathrm{mg} \quad \mathrm{MgCl}_{2}$

LDH Lactate dehydrogenase

$50 \mathrm{ml}$ stain buffer III

few drops DL-lactic acid

$5 \mathrm{mg} \quad \mathrm{NAD}$

$10 \mathrm{mg} \quad$ NBT

$5 \mathrm{mg} \quad \mathrm{PMS}$

$\mathrm{MDH} \quad$ Malate dehydrogenase

$50 \mathrm{ml}$ stain buffer III

$25 \mathrm{ml} \quad 0.5 \mathrm{M} \mathrm{DL}$-malic acid, pH 7.0 (malic acid solution)

$5 \mathrm{mg} \quad \mathrm{NAD}$

$10 \mathrm{mg} \quad$ NBT

$5 \mathrm{mg} \quad \mathrm{PMS}$

malic acid solution:

$8.9 \mathrm{~g} \mathrm{DL}$-malic acid / $100 \mathrm{ml}$ distilled water $\mathrm{pH}$ adjusted to 7.0 with $1 \mathrm{~N} \mathrm{HCl}$

$\mathrm{ME}$

Malic enzyme

$50 \mathrm{ml}$ stain buffer III

$25 \mathrm{ml} \quad 0.5 \mathrm{M} \mathrm{DL}$-malic acid, $\mathrm{pH} 7.0$ (malic acid solution, as above)

$5 \mathrm{mg} \quad \mathrm{NADP}$

$10 \mathrm{mg} \quad \mathrm{NBT}$

$5 \mathrm{mg} \quad \mathrm{PMS}$

$10 \mathrm{mg} \quad \mathrm{MgCl}_{2}$

MPI Mannose phosphate isomerase

$50 \mathrm{ml}$ stain buffer II

$50 \mathrm{mg}$ D-mannose-6-phosphate.

$100 \mathrm{u} \quad \mathrm{PGl}$

$80 \mathrm{u} \quad \mathrm{G} 6 \mathrm{PDH}$ 
$\begin{array}{ll}5 \mathrm{mg} & \text { NADP } \\ 10 \mathrm{mg} & \mathrm{NBT} \\ 5 \mathrm{mg} & \mathrm{PMS} \\ 10 \mathrm{mg} & \mathrm{MgCl}_{2}\end{array}$

NP Purine nucleoside phosphorylase

$50 \mathrm{ml}$ stain buffer II

$100 \mathrm{mg}$ inosine

$100 \mathrm{mg}$ arsenic acid

$2 \mathrm{u}$ xanthine oxidase

$10 \mathrm{mg} \quad$ NBT

$5 \mathrm{mg} \quad \mathrm{PMS}$

\begin{tabular}{|c|c|}
\hline $\begin{array}{l}\text { PEP } \\
50 \mathrm{ml} \\
50 \mathrm{mg} \\
80 \mathrm{mg} \\
80 \mathrm{mg} \\
80 \mathrm{mg} \\
80 \mathrm{mg} \\
10 \mathrm{mg} \\
50 \mathrm{mg} \\
500 \mathrm{u}\end{array}$ & $\begin{array}{l}\quad \text { Peptidase } \\
\text { stain buffer II } \\
\text { O-dianisidine } \\
\text { L-leucyl-L-alanine } \\
\text { L-phenylanyl-L-proline } \\
\text { L-leucyl-L-tyrosine } \\
\text { L-leucylglycyl-glycine } \\
\text { amino acid oxidase (snake venom) } \\
\mathrm{MgCl}_{2} \\
\text { peroxidase }\end{array}$ \\
\hline $\begin{array}{l}\text { PGD } \\
50 \mathrm{ml} \\
100 \mathrm{mg} \\
5 \mathrm{mg} \\
10 \mathrm{mg} \\
5 \mathrm{mg} \\
10 \mathrm{mg}\end{array}$ & $\begin{array}{l}\text { Phosphogluconate dehydrogenas } \\
\text { stain buffer II } \\
\text { 6-phosphogluconic acid } \\
\text { NADP } \\
\text { NBT } \\
\text { PMS } \\
\mathrm{MgCl}_{2}\end{array}$ \\
\hline $\begin{array}{l}\text { PGM } \\
50 \mathrm{ml} \\
250 \mathrm{mg} \\
5 \mathrm{drops} \\
50 \mathrm{u} \\
5 \mathrm{mg} \\
10 \mathrm{mg} \\
5 \mathrm{mg} \\
10 \mathrm{mg}\end{array}$ & $\begin{array}{l}\quad \text { Phosphoglucomutase } \\
\text { stain buffer II } \\
\text { a-D-glucose-1-phosphate } \\
\text { a-D-glucose-1,6-diphosphate } \\
\text { G6PDH } \\
\text { NADP } \\
\text { NBT } \\
\text { PMS } \\
\mathrm{MgCl}_{2}\end{array}$ \\
\hline $\begin{array}{l}\text { SORDH } \\
50 \mathrm{ml} \\
100 \mathrm{mg} \\
5 \mathrm{mg} \\
2.5 \mathrm{mg} \\
10 \mathrm{mg}\end{array}$ & $\begin{array}{l}\text { Sorbitol dehydrogenase } \\
\text { stain buffer I } \\
\text { D-sorbitol } \\
\text { NAD } \\
\text { MTT } \\
\text { NBT } \\
\text { PMS }\end{array}$ \\
\hline
\end{tabular}

